\section{6,2 ,}

\section{xis}

(3)

30 a costors

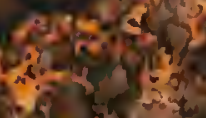
ats

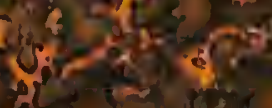

aㅏㄴ. 4 th as (x)

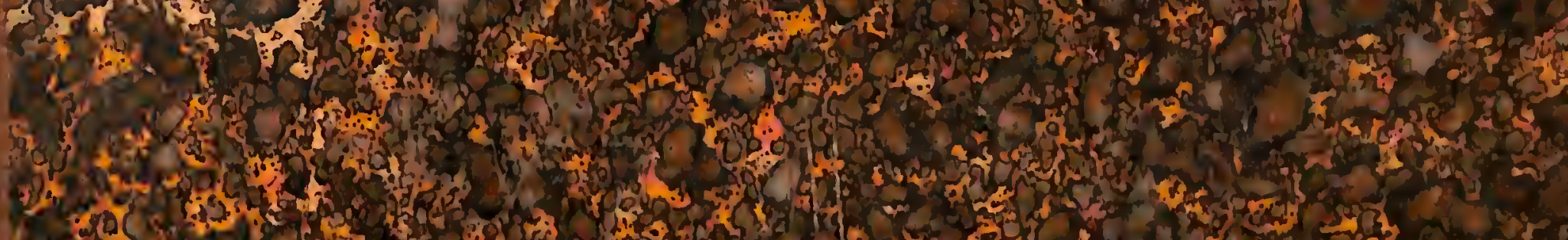

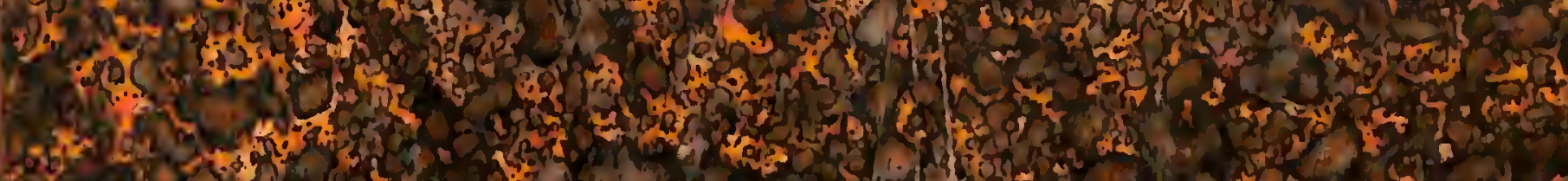

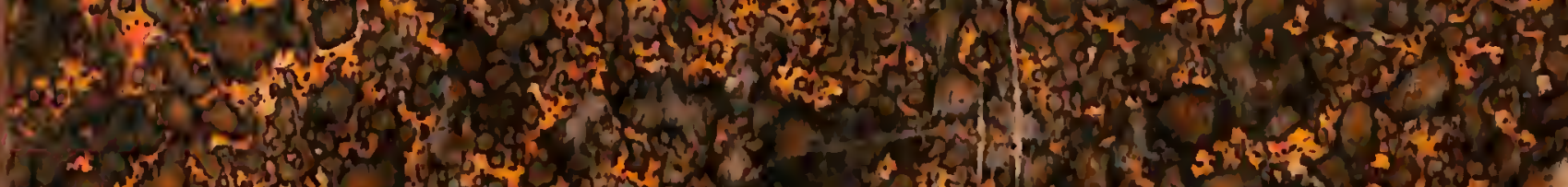

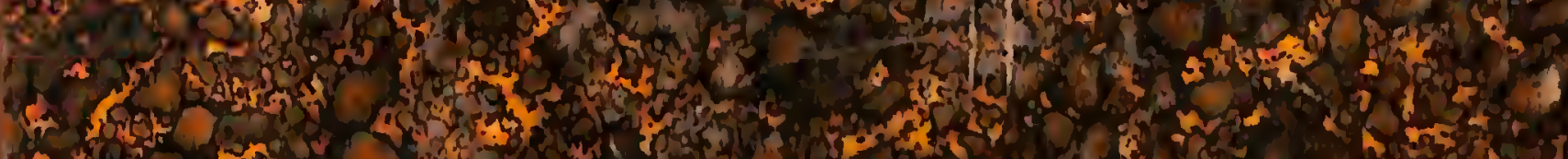

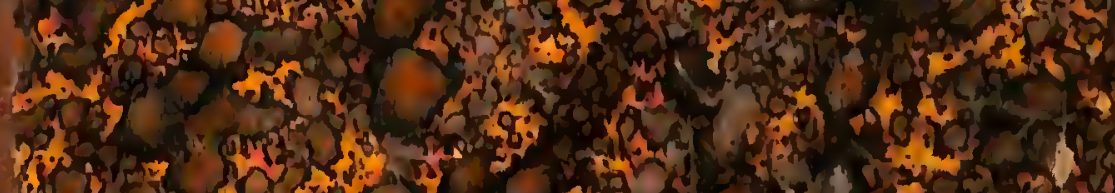

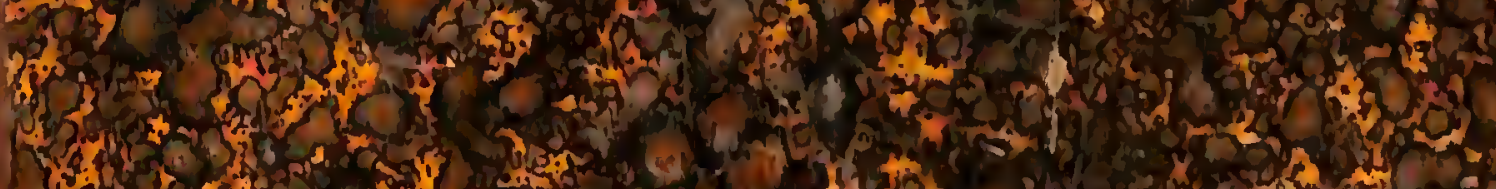
3.7. H.

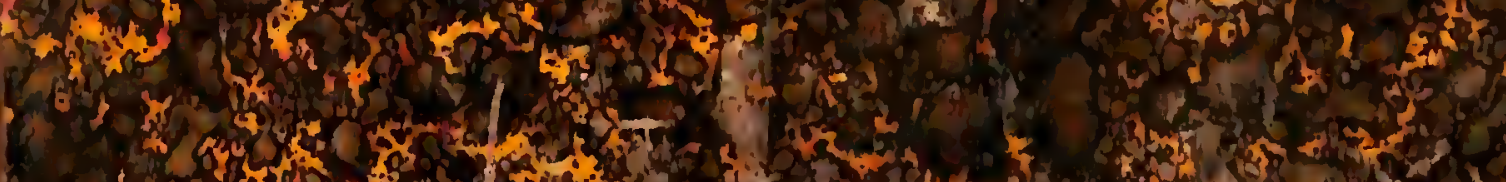
$4 x^{2}+x^{2}+5$ 17.

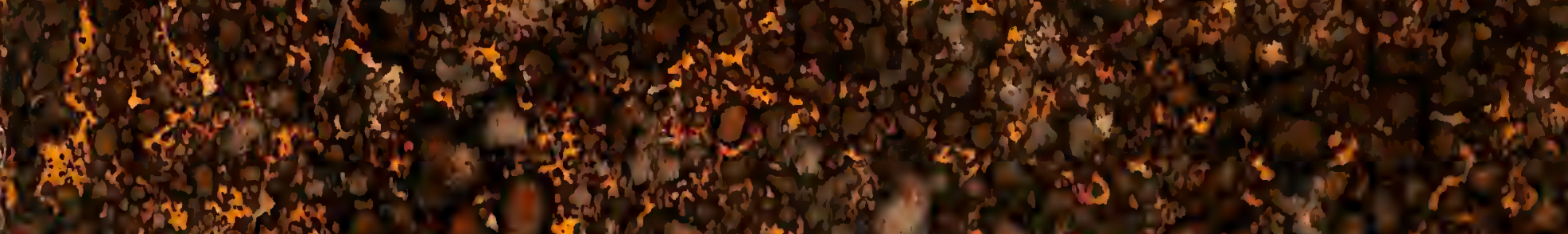
H.

UNAM INSTITUTO DE GEOLOGÍA - CU

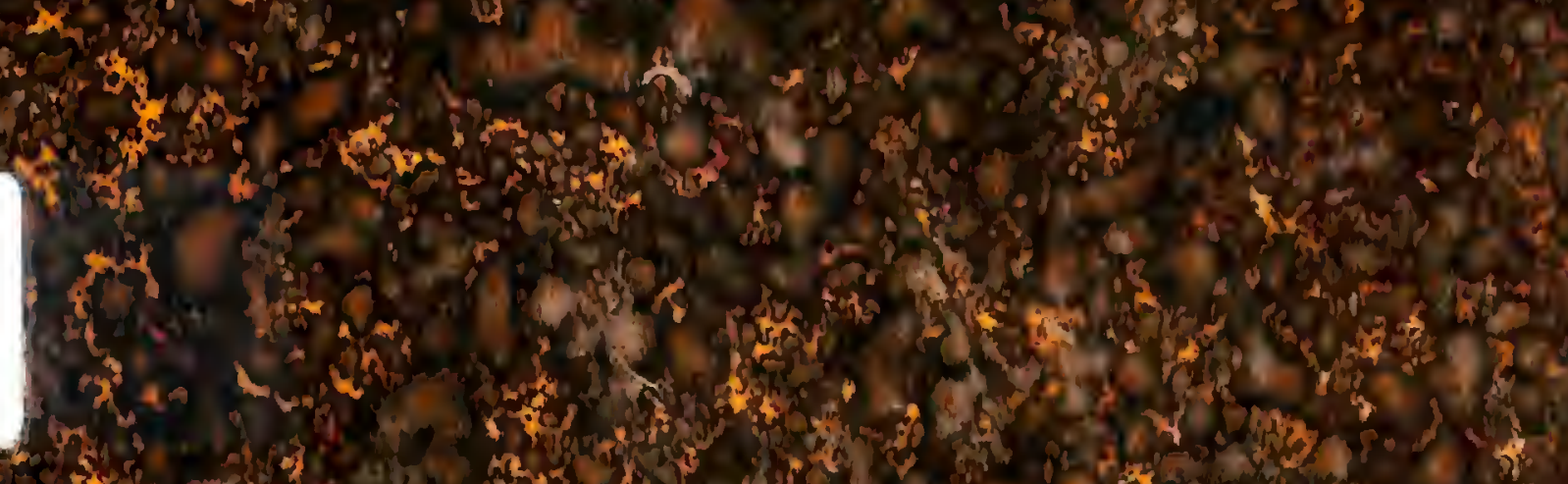

4)

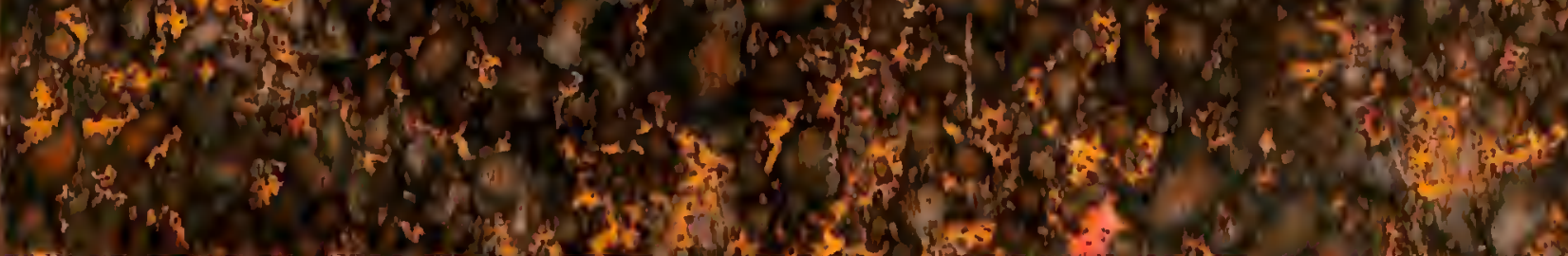

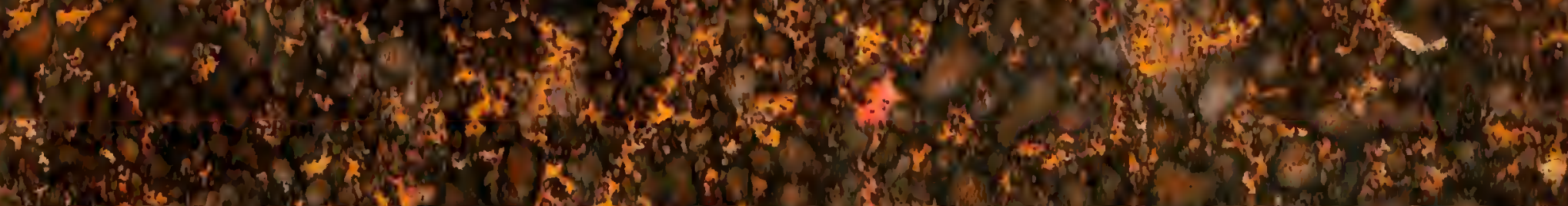




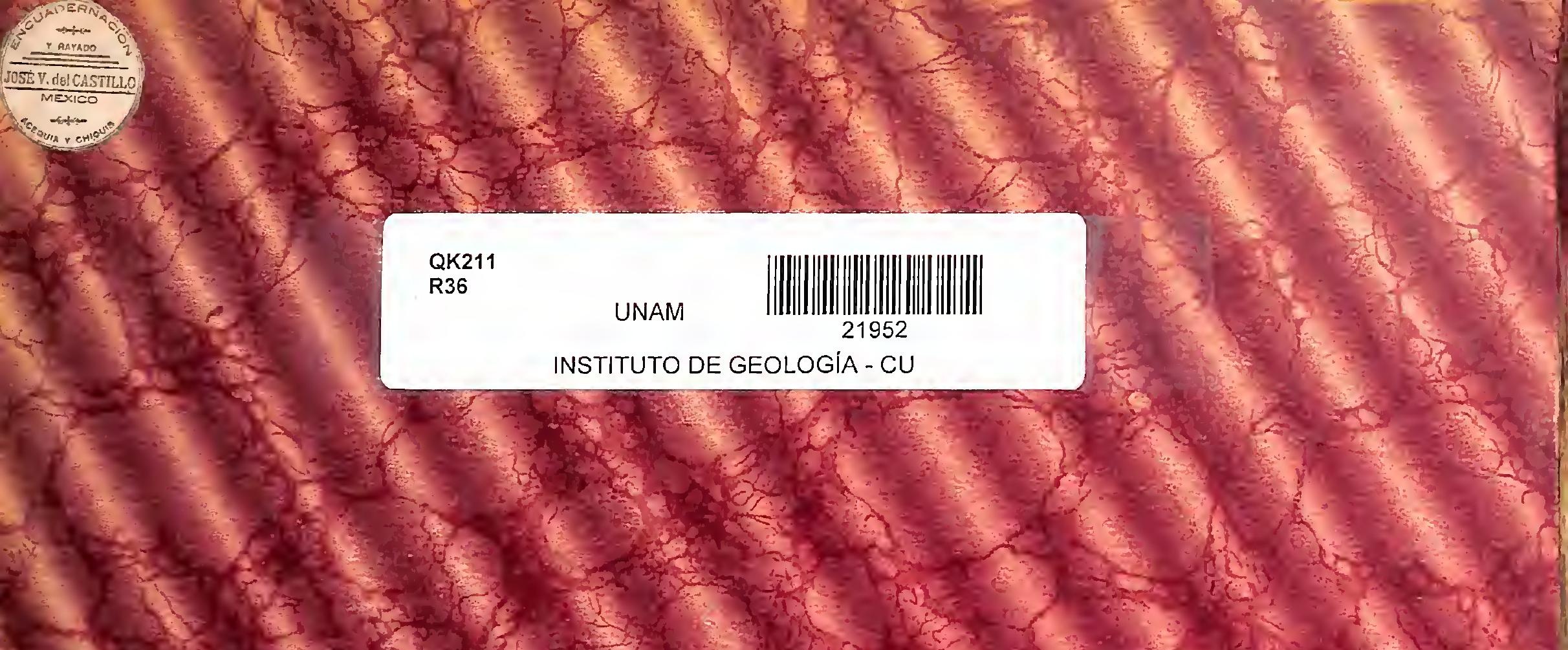




\section{Wh wo?}

Y I N W

- a war $n$,

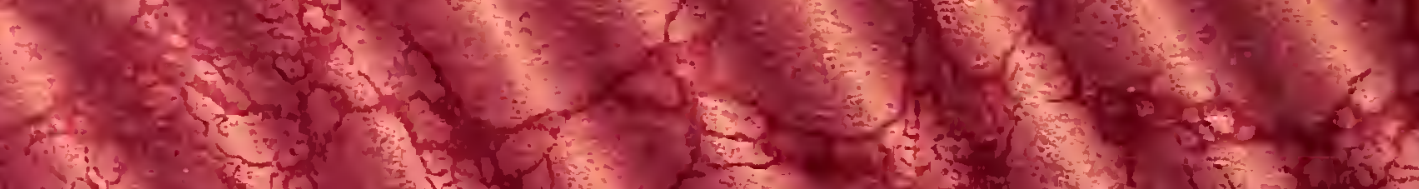

H) W. W

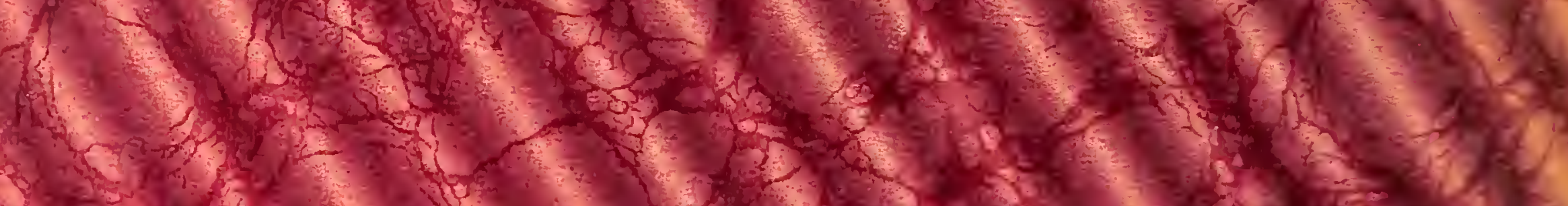

Woras

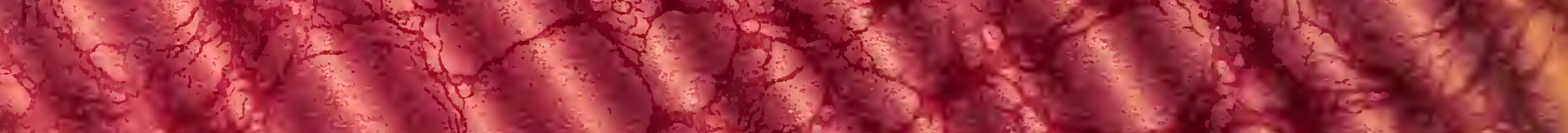
1. W

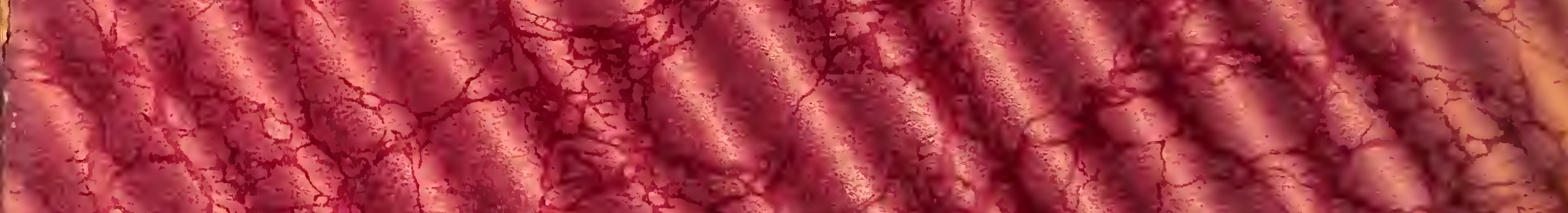

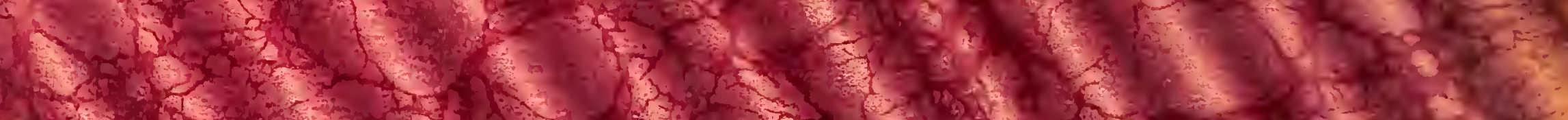

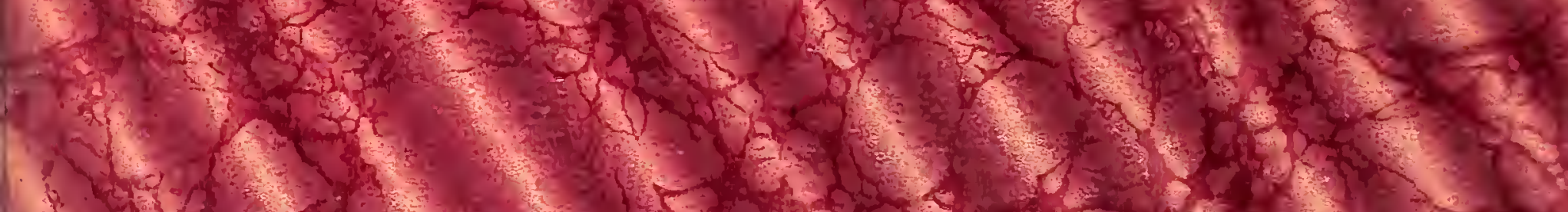
a 1 tor.

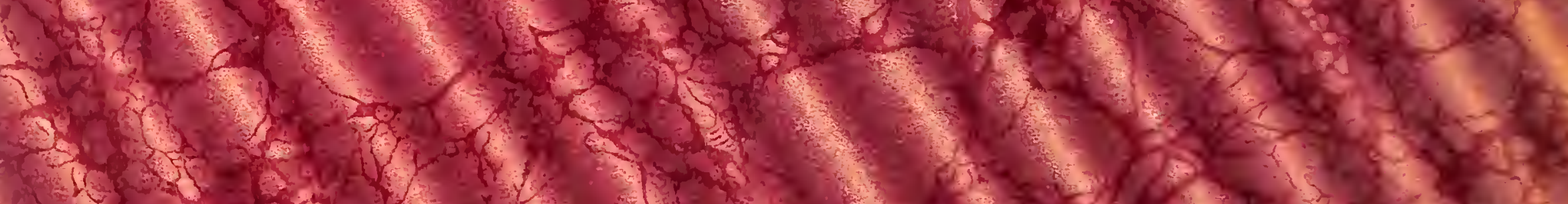
1.1. W

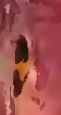
ex $x^{2}+x^{2}$ 8

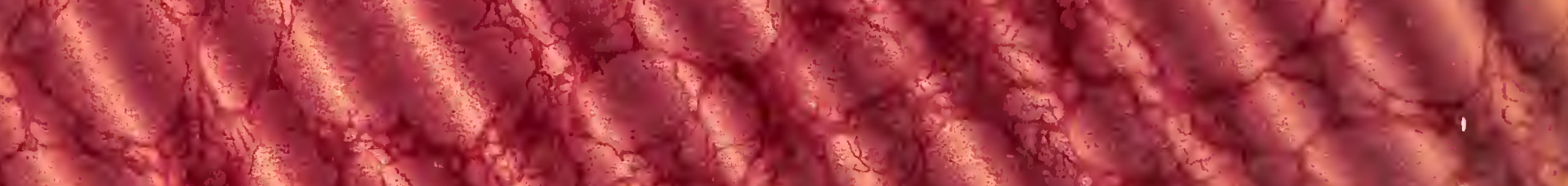

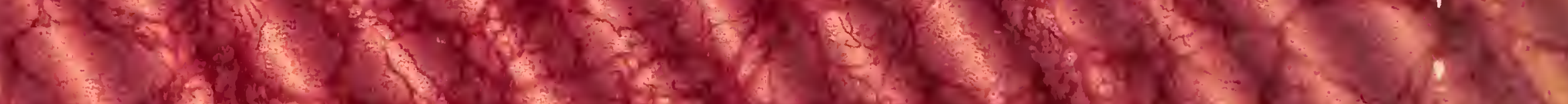
a $x$ 1 19 . dines and a) $x+101$ an 
$\sin 2$

$-8)^{\circ}$ 
$\frac{\text { Chan } 74325}{I-3-7-4}$

SINOVIIIIA VULGAR Y CIBTIFICG

DE LAS

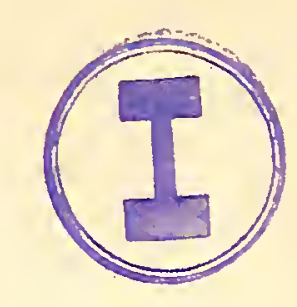

\section{PLANTAS MEXICANAS}

AREEGIADA

\section{POR EL DR. JOSÉ RAMÍREZ}

MIENBRO DEL INSTITUTO HEDICO NACIONAL IDE MEXICO,

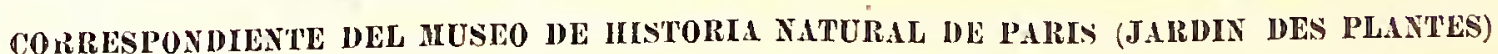
Y CABLLEERO DE LA LEGION DE HONOR.

CON LA COLABORACION DEL

SR. GABRIEL V. ALCOCER

Encargado

del Herbario del Instituto Médico Nacional de México.

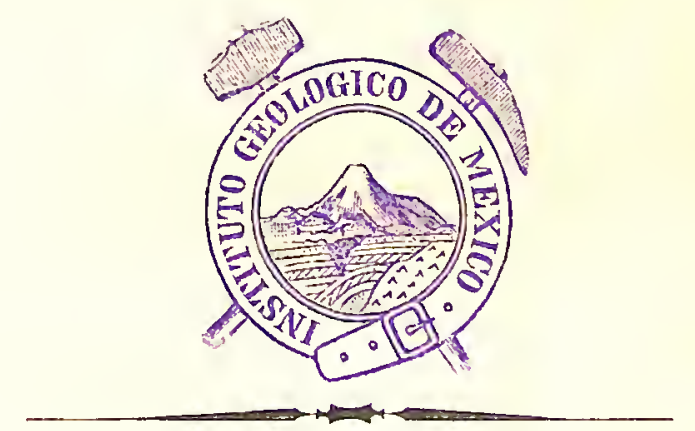

\section{MEXICO}

OFICINA TLPOGRÁFIOA DE LA SECRETARIA DE FOMENTO.

Oalle de san Andrés, núm. 15. (Avenida Oricnte 51.)

$$
1902
$$


12 MAR 2013

$$
\begin{gathered}
\underset{1}{\text { DONACION Verna }} \\
\text { QK211 } \\
\text { R36 } \\
\text { I-21952 }
\end{gathered}
$$




\section{INTRODUCCION.}

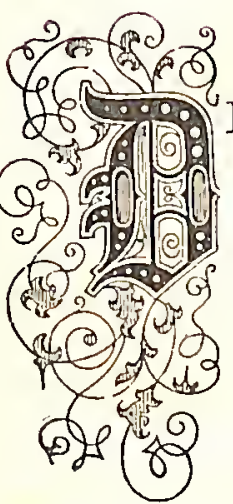

ESDE hace inuchos años ha sido preocupación constante de la Sociedad de Historia Natural, así como del Instituto Médico, reunil en un solo volumen la sinonimia vulgar y científica de las plantas más comunes de México. Las flórulas, los calendarios botánicos y las clasificacio. nes que contienen "Lat Naturaleza" y los "Anales" son las primeras manfestaciones de aquel deseo, que nosotros afortunadamente hemos podido realizar.

El Profesor Alfonso Herrera, tomando por base el Diccionario de Colmeiro y reuniendo por primera vez en 1873 los nombres vulgares de las plantas con sus identificaciones botánicns, hechas anteriormente por Cervantes, Cal, Oliva, González, los Dondé, etc., etc., y agregando los que él había puesto en la segunda edición de la Farmacopea mexicana, prestó bastantes servicios, facilitando el conocimiento de nuestra flora.

Después han aparecido las clasificaciones de Villada, Bárcena, Altamirano, Urbina, Rovirosa, Dugès, Alcocer y Ramírez, al mismo tiempo que las de las publicaciones de los botanistas y colectores que se han ocupado de la flora de México, ó que han recorrido el país en estos últmos años, como Palmer, Parry, Pringle, Brandegee, Soler y señora, Nelson, Rose, Duget, Rusby, Eisen, Donel Smith, Consati, Fernald, Greenman, Poisson, Bureau, Seaton y Robinson.

En 1889 publiqué en Paris, con la colaboración del Sr. G. Crespo, una lista de los nombres vulgares y científicos de los árboles de los cuales se habían enviado colecciones de maderas á la Exposición Universal que se verificó en aquel año. Aquellia lista, que acompañaba á las colecciones, contenía los nombres de las plantas identificadas hasta esa fecha, y los pocos de las que nosotros habíamos clasificado.

Con motivo de la misma Exposición de Paris, el Sr. Dr. F. Altamirano, como empleado de la Comisión mexicana, comenzó una recopilación de las sinonimias publicadas; ordenando que en un libro se copiaran aquéllas, en orden alfabético; tomando los nombres de las publicaciones de que se pudo disponer entonces. Este libro, que permaneció inédito, pasó á la Biblioteca del Instituto Médico, cuando se creó en 
1890. En él se fueron agregando los nombres de todas las plantas que yo clasificaba, así como los nombres que se encontraban en las publicaciones de botanistas competentes. Estil recopilación nos lia prestado mu. chos servicios, facilitando nuestros trabajos habituales en el Instituto Médico, y de ella nos servimos cuando se nos han pedido los nombres vulgares de las plantas, acompañados de sus sinónimos científicos.

En 1891, el Sr. M. Bárcena publicó una noticia histórica del Estado de Jalisco, en la que apareció una lista de las plantas más comunes de aquel territorio, siendo las clasificaciones de los Sres. Bárcena, Villada y Urbina.

En 1894, publiqué con el Dr. F. Altamirano, una lista de todus los nombres vulgares y botánicos de árbules y arbustos propios para repoblar los bosques de la Repíblica, aprovechando la lista que hice en Paris, y agregándole las numerosas clasificaciones que habían aparecido durante los cinco años transcurridos; tanto las de los botinistas mexicanos como las de los extranjeros.

En 1895, al concurrir el Instituto Médico á una de las Exposiciones agrícolas que se verificinl anualmente en Coyoacán, publiqué, en unión de los Sres. Altamirano y Alcocer, un catálogo con la sinonimia científica y vulgar de muchas plantas del herbario, la de 238 drogas y la de 212 maderas de la colección de dicho Establecimient.. Entre las maderas figuraban varios ejemplares de una colección perfectamente clasificada por el inteligente botanista, Sr. José N. Rovirosa y que estuvo en la Exposición de Chicago, enviada por el Estado de Tabasco.

En 1897, el Sr. Dr. M. Urbina publicó un catálogo de las plantas mexicanas existentes en el Ḿuseo Nacional, que contiene numerosas clasificaciones perfectamente determinadas, con sus correspondientes nom-
bres vulgares.

Acumulados todos estos elementos, á los que reunimos, como hemos dicho, los que nos han proporcionado los botanistas extranjeros, varias veces intentamos su arreglo definitivo, rectificando, siempre que había oportunidad, los numerosos errores de que adolecían muchas de las clasificaciones publicadas antes de
de 1870, y que habían pasado como correctas.

Nuestro propósito era publicar un cuaderno que facilitara el conocimiento de la sinonimia de nuestras plantas que, aunque incorrectu, pudiera fácilmerite perfeccionarse en muy poco tiempo, una vez que se tuviera reunido tolo lo publicado; pero la falta de tiempo, debido á las numerosas ocupaciones que tenemos en el Instituto Médico, nos habían impedido por completo dar término á nuestro trabajo, hasta que, con motivo de la Exposición Universal de Paris de 1900, como miembro de la Comisión que preparó el contingente de México para dicho Certamen, cınseguí que la Secretaría de Fomento me nombrara un escribiente, que dediqué al trabajo exclusivo de copiar en orden alfabético todos los materiales que tenía acumula-
dos. Terminada esta laboriosa tarea, hice nes notoriamente malas, y después emprendí formar revisión del manuscrito, para clesechar las clasificacio* una lista alfabética de los nombres científicos de las plantus parte de esta sinonimia, que consiste en de las familias, y la serie de nombres vulgares que les corre que forman la primera parte, con indicación de una sinonimia, no se había hecho hasta ahora para los ponden. Este trabajo, que es el complemento

Concluídos estos trabajos, envié el manuscrito á las plantas de México.

bas iba corrigiendo todas las clasificaciones que comprobaba que eran orón que me entregaban las pruelos elementos par'a adquirir la certidumbre de que mi corrección era la exáneas; es decir, cuando tenía meros pliegus, cuando tuve que emprender mi viaje á Europa, como exacta. Habían aparecido los pripar'a la Exposición de Paris de 1900, y entonces hube de aon como miembro de la Comisión mexicana el Sr. G. Alcocer, quien con una dedicación extraordinatia lo dese trabajo de revisión á mi buen amigo 
fines de Diciembre del mismo año. Después juntos hemos seguido la labor hasta que ésta ha terminado.

La enumeración de los trabajos preparatorios, que fué necesario emprender para publicar esta obra, demuestra la libor considerable que ha costado y que jamás sospecharán las personas que ignoren la materia.

En cuanto á la importancia que tiene una sinonimia para el adelanto de la botánica de determinada región, es un lecho que todo el mundo reconoce. En México, son evidentes las ventajas que proporcionará una recopilación de esta naturaleza á las personas que deseen ocuparse de su flora desde cualquier punto de vista. Paral los que no estén versados en la botánica, como acontece á la mayor parte de nuestros agricultores prácticos, ahora les será fácil identificar cualquier planta por medio de esta sinonimia, ó guiarse con ella siempre que tengan necesidad de saber el nombre científico de algín regetal del que no conozcan sus propiedales, por ignorar su clasificación. Pero aun el misno botanista mucho ganará cuando pueda hacer rá. pidamente una illentificación segura, valiéndose de esta sinonimia. El colector, que generalmente no clasifica sus plantas, encontrará también un buen auxiliar en esta recopilación, y no menos ńtil será para que lleguemos á tener un conocimiento perfecto de la distribución geográfica de las plantas que forman nuestra flora.

Si alguna persona considerara exageraria nuestra opinión sobre el valor de una sinonimia, es induilable que no calificará de igual manera el concepto que tenía sobre el mismo asunto el eminente botanista A. De Candolle, quien se expresó en los siguientes términos al enumerar los elementos de que dispuso para describir las especies correspondientes á los dibujos de la Flora mexicana inédita de Mociño y Sessé:

"Lo que pude sacar de estos manuscritos la sido: 19 indicación un poco más detallada de la patria de eiertas plantas y algunas notas sobre la época de las floraciones; $2^{\circ}$ el conocimiento de algunos usos locales; $3^{\circ}$ el de los nombres vulgares y de los sinónimos de Hernández. Esta última porción ha sido de un valor considerable á mi juicio. Antes de que me hubieran comunicado los trabajos de que doy cuenta, parecía imposible referir las descripciones abreviadas de Hernández á las especies y aun frecuentemente á los géneros conocidos; aun era difícil tener una verdaclera confianza en una obra que presentaba tantas figuras de objetos desconocidos y extriordinarios. Uno de los primeros resultados de mi trabajo fué, pues, el aumentar mi estimación por el Thesaurus rerum medicarum Novie-Hispanie, y el darme algunos medirs para referir á sus géneros una parte de las especies que se encontraban designadas. Los nombres vulgares, lo repito, me han sido de una grande utilidad para este objeto, y no podría repetir demasiado, cuánto iumporta no despreciarlos en los viajes lejanos, como medio de poner á los naturalistas en relación con los habitantes de los países mismos y con aquellos viajeros que ignoran los términos de la ciencia y se sirven de estas nomenclaturas populares. Casi todos los nombres de la Nueva España están compuestos de diversas palabras que expresan su naturialeza ó semejanza, así son de una dimensión que espanta á los oídos europeos. Pertenecen á tres dialectos principales: $1^{\text {? }}$ el verdadero mexicano que se habla entre los indígenas de lus alrededores de México y Tlaxcala; $2^{\circ}$ el dialecto otomí, que se habla entre los indígenas de una parte de la provincia de Valladolid y en las de Querétaro y Zacatecas; 3o el dialecto tarasco que se babla en Sonora y Durango. Hay que agregar á estas tres lenguas los nombres que los españoles han diado á los productos que han encontrado ell sus nnevas posesiones; nombres formados, ya según contrataciones con objetos europeos, ya formados en parte con los nombres indígenas más ó menos alterados."

In esta recopilación, que contiene más de cuatro mil nombres, hentos tonido especial cuidaclo de indi. car quién es el autor de la identificación, así como el lugar ó pueblo en donde se ha recogido la planta y lle- 
va el nombre señalado. De esta manera, en lo venidero, ya se evitarán las confusiones á que han dado lugar los nombres vulgares, cuando se les ha admitido sin la precaución indicada. No obstante los errores que debe tener esta recopilación, creemos firmemente que prestará numerosos é importantes servicios á las personas que se entreguen al estudio de la flora mexicana. Esta consideración es la que nos ha animado á pu. blicar la sinonimia.

México, Marzo de 1902.

Dr. José Ramírez. 


\section{BIBLIOGRAFIA}

DE LAS

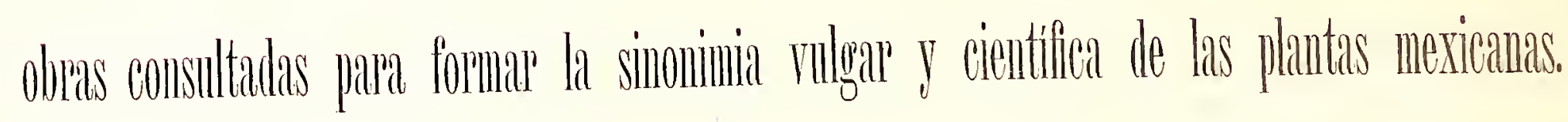

Altamirano Dr. Fernando.-Véase Ramírez.

Bárcena Mariano.-I. Calendario botánico del Valle de México. En "La Naturaleza." Periódico científico de la Sociedad Mexicana de Historia Natural. Tomo IV, 1880-1881.

Contiene una enumeración de las plantas que florecieron en los meses del año de 1879. Muchas de las clasificaciones le fueron comunicadas al Sr. Bárcena por los Sres. Manuel Villada y Alfonso Herrera. Casi todas las determinaciones son exactas.

II. Sinonimia vulgar y científica, propiedades y usos de las plantas que caracterizan climas y terrenos en el Estado de Jalisco. En el "Ensayo estadístico del Estado de Jalisco." Of. tip. de la Secretaría de Fomento. 1891.

Contiene 634 nombres. Muchas de las clasificaciones le fueron proporcionadas por los Sres. Villada y Urbina

Barroso Agustín.-Apuntes sobre la vegelación del Istmo de Tehuantepec. 1874. Como apéndice del "Informe sobre et reconocimiento del Istmo de Tehuantepec," presentado al Gobierno Mexicano por Manuel Fernández, Ingeniero topógrafo y Jefe de la Comisión que practicó el reconocimiento. México. Imprenta de F. Díar de León. 1879.

El Sr. Barroso fué un ingeniero muy reputado, pero no se le conoció como botanista. Se ignora quién le proporcionó las clasificaciones de las plantas que forman su lista ó si él las hizo. Es de sospechal', que á los nombres vulgares que recogió en su viaje les aplicó las clasificaciones bolánicas ya publicadas por aquella época. La lista contiene 125 nombres, con algunas determinaciones erróneas y otras que corresponden á plantas que no son de México.

Brandegee Tounshend S.-I. A collection of plants from Baja Californiil. 1889. En los "Proceedings of the California Academy of Sciences." Ser. 2. Vol. II. 1889.

II. Flora of the Cape region of Baja California. En los "Proceeỏings of the California Academy of Sciences." Ser. 2. Vol. III. 1891.

Cal y Bracho Antonio.- Ensayo para la Materia Médica Mexicana, arreglado por una Comisión nombrada por la Acade- 
demia Médico-Quirúrgica de esta Capital, quien ha dispuesto se imprima por consillerarlo úlil. Puebla. 1832.

Esta obra se reimprimió en "El Estudio," periólico de medicina, que rlespués tomó el título de "Anales del Instituto Médico Nacional." Los principales artículos de este Ensayo se encnentran reproducidos en el Apéndice al Diccionario de Geografia é Hisloria, publicado en México en 1855-56, biajo la dirección del Sr. Manuel Orozco y Berra. Contiene algunas de las clasificaciones de la Flora Mexicina de Mociño y Sessé, lo que hace suponer que el Sr. Cal conoció la obra inédita ó una copia. Las 118 plantas que menciona están en orden alfabélico y los dalos relativos á sus aplicaciones vulgares son de imporlancia, pues además de reproducir lo que consignó Ciervantes el Sr. Cal agrega olros de su cosechat.

Cervantes Vicente.-I. Ensayo á la Materia Médica vegetal de Mćxico. Edición de "El Estudio," órgano del Institulo Médico Nacional. 1889.

Esta obra la publicó el Instituto Mlédico, valiéndose de un MS. que poseía el Sr. Alfonso Herrera y que obsequió al Sr. Dr. F. Altamirano, quien ásu vez lı) cedió al Instituto.

Esta obra no se encuentra mencionada con este título en la bibliografia de Colmeiro, titulada: "La Botánica y los Botánicos de la Península Hispano-Lusitana."

Nosotros opinamos por que corresponde á la Oración que pronunció en México el Sr. Cervantes, con motivo de la apertura de su curso en el Jardín Botánico. el día 28 de Mayo de 1791, y que se encuentra mencionada en la citada obra de Cólmeiro, tomando este dato del Prólogo del "Ensayo para la Materia Médica Mexicana," publicado en Puebla en 1832. Además, lıemos adquirido la convicción de que, como lo supone el mismo Colmeiro, de esta Oración copió Neé la "Lista de las plantas oficinales que se hallan en el reino de México," y que por cl año de 1858 estaba en poder de la filmilia Boulelou de Sevilla. En efecto, esta lista contiene 308 plantas y poco más ó menos este es el número de las plantas de que se ocupa el "Ensayo" de Cervantes.

José Mariano Beristain, en su "Biblioleca Hispano-Americana Septentrional," cita esta obra con el título algo cambiado, pues enumerando los escritos de Cervantes, dice: "Linsayo á la Maleria Médica del Reino vegetal de la Nueva España. Disertación leída en el Jardín Bolánico de México el año de 1791. En esta Disertación se da una razón extensa de todas las plantas medicinales que se críin en los contor-
nos de México."

Es, pues, evidente que la obra corresponde también con la citada por Berislain, aun cuando haya una pequeña diferencia con el título que scñala este bibliótılo, y el que tiene el MIS. de que se sirvió el Instituto Médico para hacer su edición.

Esta obra es de mucha importancia, tanto por los dalos relativos al uso medicinal de las plautis, como porque facilitó la identificación de numerosas especies de la Flora Mexicana de Sessé y Mociño. También co-Quirúrgica de Puebla, pues en al se del Ensayo de Materia Médica que publicó la Academia Médi(entonces inédita), que tuvieron á se dice que reproducen lo que consignó Cervantes en su Oración

II. Notas en la edición española vista para escribir su obra, como lu aseguran en el Prólıngo.

México," traducción del Sr. Dr. Fiance obra del Abate Francisco Clavijero, titulada: "Hisloria antigua de

Las notas que se refieren a lis phancisco P. Vázụuez.

podemos exponer, hemos supuesto que pertáı autorizadas con la letra C. que, por razones que aquí no actaratorios sobre aplicaciones y usos de las ptantas, afervantes. Estas notas contienen multitud de datos Todas las plantas que cita Clavijero las idcutifico así como relativos á botánica y elimologías. ren más valor.

Colmeiro Miguel.-Diccionario de los diversos nombres vulgares de muchas plantas usuales ó vo mundo, con la correspondencia científica y la indicación aluntas usuales ó nolables del antiguo y nuc- 
milia á que pertenece cada planta. Complemento del Curso de Botánica. Madrid. Imprenta de Gabriel Alhambra. 1871.

Recopilación inuy importante de los nombres vulgares españoles de las plantas, así como los nombres usados en América. En la parte que se refiere á México, los 441 nombres vulgares y las identificaciones, están tomalos en su mayor parte de los autores mexicanos anteriores á Colmeiro.

Conzatti C. y Smith I. C.-Flora sinóptica mexicana. Oaxaca. Imprenta de Lorenzo San-Germán. 1897.

Esta importante obra, que desgraciadamente está sin concluir, contiene 134 identificaciones de plantas, unas originales y otras tomadas de los autores anteriores, pero todas exactas.

Coulter John M.-I. Botany of Western Texis. A Manual of the Phanerogames and Pteridophytes of Western Texas. Washington Governement Printing Office. 1891. En las "Contributions from the U. S. National Herbariunl." Vol. II.

En esta Flora de Texas, cncontramos 48 nombres vulgares de plantas con su correspondientes identificaciones. Aunque las plantas son de Texas, como se sabe ese Estado perteneció á México, y los nombres corresponden al idioma español ó á los provincialismos usados por los mexicanos.

II. Preliminary revision of the North American species of Echinocactus, Cereus and Opuntia. En las "Contributions from the U. S. National Herbarium." Vol. III.

En esta memoria nos encontramos 18 nombres vulgares, siendo algunos repetidos de los que se señalan en la Flora de Texas.

Dodge Chas. Richards. - A descriptive catalogue of useful fiber plants of the World, including the structural and economic clasifications of fibers. Washington Governement Printing Office. 1897.

Algunas de las clasificaciones de liıs plantas mexicanas le fueron proporcionadas al Sr. Dodge por el Dr. Ranírez, según lo manifiesta el autor en el Prefacio de su importantísima obra, y única hasta la fecha, relativa á la materia.

Dondé Joaquín y Dondé J. I. - Apuntes sobre pliantas de Yucatán. Edición de la Sociedad Médico-Farmacéutica. Méridit. 1874.

Esta útil obrita contiene, además de los nombres vulgares castellanos y provinciales, los correspondienles á la lengua maya.

II. Lecciones de botánica. Méridal de Yucatán. Imprenta de Juan F. Molina Solís. 1876.

Conticne al fin un índice alfabético de las plantas citadas en la obra, con sus nombres botánicos y los de las familias á que pertenecen. En este índice hay muchos nombres de la lengua maya, y está dividido ell tres columnas, perteneciendo los nombres vulgares de la primera al Sr. Lic. Tumás Barbachano. Todis los autores posteriores han copiado la sinonimia de los nombres mayas señalados por los Sres. Dondé.

Dugès Dr. Alfredo.-Lista de las plantas más comunes en el Estado de Guanajuato, con sus clasificaciones respectivas, publicada en la "Meınoria sobre la administración del Estado de Guanajuato," presentada al Congreso del mismo por el Gobernador constilucional Lic. Joaquín Obregón González el 10 de Abril de 1895.

Esta iista es muy importante porque todas sus clasificaciones son exactas. Todos los nombres se encuentran en nuestra sinonimia, habiéndoles agregado los nombres de los autores, que se omitieron en la. lista publicada en la Memoria.

González Eleuterio.-Apuntes que pueden servir de base para la formación de la Flórula de la ciudad de Monterrey y sus inmediaciones. En "La Naturaleza." Periódico científico de la Sociedad Mexicana de Historia Natural. Tomo III. 1874-1876.

Esta lista enumcra 314 nombres vulgares. Muchas de las clasificaciones se han corregido posteriolmente, pero en general las determinaciones son exactas.

Grisard J. et Vandenberghe M.-Les bois industriels indigènes et exotiques. Synonymie et descriptions des espèces, proprietés physiques des bois, qualités, défauts, usages et emplois. Deuxiéme édition. Tome premier. Paris. 
En esta obra excelente, hay 25 nombres vulgares de plantas mexicanas, escritos correctamente y lomados de la lista que publicaron en Paris los Sres. Crespo y Ramírez en 1889, con motivo de la Exposición Universal que se verificó en ese año.

Herrera Alfonso.- I. Sinonimia vulgar y científica de algunas plantas silvestres y de varias de las que se cultivan en México, dispuesta en orden alfabético. En "La Naturaleza." Periódico de la Sociedad Méxicana de Historia Natural. Tomos II, III, IV, V y VI. 1873-1884.

Esta sinonimia es la segunda recopilación de nombres vulgares y científicos de plantas mexicanas, y lia prestado muchos servicios á los botanistas que estudian la Flora de México. El Sr. Herrera enumera 1825 nombres, de los que 316 corresponden á plantas cultivadas.

II. Clasificaciones y nombres vulgares de las plantas mexicanas mencionadas entre los productos naturales vegetales de la segunda parte de la "Nueva Farmacopea Mexicana" de la Sociedad Farmacéutica de México. Tercera edición. 1896.

En esta obra se mencionan 546 plantas. Las clasificaciones identificadas pertenecen también al Sr. Dr. F. Altamirano, que ayudó á escribir el Apéndice publicado en 1890, así como á los Sres. Alcocer. Dugès, Ramírez, Urbina y Villada.

La Llave Pablo y Lezarza J.-Novarum vegelabilium descriptiones. In lucen prodeunt opera Paulli de la Llave et Joanis Lexarza. Reip. Mexic. CIV.

Fascículos I, II. Mexici. Apud Martinum Riveram. Ann. Dom. MLGGGXXIV.

Estos fascículos enumeran 40 nombres vulgares, de los cuales algunos son de los que menciona Hernández. Algunas clasificaciones pertenecen á Cervantes y están marcadas con la inicial G.

Losener Th._Plantæ Selerianæ die von Dr. Eduard Seler uıd Fran Cœeille Seler in México, etc., etc. En el "Bulletin de Herbier Boissier." Tome II et tome III. 1894-1895.

El Sr. Seler enumera 85 nombres vulgares, algunos están impresos incorrectamente, pero este defecto es probable que dependa del corrector, pues es conocida la competencia linguíística del colector.

Oliva Leonardo.- - I. Lecciones de Farmacología dadas por el catedrático del ramo en la Universidad de Guadalajara. Tip. de Dionisio Rodríguez. Tomo I-II. 1853-1854.

II. Flórula del Departamento de Jalisco en el año de 1859. Comunicada á la Sociedad Mexicana de Historia Natural por el Dr. Alfredo Dugès. En "La Naturaleza." Periódico científico de la Sociedad Mexicana de Historia Natural. Tomo V. 1880.

Esta flórula enumera 406 nombres vulgares.

Ramírez Dr. José. - I. Sinonimia vulg^r y científica de varias de las "Plantas de Nueva España" de M. Sessé y J. Mociño Estudio." Tomo IV, pág. 220. México. Imprenta de la Secretaría de Fomento. 1892-1893.

Esta lista enumera 258 nombres vulgares, la mayor parte identificado; después de un estudio cuidadoso.

II. Enumeración de las principales plantas colectidas en las montañas del Ajusco y Sierra de las Gruces. En el "Informe que rinde á la Secretaría de Fomento" el Director del Instituto Médico Nacional, Dr. Fernando Altamirano, sobre algunas excursiones á las montañas del Ajusco y Serranía de las Cruces. Méxi-
co. 1895 . III. Sinonimia vulgar y científica de las plantas enumeradas en la obra titulada "Datos para la Materia
Médica Méxicana." Primera, segunda y tercera parte. 1894-1898-1900. Publicación del Iustituto Médico
Nacional de México.

Se enumeran 182 sinónimos con sus clasificaciones respectivas.

Ramírez Dr. J. y Crespo G. - Glasification des principales bois de la République mexicaine qui sont employés pour la construction, l'ébénisterie et autres industries. Paris. 1889. Exposition du Mexique à Paris. Groupe V.
Ćlasse 42.

Este folleto contiene la clasificación de 500 plantas, que revisadas están incluídas en la Sinonimia.

Ramúez Dr. J. y Altamirano F-Lista de nombres vulgares y botánicos de árboles y arbustos propios para repoblar 
los bosques de la República, acompañados de la indicación de los climas en que vegetan y de la manera de propagarlos. México. Of. tip. de la Secretaría de Fomento. 1894.

Muclios de los nombres están tomados de la lista publicada en Paris por los Sres. Ramírez y Crespo.

Ramírez Dr. J., Altamirano F. y Alcocer G.-Catálogo de los productos que exlibe el Instituto Médico Nacional en la Exposición de Coyoncán. México. 1895. Of. tip. de la Secretaría de Fomento.

Este catálogo contiene listas de drogas, de plantas de Herbario y de maderas, con sus clasificaciones

Ramírez Lic. Ricardo.-Indice de nombres vulgares é indígenas. 1894.

Este índice, con sus correspondientes nombres botánicos, se agregó por su autor á la edición de la Secretaría de Fomento de la "Flora Mexicana," de Martín Sessé y Mariano Mociño. En este índice se han compilado 761 nombres vulgares que enumeran Sessé y Mociño en la "Flora," así como en su otra obra, "Plantæ Noræ Hispanix." Muchos de los nombres de la lengua Nahuatl tienen su significado en castellano según el autor del índice.

Rose J. N.-I. List of Plants collected by Dr. Eduard Palmer in Western Mexico and Arizona in 1890. En "Contributions from the U. S. National Musseum." Vol. I.

Esta lista contiene 21 nombres vulgares.

II. Report on a collection of plants made in the States of Sonora and Colima, México, by Eduard Palmer in the years 1890 and 1891. En "Conrtibutions from the U. S. National Herbarium." Vol. I.

Esta lista contiene 36 nombres vulgares.

III. Notes on useful plants of México. En "Contributions from the U. S. National Herbariun." Vol. V. 1889.

Obra muy importante, con todas sus clasificaciones exactas, y muchas nuevas de su autor. Contiene 152 nombres vulgares, la enumeración de muchas plantas, numerosos datos relativos á sus usos en México, que no se encuentran en ninguna otra obra.

Rovirosa Ing. José IV.-I. Calendario botánico de San Juan Bautista y sus alrededores. En "La Naturaleza." Periódico cientifico de la Sociedad Mexicana de Historia Natural. Tomo II. Segunda serie. 1891.

Todas sus clasificaciones son exactas y se encuentran en nuestra Sinonimia.

II. Catálogo de las maderas del Estado de Tabasco. En el "Catálogo de los productos que exhibe el Instituto Médico Nacional, en la Exposición de Coyoacán." 1895.

Este catálogo se formó con la lista de las maderas que remitió el Estado de Tabasco á la Exposición de Chicago de 1893 , así como con los datos que constaban en los membretes de cada ejemplar, recogidos por el mismo Sr. Rovirosa.

San Giorgio Contesa Di.-Catalogo poligloto delle Plante. Firenze. 1870.

Esta obra muy útil, pero poco conocida, contiene los nombres de las plantas de España, tomados de Colmeiro y en consecuencia no apróvechamos ninguno, y si hacemos la cita, es porque consideramos la obra de uso necesario para los botánicos.

Tena Miguel. - Calendario botánico de Michoacán, presentado á la "Suciedad Unión Médica" de Morelia. 1893.

Contiene datos relativos á la época de floración de las especies que se mencionan.

Urbina Dr. Manuel. - Catálogo de plantas mexicanas. (Fanerógamas). México. Imprenta del Museo Nacional. 1897.

Este importante catálogo contiene una abundante sinonimia vulgar y científica de muchas de las plantas que forman actualmente el Herbario del Museo Nacional. 'Todas las clasificaciones son correctas y están pasadas á nuestra Sinonimia.

Vasey Geo, and J. N. Rose.-List of planls collected by Dr. Eduard Palmer in Lower Galifornia and Western Mexico in 1890 .

Esta lista contiene 27 nombres vulgares identificados.

Villada Dr. Manuel.-I. Estudios sobre la Flora de Pachuca, Mineral del Cilico, Real del Monte, Huesca y Barranca Hon- 
da. En la "Memoria de los trabajos ejecutados por la Comisión Científica de Pachuca" dirigida por el Ingeniero Ramón Almaraz. México. Imprenta de J. M. Andrade y F. Escalante. 1865.

Enumera 292 plantas.

II. Clasificación científica de los objetos del Grupo 8, Clases 69 y 79 en la Exposición de Toluca. Leña, maderas finas, maderas de construcción, plantas medicinales y de recreo é instrucción, sobre sus propiedades y lugares de producción. En la "Memoria de la primera Exposición de la Capital del Estado de México, Toluca. Impresa en México por Miguel Ulloa. 1883.

Numerosos son los trabajos del Sr. Dr. Villada sobre la Flora de México, y bien conocidos de los naturalistas. Sus clasificaciones, siempre corectas, están reproducidas en nuestra Sinonimia.

Watson Sereno.-I. List of plants collected by Dr. Eduard Palmer in South Western Chihuahua, México in 1885. En los "Proceedings of the Amer. Acad. of Arts \& Sciences." Vol. XXI.

II. List of plants collected by Dr. Eduard Palmer in the State of Jalisco, México in 1886. En los "Proceedings of the Amer. Acad. of Arts \& Sciences." Vol. XXII. 1887.

III. Upon a Collection of Plants made by Dr. Eduard Palner in 1887, about Guaynuas, Mexico, at Mulegé and Los Angeles Bay in Lower Galifornia, and on the Island of San Pedro Martin in Gulf of California. En los "Proceedings of the Amer. Acad. of Arts \& Sciences." Vol. XXIV. 1889.

La primera lista contiene 18 nombres vulgares, la segunda 42 y la tercera memoria 45 . Muchos de tos nombres están escritos de una manera incorrecta, como sucede generalınente en las obras de todos los autores que se han ocupado de nuestra flora, pero no debe olvidarse que Watson y Asa Gray han sido los botanistas americanos modernos que han despertado el gusto por conocer lil Flora de México.

México, Marzo de 1902.

\author{
Dr. José Riamírez.
}




\section{(2) EXPLICACION}

DE

algunas de las abreviaturas usadas enl los nombres de la charta columna de la sinonimia mulgar y cientifica.

Alt. et Ramz.-Altamirano y Ramírez.

B...... $\}$ Brandegee.

B., V. et Urb.-Bárcena, Villada y Urbina.

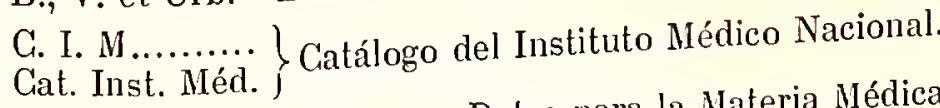

Cerv., M. M.-Ciervantes y Datos para la Materia Médica Mexicana.

Colm.-Colmeiro.

Goult.-Coulter.

DC.-De Candolle.

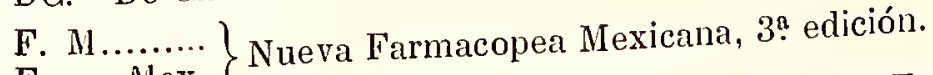

Farm. Mex. M.-Flora Mexicana. Edición de la Secretaría de Fomento.

Herb. Pringle.-Herbario Pringle del Instituto Médico Nacional.

Hernz.-Hernández.

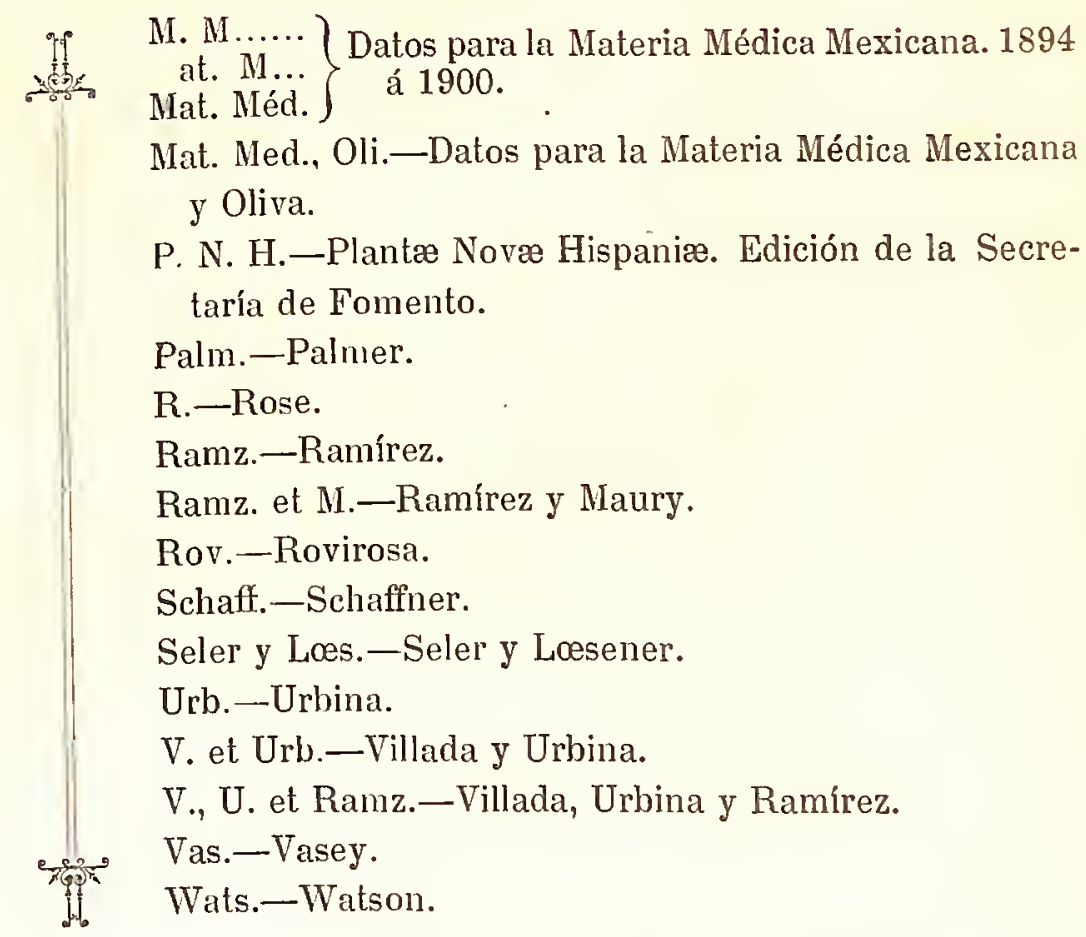

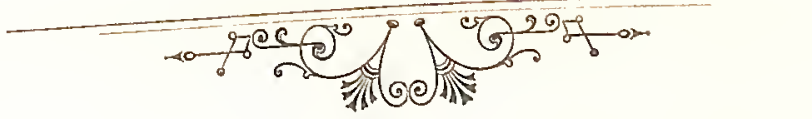




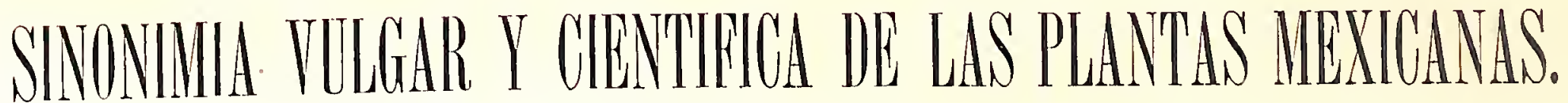

\section{PRIMERA PARTE.}

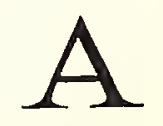

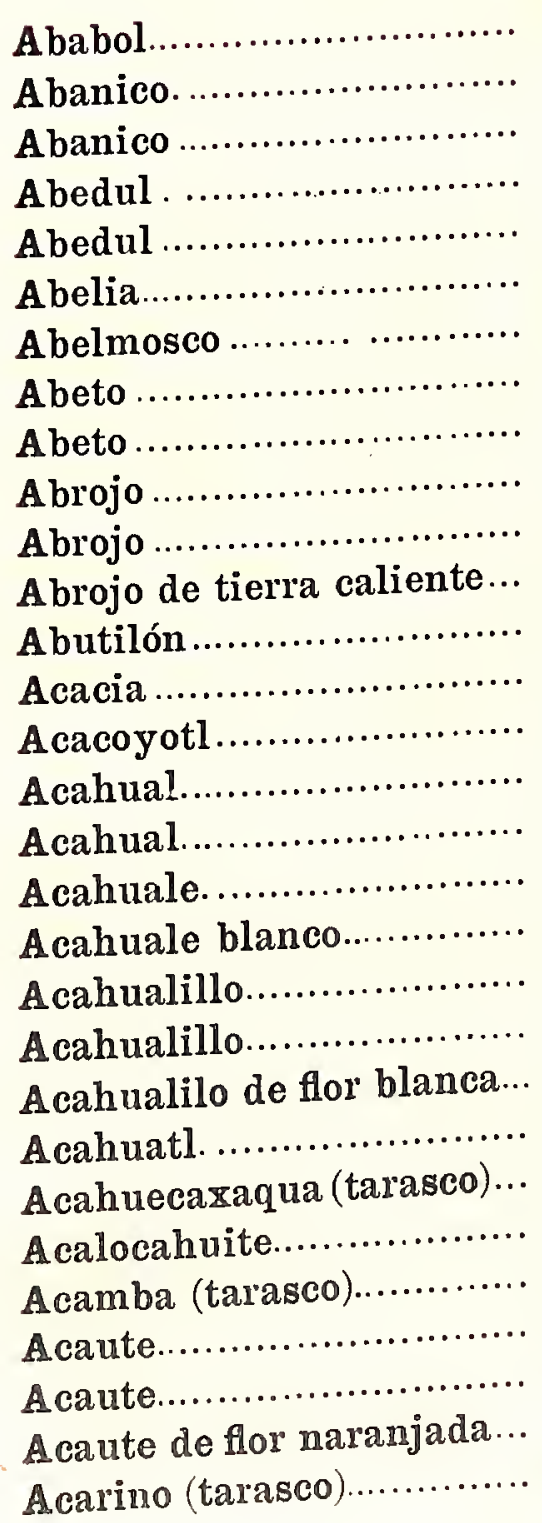

Papacer vhates I.

Alriplex, sp?...

Celosin cristata. I

Alines aenminata H. B. K

Betula lentr. L

Abelia floribruma Dene

Hibiscus abelmoschns L.

Pseudotsuga douglesii Ciurr.

Abies religiosa Cham. et Schl.....

Opnntia tunicata Lehm.

Xanthium strumarium L...

Tribulus cistoides L.

Abutilon, sp?...

Robinia psendoacacia L.

Lilhospermum, sp?.......

Helianthus annuus L....

Encelia mexicana Mart.

Tithonic tubceformis Cass.

Bidens leucantha Willd...

Bidens helianthoides H. B. K......

Birlens tetragona DC

Billens pilosa L.

Heterolheca inuloides Cass

Portuluca oleracea I.

Pinns ryacaluite Ehrenb..

Agave potatorum Zucc.....

Helianthus. spe?

T'ithonia tubeformis Cass.

Tilhonia tagetiflora Desf.

Siryos mgrulatus. L.
Papaveráceas....... Amaranláceas...... Amarantáceas..... Cupuliferas Cupuliferas ........ Caprifoliáceas ......

Mal váceas ...........

Coníferas

Coníferas

Cácteas.

Compuestas

Zigofleas.............

Malváceas ..........!

Leguminosas.......

Borragineas .........

Compuestas .........

Compuestas .........

Compuestas .........

Compuestas .........

Compuestas.........

Compurstas .........

Compuestas........

Compuestas........ Valle de México.

Portuláceas......... Mich., N. León...

Coníferas........... Veracruz.

Miclı., N. León...

Compuestas ......... Jalisco............

Compuestas........ Jalisco............ B. V. et Urbina.

Compuestis ........ Jalisco........... B. V. el Urbina.

Ciucurbiláceas..... Michoacán........ N. Teón.

M. Tena.

Farm. Mex.

Ramírez.

Ramírez.

Rovirosa.

Farm. Mex.

M. Tena.
Mat. Med.

N. León.

Ramíre\%.

N. León.

I 


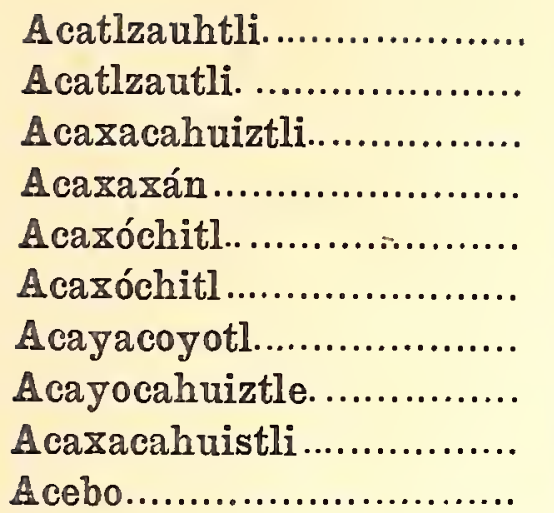

Acecé............................

Acedera.........................

Acedera..........................

Acederilla........................

Aceitilla...

Aceitilla..

Aceitilla

Aceitillo.

Aceituno.

Acelga..

Acelga .

Acezintle.

Acitz (maya)

Acocote

Acocote

A cocotli de Hernández.....

Acónito

Actimpatli...

Acuitze variqua (tarasco)..

Acuyo..

Aczoyátic.

Acroyatl.

Achicoria

Achicoria amarga

Achicoria dulce.

Achicoria dulce.

Achichil

Achiote

Achiotillo.

Achiotl.

Achiotlín.

Adelaida..

Adelaida.

Adelaida.

Adelaida.

Adelfa.

Adelfilla..

Admirable.

Adormidera

Adormidera china............

Agapando

Agare palo.

Agrimonia

Agrimonia

Agritos.

Agritos.
Cranichis tubularis Llav. et Lex... Cranichis speciosa Llav. et Lex.... Cynodon dactylon Pers....

Potamogeton nutans L.

Escobedia linearis Schl......

Lobelia laxiftora H. B. K.

Crix lacryma 1 ,

Solknum cornutem Lam.

Triticun repens $\mathrm{L}$.

Ilex: sp?.

Euchliena luxurians Dur. et Asch.

Oxalis corniculata L.

Rumex acetrowe $\mathrm{L}$....

Rumex acetosella $\mathrm{L}$.

Bidens leucontha Willı.

Billens pilosa. L

Tugetes, sp?

Bursera mexicana Eugl.

Olea enumpera I.

Beta cycla L.

Bela vulgaris Moy...................

Negnudo mexicanum DC.

'ithevetia neriifolia Juss.

Arracercia retropurpuren B. et H.

Lagenuria enlyaris Ser.

Birlens leucantha Willi.

Aconilum nopellus I,

Echites cimicirla Mnc. ot Sessé.

Rutu graveolens I.

I'iper scenctum Schl.

Iprmace capillacea Don.

Abies religiosa Ch. el Sihl

Cichorium intybus L.

Turaxacum officinale Wigg.

Sonchus cilialus L

Sonchus oleraceus L.

Viburnum glabratum H. B. K.....

Bixa orelluna L.

Bixa mellana $\mathrm{L}$

Bixa orellena L.

Piper angustifolium Ruiz et Pav..

Fuchsia urborescens Sims.

Fuchsia coecinea Ail.

Fuctesia corymbifolia Pilv.

Fuchsia fulyens DC:

Nerium oleander 1.

Daphue laureola $\mathrm{L}$.

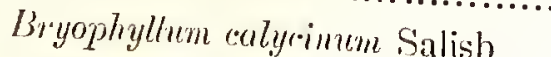

l'upuver rhoens I,.

l'apever somniferum L

Agrevanthes rombellifereses L'Ilér.

'Iillandsia recurvala $\mathrm{L}$.

Agrimonia eupaloria $\mathrm{L}$

Ayrimonia parvifloma Ail.

Oxalis latifolia H. B. K.

Oxulis, sp?...
Orquídcas

Orquídeas.

Graníneas

Nayadáceas

Escrofularíneas.....

Lobeliáceas

Gramíneas

Solináceas ....

Gramíneas

Ilicineas.

Gramíneas

Geraniaiceas

Poligonáceas.

Poligonáceas

Compueslas

Compruestas

Compuestas

Burseráceas.

Oleáceas.

Quenopmdiáceas ..

Quenoprodiáceas ...

Acerá cas..

Apocinácears.

Umbeliferas ...

Cucurbiláceas

Compuestas.

Ranunculácesıs...

Apocinácens.

Putáceas ...

Piperáccas.

Convolvuláceas....

Coníferas.

Cimpuestas

Comprestas.

Compuestas

Compueslas.

Caprifoliáceas.....

Bixíneas.

Bixíneas.

Bixíneas.

Piperáccas.

Onagrar icas.

Onagraricas

Onagrarieas.

Onagruritis.

Ajocináceas.

Timeleáceas

Ciłasuláceas .

Papaveríccis......

Papkiveráceas.......

Liliáceas.

Bromelíne.........

Rinsícens.......

Pisácas ...........

Riosáceas...

Gelaniáceas.

Ceraniáceas.
Michoacán

Naluralizada .....

S. L. P., Glo.....

Valle de México.

Valle de México. Mal. Mird.

Ciosliuila.

Vallı: de México.

Cullivada.........

Jalisco

Jalisco

Michoilcán

Cullivada...........

Cullivada...

Valle de México.

Jallisco, Michl....

Miclnoarcín

Verileruz..........

Cultivarla...

Jilist:o.

Ciultivada.........

Valle de Méxien.

Veracrur.

Varios lugares...

Jilisco.

Valle de México.

Cullivida..

Tepechicollán ...

Mi.hoacán ........

Veracruz......

Valle de Méxiro.

D. Federal, Mor.

Cultivada.........

Naturalizada .....

Valle de México.

Cullivada.

Chiapas...

Istmo Tehc., Jal.

Lug. calienles....

Islmo Telie., Jal.

Veracruz.

Veraciuz, Oax...

Cultivadia.

Cultivada.

Michoacán.......

Ciulivada.

Cullivada.

Veracruz, Hgo...

rullivadir.

Cullivarla........

Jillisco............

Valle de México. Kunlh.

Cullivada.........

Valle de México.

Jalisco.

Ville de México.

Colmeiro.
Colmeiro.

Riıníre\%.

Urbina.

M. T'ena

Firm. Mex.

Mart. Mérl.

B. V. et Urbina.

M. Tewa.

liamirez.

B. V. el Urbina.

Ramirez.

Mall. Mérd.

Farm. Mex.

P. N. H.

Urina.

Firm. Mex.

Colmeiro.

Farm. Mex.

B. V. et Urbina.

Farm. Mex.

P. V. el Urbina. 
Agritos color de rosa Agritos de maceta Agua de azahar.

Aguacachile.

Aguacate

Aguacate oloroso

Aguacatillo.

Aguacatillo.

Aguacatillo.

Aguacatillo blanco.

Aguacatillo colorado

Aguatle

Aguava

Aguiejote.

Aguileña

Aguinaldo.

Aguja del pastor.

Ahate

Ahate.

Ahoacaquáhuitl

Ahoaquáhuitl.

Ahoaq uáhuitl.

Ahoaquáhuitl.

Ahoatl..

Ahoehuetl.

Ahuacate.

Ahuacachile.

Ahuacaquahuitl

Ahualtzocotl.

Ahualzocotlque

Ahuatezón.

Ahuatle.

Ahucepitzáhuac

Ahuehuete.

Ahuehuetl.

Ahuejote...

Ahuejote...

Ahuichichi

Ahuilote

Ahuiron.

Ahuitule

Aile.

Aile.

Ajenjo de México

Ajenjo del país.

Ajo

Ajocopaque

Ajonjolí.

Akec.

Ala de ángel.

Ala de perico

Alacate....

Alacrancilio

Alamo

Alamo.

Alamo.
Oxalis decaphylla H. B. K...........

Oxalis corniculata L.

Qhoothera rosec Ait.

Persea, sp?

Persea gratissima Grertn............. I'erse't diymifotic Chanı. el So:hl.

Lorrenthess, sp??

Persea grutissima Gerln...........

I'crsen, sp?

l'ersea, sp?

Persea, sp?

Quereus acutifolia Nee..............

Bromelia pinguin L.

Teeoma, sp?.

A quilegia vulgaris $\mathrm{L}$

C'ampanula rapunculoides (?) L...

Erodium cicuturium Leman........

Anona purpurea DC.................

Anona, sp?.

Persen gratissima Gertu............

Querens insignis Nart. et Gal......

Quevens reticulute Humb. "t Bonll.

Quercus stromboearpa Liebnil.....

Queiens arutifoliu Nee.

Taxodium mueronatum Ten........

Persec gratissimu Giertn...........

Persea, sp

Persea gratissima Gær 1 ............

Malphigia ureus L.

Malphigia nerens $\mathrm{L}$.

Quercus, sp?

Quevers aculifolin Nee.

Quercus laurina Huml). et Bon|l..

Taxodium mucronatum Ten.......

Thaodium nucronatum Ten.......

Teeoma, sp?

Saliz lesiolepis Benth..............

Trianosperma racemosa (?) Hemsl.

Vitex mollis H. B. K

Ciehorium intybus L......

Vernonia salicifolia Schz.

Aluas acrmineata H. B. K

Alnus cordifolia Ten.

Artemisica laciniata IVilld...........

Artemisia mexicana Willa..........

Allium sativum L

Gaultheria, sp?..

Sescomum indicum L.

Copania akesia Chamb.

Begonia gracilis H. B. K.

Amarentlus trieolor L..

Lagenuria vulgaris Ser.

Heliotropiun indienen $\mathrm{I}$..............

Platemus lindeniena Misrl. et Gal.

I'latanus occidentalis L.

Popuhus, sp?...
Geraniáceas.........

Geraniáceas.........

Onagrarieas.........

Liuríneas............

Lauríneas............

Lauríneas............

Lolinláceas. .........

Lilulíneas.

Laulineris............

Laurineas...........

Lauríneas............

Cupulíferas..........

Bromeliáceıs .......

Bignoniáceas........

Ranunculáceras.....

Cilmpanuláceas.....

Geraniáceris.........

Allonáceaıs ..........

Anonáce:ıs ..........

Ialurineals............

Cupulifer:ıs..........

Cupuliferas..........

Cinpuliferas.........

Cupulíferis..........

Coníferas ...........

Lauríneas

lamrineas.

Laulíncis ...

Mislpigiáceas.........

Milipigiéceas.........

Cinpulíferis.........

Cupulíferis..........

Cupulíferas..........

Coníferas....

Coníferas.

Bignoniáceas.......

Salicineas...........

Cucurbiláceas .....

Verbenáceas ........

rompuestas .........

Compuestas .........

i iupulíferas.........

Citpuliferis.........

Compuestas .........

Compuestas........

Liliaceas............

Er ¡icáceas...........

Pedalíneas ..........

Sapindaceas........

Begoniáccis .........

Amarantáceas.....

Cucurbiláceas .....

Borragíneas........

Plalanáceas........

Platanáceas ........

Salicineas............
Jalisco......

Jalisco.

B. V. et Urbina.

Jalisco.

Horelos, México. Ramírez.

Regs. calienles...

Veracru\%......... Ramírez.

Miclıoacán........ M. Tena.

Morelia

Vericruz......... Ramírez.

E. de México.... Ramírez.

E. de Méxiro..... Ramírez.

Veraciuz.

Colm., Kunth.

Lugares cálidos..

Oaxilca........... Cuit. Inst. Méd.

Cultivadil........ P. N. H.

Cultivarla.........

Valle de México.

Jalisco............ Oliva.

Luga:ı•e- cálidos.. Ramírez.

Morelos, Mich...

Veracru\%.........

Guanijuito, elc..

Veraclus..........

Veracruz..........

Valle de México.

Mol., Jalisco, etc.

Morelos, México. Ramírez.

Mor., Jalisco.....

J.ugarres cálidos.. Colmeiro.

.................... Colmeiro.

Morelos, México. Alt. el Ramz.

Veracruz......... Alt. et Ramz.

E. de México.... Alt. et Ramz.

Valle de México.

Valle de Mléxico.

Jilisco........... Oliva.

Jal., V. de Méx.. Ramírez.

Regs.templadas. Falrm. Mex.

Jalisco........... Rismírez, etc.

Naluralizada .....

E. de México.....

Jilisco.

Cultivadi........

Cultivada.........

Valle de México.

ciultivada.........

E. de México....

Cultivada.........

Veracruz..........

Jilisco.

Jalisco.

Natmalizada ....

Veraciuz..........

Cullivarla........ Ramíre\%.

México........... Farm. Mex.
Urbina.

B. V. et Urbina.

Ranuírez.

Colmeiro.

Mat. Méd.

Farn. Mex. Alt.

Farm. Mex.

\section{B. $V$. et Unjina.}

Farm. Mex. 
Alamo

Alamo blanco.

Alamo blanco.

Alamo negro.

Alamo plateado.

Alamo temblón.

Alampepe

Alantana.

Albaca..

Albaca fina.

Albacarón

Albahaca.

Albahaca de la tierra.........

Albaricoque.

Albérchigo.

Alcabucil

Alcachofa

Alcanfor de maceta.

Alcanfor de mata

Alcanforada....

Alcaparrillo

Alcaravea .

Alcartaz ó Alcatraz

Alcaucil.

Alcornoque.

Alegría.

Alegría

Alejandría..

Alfalfa.

Alfilerillo

Alfombrilla del campo......

Alfombrilla hedionda.

Alfombrilla de jardín........

Alfónsigos de tierra.

Algalia.

Algarroba

Algarrobilla

Algodón.

Algodón. .

Algodón.

Algodón de árbol.

Algodón común.

Algodón coyuche.

Algodoncillo.

Algodoncillo.

Algodonero arbóreo

Algodonero herbáceo

Alhelí

Albelí blanco y encarnado.

A.lhelí camaleón

Almendra.

Almendro

Almizclillo.

Almoraduj ó Almoraduz..

Aloloé amarillo.

Alpiste ó Alpistera
Populus nigra $\mathrm{L}$

Populus alba L

Platanus mexicana Moric

Populus nigra $\mathrm{L}$.

Populus heterophylla L.

Populus tremulre L....

Dolichos, sp?.

Lantana camara L.

Ocinum basilicum L.

Ocimum mininum L...

Ocimum basilicune I,.

Ocimum basilicum 1 ,

Ocinonm micranthum Willd.

Prunus armeniaca L.

Prunns persica Benlh. el Hook..

Cynara cardunculus L.

(ymaina scolymus L.

Camphorosmu. monspeliaca L.......

Heterothecr. leptoglosa. DC.

Camphorosma monspeliar:a I.......

Cossin bicupsularis L.

Crumeme carri I

Richerrtia africana Kunth.

Cynara cardumanlus L.....

Quercus, sp.?.

Amaronthus hypochondriacus L...

Amaranthus leucospermus Watson

I'luneria rubro I.

Medicugn sntim L

Erodium cicutarium Lemo.......

Verbcun cilirtu Benlh

Lerntena horrida H. B. K.

Verbena corymbosa Rui\% et Pav..

Arachis hypogoea L.

Hibiscus abelmoschus L....

Acercio pennatula Benth.

Vicia sitiva L.

Gossypium arboreum $\mathrm{L}$....

Gossypium herbaceum L

Gossypium religiosum L....

Gossypium aboveum L.

Gossypium barbadense L...

Gossypium herbuceum L.

Inga, sp? .

Asclepirs incarnata L

Cossypium crboreum I

Gossypinm herduceum L

Cheirunthus wheiri L

Mothiold annule Siveet.

Cheiranthus scoporims Willd.

T'erminalin sorleppose L.

Prumes comygdalus Hook. fil......

Datura suaveolens Humb. et B..

Saleia patens Cilv.

Jurobinia anren Femsl

Ihalaris comoriensis $I_{\alpha}$
Salicineas.

Salicineas.

Platanáceas

Salicineas.

Salicineas.

Salicineas.

Leguminosas.

Verbenáceas

Labiadas.

Labiadas.

Labiadas.

Labiadas.

Labiadas.

Rosáceas

Rosáceas

Compuestas.

Compuestas

Quenopodiáceas ...

Compueslas.

Quenopoliáceas ..

Leguminosas.......

Umbelíferas

Aroideas.

Compuestas

Ciupulíferas.

Amarantáceas

Amaranláceas

Apocináceas.

l.eguminosas.

Geraniáceas

Verbenácens

Verbenteeas....

Verbenácens ...

Leguminosas.

Malváceas

Leguminosas.

Leguminosas.

Mal váceas

Malváceils

Malváceas

Malváceas

Malváceas

Nalváceas

Leguminosas

Asclepiareas

Malváceás ....

Malváceas

Cruciferas.

Ciruciferas.

Crucíferas.

Combretáceis

Rosáceas

Solanáceas.

Labiadas....

Acantácens

Gramíneis.
Jalisco

Cultivada.

Hidalgo, N. L..

Cultivada

Cullivada...

Cultivada

Puebla

Distrito Federal.

Cultivada.

Cultivada.

Michoacán.e....

Jalisco.

Tabasco.

Cultivada

Cultivada

( Cultivada

Cultivadi

Cultivada

Jalisco.

Cultivadil.........

Lug. calientes...

Cultivada

Cultivada.........

Cullivada.

Michoacán.

E. de Méx., Jal..

Jalisco.

Morelos

Cultivad ...............

Ville de México.

Jalisco.

Michoacán.......

Cultivada.

Guanajualo, etc.

Cullivada.

Sonora...

Naturalizada .....

Cultivada.

Cullivada...

Cullivada.

Cultivada

Cultivada.

Cultivadi

Oixaca.

Jalisco.

Cinltivada.

Cultivada

Cullivadil.

Cullivarla

Cullivada.

Baja Califounir.

lultivada.......

México

S. Luis Potosí, \&

Jalisco

Cullivala...
B. V. et Urbina.

Ramírez.

Urbina.

Ramírez.

Ramílez.

Cat. Inst. Med.

Rovirosa.

P. N. H.

Firm. Mex.

II. Tellik.

B. V. el Uphina.

B. V. et Ushina.

B. V. et Urbina.

B. V.el Upbina.

M. Telli.

B. V. el Urbina.

Barroso.

Colm. et Oliva.

Rose. 
Alquimila

Alquimila del país.

Alquimila del país.

Alquitira del país......

Altamisa ó Altemisa

Altamisa

Altamisa

Altea.

Altea.

Alverja ó Alverjón

Alzoyatic.

Amacayo.

Amacostic.

Amamalocotl

Amamastla.

Amantillo

Amapa.

Amapola.

Amapola amarilla

Amara amarilla...........

amarilla de B. C.

Amapola amarilla de B C.

Amapola grande........

Amapolita morada.

Amaranto

Amargoso

Amargoso

Amate

Amate.

Amate amarillo

Amate blanco

Amate prieto

(n).......

Amatzauhtli..................

Ambar...............................

Ambar, Ambarilla ó Ambarina

Ambástaqua (tarasco)........

Ambrosia......................

Amesquitea .....................

Amiga de noche ..............

Amistad.........................

Amole ............................

Amole ó Aniolli.

Amole

Amole..

Amole

Amole

Amole de bolita

Amole de bolita

Amole lechuguilla

Amole de raíz

Amole de raíz

Amolilla

Amolquelite.

Amolquelite ó Amolquilit]

Amor de estos tiempos.....
Geranium mexicanum H. B. K.....

Geranium hornandezii DG.

Geranium mexicanum H. B. K.....

Opuntia tuna Mill ?...

Ambrosia artemisiafolia L.........

Mhaithenium hysterophorus L......

Pyoeth'um parthenium Smith....

Althace officinalis L.

Anode hastata Cav.

Pisum sativum L.

Mirebilis longiflora L...............

Spretelic formosissima Herb. App.

Ficus nymphceifolia L.

Soliclago paniculata DC.

Rumox mcxicanus MLeissn...

Abutilon triquctrum Presl...

T'abebuiu palmeri Rose.

Papaver rhoces L.

Cholidnniun majus Mill...

Eschscholtzia californica Chann...

Hunnemannia fumaricefolia Sweet.

Althera rosea Cav.

Anoda triangularis $\mathrm{DC}$.

Amaranthus sanguineus $\mathrm{L}$.

Castcla nicholsoni Hook.

Piscidia sp?...

Ficus nymphaifolia L...............

Ficus $\mathrm{sp}_{\mathrm{p}}$............................

Ficus petiolaris H. B. K............

Ficus bonplandiana Mic............

Ficus tecolutensis Miq................

Ficus sp?...........................

Epidendrum pastoris Llav. et Lex.

Hibiscus sp?:

Seabiosa atropurpurea L..........

Eryngium serrutum Cav..

Ambrosia artemisicefolia L..........

Ficus complicota H. B. K..........

Polianthes tuberosa L.

Hibiscus mutabilis L.

Agave sp?

Polianthes tuberosa L

Rhammus sp?

Stegnosperma halimifulia Benth...

Yucca baccata Nutt.

Zizyphus mexicana Rose...........

Prochnyanthes viridescens Wats...

Sapindus marginatus Willd ?.....

Sapindus sp?....

Ague biachystachys Ciav

Agave moxicana Liam.

Furcerea sp?

Prochnyanthes viridescons WVats...

Michrosechium helleri Cogn.........

Phytolacea icosandra L..............

Hibiscus mutabilis L...
Geraniáceas.

Geraniáceas.

Geraniáceas.

Cácteas

Compuestas.

Compuestas.

Compuestas.

Malváceas..

Malváceas

Leguminosas........

Nictagineas..........

Amarilídeas.

Urticáceas

Compuestas........

Quenopodiáceas ...

MLalváceas............

Bignoniáceas.

Papaveráceas......

Papaveráceas.......

Papaveráceas.......

Papaveráceas.......

Malváceas

Malváceas

Amarantácens

Simarubáceas.......

Leguminosas........

Urticáceas ...........

Urticáceas ...........

Urticáceas ...........

Urticáceas ...........

Urticáceas ............

Urticáceas ...........

Orquídeas............

Malvác eas............

Dipsáceas ............

Umbelíferas.........

Compuestas. ........

Urticáceas ...........

Amarilídeas.........

Malváceas............

Anıarilídeas.........

Amarilídeas.........

Ramneas............

Fitolacáceas .........

Liliáceas............

Rhamneas..........

Amarilídeas........

Sapindáceas.........

Sapindáceas .........

Amarilídeas........

Amarilídeas........

Amarilídeas........

Amarilídeas........

Ciucurbitáceas ......

Fitolacáceas........

Malváceas...........
Morelos....

Valle de México. Farm. Mex.

Valle de Mléxico. Farm. Mex.

San Luis Potosí.. Mat. Méd.

Valle de México.

Tabasco.

Naturalizada .....

Cultivada......... Rose, Farm. M.

Valle de México. Farm. Mex.

Cultivada.........

Hidalgo.......... Colm., Ram.

Valle de México. Colmeiro.

Morelos .......... Colm., P. N. H•

Valle de México.

Valle de México. Ramírez.

Jalisco ............ Oliva.

Sonora........... Rose.

Cultivada......... Farm. Mex.

Cultivada........ V. et Urb.

Son., Ghih., B. G. Farm. Mex.

Oaxaca, B. C..... Farm. Mex.

Cultivada....

Jal. V. de Méx... V. et Urbina.

Cultivada.

Nuevo León...... Ramírez.

Regiones cálidas

Oaxaca.

Tabasco.

Morelos, Gro....

S. L. P., Morelos

Jal., Morelos......

Tabasco ...........

Mich oacán........

Jalisco

Cultivada

Michoacán

Valle de México.

Morelos ...........

Guanajuato ......

Gullivada.........

Sinaloa ........... Rose.

Guanajuato ......

................. P. N. H.

Baja California... Rose.

Jalisco........... Rose.

Jalisco............ Rose.

Jalisco............ Rose.

Lugs. calientes... Farm. Mex.

Jalisco........... V. et Urb.

Jalisco ........... Rose.

Coah., V. México Farm. Mex.

Jalisco........... V. et Urb.

Jalisco............ Rose.

Morelos .......... Urb., Ram.

Valle de México. P. N. H.

Cultivada......... 
Amor de hortelano

Amor seco.

Amor seco.

Anor tras de la puerta.....

Anor al uso

Anacahuite ó Anacahuit1..

Anagálide.

Anagua

Ancoas.

Andani (tarasco).

Andúmuqua (tarasco)...

Andímuqua (tarasco)........

Angólica del país..

Angiolote.

Anisillo.....

Anisilio ó Anisillo cimarrón

Anisillo

Anisillo

Anisillo

Anona.

Anona

Anona.

Anona.

Anona amarilla.

Anona blanca.................

Anona colorada...............

Anona morada.

Anona silvestre.

Anonilla....

Anonilla....

Añil.

Añil.

Añil blanco.

Añil colorado.

Añilillo

Aoatl.

Roatl.

Aoatl.

Aparequa (tarasco).

Apáczequa (tarasco).........

Apancholoa.

Apio

Apitzalpatli

Apompo

Apompo

Apoyamatii.

Apupu (tarasco)

Aqualacti...

Aralia.

Aralia

Arámicua

Araña.

Ara年.

Arbol del algodón.

Arbol de las calabazas.....

Arbol de la cer'a..
Galium mexicanum H. B. K

Gomphrenc dccumbens Jacq.

Gomphrena globosa L..............

B.ynphyllum ealycinum Salisb....

Hibiseus nutabilis L...

Cordir boissievi A. DG....

Anagallis arvensis L....

Eteretic elliptica DC.

Zingiber officinale Roscoe.....

Helicenthus annures L.....

Mentha sp?

Nicotiana rístiea L.

Angelian mexicana Vafke...........

Lennoc cornulea Fourn...............

Elylravia calicosa Bucks....

Schluhria abrotanoides Roth.....

Schliuhria virgata DC..

Tagetes micrantha Cav....

Tagetes pussilla H. B. K.

Anona squamosa I....

Anona glabra L.

Anona reticulala 1

Rollinia mucosa Baill.

Anone muricata L.

Anona squamosa L.

Anona reticulata L....

Anona parpurea DC.....

Anona sp?...

Asimina sp?..

Magnolia grandiftora L....

Indigofera añil L.

Indigofera argentea L

Indignfera tinctorea L....

Indigofera cuñl I,

Indigofera mueronala Spreng.....

Quercus insignis Mart. et Gal.....

Quereusreticulata Humb.et Bonpl.

Quercus strombocarpre Jicbm.....

Urtica urens L....

Trygtes evecta L.....

Cuphea aprenareloa DC:

Apinm graveolens L.

Mature sp?.

Practiva insignis Savign

Pachiva macrocarpa Ch. et Schl..

Cyperns articulatus L.

Scchium edule Siv....

Trianosporma attemuta Hemsl

Arelire humilis Cav.

Arelice pubescens DC.

Xunthosemet robustum Schoot....

Nigella demascena l.s....

Panciatiom illiricum L..

thiorlendron orcidenterle $\mathrm{T} \mathrm{l}$. et......

Crescentic oujcte L

Myrica ralupensis IT. B. K.
Rubiáceas .

Amarantáceas

Amarantáceas.

Crasuláceas....

Malváceas.....

Borragíneas.

Primuláceas.

Borrogíneas.

Zingiberáceas....

Compuestas.

Labiarkas. .

Solanáccas.

Umbelíferas.

Lennoáceas...

Acantáceas.

Ciompuestas.

Compuestas

Compuestas.

Compuestas.

Anonáceas.

Anonáceas.

Anonáceas.

Anonáceas.

Anonáceás.

Arronáceas

Anonáceas

Anonáccas.

Anonáceas

Anonáce:ı

Magnoliáceas

Legumin osas...

Leguminosas.

Leguminosas ...

Leguminosàs

Leguminosas

Cupulíferas...

Cupulíferis.

Cupulíferas.

Uılicáccas

Compruestas.

Litrarieas.

Umbelíferas

Malváceas.

Malváceas.

Malváceas

Ciperáceas.

Ciucurbiláceas .....

Cuculbiláceas.

Araliáceas

Araliácéss

Aroideas.

Ranunculáceas....

Amaralídens.

Malváceas ...

Bignoniáceas........

Miricáceas
Gto., V.Mex., Ver. Farm. Mex.

Mesa Central....

Naturalizada ....

Morelos

Cultivada...

Hgo., N. L., 'Tim. Ramírez.

Naturalizada......

N. L., Chih........

Cultivada....

Michoacán ........

Mirhoacán ........

Michoacán.......

Valle de México.

Mor., S. L. P....

Chihuahua .......

Valle de México.

Valle de México.

Valle de México.

Valle de México.

Iug. calientes...

Jilisco.

México

Veraciuz...

'Tabasco

Cliiapas.

Chiapas.

Jalisco

Jalisco

Cullivada

Valle de México.

Jalisco .

Tabasco

Tabasco

Tábasco....

Veracruz.

Oaxaci, Ver.....

Veracruz.

Michoacán ...

Michorcán...

Veracruz.

Cultivida.

Ver., Mor., Méx..

Tab., Yuc., Ver... G!o., Veracruz...

Michoacán.......

Acapulco

Sonora....

Morelos

Veracruz

Cultivada...

Cullivada.

Morelos..

Varios lugares... Hgo., Oax., Ver..

N. León.

N. León.

N. León.

Ram., Villada.

Ul).

Rose, Ramz.

Farm. Mex.

Cat. Inst. Méri.

Oliva.

Oliva.

Mat. Méd.

$\mathrm{V}$. et Urb.

Rovirosa.

Rovirosa.

Rovirosa.

Farm. Mex.

N. León.

N. León.

N. Lcón.

Mociño.$$
\text { ... }
$$

$$
\ldots
$$

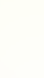

.

Caat. Inst. Méd.

Cat. Inst. Méd. 
Arbol del copal.

Arbol de las cruces.

Arbol del cuerno.

Arbol del cuerno.

Arbol del chicle.

Arbol del diablo.

Arbol del diablo.

Arbol del hule

Arbol del hule.

Arbol de las manitas

Arbol-Miaría

Arbol del muerto

Arbol del pan..

Arbol del Perú.

Arbol santo

Arce....

Archipín goma de...

Arete ó Aretillo.

Aretillo

Aretillo

Aretillo

Aretillo

Aretitos

Arí.

Aristoloquia mata-culebra

Arnica

Arnica del país

Arnica del país.

Armuelle.

Armuelle silvestre

Aro...

Aroma ó Arromo...

Aróraqua (tarasco)

Arrayán.

Arrayán.......

Arrebolera

Arrebolera

Arroz.

Artemisa ó Artemisia........

Asmodeo.......

Astras.

Astronómica

Atanasia amarga.

Atecuirtli (mexicano).......

Ateruchil

Atlacote ó A tlacoti.. ..... .. Atlalzopillin ó Atlatzompillin

Atlancán.

Atlanchán ó Atlanchana...

Atlanchana.

Atlanchane....

Atmosferica

Atoyacócotl

Atuto

Atzahutli ó Atzautli........

Atzapotlquahuitl...
Elaphrium copalliferem DC.

¿ Randia letracantha DG?

Acacia cornigera Willd.

Acacia melanoceras Beurling ?.....

Jat"opha tebulosa Müll. Arg. var..

Hura crepitans $\mathrm{L}$.

ITorisonia americana $\mathrm{L}$

Castilloa cllastica Cerv....

Ficus elastiea Roxb.

Cheirostemon platenoides H. et B..

Eupalorium $s p$ ?

Ipomoca nurucoides Ræm. et Sch.

Sahagunia mexicana Liebm ?......

Schimus molle L....

Guatacum verticale Orl....

Acer mexicanum A. Gray.

Busera gumifera L................

Bessera elegans Schultes............

Fuchsia arboreseens Sims...........

Fuchsia coccinea Ail................

Fuchsia corymbiflora R. \& Pav....

Fuchsia fulgens DC.

Lobelia laxiflora H. B. K............

Mimosa, sp?

Aristoloquia, sp?...................

Tithonia, sp......................

Helenium mexicanum H. B. K......

Heterothece inuloides Cass..........

Atriplex hortensis [................

Chenopodium, sp?..................

Richardia afrieana Kunth........

A cacia farnesiana Willd.

Callleya citrina Lindl.

Myrtus arayan H. B. K

Mryrtus ehrenbergii Berg....

Mirabilis dichotoma $\mathrm{L}$.

Mrirabilis Jalapa L.

Oryza sativa L.

Ambrosia artemisicefolia L

Sedum, sp?...

Crrllistephus chinensis Nees........

Lagerstromia indica L...............

Brickellia cavanillesii A. Grny......

Rhynchosia precatoria DC..........

Fuchsia arborescens Sims..........

Aster, sp??.

Hibiscus pentucarpos $\mathrm{L}$.

Cripher lancolate Ait....

Cuphea lanceolata Ait.

Potentilla candicans Tumb. et B. Lytlun kennerlianum H. B. K.... Largerstremia indica L.............. Spondias purpurea L.

Viter mollis H. B. K.............

Cranichis speciosa Llav. et Lex...

Lncuma mammosa Gærtn.
Burseráceas..........

Rubiáceas

Leguminosas........

Leguminosas........

Euforbiáceas........

Euforbiáceas.......

Caparideas....

Urticáceas

Urticáceas

Esterculiáceas.....

Compuestas.

Convolvuláceas....

Urticácens

Anacardiáceas.....

Zigofileas ....

Aceráceas......

Burseráceas........

Liliáceas.

Onagrarieas.........

Onagrarieas.........

Onagrar ieas.........

Onagrarieas........

Lobcliáceas .........

Leguminosas........

Arisloloquiras ......

Compuestas.

Compuestas.

Compuestas.

Quenopodiáceas...

Quenopodiáceas ...

Aroideas............

Leguminosas ........

Orquídeas.

Mirtáceas....

Mirtáceas.

Nictagineas.

Nictagineas.

Gramíneas....

Compuestas.

Cirasuláceas

Compuestas

Litrarieas....

Compuestas .................

Compinestas ....................

Onagrarieas.......

Compuestas .........

Malváceas.

Litrarieas. ...

Litrarieas....

Rosáceas

Lilrarieas....

Litrarieas

Anacardiáceas.....

Verbenáceas.........

Orquídeas............

Sapotáceas.
Morelos .

Gro., Colima......

Veracruz...

Mor., Guerrero...

Michoacán

Morelos

Colima.

Veracruz

Cultivada

Méx., Morelos...

Jalisco ............ V. et Urb.

Mor., T. de Méx.

Veracruz..........

V. de Méx., etc...

Puebla ............

Nuevo León.....

Morelos

Colim., Jalisco...

Michoacán........

Cullivada.........

Cultivada.........

Michoacán ........

Valle de México.

Jalisco ........... Oliva.

FI. MI.

Tabasco

Valle de México.

Varios Estados... Mat. Méd.

Cultivada........ Cerv., M. M.

Cerv., M. MI.

Naturalizada.... Farm. Mex.

Yucatán........... Farm. Mex.

Michoacán........ N. León.

Guerrero.........

Varios Estados...

Reg. tem. y frías

Ver., V. de Méx..

Cultivada.

Varios Estados... Farm. Mex.

Michoacán........ M. Tena.

Ciultivada.........

Cultivada.........

Jal., V. de Méx... Mat. Méd.

Gro., V. de Méx.

E. de México.... Urbina.

Valle de México.

Morelos

Hidalgo.

Jalisco..

E. de MTéxico....

S. L. P., V. Méx

Cultivada.

Norelos ...........

Michoacán....... Ramírez.

Mlichoacán....... Colmeiro.

Colm., V. et Urb.

Urb., Ramírez.

Mor., Veracruz.. 
Atzcalsóchitl.

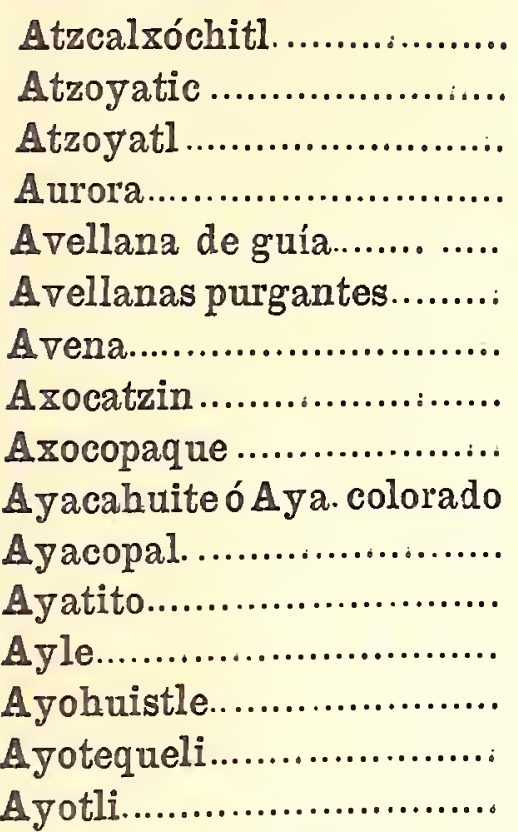

Azafrán ó Azafrancillo......

Azafrán amarillo.

Azafián de bolita.

Azafrán del campo.

Azafrancillo.

Azafrancillo de México .....

Azalea ó Azalea de arbusto

Azalea de guía

Azcalxóchitl.

Azota caballos.

Azucena amarilla.

Azucena am. ó azafranada.

Azucena blanca.

Azucena encarnada ó roja..

Azucena matizada.

Azucena del monte.

Azufaifo.
Zephyrantes carinala Herb.

Spreleelia formosissima Herb.

Ipomrea, sp?

Mirabilis longiflora L

Ipomoca purpurea Lam.

Momordica balsamina L............

Jatropha cureas L.

Avena sativa L

Sida rhombifolic L.

Gaultheria, sp?

Pinus ayacaluaite Ehrenb.

Arbutus, sp?.

Calochortus flavus Schult. f..

Alnus acuminata H. B. K.

Solanum cornutum Lam.

Manihot foeticla Pohl.

Cucurbita maxima Duch..

Carthamus tinctorius $\mathrm{L}$

Crocus sativus L.

Argithamnia heterantha Müll.....

Buddleia marrubiifolia Benlh......

Escobedia linearis Schl.....

Escobedia scabrifolia R. el Pav...

Rhododendron indicum Sweet.....

Bougaincillea spectabilis Willd...

Spreletia formosissina Herb.

Cordla laxiftora H. B K

Govenia superba LindI

Hemerocallis flava $\mathrm{L}$

Lilium candidum $\mathrm{L}$

Hippeastrum equestre Herb

Hippeastrum, sp?.

Govenia liliacea LindI.

Rhamnus, spp?
Amarilídeas.

Amarilídeas....

Convolvuláceas....

Nictagineas.

Cor vol vuláceas....

Cucurbitáceas ...

Euforbiáceas

Gramíneas .

Malváceas

Ericáceas.

Coníferas

Ericáceas

Liliáceas....

Cupulíferas....

Solanáceas .....

Euforbiáceas ........

Cucurbitáceas ......

Compuestas.

Irideas

Euforbiáceas

Loganiáceas

Escrofularíneas

Escrofulal'íneas...

Ericáceas .

Nictagineas.

Amarilídeas

Borragíneas

Orquídeas

Liliáceas

Liliáceas

Amarilídeas....

Amarilídeas.

Orquídeas

Ramneas....
Hidalgo, Gto......

México, Gto......

Hidalgo, Oaxaca.

V. de Méx., Oax.

Naturalizada ....

Cultivada.........

Valle de Míxico.

Hgo., Puebla.....

Hgo., México.....

Chih., E. Méx....

Morelos

Pueb., S. L. P...

Veracruz.

Naturalizada.....

Gultivado

Cultivado.........

Guanajuato.......

Chihualıua........

Guanajuato.......

Varios Estados...

Cultivado.

Naturalizada .....

Méx., Gto. ,etc...

Tabasco...........

Oaxaca, Mich.....

Cultivada.........

Cultivada.........

Jalisco, Mich......

Michoacán........

Mich., Oax., Ver.

Fl. M., P. N. H.

Colmeiro.

Farm. Mex.

P. N. H.

Cat. Inst. Med.

Colm., Ramz.

Urb., Ram\%.

Colm., Kunth.

Ramírez.

Cat. Inst. Med.

F. M.

Rovirosa.
Chiapas .......... Ramírez.

Oaxaca........... Cat. Inst. Med.

Morelos .......... Cat. Inst. Med.

Veracruz.......... Ramírez.

Michoacán, Ver.. Cat. Inst. Med.

Oixaca........... Barroso.

Tabasco.......... Cat. Inst. Méd.

Orizaba........... González.

V. de Méx., Hgo.

Lug. elevados....

Jalisco.............

Valle de México.

Tabasco....

Tabasco.....
Farm. Mex.

Rovirosa. Rovirosa. 
Barbas de chivo.

Barbas de gato.

Barbas de gato.

Barbas de Juan de Dios....

Barbas de viejo

Barbas de viejo

Barbasco

Barbasco

Barbasquillo..

Barbona rosada.

Barbudilla.

Barbudilla.....................

Bardana del país

Barí....

Barrera.

\section{Barreta.}

\section{Batatas.}

Beeb.

Begonia blanca

Begonia imperial.

Bejuco de ajo....

Bejuco de amole

Bejuco de canoa....

Bejuco cola de iguana.......

Bejuco colorado...

Bejnco de chiquihuite.......

Bejuco de huico

Bejuco loco

Bejuco de mondongo.........

Bejnco de tachicón

Bejuco de tachicón

Bejuco tres lomos

Bejuco vaquero.

Bejuquillo.

Belén ó Belenes

Beleño negro..

Bella Inés.

Belladona del país

Belladona del país

Bellota

Bellota.........................

Bembéricua (tarasco).........

Bereugena......................

Berenjenita peludita.........

Berro .............................

Berros

Berros

Berza.............................

Berza colinabo ó de Siam..

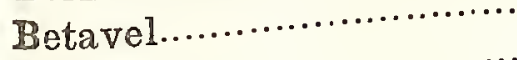

Betónica

Bicho.

Bicho de arbusto

Bignonia......

Bignonia
C'ematis sericea H. B. K.

Clematis dioica $\mathrm{L}$

Irymenocallis rotata Herb.....

Senecio barba-johanis DG.....

Clematis sericea H. B. K.

Usnea barbata L....

I'aullinia pinnata L..

Tephrosia toxicaria Pers.

Parllinia pteropoda DG.

Cleome lieptaphinglla L.

Dorstenia contrayerba L

Dorstenia drakena L.

Camna patens Ait.

Cordia gerascanthoides H. B. K...

Sinm angustifolium L

Helietta parrifolia Benth.

Ipomoca batatus Lam

I'isnnia aculcate $\mathrm{L}$

Begonia incana Lindl.

Begonia imperialis Lem.

Bignonia alincia Lam.

Entada polystachya DC.

Bignonia laurifolia Valıl.

Bignonia, sp?.

Hippocratea, sp?.

Smilax mexicana Griseb...

Pithecoctenium, sp?.

Tritis sicyoidles Baker.

Entarla polystachya DC.

Tetracera ovalifolia DC.

Tetracera volnbilis $\mathrm{L}$.

Bignonia linelleyi DC.

Malpighia, sp?.

Cephalis tomentose WVillı?

Impatiens balsamina L.

Iyoscyromus niger $\mathrm{L}$

Castilleju arvensis Cham. et Schl..

Ccstrum roseum H. B. K

Nicandra physaloides Gacrtn.....

Quercus castanca Nees.

Sterculia carthagenensis (.av........

Rhus toxicodendion L.

Solcumm esculentum Dun.

Solanum mamnosum L.

Sium angustifolinem L.

Naslutium officinale $\mathrm{Br}$.

Sium angustifolium L.

Sisymbrium nasturtinm L.

Brassica oleracea L.

Brassica oleracea $\mathrm{L}$.

Beta vulgaris Moq..

Betonica alopecmms L...

Cassia bicapsularis L.

Crssisia, spo?..

Bignonia eapensis Th.

Tecoma stans Jiss.
Ranunculáceas.....

Ranunculáceas....

Amarilídeas.........

Compuestas.........

Ranunculáceas.....

Líquenes . . .

Sapindáceas .........

Leguminosas........

Sapindáceas.

Capauídeas

Urticáceas

Urticáceas .

Zingiberáceas

Borragíneas.

Umbelíferas.

Rutáceas ...........

Convolvuláceas....

Nictagineas..

Begroniáceas.

Begoniáceas.

Bignoniáceas

Leguminosas.

Bignoniáceas....

Bignoniáceas...

Hipocrateáceas....

Liliáceas.

Bignoniáceas ........

Ampclídeas ........

Leguminosas.......

Dileniáceas..........

Dileniáceas.........

Bignoniáceas.......

Malpigiáceas.........

Rubiáceas ...........

Geraniáceas.........

Solanáceas ..........

Escrofularíncas.....

Solanáceas.

Solanáceas

Cupulíferas

Esterculiniceas....

Anacardiáceas.....

Solináceas ....

Solanáceas

Umbeliferas...

Crucíferas.

Umbelíferas.

Crucíferas.

Crucíferas.

Crucíferas

Quenopodiáceas ...

Labiadis....

Leguminosas

Leguminosas.......

Bignoniáceas ........

Bignoniáceas........
Virios lugares...

E. de México.....

Valle de Mléxico.

Valle de México.

Jalisco.

Jalisco............

Tabasco...........

Jalisco, Oasaci..

Jalisco.

Chiapas...........

Michoacán........

Jalisco.....

Jalapa .

Miclioacán, Tab..

Oaxica, Sonora.

Chilhuahua .......

Regs. calientes...

Yucatán

Veracruz.

Michoacán.

Tabasco...........

Gro., Tabasco...

Tabasco..........

Tabasco..........

S. Madre de Tep.

Tabasco...

Tepic .. ...........

Tabasco...........

Tabasco...........

Tabasco..........

Tabasco..........

Tabasco...........

Michoacán ........

Oaxaca, Tabasco

Jalisco............

Cullivada.........

Valle de México.

Hgo., Oaxaca.....

E. y V. de Méx... Farm. Mex.

lieg.tem.denéx. Farm. Mex.

Tabasco.......... Rovirosa.

Michoacán....... N. León.

Cultivada........

Tabasco...........

Jilisco..............

Cultivada........

Oaxaca, Sonora.

Aclimatada.......

Cultivada........

Cultivada.........

Cullivada.........

Cultivada.........

Jalisco............

Jillisco...

Cultivada.....

Varios lugares...
Farm.Mex.Urb.

Urbina.

. et Urbina.

Cat. Inst. Med.

Rovirosa.

Cat. Inst. Med.

Colmeiro.

Colm., V. et Urb.

Ramírez.

Cat. Inst. Med.

Cat. Inst. Méd.

Rose, Rovirosa.

Rovirosa.

Cat. Inst. Med.

Rose.

Povirosa.

Rose.

Rovirosa.

Rovirosa.

Cat. Inst. Med.

Rovirosa.

Rovirosa.

Cat. Inst. Méd.

V. et Urbina.

Rovirosa.

$\mathrm{V}$. et Urbina.

Farm. Mex.

Farm. Mex.

V. et Urbina.

$\mathrm{V}$. et Urbina 


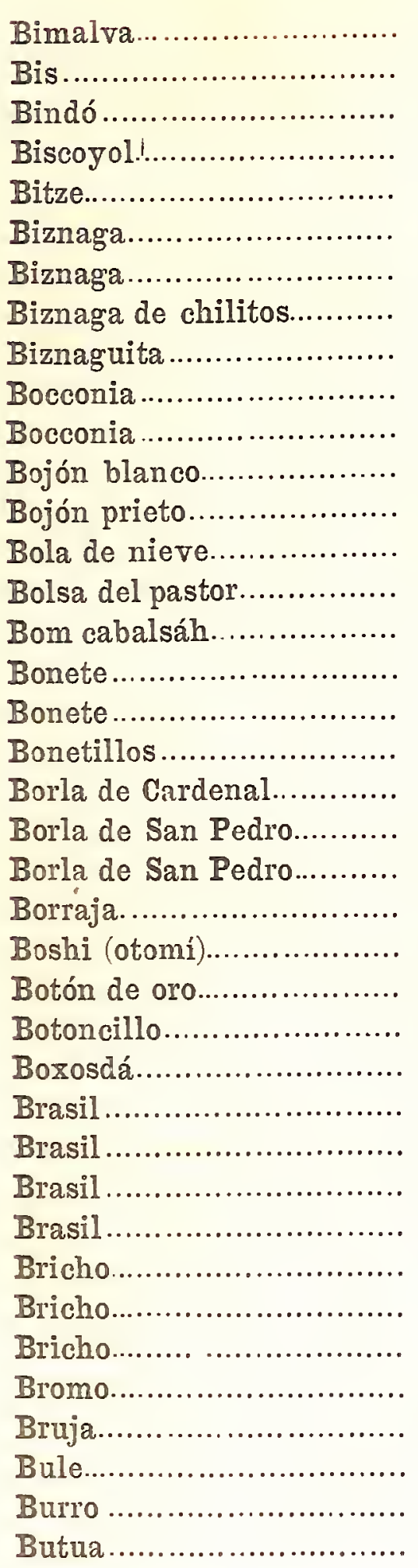

Althoca officinalis L.

Hibiscus rosa-sinensis $\mathrm{L}$.

Condalia mexicana Schl.

Cocos, sp?.

Inga spuria Humb. et Bompl......

Mamillaria pusilla DC.

Namillaria spharica Dietrick.....

Eclinocactus cornigerus DC.........

Mamillaria, sp?.

Bocconia arborea Wats.

Bocconia fintescens I

Cordia gerascantlius Jacq.

Cordia gerascanthus Jacq.

Arabis verna L.

Capsella bursapastoris L............

Chamodorea loumilis Wats.

Carica hieptaphylla Moc. et Sessé.

Carica nana L.

Cucurbita melopepo L.

Cleome cardinalis DC.

Bignonia, sp?.

Tecoma stans Juss.

Borago officinalis $\mathrm{L}$

Baccluris pterionioides DG.........

Spilantlies becabunga DC.

Centaurea cyanus Lindl.

Bigelovia veneta A. Gr.

Cosalpinia, sp?..

Condalia oborata R. et Pav

Hoematoxylon boreale Wats...

Homatoxylon campechianum L...

Cassia bicropsularis I

Cassic floribunda Cav...

Cassia occidentalis L.

Bromus sterilis L.

Bryophyllum crlycinum Salisb.....

Lagenaria vulgaris Ser.

Senecio roldana DG.

Cissampelos pareira Lamark......
Malváceas

Malváceas

Rhamneas

Palmeras....

Leguminosas.......

Cácteas

Cácteas

Cácteas

Cácteas

Papaveráceas......

Papaveráceas.......

Borrogíneas........

Borragíneas........

Crucíferas...........

Crucíferas.

Palmeras...

Pasifloráceas.......

Pasifloráceas.......

Cucurbitáceas ......

Caparídeas

Bignoniáceas.

Bignoniáceas.

Borragíneas

Compuestas.

Compuestas.

Compuestas.

Compuestas

Leguminosas...

Rhamneas...

Leguminosas.

Leguminosas.

Leguminosas.

Leguminosas.

Leguminosas.

Gramíneas

Crasulácens

Cucurbiláceas

Compuestas.

Menispermáceas ..
Cultivada

Cultivada

San Luis Potosí.

Onxaca

Tabasco

Oax., V. de Mex.

Jalisco, Onxaca.

Oaxaca.

Guanajuato .....

Michoacán, Ver.

Michoacán, Ver.

Tabasco...

Tabasco

Jalisco

Naturalizada ....

Onx., Veracruz...

Morelos

Jalisco.

Cultivada...

Jalisco

Jalisco.

Chiapas, S. L. P.

Cullivada...

E. de Méx., Jal...

V. de Méx., Jal...

Cultivada........

S. L. P., V. Méx.

Lug. calientes...

Chihuahua ......

Sinaloa.

Jalisco.

Jalisco, S. L, P.

Guannjuato ......

Jalisco, S. L. P..

Jalisco

Jalisco.

Cullivada

Jalisco.

Col., Gro., Ver..
Farm. Mex.

Uibina.

Ramírez.

Rovirosa.

Barroso.

Bitroso.

Barroso.

Ramírez.

kamírez.

Cat. Inst. Méd.

Rovirosa.

V. et Urbina.

Ramírez.

V. et Urbina.

Oliva.

V. et Uıbina.

Urbina.

Urbina.

V. et Urbina.

V. et Urbina.

Rose.

V. et Urbina.

Urbina.

Urbina.

V. et Urbina.

V. et Urbina.

Urbina.

Farm. Mex.

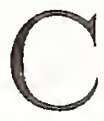

Cabalonga.....

Cabalonga de Tabasco......

Cahellera

Cabellera

Cabellitos de ángel.

Cabellitos de una vara.....

Cabellos de ángel.

Cabellos de Venus.

Cabeza de chamal.
Strychnos triplinerviu Mart.?

Strychnos triplinervia Mart. ?.......

Iopronthus venetus H. B. K

Phoralendron rubrum Griseb.

Calliandra grameliftora Benth...

Cullimandra grandiftora Benth...

Puclira insignis Savign.

Nigella damascena L

Dioon edule Lindl.
Loganiáceas.

Loganiáceas. .

Lorantáceas ......

Lopintáceas.

Legumino $=$ as......

I.eguminosas.......

Malváceas....

Ranunculáceas......

Cicadáceas
Tabasco....

Tabasco

Tabasco

Tabasco

Morelos

Morelos

E. de México...

Cullivada.

N. L., Cost. del G.
Rovirosa.

Farm. Mex.

Rovirosa.

Rovirosa.

Mat. Méd.

Farm. Mex.

Ramírez. 
Cabeza de negro.

Cabeza de negro

Cabeza de negro

Cabeza de negro.

Cabeza de negro

Cabeza de negro

Cabeza de turco.

Cabeza de viejo

Cabezona

Cabezona

Cabezuela

Cabo de hacha.

Cabrito

Cacahoanantzin

Cacahuananche...

Cacahuananchi ?..

Cacahuate.

Cacahuaxóchitl

Gacalacacaxtli.

Cacalaco.

Cacaloxóchitl

Cacaloxóchitl

Cacaloxúchil

Cacao ó Cacaoquáhuitl......

Cacao .

Cacao

Cacaoxóchitl

Cacomite

Cachalohuai.

Cachimbo

Cachisdá

Cachisdá?.

Cachisdá?

Cadillo...

Cadillo....

Cadillo de bolsa.

Café ó Cafeto.

Café del país....

Cafetillo.

Cahúas (tarasco)

Cahuichi...

Cahuinga.

Cahuitzi...

Caimito

Caimito verde.

Cajete

Calabacilla amarga.

Calabaza

Calabaza.

Calabaza india

Calabaza turbante

Calabazate

Calaguala.

Calaguala...

Calaguala

Calaguala.
Anona muricata L.

Evitrichium, sp?...

Kr.ymitakia floribunda A. Gr. ?.....

Nymphcea elegans Hook

Nymphera gracilis Zucc.............

Nymphaa moxicana Zucc.

Cucurbita melopepo L.

Cereus senilis Salm. Dyck.

Gomphrena decumbens Hort........

Helcninm mexicanm H. B. K......

Contaurea cyanus Lindl.

Trichilia spondioides Swartz.......

Theretia iccolli A. DC.

Licania arborea Seem.

Licania arborea Seem...............

Gliricida lambii MI. L. Fernald....

Arachis hipogaca L.

Iyrodia funebris Bent.

Xanthosoma robustum Schott.....

Cesalpinia cacrlaco H. B. K......

Macrosiphonia brachysiphon A. Gr.

Plumeria mbra L.

Plumeria rubra L...................

Theobroma angustifolia DC...

Theobroma cacao L.

Theobroma ovalifolia $\mathrm{DC}$

DIyrodia funebris Benth...

Tigridia paronia Ker.

Erythrea stricta Schiede

Cassia bicapsularis L...

Berberis pinnata Lag.

Bocconic arborea Wals.

Bocconia frutescens $\mathrm{L}$.

Cenchrus echinatus L...

Trizmfetta scmitriloba $\mathrm{L}$

Priva echinata Juss.

Coffeca arabica $\mathrm{L}$

Cassia lavigata Willd

Casereria nitida Jacq.

Capsicum annunum $\mathrm{L}$

Vaciuium micronthum Dunat.

Cascalpinia, sp?.

Vaccinium micranthum Dunal.....

Chrysophyllum ccimito L............

Chrysophyllum caimito $\mathrm{L}$

Tiriumfetta oxyphylla DC.

Cucurbita foctidissima Kunth......

Cucurbita maximn. Duch...

Cucurbita pepo L.

Cueurbita, sp?

Cucurbita melopepo $\mathrm{L}$

Lagenaria vulyaris Ser

Acrostichum cenoptcris Kunze....

Acrostichum sorbifolizm L.........

Polyportizm angustum Mett........

Polypodium aureum L...
Anonáceas

Borragíneas

Borragíneas.

Ninfeáceas...........

Ninfeáceas

Ninfeaceas.

Cucurbitáceas ......

Cácteas

Amarantáceas ......

Compuestas .........

Compuestas .........

Meliáceas.

Apocináceas........

Rosáceas...

Rosáceas ............

Leguminosas........

Leguminosas...

Esterculiáceas......

Aroideas

Leguminosas

Apocináceas.

Apocináceas.........

Apocináceas.........

Esterculiáceas ......

Esterculiáceas ......

Esterculiácens ......

Esterculiáceas ......

Irideas

Gencianeas..........

Leguminosas........

Berberídeas.........

Papaveráccas.......

Papaveráceas.......

Gramíneas...........

Tiliáceas. ...........

Verbenáceas.........

Rubiáceas ...........

Leguminosas........

Samidáceas.........

Solanáceas ..........

Vacciniáceas.........

Leguminosas........

Vacciniáceas........

Sapotáceas ..........

Sapotáceas .........

Tiliáceas............

Gucurbitáceas ......

Cucurbitáceas ......

Cucurbitáceas ......

Gucurbitáceas .....

Cucurbitáceas .....

Cucurbitáceas.....

Helechos ............

Helechos ............

Helechos

Helechos
Jalisco..

E. de México....

Valle de México.

Lagos del V. Méx.

Lagos del V. Méx.

Lagos del V. Méx.

Cultivada...

Sonora., Var. lug.

Lago de Chalco..

Gto., México......

Cultivada.........

Oaxaca.

Jalisco.

Gro., Mor., Mich.

Gro., Mor., Mich.

Sinaloa, Tepic...

Jalisco, Gto.......

Oaxaca.

Veracruz

Colima

San Luis Potosí.

Lug. temp. y cal.

Lug. temp. y cal.

Tabasco...........

Tab., Jal., Oax...

Tabasco.

Oaxaca, Mich.....

Mesa Central,Jal.

México, Morelos.

Tabasco

Hgo., Méx., Gto..

Lug. calientes...

Jug. calientes...

Tabasco..........

Tabasco...........

Tabasco

Cultivada.........

Veracruz..........

Tabasco...........

Michoacán........

Hidalgo, Ver.....

Michoacán........

Hidalgo, Ver....

Mor., Veracruz...

Tabasco

Chiapas............

Chiap., Gto., Tep.

Cullivada.........

Cultivada........

Guanajuato ......

Gultivada.

Gultivada.

V. M., S. L. P., \& F. M

V. M., Ver., Oax, F. M.

V.M., S. L. P., \& F. M.

V. M., S. L. P., \& F. M.
V. et U., Ramz.

Urbina.

Ramírez.

Cat. Inst. Méd.

Mat. Méd., Oli.

Ramírez.

V. et Urbina.

Alcocer.

Alcocer.

La Llave.

Villada.

Colm., Kunth.

Urbina.

F. M.

La Llave.

F. M., Urbina.

G. I. M., Rovi,

F. M.

Pringle.

Rovirosa.

Rovirosa.

Rovirosa.

Rovirosa.

N. León.

Cat. Inst. Med.

Cat. Inst. Méd.

Cat. Inst. Med.

Cat. Inst. Med.

Rose. 
Calaguala

Calaguala...

Calaguala...

Calaguala.

Calaguala

Calaguala ....

Calahuala...

Calancapatle..

Calancapatle.

Calancapatle

Calancapatli

Calicedra

Calminga

Calomopoti...

Calzón de indio

Calzoncillo..

Camalote

Camburí.

Camelia.

Camelina.

Camelote

Camemba (tarasco)

Camichín.

Camisdá.

Camochayote.

\section{Camote ó Camotic.}

Camote de cerro.

Camote del monte.

Camote del ratón.

Campanilla

Campanilla

Campanilla.

Campanilla blanca...........

Campanilla silvestre........

Campanilla silvestre........

Campechana ó Címpoloneril

Campeche.....................

Canagria ó Canaigre........

Canaria

Canatilla

Cancerillo.

Canchalagua

Canchín...

Candelero

Candelero

Candelilio ó Caneliillo.......

Candelon.

Canelilla..

Canelillo

Canelilio

Canica ó Caninana...........

Canilla de mulito..

Canónigos.

Cantemó.

Cantueso.

Caña de azúcaró Caña creolla
Polypodium calaguala R. P.

Polypodium crassifolium L.

Polypodium decumanum Willd..... Polypodium lycopodioides L......... Polypodium neriifolium Schkuhr.. Polypodium plebeium Ch. et Sch].. Polypodium vulgare L.

Baileya mulliradiata Harv, \& Gray. Soliclago mexicana Linn.....

Solidrgo velutina DC....

Solidago mexicana L

Cedrela mexicana Rom.

Acacia, sp?.

Senecio vulneraria DC.

Crestilleja tenuifolic Mart. et Gal...

Bantivia porrecta Sw.

Eichornice azurea Kunth.

Ifusa sapiontum L..

Camellia japonica L

Heliconia bihai L

Oplismenus holciformis H. B. K...

Ambrosia artemisiofolia L...

Ficus padifolia H. B. K.

Berberis pinnata Lag..

Seclium edule Siv....

Ipomoea balatas Lam.

Oncus esculcntus Lour.

Peteria scoparia A. Gr

Hoffinanseggia stricta Benth......

Campanula medium L.

Ipomsea purpurea Lam.

Thevetia neriifolia Juss.

Datura suaveolens Humb.el Bonpl.

Ipomoea capillacea Cav. Don.......

I pomoea, sp?

Phumerice rubia L

Hamatoxylon campeclionum L....

Rumex lyymonosepalus Torrey.....

Irosa sulplurea Ait.

Ephedra antisyplititica Berland...

Asclepias curressavica L....

Erythrea stricla Schiede.

Cassia spectabilis DC.

Cordia collococca L

Solanum asperum Vahl.

Pedilanthus pavouis Boiss...

Rlizophoia mangle L...

Lippra oaxacana Rob. \& Greenm.

Croton ciliato-glandulosus Ort.....

Gurpliatiun sphacelıtum H. B. K.

Chiococea recemosa Jacq. .

Mtelenthera deltoidea Rich...

Taleriena tolucanat DC.

Acaciu filicina IVilld.

Saliza longispieata Mart. et Gal...

Saccharum officinasum L.
Helechos...

Helechos

Heleclios

Helechos

Helechos

Helechos

Helechos

Compuestas

Compuestas.

Compuestas

Compuestas.

Mcliáceas.

Leguminosas........

Cömpuestas........

Escrofularíneas.....

Leguminosas........

Pontederiáceas .....

Musáceas...

Terustrœmiáceas..

Musáceas.

Gramíneas.... ......

Compuestas........

Urticáceas .

Berberídeas........

Cucurbitáceas ......

Convolvuláceas.....

Dioscorcáceas ......

Leguminosas........

Leguminosas........

Campanuláceas.....

Convolvuláceas.....

Apocináceas.

Solináceas

Cinvolvuláceas.

Convolvuláceas....

Apocináceas.

Leguminosas.

Poligonáceas

Pusáceas

Gnetáceas

Asclepiadeas

Gencianeas.

Leguminosas

Borragíneas...

Solanáceas

Euforbiáceas

Rizoforácens.

Verbenáceas

Euforbiáceas...

Compueslas.

Rubiáceas...

Compueslas.

Valerianeas

Leguninosas....

Laljiadas.

Gramíneas.
Veracruzi.........

Veracruz..........

Veracruz........ F. M.

Veracruz......... F. MI.

V. M., S. L. P., \& F. M.

Veracruz......... F. M.

Jalisco, Oaxaca.. V. el Urbina.

Chibuahua...... Cat. Inst. Méd.

V.Méx., Chiapas. C.I.M., F.M., V.

V.Méx., Chiapas. C.I.M., F.M., V.

Chiapas .......... Cint. Inst. Med.

Sur de México...

Michoacán....... Cat. Inst. Med.

V. y E. de Méx.. Uıbina.

Colimi, Mich.... U.bina.

Tabasco.

Rovirosa.

Aclimatada......

Cultivada...

Cultivada....

Sur de México...

Jalisco.

Michoacán

Jalisco. .

Hidalgo, Ver....

Varios lugares...

Lug. calienles...

Cultivada.........

Chih., S. L. P...

Chilhualıua .......

Cultivada..

V. de Méx., etc...

Morelos, Gro....

Colima...

Sonora, S. L. P..

Morelos, Puebla.

Jal., Yuc., 'Tab...

Coalıuila, Chih...

Ciultivada.

Cilihuahua

Lung. templarlos.

México, Morelos.

Tabasco, Ver....

Tilbasco...

Tilbasco....

Jalisco.

Ver., Col., Sina..

Morelos, Oaxaca.

Jalisco..

Varios Jugares...

Tabasco.

E. de México...

Tabasco..

E. y V. de Méx.

Cultivada.
Urbina.

Mat. Méd.

F. M., Urjuina.

Rovirosa.

Alt. el Ramz.

Rovirosa.

Ramírez.

Pringle, Ram.

Kunlh.

Ubbina.

Urbina.

Urbina.
Cart. Inst. Méd. 
Caña brava.

Caña coro ó Caña de cuentas Caña dulce...

Caña de javalí.

Cañafistula

Cañafistula chica.

Cañafistula grande.

Cañagre, Cañagria ó Cañaigre

Cáñamo

Cañamo del Canadá...........

Cañaveral

Cañita agria.....................

Cañuela

Cañuela

Cañuela

Caoba ó Caobo.

Caobilla.

Caoutchouc.

Capa de San José

Capire.

Capiri .

Capitana

Capitaneja.

Capitaneja.

Capitaneja.

Capitaneja.

Capitaneja.

Capitaneja de Tonila........

Capolín ó Capulín.............

Capomo.....

Capuatl-cacao

Capuchina.

Capul ....

Capulín

Capulín manso.

Capulin silvestre

Capulincillo

Capulincillo

Capulincillo

Capulincillo

Capulincillo

Capulincillo.

Capulincillo....

Capulincillo.....

Capulincillo....

Capulincillo

Capulincillo cimarrón........

Caracol ó Caracolillo........

Caracolillo.

Carámiqua (tarasco)...........

Cardenal

Cardenal de la laguna......

Cardenal de maceta..........

Cardencha.

Cardo

Cardo
Gynerizm saccharoides H. et B....

Canna indica $\mathrm{L}$

Licania arborea Seem.

Costus spicatus Swartz.

Cassia fistula $\mathrm{L}$

Cassia moschata H. B. K...........

Cassia grandis L. fil.....

Rumex hymenosapalus 'Torr ........

Cannabis sativa L.

Apocynum cannabinum L..........

Arundo donax L.

Costus spicatus Swartz..............

Equisetum mexicanum Milde.......

Equisetum ramosissimum Def......

Equisetum robustum A. Brown....

Swietenia mahogani L.............

Croton glabellus L.

Castilloa elastica Cerv.

Enothera grandiflora Ait

Ficus, sp?..

Lucuma capiri A. DC.

Verbesina pinnatifida (av...........

Bidens heterophylla Ort.............

Verbesina alata L.

Verbesina capitaneja Nees..........

Verbesina crocata Less..............

Verbesina spharocephala A. Gr....

Terbesina capitaneja Nees..........

Prunus capuli Cav...

Brosimum alicastrum Sw...........

Spondias purpurea L.

Tropceolum majus L.

Condalia obovata Hook.

IIuntingia calabura L.

Ardisia revoluta H. B.K.

Ardisia capollina A. DC.

Belotia grevicefolia A. Rich

Delotia grevicefia A. Rich.......

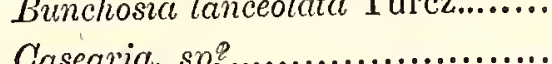

Conostegia xalapensis Don..........

Karswinskia humboldtiana Zucc..

Pernettya ciliaris Don.

Rhamnus humboldtianus Røm....

Rhamnus serratus Willd.

Ribes affine H. B. K...............

Ribes mulliflorum H. B. K........

Rhamnus humboldlianus Rœm....

Phaseolus caracalla L.............

Inga, sp? .........................

Xanthosma robustum Scholt.....

Ieterotoma lobelioides Zucc........

Lobelia fulgens Willd................

Lobelia cardinalis L.................

Dipsacus fullonum L...............

Karatas plumieri E. Morr...........

Bromelia pingrin L................
Gramíneas.

Zingiberáceas.......

Rosáceas

Escitamíneas........

Leguminosas........

Leguminosas........

Leguminosas........

Poligonáceas .........

Urticáceas ...........

Apocináceas.........

Gramíneas...........

Zingiberáceas.......

Equisetáceas ........

Equisetáceas ........

Equisetáceas........

Meliáceas...........

Euforbiáceas ........

Urticáceas ..........

Onagrarieas.........

Urticáceas

Sapotáceas.

Compuestas. ........

Compuestas ........

Compuestas.........

Compuestas .........

Compuestas.........

Compuestas........

Compuestas.........

Rosáceas...........

Urticáceas

Anacardiáceas......

Geraniáccas........

Ramneas ............

Tiliáceas............

Mirsineas...........

Mirsineas.

Tiliáceas.

Malpigiáceas

Samidáceas.........

Melastomáceas ....

Ramneas

Ericáceas..

Ramneas

Ramneas

Saxifragáceas .......

Saxifragáceas .......

Ramneas...........

Leguminosas........

Leguminosas.......

Aroideas............

Lobcliáceas .........

Lobeliáceas .........

Lobeliáceas .........

Dipsáceas............

Bromeliáceas.......

Bromeliáceas......
Tabasco.

Naturalizada....

Guerrero

Veracruz..........

Varios lugares...

Tabasco.

Tabasco...........

Chihuahua .......

México........... F. M.

Sonora.......... F. M.

Varios lugares...

Tabasco.......... Cat. Inst. Med.

Orizaba.

Valle de México. Ramírez.

Valle de México. Ramírez.

Yucatán, Gro....

Veracruz....

Veracruz, Tam...

Valle de México.

Michoacán...

Michoacán, Mor.

Jalisco............

Distrito Federal.

Jalisco.

Varios lugares...

Varios lugares...

Jalisco.............

Jalisco............

Varios lugares...

Jal., Ver., Yuc...

México, Morelos.

Cultivada.

Ghihuahua........

Tabasco..........

E. de Méx., Ver..

Veracruz.........

Chiapas.

Veracruz.........

Motzorongo ......

Puebla, S. L. P.

Querétaro.........

Valle de México.

Varios lugares...

Varios lugares...

Valle de México.

Valle de México.

Valle de México.

Cultivada.........

Tabasco

Michoacán.......

E. de México....

Jalisco.............

Cullivada.........

Cultivada.........

Veracruz..........

Veracruz.........
Rovirosa.

Rose.

Bar., B.,V.et. U.

Rovirosa.

Cat. Inst. Med.

at. Méd.

M.

at. Inst. Med.

Cat. Inst. Med.

Colm., Urbina.

F. M.

F. M.

Herb., F. II.

B. V. et Urbina.

Rovirosa.

Urbina.

Ramírez.

Cat. Inst. Méd.

Ramírez.

Alt. et Ramz.

Urbina.

Ramírez.

Ramirez.

F. M.

F. M.

Cat. Inst. Méd.

B. V. et Urbina. 


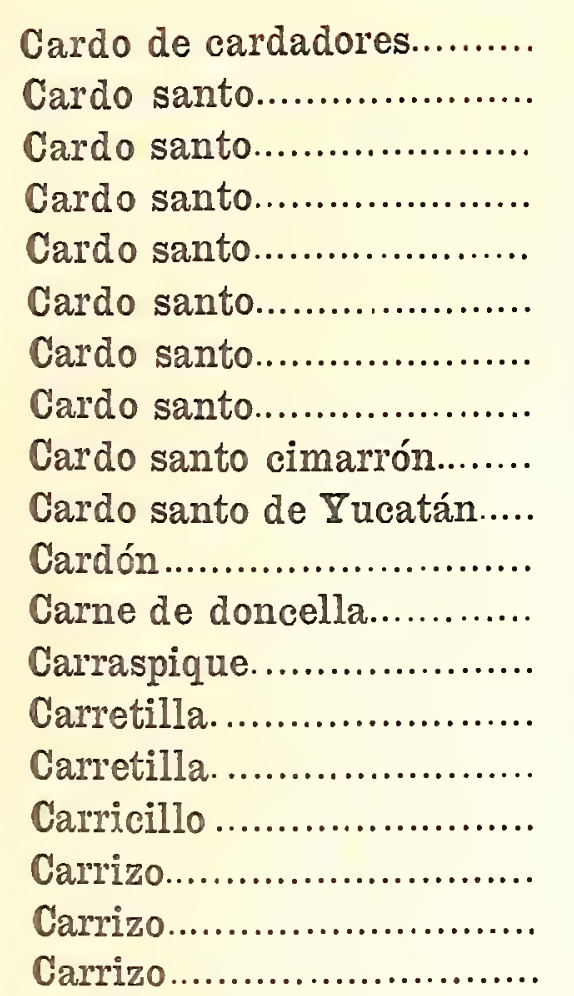

Carrizo verde...................

Casahuate blanco..............

Casahuate prieto...

Casalina....

Casanaca ó zazanaca..........

Cascalote.

Cascalote.

Cashúrequa (tarasco).... ..

Castaño...........................

Castaño de Indias............

Castarrica .......................

Castilleja........................

Castillitos.

Catalina.

Catalina.......

Catarrillo.

Catíriqua (tarasco)............

Catuche ó Catucho............

Cayolizán, layolozán ó tayoluaín.....

Cazahuate blanco..............

Cazahuate prieto ..............

Cazotl............................

Cebada.

Cebada

Cebada

Cebadilla.

Cebadilla

Cebadilla

Cebadilla falsa.

Cebadilla falsa.....

Cebolla.

Cebolla albarrana............

Cebolleja..........................

Cebolleja

Cebolleta....

Cebollino común

Cebollino francés
Dipsacus fullonum L....

Argemone mexicana L.

Carcluus tenuiflorus $\mathrm{DC}$

Centaurea grandiflora DC.

Centaurea amcricana Nutt....

Chicus mericanus Hemsl

Chicus pazcuarensis DC.

Cnicus rhaphilepis Hemsl.

Cnicus mericanus Hemsl.

Argemone mexicana L.

Karatas plumieri E. Morr.

Begonia gracilis H. B. K.....

Iberis unbellata $\mathrm{L}$.

ITedicago denticulata Willd.

Medicago lupulina $\mathrm{L}$

Phraymites communis Trin.

Arundo donaz L....

Phragmitis communis Trin.........

Chrsquea galeottiana Rupr.........

Olyra latifolia L.

Ipomoca arborescens Don............

Ipomoca murucoides Røm et Schult

Euphorbia heterophylla L

Lagascea mociriana DC....

Ccescaljinia cacalceco Humb. \& B...

Cesalpinia coriaria Willd...

Begonia bulmisiana Ruiz.

Castanea vulgaris Lam.

Eisculus hippocastanum, L........

Alibertia edulis Rich

Castilleja arvensis Ch. et Schl.....

Agrostis alba L

Euphorbia hetherophylla L.

Euphorbia pulcherrima Willd....

Melastoma, sp?

Tumfordia floribunda DC.

Anona muricata L

Buddlcia americana L....

Ipomoea arborescens Don

Ipomosa nutrucoides Rœm et Schult

Pachyrhizus angulatus Rich.....

Hordeum distichon L.

Hordcum hexastichon L.

Hordenm vulgare L.

Schrenocaulon officincle A. Gr.

Stcnanthium frigiduon Kunth.

Zygadenus mexicanus Hemsl........

Pentsicmon burbutus Nutt...

Peitstemon imberlis Traulv.

Allien ceper 1.

Itymanomellis rolate Herb.

Schrenocrution officinale A. Gr......

stencontrium frigidum A. GI...

Allim fistulosum L.

Allium stelcenoprosum L.

Alliun schenopretsun L.
Dispáccas............

Papaveráceas.......

Compuestas.........

Compuestas .........

Compuestas.........

Compuestas........

Compuestas........

Compuestas........

Compuestas .........

Papaveráccas.......

Bromeliáceas .......

Begoniáceas.........

Grucíferas...

Leguminosas ........

Leguminosas........

Gramíneas..........

Gramíneas.........

Gramíneas..........

Gramíneas....

Gramíneas.

Corivolvuláceas....

Convolvuláccas.....

Euforbiáceas

Compucstas.

Leguminosas.

Leguminosas.

Begoniáceas.

Cupulíferas....

Hipocastanáceas...

Rubiáceas ...........

Escrofularíncas....

Gramíneas ...........

Euforbiáccas.......

Euforbiáceas

Melastomáccas .....

Compuestas.

Anonáceas.

Loganiáceas.

Convolvuláceas...

Convolvuláceas...

Leguminosas.

Gramíncas

Gramíneas

Gramíncas

Liliáceas...

Liliáceas

Liliáceas.

Escrofularíneas....

Escrofularíneas.....

Liliáceas

Amarilídeas

Liliácea:

Liliáceas.....

Liliáceas.

Liliáceas.

Jiliáceas.
Gultivado

Tabasco...........

Jalisco.

Rovirosa.

Jalisco..............

Jalisco.............

Jalisco............

Méx., Ver., Jal...

Jalisco, Mich....

V. de Méx., Jal...

Tabasco, etc.....

Varios lugares...

Veracruz..........

México.

Cultivada.

Naturalizada ....

Naturalizada .....

Valle de México.

Varios lugares...

Jalisco.

Jalisco.

Tabasco.

Morelos....

Morelos

Jalisco, Chiapas..

Morelos

Oaxaca, Gro..... F. M.

Oaxaca, Gro..... F. M.

Michoacán........

Cullivada.........

Cultivada.

Tabasco.....

Distrito Federal.

Jalisco.

Jalisco.

Sierra Madre....

Michoacán .

Jalisco.

Varios lugares...

Jalisco.

Varios lugares...

Veracruz, Oax...

Cultivada.

Cultivada.

Gultivada

Veracruz.

E. de Méx., Mich. México.

Varios lugares...

Varios lugares..

Cultivada.........

Valle de México.

Veracruz.

Guanajuato, Méx

Gultivada.........

Cultivada...

Cullivada.....
B. V. et Urbina

B. V. et Urbina.

B. V. el Urbina.

Urbina.

B. V. el Urbina.

Urbina.

F. M., Rovirosa.

Alcocer.

Alcocer.

B. V. et Urbina.

Povirosa.

Cat. Inst. Med.

B. V. et Uıbina.

B. V. et Urbina.

N. Lcón.

Mat. Méd.

Farm. Mex. 


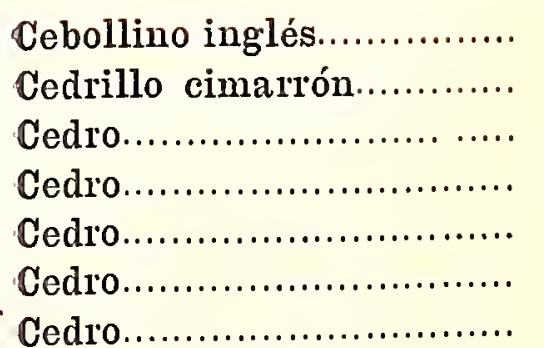

Cediro....

Cedro blanco

Cedro blanco.

Cedro colorado.

Cedro colorado

Cedro colorado

Cedro chino..

Cedro de la Habana..........

Cedro liso.

Cedro macho.

Cedro de México

Cedro rojo

Cedro de la sierra

Cedro limón...

Cedrón

Cedrón

Cedrón de Oaxaca. ...........

Cedrón de Oaxaca.

Ceiba.

Ceiba ó Ceibo.

Ceiba ó Ceibo

Celedonia

Celedonia

Celidonia

Celidonia ó Celidueña.......

Cempaxúchitl ó Cempazúchil

Cempazúchil chiquito.......

Cempazúchil sencillo........

Cempazuchilito

Cempoalehuatl.................

Cempoalxóchitl.

Cenicilla

Cenicilla

Cenizo

Cenizo........

Centaura.

Centaura menor...

Cepillo del Diablo.

Cepillo del Diablo.

Cerbatana

Cerraja.

Cicuas de Cueramo...........

Cicuta, Cicuta mayor........

Cicutilla........................

Cidra............................

Cidra cayote.

Cidra limón....

Gidracayote

Cidrelo ó Cidrero
Allium fistulosum L.....

Guarea triehilioides L....

Cedrela dugesii Watson.

Cedrela glaziovii C. DG

Cedrela mexieana Ræm....

Cupressus benthami Endl...

Cuppessus thurifera H. B. K

Cupressus, sp?

Cupressus benthami Endl...

Cupressus lindleyi Klotsch..........

Cedrela mevicana Rœm............

Juniperus flaceida Schl.............

Juniperus virginiana L...

Cedrela, sp?

Cedrela mexieana Ram.............

Cedrela, sp?....

Cedrela mexieana Roem.......

Cupressus tharifera H. B. K.........

Cedrela odomatas Le....

Cupressus thuriferc H. B. K........

Citrus mediea $\mathrm{L}$

Lippia eitriodora H. B. K...........

Verbena trificla H. B. K............

Simaba eedron Planch....

Quassia eedron H. Bn....

Bombax ellipticum H. B. K........

Eriodendron ceseulifolizm H. B. K. Eriodendron oeeidentale Tr. et Pl.

Euphorbia maculata L...

Eupterbia thymifolic Burm.......

Euphorbia maculata L..............

Chelidonium majus Mill....

Tagetes ereeta $\mathrm{L}$

Tagetes multiseta DC

Adenophyllum porophyllum Hemsl.

Bidens angustissima H. B. K.....

Ulmus mexieana Planch...........

Tagetes ereeta L. ....................

Sesuvium revolutifolium Ort........

Zaluzania angusta Schz............

Atriplex eaneseens James............

Miconia argentea $\mathrm{DC}$....

Milleria linearifolia $\mathrm{DG}$

Coutoubea spieata Aubl.

Combietum jacquini? Griseb.......

Combretun laxum LœIl.............

Montanoa, sp?....................

Taraxacum offieinale Wigg.........

Cordia boissieri $\Lambda$. DC.

Conium nueulatum L.

Parthenium hysterophorus L.......

Citrus medica L.......................

Citrullus vulgaris Schrad. var?....

Citme medica L......................

Cucurbita fucifolia Bouché ........

Citrus mediea L.
Liliáceas............

Meliáceas............

Meliácens...........

MIeliáccas...........

Meliácens...........

Conifferas ...........

Coniferas............

Coníferas

Coníferas ...........

Coníferas ............

Meliáceas....

Coníferas.

Coníferas

Meliáceas

Meliáceas...........

Meliáceas...........

Meliáceas....

Coníferas

Meliáceas

Coníferas

Rutácens

Verbenáceas.........

Verbenáceas........

Rutáceas

Rutácens

Malvácens

Malváceas...........

Malváceas...........

Euforbiácens.......

Euforbiáceas........

Euforbiáceas........

Papaveráceas.......

Compuestas........

Compuestas .........

Compuestas........

Compuestas........

Urticáceas ...........

Compuestas........

Ficoideas ...........

Compuestas........

Quenopodiáceas...

Melastomáceas...

Compuestas........

Gencianeas.........

Combretáceas .....

Combretáceas .....

Compuestas........

Compucstas. ......

Borragíneas........

Umbelíferas........

Compuestas ........

Rutáceas ...........

Cucurbitáccas ......

Rutáceas ...........

Cucurbitáceas.....

Rutáceas ...........
Cultivada......... Urbina.

Tabasco.......... Rovirosa.

Guanajuato ...... Alt. et Ramz.

Tabasco.......... Rovirosa.

Jalisco........... B. B. et Urbina.

Jalisco............ B. V. et Urbina.

Guerrero, Ver...

Sierra Madre.....

Sierra Madre.... Ramírez.

Regs. calientes... F. M.

Verncruz

Veracruz......... Ramírez.

Varios lugares...

Varios lugares...

Guerrero,

Colmeiro

Oaxaca.......... Barroso.

Guerrero, Ver... Colmeiro.

Cultivada.........

Jalisco.............

Hidalgo............

Oaxaca.

F. M., B.V. et U.

F. M.

F. M.

Oaxaca........... F. M.

Guerrero, Ver...

Mor., Tam., Yuc. F. M.

Mor., Tam., Yuc. F. M

Veracruz.

Veracruz..........

Veracruz.........

Gultivada.........

Varios lugares..

Jalisco.............

Jalisco.

Jalisco.....

Veracruz

Distrito F'ederal.

Introducida ?...

E. de Méx., D. F.

Chihuahua, Son.

Tabasco............

Jalisco............

Tabasco.........

Tabasco..........

Jalisco.

Jalisco. .

Michoacán.......

Cultivada........

Varios Estados..

Cultivada........

Cultivada.....

Cultivada.........

Cultivada.........

Oaxaca...........

F. M.
B. V. et Urbina.

B. V. et Urbina.

B. V. ct Urbina.

Alt. et Ramz.

Urbina.

Alt. et Ramz.

B. V. et Urbina.

Colmeiro.

Rovirosa.

B. V. et Urbina.

B. V. et Urbina.

Cat. Inst. Mćd.

E. I.

F. M. 
Cidro.

Cielo raso

Ciento en rama.

Cihoapactli

Cilantro

Cinco llagas....

Cinco de Mayo.

Cinco de Mayo

Cinco negritos.

Cineraria azul....

Cineraria común.

Ciprés.....

Ciprés.

Ciprés.

Ciprés común

Ciprés de Mèzico...

Ciprés de Moctezuma

Ciraroque.

Cirial ó Cirián.

Cirial..

Cirima

Ciruela

Ciruela amarilla.

Ciruela campechana..........

Ciruela colorada ó roja......

Ciruelillo.

Ciruelillo.

Ciruelillo.

Ciruelillo.

Ciruelo agrio..

Ciruelo amarillo de Cuba...

Ciruelo chico.

Ciruelo de España.

Ciruelo de Mézico...

Ciruelo del país.

Ciruelo del pais.

Ciruelo rojo......

Cirujano.

Civil..

Clagot.

Clarincillo.

Clavel encarnado.

Clavel de las Indias.

Clavel de manojo...

Clavel de la nobleza.

Clavel del poeta......

Clavelón..

Clavellina.

Clavellina.

Clavellina blanca.

Clavellina roja.

Clavillo

Clavo.

Clavo de especie.

Clavo de olor.....

Clavos del Señor.
Citrus decumana $\mathrm{L}$

Vinea minor $\mathrm{L}$

Achillea millefolium L.

Montanoa tomentosa Llav. et Lex. Coryandrum sativem L...

Tagetes lunulata Ort..

Amarantus caudatus L.....

Celosia cristata L...

Lantana hirsuta Mart. et Gal......

Solidago, sp?

Solidago, sp?

Cupressus benthami Endl

Cupressus sempervirens L............

Cupressus thurifera H. B. K........

Cupressus sempervirens $\mathrm{L}$

Cupressus benthami Endl.

Taxodium mucronatum Ten.

Rhus, sp?.

Parmentiera alata Miers

Spondias dulcis Forst. var. acida.

Tilia mexicana Benth.

Spondias, sp?.

Spondias lutea L.

Spondias purpurea L

Spondias purpurea L

Bunchosia lanceolata Turcz

Bunchosia sessilifolia ? DC.

Ribes affine H. B. K...

Ribes multiflorum H. B. K.

Spondias dulcis Forst. var. acida.

Spondias, sp?....

Spondias, sp?

Prunus clomestica L

Spondias purpurea L....

Spondias lutea L.

Spondias purpurea L.

Spondias purpurea L

Burserce, sp?....

Malrariscus arboreus Cav

Calliandra grandiflora Benth......

Senecio canicida Moc. et Sessé....

Dianthus sinensis L

Tagetes erecta $\mathrm{L}$

Dianthus, sp?

Dianthus barbatus I

Dianthus berbatus L

Tagetes erecta L

Dianthus barbatus L

Pachira insignis Savign.

Pachiva, sp?

Pachira insignis Savign

Choisya ternata H. B. K.

Pittosporum tobira H. B. K

Caryophlyllus aromáticus L........

Choisya ternata H. B. K

Pussiforce edulis Sims.....
Rutáceas.

Apocináceas.

Compuestas.

Compuestas.

Umbelíferas.

Compuestas.

Amarantáceas

Amarantáceas......

Verbenáceas

Compuestas

Compuestas....

Coníferas.

Coníferas

Coníferas.

Coníferas.

Coníferas.

Coníferas.

Anacardiáceas .....

Bignoniáceas.......

Anacardiáceas ......

Tiliáceas.

Anacardiáceas.

Anacardiáceas

Anacardiáceas

Anacardiáceas.

Malpigiáceas.

Malpigiáceas.

Saxifragáceas..

Saxifragáceas

Anacardiáceas. ..

Anacardiáceas. ....

Anacardiáceas ..

Rosáceas

Anacardiáceas

Anacardiáceas

Anacardiáceas .....

Anacardiáceas .....

Burseráceas

Malváccas

Leguminosas

Compuestas

Cariofileas

Compuestas

Cariofileas

Cariofileas

Cariofileas

Compuestas

Cariofileas.....

Malváceas

Malváceas

Malváceas

Rutáceas ....

Pitosporeas.

Mirtáceas.

Rutáceas

Pasilloráceas
Jalisco.

B. V. et Urbina.

Cultivada.

Distrito Federal.

Varios lugares...

Ciultivada.........

Valle de México.

Cultivada...

Cultivada...

Veracruz.....

Michoacán.......

Michoacån........

S. L. P., Ver. \&...

Cultivada.

Gro., Hgo., Ver...

Cultivada.........

S. L. P., Ver., etc.

Varios lugares...

Michoacán

Varios lugares...

Lug. calientes...

Jalisco, Oaxaca.

Lug. calientes...

Veracruz.

Lug. calienles....

Lug. calientes....

Veracruz.

Jalisco.

Hidalgo, Zac.....

Hidalgo, D. F....

Lug. calientes....

Lug. calientes...

Jalisco..

Cultivada.

México

Jalisco.

México

Jalisco.

Morelos

Tabasco, Ver.....

D. F., Hgo., Mor.

Jalisco.

Jalisco.

Distrito Federal.

Cultivada........

Cultivada.........

Cultivada.........

Distrito Fecleral.

Gultivada........

Jalisco, México...

Jalisco.

Jalisco.

Varios lugares.

Cultivada

Art. de comercio

Varios lugares..

Cultivada.
Mat. Méd.

Urbina.

Urbina.

Ramírez.

F. M.

F. M.

Mat. Méd.

Cat. Inst. Med.

Colmeiro.

B. V. et Urbina.

Rose.

Rose.

F. M.

B. V. et Urbina.

F. M.

V. et Urbina.

Ramírez, F. M.

V. et Urbina.

Ramírez, F. M.

V. et Urbina.

Ramírez.

Rovirosa.

Mat. Méd.

Ramírez.

B. V. et Urbina.

B. V. et Urbina.

B. V. et Urbina

F. M.

F. M. 
Clemole ó Clcmolitos.........

Clemolillo.....................

Coachipilín. ...

Coamecate.

Coamecate ó Coamecatl....

Coamecate blanco

Coamecate de cerro...........

Coamecate verde

Coanabichi.

Coanabichi.

Coanenepilli.

Coanenepilli.

Coanenepilli.

Coapatli.

Coate ó Coatl..

Coatlamitl.

Coatzontecoxóchitl

Coca.

Coclearia del país.

Coclearia del país.

Coco ó Cocotero.

Coco de aceite.

Coco de agua......

Coco de castillo...

Cocoba ó Cocobá.

Cocoite.

Cocolmecan.

Cocolmecan ó Cocolmecatl.

Cocoxíhuitl.

Cocoxíhuitl.

Cocoyul

Cocuile.

Cocuiztle...

Cocuite.

Cocuite.

Cocuite ó Cocuitl

Cocluolloxin (maya)

Cochinita .....................

Cochiztzapotl...

Codo de fraile (semilla)......

Cohombrillo

Coilotópalo

Coilotópalo.

Col

Col enana

Col de Milán.

Col rizada

Cola de borrego...

Cola de borrego.

Cola de borrego.

Cola de caballo.

Cola de iguana....

Cola de lagarto

Cola de mico

Cola de pato.

Cola de pescado
T'agetes putula L....

Wedelia hispida H. B. K.

Ilosuclita, sp?.

Antigonon leptopus Hook et Arn.

Antigonon viride Wats.

Asclepies nirea $\mathrm{L}$.

Aspicarya, sp?.

Antigonon, sp?

Hippocrater obcordeta Lamk......

Hippocratec orata Lamk....

Beerhanata riscosa Lag. et Rodr..

Passiflora, sp?

Potentilla candicans H. \& B.......

Commelinu tuberosu L...

Lyscohthardice amorphoides H. B. K.

Rubus strigowus Michx?.

Stanliopea tigrina Batem.

Erylleroaylon macrophyllum (av..

Lepiolium latifolium L.

Lepillinm virginicum L.............

Cocoss nucifera L.

Litoeis melannocen Gærnt...........

Cocos mucifera L...................

Cocos mueifera L.....................

Aristolochica orloratissima L.........

Robinia, sp....

Smile:r cordifolic Humb. et Bonpl.

Smilax rotundifolia

Bocconic arborea Wats.

Bocennia frutescens L...

Acrocomire sclerocarpa Mart......

Piscirlic erythrina L.................

Bromelia, sp?......

Bromelia. sp?

I'iscicliu, sp?

Bobinia, spl?

Ascleplars curassavica I

Asclepias curassarica L

Casimirou erfulis Llav. et Lex......

Theretia yecolli DC:

Cucu-bita foetidissima Kuntl.......

Cecropia mexicana l.

Cecropia pellater L

Brassica olereteea 1.

Brassicu olercusea Lar.

Brassica oleracea L var.

Brassice oleracea L var...........

(astilleja arvensis Ch. el Schl.....

Caslilleja canescens Benth...........

Castilleja lithospermoides, H. B. K.

liquisetum robustum A. Brown. ...

Plumbago pulchella Boiss.

A enceir pamiculata WVild.

Heliotropium anassavicum L......

Sagillarin laneifolia L. var........

Plumbayo pulchella Boiss...
Compuestas

Compuestas.

Leguminosas.

Poligonáceas

Poligonáceas

Asclepiadeas

Malpigiáceas

Poligonáceas.

Hijocrateácea =....

Hipocrateaceas ....

Nictagineas ........

Pasifloráceas........

Rosáceas .

Commelináceas....

Leguminosas.

Rosáceas

Orquídeas

Lineas.

Cruciferas.

Crucíferas....

Palmeras

Palmeras

Palmeras

Aristoloqui ras......

Leguminosas........

Liliáceas.

Liliáceas.

Papaveráceas.......

Papaveráceas.......

Palmeras

Leguminosas.

Bromeliáceas .......

Bromeliáceas .......

Leguminosas........

Leguninosas........

Asclepiadcas.......

Asclepiadeas ........

Ruláceas ............

Apocináceas.........

Cucurbiláceas ......

Urlicáceas ..........

Urlicáceas

Cirucíferris.

Crucíleras.

riruciferas.

Ciruciferas.

Escrofularíneas....

Escrofularíneas....

Escrofularíneas....

Equisetáeeas.....

Plumbagíneas.

Leguminosas....

Borragíneas.

Alismáceas.

Plumbagíneas .....
Distrito Federal.

S. L. P., V. Méx.

Chiapas .......... Urluina.

Morelos, Sinaloa

Jalisco............ Uı. Uina.

Jalisco.............

Jalisco............ Urbina.

Jalisco..............

Guerrero

B. V. et Urbina.

Valle de México.

Varios lugares...

F. M., A. \& R.

Colmeiro.

Urbina.

E. de México.....

Veracruz..........

Jalisco.

Urbina.

Valle de México.

Veracruz..........

Veracruz..........

Valle de México.

Valle de Mléxico.

Lug. calientes....

Lug. calientes....

Lug. calientes....

Lug. calientes....

Tabasco.

Morelos.

Veracruz...

Jalisco..

Jalisco...

Jalisco.

Sinaloa.

Guerrero, Sina...

Sinaloa, Jalisco..

Sinaloa, Julisco..

Veracruz, Hgo ..

Veraciuz..........

Cullivada........

Cultivada.........

México, Morelos.

Morelos..........

Guanajuato ......

V.de Méx., Mich.

V. de Méx., Mich.

Cultivada........

cultivada.........

Cullivada....

Cullivada...

D. F., Jalisco....

Varios lugares...

Solim:r, Ver.....

Veracruz, S. L. P.

Valle de México.

'Tabasco..........

Distrito Federal.

Tabasco

Valle de México.
B. $V$. et U Urbina.

Alt. et Rillnz,

F. N.

F. N.

Rovirosa.

Ramírez.

B. V. et Urbinal.

Mat. Méd.

Mat. Méd.

Rose.

Rose.

Rose.

Cat. Insl. Med.

Alt. el Ramz.

Mat. Méd.

Milt. Méd.

F.M., Golneiro.

F. M., Colmeiro.

B. V. et Urjoina.

F. M.

Urbina.

Mat. Méd.

Alt. el Ramz.

Rovirosa.

Mat. Méd. 
Cola de venado.

Cola de zorra...

Cola de zorra..

Cola de zorra..

Cola de zorra..

Cola de zorra..

Colecitas

Coliflor.

Colima.

Colinabo

Colomo delgado.

Colorín

Colorín

Colorín chiquito.

Colorín de peces.

Cóltotl.

Coma.

Comida de pajarito.

Comida de pajaritos

Comíngalo.

Comino

Comino de los prados..........

Cominos rústicos.

Commelina.

Confitilla

Confitura

Cóngora ó Congueran...

Congueran.

Conoca

Consuelda mayor.

Contra ranilla de bestias...

Contrahierba

Contrahierba

Contrahierba

Contrahierba

Contrahierba

Contrahierba aromática.....

Contrahierba blanca.........

Contrahierba del Interior..

Contrahierba de Julimes...

Copa de oro..

Copal.

Copal.

Copal.

Copal amargo

Copal amargoso

Copal blanco.

Copal chino.

Copal de penca.

Copal de santo.

Copal de santo.

Copal de Veracruz.

Copalastle (iruto)....

Copalcocote ó Copaljocote.

Copalcuaquitle de Mézico

Copalchi.
Andropogon condensatus H. B. K. Cheilanthes myriophylla Desv......

Gymnosperma, sp? ?.....

Lobelia fenestralis Cav..............

Perezia moschata Llav. et Lex....

Perezia thyrsoidea, A. Gr.....

Euphorbia radians Benth

Brressica oleracea L. var............

Zanthoxylon pterota L.

Bressica oleracea $\mathrm{L}$. var............

Sagitaria sagittifolia L......

Er.ytlerina corallorlendron L........

Erytlirina corralloides $\mathrm{DC}$

Rhynehosia precatoria $\mathrm{DC}$

Piscidir erythrina $\mathrm{L}$.

Dalea lugopms Willd....

Bumelict lucioides: Gærnl. fil.........

Lepistium virginirum L.............

Capsella burse pastoris Mœur:h....

Lucume splicerocarpa A. Dr.........

Cuminum cyminum L.

Carum carvil.

Arracacia atropurpurea B. et H...

Commelina pollida, Willd..

Parthenium hysterophorus [.........

Hyptis suareolens Poit......

Phylolacen icosandra L.

Phytolacea octandra L.....

Solanum torrum Siv.

Wigandia scorpioides Choissy

Serjania racemosa Schum

Asclepias setosa Benth.

Bouvardia triphylla Salisb.......

Dorstenia contrayerva I.

Dorstenia drukena I.

Flaveria contrayerva Pers....

Psoralea pentaphylla L.

P'soralea pentapliylla $\mathrm{L}$.

Psoralea pentiphylla $\mathrm{L} .$.

Asclepsias setosu. Benth..

Solcrimtra guttata Don.

Bursera lanuginosa Engl...

Cyrtocarpa procere H. B. K.......

Elaphrinem copalliferum $\mathrm{DC}$.......

Bursera bipinnala Engl.

Burserer bipimata Engl.

Bursera jorullensis Engl.

Bursere bipinnate Engl.

Purserr jomullensis Engl.

Bursere mexicana Engl....

Bursera jorullensis Engl.

Helincarpus americanus 1

Schinus molle I.....

Cyrtocarpa procera H. B. K.......

Rhus copallina $\mathrm{L}$.

Cyrtocarpa procera H. B. K.......
Gramíneas......... Tabasco, Ver....

Helechos ....

Compuestas

Lobeliáceas

Compuestas.

Compuestas .

Euforbiáceas

Crucifernas

Rutáceas

Girucíferas.

Alismaceas.

Leguminosas.

Leguminosas.

Leguminosas..

Leguminosas..

Leguminosas........

Sapoláceas ..........

Cirucífiras.....

Cruciferas.

Sapotáceas....

Umbelíleras

Umbeliferas

Unbelíferas

Commelináceas ...

Compuestas.

Labiarlas.

Fitolacáceas

Fiłolacáceas

Solanáceas.

Hillrofiláceas.

Sapindáceas

Asclepiadeas.

Punbiácens

Urticáceas

Urticáceas

Compuestas

Leguminosas.

Leguminosas...

Leguminosas.

Asclepiadeas...

Solanáceas....

Burseráceas .

Anacardiáceas.

Burseráceas ..

Burseráceas ....

Burscráceas.

Burseráceas.

Burseráccas ....

Burseráceas ....

Bulseráceas.

Burseráceas.

Tiliáceas.

Anacardiáceas.

Anacardiáceas .

Anacardiáceas

Anacardiáceas .
S. L. P., Oax., \&.

Chihuahua.......

Jalisco.

Michoacán.

Michoacán........

E. de México.....

Cultivada.

Chihuahua .......

cultivada.

Jalisco.............

Jalisco...........

Ville de México.

Guerrero, Mor...

Guerrero, Sima...

Jalisco.............

Ghihuahua .......

Jalisco............

cultivarla........

Jalisco....

Cultivada.

Cultivarła..

S. L. P., Hgo....

Varios lugares...

V. re Méx., ele..

Sonora.

Valle de México.

V. de Méx., etc..

Jalisco.

E. de Méx., Ver.

Veracruz...

Zacatecas.

V. de Méx., etc..

Veracruz...

Tamaulipas......

Varios lugares...

Varios lugares..

Varios lugares..

Varios lugares...

Varios lugares...

Cullivada.

Morelos....

Guerrero, Oax...

Jalisco, Oaxaca.

S. L. P., Mich...

Guerrero, S. L.P.

Morelos.

Mirhoacán.......

Morelos ..........

Michoacán, Ver..

Norelos, Mich.

Veracruz....

Varios lugares...

Guerrero, Oax...

Morelos ...........

Varios lugares.
Urbina.

Urbina.

Cat. Inst. Méd.

Povirosa.

F. M.

Cat. Inst. Méd.

B. V. el Urbina.

Ubina .

Urbina.

B. V. et Urbina.

B. V. et Urbina.

F. M., Ramz.

F. M.

F. M.

B. V. et Utbina.

F. II.

F. M.

F. M.

Mat. Med.

F. M.

B. V. et Urbina.

F. M.

F. M.

F. M.

F. M.

Urbina.

kamírez.

F. M.

All. et Ramz.

F. M.

Ubbina.

Mat. Méd.

Ramírez.

Ramírez.

Alt. et Ramz. 


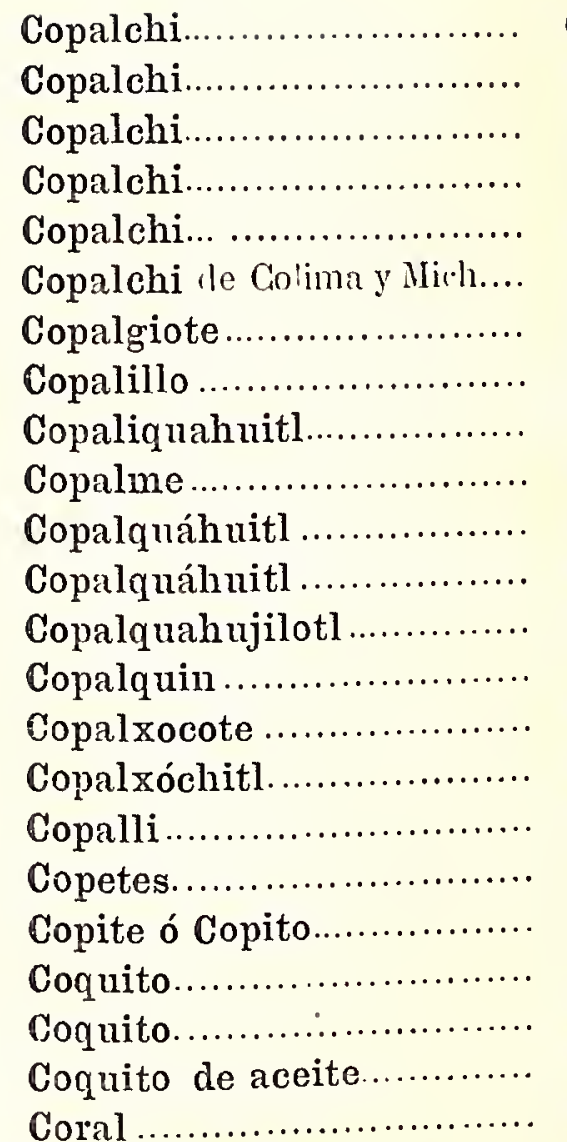

Coral de Colima ................

Coral de jardines ...............

Coral de la playa...

Coralillo.

Corazón de ángel.

Corazón de Jesús.

Corcho

Cordón de cardenal..........

Cordón de San Fraucisco...

Cordón de ó del obispo......

Cordoncillo

Cordoncillo......................

Cordoncillo.....................

Cordoncillo ....................

Cordobán.........

Cornezuelo

Corona de Cristo.

Corona de Cristo.

Corona imperial.

Corpus

Correosa .........

Corrimiento

Cortejo.....

Corteza de Chachaca........

Corteza de Chachaca........

Corteza de chicozapote.....

Corteza de Chilillo............

Corteza de Drimis.

Corteza de liquidámbar.....

Corteza del Perú..............

Corteza de Winter...........

Corteza de yoloxóchitl.....

Cortina
Coutarea latiflora DC:

Croton glabellus L.

Croton niveus Jacq.

Cioton reflexifolius $\mathrm{H}$. B. $\mathrm{K}$

Croton subfragilis Mïll. Arg........

Contarea speciosa, Aubl.............

Pseudosmodlinginn perniciosum E.

Croton, sp?.......

Elaphrim copalliferum DC....

Liquidambar stypreciflua $\mathrm{L}$.

Rhus copallina L

Schimus molle L.

Rhus, sp??

Croton, sp?

Sirpinulus, sp?...

Bursera mericana Engl..

Bursera jorullensis Engl...

Tugreses erectu L.

Cortia dodecendira A. DC..........

Attrlea colume Mirt ?..............

Prechira, sp?........................

Eleeis melenocorece Gernt............

Ririna humilis L.

Vitis tuberosn DG

Solrnum psendocupsicum L........

Russelia juncea Zucc................

Aungallis arvensis L....

Begonia fuchsioides Hook.

Begonia fuchsinides Hook.

Anona palustris L

Polygonom, sp?..

Gomphrena nilida Rothr.

Aneriantus candertus L.

l'eperomia, sp?.

Piper angustifolium Ruiz et Pav..

Priper longum L...

Piper tnberenlatum Jacq...........

Perlilanthus tomentellus Rob. et G.

Acacia sparlicigera Ch. et Schl....

linphorbia fulgens Karw.

Euphorbia sylendens Boja....

Amorphophallus Ririmi Durieu...

Magnolia, sp?

Rhus: microphylla Engelm.

Diosconea composita Hemsl........

Tibiscus mutubilis $\mathrm{L}$.................

Mnimys granalensis H. B. K.......

Drimys mexicana Moc. et Sessé..

Achras srapote L....................

Drimys granutensis H. B. K.......

Drimys granatensis H. B. K.......

Liquidambar slynaciflua L..........

Schimes molle $\mathrm{T}_{\text {......................... }}$

Dirimys granatensis H. B. K.......

Trlauma macracarpa Zucc........ Mesembryanthemum arassifolium L.
Rubiáceas

Euforbiáceas

Euforbiáceas.

Euforbiáceas....

Euforbiáceas.....

Rubiáceas....

Anacardiáceas. ....

Euforbiáceas ........

Burserácens .........

Hamamelídeas..

Anacardiáceas

Anacardiáceas

Anacardiáceas .

Euforbiáceas ........

Snpindáceas.

Burseráceas .........

Burseráceas .........

Compuestas.........

Borragíneas.........

Palmeras ............

Milváceas...........

Palmeras...........

Fitolacáceas .........

Ampelideas .........

Solanáceas ..........

Escrofularíneas....

Primuláceas .........

Begoniáceas........

Begoniáceas.........

Anonáceas .........

Poligonáceas........

Amarantáceas .....

Amarantácens ......

Piperácens.

Piperáceas..........

Piperáceas..........

Piperáceas..........

Euforbiáceas .......

Leguminosas........

Euforbiáceas ........

Eufolbiáceas ........

Aroideas............

Magnoliáceas .......

Anacardiáceas......

Dioscoreáceas ......

Malváceas

Mizgnoliáceas ........

Nagnoliáceas .......

Sapoláceas ..........

Marnoliáceaıs .......

Magnoliáceas .......

Hamamelídeas.....

Anacardiáceas .....

Magnoliáceas ...

Magnoliáceas .

Ficoideas.
Jalisco...

Tabasco

Varios lugares..

Varios lugares...

Chiapas

Ciultivada.........

Michoacán, Mor.

Michoacán........

Morelos

Veracruz........

Morelos, Gro....

Varios lugares...

Sinaloa.

Michoacán........

Colima, Ver.....

Horelos.......... F. M,

Valle de Mléxico.

Veracruz, Yuc...

Colima ........... Rose.

Oaxaca. ........... Barroso.

Guerrero, Colin. F. MI.

Varios lugares... Alcocer.

Jalisco.............

Cultivada.........

Oaxaca.

Naturalizada

Cultivada........

Cultivada.........

Tabasco...........

Jalisco............

Varios lugares...

Cultivada.........

Jalisco...

Veracruz

Chiapas

'T'abasco

Oaxaca

Tabasco.

Oaxaca, Norelos

Cullivada

Cultivada

Sinaloa, Jalisco.

San Luis Potosí.

Tabasco.....

Cultivada.

Sur de la Rep..

Sur de la Rep...

Varios lugares..

Sur de la Rep...

Sur de la Rep....

Chiapas ...........

Varios lugares...

Sur de la Rep...

Veracruz..

Cultivada.
Alt. el Ramz.

Rovirosa.

F. M.

F. M.

Ramírez.

Gat. Inst. Méd

Colmeiro.

Colmeiro.

M. Méd., Colm.

Cat. Inst. Méd.

B. V. et Urbina.

F. M.

Rovirosa.

B. V. et Urbina.

Urbina.

B. V. et Urbina.

Cat. Inst. Med.

Rovirosa.

Ramírez.

Rovirosa.

Alcocer

Rose.

Urbina.

Rovirosa.

F. . I.

F. M.

F. M.

F. H.

F. N.

Cat. Inst. Med.

Calt. Inst. Med.

F. M.

Cat. Inst. Med. 


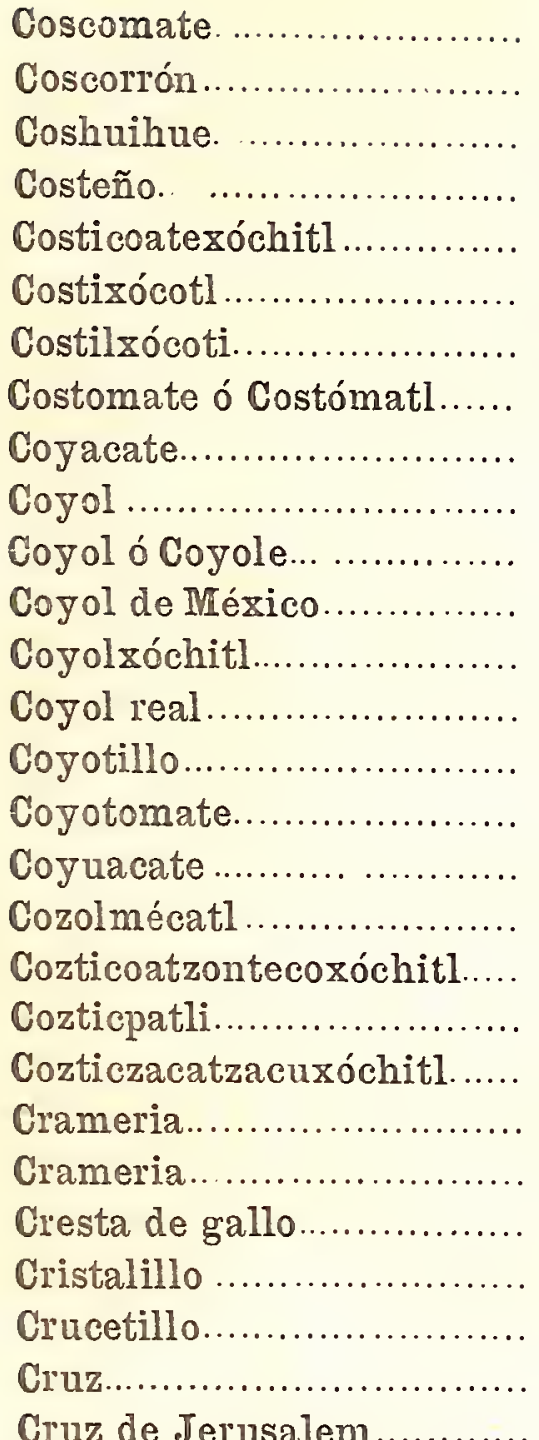

Cuachalalá ó Cuachalalate

Cuachile

Cuaguayote

Cuahualahua.

Cuahuchichi.

Cuahuchichi

Cuahuchichi.

Cuahuhuécatl

Cuahulote.

Cuahutéshuatl.

Cuajilote.......

Cuajinicuil.

Cuajiote.

Cuajiote amarillo.

Cuajiote amarillo.

Cuajiote blanco.

Cuajiote colorado.

Cuajiote chino.

Cuajiote verde.

Cuamecate..

Cuanenepile

Cuanenepile

Cuapinol ó Cuapinole.......

Cuapinole ó Cuapinoli......

Cuasia

Cuastecomate

Cuatante

Cuate.
Physalis coztomal Moc. et Sessé...

Cratceva tapia I

Cupania, sp?.

Musa regia Rumph...

Cattleya citrina Lindl.

Spondices purpurea l................

Syondias dulcis Forst. var. acida.

Plyysulis coztomatl Moc. et Sessé.

Piptadenia foetida Benll.....

Cunna indica L.

Cocos gruacoyule, Liebm............

Costus glabrotus Sw...

Bomarea hirtella Herb...

Attalea sp?

Karuinskia humboldtiana Zure.

Vitex mollis H. B. K

Ingre freticla Willd.

Sinitax cordifolic Humb. at Bonul.

Catfleya citrima Lindl.

Thetictrom hernomderai Tausch...

Govenirt snperbo lindl.............

Krameria pauciflora DC:.....

Krameria secundiftora DC.

Celosia cristata L.

Mesembryanthemum crystallinum I,

Parmentiera, sp?.

Sprelelia formosissima Herb.

Lychnis chalcerlonica L

Dioscorea, sp?.

Loeselia coccinea Don...

Carica, sp?

Heliocarpus americanus L...

Garrya oblonga Benlh....

Garrya overta Benth....

Gumya racemosa Rimírez....

Serjania mexicana IVilla.

Gucauma polybotrya liav.

Crescentia, sp?.

P'armentiera pralis Dr.

Inga jimisuil Schl.

P'seudosmortinginm permiciosum. E. Burseru faguroirles Engrl.

Bursere multijuga Engl.

Pseudesmorlingium perniciosum $\mathrm{E}$.

Bursera fraguroides Engl.

Bursera trijugu Ramírez.

Burserca aptere Ramírez.

Antigonon leptopmes Hook...

Gererdia, sp?....

Boerhaavia, sp?

Hymenced rourbril L...

Hymencer candolleana H. B. K...........

Quessia amara I. fil

Parmentiera aluta Miers.

Minosa, sp?.

Eysenhardtia cenorphoides H. B. K.
Solanáceas

Caparídeas ....

Sapindáceas

Musáceas....

Orquídeas.

Anacardiáceas .....

Anacardiáceas.....

Solanáceas ..........

Leguminosas........

Zingiberáceas.......

Palmeras...........

Zingiberáceas.......

Amarilídeas .........

Palmeras

Rammeas.

Verbenáceas ........

Legurnino-as........

Liliáceas.............

Orquírleas.

Ranunculáceis....

Orquídeas.

Poligaleas....

Poligaleas.....

Amarantáceas.....

Ficoideas

Bignoniáceas.......

Amarilídeas.

Cariofileas

Dioscoreáceas ......

Polemoniáceas.....

Pasifloráceas

Tiliáceas

Cornáceas

Cornáceas

Cornáceas

Sapindáceas.

Esterculiáceas.....

Bignoniáceas.......

Bignoniáceas.......

Legumin osas.......

Anacardiáceas ......

Burserácerus.

Purseráceas

Anacardiáceas .

Burseráceiss

Burseráceas

Burseráceas........

Poligonáceas.

Escrofularíneas....

Nictagineas.....

Leguminosas....

Leguminosas...

Simarrubáceas.

Bignoniáceas.

Leguminosas. ...

Leguminosas.
Varios lugares...

Tabasco

Tabasco...

Cultivada...

Michoacán......

Morelos

Morelos

Varios lugares...

Guerrero........

Naturalizada ....

Veracruz.........

...................

D. F., E. de lléx.

Veracruz..........

Varios lugares...

Guerrero.

Veracruz.

Veracruz....

Michoncán........

$V$. de Mćx., otc..

Miclınacáll........

Varios lugares..

Varios lugares...

Gultivada.

Gultivada...

Tabasco

E. y V. de Méx..

Cultivada....

Oaxaca.

Varios lugares...

Jalisco.

Veracruz.

Hidalgo.

Zacatecas...

Morelos

Valios lugares...

Puebla, Guerrero

Guerrero.

Jalisco, Morelos..

Veracru\%...

Hidalgo, Morelos

E. de Méx., Mor.

Colina....

Norelos, Quer..

...................

Nlorelos

Morelos

Jalisco

Jalisco

Jilisco.

Tabasco, Oaxaca

Varios lugares..

Cultivada........

Jug. calientes..

Jalisco.

Varios lugares.
F. H.

Rovirosa.

Ciat. Inst. Med.

Colmeiro.

Alt. et Rill11z.

F. MI.

Kerchove.

Colnueiro.

linck.

Colmeiro.

Ciolmeiro.

F. M., M. Méd.

Ciolmeiro.

F. M.

F. M.

Cat. Inst. Ned.

Cat. Inst. Méd.

Mat. Méd.

Alt. el Ramz.

F.M., B.V.etU.

Urbina.

Mat. Méd.

Mat. Méd.

Mat. Mléd.

Mat. Méd.

B. V. el Uıbina.

B. V. et Urbina

Cat. Inst. Med.

Barroso, Ramz.

B. $V$, et Urbina.

B. V. et Urbina. 
Cuatlatlaya ....................

Cuauchalalá ó Cuatuchalalate

Cuauchichic.

Cuauchichilli.................

Cuaulaláhuac blanco..

Cuaulote

Cuautecomate.

Cuautecomate ó 'mautecomatl.....

Cuauteteco.

Cuauxúchil.

Cucharilla...

Cueramo..

Cuernecillo de maiz...........

Cuernito de hormiguero.....

Cuernitos.

Cuernitos.

Cuerno.

Cuhrucumin (tarasco)........

Cuirindal.........................

Cuitlacoche.....................

Cuitziquiendas (tarasco).....

Culantrillo de México.

Culantrillo de ojo de agua.

Culantrillo de pozo..............

Culantrillo de pozo..............

Culantro.

Culhua.

Culhua.

Cumbaquare (tarasco)........

Cundá (tarasco).

Cundeamor.

Cundeamor.

Cundeamor.

Cundeamor....

Cundeamor.

Cundeamor

Cundeamor de Yucatán....

Cundemba (tarasco)............

Cunicho

Cupamu (tarasco).............

Cupanda (tarasco).

Cupandra

Cupu (tarasco)

Cúralo todo.

Cúralo todo.

Cúrcuma.

Cúreuma...

Curiqua (tarasco)

Cútaqua (tarasco)

Cutzis (tarasco).

Cutzis (tarasco).

Cuyotomate

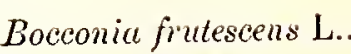

Dioscorca, sp?

Garrya raccurosa Ramírez.........

Boccorria firutescens L...............

Heliocarpus americanus L..........

Heliocarpus americanus L..........

Crescentia cujcte $\mathrm{L}$

Parmentiera alata Miers.

Heterotheca imuloidles Ciss.

Plunierie rubra $\mathrm{L}$

Dasylirion acrotrichun Zncc........

Cordia, sp?...

Ustilago maydis DC.

Mratynia firagrens Lindl.

Acacia cormigera Willa...

Acacia melenoweras Beurling?.....

Cercus flagdliformis Haw..........

Tagetes lucida Giv...

Ficus, sp?

Ustiluyo maydis DC.

Tropaolum majus $\mathrm{L}$

Adiantrem tencium Swartz.........

Adiantum cupillus-veneris L.......

Adiantum trapeziforme L....

Adiantum seabrum Raulf.

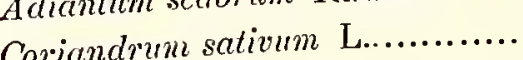

Opuntia tune Mill.

Opuntia vulgaris Hill.

Nymphere odorater Ait.

Plumeria mbra L.

Cobcea scandens Cav.

Ipomoa coceinea L....

Marrandia batelayana Lindl.....

Maurandia semperforens Ort.....

Momordica charentia L............

Iomordiea balsamina $\mathrm{L}$

Mlomordica charentia L....

Sambueus mexicrna Presl.

Schoenocaulon officinale A. GI......

Bambusa vilgaris Wendl.

Persea gratissima Gerln.

Persea gratissima Gerlil...

Lobelia, sp?........................

Lobelia laxiflora H. B. K..........

Curcuma louga L.

Curcuma tinctoria Guib.

Plumbugo pulchella Boiss.

Solandra grandiflora Sw.

Spiranthes aurantiacus Hemsl... Spiranthes cimabarinus Hemsl.. Vitex mollis $\mathrm{H}$. B, K.
Papaveráceas.......

Dioscoreáceas .....

Cornáceas

Papaveráceas.......

Tiliáceas

Tiliáceas.

Bignoniáceas........

Bignoniáceas........

Compuestas .........

Aprocinácens.........

Liliácea.s.............

Borragíneas.........

Hongos..............

Pedalíneas...

Leguminosas.......

Leguminosas........

Cácteas..............

Compuestas.........

Urlicáceas ...........

Hongos..

Geriniáceas.........

Helechos ............

Heleclios ..........

Helechos ............

Helechos

Umbelíferas

Cácteas

Cácteas

Ninfeaceas

Apocináceas.........

Polemoniáceas.....

Convolvuláceis....

Escrofularíneas....

Escrofularíneas ....

Cucurbitáceas .....

Cucurbitáceas ......

Cucurbitáceas .....

Cilprifoliácens .....

Liliáceas............

Gramíneas..........

Lautíneas... .......

Lauríneas.

Anacardiáceas .....

Lobeliáceas

Lobeliáceas

Zingiberáceas......

Zingiberáceas.......

Plumbagíneas ......

Solináceas .

Orquídeas.

Orquideas.

Verbenáceas
E. de México.....

Michoacán, Pue. F. M

Morelos

Mat. Méd

Mlichoacán........ Colmeiro

Veracruz

E. de México..... Uibina.

Oaxaca............

Varios lugares... Cat. Inst. lled.

Ville de México. A. H., M. Méd.

E. de Mexico....

San Luis Polosí.. Urbina.

Michoacán....... Ramírez.

Jalisco............ B. V. et Urbini.

Jalisco........... B. V. et Urbina.

Guerrero .........

Chiapas .......... Alcocer.

Varios lugares...

Michoacán........ N. León.

Michoacán....... Cat. Inst. Med.

Piırásila

Ciullivadil........

Mesa Cientral.... F. M.

.................. Rose.

Veracruz.........

Jalisco............

Cullivada.........

Varios lugares...

Varios lugares...

Michoacán.......

Michoacálı.......

Ville de México.

Valle de México.

S. L. P., D. F....

Hidalgo, Oaxaca.

Varius lugares...

(Liapas ...........

Tabisco, Yuc....

Mirhoacán.......

Veracruz.........

Michoacán........ N. Leon.

Miclıoncán........ N. León.

Morelos ...........

Mlichoacán........

Jillisco.............

Jalisco

N. León.

B. V. et Urbina.

Urbina.

Cullivada........ F. M.

Cultivada........ F. M.

Michoncán....... N. León.

Miclroacán....... N. León.

Oax., Mich., Ver.

Oax., Mich., Ver.

Michoacán....... Ramírez. 


\section{$\mathrm{CH}$}

Chacalzóchitl

Chacanguarica.

Chacate.....

Chacmol

Chachaca

Chachalma

Chachalcahuite.

Chachamole.

Chachisdá.

Chaiotl.

Chalchuán.......

Chamal.

Chamico....

Chancarro

Chancle.

Changungo.

Chanznuc (maya).

Chaparro prieto.

Chapón.

Chapulizctli

Chapuz

Chapuz

Chapuz

Chaquira

Chaquira ó Chaquirilla......

Chaquira ó Chaquirilla......

Charahuesca (tarasco) ........

Charahueso.

Charaitzicua (tarasco)........

Charamusco..................

Charás petequa (tarasco)....

Charin.

Charraspique..

Chautle

Chautle

Chavacano

Chayote

Chayotillo.

Checámequa (tarasco).......

Checámeti (tarasco)..

Chelele.

Chía.....

Chía

Chia

Chía.

Chia azul grande.

Chía cimarrona

Chía gorda
Casalpinia pulcherima Sw.

Bixa orellana L...

Krameria canescens A. Gr. Gomphrena globosa L...

Prosopis juliflora DC.

Phoradendron, sp?

Mimosa, sp?.

Nymphcea, sp?.

Berberis pinnata Lag

Sechium edule Siv...

Erigerm affinis DG.

Dioon erlule Lindl.

Datura stramonium L...

Cecropia mexicana Hemsl.

Canna, sp.

Malpigia, sp?......

Tribulus cistoides I

Mimosa, sp?.

Taberncemontana, sp?

Dodoncea viscosa $\mathrm{L}$

Helenium autumnale $\mathrm{L}$

Helenium integrifolium B. et H...

Helenium mexicanum H. B. K.....

Colubrina alamani DC.

Ceanothus azureus Desf....

Ceconothus cerruleus I ag.

Dahlia, sp?

Jahliar erariabilis Dest

.Jacobinia moluintli Hemsl....

Calliandra houstori Benth.........

Castilleja scorsonercefolia H. B. K.

Bignonia buceinatoria Mairet.....

Theris inlermedia Guer...

Bletia e:cmpamulata. Lliv. et Lax... Cranichis specinsu Islav. et Lex..

Irunus armeniaca l....

Sectivim edule Sw.

sycios angulatus I,

Cnicus mexiranus Hemsl...

Eiyngium seriontum (iav.

Inga, spo........

Hyptis spreceter Poit.

Saleice hisyaniora I.

seleve titicefolice Vahl.

Salvia polystachya Ort

Salvia oymanca Bentli...

Sulviu anguslifolia Cav

Plankago, sp.
Leguminosas........

Bixíneas.

Poligaleas.

Amarantáceas.....

Leguminosas........

Lorantáceas.

Leguminosas.

Ninfeáceas.

Berberídeas

Gucurbiláceas ......

Compuestas.

ricadáceas

Solanáceas .

Urticáceas

Zingiberáceas

Malpigiáceas.

Zigofileas...

Leguminosas.

Apocináceas.........

Sapindáceas...

Compuestas....

Compuestas.........

Compuestas .........

Ramneas

Ramneas....

Ramneas

Compuestas

rompueslas

Acantáceas

Leguminosas.

Escrofularíneas.

Bignoniáceas.

Gucíferas

Orquídeas.....

Orquíleas.

Rirsáceas

Cucurbiláceas

rucurbiláceis

Compueslas.

Umbeliferns ... ....

Leguminosas......

labiulalas.

Libindas....

Labiudas.

I abiardas.

Labiadas...

Labiadas....

Plantagíneas
Jalisco..

Morelos

Chihuahua......

Naturalizada ....

V. de Méx., etc..

Guerrero

Jalisco.

Michoacán.....

V. de Méx., Gto..

Varios lugares...

Valle de México.

Nuevo León.....

Tabasco...........

Veracru\%.........

Tiabasco..........

Michoacán.......

Yucatán.

Chihuahua .......

Chiapas

México ............

Nuevo Leólı......

Guerrero .........

Miclıoacán.

Veracruz.

Varios lugares...

Varios lugares...

Michoacán.......

Varios lugares...

México ............

Tabasco..........

Miclıoacáı........

Varios lugar es...

Cultivadiı........

Veracruz, Mich.

Michoacán........

Cultivada.........

Varios lugiles...

Valle de México.

Michoacálı

Micluoacain......

'labarseo..........

Jilisco.............

V. de Móx., Ver.. Rose.

V. de Méx., etc.. Rose.

V. de Méx., Gro.

Jalisco.

Varios lugares..

Jilisco.
B. V'. el Urbina.

Cal. Inst. Méd.

(at. Inst. Med.

Cal. Inst. Med.

Cat. Inst. Méd.

Alcocer.

Mat. Med.

Mal. Méd.

Farm. Mex.

larm. Mex.

N. León.

Urlina.

Rovirosa.

N. Léóll.

Falli. Hex.

Farm. Hex.

N. Leó!ı.

Cal. Inst. Méd.

Rose. 
Chiantzozolli.

Chicalote ó Chicallotl.

Chicalote ó Chicallotl.

Chicalote ó Chicallotl.

Chicalote de árbol.

Chicalote grande.

Chicle.

Chicochuchi de México...

Chicozapote ó Chiczapotl...

Chicharo.

Chícharo de comer.

Chícharo de olor.

Chichi de gato.

Chichibé (maya)

Chichicahoatzón.

Chichicamole ó illichicamolli...

Chichicaquilitl

Chichicaquilitt

Chichicaxtli

Chichicuahuitl

Chichillotl

Chichiquálhuitl

Chichiquáhuitl......

Chichiquáhuitl......

Chichiquáhuitl. .

Chichiquelite ó Chichiquilitl.

Chichón blanco.

Chichón colorado

Chijol

Chilacaxtli.

Chilacayote

Chilacuate

Chilacuate

Chile.

Chile.

Chile verde.

Chile ancho

Chile ancho

Chile cara

Chile colorodo boludo........

Chile cuachili

chile mirasol.

Chile mulato

Chile pasilla

Chile piquín

Chile piquín

Chile poblano.

Chile tzincuayo

Chile valenciano

Chilillo

Chilillo

Chilillo

Chilillo

Chilillo

Chilillo

Chilillo de la Huaxteca...
Salvia tilicefolia Vahl.: Argemone grandiflora Sweet....... Argemone mexicana L...

Argemone oehroleuea Sweet.........

Boeeonia frutescens L.

Argemone grandiftora Sweet.......

Achras sapota L.

Bombax elliptieum H. B. K.........

Aehras sapota L.....

Pisum sativum L.

Pisum sativum L

Lathyrus odloratus L................

Physalis, spp?......................

Sirla, sp?...

E.yngium, sp?....

Microsechium helleri Cogn...........

Sonchus ciliatus L.

Sonchus olercreeus L...

Gronovia scandens L...

Garrya racemosa Ramz.....

Argemone mexieana L.

Garrya laurifolia Hartw.

Garrya oblonga Bentls.

Garrya ovata Benth....

Garrya racemosa Ramz.

Solanum nigrum L.

Cupania, sp?.....

Cupania americana $\mathrm{L}$

Piscidia erythrina L.

Azolla earoliniana IVilld....

Cucurbita ficifolia Bouché...

Styrax argentem Presl...

Styrax Ramircrii Greenman......

Capsicum annuum L.

Capsicum ammum longum Sendt.

Capsicum anmum aeuminatum $\mathrm{F}$.

capsicum anmuim grossum Sendt.

Capsicum cordiforme Mill....

Capsicum anmum longum Sendl.

Capsicum axi Vell..

Capsicum fintescens L.............

Capsicum, sp?....

Cupsicum cordiforme Mill..........

Capsicum longum DC...............

Capsicum ammum cerasiforme $\mathrm{M}$.

Capsicum frutescens L.............

Coppicum ammum grossum Semull

C'apsicum violuceum H. B. K.......

Capsicem dule Horl

Clementios sericea H. B. K

Drymis mericana Moc. el Ses=t..

J'umbago puldehella Boiss..........

Polygonume are H. B. K...........

Polygonum hylropiper L...........

Polygonm hydropiperoides Michx

Ronres oldongifolia Hook el Arn.
Labiadas........... V. de Méx., Ver.

Papaveráceas...... Varios lugares...

Papaveráceas...... Varios lugares...

Papaveráceas...... Varios lugares...

Papaveráceas....... Jalisco...........

Papaverááeas...... Jalisco............

Sapotáceas......... Yucatán......... Farm. Mex.

Malváceas ......... Guerrero ........ Ramírez.

Sapotáceas......... Varios lugares..

Leguminosas........ Cultivada.........

Jeguminosas........ Cultivada.........

Leguminosas....... Cultivada........

Solanáceas ......... Jalisco...........

Malvácens .......... Yucatán..........

Umbelíferas ........ ..................

Gucurbitáceas..... Varios lugares...

Compuestas........ Introducida......

Compuestas........ Introducida.....

Loaseas............ Tam., Camp.....

Cornáceas .......... Valle de México.

Papareráceas...... Varios lugares...

Cornáceas ......... E. de Méx., etc..

Cornáceas.......... Hidalgo, E. Méx.

Cornáceas ......... E. de Méx., Zac..

Cornáceas .......... Valle de México.

Solanáceas.......... Jalisco, V. Méx..

Sapindáceas........ Tabasco.........

Sapindáceas........

Leguminosas.......

Rizocarpeas........

Cucurbitáceas ......

Estiráceas

Estiráceas...........

Solanáceas ..........

Solanáceas.....

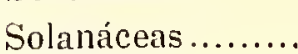

Solanáceas .........

Solanáceas ..........

Solanáceas ..........

Solanáceas..........

Solanáceas ..........

Solanáceas ..........

Solanáceas ..........

Siolanáceas..........

Solanáceas ..........

Solanáceas..........

Solanáceas.........

Solanáceas.. .....

Solanáceas.

Ranunculáceris ....

Mignoliáceas .......

Plumbagineas ......

Polignneas. .........

Poligoners ..........

Poligonáceas.......

Commiraceas........

Tabasco...........

Tamaulipas......

Valle de México.

Cultivada........ Rose.

Guerrero, elc.....

Morelos..........

Jalisco, Veracruz

Cultivada........

Cultivada........

Cultivada........

Cültivada........

Cultivada........ Rose.

Ciultivada.........

Ciultivada.....

Jalisco.

Cultivada.

Ciltivada.

Cultivada........

rilulivida..

Cinltirada.

Cullivida.

(:ultivada.....

México Ver.

Jalise.o.

Oilxaca........... Cat. Inst. Méd.

Valle de Méxien. Ciut. Inst. Med.

Jalisco, México... Farm. Mex.

Ver, V. de Méx.

Tepie, Veracruz.

Finm. Mex.
B. V.et Urbina.

Cat. Inst. Med.

Cat. Inst. Med.

Alt. et Ramz.

Kamíre\%.

B. V. et Urbina.

Rose.

Rose.

Rose.

B. V. et Uluina.

Rose.

Rose. 
Chilillo venenoso.

Chilochuchi...

Chilpantlacol.

Chilpantlacol .

Chilpantlozolli ó (liipansóellitl...

Chiltepín ó Chiltipiquin

Chiltepiquín ó Chiltipin

Chiltipiquín de Papantla...

Chillazotl.

Chimálatl ó Chimalitl ......

China

Chinaca

Chinchayote.

Chinchiligua

Chinchín..

Chinchine aras.

Chinchis

Chinín....

Chino.

Chinos.

Chintul grande .............

Chioplé.

Chioplé

Chiopilcoite

Chipilín.

Chipilín cimarrón.............

Chiqueo

Chiqueo de monjas ...........

Chirimolla ó Chirimollo...

Chirimoya ó Chirimoyo....

Chirimoyo.

Chirivía.

Chirlo ó Chirlos

Chismes

Chivatillo

Chontal.

Chopo.

Chopo.

Chorros.

Chucata (tarasco).

Chucte

Chúmbahua (tarasco)..

Chumchintok (tarasco?).

Chuparrosa.

Chupiqua (tarasco)

Chupire, Chupireni ó Chupini.

Chupirín vitzaqua (tarasco)

Chuspata (tarasco:
Rourea oblongifolia Hook et Arn.

Bombax ellipticum H. B. K........

Pentstemon barbatus Nult....

Pentstemon imberbis Trautv.........

Lobelia lavifora H. B. K.

Copsicum microcarpum DC.

C'apsicum baccatum I.

Capsicum annuum $\mathrm{L}$

Argemone mexicana L.

Helianthus annuus L.

Snilax rotundifolia L

Stigmaphyllon humboldtianum A.J.

Sechium edule Sw

Solanum, sp?

Viburmum prunifolium $\mathrm{L}$

Astragalus humboldtii A. Gr.

Jutropha? ?.....

I'rsea, sp?.

l'ithecolobium mexicanum Ruse....

inpatiens balssmina L.

Cyperus articulatus L....

Euprtorium macrophyllum L.......

Eupetorium populifolium H. B. K.

Robinia, sp?.

Crotalaria, sp?

Crotalaria guatemalensis Benth...

Convolvulus, sp?...

Ipoincea hederifolia L.

Anona cherimolia Mill...

Anona cherimolia Mill.

Anona squamosa L.

(ichorium intybus $\mathrm{L}$

Oxalis tetraphylla Cav.

Sedum acre L

Porophyllum coloratum DC.

Erythrina, sp?

Populus fremonti Wats.

P'opulus nigra $\mathrm{L}$

Fuchsia arborescens Sims

Prosopis juliflora DC.

AIyroxylon pereirae Klotzsch.

Nymphcee, sp?

Gucaicucum, sp??

Lreselia cocciner Don

Ipomœe, sp?.

Euphorbia calyculata H. B. K.

Castilleja canescens Benth.

Cyperus, sp?.........
Connaríceas

Malváceas

Escrofularíneas....

Escrofularíneas.....

Lobeliáceas

Solanáceas ....

Solanáceas...

Solanáceas.

Papaveráccas.......

Compuestas.

Liliacuas.

Mill pigiáceas

Cucurbiláceas .....

Solinnácens

Caprifoliáceas.......

Leguminosas........

Euforbiáceas .......

Lauríneas

Leguninosas........

Geraniaiceaı.........

Ciperáceas...........

Compueshas........

Compuestas...

Leguminosas.

Leguminosas..

Leguminosas...

Convolvuláceas...

Corivolvuláceas. ...

Anonáceas

Anonáceas

Anonáceas

Compuestas.

Geraniáceas.

Crasuláceas

Compuestas.

Leguminosas.

Salicineas.

Salicineas

Onagrarieas.

Leguminosas....

Legumino:as

Ninfeáceas

Zigofileas

Polemoniáceas.

Convolvuláceas....

Euforbiaiceas

Escrofularíneas....

Ciperáceas.
Tepir, Verucruz..

Valle de México.

Valle de lléxico.

Valle de México.

Cultivada........

Chilumahual......

Cullivida.........

Varros lugrarrs...

Ville de México.

Lugrures cálidos..

Veracruz.

lléxico

'limm., Hirlalgo...

Jalisco.

Jilisco.

Tabasco.

Sonora..

Cullivada.

'lábasco.

Tabasco

'libasco

Tabasco

l:hiapas

Tabasco

Jalisco.

Jalisco, S. L. P..

México, etc.......

México, etc......

Jalisco

Gullivada

Oaxaca.

Gultivada........

Michoacán.. .....

Tabasco ..........

Chih, S. L. P...

Cullivada.

Jilisco.

Michoacán.......

Veracruz.

Michoacán.......

Michoacán.......

Valle de México.

Michoacán.......

Michoacálı......

Michoacán.......

Michoacán.......
Farm. Mex.

Colm., Kunth.

N. León.

Alt, et Ramz.

D. (.

B. V. el Urbina.

Ciat. Inst. Med.

Rovilosal.

Urbinat

Rovirosil.

Cal. Inst. Méd.

Ciit. Inst. Mled.

Rovirosa.

B. V. el Urbina.

Urbina.

B. V. el Urbina.

Cilt. Mnst. Méd.

Cat. Inst. Méd.

Ubbina.

B. V. el Urbina.

Mat. Méd. 


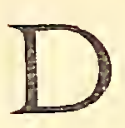

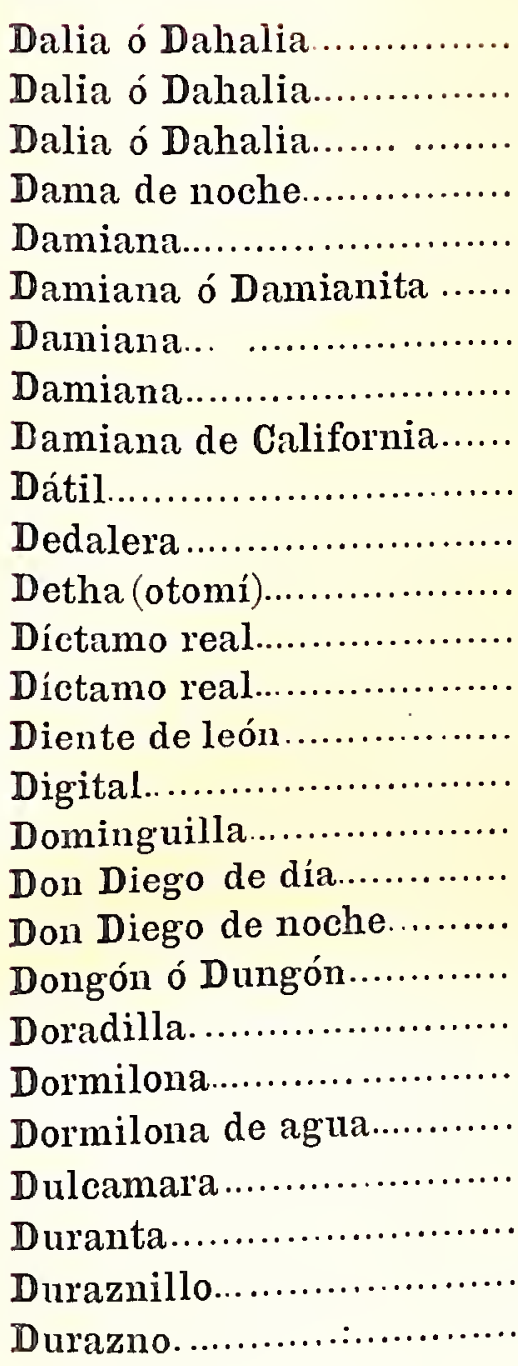

Dahlia coccinea Cav

Dahlia impcrialis Roezl.

Dahlia variabilis Desf..

Cestrum nitidum Mart. et Gal.....

Bigelovia rencta A. Gr...

Chrysactinia mexicana A. Gr...... Senceio, sp?.

Turnera humifusa Endl..

Turnera aphrodisiaca IVard.

Phonix dactylifera L.

Digitalis purpurea 1 .................

Zea mays L....

Passiflora dictamo DG

Passiflora mexieana Juss

Taraxacum officinale Wigg.........

Digitalis purpurca L................

Uitica dioica L......................

Fuchsia arborescens Sims...........

Mirabilis jalapa L..................

Sterculia, sp?...

Sclaginella rupestris Spring.........

Mimosa pudica L...... .............

Ncptunia oleracea Lour.............

Solanum dulcamara L. var.......

Duranta phumieri Jacq.............. Verbenáceas........

Acalypha phleoides Cav. var....... Prunus persica Benth. et Hook...
Compuestas........ Valle de Néxico.

Compuestas ........ Veracruz.........

Compuestas........ Valle de México.

Solanáceas......... Tabasco..........

Compuestas........ V. de Méx., Ver..

Compuestas........ V. de Méx., etc..

Compuestas.........

Turneráceas.........

Turneráceas.........

Palmeras.

Escrofularíneas....

Gramíneas.

Pasifloráceas........

Pasifloráceas........

Compuestas........

Escrofularíneas....

Urticáceas

Onagrai ieas.

Nictagineas

Esterculiáceas ......

Selagineláceas......

Leguminosas.......

Leguminosas........

Solanáceas ....

Euforbiáceas

Rosáceas.
Rovirosa.

Cat. Inst. Méd.

Gat. Inst. Méd.

Guerrero........ Rose.

San Luis Potosí.. Farm. Mex.

Lug. calientes...

Cultivada.........

Cultivada.........

Jalisco, Morélos..

Jalisco, Morelos., Farm. Mex.

Introducida ......

Cultivada.........

Jalisco.

E. de México.... Urbina.

Veracruz, ctc....

V. de Méx., Jal... F. M., B. V.et U.

Veracruz..........

Tabasco.......... Rovirosa.

Cultivada........ Farm. Mex.

Orizaba...........

Michoacán........

Cultivada.........

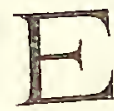

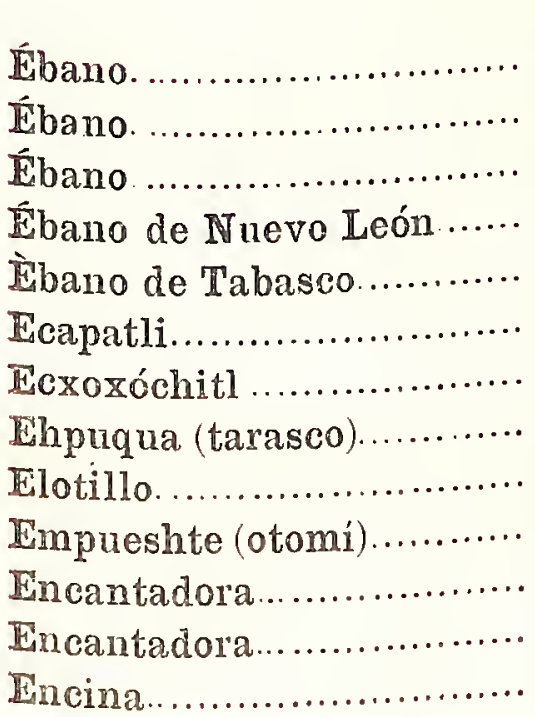

Calliandra formosa Bentlı....

Brya ebenus DC

Diospyros velutina Hiern ?..........

Dimosa, sp?

Calliandra formosa Benth.........

Cassia occidentalis L...............

Helenium mexicanum H. B. K......

Agave mexicana Lam...............

Conopholis americana Wallr...

Piqueria trinervia Cav..............

Hibiscus, sp?.......

Howhousia triloba Moc..........

ingenhousia triloba Nercus acutifolia Nee...
Leguminosas.

Leguminosas....

Ebenáceas.

Leguminosas

Leguminosas........

Leguminosas........

Compuestas .........

Amarilídeas ........

Orobancáceas .....

Compuestas. ........

Nalváceas..........

Malváceas..........

Cupuliferas.
Tabasco. Introducida.....

Oaxaca.

Nuevo León.....

Tabasco........... Méx., Oax., Ver.

Michoacán........

Michoacán...... N. León.

Hidalgo, Mich.... Kamz., Villada.

Jalisco, E. Méx... Ulbina.

Jalisco............ B. V. et Urbina.

Jalisco, Sonora...

Veracruz......... Ramírez. 
Encina

Encina

Encina.

Encina

Encina

Encina

Encina

Encina

Encina

Encina

Encina

Encina

Encina

Encina

Encina

Encina

Encina

Encina de mar

Encina memolito

Encina memelito.

Encina memelito.

Encina memelito.

Encina memelito.

Encina memelito.

Encina memelito

Encina memelito.

Encina memelito

Encina memelito.

Encina memelito.

Encina de miel

Encina negra.

Encina negra.

Encina roble

Encinilla

Encinilla.

Encinilla

Encino aguacapuli.

Encino blanco.

Encino capulincillo

Encino colorado.

Encino delgado

Encino negro..

Encino trompillo..

Enchiladitas.....

Enchiladora

Enebro

Eneldo....

Engorda-cabras.

Inguambo ó Engüiemba....

Engüemba ..........................

Ensalada......

Epazote ó Epazot1............

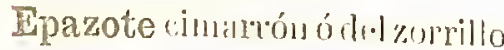

Epazote del zorrillo..

Escabiosa

Escarola.
Quereus barbinervis Benth.

Querens castunea Nee.

Quercus confertifolia Humb. el B.

Quercus cortesii Lichm.

Quercus crassipes IIumb. el B.... Quercus crassifolia Humb. el B... Quercus depressa Hunb. et B..... Quercus insignis Mart. el Gal...... Querens obtuseta Humb. el Bonpl. Quercus polymorpha Cilam. el S.. Quercus pulchella Humb. el Bonpl. Quercus repanda Humb. el Bonpl. Quercus reticulata Humb. el B.... Quereus rugulosa Niart. el Gial..... Quercus splendens Nee. Quercus strombocurpa Liebm..... Quercus tomentosa Willa

Fucus resiculosus L

Quercus acutifolic Nec

Quevens agrifolia Nec.

Quercus castanea Nee.

Quercus chrysophylla IIumb. ct B. Quercus glaucescens Hurnt). el B.. Quercus lanceolata Humb. el B... Quercus laurina Humb. el Bonpl. Quercus obtusata Humb. et Bonpl. Quercus reticulata Humb. el B... Quercus sideroxyla. Humb. ct B... Quercus skinneri Benth.

Quercus reticulata Humb. el B... Quercus crassipes Hunb. el B.... Quercus repanda Humb. et Bonpl. Quercus ralapensis Humb. el B... Crolon corymbulosus Engelm...... Croton fruticulosus Torr.

Krameria, sp?.

Quercus, sp?

Quercus, sp?

Quercus microphylla Nec

Quercus, sp?

Quercus laurina Humb. et Bonpl. Querous virens Ait...

Quercus, sp??.

Custilleja arvensis Cham. et Schl.. Croton ciliato-glandulosus Ort... Juniperus virginiana $\mathrm{L}$.

Peucedlamam anethum Bn.... Dulea tuberculctuta Lag Bocconia frutescens L. Bocronice arborea Wals. Plumeria tricolor Ruíz et Pav Chenopodium ambrosioides Chenopodium foetidum Schrad. Chenopodium incisurn Poir. Scobiosa atropurpurea $I_{\text {. }}$. Cichorium endivia 1
Cupuliferus Copulifuras

Cupuliferas.

Ciupruliferas.

Cupulíferis.

Ciupulíferis.

Cupuliferas.

Ciupulíferas.

Colpulíferens.

Capuliferis....

Capulíferas

Cuprulíferts.

Gupuliferas

Cupulíferas.

Ciupulíferas

Cinpuliferas.

Cupuliferas....

Algas

Cupulíteras

Cuy uliferis

Cupulíferas....

Cupuliferas.

Ciupulíferis.

Cupuliferas.

Cumpulíf(cris....

Cupulíferas.

Cupuliferas.

Cupulíferas.

Ciupulíferas.....

Cupulíferers....

Cupulíferas.

Cupulíferas.....

Cupulíferas....

Euforbiáceas

Euforbiáccils.

Poligaleas.

Cupulíferas.

Cupuliferis.

Cupuliferas.

Cupuliferas

Ciupulíferas.

Cupulíferas....

Cupulíferas....

Escrofularíneıs.....

Euforbiáceas....

Coníferas

Umbelíferas

Leguminosas.

Papaveráceas...

Papaveráceas......

Apocináceas.

Quenopodiáceas...

Quenopodiáceas...

Quenopodiáceas ..

Dipsáceas.

Compuestas.
Hidiallyo.

IIidinlgo.

fiunmijuilto .....

Vericluz, Pue...

Valle de México.

Guerrero, Ver...

IIidalgo....

Vericluz.........

Michoncín, Ver..

Sonola, Vel......

Guanajuallo .....

Ilidalgo....

Guamijuito, ele..

Ilirlalgo

Guermo

Veracius

Velicru\%

Golfo le Hexice

Vericrie........

Vericruz.........

Cihiluuahua .......

Midalgo, Ver.....

Hidalgo.

Iliclillgo.

Hidalgo.

Hiclialıro.

Mlichuacán, Ver. Guaniljualo, Oax. Hidlalgro............ Oaxaca, Hidalgo E. de Méx., elc.. Guan ijuato ...... Hidallgo.... Veracruz, Oax... Cililiuahua, N. L. Chilıuahua ...... Nuevo Leór...... E. de Mexico..... E. de Méxiro..... E. de lléx., elc... E. de México.... E. de Méx., Hgo. Oaxaca, Ver., ele. E. de México.... Dislrito Federil. Oixaca, Ver., elc. Jalisco............. Cultivida........ Sau Luis Polosí.. Michoncán....... Michoacál ....... Cinllivada......... Varios lugares... Varios lugares...

Distrito Federal. Rose. Cultivada........

Cultivada.........

Finul. Mex. Film. Mex.

Farm. Mex.

Fillm. Mex

Urbina.

Ciil. Inst. Med. Cat. Inst. Ned.

Barroso.

B. V. el Uibilla. Farm. Nlex.

Ullina.

Mat. Méd. 
Escila del país

Escoba amarga.

Escoba amarga

Escoba colorada.

Escoba colorada

Escoba colorada

Escobilla

Escobilla

Escobilla

Escobilla

Escobilla

Escobilla

Escobilla

Escobilla

Escobilla amarga

Escobillo.

Escobillo

Escobillo colorado.

Escorzonera

Escorzonera

Espadaña

Espadaña

Espanta-lobos ó Ispanta-raqueros

Espárrago

Espinaca.

Espino.

Espino blanco

Espino blanco.

Espino blanco.

Espino de Judea.

Espino herrero.

Espinosilla..

Esponjilla.

Espuela de caballero.

Estafiate.

Ester ó Esther.

Estoraque

Estrella

Estrella del mar.

Estrella de San Nicolás....

Estrellita

Estropajos.

Etzemo (tarasco).

Etzquáhuitl

Eucalipto ó Eucaliptus.....

Euforbia brillante.

Expujuj (maya)
Hymenocallis rotatr Herb....

Flaveria angustifolia Pers...

Milleria, sp? .

Dalea diffusa Moric.

Dalcu gracillima Wats.

Mimosa, sp?.

Aster panciflorns Null..............

Baccharis conferta II. B. K........

Baccharis heteroplylla H. B. K.....

Buddleiu scordioides H. B. K......

Millerie quinquaflord L.

Scabiosa atropurpurea L...........

Schlinhria abrotenoides Roth.......

Solidlago velutine DC.

Scoparia dulcis L.

Eugenia guayaquilensis DC........

Malpighio glabra L.

Malpighia, sp?

Picridium vulyare Desf.

Pinaropappus roscus Less..........

Typha angustifolice L.

Typha latifolia L...

Ipomece stans Cav..............

Asparagus officinalis L.............

Spinacia oleracea L...

Acacia paniculata Villa.

Acacia, sp?.

Mimosa: sp?.

Randia, sp?

Euphorbia, sp?

Randia, sp?......

Laselia coccinea Don.

Luffa operculata Cogn...............

Delphinium ajacis L...............

Artemisia mexicana Willd.........

Callistephus hortcrisis Cass........

Liquidambar styraciflua L.........

Milla biflora Ciav..

Nigella damascena L................

Hymenocallis rotata Herb..........

Gallinsoga parviflora Cav..........

Luffa cylindrica Roem..............

Schonocarulon officinale A. Gr. ..... Eucalyptus globulus Labill.......... Euphorbia fulgens Karw...........
Amarilídeas.

Compuestas.

Compuestas

Leguminosas.

Leguminosas.

Leguminosas.

Compuestas.

Compuestas.

Compuestas.

Loganiáceas.

Compuestas.

Dipsáceas....

Compuestas .........

Compuestas........

Escrofularíneas....

Mirtáceas.

Malyigiáceas

Malpigiáceas

Compuestas

Compuestas

Tifáceas....

Tifíceas.

Convolvuláceas....

Liliáceas............

Quenopodiáceas ...

Leguminosas........

Leguminosas........

Leguminosas........

Rubiáceas ..........

Euforbiáceas ........

Rubiáceas ...........

Polemoniáceas......

Cucurbitáceas ......

Ranunculáceas.....

Compuestas .........

Cimpuestas.........

Hamamelídeas......

Liliáceas

Ranunculáceas....

Amarilídeas........

Compueslas........

Cucurbiláceas......

Liliáceas..

Euforbiáceas........

Mirtáceas....

Compuestas ....
Valle de México. Farm. Mex. Oaxaca, Jalisco..

Valle de México. Farm. Mex.

Jalisco........... Urbina.

Jalisco............ Urbina.

Hidalgo, Dist. F.

E. de México.... Alcocer.

Valle de México. Urbina.

Valle de IIéxico.

Veracruz, Jalisco

Cultivada.

Oaxaca, Ver., etc.

Hidalgo, etc......

Varios lugares... Kunth.

Tabasco.......... Provirosa.

Tabasco.......... Rovirosa.

Tubasco......... Cat. Inst. Méd.

Valle de México. Urbina.

Valle de México.

Tabasco.......... Rovirosa.

Valle de México.

Valle de México.

Cultivada.........

Cultivada.........

Tabasco..........

Tabasco...........

Jalisco.............

E. de México.....

E de México....

Querétaro, etc

Méd

Guerrero........ Farm. Mex.

Gultivada.........

Valle de México.

Cultivada.........

Veracruz..........

Ghihuahua, etc..

Veracruz.........

Valle de México.

Morelos.....

Veracruz..........

Oaxaca.....

Cultivada.....

Oaxaca

Yucatán

N. León.

Colmeiro.

Farm. Mex.

Urbina.

Rose. Croton gossypiifolius Valıl. var.... Tagetes patula L.

Euforbiáceas ........

Cultivada........

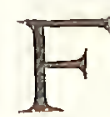

Falsa alcaparra

Falsa árnica.

Larrea mexicana Moric...

Zigofileas .

Sonora, S. L. P.

Compuestas .........
Valle de México. Mat. Méd. 
Falsa árnica.

Falsa árnica.

Falsa damiana.

Falsa quina.

Falsa quina.

Falso hipericón...

Falso zacatechichi.

Farolitos.

Felvira.

Filipéndula.

Flecha de agua

Flor de arete.

Flor de arete.

Flor de arete.

Flor de arete.

Flor de borla.

Flor de cabeza.

Flor de cacao.

Flor del camarón.

Flor del camarón.

Flor de la campana.....

Flor del cangrejo.

Flor de cera..

Flor de las cinco llagas......

Flor del clavo.....

Flor del corazón.

Flor del corazón.

Flor del corazón.

Flor de Corpus.

Flor de Corpus.

Flor del cuerno.

Flor del cuervo.

Flor de día.

Flor de encinillo.

Flor de encino..

Flor de estrella.

Flor del gallito

Flor de gloria....

Flor de hielo..

Flor de huevo

Flor de hueso..

Flor del incienso..

Flor de invierno.

Flor de invierno

Flor de Jamaica

Flor del látigo.

Flor de la manita

Flor de Mayo.

Flor de Mayo.

Flor de Mayo.

Flor de Mayo.

Flor de Mayo

Flor de Mayo.

Flor de Mayo

Flor del muerto

Flor del muerto
Heterotheca leptoglosa DC..

Trixis angustifolia DG.

Bigelovia reneta A. Gr.

Contarea latiffora DC.....

Exostemma earibceum Rom. el S.

Tagetes lucida Cav..

Conyza (lonnecia) filaginoides DG

Cardiospermum haticacabum L.....

Tinantia fugax Scheidw.

Chrysanthenum coronarium L.....

Sugitlaria sagitlifolia L

Fuchsia arborescens Sims

Fuchsia coecinea Ait..

Fuchsia corymbiffora Ruíz et Pav.

Fuehsia fulgens DC.

Vernonia schicdeana Less....

Stanhopea tigrina, Batem

Myrodia funebris Benth...........

Casalpinia pulcherrima Sw........

Jussiceu octofila DCi...

Cobcea scandens Cav...

Canna indica $\mathrm{I}$

Hoya earnosa R. Br..

Tagetes Iumulata Ort.

Choisya lernata H. B. K

Talauma mexieana Don.

Magnolia glauca Moc. et Sessé..

Iragnolia grandiftora L

Craniehis speeiosa Llav, et Lex..

Leelia grandifolia Lindl.

Cereus flegelliformis Haw....

Plumeria rubra L

Teeoma, sp?.

Zanlleoxylon, sp?

Tillandsia, sp?

Hymenoeallis rotata $\mathrm{II} \mathrm{erb}$

Sulvic patens Gav.

Solanum duleamara L. var......

Genliana spathacea H. B. K

Solanum ovigerum Dun

Polianthes luberosa L....

Iillandsia Timbata Schl?

Dahlia coccinea Clav.

Dahlia variabilis Desf.

Hibiscus sabdariffa L

Cerens flagelliformis Hw

Cheirostemon platanoides

Zepluyranthes earinatas $\mathrm{H}$. et B.

I'lumeria bicolor Ruíz el Pav......

I'lumeria trieolor Ruíz et Pav......

I'humeria prudica Jacr.

P'humeria purpurea Riuíz et Pav...

Plumeria mubra $\mathrm{L}$

Spreketia formosissima Herb.

Datura stramonium L

Dysodia chrysanthemoides Lag.....
Compuestas.

Compuestas .........

Compuestas .........

Rubiáccas ....

Rubiáceas ....

Compuestas.

Compuestas.

Sapindáceas.

Commelinácerıs....

Compuestas.

Alismáceas.

Onagrarieas...

Onagrariens...

Onagrarieas...

Onagrarieas...

Compuestis.

Orquícleas....

Esterculiáceas......

Leguminosas........

Onagraricas.........

Polemoniáceas.....

Zingiberáceas.......

Asclepiadeas........

Compuestas .........

Rutáceas

Magnoliáceas ......

Magnoliáceas .......

Magnoliáceas .......

Orquídeas.

Orquídeas.

Cácteas.

Apocináceas.

Bignoniáceas.

Rutáceas

Bromeliáceas.......

Amarilídeas........

Labiadas.

Solanáceas

Genci.nneas

Solanáceas

Amarilírtea.

Bromeliáceiss......

Compuestas.

Compuestas.

Malvácens.

Cácteas.

Esterculiácens.........

Amarilídeas.

Apocináceas

Apocináceas

Apocináceas

Apocináceas

Apocináceas

Amarilídeas

Solaníceas.

Compuestas
San Luis Polosí..

Querétaro, etc...

Valle de México.

Michoacán........

Lugares cálidos..

Valle de Mléxico.

Valle de MIéxico.

Tabasco.

Valle de México.

Cultivada.

Varios lugares...

Veracruz, etc.....

Ciultivada.........

Cultivarla........

Michoacán

Cihiapas, Ver....

Veracruz

Oaxisca, Ver.....

Guerrero .........

Tabasco...........

E. y V. de Méx...

Nalurilizida ....

Cultivada........

Valle de México.

Valle de Mléxico.

Morelos, México.

Morelos...

Cultivada........

Michoncán.......

Michoacán........

Varios lugares...

Morelos, Puebla.

.................

E. de MIéxico

Guanajuito, etc.

Cullivada........

E. de Méx., etc..

Cultivada........

Guanajualo .....

Veracruz..........

Varios lugares...

Varios lugares...

Cullivada...

Varios lugares...

E. de México....

Hiclalgo, Gto....

Ciultivada...

Cultivarla........

Gultivarla........

Cultivada.

Puebla, Morelos.

Varios lugares...

Varios lugares..

Contruila, elc...
Urbina.

Ulbina.

Upbina.

Ramírez.

All. et Ram\%.

Mat. Méd.

Rovirosa.

Uıbina.

Urbina.

Rovirosa.

Colmeiro, etc.

Mat. Méd.

Mal. Méd.

Colmeiro.

Ciolmeiro.

Urbina.

Urbina.

Golneiro.

Farm. Nex.

Alcocer.

Urbina. 
Flor de muertos.

Flor de muertos.

Flor negra.

Flor de nieve..

Flor de Nochebuena.

Flor de oro..

Flor de papagallo

Flor de Pascua

Flor de la pasión

Flor del pato

Flor del pelicano

lor de piedra

Flor de San Andrés...

Flor de San Cayetano........

Flor de San Diego.

Flor de Santo Domingo......

Flor de San Juan

Flor de San Juan.

Flor de San Pedro

Flor de San Francisco.......

Flor de Santa Rita...........

Flor de Santa María.........

Flor de Santiago.............

Flor de los santos.............

Flor del secreto.

Flor del soldado.

Flor del tigre.

Flor de tuna blanca

Flor de venadillo

Florecilla

Floricuerno

Floripondio.

Floripondio blanco

Floripondio rojo.

Fresa.

Fresno

Fresno

Fresno

Fresno

Fresno amarillo.

Fresno azul

Fresno blanco.

Frijol almendro.

Frijol amarillo

Frijol ayocote

Frijol ayocote morado......

Frijol blanco.

Frijol blanco.

Frijol colorado

Triol colorado bolita.

Frijol judio

Frijol meco

Frijol moro.

Frijol negro

Frijol parraleño.
Bletia campamulata Llav. et Lex.. Oneidium tigrinum Llav. et Lex... Yanille planifolia Andrews.

Gentiona calyeulata Llav. et Lex.. Enpharbia molcherrima Willd....

Chrysanthemem coronerinm l...... Piscillia erythrina I.

Euphorbia pulcherrima Willd......

Pressiftora edulis Sims...............

Aristolochia grandiflora Sw.......

Cypripedium irapermum Ll. et l...

Seluginella mprestris Spring.......

Lennoa madreporoides Lailv. et L.

Lentuma hirsuta Mart. et Gall.....

Antigonon cordutum Mart. et Gall.

Gentiana eulyeulata Llav. et Lex.

Honstonia longiflora A. Gray.......

Maerosiphonia hypolenea Müll. A.

Tecoma mollis H. B. K.....

Comphrena globosa L.

Petrece volubilis Jaca.

Tragetes lucida Cav....

spreleclie formosissima Herb.

Lelia antumnalis Lindl............

Cassia alata L....

Cestrum elegans Schl...

Tigridia pavonia Ker.

Opuntia tuna Mill ?...

Swietenia hmilis ? Zucc.

Helenium quadridentatum Labill.

Cereus flagelliformis Haw.

Datura arborea L....

Datura suareolens Humb. et B....

Datura samguinea Ruíz et Par....

Fragaria mexicama Schl.

H'raximus amerieana L ?............

Fraximus juglundifolia Litm.......

Fraximus schiedleana Chan, et S..

Freximes vividis Micl..............

Fraximes, sp?...

Hraxinus, sp?.

Fraxinus, sp?

Phuseolus, sp?

l'huseolus vulgaris L.

Phaseolus, sp?

Phaseolus multiflon

Pherseolus mulliflorns Willd........

Phaseolus lunatus L.

Phasenhes vulgaris L. var..........

l'haseohes vulgaris L. var'..........

I'huseolus velguris $\mathrm{L}$. var...........

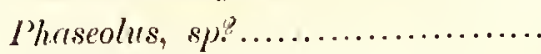

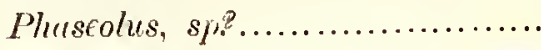

Phuseolus, sp.?.

Phaseolus vulgaris L. var...........

Phaseolus vilgaris L. var..
Orquideas

Orquídeas

Orquídeas

Gellcianeas.

Euforbiáceas

Compuestas

Leguminosas........

Euforbiáceas........

Pasifloráceas.

Arisloloquieas.....

Orquídeas ...........

Selagineláceas.....

Lennoáceas.........

Verbenáceas ........

Poligonáceas ........

Gencianeas.........

Rubiáceas ..........

Apocináceas........

Bignoniáceas.......

Amarantáceas......

Verbenáceas .......

Compuestas. ........

Amarilídeas........

Orquídeas ...........

Leguminosas........

Solanáceas .........

Irideas.....

Cácteas.

Meliáceas

Compuestas.

Cácteas.

Solanáceas

Solanáceas

Solanáceas

Rosáceas

Oleáceas.

Oleáceas.

Oleáceas.

Oleáceas.

Oleáceas

Oleáceas...

Oleáceas.............

Leguminosas.. .....

Leguminosas.......

Leguminosas...

Leguminosas.........

Leguminosas........

Leguminosas........

Legıminosas........

Leguminosas.......

Leguminosas.......

Leguminosas........

Leguminosas......

Leguminosas........

Leguminosas........

Leguminosas........
Veracruz..........

Michoacán........

Veracruz..........

Michoacán........

Sonora, Chil.... Far. Mex

Cultivada.........

Lugares cálidos..

Varins lugares...

Cultivada.

Jalisco, Veracruz

Veracruz, Chiap.

D. Federal, etc...

Michoacán........

Veracruz, Puebla

Veracruz, Oax...

Michoacán........

Puebla, etc...... Farm. Mex.

S. L. P., Jalisco.. Uibina.

S. L. P., V. $d \in$ M..

Guerrero

Tabasco

Dist. F., Jalisco..

Guanajuato, Pba.

Oaxaca, Mich...

Yucatán .

Veracruz.

Varios lugares...

Mesa Central....

Sinaloa ?..........

Tabasco..........

Varios lugares....

Jalisco, Veracruz

México ............

E. de Méx., Mich.

Cultivada.

Cultivada.........

Cultivada.

Veracruz.

MesaCentral,ete. Farm. Mex.

Cultivada....

Cultivada....

Cultivada....

Cultivada....

Gultivada....

Cultivada.

Cultivada....

Cultivada.

Cultivada....

Cultivada....

Cultivada....

Cultivada.

Cultivada.

Cultivada....

Unhina.

Alcocer.

Rovirosa.

B. V. et Urbina.

Far. Mex.

Farm. Mex.

Rose.

Rovirosal.

Fallu. Mex.

( colneiro.

Rose.

.

...

........

....... 
Frijol prieto.

Frijol prieto garrapata.....

Frijolillo

Frijolillo

Frijolillo

Frijolillo

Frutilla .

Frutilla

Fulmina.

Fumaria

Fuscia

Fuscia

Fuscia

Fuscia
Phaseolus vulgaris L. val.....

Phaseolus, sp?

Camavalia villosa Benth............

Lathyrus, sp?

Phaseolus multiflon Willd .......

Sophora secundiftora Lagg....

Arctostaphylos, sp?.

Canna indica L.

Antigonon leptopus Hook el Arn..

Fumaria parviflora Lam.

Fuchsia arborescens Sims.

Fuchsia coccinea Ait.

Fuchsia corymbiftora Ruíz et Pitr.

Frehsica fulgens Dr..
Leguminosas.......

I.eguminosas........

Leguminosas.......

Leguminosas.......

Leguminosas.......

I.rguminosas........

Ericácens

Zingiberáceas......

Poligonáceas .......

Fumariáceas........

Onagrat icas.........

Onagrarieas........

Onagririens...

Onagraricas....
Cultivarla

Cultivarla.

Tabasco

Hidatgo, Oaxaca

Nuevolseóu, elc. Valle de México.

Naluralizada....

Morelos

Naluralizada ....

Mlichoacán, Ver..

Cultivada........

Cultivada...

Miclioarán.....
Povirosa.

B. V. et Uhina.

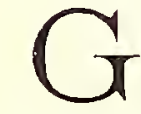

Gabía

Gallitos

Gallitos

Gallitos

Garabato.

Garambullo

Garambullo

Garambullo.

Garbancillo.

Garbanzo

Gardenia

Garrapata ó Garrapatilla...

Gateado.

Gayuba del pais

Gelsemio.

Genciana...

Gengibre.

Geranio

Geranio de olor ó G. rosa..

Geranio de la reina.

Gigante

Gigantón

Girasol de agua

Girasol amarillo

Girasol morado.

Girasol rojo.......

Gitomate.

Gitomate guajito

Gitomate redondo.

Globularia.

Gloria

Gobernadora

Gobernadora de Mézico.

Gobernadora de Puebla.
MTimosa, sp?...

Cynodon dactylon Pers...

Tillandsia brachycaulos Schl.....

Tillandsia recurvata $\mathrm{L}$....

Mimosa, sp?

Arctostaphylos arguta Zucc.

Cereus geometrizans Mart....

Rosa monterumce Red.

Lupinus eleguns H. B. K...........

Cicer arietinum $\mathrm{I}$

Gardenia forirla I.

Symplocos martinicensis Jar.q......

Srcietenia humilis Zuce?

Arctostaphylos pungens H. B. K...

Gelsemium sempervirens Ait

Gentiana, spe.

Zingiber officinale Rosco

Geranium robertianum L

Pelargonium odoratissimun Ait....

Pelurgonium speciosum Willd.

Encalyptus globulus Labill.

Helianthus annums L......

Bidlens heliunthoides H. P. K.......

Cosmos sulphweus fiav... .........

cosmos purpureus Benth.

Jycopersicum esculentum Mill.

Lycopersicum pyriforme tru.......

Iycopersicum cerresiforme 1) III.....

Globularia vulgaris L....

solenum dulcremare L. vilP....

Coviller tridentata (DC), Vail.....

Larrea mexicana Moric.

Brickellia reroniccefolia $A$. Gi.....
Cosmos bipinnatus Gilv....
Leguminosns.......

Círamíneas.

Bromeliácens.

Bromeliaíccas

Leguminosas......,

Ericáceas.

Cácteas

Rosáceas ....

Leguminosas.

Leguminosas.......

Rubiáceas

Estiráceas.

Meliáceas.

Ericáceas....

Loganiácens....

Genci.ulears ar.....

Zingiberáceas......

Gieraniácciss. ......

Geraniáceas........

Geraniáceas........

Mirtáceas.

Ciompuestas.......

Ciompuestas.......

Compuestas

Compuestas

Compuestas

Solanáceas

Solanáceas.

Solanáceas

Selagineas.

Sol.nnáceas

Zigofilens....

Zigofileas....

rompuestas
Varios lıgares...

Tabasco.......... Povirosa.

Valle de Mćxiın.

Jalisco............

Y. de Méx.. Mr.. Mat. Mérl.

Hidalgo.......... Rose.

Varios lugares...

Varios lugares...

Cultivada........

Cultivada........

Veracril..........

Veracruz..........

Hidalgo, Dist. F.

Frontera N., et.

Puebla.....

Cultivada.........

(:ultivada.........

Cultivada.........

rillivada.........

Cultivada.........

Cinltivarla........

Valle de Mrixico.

Norelos, Jaliseo..

$\checkmark$. de Méx.. Jal..

Morelos, Julisero..

Cinltivada.......

Cinltivada...

riultivada.

rinltivada.........

Cinllivarda........

Acaponeta.......

S. L. P., Sonori.

V. de Méx., Jgon..
B. V.el Urbina.

B. $r$. el Urbina.

B. Y.el Urbina.

Pinse.

Farm. Nos. 
Goco dulce.

Golondrina.

Golondrinera

Gordolobo

Gordolobo

Gordolobo del monte ........

Gordolobo del país.

Gordolobo del país.

Gordolobo de la sierra......

Grama.

Grama

Grama de agua.

Grama de pie de polla......

Gran cuerno

Gran duque

Granada ó Girinadila de China

Granadillo

Granadillo...

Granadillo

Granadita fétida

Granado.

Granado dulce

Grano de oro

Grano de oro

Granjeno.

Guacanalá.

Guácima ó Guácimo

Guácima.

Guaco.

Guaco.

Guaco.

Guaco.

Guaco de Gurtemila ó de 'Tab.

Guaco de Jalapa

Guacóporo

Guacoyul

Guachilli.

Guachilli

Guajaje

Guaje...

Guajilote

Guajilote.

Gualda.

Gualdaperra..

Guamachi.

Guamara.

Guamis

Gruanábana ó Guanábano..

Guano de sombrero..........

Guapaque .....................

Guapilla...

Guapilla

Guapilla

Guapilla.

Gruardalagua

Guarima, fillarumbo if fillarmma......
Salacia elliptica Peir...

Pilea microphylla Liebm.

Chelidonium majus Mill..

Graphalium canescens DC.

Gnaphaliun sphercelalum H. B. K.

Terbesina virgula Cav.

Gnaphalium berlandieri DC.......

Gnaphatium conoideum H. B. K...

Solidlıgo, sp?.

Cynodon dactylon Pers.

Paspalim pusillum Vent...........

Oplismenus crus-galli Kunth......

Cumodon elactylon Pers....

Cereus, sp...........................

Jasminum sambac Ait.

Passiflora edulis Sints.

Brya ebenus DC.?.

Nesere salicifolia H. B. K

Pithecolobium multiflorum Benlh...

Petsisflora sexflore Juss.

Punica grenatum L.

Punica gronatum L.................

Galphimia glandulosa Cav.........

Spilunthes mutisii H. B. K...........

Celtis pallide Torrey.................

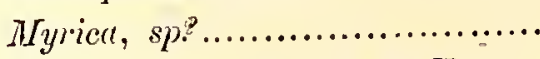

Gnazuma tomentosa H. B. K......

Guctzuma polybotiya Cav.....

Aristolochia odoratissima L..........

Mikania gonoclada DG.

Mikania guaco Humb. et Bonpl...

Mrikania scandens Willd...........

Mikania graco Humb. et Bonpl..

Mikania denticuluta Willd.........

Purkinsonia aculeuta L.

Cocos guacoyule Liehm...

Bocenia arborea IVials

Bocconia frulescens $\mathrm{J}$.................

Solidago, $s p q^{2 . . . . . . . . . . . . . . . . . . . . . . . ~}$

Leuccena esculentu bentli...........

Parmentiern eddutis DC...

Pseudosmodingium proniciosum E.

Resecla luteola 1 .

Digitalis purpurea L................

Pithecolobium dul.r Benlh........

Bromelia pinguin L.................

Larvea mexicana Moric............

Anona muricala L....................

Coryphe sp?

Ostrya virginica Willd

gave falcata Engelm..............

Agreve striata Zir..................

Hechtia ghiesbreghtii, Lem.ıire.....

Hechtic glomereter. Zuce...

Rhus toxicodendron L..............

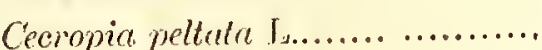

Celastrineas

Urticáceas

Papaveráceas.......

Compuestas .........

Compuestas.

Compuestas.

Compuestas........

Compuestas .........

Compuestas .........

Gramíneas..........

Gramíneas....

Gramíneas

Gramíneas

( 'ácteas..

Oleácens ............

Pasifloráceas.......

Pasifloráceas........

Leguminosas.......

Leguminosas........

Pasifloráceas.......

Litrarieas............

Litrarieas....

Valpigiáceas........

Compuestas .........

Urticáceas ...........

Miricáceas ...........

Esterculiácens......

Esterculiáceas ......

Aristoloquieas......

Compuestas........

Compuestas. ........

Compuestas........

Compuestas.........

Compuestas.........

Leguminosas........

l'almeras............

Papaveráceas......

Papaveráceas......

Compuestirs.

Leguminosit........

Bignoniáceas ........

Anacardiáceas......

Resedáceas ........

Escrofularínea-.....

Leguminosas........

Bromeliáceas .......

Zigofileas ............

Anonáceas ..........

Palmeras...........

Cupulíferas.........

Amarilídeas........

Amarilídeas........

Bromeliácéss.....

Bromeliáceas.....

Anacardiáceas .....

Urticáceas ...........
Tabasco

Tabasco......

Cultivada.........

Varios lugares...

Jalisco....... ...

Varios lugares...

S. L. P., V. de M. Farm. Mex.

S. L. P., V. de II. Farm. Mex.

Puebla.

Varios lugares.

Tabasco.

Tabasco.

Varios lugares...

Jalisco............

Cullivada.

Lug. calientes...

Oaxaca...........

Varios lugares...

Lug. calientes...

Jalisco, Veracru\%

Cullivada.

Cultivada.........

Jalisco, S. L. P...

Tabasco..........

Chihuahua, etc..

Lug. calientes....

Lug. calientes....

Lug. calientes....

Región Sur.....

Veracruz, ete.....

Jalisco............

Lugares cálidos..

Tabasco.

Verncruz..

Sonora, Tilll.. \&

Oaxaca. ..........

Lug. calienls....

Lug. calienles....

México, Morelos.

Oaxaca...........

Guerrero, Mor...

Naluralizadi ....

Cultivarla........

Guerrero, elc....

Jug. calienles...

S. Luis Potuxí, «

Chiapas ...

Tabasco.

Tabasco.

Jalisco...

Jalisco.............

Querétaro..............

Querétaro, ete...

Varios lugares...

Tabasco, Chiap..
Alt. et Ramz.

Rovirosa.

B. V. el Urbina

F. M., B. V. et U.

Rovirosa.

Rovirosa.

B. V.et Urbina.

Ramírez.

Urbina.

Ramilez.

Farm. Mex.

Rovirosa.

Cat. Inst. Méd

Farm. Mex.

Urbina.

B. V. et Urbina.

Cat. Inst. Med.

Pamfrez.

Barroso.

Ramílez.

Cat. Inst. Méd.

Cat. Inst. Méd.

Alt. et Ramz.

Rose.

Rose.

Mat. Méd.

Ciil. Inst. Med.

(.. ]. M., Rov. 
Guarumbo

Guatope.

Guau.

Guau

Guayaba ó Guayabo.........

Guaya de bajo

Guaya de cerro.

Guayabillo.

Guayabillo.

Guayabo agrio

Guayabo de las sabanas...

Guayabo rojo y blanco....

Guayacán

Guayacán

Guayacán

Guayacán

Guayacán

Guayacán

Guayita.

Guayo.

Guázima ó Guázuma........

Guázima ó Guázimo.........

Guázima

Guía de jazmincillo.........

Guinda ó Guindo..

Guineillo prieto

Güiro

Güiro de Yucatán.

Guisante

Güizapol de borrego

Guoguo.

Gusanillo.

Gusano amarillo

Gusano prieto.
Cecropia mexicana Hemsl.

Inga spuria Humb. et Bonpl......

Rhus toxicodendron 1 .

Iitis quinquefoliu Michx....

Psidium pomiferum L..............

Chamedorect ernesti-augnesti Wenl.

Chamedoren elatior Mart...

Cordia dodecrndra A. DC.....

Mlelpiglia, sp? ......

Myrtus, sp?.....

Psidium pomiferum $\mathrm{L}$.

Psidium pomiferum L....

Guninenm coulteri A. Gir.

Guaiacum verticale Ort.

Guaiacum officinali $\mathrm{L}$.

Gucciacum sanctum I

Guazuma ulmifolia lamk.

Tabebuia guayacan Hemsl.

Chamcedoren elatior Mart.

Melicocea bijuga. L.

Guazuma polybotrya Cav

Guazuma tomentosa H. B. K.......

Gueazuma ulmifolia Lamk.

Solconem dulcrmara $\mathrm{L}$. var........

Cerasus capioniana DC.

Cymbopetalon penduliftorum Baill.

Parmentierc alata Miers.

Crescentia crijete L

Pisum sativum $\mathrm{J}$.

Triumfetta, sp?

Salasia elliptica Peyr.

Lobelia fenestralis Cav.

Lonchneorpus. sp?

Lonchocerpuss sericeus H. B. K.....
Urlicáceas ....

Leguminnsas........

Anacirdiáceas....

Ampelícleas

Mirtáceas....

l'almeras

Palmelas....

Borragíneas.....

Malpigiáceas....

Mirtáceas.

Mirtáceas.

Mirtáceas.

Zigofileas

Zigofileas

Zigofieas

Zigofileas....

Esterculiácras ....

Bignoniácens........

Palmeras ...........

Sapindàceas.........

Esterculiáceıs ....

Esterculiácuas.....

Eslerculidereas.....

Solanáceas...

Rosáceas . .

Anonácens

Bignoniácers......

Bignoniáceas.......

Legumin osas.......

Tiliáceas.............

Cielastrineas .........

Lobeliáceas

Leguminosas.

Legunıiıosas....
Valle do México.

'Tabasco..........

Varios lugiales...

San L Inis Polosí..

Jalisco, Mol', elre

Tabasco. .........

'Tabasco...........

Verncruz, cte.....

Michoacán........

T'abiseo.

Vericruz, Gro....

Velicruz, Gro....

Sonora ............

Jalisco, Méxic:o..

Veriacruz ?.........

Oaxalar ..........

Jalisco

'Pabasco, Minh...

'labasco ..........

Tabasco.

lugr. entlenles...

lug. calientes...

lug. calientes...

Cultivada.

Cultivada...

Oaxaca.

Sinaloa, Gro......

Yucilán.

Cultivadi...

Jalisco...

'labasco.....

E. de Méx., Jall..

Thbasco

Tibilseo
All. ol Rialluz.

(ait. Just. Méd.

Irinal.

Giit. Inst. Med.

Ciit. Inst. Méd.

villada.

Giit. Inst. Méd.

Ciit. Inst. Mled.

Farm. Mex.

Farm. Nex.

Rose.

B. V. et Utbillil.

Ramíre\%.

Barroso.

liose.

('ilt. Inst. Méd.

Rovirosa.

NIt. et Rilluz.

Vial. Inst. Hed.

Ramíle\%.

Urbina.

Rovirosal.

Unbina.

Gilt, Inst. Med.

Rovirosa.
$\mathrm{H}$

Haba

Haba

Haba de indio...

Haba de la costa .............

Haba de San Antonio.......

Haba de San Ignacio........

Habichuela

Fiabilla.

Hachichinoa

Hachinal ó Fauchinal.....

Hachogue.

Hàuchinal.

Hauchinol ó Hauchinoli...

Hauchinol ó Hauchinoli.
Fintuda scandens Benlh.

Vicia faba L

Hurc crepitens L. ....

Entada scandens Benth.

Casalpiniu bonducella Roxh....

Hure crepituns I

Phaseolus nulgaris I

Hura crepiturs L.

Tournefortia, sp?

Nesrea syphilitica DC.

Piper pulmeri (. Dr:

Nesced salicifolin II. B. K

Nescea salicifolia H. B. K.

Nescen syphilitica DC.
Leguminosas

Leguminosas...

Euforbiáceas

Leguminosas.

Leguminosas

Euforbiáceas

Leguminosas

Luforbiáceas

Borl'agíneas...

litrallinas

Piperáceas.

Lit]arieas.

Litrarieas.

Litrarieas.
Tabnsco....

Ciultivada........

sonora...........

'labasco..........

Veracru\%..........

Morelos, (iro....

Cullivada...

Morelos

E. de México....

Norelos, Méxiro.

Colima ............

Morelos, México.

Morelos, México.

Morelos Méxiro.
Rovirosa.

Colmeino, Rose

Dugés, F. M.

Colmeiro. F.N.

liose.

Farm. Mes.

Finm. Mex.

Farn. Mex. 
Haya.

Haya.

Hediondilla

Hediondilla

Hediondilla

Hediondilla..

Helecho hembra

Helecho macho.

Heliotropo

Heliotropo del cerro.

Heliotropo cimarrón

Heliotropo cimarrón

Henequén

Henequén blanco..

Heno.

Heno largo.

Heno pequeño.

Hicaco.

Hiedra extranjera...........

Hiedra morada..............

Hiedra roja

Hierba del aguardiente....

Hierba del alacrán...

Hierba del alacrán...... ....

Hierba de la alferecía.. .....

Hierba amargosa............

Hierba amarilla.

Hierba del ángel... ..... ....

Hierba del ángel.............

Hierba del ángel...

Hierba de las ánimas.. . ...

Hierba de San Antonio ....

Hierba añil.

Hierba añil. ................

Hierba de la barranca. ....

Hierba del becerro ..........

Hierba del becerro ..........

Hierba buena.. ..............

Hierba buena.. ..............

Hierba buena.. ....... .....

Hierba buena.. ............

Hierba buena................

Hierba del bney .............

Hierba del buey.............

Hierba del buey.............

Hierba del burro............

Hierba del burro .. ..........

Hier'ba cabezona

Hierba del cáncer.

Hierba del cáncer.

Hierba del cáncer.

Hierba del cáncex.

Hierba del cáncer.

Hierba del cáncer.

Hierba del carbonero.

Hierba del carbonero
Cesalpinia mexicana A. Gr.

Platanus, sp?

Cestrum roseum, H. B. K

Chenopodium vulvaria L..........

Heliotiopinm curassavicum L......

Senecio sinurtus H. B. K.

I'teris aquilina L

Ncphrodium filix-mos Rich........

Heliotropium peruvianum L........

Heliotropium corymbosum R. et P?

Heliotropium curassavicum L......

Heliotropium limbatum Benth......

Agave rigida Mill.

Agave americana L................

Tillundsia usneoides L.

Tillendsia usncoides L..............

Tillandsia recurvata $\mathrm{L}$.

Chrysobalumus icaco L

Hedera helix L.....

Cobrea scandens Cav.

Ipomen quamoclit L.

Viola odorata L.

Plumbago pulchella Boiss.

Plumbago scumlens L...

Cereus flagelliformis Haw.

Ambrosia artemisicefolia L..........

Heliopsis buphthalnoides Dun......

Calliaudra grandiflora Benth......

Eupatorium collinum DC.

Eupatorium, sp?......

Helenium mexicanum H. B. K......

Sulmer grandiceps Calss............

Tugetes lucida Carv.

Jacobinia mohintii Hemsl.

Flaveria angustifolia Pers..........

Brickellia cavanillesii A. Gr ........

Calea scabrifolia Benth. et Hook.

Enpatorium, sp?

Hedeoma piperita Benth............

Mentha rotundifolir. L.

Mentha viridis L

Micromeria douglasii Benth.......

Catssia, sp?.........................

Solanum, sp?......................

Titis incisa Nutt.

Schonocculon, sp?..

Spigelic longiflore Miat. et Gal.

Helenium ooclinium A. Gr.........

Acalypha plleoides? Cilv.

Castilleju tenuiflora Benth.........

Gomphrena globosa L...............

Lythrum alutum Pursh.. ..........

Lythrum album H. B. K...........

Salvia indica L.

Baccharis alcmani DG..............

Baccharis conferta H. B. K........
Leguminosas........

Platanáceas

Solanáceas ..........

Quenopodiáceas ...

Borragíneas.

Compuestas ........

Helechos

II elechos ....

Borragíneas........

Borragíneas.........

Borragíneas.........

Bor rigíneas.........

A marilídeas.........

Amarilídeas........

Bromeliáceas......

Bromeliáceas .......

Bromeliáceas. ......

Rosáceas

Araliáceas.

Polemoniáceas ....

Convolvuláceas....

Violáceas ............

Plumbagílleas .....

Plumbagíneas ......

Cácteas..............

Compuestas........

Compuestas.........

Leguminosas........

Compuestas........

Compuestas. ........

Compuestas ........

Compuestas .........

Compuestas ........

Acantáceas..........

Compuestas. ........

Compuestas........

Compuestas. ........

Compueslas .........

Labiadas.

Labiadas. ...

Labiadas.

Lubiadis............

Leguminosas........

Solnnáceas ..........

Ampelídeas ........

Liliáceas.

Loganiáceas.

Compuestas.......

Euforbiáceas........

Escrofularíneas....

Amarantáceas......

Litrarieas..........

Litrarieas...

Labiadas.

Compuestas

Compuestas.
Chihuahua, etc.. Alt. et Ramz.

Nuevo León..... Alt. et Ramz.

Hidalgo, Oaxaca

Introducida......

Mesa Central.....

Guanajuato, etc..

Varios lugares...

Varios lugares...

Cultivada.........

Jalisco............

Mesa Central.....

Veracruz..........

Yucatín

Vurios lugares...

Varios lugares...

Jalisco.

Jalisco......

Yucatán

Cultivada.........

Valle de México.

Varios lugares..

Jalisco........... B. V. et Urbina.

Zacatecas, etc... Mat. Méd.

S. L. P., Gto.... Farm. Mex.

Varios lugares... Golmeiro.

S. L. P., Jalisco.. Urbina.

Querétaro, etc... Urbina.

Varios lugares... Mat. Méd.

Varios lugares...

E. de México....

Varios lugares...

Puebla, Veracruz

S. L. P., V. de M.

Varios lugares...

Jalisco, Oaxaca.

S. L. P., V. de M.

México, etc......

Valle de México.

Hidalgo.

Nalluralizada .....

lultivada

Birja California..

Chihualıua......

Valle de México.

Cilihuahua, Son.

Norte de México

Hidalgo...

Chih., Qro.......

Jalisco

S. L. P., Ghih.....

Naturalizada....

México, Puebla.

México, Puebla.

Cullivada........

Valle de México.

Valle de Méxieo.
Colmeiro, F.M.

B. V. et Urbina.

B. $V$. et Urbina.

Cat. Inst. Med.

Cat. Inst. Med.

Rose.

Cat. Inst. Méd.

Mat. Méd.

Cat. Inst. Méd.

Cat. Inst. Med.

Farm. Mex

Rose.

Urbina.

Farm. Mex.

Alcocer.
Mat. Méd. 
Hierba del carbonero.

Hierba del carbonero.

Hierba del clavo.

Hierba de Corpus..

Hierba del coyote..

Hierba del coyote..

Hierba de la Cruz..

Hierba de la cucaracha....

Hierba de la cucaracha....

Hierba del cuiji...

Hierba del cura.

Hierba del cura.

Hierba del cura.

Hierba de la doncella.

Hierba de la doncella.

Hierba dulce.

Hierba dulce.

Hierba dulce de México..

Hierba escarchada...........

Hilerba del espanto......

Hierba de la flecha...

Hierba de las gallinitas....

Fierba del gato.

Hierba del gato.

Fierba do la Coberuadora de Puebla.

Fierba de la golondrina.

Hierba de la golondrina

Hierba de la golondrina

Hierba de la golondrina

Flierba de la golondrina estranjera ....

Hilerba del gol je.

Hierba del golpe..

Hierba hedionda.

Hierba del histérico.

Hierba del indio.

Hierba del indio

Iilerba del indio.

IHierba del jabalí.

Hierba de San Juan.........

Hierba loca.

Hierba loca.

Hierba Luisa

Hierba lumbre

Iierba del manso.

Hierba Martín.

Hierba de las mataduras

Hierba mora.

Hierba de la mula

Hierba de la mula

Hierba del mulato

Hierba del negro...

Hierba de San Nicolás

Fierba de San Nicolás

Hierba de San Nicolás

Hierba de San Nicolás

Hierba del oso
Baccharis mucronata H. B. K...... Baccharis multiflora H. B K...... Choisye ternata H. B. K.

Lcelia grandiffora Lindl. Euphorbia campestris Chan. el S. Polanisia uniglandulosa Cav....... Brickellia, sp?.

Macrosiphonia hypolence Müll....

Truchelospermum stans A. Gr.....

Aristolochia, sp?.

Iernstromia Altamirania Schiede

Ternstrcemia sylvatica Ch. el Schl.

Ternstrcemia tepezapote Ch. et Schl.

Begonia gracilis H. B. K.

Begonia tuberosa Moc. et Sessé...

Lippia dulcis Trevir....

Lippia graceolens II. B. K

Brickellia cavanillesii A. Gr.....

Mesembryanthemum christulinum L.

Micromeria brownei Benth.........

Sebastianir Palmeri Rosi.

Petiveria allincea $\mathrm{L}$

Valerina mexicana DC.

Veleriana tolucana DG.

Brickellia veronicafolia A. Gr.....

Euphorbia muculata L.

Euplorbia mendezii Boiss...

Euphorbia prostrata Ait..

Enphorbia thymifolia Burm...

Chelidonium majus Mill.

Allionia incarnata $\mathrm{L}$.

Enothera simsiana Ser.

Cestrum noctu'num L

Conyza, sp?

Aristolochia fretide H. B. K.........

Aristolochia mierophylla Will.1....

Aristolochica outoratissima I

Cioton maritimus, Walt

Verbena, sp?.....

Croton fruticulosus Tor'r. valt.....

Oxylropis lamberti Pursh.

Lippice citriodor H. B. K.....

I'lumbago pulchella Boiss....

sputhiphyllum, sp??....

Hyptis verticilluta, Jacy

Talauma mexicana Don.

Sollennm nigrum L....

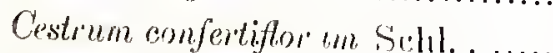

Monnina Ocompi Herr. et Vill.

Tomentortire, sp?.....

Spheralcert angustifolia St I Iill

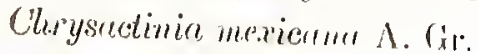

Piqueria trinervic Cilv.

Sedum suxatile Willd.

Ionidium angustifolium H. B. K...

Arracacia atropuppurea B. of H...
Compuestas.

Compuestas.

Puláceas

Orquídeas

Euforbiáceas

Caiparideas

Compueshis.........

Apocináceas.

Apocináceas.........

Arisloloquieas......

Ternstrœmiáceas ..

Ternstrœmiácnas..

T'ernstrœmiáceis .

Begoniáceas .........

Begoniáceas........

Verbenáce:ıs .......

Verbenáceas ........

Compuestiı.........

Ficoideas

Labiadas....

Euforbiáceas

Fitolacáceas

Valerianeas

Valerianeas

Compuestas.

Euforbiáceas.

Euforbiáceas. .

Euforbiáceas

Euforbiáceas ........

Papaveráceas......

Nictagineas ........

Onagrarieas.

Solanáceas

Compuestas...

Aristoloquieas......

Aristoloquieas......

Aristoloquiea =.....

Euforbiáceas....

Verbenáceas...

Euforbiáceas.

Leguminosil=...

Verbenáceas

Plumbagineas.

Aroideas.

Labiadas...

Magnoliáceas

Solanáceas

Solanáceas ...

Poligaleas.

Borrogíneas

Malváceas

Compuestas.

Compuestas

Crasulácerts

Violarieas.

Umbelíferas.
México, S. I. P..

E. de México....

S. L. P., V. de M. Farm. Mex.

Michnacán.......

Hidalgo, S. L. P.

Nuevo León, elc.

Hidalgro.

S. Luis Potosí, \&

México, Cllih....

Jalisco............

Veracruz...

Veracruz..........

Sinaloa, Mrrelos

V. de Méx.. Puel

V. de Méx., Pueb

Jalisco, Verarrur

Hidalgo, Ver.....

Varios lugares...

Cultivarla........

Veracruz.........

Sonora

Oaxaca, Yuc......

Valle de Mćxico.

S. Luis Polosí, \&

Puebla

Jal., N. de Méx...

Jalisco, Gto., etu'.

Valle de México.

Veracruz

Cullivada...

Valrios lugarr's...

E. de México....

Varios lugilres...

Oaxaca............

Guanajuato, etr..

Guanajuato, ete..

Jalisc'o.....

Veracruz .........

Valle de Méxiro.

Chihuahua......

Chihuahua ......

Cultivada.........

Zacatecas, Oax.

Tabasco

Morelos, México.

Varios lugares...

Valle de Móxico.

Valle de Méxion.

México

Varrios lugares ..

San Luis Polosí.

$V$. de Méx., Jall...

Tamaulipas.....

Varios lugares...
Cat. Inst. Méd.

Cat. Inst. Med.

Cat. Inst. Méd.

Firm. Mex.

Urbina.

Oliva.

Colmeiro, F. MI.

Finm. Mex.

Unina.

Colmeiro, F. M

Colmeiro, F. M.

Farm. Mex.

Farm. Mex.

Upbina.

Ciit. Inst. Méd.

Colmeiro.

F. H., B. V.et U.

Upbina.

Uıb., Ramírez.

Ciit. Inst. Med.

Colm., F. M.

Firm. Mex.

B. V. et Urbina.

Uibina.

Cat. Inst. Méd.

Alcocer.

Malt. Mérl.

Rovirosa.

Farm. Mex.

Villatia.

Cat. Inst. Med.

Firnı. Mex.

(iil. Inst. Med.

Mat. Méd.

Colmeiro.

Villada.

Cat. Inst. Méd. 
Hierba del pájaro.

Hierba de la paloma

Hierba del pastor...

Hierba de San Pedro

Hierba de la peña.

Hierba de las perlas

Hierba del perro

Hierba del perro

Hierba del perro

Hierba de la playa

Hierba de la playa

Hierba del pollo.

Hierba del pollo

Hierba del pollo

Hierba del pollo

Hierba de la princesa .......

Hierba de la Puebla.

Hierba de la pulga.

Hierba de la pulga

Hierba de la punzada

Hierba de la punzada.

Hierba de la punzada........

Hierba de la rabia...........

Hierba de las reumas.......

Hierba de rosa.

Hierba de Santa Rosa.......

Hierba del rosario.

Hierba del Sagrado Corazún de Jesír...

Hierba santa

Hierba de Santa María.....

Hierba de Santa María ....

Hierba del sapo.

Hierba del sapo.

Hicrba del sapo.

Hierba del sapo.

Hierba del sapo.

Hierba del tabardillo

Hierba de Tata-Lencho...

Hierba de Tata-Lencho.. ..

Hierba del toro.................

Hierba de la Trinidad .......

Hierba del venado............

Hierba de la víbora

Hierba de la víbora

Hierba de la Vírgen..........

Hierba de la viruela.........

Hierba de la viruela ........

Hierba del zopilote ...........

Hierba del zopilote ............

Hierba del zopilote ...........

Hierba del zopilote

Hierba del zorrillo

Hierba del zorrillo...........

Hierba del zorro.

Hierbabuena.....

Hiierbabuena cimarrona...
Anagalis arvensis $\mathrm{L}$.

Calea urticcefolia $\mathrm{DC}$.

Acalypha phleoides Cav. vir........

Buddleia, sp?.

Cheilanthes lindheimeri Hook.....

Lithospermum, sp?.

Cestrum hartwogii Dun..............

Solanum candidum Lindl...

Solanum Cervantesii Lag....

Buddleia verticillata H. B. K. (?)..

Salvia lencantha Calv....

Commelina pallida IVilld.

Commelina tuberosa $\mathrm{L}$

Tinantia fugax Scheirlw...........

Tradescantia geniculata Jilcq......

Isippia citriodora H. B. K .........

Senecio canicida, Moc. el Sessé...

Galium, sp?

Stevia viscida H. B. K

Hyoscyamus niger L...

Nama palmeri A. Gr..

Nance undulation H. B. K

Calea axillaris DC.

Frankenia grandifolia Ch. et Sclıl.

Solanum amictum Moric...........

Antigonon leptopus Hook. el Aru.

Canna indica L

Elytraria tridentata Vah...

I'iper sanctum Schl...

Chrysanthemum parthenium Smi I! Tagetes lucida Gav....

Eryngium beecheyanum H. et $\Lambda .$. Eryngium phyteumatos: D. Eryngium comosum Delar........... Eryngium gracile Delar........... Eryngium serratum Cav............ Piqueria trinervia Cav. Gymnosperma multiflorum DG..... Gymnosperma corymbosu n I) C...... Tinnia linerris Benth.

Tigridia pavonia Ker.............. Porophyllum macrocephalum D( :.. Calophanes linearis A. Gi.

Helenium ooclinium A. Gr...........

Loeselia coccinea D.11.

Pilea falcutu Liehm ...

Pilea mierophylla Liebm........... Cestrum aurantiacum Limill........ P'erezia moschata Llav. et I ex..... Solunum, sp?......

Stevia, sp?......

(n)

Perezia thyrsoidea A. Gr............

Gurdichandia cynun hoiles H.B.K

Mentha rotundifolia L.

Lippia geminata H. B. K.
Primuláceas......

Compuestas

Euforbiáceas

Loganiáceas.

Helechos

Borragíneas....

Solanáceas.

Solanáceas.

Solanáceas .

Loganiáceas.

Labiadas.

Commelináce

Commelináceas ....

Conmelináceas...

Commelináceas ...

Verbenáceas... ....

Compuestas .........

Rubiáceas ..........

Compuestas .........

Solanáceas..........

Hidlofiláceas........

Hidrofiláceas........

Compuestas .........

Frankeniáceas .....

Solanáceas

Poligonáceas.......

Zingiberáceas.......

Acantáceas ........

Piperáceas..........

Compuestas.........

Compuestas ........

Umbelíferas........

Umbelíferas .........

Umbelíferas .........

Umbelíferas ......

Umbelíforas ........

Compuestiı .........

Compuestas. ......

Compuestins........

Compueslas.........

Irideas..............

Compuestas.........

Acantáceas.........

Compuestas .........

Polemoniáce:is......

Urticáceas ..........

Urticáceas ...........

Solanáceas..........

Compuestas .........

Solanáceas ..........

Compueslas........

Euforbiáce:ı.......

Compuestas .........

Malpigiáceas........

Labiadas............

Verbenáceas
Naturalizada .....

Ver., E. de Méx.. Urbina.

Valle de México. Farm. Mex.

Chihuahua....... Cat. Inst. Med.

S. L. P., Chill.... Cat. Inst. Méd.

Valle de México.

Oaxaca, Puebla..

Hidalgo

S. Luis Polosí, \&

Talbasco.......... Rovirosa.

Oaxaca, Ver......

Valle de México. Cat. Inst. Méd.

V. de Méx., Jal... B. V. et Urbina.

Varios lugares... Cat. Inst. Med.

Oaxaca............

Cultivadi.........

Puebla ............

E. de Héxico.....

México, Jalisco...

Cullivada.........

S. L. P., Coah...

S. Luis P., etc...

Chiapas ...........

Sonora............

Morelos, Ver....

Morelos...........

Naturalizada .....

Chihuahua.......

Jalisco, Veracruz

Cultivada.........

V. de México, \&.

Jalisco, Ver., etc.

E. de Mexico....

Valle de México.

Varios lugares...

S. Luis Potosí, \&

Jalisco, etc....

Valle de México.

Veracruz..........

Oaxaca.

Varios lugares...

Virios tugares...

Chihuilhua......

Gtrihuahua.....

Vilrios lugares...

Oaxaca...........

Oaxaca.

('hiapas ?.........

Michoacán, Jal..

Querétaro

V. y E. de Méx.

Michoacán......

Gto., Michoacán.

Naturalizada ....

Jalisco, Veracruz
Farm. Mex.

Cat. Inst. Med.

Urbina.

Urbina.

Urbina.

Urbina.

B. V. et Urbina.

Farm. Mex.

Farm. Mex.

B. V. et Urbina.

F. M., M. Méd.

Far. Mex.

Rose.

Oliva.

Urbina.

Mat. Méd.

Cat. Inst. Med.

Alcocer.

E.M., B.V.etU.

Cat. Inst. Med.

Urbina.

B. V. et Urbina. 
Higuera

Higuera ó Hignerón ó Higuerote.

Higuera del diablo

Higuera de higo

Higuerilla.

Higuerilla pequeña

Hikori....

Hikori

Hincha huevos

Hinojo

Hinseseli.

Hipericón

Hipericón

Hipericón

Hisopo.

Hisopo del país..

Hisopo de Puebla.

Hisopo de Puebla..

Ho.

Ho.

Hoacazóchitl..

Hoazacán

Hoaxin (mexicano)

Hoaxinus

Hobo.

Hobo

Hoitzilózit]

Hoitzcolotli

Hoitzitzilteutli

Hoitzmamazali

Hioja blanca.

Hoja de aján.

Hoja peluda

Hoja ú hojas de pescado

Hioja sen del país.

Hojarasca

Hiojas de San Pedro.

IIojasén

IIolquáhuitl.

Hombrecillo.

Hongo.

Hilongo de huevo

Honoba.

Hormiguera.

Hortensia

Hotzachinquáhuitl...

Hotzquáhuit1.

Hoztilxócotl

Huacalillo...

Huacamote is Hracamot ó Hliacamnlti

Huacanalá ....

Huacux (tarasco)...

Huachacata.

Huahnauchi...

Huaje.....

Huajericián
Ficus carica $\mathrm{L}$. var.

Ficus nymphceifolia $\mathrm{L}$

Ricinus commenis L.

Ficus carica L. var....

Ricinus commenis L. var

Ricinus communis L. var

Anhalonium Lewinii Hennings....

Anhaloninu Williemsii Lem......

Rluus, sp?

Foniculum vulgare Grertn

Loeselia, sp?

Hypericum denticulatum H. B K..

Hypericum fastigiatum H. B. K...

Hypericum formosum H. B. K....

Salvia polystachya Ort.

Salvia axillaris Moc. et Sissé.....

Salvia axillaris Moc. et Sessé....

Salvia polystachya Ort....

Anlalonium Lewinii Hennings...

Anhalonium Willamsii Lem......

Philodendron, sp?.

Guaiaenu verticale Ort

Leuccena csculenta Benth.

Tamarindus indica $\mathrm{L}$.

Spondias dulcis Forst. var. acida..

Spondias purpurea L.

Myroxylon pereirce Klotzsch.......

Eryngium foetidum $\mathrm{L}$....

Lobelia laxiflora H. B. K. var....

Acacia cornigera Willd.

Heliconia caribea Lamk.

Piper senctum Schl.

Brachistus stramonifolius Miers...

Limnanthemum lumboldtianum G.

Ccesclininia exostennu D:

Pereaia uduata A. GI:

Daplnopsis salicifolia Meissn.....

Cecsalpinia cxostemina DC.

Castilloa elastica Gerv

Humuhes lupulus L.

Agaricus campestris t

Iycoperdon boxista $\mathrm{L}$

Rudbectia mexicana Vints..........

Cressia leiopleylla Vog..

IIydirangea hortensice DC.

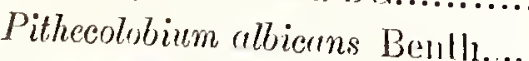

C'esalpiniue celinatu? Lam.

Spondias lutere I.

Ocotert, sp:.

Mroniliot utilissima Poht

Myrice melapensis II. B. K.

Lucume, sp.

Lecoma struns Juss.

Pterocarpus draco? L.

Leuccena esculenta Benth....

Parmentiera alata Miers
Urticáceas

Urticáceas

Euforbiáceas........

Urticáceas

Euforbiáceas ........

Euforbiáceas ........

Cácteas.

Cácteas.

Anacardiáceas .

Umbelíferas.

Polemoniáceas ....

Hipericíneas

Hipericíneas

Hipericíneas.

Labiadas.

Labiadas.

Labiadas.

Labiadas.

Gícteas.

Cácteas.

Aroideas

Zigofileas

Leguminosas..

Leguminosas..

Anacardiáceas.

Anacardiaceas.

Leguminosas..

Umbelíferas.

Lobeliáceas .

Leguminosas..

Musáceas.

Piperáceas.

Solınáceas

Gencianeas.

Leguminosas

Compuestas

Timeleáceas

Leguminosas..

Urticáceas ...

Urlicáceas

Hongos

Hongos.

Compuestas

Leguminosas.

Saxifragáceas.

Leguminosas.

Leguminosas.

Anacardiáceas .

Lisuríneas.

Euforbiácens .......

Miricáceaı

Sapotáceas.

Bignoniáceas.

Leguminosas.

Leguminosas.

Bignoniáceas.
Cultivarla.

Sinaloa.

Varios lugares...

Cultivada.

Varios lugares...

Varios lugirres...

N. de Méx., Quer.

N.de Méx., Quer.

Michroacán........

Naturalizada ....

Tepic ..............

Guanajuito, cle..

Guanajuato, etc..

Guanaijuato, etc..

Varios lugares...

Jalisco.

Jalisco.

Varios lugares...

N.de Méx., Quel.

N. de Mćx., Quer.

Miclıoacán.......

E. de México....

México, Morelos.

Naturalizada ....

Mor., Gro., Ver...

Mor., Gro., Ver'..

Tilbasco, Ver....

Varios lugares...

Varios lugares...

Morelos

Tabasco.

Vericruz.

Veracruz.....

Varios lugares...

Jalisco.

Guerrero, Mich.

Veracruz, Mor...

Jilisco.

Lugares cálidos.

riultivada...

Varios lugares...

Varios lugares...

Chihuahua ......

Tabasco

Cultivada...

Ciampech...

Lugires cálidos.

Morelos:

Veraciuz...

Ciullivadia...

Veracruz, Oax...

Michoacán.......

Varios lugares...

Michoacán.......

México, Morelos.

lugares cálidos.
Cat. Inst. Med.

Far. Mex.

Rose.

Fir. Mex.

Far. Med.

Far. Mex.

Colm., Far. M.

B. $V$. et Urbina

Mat. Méd.

Ramírez.

Rovirosa.

Urbina.

Colmeiro.

Oliva.

Gal. Inst. Med.

Firm. Mex.

B. V. et Urbina.

Villada.

C. I. M., Herb. Rovirosa.

Alt. et Ramz.

Farm. Mex. 
Hualhua

Huamuchil

Huanita

Huano.

Huas (maya)

Huauzontle

Huaxaten (tarasco)...........

Hueinacaztli ..................

Huejocote ó Huexotl .........

Huele de noche

Hueso.

Hueso.

Hueso ó codo de fraile ....

Huevos de toro ...............

Huey tochtlinacaztli .......

Huicicilo.

Huicumo (tarasco)

Huichichile

Huichín.

THuila

Huinar ó Huinari ............

Huinari (tarasco).............

Huiquilitl

HLiro

Huiro de montañas .........

Huisache.

Huisache ó Huizache.......

Huisache blanco

Huisache prieto

Huiscoy ul.

Huitlacoche

HLuitzitziltzin ó Huitzitzil|róclitl..

Huitzquáhuitl...............

Huitztomatzin..............

Huizache.

Huizapotillo

Hulándini.

Eule.

Huriata erauche (tarasco).

Hustlacoche
Talauma mexieana Don

Pithecolobium dulce Benth.

Bourreria huanita Hemsl...

Latania borbonica Willd.

Creseentia cujete L

Chenopodium bonus-IIenricus L...

Solidago, sp?

Enterolobium cyclocarpum Griseb..

Salix lasiolepis Bentlı.

Cestrum nocturnum L.

Exostemma, sp?

Framea odoratissima DG...........

Thevetia yoyotli A. DG...............

T'aberncemontana acapulcencis M...

Asclepias cormuti. Done.

Locselia coceinea Don.

Luenma salicifolia H. B. K........

Loeselia eoceinea Don.

Verbesina pinnatifida Gav

Agave, sp?

Sida rhombifolia L..

Malva scoparia L'Herit............

Indigofera anil L.

Parmentiera alata Miers...........

Crescentia macrophylla Seem......

Mimosa, sp?.

Pithecolobium albicans? Benth....

Acacia, sp?

Acacia, sp?

Cocos guacoyule Liebm

Ustilago mayclis $\mathrm{DC}$..

Loselia coccinea Don.

Casalpinia echinata? Lam.........

Solanum hornandezii Moc. et Sessé

Piptadenia foetida Benth.

Triamfetta semitriloba L.............

Inga, sp? ....

Castilloa ellastica Cerv.............

Malva, sp?.....

Ustilayo maydis, DG.
Magnoliáceas ....... Morelos, México.

Leguminosas....... Mor., Ver., etc...

Borragíneas s....... Veracruz, Mich.

Palmeras.......... Cultivada

Bignoniáceas....... Yucatán..........

Quenopodiáceas ... Naturalizada ....

Compuestas........ Michoacán........

Leguminosas........

Salicinens........... Valle de México.

Solanáceas ......... Veracruz, Jalisco

Rubiáceas .......... Tabasco....

Rubiáceas .......... Veracruz.........

Apocináceas......... Morelos ..........

Apocináceas........ Guerrero, Oax...

Asclepiadeas ....... ...................

Polemoniáceas...... Varios lugares...

Sapotáceas ......... Michoacán.

Varios lugares...

$\begin{array}{ll}\text { Compuestas........ Michoacán, Jal... } & \\ \text { Amarilídeas........ Jalisco............ Rose. }\end{array}$

$\begin{array}{ll}\text { Compuestas........ } & \text { Michoacán, Jal... } \\ \text { Amarilídeas........ Jalisco........... Rose. }\end{array}$

Malváceas .......... E. de Méx., etc...

Malváceas .......... Michoacán.......

Leguminosas........ Jugares cálidos.

Bignoniáceas....... Lugares cálidos.

Bignoniáceas........ Tabasco..........

Leguminosas........ Oaxaca ..........

Leguninosas........ Hidalgo, Camp...

Leguminosas....... Michoacán.......

Leguminosas........ Michoacán.......

Palmeras ........... Lugares cálidos.

Hongos............. Sobre el maíz...

Polemoniáceas..... Varios lugares...

Leguminosas....... Morelos........

Solanáceas......... Zacatecas........

Leguninosas........ Guerrero, Jalisco

Tiliáceas............ Jalisco...........

Leguminosas....... Michoacán.......

Urticáceas .......... Tabasco.........

Malváceas .......... Michoacán........

Hongos............. Parásita.........

B. V. et Urbina.

N. León.

Cat. Inst. Med.

Barroso.

Farm. Mex.

Cat. Inst. Méd.

Cat. Inst. Méd.

Farm. Mex.

Mat. Méd.

B. V. et Urbina.

Urbina.

N. León.

N. León.
Rosáceas.......... Guerrero, etc.....

Rosáceas........... Tabasco..........

Orquídeas .......... Michoacán........

Liliáceas ............ Valle de México.

Bignoniáceas....... Varios lugares...

Dioscoreáceas ...... Cullivada.........

Anonáceas......... Lugares cálidos.

Aroideas........... Gultivada.......
Rovirosa.

Alcocer: 
Incienso

Incienso del país...

Indigo.

Ingerto.

Inguande.

Inguande.

Inmortal

Iocroma

Ipecacuana blanca..

Ipecacuana de Jalapa ......

Ipecacuana de Mézico.....

Ipecacuana de México.....

Iscoque

Isote..

Istactzapotl

Itamo real.

Itamo real

Itamo real

Itavio

Itzamaqua ó Itzmaqua......

Itzcuimpatli.

Itzcuimpatli.

Itzmetl

Itzmetl.

Itztamatl

Itzmaqua

Ixcle.

Ircuicuil.

Ixpepe.

Ixquixóchitl

Irtauhyatl ó Iztauhyal....

Ixtle..

Ixtle ó Ixtli

Iyamolle.

Izote.

Izote..

Izote ó Izotl

Izote ó Izot]....

Izqua

Iztacpatli

Iztactepetzacuxóchit

Iztactepetzacuzóchit
Hecluvigia balsamifera? Sw.

Bursera bipinnata Engl.

Indigofera anil $\mathrm{L}$

Loranthus americanus Jacq.

Bocconia arborea Wats..

Bocconia fintescens L

Gomphrena globosa L.....

Iochroma tubulosum Benth....

Richardsonia scabra A. St. Hil....

Psychotria excelsa H. B. K.

Ionidium polygaloefolinm Vent.....

Psychotria mexicana Willd.

Tragetes patula L

Yucca treculeana? Carr.

Casimiror edulis Llav. et Lex.....

Passiffora dictamo DG.

Potertilla, sp?.

Pellcea cordata J. Sm.

Eupatorium, sp?...

Lelia grandiflora Lindl ...

Senecio canicida Moc et Ses.é....

Schoenocaulon officinale $A$.

Sedum quitense H. B. K

Sedum roseuni Stev....

Bombax ellipticum H. P. K......

Icelia majalis Lindl

Agave rigida .lill

Anagallis arvensis? L

Trema micrantha $\mathrm{BI}$.

Bourreria hucenita Benlh.

Artemisia mexicana WVilld

Agare hetheracantha Zucc...

Agave rigida Mill...

Sapindus, sp?.

Yucea aloifolia L...

Yucca breccata Torr.

Yucca filamentosa $\mathrm{L}$

Yucea treculeana ci..............

Rumex obtusifolius $\mathrm{L}$

Apocinum, sp?

Cissampelos pareira Lamark

Govenia liticacen LindI.
Anacardiáceas ......

Burseráceas.

Leguminosas:

Lorantáceas.

Papaveráceas

Papaveráceas

Amarantáceas

Solanáceas ...

Rubiáceas

Rubiáceas

Violarieas.

Rubiáceas

Compuestas

Liliáceas.

Rutáceas

Pasifloráceas.......

Pinsáceas

Helechos

Compuestas

Orquídeas

Compuestas

Liliáceas.

Crasuláceas

Crasuláceas

Malváceas

Orquíleas

Amarilídeas.

Primuláceas.

Urlicáceas

Borragíneas

Compuestas.

Amarilídeas.

Amarilídeas.

Sapindáceas.

Liliáceas

Liliáceas

Liliáceas

Liliácens.

Poligonáceas

Apocináceas.

Menispermáceas

Orquídeas.
Oaxaca.

Norelos

Regiones cálidas

Michoacán.

Michoacán

Michoacán

Naturalizada ....

Cultivada

Veracruz

Veracruz.

Valle de MIéxic

Veracruz

Michoacán........

Valle de México.

Lug. templados.

Varios lugares...

Veracruz..

Oaxaca, etc......

Oaxaca.

Michoacán

Puebla ............

Veracruz..........

Cultivada...

Cultivada........

Guerrero, Ver...

Michoacán

Varios lugares...

Naturalizada .....

Varios lugares...

Michoacán........

Varios lugares...

Frontera Norle.

Varios lugares...

Morelos

Valle de México.

Jalisco.

Chiapas

E. de Méxicu

Frontera Norte.

Lug. calientes....

Veracruz..........

Michoacán, Ver.
Barroso.

Ramírez.

Mat. Méd.

Mat. Méd.

Mat. Méd.

Farm. Mex.

Farm. Mex.

N. León.

Rose.

Mat. Méd.

Cat. Inst. Med.

Ciat. Inst. Med.

Mal. Mléd.

Ramirez.

Lejarza.

Mat. Méd.

Pamirez.

B. V. el Urbina.

Alcocer.

De Candolle.

\section{J}

Jabí ó Jabin

Jaboncillo.

Piscidia erythrina $\mathrm{L}$

Jaboncillo.

l'sendotsuga douglassi Citrr........... Sapindus necryinutus Willd. .......

Jaboncillo

Jabonera. Symplocos citrea? Llav. el Lex...

Jacalozúchil blanco

Phytolacea octendra L.. ..........

Themeria rubra L. var.

Leguminosas

Coníferas

Sapindáceas .....

Estiráceas

Fitolacáceas.

Apocináceas.
Tabasco.

Nichoacán......

Tabasco ............

México.

Vulle de México.

Jalisco.
Rovirosa.

Villada.

Cat. Inst. Med.

Colmeiro.

Urbina.

B.V. el Urbina. 
Jacalozúchil rojo......

Jacinto.

Jagua ó Jagua blanca........

Jalapa

Jalapa

Jalapa limoncillo

Jalapa de Tampico

Jalocote

Jalocote

Jalocote.

Jaltomate

Jaltomate

Jaltomate

Jamaica.

Janacahuate

Jara....

Jara ó Jaral amarillo.

Jara amarga.

Jaral blanco.

Jaralillo amarillo.

Jarana de pitahayita ........

Jarilla

Jarilla

Jarilla.

Jarilla

Jarilla

Jarrilla

Jarritos

Jarritos.

Jarritos

Jarritos

Jaujas

Jaspalache.

Jazmín

Jazmín.

Jazmín amarillo

Jazmín de Arabia

Jazmín blanco.

Jazmín mosqueta

Jazmín de olor.

Jazmín de perro

Jazmincillo

Jenequén

Jengibre.

Jicaco.

Jicama......

Jícama

Jícama del cólera..

Jícama contra el tifo

Jicamilla

Jicamite.

Jicamite.......

Jícara ó Jícaro.................

Jicara de Yucatán...........

Jimba

Jinicuil
Plumeria rubra L.

Iyacinthns orientalis $\mathrm{L}$.

Genipa carulo H. B. K

Ipomcea purga Hayne.

Jatropha macrorhiza Benth........

Ipomeea purga Hayne.

Ipomoea simulans Hanbury......... Abies religiosa Cham. et Schl.....

Pinus teocote Cham. et Schl.......

Prionosciadium mexicanum IVals.

Physalis mollis Nutt.

Saracha jaltomata Schl...

Suracha umbellata \& G. Don........

Hibiscus sabdariffa L....

Brongnintia thermoides Spr.......

Lobelia, sp?

Senecio saligmus DG.

Vicia sativa L

Baccharis pteronioides DG....

Lythrum gracilc Benth....

I'hyllocactus angnliger Lem........

Calca cacosmoides Less.

Eupatorium, sp?.

Senecio calcarcus H. B. K...........

Senceio salignus DC....

Stevia salicifolia Gav.................

IIocinna heterophyla Llav. et Lex.

Lobelia laxiflora $\mathrm{H}$. B. K. var.....

Loselia ccernlea Don.

Pentstemon barbatus Nutt..........

Pentstemon imberbis Trautv........

Sucula diffusa Wats....

Seclum acre L...

Clematis triloba Roth

I'etrcee volubilis Jarq.

Jasminnm humile L...

Jasminum sambac Ait.

Jasminnm officinale L...

I'hiladelpluns coronarins L....

Jasninum grandiflornm L............

Tabernamontana acapnlcensis M..

Pisonia hivtella H. B. K...........

Agave rigida Miller....

Zingiber officinale Roscoe.

Chrysobalanus icaco L

Pachyrhizus angulatus Ricl........

Pachyrhizus palmatilobus B. et H.

Dallia coccinea Gav....

Valeriana ceratophylla H. B. K....

Jatropha, sp?......................

Dahlia coccinea Gav................

Dahlia variabilis Desf́.

Cresecntia erjete L

Crescentia cujete L..................

Bambusa gradua L.

inga jinicuil Schl...
Apocináceas.

Liliáceas.

Rubiáceas

Convolvuláceas. ..

Euforbiáceas

Convolvuláceas. ...

Convolvuláceas. ...

Coníferas....

Goníferas.

Umbelíferis

Solanáceas

Solanáceas....

Solanáceas

Malváceas.....

Leguminosas.....

Lobeliáceas

Compuestas.

Leguminosas.. .....

Compuestas........

Lilrarieas....

Cácteas.

Compuestas

Compuestas

Compuestas.

Compuestas...

Compuestas.

Pasifloráceas

Lobeliáceas

Polemoniáceas ....

Escrofularíneas....

Escrofularíneas....

Quenopodiáceas...

Crasuláceas........

Ranunculáceas....

Verbenáceas ........

Oleáceas............

Oleáceas.

Oleáceas.

Saxifragáceas ......

Oleáceas............

Apocináceas........

Nictagineas .........

Amarilídeas........

Zingiberíceas.......

Rosáceas ...........

Leguminosas........

Leguminosas........

Compuestas........

Vialerianeas..........

Euforbiácens ........

Compuestas .........

Compuestas.......

Bignoniáceas........

Bignoniáceas........

Gramíneas.........

Leguminosas.......
Jalisco...

Cultivada

Tabasco.

Veracruz

Zacatecas, Ghih.

Veracruz.........

Tamaulipas......

D. Federal, etc...

Oaxaca.

Distrito Federal.

San Luis Potosí..

Valle de México.

E. de Méx., Jal.

Gultivada........

Jalisco.

Valle de México.

Cultivada.

Jalisco.

Jalisco.

Jalisco.

Veracruz.........

E. de Méxi‘o....

E. de México.....

V. de Méx., Gto..

Distrito Federal.

Jalisco.

Querétaro, etc..

Puebla ............

Distrito Federal.

S. Luis Potosí, \&

Matamoros......

Cullivada

Cultivada.........

Jalisco, Verncruz

Cultivada.........

Gultivada........

Cultivada.........

Cultivada....

Gullivada.........

Guerrero, Oax...

Valle de México.

Yucatán, Ver....

Cultivada.....

Guerrero, Yuc..

Veracruz, Oax..

Veracruz, Oax...

Valle de México.

Valle de México.

Chihuahua....

Valle de México.

E. de México....

Tabasco

Yucatán.

Tabasco...

Veracruz.........
B. V. et Urbina

Farm. Mex.

Urbina.

Ramírez.

Urbina.

Firm. Mex.

Urbina.

Farm. Mex.

Urbina.

B, V. et Urbina.

B. V. et Urbina.

B. V. et Urbina.

Urbina.

Gat. Inst. IIéd.

Urbina.

Urbina.

Urbina.

Ramírez.

Urbina.

Cat. Inst. lléd.

Ramírez

Urbina

Alcocer.

Alcocer.

Alcocer:

Farnt Mex.

Ramírez.

Farm. Mex.

Cat. Inst. Med.

Rovirosa. 
Jipijapa de Panamá.

Jiquilite.

Jiricua...

Jitomate

Jobo..

Jobo...

Jobo espino

Jobo liso.....

Jobo roñoso.

Jocoyol.

Jocoyol.

Jocoyol.

Jocoyol.

Jocoyol color de rosa.

Jocuiste majahua

Jocuistle.

Jolocín.

Jolocín.

Jolocín blanco

Jonote.

Juanita...

Judía......

Jujito amarillo

Jujito colorado.

Jujito peludo...

Jumete.

Junco.

Junco.

Junco.

Junco.

Junco.

Junco espinoso
Carludovica palmata R. et Pav... Indigofera anil $\mathrm{L}$

Plumbago pulchella Boiss

Lycopersicum esculentum Mill......

Spondias duleis Forst. var. acida.

Spondias purpurea L.

Spondias lutea L

Spondias, sp?.

Spondias lutea L

Oxalis tetraphylla Cav...

Oxalis verticillata $\mathrm{DC}$

Oxalis angustifolia H. B. K

Oxalis corniculata $\mathrm{L}$

Begonia gracilis H. B. K

Karatas plumieri E. Morr.

Bromelia pinguin $\mathrm{L}$

Heliocarpus americanus L.....

Heliocarpus arborescens Scen.

Heliocarpus americanus I.

Heiiocarpus americanus L....

Helianthemun glomeratum Lilg....

I'haseolus vulgaris L.

Passiflora serratifolici I.

Passiflorc ciliata Ait.

Passiflora fostida L.

Pedilantlus pavonis Boiss.

Cereus flagelliformis Haw.

Cassia, sp?

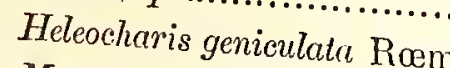

Mamillaria coronaria Haw.

Koeberlinia spinosa Zucc.

Cereus serpentinus Lagasc.
Ciclantáceas

Leguminosas.

Plumbagíneas

Solanáceas...

Anacardiáceas

Anacardiáceas

Anacardiáceas

Anacardiáceas

Anacardiáceas.

Oxalínleas

Oxalídeas

Oxalídeas

Oxalídeas

Begoniáceas.

Bromeliáceas

Bromeliáceas ...

Tiliáceas

Tiliảceas.

Tiliáceas.

Tiliáceas.

Cistineas.

Leguminosas

Pasifloráceas

Pasifloráceas.

Pasiflo ráceas........

Euforbiáceas ..........

Cácteas.

Leguminosas

Ciperáceas

Gácteas.

Simarubáceas

Cácleas
Tabasco

Ciniapas

Valle de México.

Cullivada......

Lug. calientes...

Lugr. calientes...

Tabasco.

Tabasco

Tabasco....

Jalisco...

Valle de Mréxico.

Guerrero

Veracruz.

Jalisco.

Veracruz, Mor..

Jalisco.

'Tabasco...

Tabasco....

Tabasco.

Veracruz, Mor.

V. de Méx Jal.

Cultivada.

Tabasco

'labasco.

Tibasco

Jalisco.

Varios lugares...

Jalisco.

Tabasco..

México

Cihihuahua...

Jalisco.
Rovirosa.

Mat. Méd.

Rovir., Rose.

Cat. Inst. Med.

Cat. Inst. Méd.

B. V. et Urbina.

B. V. et Urbina.

B. V. et U. Ram.

Rov., Villada.

Rovirosa.

Cat. Inst. Ned.

Cat. Inst. Med.

Ciat. Inst. Med.

Rovirosa.

Rovirosa.

Rovirosa.

Farm. Mex.

Colmeiro, F. M.

Rovirosa.

B. V. et Urbinil.
Kentó.

Kopte (maya)

Kuntz (maya)
Thalia geniculata

Cordia dodecandra A............. Zingibericens.

Nicoliona tabacum A. DG
Zingiberáceas Solanáceas
Tabasco.

Yucatán

Cultivada.
Rovirosa.

Dondé.

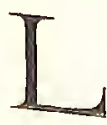

Labitz (huasteco)

Lágrimas de Job ó de S. Pedro....

Lama.

Lama...

Lambrisco.
Zingiber officinale Roscoe.

Coix lackryma L.............. Zingiberáceas.

Utricularia vulgaris ............. Gramínèas

Selaginella, sp?..................

Rihus sempervirens Scheele..........
Lentibulari........ Jalisco............

Selagincláceas..... 'T'abasco.......... Anacardiáceas.... Silios húmedos.. San Luis Polosí.
B. V. el Urbinta

Rovirosa.

Unbina. 


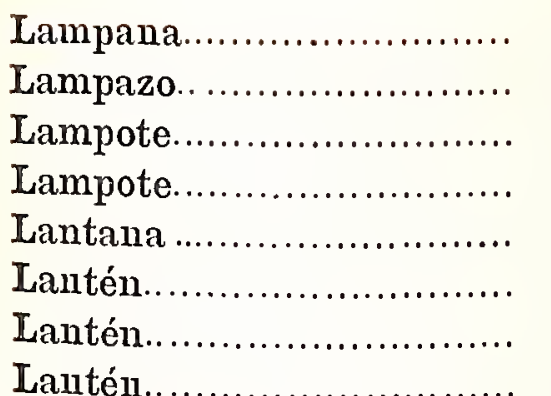

Laurel de poeta..

Laurel.

Laurel.

Laurel de chile

Laurel de India.

Laurel pimienta ó pimiento

Laurel de la playa......

Laurel rosa

Laurel tulipán.....

Laurelillo

Laurelillo agrio.

Lechemaría.

Lecherillo

Lechuga....

Lechuga romanita

Lechuguilla

Lechuguilla ....

Lechuguilla

Lechuguilla

Lechuguilla

Lechuguilla

Lechuguilla

Lechuguilla de agua

Lele.

Lengua de buey

Lengua de ciervo

Liengua de pájaro.

Lengua de perro

Lengua de vaca.

Lengua de vaca.

Isellgua de vaca.

Lengua de víbora

Leuteja

ILentejilla.

Lentejilla

Lentejilla de agua

Lentejuelilla.

Lentisco.

Lentisco de $\mathbb{N}$ uevo León...

Leño colorado

Leño nefrítico

Lepidio.

Libonia

Iicopodio

Liga.

Liga..........

Lila,

Lila.
Lantana camara L...

Nymplace odorata Ait.

Helianthus ammens L................

Helianthus comifolius H. B. K.....

Lantana camara L.

Plantago galeoltiana Dcne.........

Plentago major L...................

Plantago mexicana Link...........

Laurus nobilis L...................

Litsea glancescens H. B. K. var....

MIsisinteca capitata Cham. et Schl.

Nectandra polita Nees. var...

Fieus religiosa L....

Nectundia mollis Nees...

Ocotea, sp?....

Nerium oleander L...

Tulauma mexicana Don.

Mralphigine, sp?.

Nectandra, sp?

Cordia geraseanthoides II. B. K...

Tabencemontena grandiflora Jaeq

Lactuca sutiva L.

Lactuea sativa L. var....

Agave heteracantha Zucc....

Hieracium mexicanum Less........

ITieracium premorsiforme Schz. B.

Solidago mexicana L....

Solidago velutina DC....

Sonchus ciliatus Lam....

Sonchus olcraceus L.?

Pistic stratiotes L....

Calliandira grandiflora Benth......

Buddleia, sp?....

Polypodium lanceolatum L...

Polygonum avieulare L....

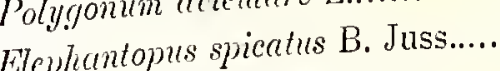

Rumex mexicanus Meissn..........

Rumex obhusifolius $\mathrm{L}$.........

Rumex patientia L

Philibertia, sp?...

Lene esculenta Mrench.

Lemna gibba, L.....................

Lepidium virginicum L.............

Lemna minor L......................

Arabis menicana Wats.

Acacia? lentiscifolia Desf..

Pistacin mexicana H. B. K........

Aretostaphylos puengens H. B. K..

Eysenhardtia amorplooides H. B. K.

Lepidium virginicum L..............

Jacobinia floribunda Hort.........

Lycoportinm elavatum L............

Loranthus calyeutatus DC...........

Phoradendion tomentostm D. Oliv

Irelia sempervirens Sw.

Pittosporum tobira Ait...
Verbenáceas ....... Varios lugares...

Ninfeáceas.......... México........... Colmeiro.

Compuestas........ Varios lugares...

Compuestas........ Guerrero .........

Verbenáceas ........ Tarios lugares...

Plantagíneas ........ Oaxaca...........

Plantagíneas ........ Tarios lugares... Farm. Mex.

Plantagíneas ....... S. L. P., V. de M. Farm. Mex.

Lauríneas... ........ Cullivada........ Alcocer.

Lauríneas.......... Veracruz, Oax... Ramz., Rose

Lauríneas........... Veracruz......... Cat. Inst. Méd.

Lauríneas........... Tabasco......... Rovirosa.

Urticáceas .......... Cultivada........ Alcocer.

Lauríneas........... Tab., Ver., Oax.. Cat. Inst. Méd.

Lauríneas ........... Tabasco.......... Cat. Inst. Med.

Apocináceas........ Cultivada........

Magnoliáceas ....... Morelos ..........

MIalpigiáceas........ Nichoacán....... Cat. Inst. Med.

Lauríneas........... Tábasco.......... Cat. Inst. Med.

Borragíneas........ Tabasco.......... Rovirosa.

Apocináceas........ Chiapas ?........ Alt. et Ramz.

Compuestas........ Cultivada........

Compuestas........ Cultivada........

Amarilídeas........ Chihuahua, Jal..

Compuestas........ Talle de México.

Compuestas........ Valle de México.

Compuestas........ Valle de México.

Compuestas........ Valle de México.

Compuestas ......... Valle de México.

Compuestas........ Valle de México.

Aroideas............ V. de Méx., Jal...

Leguminosas....... Varios lugares...

Loganiáceas.........

Helechos

Poligonáceas ........

Compuestas .........

Poligonáceas........

Poligonáceas........

Poligonácens........

Asclepiadeas........

Leguminosas.......

Lemnáceas. .........

Grucíferas............

Lemnáceas.........

Cruciferas............

Leguminosas........

Anacardiáceas ......

Ericáceas

Leguminosis.......

Cruciferas...........

Acantáceas.........

Licopodiáceas ......

Jorantáceas. .......

Lolintácens.........

Meliảcens............

Pitosporeas.........
Sonora

Valle de México.

Valle de México.

Tabasco...........

Jalisco............

Naturalizada .....

Cultivada........

Puebla...........

Cultivada.........

Valle de México.

Valle de México.

Valle de México.

Guanajuato .....

Jalisco............

Nuevo León.....

Son., S.L.P. Hgo.

Varios lugares..

Talle de México.

Cultivada....

Tialle de Atéxico.

Valle de México.

Valle de México.

Cultivada........
Cultivaril.
Urbina.

B. V. et Urbina.

Ramírez.

Ramírez.

Ciat. Inst. Méd.

Cat. Inst. Med.

Hemsley.

Ramírez.

Mat. Med.

Cat. Inst. Med.

F. M., Rose.

Alcocer.

Rovirosa.

B. V. et Urbina.

Alcocer.

Cat. Inst. Méd.

Urbina.

Urbina.

Dugés.

B. V. et Urbina.

Ramírez.

Mat. Méd.

Alcocer.

Alcocer. 
Lima dulce

Lima agria

Iima chichona

Limero

Limón

Limón dulce

Limón real

Limón real.

Limoncillo

Limoncillo

Limoncillo

Limoncillo

Iimoncillo

Limoncillo amarillo.

Limoncillo blanco

Limoncillo colorado

Limoncillo de Córdoba.

Limoncillo de ILextitlán..

Limonero

Limpia-tunas.

Limpia-tunas.

Limpia-tunas.

Timpia-tunas.

Linaloé

Linaloá de Mézico

Linaza.

Linda tarde.

Linda tarde.

Lino.

Lino de Nueva Zelanda Lino rojo

Liquidámbar.

Lirio.

Lirio blanco

Lirio dorado

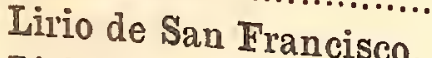

Lirio del Japón.

Lirio listado

Lirio parásito.

Lobelia.

Lobelia.

Iobelia.

Lobelia

Lobelia morada

Lombricera blanca

Liúpulo.
Cilrus limetla Risso.

Citrus limetta Risso. var.

Citrus limetta Risso. var.

Citrus limetta Risso

Citrus limonum Risso.

Citrus limonum Risso. var.

Citrus limonum Risso. var.

Citrus medica Risso. var.

Dalea citriorlora Willd.

Ipomoa purga Hayne.

Pectis difusa Hook. et Arn

Symplocos martinicencis Jicq......

Siparuna riparia A. DG

Symplocos, sp?.

Spmplocos, sp?

Symplocos, sp?.

Calophyllum, sp?.

Teinstromia, sp.

Citrus limonum Risso.

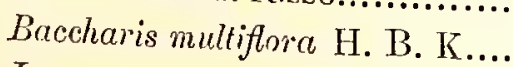

Ipomeer stuns Cav.

Spilanthes corymbosus Moc. et S...

Zaluzania angusta Schz. Bip...

Bursera alcexylon Engl....

Bursera delpechiana? Poisson...

Linum usilatissimum

Gaura coccinea Nutt.

Enothera tetraptera Cav..........

Linum usitatissimum L...

Phormium tenax Folst

Linum, sp.?.

Lineas

Liquidambar styraciflua L.

Iris germanica L...

Hymenocallis rotata Herb.

Litium auratum Lindl.

Loelia autumnalis Lind...

Lilium tigrinum $\mathrm{Ker}$.

Crinum cruentum Ker.

Lotia untumnalis Lind

Lobelia cardinalis $\mathrm{I}$.

Lobelia fulgens Willd.

Lobelia laxiflora H. B.

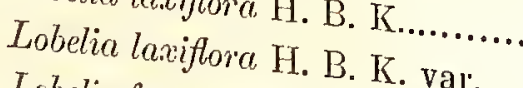

Lobelia fenestralis Ciav

Spigetia anthelmia I

Humulus lupulus L.
Rutáceas

Rutáceas

Rutáceas

Rutáceas

Rutáceas

Rutáccas

Ruláceas

Rutáceas

Leguminosas........

Convolvuláceas. ...

Compuestas...

Estiráceas.

Monimiáceas

Estiráceas.

Estiráceas

Estiráceas

Gulíferas

Ternstrœmiáceas .

Rutáceas

Compuestas.

Convolvuláceas. ..

Compuestas.

Compuestas

Burseráceas.

Burseráceas.

Lineas..

Onagrarieas.

Oṇagrarieas.

Lineas

Liliáceas

Lineas

Hamamelídeas

Irideas.

Amarilídeas

Liliáceas.

Orquídeas

Liliáceas.

Amarilídeas

Orquídeas

Lobeliáceas

Lobeliáceas.

Lobeliáceas

Lobeliáceas ....

Lobeliáceas ....

Loganiáceas.

Urlicáceas
Cultivada.

Cultivada.

Cultivada

Cultivada...

Gultivada.

Cultivada...

Cultivada..

Cultivada...

V. de Méx., Jal..

Veracruz...........

Jalisco.

Veracluz.

Veracruz

Veracruz.

Veracluz

Velaeruz

Veracruz

Hidalgo.

Cullivada..................

Valle de México.

Valle de México.

Querétaro.

E. y V. de Méx..

Regiones cálidas

Guerrero

Cullivada.

S. Luis Potosí, \& Jal., V. de Méx...

Cultivada.

Cultivada.

Jalisco...

Jalisco, Veracruz

Cultivada.

Valle de México.

Cultivada...

Jalisco.

Cultivada...

Veracruz

Jalisco.

Cullivada.

Valle de México.

Varios lugares..

Varios lugares..

E. de Mexico

Tabasco...

Cultivada.
Far. M., Colm.

Bárcena.

Colmeiro.

Mat. Méd.

P.N.H., et E.M.

Farm. Mex.

Urbina.

Alcocer.

B. V. el Urbina.

Ramírez.

B. V. et Urbina.

Alcocer.

Alcocer.

Alcocer.

Cat. Inst. Med.

Cat. Inst. Med.

Alcocer.

Rovirosa.

Llantén

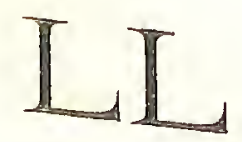

Llantén

Plantago galeottiana Dene.

Plantago major I

Plantagíneas

Plantagíneas.

Hidalgo, Oaxaca

Varios lugares... 
Llellepaxtle

Llora sangre.

Llora sangre.
Chrysaetinia mexicana A. Gr..... Bocconia arborea Wats. Boceonia frutescens L
Compuestas

Papaveráceas: Papaveláceas....
Puebla

Michoacán, Ver.. Mat. Méd.

Michoacán, Ver.. Mat. Méd.

M

Maca colorada

Macallo ó Macayo

Macpalxóchitl

Macuile.

Maculi..

Maculiz ó Maculiz prieto...

Maclura

Madera de pasta

Madre brava..

Madre de cacao.

Madreselva.

Madreselva.

Madroño

Madroño

Madroño

Madroño

Madroño de árbol

Madroño borracho

Madroño del campo ó rastrero

IMadroño chino.

Mafafa

Magnolia.

Magnolia.

Mago

Maguey

IMaguey.

Niaguey.

Maguey

Maguey

IMaguey.....

Maguey lechuguilla

Maguey de maceta.

Maguey manso

Maguey manso

Maguey meco.

Maguey de mezcal

Maguey de pulque...

Maguey de pulque.

Maguey de pulque

Maguey zhizhi...

Maguey Teometl.

Maguey de Tequil.........

Maguey de Tequila.

Magueyitos parásitos.......

Maicillo
Andira ereelsa H. B. K.

Andira execlsa H. B. K

Clecirostcmom platanoides $\mathrm{H}$. et $\mathrm{B}$.

Tecoma, sp?

Quereus virens Ail

Tabebuia leueoxyla $\mathrm{DC}$

Machera aurantiace Nutt..........

Coehlospermum hibiscoides $\mathrm{H}$. et B.

Erytlurina, sp?.

Errythrina, sp?

Lonicera caprifolizm L..............

Lonicera sempervirens L.............

Arbutus xalapensis H. B. K....... Aretostaphylos tomentosa Dougl...

Cclosia moquini Guillem

Gomphrena globosa L.

Aretostaphylos tomentosa Dougl.... Aretostaphylos arguta Zucc.

Gomphrena deeumbens Jacq.......

Arctostaphxlos, sp?.......

Xanthosoma robustum Schott.....

Magnolia grandiftora L....

Talauma mexicana Don............

Hernandia sonorc L................

Agave amerieana L.

Agare heteraeantha Zucc..........

Agcere rigida Miller.

Agave lechuguilla Torr.

Agave lophantha Schiede..........

Agave univittata Haworth...

Agare lechugrilla Torr...

Agave filifera Salm. D.

Agave potatoram Zucc.

Agare salmirna Ollo..

Agave americana $\mathrm{L}$. var............

Agave wislizeni \& Engelm...........

Aqare amerieana L.................

Agure atrovinens Karw.

Gave cochlearis . Jacobi..........

Agave brachystaehys Cav..

Agro brivinara $[$.....................

Algute, sp?..........................

Agare rigida, Miller.................

Trillandsia recurvata L..............

Authurizm seandens Engler var..
Leguminosas........................

Leguminosas...

Esterculiáceas......

Bignoniáceas

Cupulíferas ........

Bignoniáceas.

Urticáceas

Bixíneas.

Leguminosas.......

Leguminosas........

Caprifoliáceas ......

Caprifoliáceas.....

Ericáceas

Ericáceas.

Amarantáceas.....

Amarantáceas.....

Ericáceas

Ericáceas..

Amarantáceas.....

Ericáceas

Aroideas.

Mannoliáceas ......

Magnoliáceas .......

Lauríneas.

Amarilídeas.

Amarilídeas

Amarilídeas

Amarilídeas.

Amarilídeas.

Amarilídeas.

Amarilídeas.

Amalilídeas...

Amarilídeas.

Amarilídeas.

Amarilídeas

Amarilídeas.

Amarilídeas...

Amarilídeas.

Amarilídeas.

Amarilídeas.

Amarilídeas

Amarilídeas.

Amarilídeas. .

Bromeliáceas.

Aroideas.
Tabasco.

Tabasco

E. de México.....

Nuevo León, Vel.

Tabasco

Cultivada

Veracruz.........

Tabasco...........

Tabasco............

Cultivada.........

Cullivada.........

S. L. P., Ver.....

Frontera Norte.. Gat. Inst. Med.

México............

Naturalizada.....

Jalisco........... B. V. et Urbina.

Valle de México. Ramírez.

S. L. P., Jalisco.. B. V. et Urbina.

E. de México....

Veracruz......... Alcocer.

Cultivada.........

Morelos, México. Mat. Méd.

Chiapas ?.........

Chil., Coahuila.

Tamaulipas..... Rose.

Varios lugares...

Chihuahua....... Rose.

Tamaulipas..... Rose.

Chihuahua...... Rose.

Chihuahua.......

Jalisco............

V. de México, \&. Farm. Mex.

V. de México, \&. Farm. Mex.

Varios lugares...

Jalisco............

Jalisco.

V. de México, \&. Ramirez.

Tamaulipas..... Rose.

Hidalgo............

Sinaloa........... Rose.

Jalisco.

Yucatán

Jalisco.

Tabasco.

B. V. et Urbina.

Rovirosa. 
IVaíz.

Maíz amarillo

Maiz apastillado.

Maíz blanco ancho.

Maíz cacahuazintli.

Maíz colorado.

Maíz cruzado

Maíz cruzado fino.

Maíz chalcayote

Maíz de China.

Maíz chino

Maíz dulce.

Maíz de elote rojo.

Maíz forrado

Maíz liso.

Maíz mala cara

Maíz momochite cruzado...

Maíz pepitilla cruzado...

Maiz pinto

Maíz prieto.

Maíz primitivo

Maíz rojo.

Maíz de Tezas.

Maíz temitzi amarillo........

Maíz temitzi blanco..

Maiz tolonti blanco.

Maíz tolonti rojo...

Maíz veteado..

Majagua...

Majagua..

Majagüilla

Majahua..

Majahua..

IVajas grande..

Mal de ojo...

Mal de ojo.

Mal de ojo.

Mala mujer.

Mala mujer.

Malojo.

Malojo

Malva...

Màlva.

Malva.

Malva.

Malva.

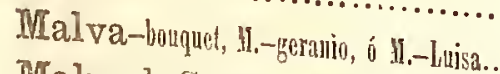

Malva de Cutstilla, M. medicinal

Malva de olor.

Malva rastrera...

Malva rosa.

Malva rubí.

Malva de Yucatán.........

Malvavisco.

Malvavisco.

Malvón....
Zer mays L.....

Zea mays L. var.

Zec mays L. var.

Zea mays L. var..

Zea mays L. var...

Zea mays L. var..

Zea mays L. var..

Zerr mays L. var.

Zea mays L. var.

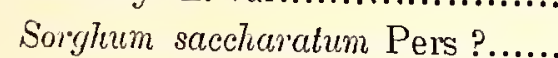

Zea mays L. var..

Zea mays L. var.

Zea mays L. var.

Zea mays L. var.

Zea mays L. var.

Zéa mays L. var.

Zea mays L. var.

Zea mays L. var....

Zea mays L. var.

Zea mays L. var.

Euchlcena luxurians Dur. et Asch.

Zea nucys L. var..

Helicenthus annuus L....

Zea mays L. var.

Zea mays L. var..

Zea mays L. var.

Zea mays L. var.

Zea mays L. var.

Hampea integerrina Schl.

Hibis'cus tiliaceus $\mathrm{L}$

Helicteres guazumcefolic H..........

Heliocarpus americanus I

Thespesia populnea Ciorr.

Cordia, sp?.

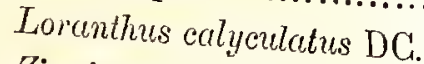

Zinnia elegans Jacq.

Zinnia tenuiflora Jacq

Rhus toxicodendron L

Solanum rostratum Dun.

Loranthus calyculatus L.

Zimnia pauciftora L.

Malachra palmata Moench........

Mulva rotunclifolia L...

Malva borealis Walh....

Malia parviftora L.

Malvastrum vitifolium I.............

Pelargonium inquinans Ait.

Malua rotendifolia $\mathrm{L}$.

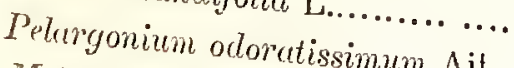

Irelea leprosa Ort.

Althea rosea Cav.

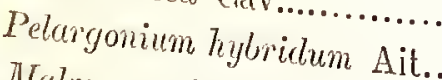

Malva americana L.

Althere offecinalis L.

Sida carpinifolice I

Pelargonium inquinans Ait.
Gramíneas..........

Gramíneas...........

Gramíneas

Gramíneas

Gramíneas.

Gramíneas

Gramíneas

Gramíneas

Gramíneas

Gramíneas

Gramíneas....

Gramíneas.

Gramíneas...

Gramíneas

Gramíneas

Gramíneas

Gramíneas.

Gramíneas

Gramíneas.

Gramíneas.

Gramineas.

Gramíneas.

Compuestas

Gramíneas.

Gramíneas...

Gramíneas.

Gíramíneas.

Gramineas.

Malváceas

Malváceas.

Esterculiáceas.....

Tiliáceas.

Malváceas ...

Borragineas

Lorantáceas.

Compuestas.

Compuestas

Anacardiáceas ......

Solanáceas

Lorantáceas.

Compuestas

Malváceas

Malváceas

Malváceas

Malváceas

Malváceas

Geraniáceas.

Malváceas

Gelaniáceas....

Malváceas

Malváceas

Geraniáceas

Malváceas

Malváceas

Malváceas

Geraniáceas.
Cullivada...

Cultivada...

Cultivada

Cultivada.

Cultivada...

Cultivada.

Cultivada

Cultivada...

Cullivada

Gultivada.

Ciultivada.

Cultivada.

Cultivada...

Cultivada...

Cultivada.

Cultivada.

Ciultivada...

Ciultivada...

Cultivada...

Cultivada...

Jalisco.

Cultivada.

Naturalizada .....

Cultivada...

Cultivada...

Cultivada...

Cultivada

Jalisco...

'Tabasco....

Jalisco, Tabasco.

Veracruz.....

Jalisco.

Naturalizada

Tabasco...

Morelos

Jalisco, Morelos.

V. de México, \&.

Jalisco.

San Luis Polosí.

Jalisco.

V. de Méx., Chih.

Tabasco....... ..

Naturalizada ...

Naturalizada ....

Naluralizada ....

Jalisco.

Ciultivada

Jalisco..

Jalisco.

Jalisco.

Gultivada...

Cullivad.

Yucatán

Cultivada.

Tabasco.

Cultivada.
Urbina.

B. V. et Urbina.

Rovirosa.

Urbina.

B. V. et Urbina.

Cat. Inst. Méd.

Urbina.

Urbina.

B. V. et U. Ram.

Urbina.

Rovirosa.

Farn. Mex.

Hemsley.

Hemsley.

B. V. el Urbina.

B. V. et Urbina

B. V. et Urbina.

B. V. et Urbina.

Dondé.

Piovirosa. 
Mamamalhuaztli

Mamey

Mamey.

Mamey de Santo Domingo.

Maná

Mananepile

Mandarina.

Mangle.

Mangle blanco

Mangle blanco

Mangle colorado

Mangle prieto.

Manglero

Mango.

Mangostan ....

Manguita del niño Jesús...

Mano de león

Mano de león.

Manto de la Virgen

Manto de la Virgen

Manto de la Virgen

Manzanilla.

Manzanilla.

Manzanilla eomún ó de Alemania ..

Manzanilla de Jalisco

Manzanilla del río.

Manzanilla romana.

Manzanilla de Yucatán

Manzanillo.

Manzanita de amor.

Manzanito

Manzano.

Mapipitza

Maquile.

Maquilihuatl.

Maranta.

Maranta.

Marañón.

Maravilla

Maravilla de España.........

Maravilla de jardín.

Maravilla silvestre

Maravillita

Margarita, Margarita reina

Margarita

Margarita

Margarita blanca, M. olorosi

Margarita del cerro.

Marihuana

Marilópez

Mariola.

Mariola.

Maripenda (tarasco)

Marrubio

Marrubio blanco

Marta
Clethra lanata Mart. et Gal.

Lucuma mammosa Gærtn. fil......

Manmea americana I

Mammea americana L

Polypodium lanceolatum L..

Citrus nobilis Loureiro.

Rhizophora mangle $\mathrm{L}$

Avicennia nitida Jacq......

Avicennia tomentosa Jacq.............

Rhizophora mangle L.

Conocarpus erecta $\mathrm{L}$

Rhizophora mangle $\mathrm{L}$

Mrangifera indica $\mathrm{L}$

Garcinia mangostana L.............

Maurandia semperflorens Ort.....

Celosia cristata L.

Senecio calophyllus Hemsl...........

Ipomcea longepedunculata Hemsl..

Ipomcea uurpurea Lam.

Pharbitis violaeea Boj

Anthemis nobilis L.....

Helenium quedridentatum Labill...

Matricaria chamomilla $\mathrm{L}$

Eupatorium conyzoides Valh........

Gnaphalinm oxyphyllum DC........

Anthemis nobilis L..

Hclenium autumnale L...............

Hippomane mancinella L............

Solemun pseudocapsicum L.........

Bunchosia guadulajarcnsis Wats.

Pyrus malus L. var.

Asclepias linaria Cav.

Tabebuia pentaphylla ? Hemsl....

Quercus virens? Ait.

IIaranta metallica Lindl......

Maranta zebrina Sims.

Anacardium oecidentale $\mathrm{L}$.

IFirabilis diehotoma L.................

Vinca rosea L.

Mirabilis longiftora L. ?.............

IIrivabilis dichotoma L...............

Oxybaphus aggregatus Vahl........

Callistephus hortensis Cass..

Galphimia glandulosa Cav.........

Karwinstia humboldtiana Zucc....

Polianthes tuberosa L................

Rhammes humboldticunus R. et S...

Cannabis sativa L......

Turnera ulmifolia L................

Chrysactinia merieana A. Gr......

P'urtheninm incanum H. B. K......

Liquidambar styracifua L.........

Marrubium vulgare L..............

MLarrubirm vulgare L..............

Phyllocactus phyllantoides Salm. D
Ericáccas .

Sapotáceas

Gutíferas.

Gutífcras.

Solanáceas

Helechos .

Rutáceas....

Rizoforácens

Verbenáceas

Verbenáceas.........

Rizoforáceas ........

Combretáceas ......

Rizoforáceas ........

Anacardiáceas .....

Gutíferas.

Escrofularíneas....

Amarantáceas .....

Compuestas ....

Convolvuláccas...

Convolvulácens....

Convolvuláceas....

Compuestas .........

Compuestas. ........

Compuestas .........

Compuestas

Compuestas

Compuestas

Compuestas

Euforbiáceas .......

Solinnáceas ....

Malpigiáceas

Rosáceas

Asclepiadeas........

Bignoniáceas.......

Cupulíferas ....

Zingiberáceas.

Zingiberáceas.

Anacardiáceas .....

Nictagineas ........

Apocináccas.........

Nictagineas .........

Nictagincas.........

Nictagineas.

Compuestas

Malpigiáceas.

Ramneas.

Amarilídeas........

Ramneas...........

Urticáceas ..

Turneráceas

Compuestas

Compuestas

Hamamelídeas....

Labiadas....

Labiadas.

Cácteas.
Oaxaca.

Mor., Oax., Gro...

Tabasco.

Veracruz..........

Cultivada....

Valle de México.

Cultivada.........

Oaxaca........... Ramírez.

Oaxaca........... Barroso.

Tabasco.......... Cat. Inst. Med.

Tab., Gro., Ver... C Cat. Inst. Med.

Tabasco.......... Cat. Inst. Med.

Jalisco............ B. V. et Urbina.

Cultivada.........

India oriental....

Jalisco........... B. V. el Urbina.

Cullivada.........

Jalisco............ B. V. et Urbina.

Hid., Dist. Fed... Ramírez.

D. Federal, etc... Ramírez.

Cullivada.........

Gultivada.........

Tabasco..........

Cultivada.........

Jalisco............ Oliva.

Chihuahua.......

Cultivada.........

Yucatán...........

Veracruz, Jalisco

Cultivada.........

Jalisco.

Cultivada

V. de M., S. L. P.

Orizaba...

Veracruz.

Cultivada....

Cullivada....

Oaxaca, Yuc.... Farm. Mex.

Mesa Central.... Farm. Mex.

Cultivada......... Alcocer.

Jalisco............ B. V. et Urbina.

Jalisco............ B. V. et Urbina.

Jalisco............ Bárcenà.

Cultivada......... Alcocer.

Morelos..........

Jalisco............

Lug. calientes...

Jalisco...........

Naluralizada ....

Oaxaca...........

D. F., E. de Méx.

Chih., Dist. Fed.

Michoacán.. ....

Mcsa Central....

Jalisco............

Jalisco...

Gat. Inst. Méd.

Rose.

B. V. et Urbina. Gat. Inst. Med.

Ciat. Inst. MIćd.

Caat. Inst. Méd.

N. León.

Rose.

B. V. el Urbina.

B. V. et Urbina. 


\begin{tabular}{|c|c|}
\hline$\cdots$ & Hibr \\
\hline Mastranzo..... & Lippia, sp... \\
\hline Mastranzo..... & Mentha rotundifolia $\mathrm{L}$. \\
\hline Mastranzo............... & Mentha silvestris L..... \\
\hline Mastuerzo................ & Tropceolum majus L.... \\
\hline Mata pez de México... & Piscidia erythrina L... \\
\hline Mata pulgas............. & Stevia viscida H. B. K.......... \\
\hline liz................... & Tradescantia geniculata Jacq... \\
\hline Matambilla.............. & Desmoncus rudentum Mart. \\
\hline Mataril ó Matarique... & ervaricefolius $\mathrm{He}$ \\
\hline Matarique.................. & Senecio grayanus Hemsl. \\
\hline$\cdots$ & Trixis corymbosa Don. \\
\hline ............. & Piper angustifolium Ruiz et Pa \\
\hline IV & Acacia farnesiana Willd \\
\hline la............... & Lantana camara L.... \\
\hline c.............. & la tuberosa $\mathrm{L}$. \\
\hline Luitl........ & Guaiacum verticale Ort. \\
\hline$\ldots \ldots \ldots$ & herimolia Mill... \\
\hline (.............. & ativus Schult. \\
\hline a & tifolia L. ?... \\
\hline ..... & a tolucana DG. \\
\hline la & Tecom \\
\hline la. & Pliytol \\
\hline cap & Ipomot \\
\hline Mecax & Smila: \\
\hline Mejora & Piper amala \\
\hline Mejoran & a veronicafolia A. Gr. \\
\hline Mejorana. & Lantana, sl \\
\hline Mejorana. .. & $m$ majorana \\
\hline Mejorana del. & ballotoeflora Benth.. \\
\hline Mejorana del p & Salvia ballotceflora Bentl.. \\
\hline Meliloto......... & Cav............ \\
\hline Melindr & parviflora De \\
\hline Melisa.. & Ipomo \\
\hline Melo & Melis \\
\hline elón. & persica Benth. et Hook \\
\hline blanco. & L......... \\
\hline Lelón loco.... & Cucun \\
\hline 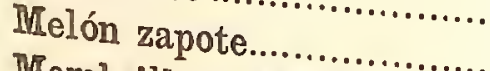 & hera? undulata A. Gr. \\
\hline ero, Membrillo. & $a \mathrm{~L} \ldots \ldots \ldots \ldots \ldots$ \\
\hline 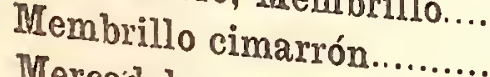 & $\begin{array}{l}\text { Cydon } \\
\text { Cotor }\end{array}$ \\
\hline dela. & ster denticulata H. B. \\
\hline Mercu & ula officinalis L.......... \\
\hline let]. & rviflora Cav. \\
\hline $1 \mathrm{~W}$ & ana $L . . . . . .$. \\
\hline Metl.... & $\begin{array}{l}\text { Agave atrovin } \\
\text { Agave notatu }\end{array}$ \\
\hline sis. . & um Zucc... \\
\hline . & L Lam.. \\
\hline Mezcal.................. & $\begin{array}{l}\text { Agare lechu } \\
\text { Agare, sp? }\end{array}$ \\
\hline uite.... & Aga \\
\hline 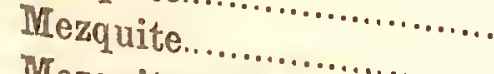 & Inga \\
\hline ite ó Merquitl. & Prosopis cinen \\
\hline an............. & gora DG.......... \\
\hline & Inosopis pubescens Ber \\
\hline
\end{tabular}

Malváceas ........... Verbenáceas ........ Labiadas.

Labiadas.

Geraniáceas.........

Leguminosas.. .....

Compuestas .........

Commelináceas ....

Palmas .

Compuestas.

Compuestas

Compuestas

Piperáceas.

Leguminosas.

Verbenáceas

Commelináceas. ... Zigofileas.

Anonáceas.

Bromeliáceas .......

Tifáceas.

Valerianeas

Bignoniáceas.

Fitolacáceas.

Convolvuláceas.

Liliáceas.

Piperáceas.....

Compuestas.

Verbenáceas

Labiadas.

Labiadas...

Labiadas.

Labiadas.

Leguminosas.......

Gonvolvuláceas. ...

Labiadas.

Rosáceas

Cucurbiláceas

Cucurbitáceas

Cucurbitácens

Pasifloráceas.

Posáceas

Rosáceas

Compueslas.

Compuestas.

Amarilídeas

Amarilídeas

Amarilídeas.

Convolvuláceas....

Amarilídeas

$\Lambda$ marilídeas.

Amarilídeas

Leguminosas.

Leguminosas.

Leguminosas.

Leguminosas

Leguminosas......
Oaxaca.

Michoacán.

Barroso.

Naturalizada ....

Naturalizada ....

Cultivada.

Guerrero, Ver...

Jalisco.............

Tabasco..........

Tabasco....

Chih., Oax., etc..

Chilı., Oax., etc..

Aguascalientes..

Colima, Jalisco...

Varios lugares...

Jalisco.............

Valle de México.

México, Jalisco...

México, Morelos.

Cultivada...

Valle de México.

E. de Méx., Mich.

Varios lugares...

Varios lugares...

Disl. Fed., Oax...

Veracruz, Hid...

V. de Méx., Zac..

Sinaloa.

Cultivada.

Chihuahua. ......

Glihuahua. ......

Valle de México.

Naturalizada .....

Varios lugares...

Gultivada.

Cultivada.

Oaxaca.

Jalisco, Gto.......

Chihuahua, etc. Jal., Pueb., Mor.. Cullivada........ Valle de México. Cultivada.

Varios lugares... Chih., Coah., etc. $V$. de Méx., \&.... V. de Méx., \&.... Dist. Fed., Oax.. Chihuahua....... Jalisco

Chilıuahua ....... Lug. calientes.... Frontera Norte.. Jalisco......

Frontera Norte.. Lug. calientes...
Cat. Inst. Med.

Farm. Mex.

Colmeiro.

B. V. et Urbina.

Rovirosa.

Rovirosa.

Farm. Mex.

F. M., M. Méd.

Farm. Mex.

Urbina.

Farm. Mex.

Colmeiro.

Cat. Inst. Med.

Rose.

Farm. Mex.

Farm. Mex.

Farm. Mex.

Urbina.

Farm. Mex.

Farm. Mex. B. V. et $\mathrm{Ur}^{\mathrm{bi} \mathrm{n}^{\mathrm{a}}}$ Farm. Mex. 


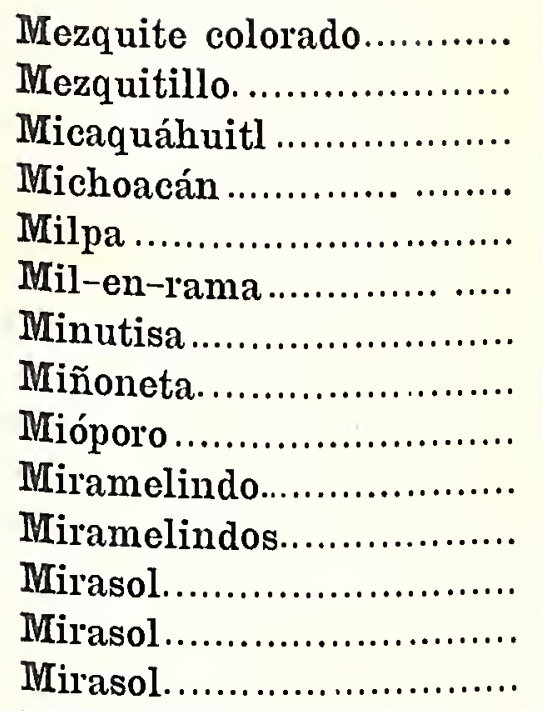

Mirasol amarillo.

Mirasol anaranjado...

Mirto.

Mirto.

Mirto silvestre.

Mispatle

Mispatle

Misquicopalli.

Misquicopalli ó Misquicopatti......

Mitihuaxaqua (tarasco)......

Mitlalzíhuitl

Mitra.

Mixtlácotl

Moca.

Moco de guajolote

Moco de guajolote

Moco de pavo.

Moitli ó Ilolitli, Molintle ó Mlolintli....

Molonqui.

Molle.....

Mombin

Momo.

Momo de zopilote.

Monacillo

Monacillo

Monacillo

Monacillo amarillo

Monacillo blanco.

Monacillo del río

Monapesto

Monilla

Monte de oro

Moquillo blanco

Moradilla

Moradilla

Moral amarillo

Moral blanco.

Moral de clavo

Moral liso.

Ihoral negro

Mosqueta.

Mostacilla.
Inga, sp?

Cassia occidentalis L..

ceguminosas.......

Leguminosas........

Convolvuláceas....

Convolvuláceas...

Ipomaca jalepa Pursh.

Zea mays L..

Achillea millefolium L.

Dianthus barbatus L.

Reseda odorata $\mathrm{L}$

Myoporum ellipticum R. Br........

Delphinium ajacis L....

Impations balsamina L.

Cosmos bipinnatus Cav....

Cosmos ehrysanthemifolius H. B. K

Helianthus annnns L.

Cosnos sulphureus Cav....

Ilclianthus ovatus Lehm.

Salvia coccinea L....

Salvia microphylla H. B. K.........

Loselia coccinea Don................

Buddleia, sp?........................

Buddleia vertieillata H. B. K.....

Inga, sp?.

Prosopis juliflora DC

Sonchns oleracents L.....

Lopezia mexicana Jacq......

Cyclamen europaum L....

Enpatoriun glabratum H. B. K...

Andira excelsa H. B. K.

Polygonum pennsylvanicum L.....

Polygonum persicarioides H. B. K.

Amaranthus caudatus L.

Jacobinia mohintli Hemsl..........

Cissus tiliacea H. B. K...............

Schinus molle L....

Spondias dulcis Forst. var..........

Piper auritum H. B. K..............

Piper peltatum L....................

Abutilon, sp?...

IIalvaviseus arboreus Cav.........

Malvaviseus pentacarpus DC.......

Abutilon striatum Hort.

Hibiscus, sp?.

Hibiscus tubiflorus DC.

Tecoma radieans Juss.

Paullinia, sp?...

Jacobinia anrea Hemsley...........

Gardiospernnm, sp?

Senecio helodes Benth.

Chlorophora tinctoria Gaud. var...

Chlorophora tinctoria Gaud. var..

Norrts alba $\mathrm{L}$.

Chlorophora tinctoria Gaud. var... Chlorophora tinctorea Gaud. var.. Morus nigra L...

Philadelphns coronarius L..........

Wruea sativa Lamk.
Gramíneas.

Compuestas.

Cariofileas.

Resedáceas.........

Mioporineas..

Ranunculáceas....

Geraniáceas.

Compuestas ....

Compuestas

Ciompuestas.........

Compuestas.........

Compuestas........

Labiadas.

Labiadas.

Polemoniáceas .....

Loganiáceas.........

Loganiáceas. ........

Leguminosas.......

Leguminosas.......

Compuestas .........

Onagrarieas.........

Primuláceas.........

Compuestas .........

Leguminosas........

Poligonáceas........

Poligonáceas........

Amarantáceas.....

Acantáceas..........

Ampelídeas..

Anacardiáceas ......

Anacardiáceas......

Piperáceas...........

Piperáceas..........

Malváceas ...........

Malváceas ...........

Malváceas ..........

Malváceas ..........

Malváceas ...........

Malváceas ...........

Bignoniáceas.......

Sapindáceas........

Acantáceas.........

Sapindáceas .........

Compuestas.........

Urticáceas ...........

Urticáceas..........

Urlicáceas ...........

Urticáceas ..........

Urticáceas .

Urticáceas ............

Saxifragáceas.......

Crucíferas.......
Lug. calientes....

Oaxaca, Jalisco.

Mor., Dist. Fed...

Veracruz.......... Farm. Mex.

Jalisco............ B. V. et Urbina.

Jalisco, etc....... Farm. Mex.

Cultivada.........

Cultivada.........

Cultivada.........

Cultivada.........

Cultivada........

Valle de México.

Michoacán........

Valle de México.

Jalisco............. Oliva.

Chiapas .......... Cat. Inst. Méd.

Tabasco.......... Rovirosa.

Jalisco............ B. V. et Urbina.

Varios lugares... Mat. Méd.

E. de México.... Cat. Inst. Med.

Dist. Fed., Méx.. Farm. Mex.

Lug. calientes...

Varios lugares...

Michoacán....... N. León.

Varios lugares...

Cultivada.........

E. de Méx., etc.. Urbina.

Tabasco, Chiap.. Farm. Mex.

S. L. P., Jalisco.. Urbina.

Hidalgo, Ver.....

Cultivada.........

Chiapas, etc......

Valle de México.

Naturalizada....

Lug. calientes....

Tabasco...........

Tabasco...........

Oaxaca ...........

Oaxaca............

Veracruz..........

Cultivada.........

Jalisco............ B. V. et Urbina,

Valle de México. Urbina.

Cullivada.

Nuevo León......

Veracruz, Oax...

Chiapas ...........

E. de Mexico.....

Ver., Camp.......
Cat. Inst. Med.

Ciat. Inst. Med.

C. I. MI., Herb.

Alcocer.

Cat. Inst. Méd.

Urbina.

De Candolle.

Rovirosa.

Rovilosa.

Barroso.

Farm. Mex.

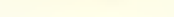

Ver., Ciamp.......

Gultivada........

Tabasco

Tabasco..........

Cultivada.........

Cultivada.........

Cullivada........ 
Mostaza

Mostaza negra

Mota morada.

Motita, Motita morada

Motitas.

Motitas de la playa.

Muela de San Cristóbal.

Muérdago

Muérdago.

Muicle ó Muitle.

IIulinillo

Mulito

Mulito.

Munditos.

Murciélago.

Musgo..

Muste.
Brassica nigra Koch.

Brassica nigra Koch.

Ageratum corybosum Zucc.

Pinaropappus roseus Less.

Chaptalia nutans Hemsl.

Tridax trifida A. Gr.

Aquilegia vulgaris $\mathrm{L}$....

Loranthus calyculatus L....

Loranthus mexicanus Presl

Jacobinia mohinilli Henısley

Cyperus luzulce Poltb.

Bidens leucantha Willd

Melanthera deltoidea Pich

Cardiospermum molle H. B. K.........................

Passiflora difformis H. B. K.

Bryun, Polytrichum, \& species..

Clerodendron ligustrinum R. Br...
Crucíferas.

Crucíferas.

Compuestas.

Compuestas .

Compuestas

Compuestas

Ranunculáceas

Lorantáceas.

Lorantáceas.

Acantáceas.

Ciperáceas.

Compuestas.

Compuestas.

Sapindáceas

Pasifloráceas

Musgos

Verbenáceas
Cultivada

Cultivada

Varios lugares...

Valle de México.

Tabasco

Jalisco.

Cultivada.........

Morelos

Oaxaca...........

V. de Méx., Jal..

Tabasco

Tabasco

Tabasco

D. F., S. L. P...

Tabasco

Lug. húmedos...

Tabasco.
Urbina.

Rovirosa.

B. V. et Urbina.

Farm. Mex.

Colmeiro.

Rovirosa.

Rovirosa.

Urbina.

Farm. Mex.

Rovirosa.

Rovirosa.
N

Nabá

Nabo

Nabo

Nabo colorado de Jarava...

Nabo comestible.

Nacascul.

Nacascul.

Nacaztle ó Nacaztle

Nacazcul.

Nahuitiput.

Nanabuapatle

Nanahuapatli.

Nanahuapatli.

Nanahuapatli.

Nananche.

Nance, Nancis ó Nanche.

Nance agrio.

Nancenes.

Nancenes.

Nanche.

Nanche de perro

Nanzinguahut]

Nanzinzócotl.

Naranjillo.

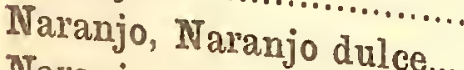

Naranjo agrio

Naranjo amarillo.

Naranjo amarillo.

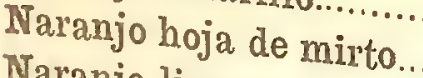

Naranjo lima

Narciso.

Cosalpinia cacalaco H. et $\mathrm{B}$
Myrospermum frutescens Jacq.?...

Brassica campestris L. var.

Brassica oleracea L. var.

Beta vulgaris Moq. var.

Brassica napus L. var.

Cosalpinia coriaria Willd.

Enterolobium, sp

Datura stramonium L...........

Verbesina crocata Less.

Smilax jalapensis ? Schl

Smilax medica Cham. Schl........

Solidago mexicana $\mathrm{L}$. et Sohl.....

Solidago vexicana L.

Byrsonima velutina DG.................

Byrsonima cotinifolia H.............

Byrsonima cotinifolia H. B. K....

Byrsonima karwinskiana . K.....

Bueltneria lanceolala

Malpighia glabra L

Banchosia lanceolata.............. Malpigiáceas...

Byrsonima cotynifoli Turcz....

Byrsonima, sp?....

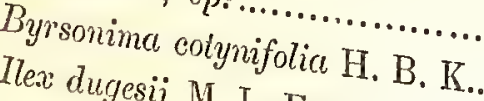

Ilew dugesii M. L. Fernald........

Citrus vulgaris Risso

Thevetia neriifolia Juss....

Citrus ovala A. DC.

Citrus vulgaris Risso. var..........

Narcissus liftum Risso. var.......
Leguminosas.

Crucíferas..

Crucíferas

Quenopodiáceas...

Grucíferas

Leguminosas.

Leguminosas.

Leguminosas.

Solanáceas ....

Compuestas.

Liliáceas.

Liliáceas.

Compuestas.

Compuestas.

Malpigiáceas.

Malpigiáceas

Malpigiáceas.

Malpigiáceas

Malpigiáceas.

Malpjgiáceas.........

Malpigiáceas........

... Lug. calientes...

Rutáceas ......... Guanajuato .....

Rutáceas .......... Cultivada........

Apocináceas...... Cullivada........

Apocináceas...... San Luis Polosí.

Rutáceas ........ Ciultivada........

Rutáceas ......... Cultivada........

Amarilideas...... Cultivada........

Cullivada........
Gat. Inst. Méd.

Finck.

Urbina.

Cat. Inst. Med. Cat. Inst. Med.

Alcocer.

Cat. Inst. Med.

Dondé.

Cilt. Inst. Med. 
Narciso

Narciso.

Narciso amarillo

Narciso amarillo

Nardo

Navajuela..

Navanché de Yucatán.......

Nazareno.

Negrillo.

Negritas

Negritos

Nela..

Neman (maya)

Neuctixihuitl.

Neuctixihuitl.

Nexmitzi (otomí).

Ngedri ó Ngidri (otomí)....

Nicua...

Nigela....

Nigua

Ninfa

Ninfa

Ninfa

Ninfa

Níspero

Níspero

Nixtamaxóchitl.

Nocheznopalli .

Nochtli.

Nochtli.

Nogal......

Nogal

Nogal morado.

Nogal motudo ó N. rayado.

Nogal negro..................

Nogal de Uriqui.

No-me-olvides...

Nopal

Nopal

Nopal

Nopal

Nopal de cochino..

Nopal comúu..

Nopal real.

Nopalillo color de rosa.....

Nopalnocheztl.

Nopalzochicuezaltic........

Noriten (tarasco)........

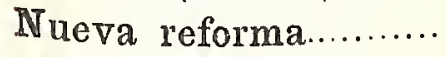

Nueve hojas.

Nuez de Calatola.

Nuer de Castilla.

Nuez chiquita ó encarceladi

Nuez meca

Nuez moscada

Nuez purganie..

Nurite..
Narcissus poeticus L.

Narcissus psendo-narcissus $\mathrm{L}$

Thevetia yoyotli A. DC..

Theretia orata A. DG.

Polianthes tuberosa L.

Rynehospora aurea Vahl.

Sassafias offieinale Nees.

Brosimum alicastium? Sw.

Ulmus mexicana? Planch.

Rhynrhosia preeatoria DC.

Rhynchosia phasevloides DG.......

Euphorbia heteroprlylla L.

Heliotropium previflorum L........

Lippia dulcis Trevir.

Lippia graveolens H. B. K... ...

Artemisia mexieana Willd..........

Elrophrium copalliferum DG.........

Ipomoca bona-nox L.....

Nigella damascena L......

Conosteria xalapensis Don.........

Limnanthemun humboldtianum G.

Nymphcea elegans Hook............

Nympheca gracilis Zucc............

Nymphara mexicana Zucc...........

Eriobotrya japonica Lindl.........

Mespilus germanica L..............

Tecoma strus Juss..

Opuntia hernandezii $\mathrm{DC}$............

Upuntia tuna Mill...................

Opuntia vulgaris Mill.

Juglans regia L...

Juglans rupestris Engelm

Carya oliveformis Nutt..

Carya mexieana? Engelm.......

Juglans nigra L...................

Juglans, sp.

Myosotis palustris With.

Opuntia cagelnamii Salm. Dyck.

Opuntia pseudo-tuna Salm. Dyck.

Opuntia tuna Mill...................

Opuntia vulgaris Mill..............

Opuntia tuna Mill.

Oprntia hernandezii DC.

Opuntire microrlasys Schm. var....

Phyllocactus phyllanthoides S. D...

Opuntia hernandezii DC.

Phyllocactus phyllanthoides S. D... Cedronclla mexicana? Benth......

Veronica spicata L. ................

Serjania racemosa Schum.........

Juglans, sp.

Juglans regia L.

Carya olivaformis Nutt.

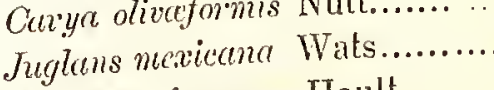

Myristica fragrans Hoult..........

Jatropha multifida L................

Culamintha mucrostema Bentl.....
Amarilídeas...

Amarilídeas.

Apocináceas..

Apocináceas

Amarilídeas.

Ciperáceas..

Lauríneas.

Urticáceas

Urticáceas

Leguminosas.

Leguminosas.

Euforbiáceas

Borragíneas.....

Verbenáceas

Verbenáceas

Compuestas ....

Burseráceas........

Convolvuláceas....

Ranunculáceas..

Melastomáceas ...

Gencianeas.........

Ninfeáceas..........

Ninfeáceas..........

Ninfeáceas..

Rosáceas .

Rosáceas

Bignoniáceas..

Cácteas.

Cácteas.....

Cácteas. .

Juglandeas

Juglandeas

Juglandeas.

Juglandeas. .........

Juglandeas. .........

Juglandeas..........

Borragíneas........

Cácteas

Gicteas.

Gácleas

Cácteas

Cácteas.

Cácteas...

ráctens.

Cácteas.

Cácteas

Cácleas

Labiadas....

Escrofularíneas..

Sapindáceas........

Juglandeas ...........

Juglandeas. .........

Juglandeas. ........

Juglandeas ..........

Miristicens..........

Eufolbiáceas ........

Labiadas.
Cultivada.........

Cultivada.........

Morelos, Chiap...

Michoacán......

Guanajuato, Jal..

Tabasco......

Yucatán.......

Veracruz.

Veracruz.

Guerrero, etc..... Herrera.

S. L. P., Jalisco.. Urbina.

Jalisco, Chiapas. Alcocer.

Yucalán........... Dondé.

Oaxaca, Ver.....

Coahuila, Ver...

Valle de México.

Michoacán.......

Valle de México.

Cultivada........

Puebla, S. L. P.. Urbina.

Varios lugares...

Valle de México. Golneiro.

Valle de México.

Valle de México.

Cultivada.........

Cultivada.........

Méx., Dist. Fed..

Jalisco.

Colmeiro.

Varios lugares...

Varios lugares...

Cultivada.........

Chihuahua, Son.

S. L. P., Sonora..

San Luis Potosí..

Frontera Norte..

Jalisco............ Oliva.

Cultivada.........

Chihuahua......

Jalisco.

Varios lugares...

Varios lugares...

Jalisco..

Jalisco.

Jalisco

Jalisco

Jalisco.

Jalisco.

Michoacán.

Jalisco..

Veracruz.........

Puebla.

Cultivada........

S. Luis Potosí, \&

San Luis Potosí.

Dioga medicinal.

Guerrero...

Mich., V. de M..
Rovirosa.

Co!mciro.

Alcocer:

(

B. V. et Urbina.

B. V. et Urbina.

Colneiro.

B. V. et Urbina.

Urbina.

Cat. Inst. Méd.

Farm. Mex.

Urbina.

Fanm. Mex.

Firm. Mex.

Ciat. Inst. Méd. 


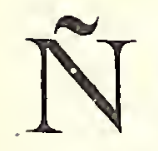

Ñamole

Phytolacea octandica L.

Fitolacáccas

Valle de México.

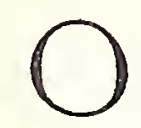

Obelisco.

Oca del Perú.

Hibiscus rosasinensis L.

Oceloxóchitl.

Ocopetate.

Ocopiaxtli.

Ocote.

Ocote.

Ocote amarillo.

Ocote blanco

Ocote chino.

Ocote hembra

Ocote macho.

Ocotilla ú Ocotillo

Ocotillo

Ocotillo

0 cotl.

Ocozóchitl.

0 cosotl

Oxalis creneta Jacq

Tigridir pavonia Ker.

.................

Malváceas

Oxalídeas

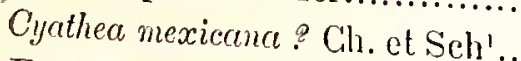

Irideas.

Helechos

Eiryngium beceheyanum? H. el A.

Pinus patula Schiede et Deppe.

Pinus teocote Cham. et Schl..

Pinus, sp?

Pinus ayacahuite Ehrenb.

Pinus leiophylla Schied et Dep...

Pinus montezumce Lamb. var.....

Pinus patula? Seh. et Dep.

Fouquiera splendens Engelm

Cardiospermum molle H. B. K.

Verbesina virgata Cav....

Pinus teocote Cham. et Schl......

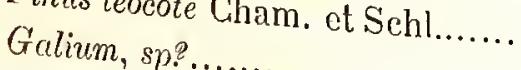

Ojite.

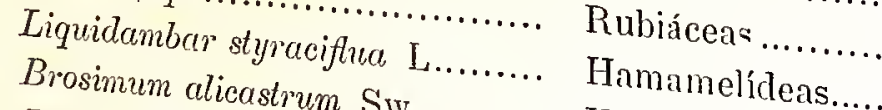

Ojitos de picho.

Ojo de bolrico

Ojo de cangrejo.

Ojo de gallo.

Ojo negro.

Ojo de perico.

Ojo de perro.

Ojo de venad.o.

Ojo de Venus.

Olcacatzin.

Olivo.

Olmo.

Olmo del país.

Ololiuhqui.

Ombligo de Venus

Ombligo de Venus.

Omixóchitl..

Omizóchitl

Orchilla

Orégano

Phynchosia phaseoloidi......... Urticáceas...

Mucuna urens DC

Rhynchosia prec.............. Leguminosas...

Umbelífera:

Coníferas

Coníferas

Coniferas

Coniferas

Coniferas

Coníferas

Coníferas ....

Tamariscineas.....

Sapindáceas

Compuestas ............

Coníferas.

Rubiáce....... Distrito Federal.

Jalisco

B. V. et Urbina.

Cultivada.........

Alcocer.

Veracruz...

Farm. Mex.

Varios lugares... Alcocer.

Mćx., Hgo., Ver..

Dist. Fed., etc....

Oaxaca.

\section{Barroso.}

Oaxaca.

Barloso.

Distrito Federal. Ramírez.

Veracruz, Puebla

Méx., Hgo., Ver..

Chihuahua...... Ramirez.

Dist. F., S. L. P..

Jalisco.

Distrito Federal.

Veracruz....

Veracruz......... Gat. Inst. Méd.

Tibasco.......... Rovirosa.

Sanvilalia precatoria Dr......... Leguminosas.... Veriaruz........ Farm. Mex.

Sanvitulia procumbens Lam ....... Compuestas...... Gro., Mor., D. F. Melampodiunbens Lan........ Compuest....... Valle de México.

Paullinia perfoliatum H.B.K. Compuestas........ Valle de México.

Mrucuna brorbadensis Jiıeq.........

IIucuna urens DG.

Thunbergia alata Ho..............

Smilar cordifolia H. et B.........

Olea curopea L...... et B. var'....

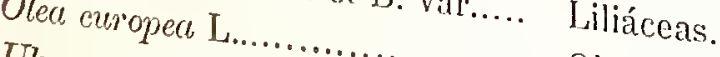

Ulmus mexicana Planch............. Olcáceas.

Sapindáceas............ Jalisco...........

Leguminosas...... Tubasco..........

Veracruz..........

Cultivada.........

Veracruz..........

$\begin{array}{ll}\text { Urticáceas ........... } & \text { Cultivada........ } \\ \text { Gupulíferas ................ }\end{array}$

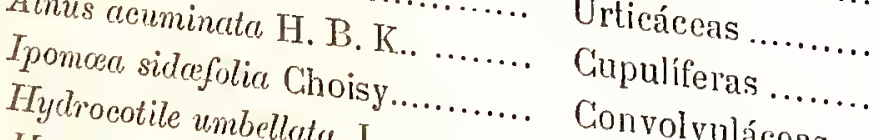

Iydrocotile ranunculoides L. fil....

tris germanion ranculoides I fil... Umbeliferas.

Polianthes tuberosa...............

Unbeliferas

Ver., Hidalgo.....

Gto., Jalisco..... Ramírez.

$\mathrm{V}$. de Méx., 'Tab.

F. M., Rov.

Irideas ........... Ville de México.

Farm. Mex.

Amarilideas....... Gultivada....

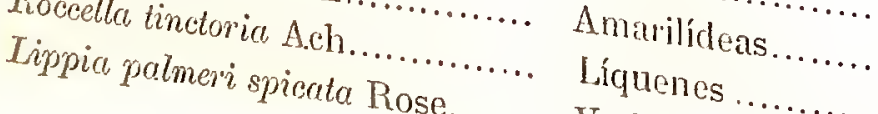

Verbenáceas ....... Baja California..

Baja California.

Urbina.

Rose. 


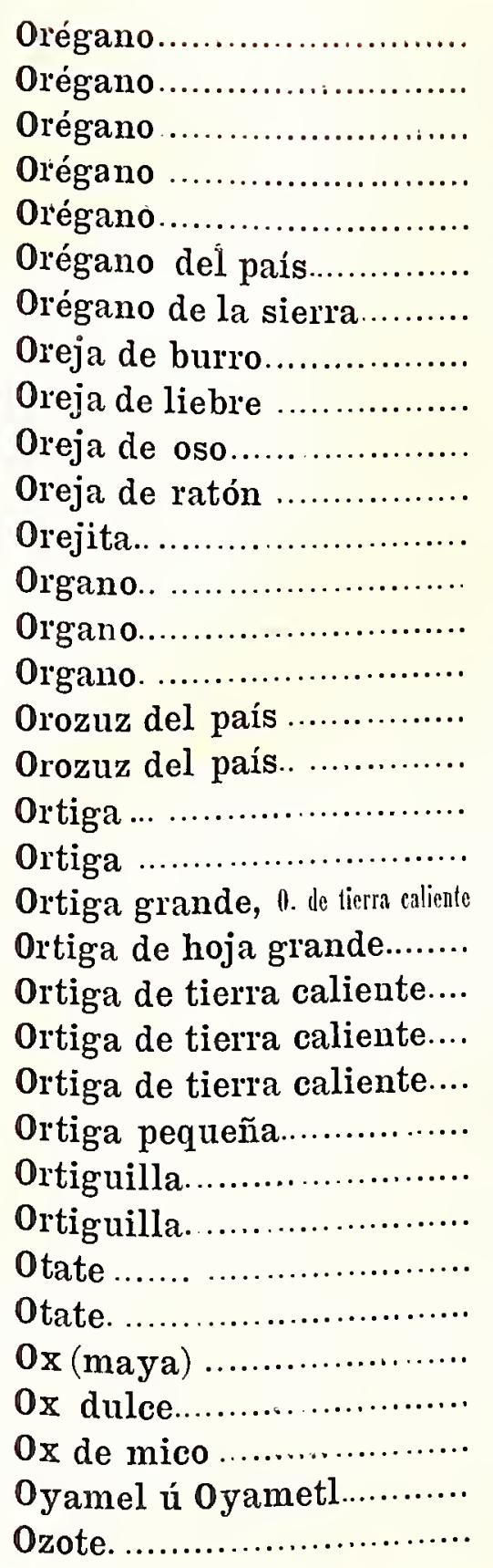

Lippia purpurea Jacq.. Lippia berlandieri Schauer... Origanum majorania $\mathrm{L}$ Origanum vulgare $\mathrm{L}$ Salvia, sp?. Lippia origanoides H. B. K......... Calamintha potosina Schaffner.... Echeverria coccinea DC.

Ipomcea, sp?.

Saxifraga erassifolia L. Dichondra argentea $\mathrm{H}$. et B. Echeverria pullerulenta Nutt. Cereus marginatus $\mathrm{DC}$

Cereus speciosissimus Desf.. Coreus stranineus Engelm..........

Lippia dulcis Trevir. Lippia graveolens H. B. K........ Uiera caracasana Griseb.......... Urtica dioiea L. var................ Wigfandia kunthii Choisy. Tournefortia hirsutissima L......... Wigandia caracasana H. B. K..... IVigandia seorpioides Choisy.... Wigandia urens Choisy...

Uitiea urens L. var.

Ton?nefortia hirsutissima L........ Urtica, sp?

Bambusa arundinaeca Roxb...... Guadua amplexifolia Presl........ Brosimum alieastrum ? Sw.........

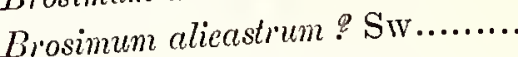
Brosimum, sp?...... Abies religiosa Cham. et Schl...... Ipomea murueoides Rœm. et S....
Verbenáceas

Verbenáceas

Labiadas.

Labiadas.

Labiadas.

Verbenáceas ........

Labiadas.

Convolvuláceas....

Convolvuláceas ....

Saxifragáceas

Convolvuláceas...

Crasuláceas........

Cácteas.....

Cácleas

Cácteas.

Verbenáceas

Verbenaceas .......

Urticáceas ..........

Urticáceas ...........

Hidrofiláceas........

Borragineas .........

Hidrofiláceas.......

Hidrofiláceas........

Hidrofilaceas.......

Urticáceas ...

Borragíneas.........

Urticáceas

Gramínea:

Gramíneas.... ......

Urlicáceas

Urticáceas

Urticáceas

Coníferas

Comvolvuláceas ..
Oax., Mor., Ver.. Rose.

Guerrero........ Rose.

Cultivada.

Cultivada.

Zacatecas

Tabasco...........

San Luis Potosí..

Valle de México.

Gultivada....

Cullivada......... Alcocer.

V. de M., S. L. P.. U.b., Ramz.

Cultivada......... Alcocer.

Valle de México. Ramírez.

Cihiapas.

Chiluuahua

Tabasco......... Rovirosa.

Varios lugares..

Tabasco.......... Rovirosa.

Valle de México. Farm. Mex.

Oax., V. de Méx.

Jalisco.

B. V. et Urbina.

Oaxaca............

Veracruz, Oax...

Guanajuato ......

Jalisco. .

B. V. et Urbina.

Varios lugares..

Valle de México.

Cultivada.

Jalisco........... B. V. et Urbina.

Yucatán.......... Dondé.

Tabasco.......... Cat. Inst. Med.

Tabasco.......... Gat. Inst. Méd.

Dist. Fed., etc...

Jalisco.

B. V. et Urbina.

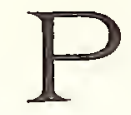

Pabellón mexicano

Pacay.

Pajarillo.

Pajaritos.

Pajaritos

Pajaritos del Norte ...........

Pájaro azul

Pajiza

Pakmul (maya).

Palancapatle ó Palaneapalli.

Palancapatli.

Palancapatli...................

Palancapatli...................

Palancapatli de Puebla....
Salvia mierophylla H. B. K......... Labiadas. Andira excelsa H. B. K.. Frumaria parviftora Lant.... Aquilegia corulea Hort. Tropceolum peregrinum L........... Lobelia fulgens Willd...... Strelitaia regince Ait. Hibiscus manihot $\mathrm{L}$ Physalis angulata L................ Baileya multiradiata Harw........ Senecio vulneraria $\mathrm{DG}$ Solidago mericana L................. Solidago velutina DCi................ Grindelic inuloides Willd. Leguminosas. Papaveráceas. Riınunculáceas.... Geraniáceas.

Lobeliáccas .........

Musáceas.....

Malváceas

Solanáceas ....

Compuestas

Compuestas .........

Compuestas .........

Compuestas........

Compuestas ........
Valle de México, Ciat. Insl. Med. Tabasco.......... Rovirosa. Naturalizada..... Hemsley. Cultivada......... M. Tena. E. de Mexico.... Urbina. Ghilı, V. de Méx. Cultivada........ Cultivada......... Yucatán....... Ghihuahua...... Urbina. E. y V. de Méx.. Urbina. Valle de México. Farm. Mex. Valle de México. Farm. Mex. Valle de México. Urbina. 
Palencano

Palillo.

Palillo de Guanajuato..

Palma

Palma

Palma

Palma

Palma abanico

Palma apache.

Palma barrigona.

Palma de coco.

Palma común.

Palma coyol

Palma crioya.

Palma culona

Palma Christi...

Palma del desierto

Palma dulce...

Palma enana..

Palma gladiola.

Palma loca.

Palma real...

Palmadesombrero, $\mathbb{P}$ soyal

Palmacte.

Palmilla ó Palmillo..........

Palmira

Palmita

Palmitas.

Palmito.

Palo amargo.

Palo amarillo.

Palo amarillo.

Palo amarillo.

Palo de bálsamo.

Palo baria.

Palo blanco.

Palo blanco.

Palo blanco.

Palo bobo.

Palo del Brasil..

Palo del Brasil..

Palo del Brasil..

Palo bronco....

Palo de Campeche

Palo cenizo.....

Palo del chilillo.

Palo dulce..

Palo dulce.

Palo de fierro...

Palo de la flecha.

Palo guaco

Palo hediondo............

Palo jiote.

Palo loco.

Palo madera.

Palo Maxía.
Belotia grewiafolia A. Rich

Croton morifolius Willd. var.

Croton morifolius IVilld. var.

Astrocaryum mexicanum Liebm...

Attalea cohune? Mart.

Attalea, sp?

Ceratozamia mexicana Brongn.....

Latania borbonica W.

Brethea dulcis Mart.

Dasylition incrmis Wals.

Cocos nucifera L..

Phoenix dactylifera $\mathrm{L}$.

Attalea, sp?...

Yucca baccata Torr.

Dasylirion inermis Wats.

Ricinus communis L. var.

Draccena draco? L

Brahca dulcis Nart.

Chamcerops humilis I.

Gladiolus communis L

Yucea filifera Chabaud.

Oreodoxa regia H. B. K

Brahea dulcis Mart.

Oreodoxa regia H. B. K.

Copernicia nana Liebm.

Ipomcea versicolor Meissn....

Draccena, sp?.....

Polypodium, sp?...

Chamcerops humilis L.........

Carya, sp?.

Berberis pinnata Lag...........

Bocconia arbrea Wats.

Bocconia frutescens $\mathrm{L}$

Myroxylon pereirce Klotszch.........

Cordia gerascanthoides H. B. K...

Celtis occidentalis I.

Lysiloma candida Brau...........

Piscidia mollis Rose.

Ipomcea molls Rose.

Homatoxylon oriles Ricm. et S...

Hornatoxylon campechianum L....

Ccesalpinion boreale Wats

Ccesalpinia, sp?

Malphigia urens L

Iromatoxylon C.................

Verbesina serrato Campechianum L.....

Rourea serrata Ciav.

Rourea oblongifolia Hook et Arn.

Eysenhardtic amorphoides H. B.K.

Eysenhardtia orthocarpa W.B.K.

Sebastiana Da.....................

Mikania palmierii Pose.

Gyrocerpustoris Willd.

Burocurpus jucquini Roxb.

Sensecio grunmifera Jacq............

I'ecoma, sp?.. Achras sapota L.
Tiliáceas.

Euforbiáceas

Euforbiáccas

Palmas

Palmas

Palmas

Cicadáccas.

Palmas

Pálmas

Liliáceas

Palmas

Palmas

Palmas

Liliáceas.

Liliáceas.

Euforbiäceas

Liliáceas.

Palmas

Palmas

Irideas

Liliniceas.

Palmas

Palmas

Palmas

Palmas

Conrolvu'áceas.

Liliáccas.

Helechos

Palmas

Juglandeas.

Berberídeas.

Papaveráceas

Papaveráceas

Leguminosas.

Borragíneas.

Uiticáceas..

Leguminosas.

Leguminosas.

Convolvuláceas...

Leguminosas.

Leguninosas.

Leguminosas.

Malpigiáceas

Leguminosas.

Compuestas

Cionnaráceas

Leguminosas.

Leguminosas.

Leguminosas.

Euforbiáccas

Compuestas.

Combretáceas

Burseráceas.

Compuestas

Bignoniáceas

Sapotáceas
Tabasco...

Oaxaca .

Guanajuato ......

Oaxaca.

Chiapas

Veracruz

Veracruz

Cultivada.

Hidalgo, Puebliı.

Chihualıua......

Lug. calienles....

Lug. cillentes...

Veracruz.

Gliihuahua .......

San Luis Potosí..

Varios lugares...

Cultivarda........

Pucbla, Gro.....

Cultivada.

Cultivada.

Tamaulipas....

Oaxica.

Guerrero, Ho

Tabasco.

Guerrero.

San Luis Polosí.

Cultivada.

Valle de México.

Cultivada.

Chihualuua, etc.

Michoacán.

Michoacán.

Veracruz, Jalisco

Oaxaca.

Cihihuahua......

Sonora, Baj. Cal.

Sonora............

Morelos..

Tabasco, elc.....

Jalisco...

Lug. calientes...

Lug. calientes...

Tabasco, etc.....

Michoacán.......

Puebla

Dist. Fed., Hgo.

Julisco.

Introducida

Sonora

Ver, Yuc., Tab.

Morelos

Ciniapas

Valle de México.

Jalisco..

Yuc., Chiap., etc.
C. I. M., Rov.

Mat. Méd.

B. V. et Urbina.

Colmeiro.

Rose.

Kunlh.

Cilt. Inst. Méd.

Urbina.

B. V. et Urbina.

Schaffncr.

Ramílez.

Cat. Inst. Med.

Ramírez.

B. V. et Urbina.

Barroso.

Rose.

Rose.

Ramírez.

Rose.

Urbina.

Cat. Inst. Med.

Ciat. Inst. Med.

Herb, Príng.

Urbina.

Villada, liamz.

Cat. Inst. Med.

Oliva. 
Palo María

Palo María

Palo misanteco

Palo moral.

Palo del muerto.

Palo del muerto.

Palo de la mula.

Palo mulato

Palo mulato

Palo mulato

Palo mulato de Córdoba.....

Palo mulato de Mazatlán..

Palo mulato de México......

Palo mulato de Michoacán.

Palo mulato de Chiapas....

Palo negro.

Palo de peine amarillo.....

Palo de peine blanco

Palo de pingüica

Palo de rosa.

Palo de rosa.

Palo de rosa.

Palo de rosa...

Palo santo

Palo santo

Palo santo

Palo santo

Palo santo

Palo santo

Palo tambor.

Palo de tinte.

Palo de la vida.

Palomas

Palomas

Palomillas

Palomitas

Palomitas

Palto.

Pambazo..

Pambotano

Pamita

Pan caliente.

$\mathbb{P}$ an de manteca

Pan de puerco

Pan de tiorra caliente...

a callente........

Panal ó Panalillo.

Pananex (trrasco).

Pancololote.

Pangagé

Paniquesillo

Panzóchits

Pañati ó Pañete

Paño de Folanda.

Papa ó Papas.

Papaconi.

Papagallo del Brasil........
Cordia gerascanthus Jacq.

Ficus nympherifolia L.

Misanteca capitata Clıam. et Schl. Morus celtidifolia? H. B. K. Galphinia humboldtiana Bartl..... Ipomoea murucoides Rœm. et.S... Monnina ralapcnsis H. B. K....... Bursera gummiferca Jacq. Schimus ?......

Zanthoxylon pterota $\mathrm{L}$.

Simaruba, sp?...

Zanthoxylon, sp?.....

Zanthoxylon pentanome $\mathrm{DC}$

Zanthoxylon affine H. B. K.....

Bursera gummifera Jacq....

Cordia, sp?.

Posoqueria, sp?

Posoqueria latifolia Rœm. et S...

Arctostaphylos pungens H. B. K...

Guaiaenm officinali L.

Pterocarpus, sp?

Tabebuia rosea? DC.

Tecoma, sp?.

Diphysa suberosa Wats.

Fouquieria formosa H. B. K.......

Guaiacum sanctum L...............

Guaiacum verticalc Ort.

Ipomeea glabrata A. Gr.

Ipomoa murucoides, Rœm. et S...

Rhus, sp?

Hcematoxylon campechianum L....

Verbena, sp?.....

Aquilegia vulgaris $\mathrm{L}$.

Aristolochia foetens Lindl...........

Tropceolum peregrinum $\mathrm{L}$

Bignonia buccinatoria Mairet..... Pithecoctenium heragonum DC....

Perser gratissima Gæertn. var.....

Boletus echulis Bull..................

Calliandra grandiflora Benth.....

Sisymbrizm cancscens Nult........

Eragrostis reptans Nees........... Iimnanthemum humboldtianum G. Cyclamen persicum Mill....

Manihot utilissina Polıl.

Alyssum maritimum Lam.

Arbutus, sp?...

Gonolobus, sp?

Cornutia pyramidata L

Capsella bursa-pastoris L.

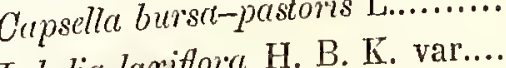

Lobelia laxiflor H. B. K. var....

Plumbago pulchella Boiss..........

Euphorbia pulcherrima Willd.....

Solanum tuberosum L............... Gnaphalium canescens DC.

Caladium bicolor, Vent...
Borragíneas.........

Urticáceas

Lauríneas

Urticáceas

Malpigióceas....

Convolvuláceas....

Poligaleas.

Burseráceas.........

Anacardiáceas .....

Rutáceas ............

Simarrubáceas.....

Rutáceas

Rutáceas

Rutáceas ....

Rutáceas ....

Borragíneas........

Rubiáceas. .

Rubiáccas..

Ericáceas

Zigofileas

Leguminosas.......

Bignoniáceas........

Bignoniáceas.......

Leguminosas........

Tamariscíneas.....

Zigofileas

Zigofileas...........

Convolvuláceas....

Convolvuláceas....

Anacardiáceas. ..

Leguminosas........

Verbenáceas ........

Ranunculáceas....

Aristoloquieas.....

Geraniáceas.........

Bignoniáceas.......

Bignoniáceas.......

Lauríncas.

Hongos..

Leguminosas........

Crucíferas............

Gramíneas

Gencianeas

Primuláceas

Euforbiáccas........

Crucíferas............

Ericáceas............

Asclepiadeas ........

Terbenáceas ........

Crucíferas....

Lobeliáceas

Plumbagineas ......

Euforbiáceas........

Solanáceas ..........

Compuestas........

Aroideas.............
Veracruz, Gro...

Sinaloa.

Veracruz.

Oaxaca........... Barroso.

E. de Méx., Jal.. Urbina.

Valle de México. Farm. Mex.

Varios lugares... Cat. Inst. Med.

Tabasco.......... Rovirosa.

Oaxaca .......... Barroso.

Jalisco............

Veracruz......... Ramírez.

Sinaloa

Valle de México. F. M., Ramz.

Michoacán....... Ramírez.

Chiapas .......... Rovirosa.

Tabasco.......... Cat. Inst. Méd.

Tabasco.......... Alt. et Ramz.

S.L.P. Hgo., Gto.

Veracruz.

Oaxaca........... Barroso.

Tabasco.......... Alt. et Ramz.

Veracruz......... Fink.

Jalisco............ B. V. et Urbina.

Puebla ........... Cat. Inst. Med.

Oaxaca............

Jalisco.............

Sonora............

Sonora

Oaxaca

Gultivada.........

Tabasco.......... Rovirosa.

E. de México.... Urbina.

Puebla, S. L. P..

San Luis Polosí.. Urbina.

Puebla, México..

Valle de México.

$\checkmark$. de Méx., Ver..

Sonora............

Tabasco

Tabasco.

Ciullivada.........

Cultivada.........

Cultivada.........

Michoacán.......

Morelos

Tabasco.......

Introducida.....

Valle de México.

Valle de México.

Sierra Madre...

Cullivada........

San Luis Potosí.

Cultivada........
Ramírez.

Mat. Méd.

F. M., C. I. M.

Rovirosa.

Rovirosa.

Colmeiro.

Alcocer.

Rovirosa.

Mat. Méd.

Mal. Méd. 
Papalocuahuite.

Papalohuiteconi

Papaloquelite.

Papalote.

Papas cimarronas.

Papas cimarronas.

Papaya, Papayo

Papelillo.

Parácáta (tarasco)

Parácáta (tarasco).

Paraíso, Paraíso morado..

Paramu (tarasco).

Pareira brava

Paré (tarasco)

Parí (tarasco)

Pari (tarasco)

Pari (tarasco)

Parietaria

Parota.

Parqui

Parqui

Parra.

Parra silvestre

Parra silvestre

Pasas.

Pascua

Pasionaria

Pastle.

Pasto inglés.

Pastora, Pastoral, Pastorcill.

Pata de león

Pata de león....

Pata de res.

Pata de vaca

Pata de vaca

Patamba

Patata ó Patatas.

Patatas cimarronas...........

Patatas cimarronas

Patatte ó Patarte..

Patartillo.

Patilón.

Patitos.

Patlahuac.

Patoles.

Patzueni..

Payenaltzi

Pebete

Pecan.

Pecetas.

Pedo del diablo

Pegajosa

Pegajosa

Pegajosa de las pa..........

Pegajosa del suelo

Pegarropa
Bauhinia, sp?.......

Perezia alamani Hemsl.

Porophyllum coloratum DG.

Ulmus mexicanc Planch.

Solanum bulbo-castanum Dun.....

Solanum tuberiferum Dun.

Carica papaya L

Euphorbia, sp?....

Oncidium tigrinum Llav, et Lex.

Odontoglossum, sp?

Melia azedarach L....

Fraxinus, sp?

Cissampelos pareira Lamark

Opuntia tuna Mill.

Amarantus, sp?..

Opuntia tuna Mill....

Opuntia vulgaris Mill.

Parietaria pennsylvanica

Enterolobium cyclocarpum Griseb.

Cestrum divirmom $\mathrm{L}$.

Cestrum nocturnum $\mathrm{L}$.

Vitis vinifera $\mathrm{L}$......

Titis caribcea DC

Vitis sicyoides? Baker

Vitis viniferc L.......

Ipomoea sidcefolia Choisy.

Passiftora edulis Sinis

Tillandsia usneoides $\mathrm{L}$

Lolium perenne $\mathrm{L}$.

Tagetes patula L.....

Geranium mexicanum $\mathrm{H}$........

Ranunculus hookeri Schl

Bauhinia divaricata Maury.

Cleome spinosa $\mathrm{L}$

Ipomoea pes-caprce Sweet

Bambusa vulgaris Wendl ?.

Solunum tuberosum L....

Solanum bulbo-castanum Dun.....

Solcunum tuberiferum Dun

Theobroma bicolor. H. et B.

Luker platypetale A. Rich

Pereskica calundrincefolia? Horl...

Centrosema plumieri Benth

Tournefortin. sp?

Phaseolus multiflorus..............

Tillandsia usnenides L

Sulvica, sp?.......

Mirabilis longiftora $\mathrm{L}$.

Carya olivceformis Nult

Zinniu elegans Jicq..

Lyeoperdon?

Ipomece stans Cav.

Mentzelia hispicla Willd.........

Itentzelia seabra H. B. K

Mentzeliu strigosa H. B. K.

Gatium mexicasum H. K.........
Leguminosas

Compuestas

Compuestas ....

Urticáceas....

Solanáceas

Solanáceas

Pasifloráceas

Euforbiáceas.

Orquídeas

Orquídens ....

Meliáceas

Oleáceas.

Menispermáceas...

Cácteas

Amarantáceas.....

Cácteas.

Cácteas.

Urticáceas

Leguminosas.....

Solanáceas

Solanáceas

Ampelídeas...

Ampelídeas.

Ampelídeas.

Ampelídeas....

Convolvuláceas....

Pasifloráceas.

Bromeliáceas.......

Gramíneas .

Compuestas.

Geraniáceas

Ranunculáceas .....

Leguninosas.

Ciıparideas

Convolvuláceas...

Gramíneas

Solanáceas

Solanáceas

Solanáceas

Eslerculiáceas....

Tiliáceas.

Cáctens.

Leguminosas..........

Borragíneas.

Leguminosas.....

Bromeliáceas

Labiadas

Niclagineas.

Juglanileas.

Compuestas.

Hongos.

Convolvulác......

Loaseas.

Loaseas

Loaseas

Rubiáceas
San Luis Potosí. Maury.

Guerrero......... Cat. Inst. Méd.

Coah., V. de M... Urbina.

Veracruz......... Alt. et Ramz.

Varios lugares...

Varios lugares...

Oaxaca, etc......

Michoacán

Michoacán........

Michoacán

Cultivada.

Michoacán

Veracruz.

Miclıoacán.

Michoacán

Mirhoacán

Michoacán

Varios lugares...

Mich., Jal., Gro..

Cultivada.........

Ver., Jal., Méx...

Cultivada.........

Tabasco.

Jalisco.

Cultivada.

Tabasco.

Cultivada.

Varios lugares...

Cultivada.

Jalisco.

Norelos.

Mich S. L. P....

San Luis Potosí.

Tabasco.

Ver., Tab. Oax...

Michoacán

Cullivada.

Varios lugares...

Varios lugares...

Tabasco

Tabasco

Jalisco.

Tabasco

Cultivada.......

Ciultivarla........

Cultivada........

Michoacán........

Puebla.

Ilidalgo, Oixaca

Chih., S. L. P....

Jalisco.

Hidalgo...

S. L. P., Hgo, etc

Dist. Fed., Hgo..

Oaxaca... ........

Oaxaca ............

Dist. Fed., Glo...
Cat. Inst. Med.

N. León.

N. León.

Farm. Mex.

Rovirosa.

Farm. Mex.

Rovirosa.

Colmeiro.

B. V. et Urbina.

Urbina.

Rovirosa.

Cat. Inst. Med.

Rov., C. I. MI.

Rovirosa.

Colmeiro.

N. Leon.

Cat. Inst. Med.

B. V. el Urbina.

Villada.

IIat. Méd.

González.

González. MI.
Nat. Med. 
Pegarropa amarilla.

Peine de bruja.

Peine de mico.

Peinecillo

Pelitre

\section{Pelitre}

Pelón, Pelonchili.

Pelonmexixquilitl

Pelonquáhuitl

Pentamón ó Pentimu, tarasco

Pensamiento

Peonía

Peonía

Peonía de botica

Peonía de niños

Peonía de Jalisco

Peonía de jardines.

Peonía del país

Pepino

Pepino de maceta

Pepino silvestre.

Pepita de San Ignacio.......

Pera, Peral.....................

Peral común..

Pereira brava

Perejil, Perejil común.......

Perejil

Perejil.

Perejilillo

Pericos.

Perifollo

Periquillo

Peritre

Peritre extranjero

Peritre del país.

Perlilla

Perlilla ó Perlitas

Permanente

Perón

Perrito silvestre

Perritos.

Persicaria

Perú cimarrón

Pesto.

Petaquilla

Petatillo

Petunia

Petzanxóchitl

Peyote, Peyotl.

Peyote ó Peyotl

Peyote ó Peyotl

Peyote.

Peyote

Peyote

Peyote

Peyote cimarrón
Mentzelia hispida, Willd.

Erodinn eicutarium Leman........

Apeiza tibourbon, Aubl.

Combretum farinosum H. B.K.....

Ancayelus pyrethrum DC.

Erigeron affinis DC.

Tropocolum majus L

Tropcoolun majus L

Schinus molle L......

Tarodium mueronatum Ten.......

Viola tricolor L. var..................

Cyperns thyrsiftorus Sch...........

Rynchosia phuseoloirles DC........

Cyperus escutentus L.................

Rynchosia precatoria DC...........

Eingthrina coralloides DC............

Pronia montana Sims..............

Cyperns esculentus L................

Cucumis sativus L....

Solanum muricatum Ait. var........

Cucumis anguria L.................

Irura crepilans $\mathrm{I}$

Pyrus communis. L. var..............

Pyrus eommumis L. var.............

Cissampelos pareira Lamarck.....

Apium petroselinum L..............

Carum petroselinum Benth........

Coriandrum sativum L..............

Eryngium nasturtiifolinm Juss......

Pedilanthus, sp?...... .............

Anthriscus cerefolizm Hoffm........

Tagetes lueida Cav...................

Spilanthes becabunga DC.

Anacyelns pyrethrum DCi...........

Erigeron affinis DC.

Lopezia mexieana Jacq............. Symphoricarpos mierophyllus H. B. Gomphrena globosa L.

Pyrus malus L. var.

Pentstemon imberbis Trautv........

Anthirrinum majus L...............

Polygonum persiearioides? H.B.K.

Solidago velutina $\mathrm{DG}$.

Stevia, sp?......

philibertia cumanensis Hemsl......

Thenardiu floribunda H.B.K.......

Petunia myctaginiflora Juss.........

Govenia liliacea Lindl..............

Anhaloninum fissuratum Engelm... Anhaloninm lewinii Hennings..... Anhaloninm villiamsii Lem........ Senccio calophyllus Hensley....... Senceio eardiophyllus IIemsley.... Cotyledon cerespitosa Haw........... Senecio pelasitis Dr. Senecio hartwegii Bentl.
Loaseas.

Geraniáceas.

Tiliáceas.

Combretáceas ....

Compuestas .........

Compuestas .........

Geraniáceas.........

Geraniáceas.

Anacardiáceas ......

Coníferas...........

Violarieas...........

Ciperáceas...........

Leguminosas.......

Ciperáceas...........

Leguminosas........

Leguminosas........

Ranunculáceas.....

Ciperáceas..........

Cucurbitácens......

Solanáceas .....

Cucurbitáceas ...

Euforbiáceas ........

Rosáceas ...........

Rosáceas ............

Menispermáceas...

Umbelíferas. ......

Umbelíferas .........

Umbelíferas .........

Umbelíferas ... .....

Euforbiáceas ........

Umbelíferas. ........

Compuestas ........

Compueslas.........

Compuestas ........

Compuestas .........

Onagrarieas.........

Caprifoliáceas......

Amarantáceas.....

Rosáceas

Escrofularíneas....

Escrofularíneas....

Poligonáceas........

Compuestas .........

Compuestas........

Asclepiadeas.......

Apocináceas.........

Solanáceas ..........

Orquideas

Cácteas

Cácteas...

Cácteas

Compuestas

Compuestas

Cirasuláceas

Compuestas.

Compuestas
Dist. Fed., etc....

Valle de México.

Veracruz

Veracruz......... Urbina.

Cultivada......... Alcocer.

Valle de México.

Cultivada.........

Cultivada........

Valle de México. Mat. Méd.

Michoacán........ N. León.

Cultivada.........

Jalisco............ Bárcena.

Jalisco., S. L. P.. Urbina.

Varios lugares...

Jalisco............ Urbina.

Jalisco............

Cultivada.........

Varios lugares..

Cultivada.........

Cultivada........

Oaxaca. ....

Morelos

Cultivada.........

Cultivada.........

Veracruz..........

Cultivada........ Farm. Mex.

Cultivada........ F. M., Rose.

Cultivada........ F. M., Rose.

Tabasco.......... Rovirosa.

Guanajuato ...... Dugés.

Cultivada.........

Valle de México.

Dist. F., E. de M

Cultivada........

Valle de México.

V. de Méx., etc...

E. de Méx., Mich.

Naturalizada ....

Cultivada.........

Jalisco............ Alcocer.

Cultivada.........

Veracruz, Hgo...

Valle de México.

Puebla

Tubasco....

Oaxaca. Cultivada......... Michoacán........ Ghih., Querétaro Cihih., Querétaro Chil., Querétaro Dist. Fed., Hgo. Dist. Fed., Hgo. Puebla........... Dist. Fed., Vel.. Sierra Madre...
Urbina.

Urbina.

Cat. Inst. Méd.

Rovirosa.

Ramirez.

Cat. Inst. Med. 
Pahcas, tarasco

Pehhuame, tarasco.

Pica-pica.

Picea.

Picea común.

Picietl......

Picietl.

Pico de pato.

Pico de pato

Picosa

Pichaga, Pichaguilla.........

Piche.

Pichichagua

Pie de cabra

Pie de cabra.

Pie de paloma

Pie de venado.

Pimienta de América.......

Pimienta gorda.

Pimienta de isézico.

Pimienta de tierra ó Pimientillt.

Pimienta de Tabasco

Pimienta de la tierra

Pinabete..

Pinahuihuirtle.

Pingüeca ó Pingüica

Pingüeca ó Pingüica

Pinicua.

Pino..

Pino.

Pino.......

Pino..

Pino..

Pino

Pino..

Pino.

Pino...........

Pino...

Pino.......

Pino..

Pino..

Pino.

Pino.

Pino.

Pino

Pino..

Pino..

Pino

Pino...

Pino blanco ó com............

Pino de corcho

Pino oyamel

Pino piŕón.

Pino real.....

Pintzan, tarasco.

Pinzan
Smilax cordifolia H. B. K. var...

Aristolochia, sp?.

Mucuna urens $\mathrm{DG}$

Pinus pinea $\mathrm{L}$

Abies religiosa Cham. et Schl......

Nicotiana rustica L....

Nicotiana tabacum L.

Amiphilophium paniculatum H.B.K

Eichornia azurea Kunth.

Croton ciliato-glandulosus Ort.....

Trixis cabreriana Schaff.

Enterolobium cyclocarpum Griseb.. Senceio cervaricefolius Hemsl......

Bauhinia pes-capree Cav

Eugenia, sp?

Iresine latifolia Benth. et Hook..

Bauhinia, sp?.

Schinus molle L.

Pimenta officinalis Berg. var...........................

Eugenia pseudo-caryophyllus DC.

Peperomia umbilicata Ruiz et Pav.

Pimenta officinalis Berg. var.....

Pimenta officinalis Berg. var......

Abies religiosa Cham. et Schl.....

IIimosa pudica L

Arctostaphylos mucronifera $\mathrm{DC}$...

Arctostaphylos pungen Jacquinia pungens A. Gr.

Pinus arizonica Engelm

Pinus ayacahuite Elrenb.

Pinus cembroides Zucc.

Pinus chiluahuana Engelm

Pinus edulis Engelm.

Pinus engelmanni Carr

Pinus filifolia Lindl

Pinus flexilis James.

Pinus greggii Engelm

Pinus hartwegii Lind

Pinus lamberticna Dougl..........

Pinus lawsoni Roezl

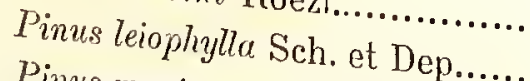

Pinus montezumce Lamb. var.....

Pinus oocarpa Schied.

Pinus patula schiede et Deppe...

Pinus pinceane Gord.

Pinus ponderosa Dougl.

Pinus pseudostrobus Lindl.

Pinus teocote Cham. et Schl....

Abies religiosa Chan. et Scht.

Pinus montezumce Lamb. var.

Abicetsuga douglasii Carr.

Avies religiosa Cham. et Schl......

Pinus cembroides Zucc.

Pinus ayacahuite Ehrenb.

Inga unguis-cati? Willd.

Pithecolobium dulce Benth.
Liliáceas.

Aristoloquieas......

Leguminosas.

Coníferas

Coníferas .

Solanáceas.

Solanáceas

Bignoniáceas.

Pontederiáceas....

Euforbiáceas

Compuestas.

Leguminosas.

Compuestas

Legurninosas.

Mirtáceas

Amarantáceas.

Leguminosas.

Anacardiáceas .....

Mirtáceas.

Mirtácens

Piperáceas

Mirláceas

Mirtáceas

Coníferas.

Leguminosas.

Ericáceas.

Ericáceas

Mirsineas

Coníferas

Coníferas

Conífuras

Coníferas

Coníferas

Coníferas.

Coníferas

Coniferas.

Coníferas

Coníferas.

Coníferas.

Coníferas

Coníferas.

Coníferas.

Coníferas.

Coníferas.

Coníferas.

Coníferis

Coníferas

Coníferus.

Coníferas

Confferas

Coníferas

Conf́eras

Coníferas

Coníferas....

Leguminosas

I teguminosas
Veracruz......

Michoacál........

Jalisco, Veracruz

Cultivada

Varios lugares...

Naturalizada .....

Cultivada.

Tabasco

Tabasco

Querétaro, etc...

Tabasco

Oaxaca, Ghilh.....

Guerrero

Jal isco.............

Valle de México.

Chiapas.

Naturalizada

Tabasco..........

México

Varios lugares..

Tabasco.......

Tabasco...........

Varios lugares...

Veracruz.

Morelos

Varios lugares...

Sonora.

Chihuahua ......

Varios lugares...

Varios lugares...

Chihuahua ......

Ghihuahua.......

Sonora.

Chiapas

Chihuahua.

Nuevo León

Varios lugares..

Chihuahua. ......

Chihuahua ......

Varios lugares...

Varios lugares...

Mich.,Oax.,Pueb

Varios lugares...

NIorelos, Hgo....

Cihiliuahua.

Veracruz....

Varios lugar...

Varios lumar...

Varios luarares.

Hidalgo.....

Varios lugares...

Varios lugares...

Varios lugares...

Michoacán

Gro., Oax., Ver..
Rovirosa.

Rovirosa.

Farm. Mex.

Rovirosa.

Colmeiro.

Oliva.

Cat. Inst. Méd.

Mat. Méd.

Cat. Inst. Méd.

Colmciro.

Farm. Mex.

Rovirosa.

Mat. Méd.

Ramírez. 
Piña

Piña anona.

Piñón

Piñón de Indias..

Piñoncillo

Piñoncillo.

Piñuela

Pío.

Piocha.

Piote.

Piote..

Piote.

Pipa....

Piperita de México

Pipilote

Pipiloxihuitl

Pipiloxóchitl

Pipiloxóchitl

Pipiloxóchitl

Pipitzahoac ó Pipitzahuac.

Piquáremu (tarasco).

Piquin

Pirimu (tarasco)

Pirú, Pirul.

Pistón.

Píta.

Pitahaya

Pitahaya

Pitahaya

Pitahaya

Pitahaya

Pitahaya de Yucatán.......

Pitajaya.

Pitasábida

Pitayita de agua

Pitayita de agua

Pitazábida.

Pititache

Pitón.

Pitzequa, tarasco.

Pixoy, maya.

Planta de la abeja

Platanillo

Plátano

Plátano

Plátano

Plátano

Plátano de Abisinia.

Plátano chico

Plátano chico

Plátano dis 'osta lici, eosteino í domínico

Plátano enano...

Plátano de flor.

Plátano gigante.

Plátano grande.

Plátano guineo.
Ananas sativus Schultes.

MIonstera deliciosa Liebm

Pinus ccmbroides Zucc.

Jatropha curcas L.

Euphorbia lathyris L...

Jutropha cureas L.

Bromelia pinguin L.

Hirtella dodccandra DG.

Melia azedarach L

Senecio cardiophyllus Hemsl........

Senecio calophyllus Hemsl...........

Cotyledon ccespistosa Haw....

Aristolochia, sp?..

Hedeoma piperita Benth.

Asclepias, sp?......

Cestrum nocturnum L...

Cestrum cliurnum? L

Lobelia luxiflora. H. B. K. var......

Silene cutesbai? Talt

I'erezin adnata A. Gr..

Phragmites communis Trin.

Capsicum microcarpum DC........

Dodoncea riscosa L..................

Schinus molle L...

Amaranthus, sp?.

Fourcroya ?.......

Cereus giganteus Engelm...........

Cereus stramineus Engelm.........

Ccreus thurberi Engelm............

Cereus variabilis Pfeiff......

Phyllocactus, sp?.....

Cereus trigonus Haw.

Cereus trigonus Haw.

Aloe variegata $\mathrm{L}$.....

Phyllocactus latifions? Salm Dick

Percokia, sp?...

Aloe vulyaris Lillm.

Pereslice culand iniafolia Hort. B.

Pachira insignis Savign............

Solanum, sp?....................... Gurazma tomentosa H. B. K...... Canna indiea L.

Mnsa eoccinea Andr.

Musa pruratisiaca L.

Whesa cavendishii Lambert........

Mrusa sapientum I,

Musa ensete Gmelin

Musat sapientum L....

Jusa roceinen Antr.................

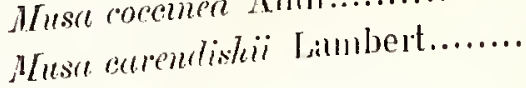

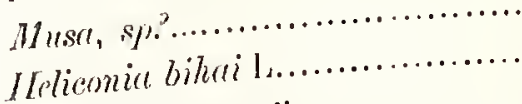

Musa ensete Gmelin.

Mrusa paralisiaed L

Musa sapientum L.
Bromeliáceas...... Cultivada........

Aroideas........... Jal., Mor., Gro...

Conifferas............ Jalisco...........

Euforbiáceas........ Veracruz, elc.... Farm. Mex.

Euforbiáceas ....... Naturalizada.....

Euforbiáceas....... Veracruz, elc.... Farm. Mex.

Bromeliáceas...... Oaxaca, Ver....

Rosáceas .......... Tabasco.

Meliáceas........... Gultivada.........

Compuestas ........ Dist. Fed., Hgo..

Compuestas........ Dist. Fed., Hgo..

Crasuláceas........ México, Puebla..

Aristoloquieas.....

Jalisco.

Hidalgo............

Asclepiadeas ....... Valle de México.

Solanáceas......... Ville de lléxico.

Solanáceas .......... Valle de MLéxico.

Lobeliáceas.......... Jilisco....

Mat. Méd.

Cariofileas.......... Frontera Norte..

Compuestas........ Hgo., Mich., etc.

Gramineas.......... Michoacán.......

Solanáceas......... Cultivada........

Sapindáceas........ Michoacán........

Anacardiáceas ..... Naturalizada .....

Amarantáceas.....

Amarilídeas....... Jalisco....................

Cácteas............. Jalisco............

Cácteas.............. Ghihuahua......

Cácteas............. Jalisco............

Cácteas............. Jalisco...........

Cácteas.............. Jalisco............

Cácteas.............. Yucatán..........

Cácteas ............ Jalisco...........

Liliáceas............ Naturalizada ....

Cácteas............. Jalisco...........

Cácteas .............. Jalisco....

Liliáceas........... Naturalizada.....

Cácteas............. México...........

Malváceas .......... Méx., Ver., Mor..

Solanáceas......... Michıacán.......

Esterculiáceas..... Y Yuc., Tab........

Compuestas ........ Cultivada........ Urbina.

Zingiberáceas...... Naturalizada .....

Musáceas ............

Musáceas ...........

Musáceas...........

Musáceaa ............

Musáceas....

Musáceas.

Musáceas.

Musáceas

Musáceas.

Musáceas.

Musáceas.

Musáceas

Musícens.

México.....

Cialtivada...

Cultivada..

Cultivida.....

Cultivada.

Cullivada.

Cultivada.

Cultivarda....

Jalisco...........

Jalisco............

B. V. et Urbina.

P. T. el Urbina.

F. M., Mat. M.

N. León.

N. León.

Oliva.

Rose.

B. V. et Urbina.

B. V. et Urbina.

Dondé.

B. V. et Urbina.

Alcocer.

B. V. et Urbina.

Mal. Méd.

Farm. Mex.

Farm. Mex.

Firm. Mex.

Ciultivadia...

Cultivada.

Cultivada....... 


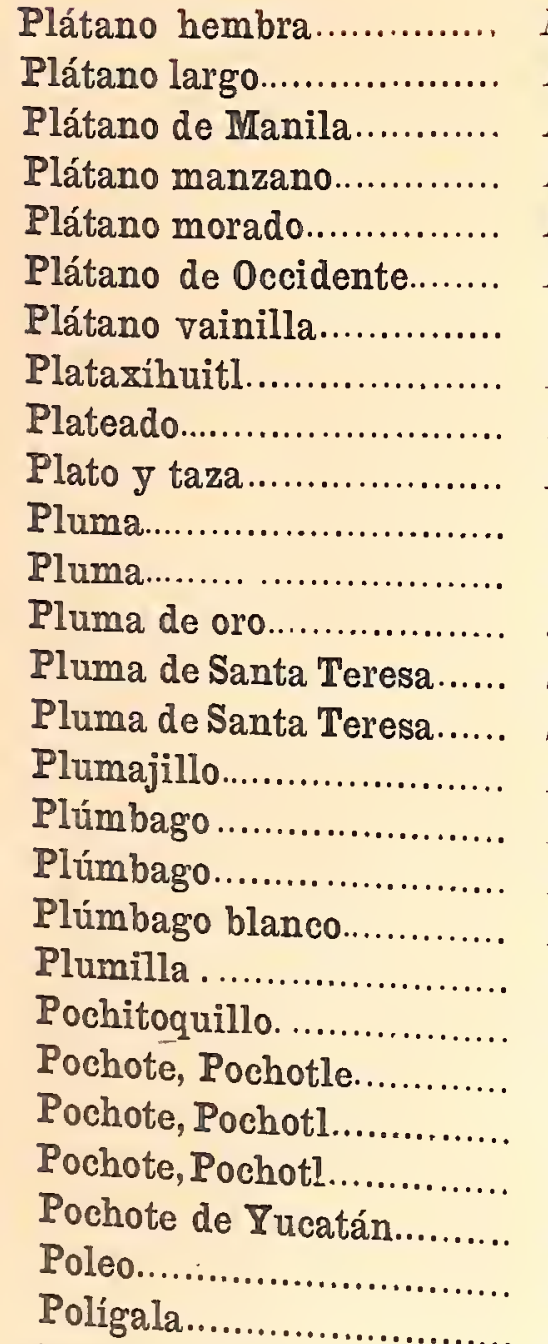

Polígala falsa.

Polígala de jardines.

Polígala de Mérico

Polígala de Mérico

Polígala medicinal.

Polipodio.

Polipodio.

Poma rosa

Pompoqua, Popoaqua......

Ponchishui.

Ponchishui

Popiste, Popiste colorado...

Popoiol.

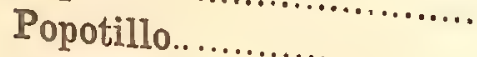

Popotule.

Presera.

Primavera.

Primavera.

Prisco.

Prodigiosa.

Prodigiosa

Pucté

Pucté

Puerro.

Pulguitas.

Pulmonaria

Pulmonaria de herbolario.

Pumu, tarasco.
MIusa paradisiaca L.

II usa paradisiaca L.

Musa textilis Nees.

Musa, sp? ?.

Mrusa rosacea Jacq

Platanus occidentalis Lindl.

Vanilla pompona Schiede.

Plantago galeotticna Dene

Tinantia fugax Scliede.

Asclepias curassavica L

Gladiolus communis L

Gladiohts byzantinus Mill.

J Jacobinica aurea Hemsl.

Salvia eriocalyx Bert.

Salvia lencantha Cav.

Achillea millefolium 1.

I'lumbrigo capensis Thunb.

Plumbrigo europera L.

Plumbago scandens L

Trixis ungrastifolia DC:

Casearia, sp?.

Cochlospermum hibiscoides $\mathrm{H}$..... $\mathrm{B}$

Eriodendron occidentale Tr. et PI.

Eriodendron cesculifolium. H. B. K.

Eriodendron occidentale Tr. et Pl.

Nentha pulegium L

Polygala galioides Poir.

Spermacoce? diversifolia H. B. K.

P'olygala myptifolin $\mathrm{L}$

Polygala mexicana DC.

Polygale scoparic H. B. K.

Polygula scoparia H. B. K.

Polypodium antreun L.

Pteris aquilina L

Jambosa vulgaris DC.

Spondias lutea. L.

Asclepias currassavica..........

Calea axillaris DC:

Condamina?

Ustilago mayrlis DC.

Buccharis pteronioides DC

Agare, sp?

Galium mexic.....................

Primula auricum H. B. K.......

ricula L.....

Primula sincinsis Lindl.

Prumus persicr Benth...........

Buccharis, sp? Benth. et Hook.

Bricketire cercentillesii A............

Terminulic bucessi A. Gr.

Terminaliu buceras Benth. et H...

Allium porrum L............

Shlinnchosia precatoria DC.

Sticte pulmonaceet Ach.....

Picridium vulyare Desf..

Bixa orellana L...

Oreodaxa tregire? H. P. . .
Musáceas............

Cultivada.........

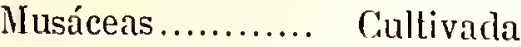

Musáceas........... Cultivada

Platanáceas........ Cultivada

Orquídeas ........... Veracruz.

Plantagíneas ........ Jalisco......

Commelináceas. ...

Asclepiadeas........

Irideas

Irideas

Acantáceas.

Labiadas

Labiadas.

Compuestas .........

Plumbagíneas ......

Plumbagíneas ......

Plumbagineas ......

Cimpuestas .........

Samidáceas

Bixíneas.

Malváceas

Malváceas

Malváceas

Labiadas.

Poligaleas

Rubiáceas

Poligaleas

Poligaleas.....

Poligaleas.

Poligaleas

Helechos

Heleclios

Mirtáceas

Anacardiácens .....

Asclepiadeas...

Compuestas

Rubiáceas ....

Hongos...

Compuestas.

Amarilídeas.

Rubiáceas

Primuláceas.

Prinuláceas

Puisáceas

Compuestas.......

Compnestas

Combretáceas

Combretáceas

Liliáceas.

Leguminosas......

Líquenes.......

Compuestas

Bixíneas.

Palmas

Cultivada.

Cultivada....

Chiapas

Tabasco.

Tabasco..

Morelos..

Jalisco...

México.

Cultivada..

Hidalgo.

Jalisco...

Jalisco.
Varios lugares...

Veracruz...........

Cullivada.........

Cultivada.........

Veracruz, Oax...

Cultivada.........

Varios lugares...

Distrito F'ederal.

Cultivada.....

Regs. calientes...

Camp., Yuc.....

Cultivada.

S. L. P., Ver., etc

S. L. P., Ver., etc

S. L. P., Ver., etc

Virrios lugares...

Varios tugares...

Cultivadia....

Michoacán.

Veracruz, Yue..

Chiapas

Tabasco....... ..

Parásita..........

E. de Méx., Jal..

Valle de México.

Gultivada.

Cultivara.........

Cultivada.

Valle de México.

Jal., V. de M., elc

Tabisco..........

Veracruz..........

Cultivada.........

Guerrero

Morelos.

Michoacán.......

\section{N. León.}

Farm. Mex.

Cat. Inst. ned.

Oliva.

Urbina.

Alcocer.

Cat. Inst. Méd.

Cal. Inst. Med.

Rovirosa.

Farm. Mex.

B. V. el Urbina.

De Candolle.

Farm. Mex.

Farm. Mex.

Farm. Mex.

F. M., U.bina.

Urbina.

Gat. Mnst. Méd.

Upbina.

Farm. Mex.

B. V. et Urbina.

B. V. el Urbina.

N. León. 
Punchuhuiche...

Purenchequa, tarasco..

Puregue.

Purensapichu, tarasco......

Purga

Purga de las ánimas........

Puro Cecilia...

Purú, tarasco.

Puscua.

Pusqua, tarasco............

Putzuteni, talasco..

Puxcanxenec, tarasco
Asclepias curassavica L.

Ev uthrina coralloides DG.

Erythrina coralloides $\mathrm{DC}$...

Rhynchosia precaloria DC.

Ipomaca purga Hayne...

Ipomca, sp?

Randia, sp?.

Cucurbita pepo L.

Ipomoea jalapa Pursh....

Ipomœa, sp?.

Schliuhria abrotunoides Roth......

Ruellia macrophylla \& Vahl........
Asclepiadeas

Leguminosas

Leguminosas.

Leguninosas.

Convolvuláceas....

Convolvuláceas. ...

Rubiáceas..

Cucurbitáceas ......

Convolvuláceas....

Convolvuláceas...

Compuestas .........

Acantáceas
Veracruz, Yuc..

Michoacán.

Michoacán.......

Michoacán

Ver., Hidalgo.....

Querétaro........ Cat. Inst. Med.

Sonora

Cultivada......... N. León.

Veracruz, Hgo...

Michoacán.....

Nichoacán........ N. Leon.

Mlichoacán........
Farm. Mex.

N. León.

N. León.

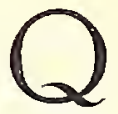

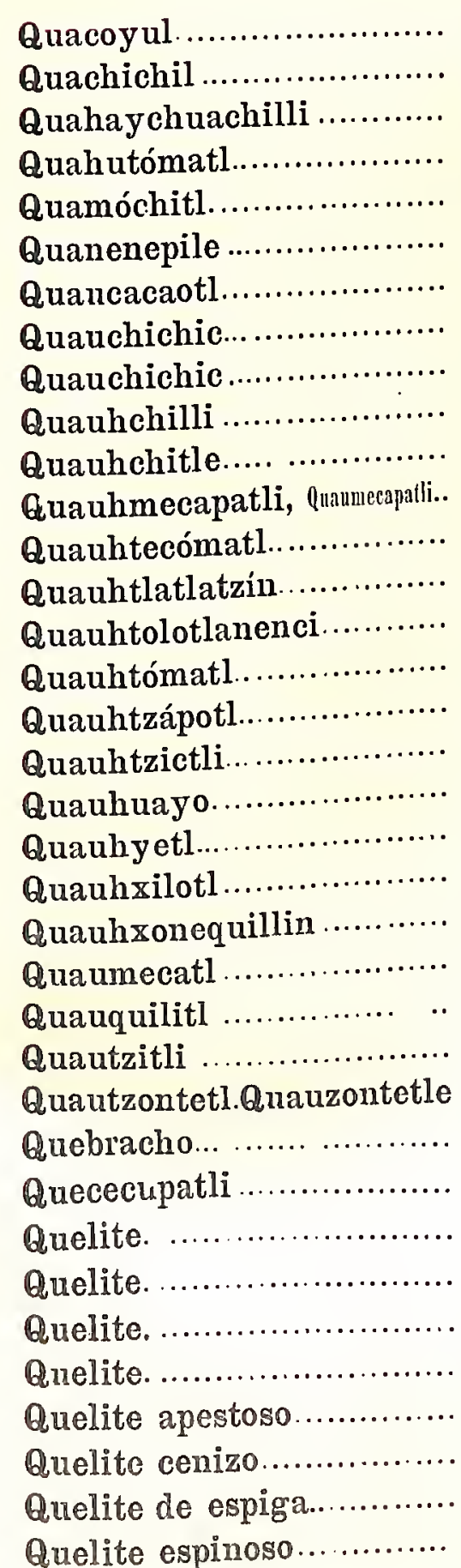

Cocos gracoyule Liebm...

Loeselia coccinea Don.

Jatropha curcas L.....

Quercus, sp?.

Pithecolobium dulce Benth........

Gerardia purpurea? L

Juglans regia L.

Garrya ovata Benth.

Garrya racemosa Ramírez........

Capsicum frutescens L.............

Loranthus mexicanus Presl.........

Smilax medica Cham. et Schl. var.

Parmentiera alata Miers..

Hura crepituns L.

Iostephane heterophylla Benth......

Quercus, sp?...

Anone reticulata L..... ...........

Loranthus calyculutus DC...........

Cassia fistulu L..

Nicotiana tabacum L..

Parmentiere edlulis DC..

Inga, sp?.

Serjania mexicana Willd.

Portulaca oleracea L. var...

Loventhus calyculatus DC.

Blitum bonus-Henricus, Reich....

Coprifere?

Mimosa predica L................... Amarantus hypochondriacus L.... Amarentus palmeri Wats.......... Cassic bacillares L. fil............... Chenopodium album Moq. val'...... Chenopodium foetidum Schrad.... Chenopodium mexicanum Moq.... Anarantus Mypochondriacus L.... Amareantus spinosus $\mathrm{L}$.
Palmas Polemoniáceas ....

Euforbiáceas......

Ciupulíferas ........

Leguminosas.

Escrofularíneas.....

Juglandeas

Cornáceas

Cornáceas

Solanáceas .....

Lorantáceas.

Liliáceas.

Bignoniáceas........

Euforbiáceas ........

Compuestas .........

Ciupulíferas.........

Anonáceas ..........

Lorantácens.........

Leguninosas........

Solanáceas ..........

Bignoniáceas........

Leguminosas.......

Sapindáceas.........

Portuláceas........

Lorantáceas.........

Quenoporliáceras...

Leguminosas........

Leguminosas........

Amarantáceas.....

Amarantáceas.....

Leguminosas.......

Quenopodiáceas...

Quenopodiáceas...

Quenopodiáceas...

Amarantáceas.....

Amarantáceas ......
Oaxaca.

Varios lugares... Mat. Méd.

Veracruz, Mor...

Distrito Federal. Cat. Inst. Med.

Mor., Ver., Gro..

Veracruz, Puebla Cultivada.........

E. de Méx., Zac... Farm. Mex.

E. de Méx., Zac...

Varios lugares...

México............ Colmeiro.

Veracruz..........

Varios lugares...

Morelos, Gro....

V. de Méx., Hgo.

Distrito Federal.

Lug. calientes...

Morelos...........

Cimpeche, Ver.. Cullivada........ Mor., Igo.,Camp Veracruz, Mor... Reg. cal. y húm.. Cultivarla........

Morelos ...........

Naturilizada ....

Veracruz..........

Veracruz.........

B. V. et Urbina. V. de Méx., Jal...

C'dihuahur, etc.. Urbina.

Tabasco.......... Rovirosa.

Varios lugares...

Vitrios lugares...

Valle de México.

Jalisco...

Altamirano.

Farm. Mex.

Teracruz. 
Quelite manchado

Quelite morado.

Quelite morado.

Quelite de puerco

Quelite de trigo.

Quelite de zorrillo.

Quequeste.

Quesadillas.....

Quesadillitas..

Quesadillitas..

Quetzalichtli.

Quetzelinzochitl.

Quiebra-hacha

Quiebra-hacha.

Quiebra-muelas

Quiebra-plato

Quiebra-plato

Quiebra-plato

Quiebra-plato

Quiebra-plato

Quiebra-platos.

Quilitl

Quiltonile.

Quingombo.

Quimichpatli.

Quina

Quina.

Quina calisaya

Quina roja.

Quiquiriquí.

Quirote-culebra

Quisquirin din 0 Quisquiringnini....

Quitlacoctli ó Quitlacotli.
Amarantus hypochondriacus L....

Amarantus hybridus L

Achyranthes verschaffeltii Lem....

Atriplex, sp?

Chenopodium allum Moq. var.....

Chenopodium foetidum Schrad......

Colocasic antiquorum Schotl. var.

Commelina pallida Willd.

Commelina graminifolia H. B. K..

Commelina tuberosa L.

Agave, sp?

Guaiacum, sp?.

Hymencer, sp?.

Asclepias curassavica L.

Datura stramonium L.

Ipomoer longepedunculate Hemsl.......

Ipomcen purpurea Lam

Salvic tilicefolia Vahl

Solamum cornutum Lam.

ITeliotropinm curassavicum I.....

Chenoporlium album Moq. var.... Amarantus hypochondriacus L.... Hibiscus esculentus L.

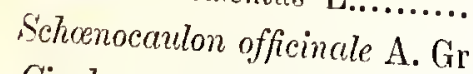

Cinchona officinalis L.

Exostemma, sp?

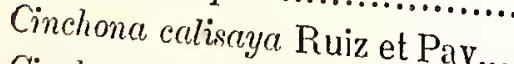

Cinchona succirubra Pav

Salvia patens Ciav

Serjania mexicana Willd......

Berberis pinnatr Lag..

Gyvocurpus jacquini Poxb.

R

Rabanillo

Rabanillo.

Rábano.

Rábano rusticano...............

Rábano de mico.....

Rábano de mico.

Rábano de mico ó de Yuca.....

Ray-grass

Raiz de Abadian...........

Raíz de Chinan

Raiz de la cucar...........

Raíz de Cuculillo

Raíz del desierto

Raiz del gato.

Raíz de granado.
Senecio sanguisorbe DC.

Senecio tolucanus DC

Raphamus sativus L. var..........

Cochlearice armoracia

Heliotropinm curassavicum L......

Heliotropinm iudassavicum L.....

Lolium perenne paftorum L.........

Illicium anisrtiun L

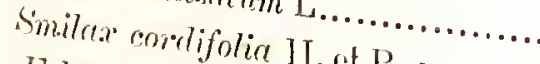

Eethites, sp? ?..... II. et B. var.....

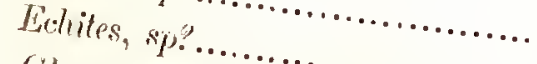

V́aleriana mexicana Dr.

Punien arranatum $\mathrm{L}$
Heliotropiam parum L.

Illicin perenne I

Glyeyrohiza lepidota Nu...........
Amarantáceas......

Amarantáceas.....

Amarantáceas.....

Quenopodiáceas...

Quenopodiácens...

Quenopodiáceas...

Aroideas.

Commelináceas ....

Commelináceas ...

Commelináceas...

Anrarilídeas.

Zigofileas

Leguminosas.. ....

Asclepiadeas........

Solanáceas

Convolvuláceas....

Convolvuláceas..

Labiaras.

Solanáceas

Borragíneas.

Quenopodiáceas...

Amarantáceas

Nalváceas ....

Liliáceas.

Rubiácens

Rubiáceas..

Rubiácens

Rubiácens

Labiadas.

Sapindáceas

Berberídeas.

Combretáceas...
Oaxaca.

Oaxaca.

Cultivada

Fronteri Nolle.

Jalisen

Varios lugares...

Cultivada.

Distrito Federal.

Guanajuato, Hgo

Ver., Dist. Fed...

Distrito Federal.

Oaxaca.n..................

Tabasco....

Naturalizada....

Jallisco, Dist. F...

V. de Méx., etc..

Jalisco...........

S. Luis Potosí, \&

Varios lugares...

Varios lugares...

Oaxaca

Cultivada

Veracruz.

Cullivada.

Michoacán

Cultivada

Cultivada

Guanajuato, Hgo

Sonora.............

Hidalgo, Dist. F..

Varios lugares...
González.

González.

Alcocer.

B. V. et Urbina.

Mat. Méd.

Barroso.

Rovirosa.

Ramírez.

Pamirez.

Oliva.

Gonzále\%.

A. De Candolle.

Farm. Mex.

('at. Inst. Mert.
Compuestas.

Compuestas

Crucíferns.

Crucíferas

Borrngíneas.

Borragineas.

Borragíneas

Gramíneas

Magnoliáceas .............

Liliáceas.

Apocináceáls........

$\Lambda$ pocináceas.......

Lrguminosas........

Valerianeas.

Iil raricas
Michoacán........

E. de M., S. L. P.

Cultivada.

Cultivada.........

Mesa Cientral....

Tabasco

Yucilán.

Cultivida.

Cinllivarla.

.

Daxaca.

Dixica .....

Chihuahua ......

Valle de México.

Collivarla
Firm. Mex.

Firm. Mes.

Rovirosil.

Donclé.

Firm. Mex. Giat. Inst. Mérl. Ciat. Inst. Med.

(.. J. M., F. M. 
Raíz del indio

Raíz del indio

Raíz de maguey

Raíz del manso

Raíz del manso

Raíz del manso

Raíz del oso.

Raíz del sapo.

Raíz de la víbora

Raíz de yerba del burro....

Raíz de zacatón

Ramié

Ramo de oro

Ramón.

Ramón, Ramoncillo.

Ramón.

Ranúnculo.

Ranúnculo amarillo

Rasca viejo..................

Ratón

Reina de las hermosas.....

Reina de la noche............

Reinita

Remolacha...

Repoyo.

Resedá.

Resina tescalama.............

Retama

Retama.

Retama delgada ó de China..

Retama de escobas..........

Retama del país....

Retama de tierra caliente..

Retamilla

Revienta muelas.

Reyan.

Ricino

Robinia

Roble

Roble amarillo

Roble blanco

Roble blanco de San

Roble de duelas..

Roble de San Luis.

Roble serrano.

Rocío.

Romerillo

Romerillo

Romerillo

Romerillo

Romerillo

Romerillo

Romeritos

Romero

Romero cedro.

Romero extranjero
Aristoloelia anguieida L. Rumex hymenosepalus Torrey..... Agave salmiana Ollo.

Iostephane heterophylla Benth...... Houttuyna californiea B. et H...... Tiguiera excelsa Benth. el Hook.. Taleriana ceratophylla H. B. K... Eryngium eomosum Delnr........... Zornia diphylla Pers................ Spigelia longiflora Mart. et Gal... Epicampes macroura Benth....... Bolmeria nivea Hook, et Arnolt. Galphimia hiv'suta Ciav............. Cereocarpus fothergilloides H. B. K Trophis amerieana $\mathrm{L}$. var......... Trophis mexierma Bureau.

Ramuneulus hydroeharis Spenner. Rammeulus napellifolius $\mathrm{DC}$....... Cordia boissieri A. DC.

Dalea diffisa Moric.. Amaryllis josephince Red............ I'hyllocactus latifrons Salm Dyck.. Calendula offeinalis L....

Beta vulgaris Moq. var.

Brassiea oleracea $\mathrm{L}$. var............

Reseda odoruta L....................

Fieus nymplkceifolia L..............

Cassia tagera L......................

Cassia floribunda Gav..............

Spartium jnneeun L.................

Spartium juneerm L................

Cassia lcevigata Willd.

Cassia tomentosa L. fil..............

Berberis, sp?

Aselepias curassaviea L.............

Eugenia ralapensis DC.....

Rieinus eommunis L. var.

Robinia psendo-aeaeia L...........

Quereus virens Ait...................

Citlunexylum ernedatum L....

Tabebuia pentaplyglla Hemsl. ?....

Iubebuia pentaphylla Hemsl. ?.... Querens xalupensis Humb. et B... Íabebuia pentaphylla Hemsl. ?.... Quercus virens Ait.

Mesembryanthemum crystallinum L Asclepias linaria Cav.

Buchnera elongata Sw.

Sucude torreyana Wats...

Chrysactinia mexicana A. Gr......

Salvia polystachya Ort

Viguiera linearis Schz....

Sucela torreyana Wats.

Rosmarines officinalis L..........

Covania mexicana Don....

Rosmarinus officinalis $\mathrm{L}$.
Aristoloquieas.....

Poligonáceas........

Amarilídeas.........

Compuestas.........

Piperáceas..

Compuestas

Valerianeas .

Umbelíferas....... .

Leguminosas........

Loganiáceas........

Gramíneas.

Urticáceas

Nalpigiáceas.........

Rosáceas ............

Urticáceas.

Urticáceas

Ranunculáceas.....

Ranunculáceas.....

Borragíneas.........

Leguminosas.......

Amarilídeas........

Cácteas......

Compuestas ........

Quenopodiáceas...

Crucíferas...........

Resedáceas ........

Urticáceas ..........

Leguminosas........

Leguminosas........

Leguminosas........

Leguminosas........

Leguminosas.......

Leguminosas........

Berberídens.........

Asclepiadeas ........

Mirtáceas...........

Euforlbiáceas........

Leguminosas........

Cupulíferas..........

Verbenáceas ........

Bignoniáceas.......

Bignoniáceas.......

Cupulíferas..........

Bignoniáceas.......

Cupulíferas.........

Ficoideas ...........

Asclepiadeas.......

Escrofularíneas...

Quenopodiáceas..

Compuestas .........

Labiadas............

Compuestas .........

Quenopodiáceas...

Labiadas...

Rosáceas

Labiadas....
México..

Frontera Norte..

Puebla....

Jalisco..

San Luis Potosí.. Urbina.

Distrito Federal. Urbina

Sur de México... F. M., Mat. M.

E. y V. de Méx. Farm. Mex.

Chilıuahua......

Hidalgo ............

V. y E. de Méx..

Cultivada.........

Jalisco....... ... B. V. et Urbina

Oaxaca .............

Tabasco.......... Gat. Inst. Med.

Tabasco.......... C. I. M., Rov.

Dist. Fed., Hgo..

Cultivada........ B. V. et Urbina.

Michoacán....... Cat. Inst. Med.

Varios lugares...

Cullivada........ Bárcena.

Jalisco............ B. V. et Ưrbina

Cultivada........ Colmeiro.

Cultivada........

Cultivada........

Cultivada........

Sinaloa

Tabasco.......... Rovirosa

Varios lugares... Alcocer.

Cultivada........ Farm. Mex.

Cultivada........ Farm. Mex.

Veracruz..........

Guerrero......... Urbina.

E. de México.... Cat. Inst. Med.

Tabasco.......... Cat. Inst. Med.

Veracruz..........

Varios lugares..

Cullivada........

Tabasco........ .

Veracruz..........

Oaxaca............

San Luis Polosí..

Veracruz.........

San Luis Potosí.

Varios lugares...

Cultivada.

S. L. P., V. de M.

Jal., V. de M., etc

Frontera Norte.

Coah., Hgo., etc.

V. de Méx., Gro.

Chih., V. de Méx.

Distrito Federal.

Cultivada....

Jalisco..

Cultivada.
Cat. Inst. Méd.

Colmeiro.

Urbina.

Urbina.

Urbina.

Colmeiro.

Urbina.

Villada.

Farm. Mex.

B. V. el Urbina. 
Romero del país..

Romoza

Rosa.

Rosa amarilla

Rosa anglicana..

Rosa de Bengala

Rosa de Bengala

Rosa blanca

Rosa Cambray

Rosa canela.

Rosa de Castilla

Rosa de Castilla cimarroza

Rosa china

Rosa enredadera

Rosa japonesa

Rosa de Jericó.

Rosa de Jericó.

Rosa de San Juan

Rosa laurel.

Rosa María.

Roșa María.

Rosa miniatura .

Rosa de IRoctezun..........

Rosa del Norte

Rosa panal

Rosa pimpinela

Rosa te.

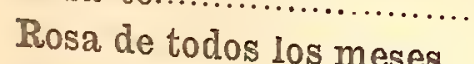

Rosa verde..

Rosadillo.

Rosadillo

Rosalillo.

Roselina.

Rosetilla

Rosilla.

Rosilla

Rosilla

Rosilla.

Rosilla de Puebla..........

Rosilla del Valle de Miérico

Rosillita.

Rta, otomi.

Rubia

Rubia

Ruda.

Ruda cabruna ó capraria...

Ruibarbo de frailes
Trichostemma lanatum Benth......

Rumex pationtia L

Tabernamontana, sp?..

Rosa sulphurea Ait. var.

Rosa, sp?.

Rosa bengalensis Pers

Rosa indica L. var

Rosa alba L. var.

Rosa alba L. var..

Rosa cinnamomea L. var.

Rosa centifoliu L

Guilleminea illecebroides $\mathrm{H} . \mathrm{B} . .$.

Hibiseus rosa sinensis L.

Rosa multiflora Thunb. var...

spircea hypericifolia DC.

Rosa canina L. var.

Rosa gallica L. var.

Senecio cinerarioidles H. B. K.

Nerium oleander. L...

Cannabis satira $\mathrm{L}$

Eupatorium, sp?.

Rosa parviflora Elırb

Rosa montezumce Humb. et Bonpl
Rosa gallica L. var...................

Eupatorium, sp?.

Rosa pimpinellifolia L...

Rosa indica L. var..

Rosa damascena Mill........

Gonolobus uniflorus H. B. K.

Cedrela, sp?.

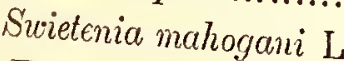

Fouquiera formosa H. B. K.

Stevia clinopodia DG..

Cenchrus tribuloides L.

Commelina pallida Willd....

Commelina tuberosa L.

Helenium mexicanum............

Rosilla lutea Lnum H. B. K.....

Helenium mexicanum H. B. K................

Bidens leucantha Willd..........

Stevia clinopodia DC.

Juglans regia I

Rubia lovigata DG.

Rubia tinctorum

Ruta graveolens I

Dalea citriodora Willd........

Rumex patientia L.
Labiadas....

Poligonáceas

Apocináceas.

Rosáceas

Rosáceas

Rosáceas

Rosáceis

Rosáceas

Rosáceas

Rosácens

Rosáceas

Amarantáceas.....

MLalváceas

Rosáceas

Rosáceas

Rosáceus

Prosáceas

Compuestas

Apocináceas

Urlicáceas

Compuestas.......

Rosáceas.

Prosáceas

Rosáceas

Compuestas.

Posáceas

Rosáceas

Rosáceas

Asclepiadeas

Meliáceas...

Meliáceas.

Tamariscíneas

Compuestas.

Gramíneas.

Commelináceas.

Commelináceas. ..

Ciompuestas.

Compuestas

Ciompuestas

Cimpuestas.

Compuestas

Juglandeas.

Rubiáceas ...

Rubiáceas

Rutáceas

Leguminosas.....

Poligonáceas
Jalisco.

Cullivada.

Jalisco.

Cullivada...

Michoacán

Cullivadi.........

r:ultivada........

Cullivada.........

Cultivada.........

Cullivada.........

Cultivada........ Farm. Mex.

Dist. Fed., Chih..

Cullivada........

Ciultivada.

Cultivada.

Jalisco.

Cultivada.

Jalisco. .

Cullivada.........

Naluralizada ....

Jalisco.

Gullivada......

Hidalgo, Dist. F.

Cullivada.

Jalisco.

Cultivada.

Cultivada...

Cultivada...

Varios lugares...

Veracruz.

Gro., Táb., Yuc.

Jalisco.

Dist. Fed., etc...

Veracruz...

Valle de Méxic.

$V$. de Méx. Jal..

Jalisco

Puebla .......... Mat. Méd.

Distrilo Federal. Mal. Méd.

Dist. Fed., elc...

Cultivada

Ciultivada.

Cullivada

Cultivada

Distrito Federal. Cultivarla........
Oliva.

B. V. et Urbina.

NI. Tena.

B. V. el Urbina.

Bílcena.

Farm. Mex.

B. V. et Urbina.

Oliva.

Fink.

Farm. Mex.

Colmeiro.
Sabacuite

Sabano.
Plumerice rubru L

Miconia urgentea DC.
Apocináceas

Melastomáccas ..........
Luug. temp. y cál.

Tabasco....
Rovirosa. 
Sábida

Sabinò

Sabino

Sabino

Sabino de arroyos............

Sabino de Oaxaca

Sacachichic ó Sacatechichi

Sacalacahuite

Sacamecate...

Sacasile

Sacatechichi.

Sacatillo

Sacatrapo

Sacaxquíhuitl

Sacci.

Sacui.

Sacxin

Saetilla....

\section{Sagitaria}

Sagú cimarrón

Salate.

Salsifi ó Salsifis.

Salvagina

Salvagina macho

Salvia amarilla.

Salvia de bolita

Salvia común.

Salvia de Guadalajara......

Salvia india..

Salvia de S. Juan de los Lagos

Salvia de México

Salvia del monte.

Salvia poblana

Salvia poblana

Salvia real...

Salvia l'eal de México

Salvia real de Puebla

Samatito

Samo prieto

Sanacoche..

Sandía

Sandia de Jalisco.

Sandiita de ratón ó del campo

Sangre de Cristo

Sangre de doncella

Sangre de drago.

Sangre de drago

Sangre de drago.

Sangre de toro

Sangregrado.

Sanglegrado...

Sangregrado

Sanguinaria

Sanjuanero.

Santa María...

Santa Marta...
Aloe varieguta L.

Bignonia, sp.?.

Juniperus mexicana Schiede. .....

Trixodium mucroncitum Ten.......

Tecoma viminalis Hensl............

Terodium mueronatume Ten........

Calea zaeatechichi Schıl.

I'ims ayacaluite Elı'enb.

Clematis, sp?.

Boussinganltiu baselloides H. B. K.

Conyza gnuphalioides H. B. K.....

Crrentria lineatro Nutt.

Helicteres gncuzumefolia H. B. K...

Cupania? dentatr DC......

Agave americana L.

Mentha rotundifolir L....

.

Bidens leneantha. Willd

Sagitteria sagjittifoliu L

Ilerrenta armalinaceu L.....

Fiens, sis?.

Tragopogon porrifolins L...........

Tillondsia usneoriles L.

Tillandsia recurvata L..............

Phlomis pungens Willd.............

Buddleic perfoliata H. B. K........

Buddleia verticillatu H. B. K.....

Salvia polystachya Ort..............

Burldleia perfulinta H. B. K.......

Sulvia, sp?

Lippia umbellata Ciav..............

Salvice microphylla H. B. K........ Lippia callicarpafolia H. B. K....

Lippia umbellata Cav..............

Lippia, sp........................

Buddleia perfoliate H. B. K.......

Lippia eallicarpeffolia H. B. K....

Ficus compplicata H. B. K..........

Coursetic glandulosa A. Gr.........

Cucubila radicans Naud...

Citrullus vulgerris Schrad....

Anguria trifoliata $\mathrm{L}$.

Melothria seabra Naud.

Melothic son conaria Lall..............

Beyonic grecilis H. B. K.. ......

Cioton gossypiffolizes? Vahl. var... Jetiopha spatulate Miill. Arg...... Pterocarpus diaco L................ Spigelia longiflora Mart. et Gall... Jutropha eurets L.................. Jetrophe plat.yphylle Nüll. Arg.. Jatrophe spetnlete Miill. A.g..... Gnaphelium berkendiriri DA...... Erblichic odorala? Seetl........... Sulvict lencantha Gav............... Cereus speciosissimus Dest...
Liliáceas

Bignoniáceas

Coníferas

Coníferas............

Bignoniáceas....

Coníferas.

Compuestas

Coníferas.

Rinunculáceas.....

Quenopodiáceas ...

Compuestas

Umbelíferas.

Esterculiáceas .....

Sapindáceas.........

Ama rilídeas........

Labiadas. ...

Malváceas

Ciompuestas

Alismaceas

Escitamíneas

Uıticácens ..........

Compuestas .........

Bromeliáceas .......

Bromeliáceas .......

Labjaddas............

Loganiáceas........

Loganiáceas........

Labiadas....... ....

Loganiáceas. ........

Labiadas............

Verbenáceas ........

Labiadas............

Verbenáceas ........

Verbenáceas .......

Verbenácens ........

Loganiáreas ........

Verbenáceas ........

Urlicáceris..........

Leguminosas........

Cucurbitáceas ....

rincurbitáceas....

Cucurbitáceas ...

Cucurbitáceas .....

Girriofileas ..........

Begoniáceas.........

Euforbiáceas....

Eufurbiáce as...

Leguminosis...

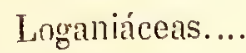

Euforbiáceas...

Euforbiáceas .. .....

Euforbiáceas ....

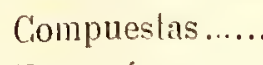

Turneráceas......

Labiadas.

Cácteas.
Naturalizada.....

Jalisco............ Oliva.

Ghih., Hgo., Méx.

Valle de México. Mat. Med.

Jalisco........... B. V. et Urbina.

Oaxaca ......... Kunth, Ramz.

Jalisco, S. L. P... UrJ., Ramz.

Villios lugares... Ramírez.

Hidalgo........... Urbina.

Morelos ...........

Ville de México.

Glıilıuahua......

Tabasco...

J.ugarares cálidos.

Chilh., Coahuila.

Naturalizada ....

Yucután........... Dondé.

Valle de México. Mat. Méd.

Varios lugares..

Tabasco..........

Ramírez.

Gultivada.........

Ville de México.

Distrito Federal.

Cultivada

Varios lugares...

Jalisco.

Jilisco......

Querétaro

Querétaro.

Varios lugares...

Varios lugares..

Puebla

Puebla.............

Puebla...

Valle de México.

Puebla

Guerrero, Mor...

Sonorn............

Néxico, Ver......

Cultivada........

Jalisco.......

Tilbasco.

Cultivada.........

V. de Méx., etc.

Jalisco............ Alcocer.

Disl. Fed., etc... Urbina.

Oaxaca.......... Barroso.

Hidalgo.......... Mat. Méd.

Sinaloa........... Rose.

Sinaloa ........... Rose.

Sinn Luis Polosí. Mat. Méd.

Zacatecas.........

Cilt. Inst. Med.

Cait. Inst. Méd.

Oaxaca, Ver.....

Jalisco. 
Saponaria.

Sapote bobo

Sapote colorado

Sapote negro ó prieto

Sapotillo

Saramulla ó Saramullo .....

Sardonia

Sargazo.

Sargazo.

Saripara, tarasco

Saruma

Saruma...

Sauce ó Sauz

Sauce ó Sauz.

Sauce ó Sauz

Sauce ó Sauz

Sauce ó Sauz

Sauce ó Sauz

Sance ó Sauz

Sauce ó Sauz.

Sauce ó Sauz

Sauce ó Sauz

Sauce ó Sauz

Sauce ó Sauz.

Sauce ó Sauz...

Sauce ó Sauz.

Sauce llorón

Sauco

Saucle ó Sautle

Sayulquelite.

Schischi.

Secocile de México.

Seda vegetal.

Segue Xeuté, totonaco..

Semilla de calabaza.

Semilla de calabaza.

Semilla de hule.

Semillas de búlsamo ó del this

Semíramis

Sempasúchil

Sempiterna

Sempiterna

Sen del país.

Sensitiva...

Señí

Señi

Señorita

Señorita

Sereno.

Shorure, tarasco

Sicajan..

Sicomoro

Sicomoro.

Sicte ó Sictillo.

Sictillo.

Sicje...
Anctgalis arrensis L.

Pachira aquatica? Aubl

Lucuma metmmosa Gærtn. fil.

Diospyros ebenaster Retz..

Erythroxylon macrophyllum Cav..

Anona squamosa L

Viburnum odoratissimum R. Br...

Cabomba aquatica Aubl.

Heteranthera graminea Valll.......

Tillandsia limbata? Schl.

Cecropia mexicana Hemsl.

Cecropia peltata I

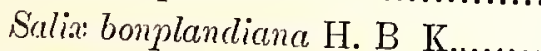

Salix cana Mart. et Gal.

Sulix humboldtiana Willd.

Salix hartwegi Benll.

Sutix latifolia Mart. et Gal

Salix nigra Marsl...

Salix paradoxa H. B. K

Salix stipulacea Mart. et Ga

Sulix taxifoliu H. B. K

Serlix irroratu And.

Salix lasiolepis Benth.

Salix longifolia Muhl...

Salix mexicana Seem

Salix pringlei Rowlee.

Salix babylonica L

Sambucus mexicana Pres

Bletia campanulata Llav. et Lex..

Mratricaria pyrethroides DC.

Agave brachystachys Cav.

Boussingaultia baselloides H. B. K.

Bahmeria nivea Hook. et A B. K.

Vanilla pompona Scliede.

Cucurbita maxima Duchartre.....

Cucurbita pepo L.

Orstilloa ellastica Cerv.

MIyroxylon pereirce Klotzch............

Magnolia grandiftora L.

Tugetes erecta $\mathrm{L}$

Gomphrena globosa L.

Helichrysum orientale Gærtn..........

Ccesalpinia exostema? DC

Mimosa pudica L

Anhalonium levini................ Leguminosas.

Anhalonium william Hennings..... Cácteas.

Asclepias curassavica Lem....... Cácteas.

A solepias incassavica

Asclepias incarnata L.

Ageraturn conyroides

Sysymbirium, sp?...

Jacquinia v

Acer pseudo-plata A. DG.

fi. pseudo-platanus L.

Ficus sycomorus I

Tabernomonte L.................

Tabernomontana litoralis H. B. K

Jacquinia mace citrifolia? L....
Primuláceas....... Naturalizada .....

Malváceas ......... Tábasco..........

Sapotáceas ......... Tabasco...........

Ebenáceas.......... Tubasco, Mor....

Lineas

Anonáceas.

Caprifoliáceas.

Ninfeáceas.

Pontederiáceas ....

Bromeliáceas....

Urticáceas . .

Urticáceas ...

Salicineas.

Salicineas.

Salicineas.

Salicineas....

Salicineas. .

Salicineas....

Salicineas.

Salicineas

Salicineas

Salirineas

Salicineas....

Salicineas

Salicineas

Salicineas.

Salicineas

Caprifoliáceas ......

Orquídeas

Compuestas

Amarilídeas.

Quenopodiáceas...

Uılicáceas..

Orquídeas.

Cucurbiláceas

Cucurbilácens

Uiticáceas

Leguminosas.

Magnoliáceas

Compuestas

Amaranláceas....

Compueslas.......

Leguminosas..

Asclepiadeas...

Asclepiadeas...

Compuestas....

Grucíferas.

Mirsiueas.

Acerineas.

Urticáceas

Apocináceas

Apocináceas.

Mirsineas
Veracruz....

Lug. calientes....

Cullivada.

Tibasco.

Tabasco.

Michoncán.

Mirh.h., V. de M...

Mich., V. de M...

Hidalgo, Ver......

Veracruz.

Guerrero, etc....

Hidalgo.

Veracruz.........

Chihuahua, Oax.

Hidalgo, México.

Hirlalgo.

Chih., Zac., Ver..

Chiluualıua .......

E. de Méxiro.....

Clıilıualıua.......

Hidalgo...

Distrito Federal.

Cultivada.

V. de Méx., ect..

Jalisco.

Jalisco.

Hidalgo, Ver.....

Morelos

Cullivada....

Oaxica, Ver......

Cullivada.

Jalisco.

Veracruz.

Guerrero, Mor..

Cultivada.

Distrito Federal.

Naturalizada .....

Cultivada...

Chiapas

Veracruz.

Querélaro, F. N.

Querétaro, F. N.

Jalisco.....

Cullivada.........

'Tibasco

Miclıoacán.....

Cihiapas

Cultivada........

Cultivada........

Tabasco.

Tabasco

Tabasco.
Cat. Inst. Med.

Cat. Inst. Med.

Cat. Inst. Méd.

Ramirez.

Rovirosa.

Rovirosa.

Colmeiro, F.M.

Colmeiro, F.M.

Herb. Pringle.

Herb. Pringle.

Herb. Pringle.

Herb. Pringle.

Herb. Pringle.

B. V. et Urloina.

B. V. et Urbina.

Cat. Inst. Med.

Cat. Inst. Med.

Farm. Mex.

B. V. et Upbina.

Oliva.

Rovirosa.

Git. Inst. Med.

Cat. Inst. Med.

Rovirosa.

Rovirosa. 
Siche.

Siegesbequia.

Siempreviva

Siempreviva.

Siempreviva

Siempreviva

Siempreviva amarilla.. .....

Siempreviva de nacimiento

Siempreviva silvestre........

Sierrilla

Siete colores.

Simonillo.

Simonillo.

Sinanché.

Sinfito

Sinhuapastle

Sinicuiche, Sinicuilcte ó Sinicril.....

Siranda, tarasco.

Siricote.

Siricote

Sirimo, tarasco.

Sirimuta, tar

Sirundaniqua,

Siruri, tarasco...............

Sirvnda vrueta. tarasco.....

Sithi, otomí.

Situni, tarasco

Sittzo, otomí...

Socoyol.

Socoyol...

Socoyol.

Sochicopal.

Sol de agua

Solano negro

Soldadillo...

Soldadillo

Solimán

Solimán

Solímán vegetal

Sombrerillo de agua.........

Sombrerillo ó Sombrerito de agaa...

Sombrerito de zote amarillo

Sonadora.

Sonoba..

Sosa.

Sosa.

Sotol.

Sotol.

Soyale.

Soyate.

Soyate.

Soyate de Mézico...........

Suchicopal.

Suchicopal.

Súchil.

Srúchil.
Jacquinia ruscifolia Jacq.

Siegesbectia orientalis L

Aizoon canaricnse $\mathrm{L}$.

Sedum quitcnse H. B. K

Sedum rosean Stev.

Sclaginella lepidophylla Spring.....

Scdum dendroideum Moc. et Sessé.

Cotyledon coceinea Ciav

Gomphrena decumbens Jacq.........

Mimosa crvendbergii A. Gr.

Lantana camara L

Calea zacatcchichi Schl....

Conyza filaginoides DC.

Acacia, sp?.

Potentilla candicans Humb, et B.

Montanoa tomentosa Llav. et Lex.

Nescea salicifolia H. B. K.

Ficus, sp?.

Cordia boissieri A. DG

Cordia dodecandra A. DG.

Tilia heterophylla? Vent

Deyeuxia, sp?

Casalpinia pulchemina Sw........

Vitis, sp?..

Diospyros cbencaster Retz............

Zingiber officinale, Roscoe..........

Rubus fruticosus L....

Salix, sp?

Oxalis angustifolia H. B. K.........

Oxalis corniculata L.

Oralis verticillata $\mathrm{DC}$..

Bursera nexicana Engl...

Nymplecea ampla, DC..

Solanum vigrum L.

Asclepias incarnata L.

Piper angustifolium Ruiz et Pav..

Asclepias linaria Cav.

Urtica dioica L. var...

Aselepias cormuti? Dene.

Hydrocotyle mexicana Ch. et Schl.

Hydrocotyle, sp?

Aristolochia, sp?

Astragalus himboldi

Astragahs hambolde A. Gr.......

Lantana camara L.

Solanum torvum Sw

Vigandia kunthii Chuissy.........

Agave, sp?.

Dasylirion, sp?.......................

Braher eluleis Mart.................

Dasylirion inerme Wats............

Nolina, sp?...

Brahea dulcis Mart.

Bursera mevicana Engl............

Elaphrieme copalliferum DC.........

Hibiscus rosa sinensis L............

Plumeria, sp?
Mirsineas

Compuestas

Ficoideas

Crinsuláceas...

Crasuláceas.

Licopodiáceas ......

Crasuláceas........

Crasuláceas..

Amarantáceas.....

Leguminosas...

Verbenáceas .. .....

Compuestas.........

Compuestas .........

Leguminosas........

Rosícens

Compuestas .........

Lilrarieas.

Urticáceas

Borlogíneas.

Borlagineas

Tiliáceas.

Gramíneas

Leguminosas........

Ampelídeas.........

Ebenácens..

Zingiberáceas......

Rosácers ...........

Salicineas.

Geraniáceas.........

Geraniáceas........

Geraniáceas........

Burseráceas........

Ninfeáceas.

Solanáceas

Asclepiadeas........

Piperáceas...........

Asclepiadeas........

Urticáceas.

Asclepiadeas.

Umbelíferas.

Umbeliferas........

Aristoloquieas......

Leguminosas........

Verbenáceas ........

Solanáccas..........

Hidrofiláceas........

Amarilídeas........

Liliáceas.

Palmas .

Liliáceas.

Liliáceas.

Palmas.

Burseráccas........

Anacardiáceas ......

Malváceas ...........

Apocinácers........
Tabasco....

Varios lugares..

Cultivada.........

Cultivada.........

Cultivada.

Chih., Coalı., Mor

Varios lugares...

Jalisco.

Tabasco.

Tabasco.

Jalisco.

Jalisco.

Valle de México.

Tabasco..........

Dist. Fed., etc....

Distrito Federal.

Nuevo León, etc.

Michoacán........

Nuevo León......

Guerrero.

Cultivada.........

Michoacán........

Michoacán........

Michoacán........

Michoacán........

Cultivada

Nichoacán.

E. de México.....

Valle de México. Farm. Mex.

Valle de México. Farm. Mex.

Valle de México. Farm. Mex.

Colima, Ver.....

Tabasco.

Varios lugares...

Cultivada........ Oliva.

Veracruz..........

Dist. Fed., etc....

Puebla............

Frontera Norte..

Oaxaca.

Jalisco...

Cihiapas..

Varios lugares...

Jalisco.

Varios lugares...

Vilrios lugares...

Sin Luis Potosí.

Zacatecas........ Rose.

Varios lugares... Colmeiro.

San Luis Potosí..

Zacatecas.

Hidalgo............

Colima, Ver......

Jalisco.............

Cultivadia........

Oaxaca.

B. V. et Unhina.
Rose.

Barroso.

Rovirosa.

Alcocer.

et Urbina.

Rovirosa.

Mat. M., Ramz.

Cat. Inst. Med.

I., G. I. M.

Fill'm. Mex.

Rovirosa.

Bárcena

Cat. Inst. MIéd.

B. V. ef Urbina

17 
Sufricago.

Sumaque.

Sumic-Ichu

Sundá.

Surumuta

Suspiros.
Lilsea glaucescens H. B. K. var..... Rhus copallina L

Stipa jarava Beauv.

Viburnum glabratum? H. B. K...

Deyeuxia, sp?....

Mirabilis longiflora $\mathrm{L}$
Lauríneas.

Anacardiáceas. ....

Gramíneas'..

Caprifoliáceas.

Gramíneas.

Nictagineas.
Varios lugares...

Chihuahua .......

Veracruz, Hgo...

Michoacán........

Michoacán........

Jalisco.
N. León.

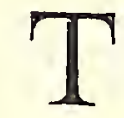

Tabaco

Tabaco amarillo...

Tabaco cimarrón.

Tabaco cimarrón

Tabaco cimarrón.

Tabaco cimarrón.

Tabaco cimarrón.

Tabaco de macuchi.

Tabachín, Tabahinino ó Tabaquín.......

Tabachín amarillo ó de Colima

Tabaquillo

Tabaquillo

Tabaquillo..

Tabaquillo.

Tabaquillo oloroso.

Tabaquillo oloroso

Tabaquito

Tacalote.

Tacamaca ó Tacamahaca...

Tacamba..

Tácari.

Taciste.

Tacopate, Tacopatle ó Tacoparile.....

Tacote.

Tacote de flor...........

Tacuache.

Tachicón.

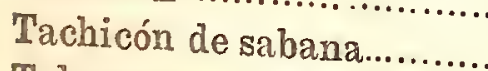

Talayote.

Talayote.

Talcacahuate ó Talcacalinitit

Talclote

Tamalayota

Tamarindo.

Tambor.

Tanchichi ........

Tanibata.

Tapate..

Tapemete.

Tapincerán, Tapintziran....

Tapioca..

Taramicua
Nicotiana tabacum

Nicotiana glanca Gral

Nicotiana mexicana Schl

Nicotiuna pilosa Dun.

Nicoticna pusilla L.

Nicotiana trigonophylla Dun........

Wigandia lianthii Choissy..

Nicotiana rustica L.

Casulpinia pulcherrima $\mathrm{Sw}$

Casalpinia pulcherrima $\mathrm{S} w$

Calamintha macrostema Bentl....

Hydrolea spinosa $\mathrm{L}$

Nicotiana glauca G....

Nicotiana phumbaginifolia Viv.....

Petunia nyctaginiflora Juss

Hedeoma piperita Benth.

Nicutiana, sp?.

Entudla scandens? Benth.

Bursera, sp?.

Chuncedorea?.

Tilleundsia usneoides L

Chamcertorea?

Aristolochic odoratissina..........

Eupatorium, sp?.....

MIontanoa arborescens DC

Ipomcea jalapa Pursh.

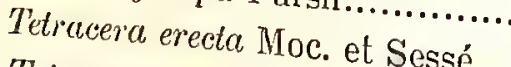

Tetracera ovalifolia ? DG

Gonolobus nummularius Hemsl.....

Gonolobus pedunculatus IIemsl.

Arcechis leypogea L

Lobelic fenestralis Cav.........

Cucurbita maxima Du............

Iramarindus indica L

Mliresulic diventhus Jacq....

Ipomcer stans Cav

Deture strarnonizum L.............

Agave vicipara L

IITimosa, sp?

Manihot utilissima................

Anthurium corsina Polnt.
Cordia gerascenthus J..............
Solanáceas

Solanáceas

Solanáceas

Solanáceas

Solanáceas

Solanáceas

Hidrofiláceas

Solanáceas

Leguminosas....

Leguminosas...

Labiadas.

Hidrofiláceas

Solanáceas

Solanáceas

Solanáceas

Labiadas.

Solanáceas

Leguminosas

Burseráceas.

Palmas

Bromeliáce fis..........

Palmas .

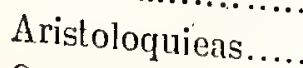

Compuestas.

Compuestas .

Convolvuláceas

Dileniáceus

Dileniáceas.

Asclepiadeas...

Asclepiadeas

Leguminosas.

Lobeliáceas

Cucurbitáceas....

Leguminosas.

Borragíneas

Compuestas.

Convolvuláceas....

Solanńceas

Amarilídeas.

Legruminosa

Euforbiácens.......

Aroideas
Cultivada.

Jalisco.

Aguasc., Ver..

Aguasc., Ver....

Aguasc., Ver.....

Coah., S. L. P....

México

Introducida ......

Guerrero..........

Jalisco.

V. de Méx., Mich

Jalisco.

Distrito Tederal.

Tabasco.

Alcocer.

Dist. Fed., Hgo..

Jalisco.

T'abasco.

Jalisco.

Michoacán

Michoacán.......

Tabasco..

Lug. calientes...

Jalisco.

Julisco................

Hiclalgo, Ver.....

T'ibasco

Tibasco.

Jalisco..

S. L. P., Mich....

Cullivada.

E. de Méx., Jal..

Cultivada...

Reg. cal. y húm..

Michoacán.......

Tabasco...

V. de Méx., IIgo.

Naturalizada....

Son., Sin., Gro... Rose.

Michoacán.......

Ciullivada........ Firm. Mex.

Michoacán..........
B. V. et Urbina.

Farm. Mex.

Farm. Mex.

Farm. Mex.

Farm. Mex.

Colmeiro.

Rose.

Urbina.

Farm. Mex.

B. V. et Urbina.

Cat. Inst. Med.

B. V. et Urbina.

N. León.

N. Leóll.

Cat. Inst. Méd.

Alcocer.

Oliva.

B. V. et Urbina.

Rovirosa.

Cat. Inst. Med.

B. V. el Urbina.

Urbina.

Farm. Mex.

(Gat. Inst. Med.

Rovirosa.

Mat. Mer. 
Tarantaqua

Tarasca de los negros

Taratana.

Taray

Taray

Tarecuen..

Tarequa.

Tarequa cahuiqua

Tarequa varirahpeni

Tarimu.

Tasajillo

Tasajo...

Tatalencho.

Tatzingueni

Tazate..

Te...

Te amarillo de milpa ........

Te de flor blanca.

Te limón.

Te de milpa

Te de milpa

Te de milpa

Te de milpa amarillo.........

Te de milpa blanco.

Te de monte.

Te de olor.

Te del país.

Te de Veracruz.

Tecats

Tecomate, Tecomatl

Tecomate, Tecomatl

Tecomaxóchitl.

Tecomaxóchitl....

Tecopalquáhuitl

Tecote prieto

Tecuampatle óTecuampatli

Tehoitztli ó Tehuixtle......

Tehuacán.

Tejocote.

Tejocote

Tejocote.

Telondilla

Temécatl

Temécatl..

Tempirque.

Ten vergüenza.

Tenaza...

Tencuanete.

Teocote, Teocotl

Teometl

Teotlale.

Teoxintli.

Tepachera

Tepame...

Tepame.

Tepeacuilotl
Castilloa elastiea Cerv

Phytolaeca icosandira L

C'assia alata $\mathrm{L}$

Cessalpinia bonducella Roxb..

Eysenhardtia amorphoides H. B. K

Qucreus, sp?...

Agarieus campestris L

Amanita muscaria L

Amanita, sp?...

Salix, sp??.

Cercus, sp?.

Cereus, sp?

Gymnosperma multiflorum DC...

Cattleya citrina LindI.

Juniperus tetragona Schl...

Lippia, sp?...

Bidens tetragona DC.

Bidens lcueantha Willd...

Andropogon citratus? DC.

Bidens heliunthoides H. B. K.......

Bidens leueantha Willd..

Bidens tetragona $\mathrm{DC}$

Bidens tctragona DC.

Bidens pilosa L..

Calamintha macrostema Benth.... Mentha viridis? L

Lippia geminata H. B. K

Bidens leucantha Willd.

Cueurbita maxima Duch...........

Crescentia cujete L.

Parmentierca alatre Miers

Solandra guttata D. Don ...........

Cochlospermum hibiscoides H. et B. Copaifera offieinalis? L.

Jatropha spatuleta Miill. Arg. var

Gonolobus, sp?

Supindus, sp?......

Lippia origanoiles II. B. K Lippia origanoides H. B. K ........ Cratagnes erus-galli $\mathrm{L}$

Crategus mexieana Dr.....

Crategus pubesecns Steud.

Jatropha spatulata Miill. Arg. var. Cissius tiliacea H. B. K ............. Iponoea emetica Choisy.

Lueuma eapiri? DC.

Mimosa pulica L.

Mimosa, sp?...

Euphorbia calyculata II. B. K ...

Pinus teocole Cham. et Schl..

Agage atrovirens Karw.............

Cupressius benhamii EnIll.........

Euchlana luxurians Dur. et Asch. Calliandra grandiftom Benth.....

Acacia cornigera Willd..

Nimosa, sp?.......

Cornus execlsa H. B. K..............
Urticáceas

Fitolacáceas

Leguminosas

Leguminosas.

Leguminosas..

Cupulíferas

Hongos

Hongos.

Hongos......

Salicineas.

Cáctcas.

Cácteas....

Compuestas

Orquídeas.

Coniferas

Verbenáceas

Compuestas

Compuestas.

Gramíneas

Compuestas

Compuestas

Compueslas.

Compuestas

Compuestas

Labiadas.

Labiadas.............

Verbenáceas ........

Compuestas .........

Cucurbitáceas .....

Bignoniáceas........

Bignoniáceas........

Solanáceas ..........

Bixíneas............

Leguminosas........

Euforbiáceas ........

Asclepiadeas.......

Sapindáceas........

Verbenáceas ........

Rosáceas ...........

Rosáceas

Rosáceas

Euforbiáceas.......

Ampelídeas .........

Convolvuláceas....

Sapotáceas..........

Leguminosas........

Leguninosas........

Euforbiáceas ........

Confferas ...........

Amarilídeas........

Coniferas............

Gramíneas..........

Leguminosas........

Leguminosas......

Leguminosas..

Cornáceas .......
Michoacán........

Tabasco....

Veracruz.

Varios lugares...

Michoacán

Michoacán........ N. León.

Michoacán........ N. León.

Michoacán........

Michoacán........

Jalisco.

Distrito Federal.

Valle de México.

Michoacán........

Durango.

Tabasco

Jalisco.

Valle de México.

Cultivada.........

Valle de México.

Valle de México.

Valle de México.

Valle de México.

Varios lugares...

V. de Méx., Mich

Cultivada........ González.

Tabasco.......... Rovirosa.

Veracruz......... Colm., Mat. M.

Cultivada......... N. León.

Lug. calientes....

Lug. calientes...

Michoacán........ Dunal.

Jalisco, Veracruz

Chiapas, Gro....

Sinaloa, Sonora. Rose.

Morelos.......... Ramírez.

E. de México.....

Yuc., Tab.........

Distrito Federal.

Hidalgo, México.

Jalisco.

Dist. Fed., etc...

Valle de México.

México.

Michoacán.......

Veracruz.

Nuevo León.

Jalisco, Mich.....

Varios lugares...

Valle de México.

Varios lugares...

Jalisco............ Uphina.

Valle de México. Mat. Méd.

Guerrero.

Jalisco

B. V. ct Urbina.

E. y V. de Méx. Farm. Mex.
Mat. Méd.

F. M., Ramz

B. V. et Urbina.

Mat. Méd.

Prodromus.

Colmeiro.

González.

F. M., Urbina, 
Tepeamatl

Tepecamichín

Tepeguaje.

Tepeguaje, Tepehoaxin......

Tepehuaje meco

Tepehuaje negro

Tepehuaje rayado.

Tepehuezote.

Tepeizquitl.

Tepejilote ó Tepepilote......

Tepemezquite.

Teperromero.

Tepexilotl.

Tepezilozóchitl.

Tepezalate.

Tepezempoalsóchitl

Tepezonzóchitl...

Teponaztle ó Teponaxtli.

Tepopote

Tepopote, Tepopot1

Tepopote

Tepopote.

Tepoza.

Tepozán.

Tepozán.

Tepozán del cerro

Tepozancillo.

Terciopelillo..

Tescilama..

Teshuate.

Tetlate, Tetlatia

Tetlatian, Tetlatín.

Tetoyezitl, Tetoyoxitl.

Texaltzapoti.

Texcalamat1.

Texcocana.

Texmole..

Tezócotl.

Texozóchitl:

Teyaqua...

Tezcapatli

Tezontzapotl

Tezopilotlzontecómatl

Thanthzanaqua.

Tharepeni

Thatzin .

Thepame.

Thucumu.

Thucupache zaqua...........

Thumbirichi zaqua

Thurá.

Thumps.

Tianquiz.

Tianquiz.

Tianquizpepetla

Tila ó Tilia.
Ficus nymphaifolia L

Ficus, sp? .

Leuccena, sp?.

Lysilomr acapulcensis Benth.....

Acacia, sp?.

Acacia, sp?.

Acacia, sp?.

Salix lasiolepis Benth.

Arctostriphylos punqens II. B. K.

Chamodorea tepejïlote Licbm...

Prosopis?.

Asclepias linaria Cav

Chamcedorea tepejilote Liehm.....

Calliandra grandiflora Benth ......

Ficus, sp?

Tagetes erecta L.

Valeriana, sp?.

Bursera, sp?

Bucchuris conferta H. B. K

Baccharis pteromioirles DC

Ephedra antisyphilitica Berland...

Ephedra trifurca Torr.

Cornus tolucensis H. B. K

Buddleia americana I

Buddleia humboldtiana Rœm. et s......

Bieddleia lanceolata Benth.

Buddleia sessiliffora H. B. K..................

Dalea lagopus WVilld.

Ficus nymphceifolia I

Melastoma, sp?

Bursera bipinnata Engl.

Bursera bipinnata Engl.

Ipomoea, sp?

Anona squamosa L.

Ficns nymphaifolia L.

Commelina tuberosa

Quercus, sp?

Cratcegnes crus-galli $\mathrm{L}$

Calliandra grandiflora Benth.....

Cheirostemon platanoides II et $\mathrm{B}$.

Senecio precox DG.

Lucumr mammosa Giertn.

Swietenia mahogani L.

Coriandrum sativum L.

QEnothera rosea Ait.

Plaseolus vulgar is L.

Acacia, sp?..

Abies, sp? ..

Solanum nirmum

Biomelia larritas L.

Altiun insignis, Savign.

Aliune cepa L

Alternanthera achyrantha..........

Plumbrigo putchella? Bois....... Alternanthera actyrantha R. Br...
Urlicáceas

Urticáceas

Leguminosas...

Leguminosas.

Leguminosas.

Leguminosas.

Leguminosas........

Salicineas...

Elicáceas

Palmas

Leguminosas.

Asclepiadeas.

Palmas

Leguminosas........

Urticáceas

Compuestas

Valerianeas

Burseráceas

Compuestas

Compuestas

Gulácens.

Gntáceas

Cornácens

Loganiáceas

Loganiáceas.

Loganiáceas.

Loganiácens

Leguminosas

Urticáceas

Melastomáceas...

Burseráceas

Burseráceas

Convolvuláco.....

Anonácens

Urticáceas

Commelináceas.....

Cuuvlíferas

Prosáceas.

Leguminosas......

Estercuilaceas.....

Compucseas....

Sampuestas

Sapotáceas

Meliáceas.

Umbelíferas ........

Onagraricas.

Leguminosas.......

Leguminosas .....

Coníferas .......

Solanácens.

Bromeliáceas.........

Malváceas

Liliáccas. .

Amarantáccas ......

Plumbaŕneas...

Amarnntáceas ....

Tiliáceas.
Sinaloa.

Jalisco.

Sinaloa

Veracruz, etc...

E. de México.....

E. de México.....

E. de Mexico.....

E. de México.....

S. Luis Potosí, \&

Morelos, Oax....

Distrito Federal.

Valle de México.

Mor., Oax., Gro..

Valle de México.

Mor., Gro., Jil....

Cultivada........

Veracruz.

Lug. calientes...

E. de México.....

Jalisco.

Cihihualiua ......

Chihuahua......

E. de México....

V. de Méx., Jal..

E. de México....

E. de Mćxico....

Valle de Móxico.

Jalisco.

Morelos, Gto., rte

Veracruz, Mor..

Morelos

Lugares cálidlos.

Morelos, Gro....

Lug. calientes...

Mor., Gto., Jal...

Valle de Mćxico.

Lugares fríos....

Valle de México.

Valle de México.

Michoacán........

V. y E. de Méx.

Lug. calientes...

Gro., Yuc., Tab.

Cultivada........

Miclioacán.......

Michoacán.......

Michoacán .......

Michoacán.......

Michoacán....... N. Jucón.

Michoacán....... N. I،óll.

Michoacén........ N. León.

Michoncán....... N. León.

Varios lugirres...

Querétaro.

Varios lugares...

Méx., Mich., Mor.
Ciolmeiro.

Oliva.

Rose.

Farm. Mex.

Allamirano.

Alcocer.

B. V. et Urbina.

F. M., Mat. MI.

Cal, Inst. Med.

B. V. et Urbina.

Farm. Mex.

Alt. et Ram\%.

Urbina.

Altamirano.

Mat. Méd.

Mat. Méd.

N. Leon.

Farm. Mex.

N. I،óll.

N. Iscón.

N. León.

Ciat. Inst. Méd. 
Tila ó Tilia

Tilixóchitl

Tilo sirimo, tarasco

Timbe

Timbe

Timbe

Timbe

Timbiriche......................

Timbrillo.

Tinco.

Tinguara.

Tinguara.

Tinguitzen

Tinto.

Tirimo.

Tiripu

Titilámatl.

Tizón de maíz

Tlacamecaxóchitl.

Tlacametl.

Tlacozóchitl

Tlacoxóchitl

Tlacochichic

Tlacopatli

Tlacopatli

Tlacopatli

Tlacozihuitl

Tlacoxihuitl

Tlacoxiloxóchitl

Tlacoxóchitl

Tlachichinoa

Tlahuitol.

Tlalacuaje

Tlalayote ó Tlalayotl.

Tlalayote.

Tlalayote.

Tlalcacahoatl.

Tlalcapolín..

Tlalcocotl

Tlalchichinolli.

Tlalchiolotl.

Tlaljicama

Tlallantlacacuitlapilli.......

Tlalmemeján.

Tlalmemeján

Tlalocopetate ó Tlialocopétlatl

Tlalpopolotl

Tlalteczolli

Tlamacazcatzotl

Tlamahuácatl.

Tlamapaquelite

Tatzalín

Tlamatzalín .................

Tlanchalagua.

Tlanepaquelite ó Tlaneparuilitil..

Tlanoquilixílhuitl.....
Tilia mexicana Schl.

Tanilla sativa Schicde.

Tilia mexicana Schl.

Acacia malacophylla? Benth.....

Bauhinia platypetala Benth...

itimosa, sp?.

Rhus, sp?

liaratas plumieri E. Morr..........

Calliandra grandiflora Benth.....

Cuassia, sp?......

Earacha umbellata? G. Don........

Solamum, sp?......

Convolvulus, sp?

IIcematoxylon campechianum L.....

Tilia mexicana Schl

Cuscuta americana $\mathrm{L}$

Bombax cllipticum H. B. K.........

Uredo maydis DC

Eupatorium, sp?

Agave salmiana Otto.

Bouvardia triphylla Salisb.

Houstonia, sp?.

Gnaphatium caneseens? DG.......

Aristoloquia, sp?.

Jostephane heterophylla Benth......

Helianthus, sp?...

Boceonia arborea IVats.

Boceonia frutescens L

Calliandra grandiflora Benth.....................

Bouvardia triphylla Salisb........

Tournefortia mexicana Vatke......

Acacia riparia H. B. K..

Piper, sp?.

Gonolobus pedunculatus Hemsl...

Asclepias, sp?.

Asclepias linaria Cav

Arachis hypogcea L...

Rluamen humboldtianus R. et

Ipomea eapillacea Don..

I'lumbago seandens L...

Lennoc madreporoides Llav. et $\mathrm{L}$.

Phaseolus, sp?

Ipomoea jalapa Pursh.

Tuphorbia thymifolia Burm.

Hephone thymifolia Burn........

Euphorbia maculata L..............

Corietria thymifolia H. B. K.......

Telianthus, sp?.

Ephedra, sp?

Calliandra grandiflora Benth......

Tiburnum elatum, Benth.

Piper sanctum Schl.

Sonchus eiliatus I ..........

Sonchus olevecers I

Erythrea tetramera Schiede.......

Piper sunctum Schl.................

Gentiuna, sp?..
Tiliáceas

Orquídeas

Tiliáceas.

Leguminosas.

Leguminosas.

Leguminosas........

Anacardiáceas.....

Bromeliácens.

Leguminosas.

Simarrubáceas.

Solanáceas.

Solanáceas..........

Cons'olvuláceas.....

Leguminosas...

Tiliáccas.

Con rolvuláceas...

Malváceas

Hongos.

Amarilídea

Rubiáceas

Ruljiáceas

Compuestas ........

Aristoloquieas.....

Compuestas

Compuestas.

Papaveráceas.

Papaveráceas......

Leguminosas.......

Rubiácens ..........

Borragíneas.........

Leguminosas.......

Piperáceas.

Asclepiadeas.......

Asclepiadeas........

Asclepiadeas.......

Leguminosas.......

Ramneas

Convolvuláceas....

Plunbagineas......

Lennoáceas.........

Leguminosas.......

Convolvulácens....

Euforbiáceas ........

Euforbiaccas ........

Coriarieas...........

Compuestas........

Gnetáccas...........

Leguminosas........

Catprifoliáceas ......

Piperáceas..........

Conpuestas.......

Compuestas........

Gencianeas.........

Piperáccas..........

Gencianeas.
Michoacán.......

Veracruz.........

Michoacán.......

Hidalgo...........

Jalisco............

Jalisco, Zac..... B. V. el Urbina.

Jalisco............ Oliva.

Regs. calienles... Farm. Mex.

Valle de México. Mat. Méd.

Tabasco.......... Cat. Inst. Med.

Jalisco, México... Uurbina.

Jalisco............ Oliva.

Michoacán........ N. León.

Tabasco........... C. I. M., Rov.

Michoacán

Michoacán........ N. I ‘’ón.

Guerrero, Ver....

Parásita..........

Valle de Méxiro.

Hidalgo........... Farm. Mex.

Distrito Federal. Farm. Mex.

Distrilo Federal. Farm. MIex.

S. L. P., Gto., d. Farm. Mex.

Lugs. calientes...

Jalisco.

México............ Colmeiro.

Jalisco, Mich..... Mat. Méd.

Jalisco, Mich..... Miıt. Méd-

Valle de México. Mat. Med.

Varios lugares...

Morelos.......... Farm. Mex.

Hidalgo, S. L. P. Urbina.

Dist. Fed., Mich.

S. L. P., V. de ML.. Urbina.

Cultivada......... Firm. Mex.

E. de México.....

Valle de México. Farm. Mex.

Guanajuato, elc. Farm. Mex.

Veracruz......... Villada.

Morelos .......... Cat. Inst. Med.

Teracruz......... Farm. Mex.

Verneruz..........

Veracruz..........

Valle de México. Mat. Mred.

Reg. calientos..

Dist.Fed., Mor.\& Farm. Mex.

Mich., Méx.......

Oaxaca, Ver..... Farm. Mex.

Valle de México. Farm. Mex.

Gultirada........ Farm. Mex.

Morelos.......... Urbina.

Oaxnca, Ver...... Colmeiro, F. M. 
Tlaolli, Tlauli.

Tlapa, Tlapatl.

Tlapahoaxpatli.

Tlapatecacáyat1.

Tlapatl.

Tlaquilin

Tlascal, Tlascale

Tlatlancuaya, Tlatlancuaye.

Tlatzcán.

Tlaxcala.

Tlaxcapán.

Tlaxizqui ó Tlaxizłle...........

Tlemole, Tlemolitos..

Tlemolli.

Tlepatli

Tlepatli?

Tlepatli

Tlilxihuitl.

Tliltzápot1.

Tlipotón.

Tlilzóchit1.

Tocó

Tocopalquáhuitl

Toje...

Toloache, Toloatzin

Toloache.

Toloache.

Tolonpat1

Tomáhuac....

Tomate, Tomatl.

Tomate, Tomatl...

Tomate, Tomatl...

Tomate cimarrón

Tomate de comer

Tomate de culebra

Tomate de culebra

Tomate de culebra

Tomatillo.

Tomillo.....

Tomillo de Jalapa

Tonalahuate.

Tonaloxóchitl....

Tonalxóchitl.

Topoza

Topoza, Topozán.

Torito.

Torito

Toritos

Tormentila

Tormentila.

Tormentila.

Tornillo.

Torongil.

Torongil

Torongil.

Torongil.
Zea mays $\mathrm{L}$

Datura stramonium L

Eysenhardtia amorphoides H. B.K.

Tagetes, sp?.

Ricinus communis L. var...

Mirabilis, sp?...

Cupressus lindleyi $\mathrm{KI}$.

Iresine, sp:.

Cupressus lindleyi $\mathrm{Kl}$.

Ipomoea, sp?.

Ipomoca stans Cav..

Cotoneaster denticuluta H. B. K...

Tagetes patula L.

Physalis angulata $\mathrm{L}$.

Plumbago pulchella Boiss.

Plumbago scandens $\mathrm{L}$.

Toun mefortia mexicana IVatke...

Sphceralcea angustifolia St. Hil....

Diospyros ebenaster Relz.

Eryngium foetidum? L

Vanitla sativa Schiede....

Coccoloba caracasana..........

Copaifera officinalis $Q$.

Roccella fusiformis? Ach.

Datura stramonium L.

Datura tatula L

Martynia fragrans? Lindl.........

Ipomoea purga Hayne.

Aristoloquia, sp?.

Physalis angulata. L.

Physalis peruviana $\mathrm{L}$

Physalis pubescens $\mathrm{L}$.

Solanum tectum Pers

Physalis coztomatl Moc. et Sessé

Nicandra physaloides. et Sessé..

Physalis angulata L

Physulis nicandroides Schl...........

Physalis angulata $\mathrm{L}$.

Thymus vulgaris L.

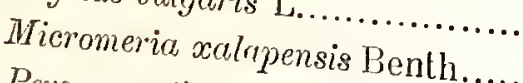

Persea gratissima Giertn.

Bletia coccinea Llav. el Lex....

Richardice africana, \& Kunth.

Cornus tolucensis II. B. K.

Buddleia americana L

Stanhopea oculata Lindl...

Stanhopea tigrina Baten....

Marlynia fragrans Lindl

Potentilla comaroides Nestl...

Potentilla candicans Humb......

Potentilla ranunculoides H. et B.

Prosopis pubescen sordes H. B. K...

Calamintha mescens Benlh.

Cedronella mexta ? Link et $\mathrm{H}$...

In'acocephadicana Benth.

Salvia microphyllavicum L.....
Gramíneas..

Solanáceas.

Leguminosas.

Compuestas

Euforbiáceas

Nictagineas

Coníferas.

Amarantáceas.

Coníferas

Convolvuláceas.

Convolvuláceas.

Rosáceas

Compuestas

Solanáceas .

Plumbagíneas

Plumbagíneas

Borragíneas.

Malváceas

Ebenáceas..

Umbelíferas.

Orquídeas.

Poligonáceas...

Leguminosas.

Líquenes.

Solanáceas .

Solanáceas

Pedalíneas

Convolvuláceas.

Aristoloquieas.

Solanáceas

Solanáceas

Śolanáceas.

Solanáceas ...........

Solanáceas

Solanáceas

Solanáceas

Solanáceas

Solanáceas.

Labiadas.

labiadas.

Lauríneas.

Orquídeas....

Aroideas.

Cornáceas ...

Loganiáceas.

Orquídeas...

Orquídeas

Pedalíneas.

Rosáceas

Piosáceas

Rosáceus

Leguminosas...

Labiadas.....

Labiadas. ...

Labiadas.

Labiadas.
Ciultivada........

Naturalizada .....

Varios lugares...

Varios lugares... Farm. Mex.

Varios lugares...

Ver., Mich., Méx.

Puebla...

Gro., Hgo., Ver..

Jalisco.

Dist. Fed., etc...

Valle de México.

Distrito Federal.

Ciultivada...

Valle de México.

V. de Méx., Hgo.

Morelos, Jal.....

Varios lugares...

Morelos

Introducida .....

Veracruz.

Tabasco.

C'hiapas.

Bija California..

V. de Méx., Jal..

Varios lugares...

Chihuahua......

Veracruz, Hgo...

Puebla...

Cultivada...

Gullivada....

Ciltivada....

México

Jalisco.

Jalisco

Gullivada...

Jalisco..

Cultivada........

Cultivada.........

Velacruz, N. L...

Morelos, México.

Michoacán

Gullivada...

Veracruz, Méx... Varios lugares... Mor., Gro., Méx. Mich., Gro., Méx. E. de Méx., Jal... Miclioacán. Ja.... Sierra de las Cruces Dist. Fed., etc... Clihuahua...... Introducida ...... Dist. Fed., ete... San Luis Polosí.. Guerrero.
Colmeiro.

Ulbina.

B. V. et Urbina.

Mat. Med.

Ramirez.

Cat. Inst. Med.

Colmeiro.

Mat. Méd.

Colmeiro.

Urbina.

Ciat. Inst. Ned.

Farm. Mex.

Farm. Mex.

Cerv. M. M.

Pl. Nov. Hisp.

Farm. Mex.

B. V. et Urbina. Urbina.

B. V. et Uıbina.

Farm. Mex.

Farm. Mex.

Altamirano.

Mat. M., Oliva.

C. I. M., Url).

Oliva.

Oliva.

Oliva.

Colmeiro.

Farm. Mex.

Urbina.

Alcocer. 
Torongil de China.

Torongil extranjero

Torongil del país

Torongil del país.

Torongil del país..

Toronja

Toronja.....

Torote verde

Tos'arza

Totolonchi

Totoncapatli.

Totoncaxoxocoyollin

Tozcuitlapilxóchitl

Tozisquint, Tozizquiuh ....

Tragacanto, goma.............

Tragacanto del país, goma.

Trébol.

Trébol oloroso.

Trementino.

Trepadora.

Trepadora amarilla..........

Trepadora bIanca.

Trepadora color de rosa.....

Trepadora hoja de murciélago

Trigo.

Trinitaria

Trinitaria

Tripa de Judas

Tripa de pollo

Tritonia.

Troeno......

Trómbita.

Tromentila

Trompetilla

Trompetilla

Trompetilla....

Trompetilla

Trompetilla

Trompillo

Trompillo

Tronadora

Tronadora

Tronadores

Trueno.

Tucuy

Tucuy liso ...... ...............

Tule...

Tule

Tule

Tule chico.

Tule grande...................

Tulillo............................

Tulillo........

Tulillo.

Tulipán

Tulipán
Nepeta calcria? L.

IIelissa officinalis L.....

Calemintha repeta? Link et H....

Cech.ouella mevicane Benth

Caltorit

Cedionella pallida Lindl

Citrus decumana L

Citrus malica Risso, var...

Fouquicra spinosa Torr.

Pinus teocotc Chlam. et Schl.......

Trianosperma racemosa? Hemsl..

Plantago galenttiana Dcne.

Begonia gracilis H. B. K

Lobelia laxiflora H. B. K. var.....

Lantana cantara L.

Astrugulus adscendens Boiss........

Opuntia vulgaris Mlill.

Trifolium arense $\mathrm{L}$.

Melilotus officinalis Willd....

Guarca, sp?......

Rosa sempervirens L. var

Rosa, sp?.

Rosa, sp?.

Rosa, sp?

Pessiftora, sp?

Triticum vulgare Villars. vars......

Tigridia pavonia Ker.

Viola tricolor L. var...

Vitis tiliacea H. B. K.

Spilanthes becubunga DC....

Tritoma uvaria Ker.

Liynstrum japonicum Thunb......

Datura arborea L.

I'otentilla tormentille? Nestl var..

Bouvardia triphylla Salisb.

Bourerdice angustifolia II. B. K...

Bonrardice quaternifolia DC........

Mirabilis julapa L...

Jacobinic molhintli Hemsl...........

Corclia boissieri A. DG.

Solanum elceagnifolium Cilv........

Crotalaria pumila Ort.

Nicotiana glauca Graham.

T'ecoma stans Juss.

Ligustrum juponicum Thunb......

I'ithecolobinm ligustrinum K........

Iithcoolobium, sp?.......

Cyperus fluvicomus Michx...........

I'ontederia cordata L...............

Scirpus, sp?.........................

Cyperus articulatus L...............

Cyperrs, sp?.......................

Cyperus csculentus L.................

Cyperns thyrsiflorus Jungh........

Juncus nexicunus IVilld............

Hibiscus roscl-sinensis L. .......

Tulipa gesneriana L....
Labiadas....

Labiadas.

Labiadas

Labiadas.

Labiadas.

Rutáceas

Ruláceas ....

Tamariscíneas...

Coníferas .

Cucurbitáceas

Plantagíneas .

Begoniáceas.

Lobeliáceas ..........

Verbenáceas ........

Leguminosas.

Ciácteas.

Leguminosas.

Leguminosas.

Meliáceas.

Rosáceas

Rosáceas....

Rosáceas

Rosácens

Pasifloráceas........

Gramíneas .... ......

Irideas .....

Violarieas.

Ampelídeas

Compuestas.

Liliáceas.

Oleáceas.

Solanáceas.

Rosáceas .

Rubiáceas .

Rubiáceas

Rubiáceas.....

Niclagineas.........

Acintáceas.........

Borregíneas.........

Solanáceas ..........

Leguminosas........

Solanáceas ..........

Bignoniáceas.......

Oleáceas.............

Leguminosas.......

Leguminosas........

Ciperáceas..........

Pontederiáceas .....

Ciperáceas...........

Ciperíceas...........

Ciperáceas...........

Ciperáceas.....

Ciperáceas.

Juncáceas.

Malváceas

Liliáceas .
Jalisco.

Gultivada.........

Introducida......

S. Luis Potosí, \& Urbina.

Glihuahua .......

Cullivada...

Oaxaca ............

Sonora...........

Michoacán........

Veracruz......... Farm. Mex.

Hidalgo, Oax.....

Valle de México. Colmeiro.

Valle de México. Mat. Méd.

Varios lugares...

Art. de comercio L. de la Vega.

Varios lugares... Colmeiro.

Jalisco..

Cultivada.........

Tabasco.....

Jalisco..

Jalisco.......

Jalisco.

Jalisco.

Chiapas ....

Cultivada........

V. de Méx., Oax.

Cultivada.........

V. de Méx, Mor'. Farm. Mex.

Hesa Central....

Cultivada.

GuJtivada........

Michoacán..............

Jalisco.

Varios lugares..

Dist. Fed., Oax..

Dist. Fed., etc...

Tarios lugares...

Veracruz, etc.....

Nuevo León, etc.

Cilihuihua......

S. Luis Potosí, \& Urbina.

Cihiluahua .......

Varios lugares...

Cultivadia....

Tilbasco

Tibasco.

Ginanajuato ......

Tabasco.

Sun Luis Potosí.

Guanajualo ......

Guanajuato .....

Varios lugares..

Jilisco............

$V$. de Méx., Hgo.

Ciultivada.........

Cultivada........
B. V. et Urbina

Cat. Inst. Méd.

B. V. et Urbina.

B. V. et Urbina.

B. V. et Urbina.

Montes de Oca.

N. León.

B. V. et Urbina.

Rovirosa.

Gat. Inst. Med.

Watson.

Rovirosa.

Watson.

Watson.

Bárcena. 
Tullidora

Tumba-vaqueros

Tumba-vaqueros

Tumbile.

Tumina.

Tuna.

Tuna.

Tuna.

Túnica de Cristo.

Tuxpata.

Tuya.

Tuzpatli...

Tuzpatli.

Tzacutli.

Tzacutli.

Tzacuxóchitl

Tzacuxóchit1

Tzat'adi.

Tzauhtli

Tzauhtli aquatica.

Tzauhtli arundinácea........

Tzauhtli florida

Tzauxilotl

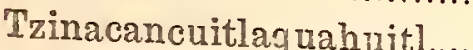

Tzinacanquahuitl

Tzinacanytlacual

Tziri.....

Tziritzequa.

Tzitzambas.

Tzivireni

Tzompantii

Tzonxóchitl.

Tzopilotláquatl.

Tzopilotláquatl.

Tzopilotlzontecómati.

Tzopotonic

Tzukix.

Truiaqua ..

Tzurumbeni?
Rhamnus lumboldtianus R. el S... Ramneas

Vitis tiliacea H. B. K.

Ipomcea stans Cav.

Pachira, sp?.

Alternanthera aehyrantha R. Br..

Opuntia ficus-indica Mill.

Opuntia tuna Mill...

Opuntia vulgaris Mill.

Passiftora edulis Sims.

Cyperus, sp?

Thuya occidentalis L.

Dorstenia eontiajerva L.

Dorstenia drakiena L.

Bletia campanulata Llav. el Lex.. Epidendrum pastoris Llav. et Lex. Bletia campanulata Llav. el Lex.. Bletia coccinea Llav. el Lex. Pinus teocote Cham. et Schl Craniehis speeiosa Llav. et Lex.. Cranichis speciosa Llav. et Lex... Cranichis tubularis Llav. et Lex... Bletia campanulata Llav. el Lex.. Arpophyllum spicatum Llav. et Lex Mimosa, sp?.

Erythrina coralloides DC.

Theretia yoyotli DC

Zea mays L. var.

Prosopis juliflora DC.

Chamcedorea?

Pinus, sp?.

Erythrina coralloides DC

Calliandra grandiftora Benth

Solanum macropliyllum? Dun

Solanum nigrum L.

Svietenia mahogani L....

Gnaphalium canescens D(

Zamia spartea A. DC:

Ccesalpinia, sp?

Pitheeolobium albieans Benth.....
Ampelídeas.........

Convolvuláceas. ...

Malváceas

Amarantáceas.....

Cácteas.............

Cácteas.

Cácteas

Pusifloráceas

Ciperáceas.

Coníferas

Urticáceas

Urticáceas

Orquídeas

Orquídeas

Orquídeas

Orquídeas

Coníferas.

Orquídeas

Orquídeas.

Orquídeas.

Orquídeas.

Orquídeas.

Leguninosas

Leguminosas

Apocináceas.

Gramíneas.

Leguminosas.......

Palmas

Conífer'as.

Leguminosas......

Leguminosas.

Solanáceas.

Solanáceas

Meliáceas

Compuestas

Cicadácens.

Leguminosas...........

Leguminosas.
Querétaro.

V. de Méx., Mor.

Dist. Fed., etc...

Michoncán.

Michoacán........

Varios lugares...

Varios lugares...

Varios lugares...

Lug. calientes....

Mlichoacán.......

Cullivada.

Velacruz

Michoacáll........

Veracruz.

Michoacán .......

Veracruz.

Veracruz.

Michoacán

Michoacán.......

Michoacán........

Michoacán........

Veracı'uz, Miclı...

Oaxara, Mich....

Ilidalgo...........

Valle de México.

Morelos, Mich...

Michoacán.

Michoacán.

Michoacán

Michoacán

Valle de México.

Valle de México.

Cullivada.

Varios lugares...

Gro., Yuc., Tab..

San Luis Polosí..

Vernciuz.

Michoncán

Miclioacán
Urbina.

Falm. Mex.

Mat. Méd.

N. León.

N. Lcón.

Film. Mex.

Ciolmeiro.

Colmuiro.

Colmeiro.

Colmeiro.

Colmeiro.

Ciolminilo.

Ciolmeiro.

Colmeiro.

N. León.

N. Leon.

Mat. Méd.

Prodiomus.

E. de Méx., Oax.

Michoacán.......

Veracruz......... Colmeiro.

Vurios lugares...

Tibasco...... .. Rovirosit.

Dislrito Federal. Farm. Mex.

Jalisco.

Varios lugares...

l'abasco.
Olivie

Rovirosa. 


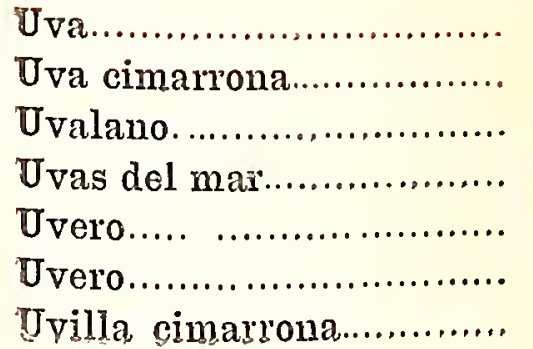

Titis viniferc $\mathrm{L}$. var.

Titis indica L....

Titex mollis $\mathrm{H}$. B. $\mathrm{K}$

Coccoloba uvifera Jaca

Cephalanthus occidentalis $\mathrm{L}$

Coccoloba uvifera Jacq.

Tritis tilicefolia Willd...
Ampelídeas

Ampelídeas

Verbenáceas

Poligonáceas

Rubiáceas.

Poligonáceas .

Ampelídeas
Cultivada.........

Chiapas, Gro.... Cat. Inst. Med.

Sinaloa........... Rose.

Tamaulipas ...... Ramírez.

Tabasco.......... Rovirosa.

'Tamaulipas..... Ramírez.

Jalisco, Veracruz Urbina.
Vacima

Vachácata

Vainilla.

Vainilla cimarrona..........

Vainilla mansi ó escarcliala.

Vainilla pompona ó boba...

Vainillo

Vainillón.

Valeriana

Valeriana

Valeriana roja

Vanita.

Vaquerillo

Vaquerillo.....

Vara ceniza...

Vara de San Francisco.....

Vara de San Francisco.....

Vara gruesa de San Miguel

Vara de Jessé.

Vara de San Joaquín........

Vara de San José.............

Vara de San José.............

Vara de justicia...

Vara de San Miguel..........

Vara de San Miguel.........

Vara de oro....................

Varal

Vauar.

Veinte reales.

Vela escamada.

Vembérequa

Venenillo

Venera de Santiago..........

Verbachina...................

Verbasco.......................

Verbena.........................

Verbena........................

Verbena.........................

Verbena..........................

Verbena de Guanajuato....

Verbena de Orizaba
Gruazuma ulnifolia Lam.

Galphimia humboldtiana Bartl... Vanilla planifolia Andrews........ Venilla sylrestris Schiede...........

Vanilla satira Schierle.

Tanilla pompona Schiede.

Cassia, sp?.....

Vanilla pompona Schiede..........

Valeriana ceratophylla H. B. K...

Valeriana mexicana $\mathrm{DC}$

Centranthus ruber DG.

Benreria hronita Hemsh...........

Bormeria haranila Hensl...........

Solenum cuneifolium Dunal.........

Spilanthes corymbosa Moc. et Sessé

Vernonia karvinskiana DC.........

Ternonia serratuloides II. B. K.....

Ternonia aschenborniana Schauer

Poliunthes tuberosa L...

Sida pechneularis DC.

Altheca rosea Cav

Polinuthes tuberos L

Jacobinia magnifica Benth et H...

Arethusa ophioglossoidea....... .

Ternonia serratuloides H. B. K....

Solidago virga-aurea $\mathrm{L}$

Cardiospermum molle H. B. K.....

Talcriana tolueana DC..............

Bidlens' ostruthioides Schz. Bip.....

Adenophora stylosa Fisch..........

Thus toxicodendron L.

Asclepias linaria Ciiv. Sprekellia formosissima Herb.. .... Phytolacea octandra L............... Gnaphalium, sp?

Verbena caroliniana L.

Terbena ciliate Benth.

Verbena officinalis $\mathrm{L}$.

T'erbena ehrenbergiana Schauel...

Verbena canescens H. B. K........

Terbena teucriifolia Mart. et Gal..
Esterculiáceas.

Malpigiáceas.

Orquídeas.

Orquídeas..

Orquídeas..

Orquídeas..

Leguminosas..

Orquídeas ...........

Valerianeas ........

Valerianeas .........

Valerianeas .........

Borragíneas.........

Solanáceas.........

Solanáceas .........

Compuestas .........

Compuestas .........

Compuestas .........

Compuestas........

Amarilídeas.........

Malváceas

Malváceas ...........

Amarilídeas........

Acantáceas.........

Orquídeas ...........

Compuestas.

Compuestas

Sapindáceas.

Valorianeas ..........

Compuestas ........

Campanuláceas

Anacardiáceas.....

Asclepiadeas........

Amarilídeas

Fitolacáceas

Compuestas........

Verbenáceas ........

Verbenáceas ........

Verbenáceas ........

Verbenáceas .........

Verbenácens ........

Velbenáceas.....
Michoacán.

Miclıoacán

Veracruz.........

Veracruz.........

Veracruz..........

Veracruz..........

Veracruz.........

Veracruz......... Farm. Mex.

Dist. Fed., elc... Mat. Méd.

Valle de México. Farm. Mex.

Cultivada........ Alcocer.

Michoacán....... Llav. et Lex.

Jalisco............ B. V. et Urbina.

Cultivada.........

Querétaro........

Jalisco............ Oliva

Jalisco........... B. V. et Urbina.

Jalisco........... B. V. et Urbina.

Guanajuato .....

Cullivada.........

Ciultivada.........

Guanajuato ......

Cultivada........ Alcocer.

Jalisco............ Oliva.

Jalisco............ Urbina.

Cultivada.........

Distrito Federal.

Michoacán....... N. León.

Valle de México.

Cultivad

Mlichoacán........

S. L. P., V. de MI..

Guanajuato, $\mathrm{Pba}$.

Valle de Héxico.

México...........

Son., Qro., Oax.

Varios lugrares...

Jaliseo, Sonora.

Sonora............

Guanajuato......

Vericruz..
N. León.

Urbina, F. M.

Colmeiro.

B. V. el Urbina. 
Verbena de Puebla..

Verdal

Verdolaga

Verdolaga acuática ó de agua

Verdolaga blanca

Verdolaga de puerco.........

Vergonzosa.

Verónica.

Verónica..

Verónica de jardines........

Veza......

Viburno

Vid.

Vid silvestre

Vid silvestre.

Vid silvestro

Vinagrera

Vinagrerita

Vinca .

Vinca pervinca

Violeta del campo.

Violeta española.

Violeta extranjera

Violeta de jardín.

Violeta de México.

Violeta de olor..

Violeta del país.

Violeta del país.

Viperina

Virginia..

Virginia.

Virreina.

Visco.

Visco cuercino.

Visco cuercino.

Vitsiqua.

Vitzaqua sipiati.

Viuda.

Viudas.

Viudas

Volantín

Volcameria.
Terbence andrieuxii Schauer.

Brassica oleracea L. var

Portulaca oleracea $\mathrm{L}$

Jussicea repens L....

Trianthema mónogyna $\mathrm{L}$

Allernanthera achyrantha $\mathrm{R} . \mathrm{Br}$

Mimosa pudica L.

Veronica peregrina. L

Veronica spicata L.....

Veronica speciosa Hook.

Vicia faba L....

Viburnum tinus L

Titis vinifera $\mathrm{L}$. var.

Vitis acupulcensis H. B. K.

Vitis rotundifolia Michx.

Vitis sicyoides Baker

Rumex acetosa $\mathrm{L}$

Rumex acetosella L.

Vinca minor $\mathrm{L}$

Vinea minor L....

Anoda triangularis DC

Viola odorata L....

Viola odorcta L

Viola odorata L

Sida triloba? Cav......

Viola odorata L

Anoda cristata Schl.

Anoda triangularis DC.

Zornia tetraphylla? Michx.

Zinnia elegans Jacq

Zinnica multiflora L

Calendula officinalis $\mathrm{L}$

Loranthus calyculatus DC.....

Loranthus calyculatus DG

Phoradendron tomentosum D. Oliv.

Marrubium vulgare L.

Plantago major L

Enotherc suaveolens? Desf

Hibiscus manihot L. var.

Scabiosa atropurpurea L

Gynandropsis speciosa DO

C'erodendron fragans Vent. var...
Verbenáceas ........ Puebla, Oaxaca..

Crucíferas............ Cultivada....

Portuláceas........ Naturalizada .....

Onagrarieas......... Jalisco............ B. V. et U.bina.

Ficoideas

Amarantáceas......

Leguminosas..

Escrofularíneas...

Escrofularíneas....

Escrofularíneas...

Leguminosas.

Caprifoliáceas

Ampelídeas

Ampelídeas

Ampelídeas

Ampelídeas.

Poligonáceas.

Poligonáceas...

Apocináceas.........

Apocináceas..........

Malváceas

Violarieas.

Violarieas

Violarieas.

Malváceas

Violarieas.

Malváceas

Malváceas

Leguminosas

Ciompuestas.

Compuestas...

Compuestas........

Lorantáceas.

Lorantáceas.

Lorantáceas

Labiadas.

Plantagíneas

Onagrarieas

Malváceas .

Dipsáceas.

Caparideas

Verbenáceas
Tabasco

Jalisco

Veracruz.

Regs. templadas.

Cultivada

Ciultivada....

Cultivada....

Cultivada.

Cultivada.

Guerrero.

Veracruz

Hidalgo, Ver.....

Cultivadi....

Cultivada

Cultivada.

Cultivada. ...

Varios lugares...

Cultivada.

Cultivada.

Cultivada......

Cultivada.

Ciultivada...

Hgo., V. de M . Jal., V. de Méx...

Tamaulipas....

Jalisco.

Hgo., Veracruz...

Cultivada

Morelos

Morelos....

Valle de México.

Michoacán...

Michoacán.

Jalisco. .

Cultivada

Cultivada.......

Veracruz

Cultivada.
Rovirosa.

B. V. et Urbina.

Farn. Mex.

Alcocer.

Farm. Nex.

B. V. el Urbina

Farm. Mex.

Farm. Mex.

Farm. Mex.

Colmeiro.

Farm. Mex.

Alcocer.

Urbina.

N. León.

N. León.

B. V. el Urbina.

Alcocer.

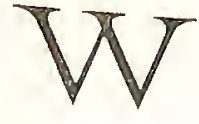

Wolkowi

Wokowi

Anthalonium lewinni Hennings....

Anfuclonium williamsii $\mathrm{L}$
Cácteas.

Cácteas
Front. N., Qro...

Front. N., Qro... 
Xahuique.

Xahuiqui.

Xalache.

Xalócotl.

Xalócotl.

Xaltomatl...

Xaltomatl.

Xalxocotl.

Xanabmucuy, maya

Xararo....

Xaricamata.

Xarimpscua

Xarimpscua

Xarimpscua

Xaté'

Xcantiris.

Xcantumbul

Xcuiche

Xeugua

Xhail, maya.

Xícama

Xícama

Xicotzápotl.

Xiloxóchitl.

Xiloxóchitl

Xiloxóchit1

Xiquite

Xitomame, Xitómat.

Xitómatl, Xitotómatl.

Xiuhquilitl....

Xiuhquilitl....

Xkambahau, maya

Xkanlol, maya

Xkantumbub, maya

Xkeu, maya

Xkoch, maya

Xmuchcoc, maya..............

Xmutz, maya.

Xnuntzutzuy, maya.........

Xoalácati..

Xoan.

Xocoac, Xoxoag, maya .....

Xococxihuitl?

Xoconochtli

Xocozóchitl

Xocoyol

Xocoyol

Xocoyol
Tigridia paronia Iser

Tigridia dugesii Wats

Sedlum acre L

Abies religiosa Chan. et Schl...... Pimus teocote Chan. et Schl.......

Physallis mollis Nutt

Saracha jaltomata Schl....

Psiclium pomiferum L. var.

Euphorbia, sp?......

Perezia hebecledn? A. Gr...........

Cosmos bipinnatus Cav.

Oxalis anguslifolic H. B. K..........

Oxalis corniculata $\mathrm{L}$.

Oxalis verticillata $\mathrm{DC}$

Argemona mexicana L.

Acacia famesiana Willd

Melampodium americanum L.....

Pachira insignis ? Savign...........

Prunus capuli Cav...

Ipomoea purpurea, Lam.

Pachyrhizus angulatus Rich.......

Pachyrhizus palmatilobus B. et $\mathrm{H}$.

Achras sapota L

Calliandra grandifora Benth.....

Casalpinia pulchervima Sw.

Pachira insignis Savign.

Eupatorium sordidum Less.

Lycopersicum esculentum Mill.....

Lycopersicum esculentum Mill......

Indigofera tinetoria L..............

Indigoferca añil L...

Dorsterica contrajerea L. var........

Tecoma sambucifolia H. B. K.......

Sanvitalia procumbens Lam.........

Loranthus ameriéanus Jacq.........

Ricinus communis L. var...........

Ceterach officinarum DC.....

Mimose pudica $\mathrm{L}$

Cercus tetragoms Haw............

Cereus speciosissimus Desf. var..... Chamedorea.

Abrus precatorius L.................

Euphorbia, sp?.....

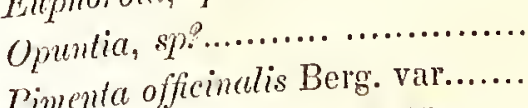

Oxalis angustifolia H. B. K.......

Orelis cominculata L.................

Oxalis rerticillata DC.
Irideas

Irideas

Crasuláceas

Coníferas

Solenáceas .........

Solanácers ........

Mirtáceas

Euforbiáceas

Compuestas

Compuestas

Geraniáceas.

Geraniáceas.

Geraniáceas

Papaveráceas

Leguminosas........

Compuestas .........

MIal váceas ...

Rosáceas

Convolvuláceas.

Leguminosas..

Leguminosas........

Sapotáceas ..........

Leguminosas.......

Leguminosas........

Malváceas ...........

Compuestas .........

Solanáceas ..........

Solanáceas..........

Leguminosas.......

Leguminosas.......

ticáceas ...........

Bignoniáceas........

Compuestas ........

Lorantáceas........

Euforbiáceas ........

Helechos

Leguminosas.......

Cácteas.

Cácteas.............

Palmas ..............

Leguminosas.......

Euforbiáceas.......

Cácteas.

Mirtáceas.

Geraniáceas

Geraniácens........

Geraniáceas........
Michoacán....... N. León.

Guanajuato...... Dugés.

Cultivada.........

Varios lugares...

Oaxaca ........... Urbina.

San Luis Potosí. Urbina.

Valle de México. Farm. Mex.

Gro., Mor., Ver...

Yucatán........... Dondé

Valle de México. Hat. Méd.

Michoacán....... N. León.

Guerrero......... Farm. Mex.

Michoacán....... Farm. Mex.

Distrito Federal. Farm. Mex.

Michoacán........ N. León.

Michoacán........

Tabasco......... Rovirosa.

Michoacán........

Michoacán........ N. León.

Yucatán.......... Dondé.

Morelos........... Alcocer.

Ver., Oax., Mor.. Farm. Mex.

Mor., Ver., Tab.

Varios lugares... Mat. Méd.

Puebla...

E. de MIéxico.

Oaxaca, Ver...... Urbina.

Cultivada........

Cultivada.......

Cultivada........

Oaxaca, Tab., etc

Yucatán.......... Dondé.

Yucatán.......... Dondé.

Yucatán.......... Dondé.

Yucatán.......... Dondé.

Yucatán.......... Dondé.

Yucatán......... Dondé.

Yucatán.......... Dondé.

Yucatán........... Dondé.

Jalisco.............

Michoacán....... N. León.

Iucatán.......... Dondé.

Dishito Federal.

Hgo., Dist. Fed..

Lugares cálidos..

Guerrero........ Farm. Mex.

Dist. Fed., Mich. Farm. Mex.

Distrito Federal. Farm. Mex. 
Xochicopalli

Xochilcorona

Xochiocotzoquahuitl.

XochiocotzotI.

Zochipipile.

Zochipitzahuac.

Zochiquáh uitl, Yochittqqáhaill....

Xolochichitl.

Xolteco.

Xonácatl

Xonequil?

Zotol.

Xoxoco

Xoxocoyollin.

Zoxocoyollin

Xoxocoyollin.

Xoxocoyollin.

Xoxonitzal.

Xoxonitztac ó Xoxonitzal

Xoxoyolpapatla?

Xpuhuc, maya

Xtez, maya

Xtohcú, maya

Xtuab, maya.

Xtuhuy, maya...

Xucul, maya

Xucúpara

Xúmet]

Xunalizase

Xunequilt ó Xunequiltzio..

Xurata
Bursera mexicana Engl....

Cornus disciflora DG

Liquidambar stypaciftua L.........

Liquidambar styraciflua L.........

Lobelia laxiflora H. B. K.

Polygala mexicana DC.

Cordia ferruginea? Rem et $\mathrm{S}$

Eupatorium deltoideum Jacq........

Dalea diffusa Moric.

Allium cepa L

Gymnosperma, sp?....

Agave, sp?

Rhus, sp?

Oxalis angustifolic Anar'ardiáccas.....

H. B. K....... Geraniáceas

Oralis corniculata L.

Oxalis verticillata DC.

Oxalis violacea? I

I'iqueria trinervia Cilv

Piqueria trinervia Ciav.

Rumer acetosa $\mathrm{L}$

Tagetes, sp?.

Amarantus spinosus I.

Datura stramonizm L

Cassia sericea Sw

Plumeria pudica Jacq.

Portulaca oleracea L

Iycopersicum eseutentun

Lycopersicum esculentum Mill...... Solanáccas..........

Sumbucus mexicana Presl.

Croton ciliato-glandulosus Ort.....

Gaudichaudia'. 'ynanchoides H.B.K Gossypium barbadense L
Burseráceas.

Ciornáceas

Hamamelídeas....

Hamamelídeas.....

Lobeliáceas

Poligaleas.

Borragíneas.

Compuestas.

Leguminosas.......

Liliáceas

Compuestas

Amarilídeas.

Geraniáceas........

Geraniáceas........

Geruniáceas

Geraniáceas

Compuestas.

Compuestas........

Poligonáceas.......

Compuestas

Amarantáceas.....

Solanáceas .

Leguminnsas........

Apocináceas.......

Caprifoliáceas.......

Euforbiáccas...

Malpigiáceas......

Malváccas
Colima, Ver......

Veracruz, Mich...

Veracruz.

Veracruz.........

V. de M., S. L. P.

Valle de México.

Veracruz.

E. y V. de Méx.

Distrilo Federal.

Ciultivada.

Distrito Federal.

San Luis Polosí.

Puebla.

Guerrero

Varrios lugares...

Distrito Federal.

Distrito Federal.

Puebla...

Mesa Centril....

Ciullivada........

Yucalán

Yucatán.

Yucatán

Tucalán...

Yucatán

Yucatán.

Michoacán

E. de México

Oaxaca.

Michoacán.......

Michoacán
Urbina.

Gutz., Lozada.

Gulz., Lozada.

Farm. Mex.

Farm. Mex.

Colmeiro.

Cat. Inst. Med.

Firm. Mex.

Farm. Mex.

Farm. Mex.

Firm. Mex.

Oliva.

Mial. Méd.

Dondé.

Dondé.

Dondé.

Dondé.

Dondé.

Dondé.

Urbina.

Cat. Inst. Néd.

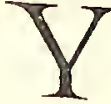

Ya, maya

Yame

Yaxci, maya.

Yaxché, maya

Yaxché, maya.

Yaxhalalché, maya.

Yayaquáhuitl.

Xedra azul.

Yedra bocina

Yedra colorada.

Tedra extranjera.

Yedra morada.

Yedra morada.

Yedra terrestre.

Yedra terrestre.

Yedrón

Yeguas
Achras sapota I.

Dioscorea alata? I

Agave rigida? Mill...

Eriodendron oecidentule..........

Eriodendron occidentule Tr. et Pl.

Pedilanthus tithymaloides $\mathrm{P}$. B. K. Monnina ocampi Herr. et Vill.......

Convolvulus arvensis L....

Bignonia buecinatoria Minirel.

Ipomea coccinea Lria Mairel.....

Hedera helix L.

Cobcer scandens Cav..........

Ipomoea purpurea Lam.....

Modiola carolineana G. Don........

Phanbitis violacer Boj.

Convolvulus, sp?.

Phaseolus formosus H. B. K
Sapoláceas

Dioscoreáccas ......

Amarilídens.

Malvácens.

Malváceas.

Euforbiáccas............

Poligaleas.

Convolvuláceas.....

Bignoniáceas

Convolvuláceas....

Araliáceas.

Polemoniríceas........

Cinnvolvuláceas...

Malváceas.

Convolvuláceas......

Convolvuláceas. ..

Leguminosas.
Yucalán

Cihiapas ....

Yucatán

Yucatán.

Yucalán

Yucatán

Valle de México.

Jalisco

S. Luis Polosí, \&

Varios lugares...

Cullivada.........

Distrito Federal.

Jalisco

Varios lugares...

Cultivada.

Jalisco.

Jalisco.
Dondé.

Dondé.

Dondé.

Dondé.

Dondé.

Herrera.

B. V. et Urbina.

Mociño.

B. V. et Uibina.

Colmciro.

B. V. et Urbina.

B. V. et Urbina. 


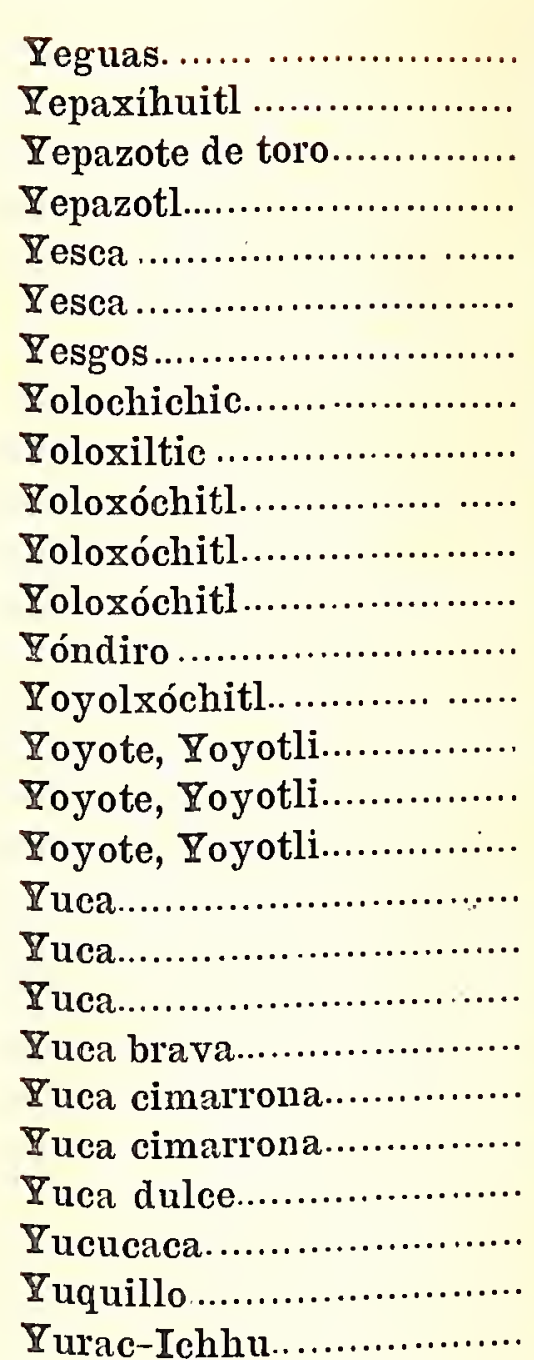

Phaseolus multiflorus Willd......... Croton dioicus Cav.................. Chenopodium fotidum Schrad...... Chenopodium ambrosioides L...... Polyporus fomentarizes Fries....

Polyporus igniarius? Per's...

Urtica mexicana Liebm............ Eupatorium deltoiderm Jacq........ Piqueria trinervia Cav.............. Eupatorizm collimun DC... Taleuma macroearpa Zucc. Talauma mexicana Don. Piptadenia foetida Benth... Commelina tuberosa L. Theretice yoyotli A. DC... Thevetia neriifolia Juss... Theretia ovata A. DC. Manihot utilissima Pohl.

Yucca filamentosa L......

Yucea aloifolia L...

Naniliot utilissima Pohl...

Manilıt foetida Pohl. Manihot cesculifolia Pohl..

Manihot aipi Pohl...

Cuphea, sp?......

Amarantus, sp?....

Stipa jarava Beauv..
Leguminosas....... Varios lugares... Euforbiáceas....... Dist. Fed., Pba... Quenopodiáceas... Varios lugares... Quenopodiáceas... Varios lugares... Hongos............. Art. de comercio Hongos.............. Zacalecas........ Urticáceas .......... Valle de México. Compueslas........ Valle de México. Compuestas ......... V. de Méx., Jal... Compuestas........ Dist. Fed., Ial... Magnoliáceas ....... Veracruz.......... Magnoliáceas ....... Mor., Jal., Ver... Leguminosas....... Michoacán........ Commelináceas .... V. de Méx., Hgo.

Apocináceas........ Mor., Gro........ Farm. Mex. Apocináceas......... Ver., Guerrero... Apocináceas........ Guerrero, Mor... Euforbiáceas........ Cultivada........ Liliáceas........... México........... Liliáceas............ Zacatecas, Mor... Euforbiáceas........ Euforbiáceas....... Euforbiáceas ........ Euforbiáceas ........ Litrarieas........... Amarantáceas..... Ciperáceas...........
Mat. Ned. Mat. Méd. Colmeiro.

Gat. Inst. Ned. Urbina, F. M.. Urbina. Mat. Méd.

Cat. Inst. Med. Mat. Méd.

Ramírez.

Mueller.

Ramírez.

Donclé.

Guerrero.........

Campeche.........

Yucatán.......... Dondé.

Oaxaca.......... Cat. Inst. Med.

Chiapas...........

Lugares fríos.... Kunth.

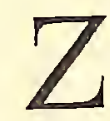

Zábida, Zábila

Zábida, Zábila

Zacachichi..

Zacachichic.....

Zacate de agua

Zacate amargo

Zacate amargo

Zacate camelote

Zacate limón

Zacate de manatí.

Zacate de la muela............

Zacate sedoso

Zacatechichi..... ...........

Zacatillo

Zacatlascal.

Zacatlazcale

Zacatlaxcalli

Zacatón

Zacatón

Zacatón

Ragalejo.
Liliáceas ............. Naturalizada ..... Liliácens............. Jalisco............. Compuestas........ Varios lugares... Compuestas........ Valle de México. Leguminosas......... Compuestas .......... Compuestas......... Gramíneas.......... Valle de México. Gramíneas.......... Naturalizada..... Gramíneas ........... Jalisco, Tabasco. Irideas .............. Chihuahua, Jal.. Gramíneas........... Compuestas......... Umbelíferas........ Convolvuláceas.... Convolvulácens..... Gonvolvuláceas.... Gramíneas........... Gramíneas........... Gramíneas........... Amarilídeas.........
Mat. M., Ramz. B. V. et Urbina. Mat. Méd. Mat. Med. Urbina. B. V. et Urbina.

Ramz., Rov.

Urbina.

B. V. et Urbina F. M., Mat. M.

Veracruz......... Chihuahua....... Mesa Central.... Farm. Mex. Mesa Central.... Farm. Mex. Mesa Central.... Farm. Mex. México, Morelos. Frontera Norte.. Frontera Norte.. Guanajuato, Pba. 
Zalate

Zanahoria

Zancudo.

Zandía

Zapalote.

Zapatito de la reina

Zapote.

Zapote amarillo.

Zapote amarillo.

Zapote blanco

Zapote borracho.

Zapote caimito

Zapote chico

Zapote de agua..

Zapote de Santo Domingo...

Zapote mamey.

Zapote de niño.

Zapote negro ó prieto..

Zapote de rata.

Zapotillo

Zapotillo

Zapdtito de San Juan..........

Zaragatona.

Zarcillos.

Zarza.

Zarza.

Zarzamora

Zarzaparrilla

Zarzaparrilla

Zarzaparrilla

Zaucle, Zautle

Zayolizán, Zayolizcán.

Zazale, Zazalic

Zazale de olor:

Zazanaca.

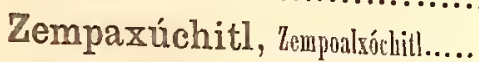

Zempoala

Zihoapatli ó Zihuapatli....

Zoapatle, Zoapatti, Zuapatli..

Zoapatle, Zoapatli, Zuapatli...

Zompantle.

Zopiloquáhuitl.

Zopilote ó Zopilotl

Zopilote colorado.

Zopilote negro

Zorrillera

Zorrillo.

Zoyacapulín.

Zoyate

Zoyate

Zoyalxóchitl.

Zumaque.

Zumaque.

Zumpantle.
Ficus, sp?.

Daucus carota L

Lopezia mexicana Jacq.

Citrullus vulgaris Schrader.

Musa paradisiaca L

Clitoria virginiana $\mathrm{L}$

Tabebuia lencoxyla DC.

Couepia polyandra Rose.

Lucuma salicifolia H. B. K.

Casimiroa edulis Llav. et Lex.....

Lueuma salicifolia H. B. K.........

Chrysophyllum caimito L

Achras sapota L

Pachira aquatica Aubl.

IJammea americana $\mathrm{L}$

Lncuma mantmosa Gærtn. fil.

Lueuma?

Diospyros ebenaster Retz

Casimiroa pubescens Ramz.

Achras sapota L.

Erytroxylon ellipticum Ramz.......

Bunchosia lanceolata Turcz..

Plantago psyllium $\mathrm{L}$

Lobelia tupa L

Buetheria carthagenensis Jacq....

Smilax medica Chan. et Schl.....

Rubus fruticosus $\mathrm{L}$

Smilax bona-nox L

Smilax medica Cham. et Schl.....

Smilax glauca Walt.

Dendrobium, sp?.

Buddleia amerieana L.

Mentzelia hispida IVilld.

Stevia salieifolia Cav.

Lagascea mociniana DA.

I'agetes erecta L.

Tagetes patula L.

Montanoa tomentosa Llav. et Lex.

Montanoa tomentosa Llav. et Lex.

Montanoa floribunda DC.

Erythrina corallorides DC

Swietenia mahogani L

Swictenia mahogani $\mathrm{L}$

Swietcnia, sp??.

Swietenia, sp?

Croton, sp?.

I'etireria alliacea L

Plicenix dactylifera L.

Brechea dulcis Mart.

Dasylirion inerme Wats........

Commetina tuberosa I.

likus mollis H. B. K

Rihns toxicodendion I.

Erythina corallodendron L..............
Urticáceas

Umbelíferas.

Onagrarieas.

Cucurbitáceas ......

Musáceas

Leguminosas

Bignoniáceas.

Rosáceas

Sapoláceas

Rutáceas

Sapotáceas.

Sapotáceas.

Sapotáceas.

Malváceas

Gutíferas.

Sapotáceas

Sapotáceas.

Ebenácens

Rutáceas .

Sapotáceas.

Lineas

Malpigiáceas.........

Plantagíneas ........

Lobeliáceas .........

Esterculiáceas...

Liliáceas.

Rosáceas

Liliáceas.

Liliáceas.

Liliáceas.

Orquídeas

Loganiáceas .........

Loaseas

Compuestas.

Compuestas

Compuestas

Compuestas.

Compuestas

Compuestas

Compuestas.

Leguminosas.

Meliáceas

Meliáceas

Meliáceas

Meliáceas....

Euforbiáceas

Fitolacáceas.......

Palmas

Palmas....

Liliáceas.

Commelináceas

Anacardiáceas.

Anacardiáceas.

Leguminosas.
Jalisco.

Oliva.

Gultivada.........

Jalisco.

B. V. et Urbina.

Cultivada.........

Cultivada........

Cultivada......... Dondé.

Veracruz......... Urbina.

Guerrero......... Rose.

Morelos

Varios lugares...

Morelos

Mat. Méd.

Farm. Mex.

Oaxaca ............ Barroso.

Lug. calientes....

Chiapas ..........

Veracruz..........

Mor., Oax., Gro..

Veracruz.........

Lug. calientes....

Querétaro........ Ramírez.

Morelos, Ver..... Farm. Mex.

Veracruz......... Cat. Inst. Med.

Veracruz..........

Cultivada.........

Cultivada.........

Tabasco..........

Veracruz..........

Cultivada........

Hgo., S. L. P...

Hidalgo, Ver......

Veracruz, Oax...

Jalisco

Valle de México.

Valle de México.

Valle de México.

E: de México.....

Cultivada.

Michoacán........

Valle de México.

Valle de México.

Hgo., Mich., etc.

Valle de México.

Guerrero..

Guerrero..........

E. de México.....

E. de México.....

Jalisco...

Tabasco.

Cultivada

Jalisco.

...

San Luis Potosí.

Distrito Federal. Querétaro, Hgo..

Varios lugares...

Farm. Mex.

Oliva.

Oliva.

Rovirosa.

Farm. Mex.

Urbina.

Farm. Mex.

Oliva.

Mat. Méd.

Mat. Med.

Farm. Mex.

Prodromus.

Farnı. Mex.

N. León.

Mat. Méd.

Mat. Méd.

Farm. Mex.

Dist. Fed., Ver... 


\section{SINONIMIA VULGAR Y CIENTIFICA DR LAS PLANTAS MEXICANAS.}

\section{SEGUNDA PARTE.}

\section{A}

Abclia floribunda Denc

Abies religiosa Cham. ct Schl...

Abies, sp?

Abrus precatorius L.

Abutilon striatum Hort...

Abutilon triquetrum Presl.

Abutilon, sp?.

Acacia cornigera Willd...

Acacia farncsiana Willd.

Acacia filicina Willd.

Acacia lentiscifolia ? Desf.

Acacia malacophylla ? Bentlı

Acacia mclanoceras? Beurling.

Acacia paniculata Willd.

Acacia pennatula Benth...

Acacia riparia II. B. K.

Acacia, sp?.

Acacia, sp?

Acacia, sp?

Acacia, sp?

Acacia, sp?

Acacia, sp?

Acacia, sp?.

Acacia, sp?

Acacia, sp?

Acacia, sp?.

Acacia spadicigera Cham. et Schl.........

Acalypha phleoides Cav. var..............

Acer mexicanum A. Gray...
Caprifoliáceas....... Abelia.

Coníferas ........... Abeto, Acxoyall, Bansú, Jalocote, Oyamel, Oyametl, Picea común, Pinabete, Pino, Pino oyamel, Xalocotl.

Confferas........... Thucumu.

Leguminosas....... Xocoac, Xoxoag.

Malváceas .......... Monacillo amarillo.

Malváceas .......... Amantillo.

Malváceas .......... Abutilon.

Leguminosas........ Albol del cuerno, Cuernitos, Hoitzmamazali, Tepame.

Leguminosas........ Aroma ó Aromo, Matitas, Xcantiris.

Leguminosas....... Cantemó.

Lcguminosas.. ..... Lentisco.

Leguminosas....... Timbe.

Leguminosas....... Arbol del cucrno, Guernitos.

Leguminosas....... Cola de lagarto, Espino.

Leguminosas....... Algarroba.

Leguminosas....... Tlahuitol.

Leguminosas........ Calminga.

Leguminosas....... Espino blanco.

Leguminosas....... Huizache blanco.

Leguminosas........ Huizache prieto.

Leguninosas........ Sinanché.

Leguminosas....... Tepehuaje meco.

Leguminosas....... Tepchuaje negro.

Lcguminosas........ Tepchuaje rayado.

Leguminosas........ Thepame.

Leguminosas....... Uña de gato de Jalisco,

Leguminosas........ Cornezuelo.

Euforbiáceas....... Durarnillo, Hierha del cancer, Hierba del pastor.

Aceráceas........... Arce. 
Acer pseudo-platanus I

Achillea millefolium L

Achras sapota L

Achyranthes werschaffeltii Lem....

Aconitum napellus L.

Acrocomia sclerocarpa Mart..

Acrostichum cœnopteris Kunze...........

Acroslichum sorbifolium L

Adenophora stylosa Fisch.

Adenophyllum porophyllum FIemsl.......

Adiantum capillus-veneris L..............

Adiantum scabrum Raulf.

Adiantum tenerum Swartz.

Adiantum trapeziforme L

Esculus hippocastanum L.

Agapanthus umbelliferus L'Her.

Agaricus campestris L

Agave americana L.

Agave americana L. var.

Agave atrovirens Karw.

Agave brachystachys Cav...

Agave cochlearis ? Jacobi

Agave falcata Engelm

Agave filifera Salm. Dyck

Agave hetheracantha Zucc...

Agave lechuguilla Torr

Agave lophantha Schiede

Agave mexicana Lam

Agave polatorum Zucc

Agave rigida Miller.

Agave salmiana Otto

Agave striata Zucc.

Agave univittata Haworth

Agave vivipara L.

Agave wizlizeni ? Engelm.

Agave, sp?

Agave, sp?

Agave, sp?

Agave, sp?

Agave, sp?

Agave, sp?

Agave, sp?

Ageratum corymbosum Zucc

Ageralum conyzoides L

Agrimonia eupatoria

Agrimonia parviflora Ait

Agrostis alba $\mathrm{L}$

Aizoon canariense L.

Alibertia edulis Rich...

Allionia incarnata $\mathrm{L}$

Allium cepa L

Allium fistulosum L....................

Allium porrum $\mathrm{L}$
Aceríneas.

Compuestas

Sapotáceas

Amarantáceas.....

Ranunculáceas....

Palmas ..............

Helechos

Helechos

Campanuláceas....

Compuestas .........

Helechos

Helechos

Helechos

Helechos

Hipocastanáceas..

Liliáceas .

Hongos

Amarilídeas.

Amarilídeas

Amarilídeas

Amarilídeas .

Amarilídeas

Amarilídeas

Amarilídeas

Amarilídeas

Amarilídeas

Amarilídeas

Amarilídeas

Amarilídeas

Amarilídeas

Amarilídeas

Amarilídeas...

Amarilídeas

Amarilídeas.

Amarilídeas.

Amarilídeas

Amarilíteas.

Amarilídeas...

Amarilídeas.

Amarilídeas.

Amarilídeas.

Amarilídeas.

Compuestas

Compuestas

-Rosáceas.

Rosáceas .

Gramineas

Ficoideas

Rubiáceas

Nictagineas.

Liliáceas.

Liliáceas

Liliáceas.
Sicomoro.

Ciento en rama, Mil-en-rama, Plumajillo.

Corteza de chicozapote, Chicle, Chicozapote, Chiczápotl, $\mathrm{Pa}-$

lo María, Xicotzápotl, Ya, Zapote chico, Zapotillo.

Quelite morado.

Acónito.

Cocoyul.

Calaguala.

Calaguala.

Vela escamada.

Cempazúchil sencillo.

Culantrillo de ojo de agua, Culantrillo.

Culantrillo de pozo.

Culantrillo de México.

Culantrillo de pozo.

Castaño de Indias.

Agrapando.

Hongo, Tarequa.

Henequen blanco, Maguey, Maguey de pulque, Metl, Sacci.

Naguey meco.

Maguey de pulque, Metl, Teometl.

Amole lechuguilla, Maguey zhizhi, Schischi.

Maguey de pulque.

Guapilla.

Maguey de macela.

Ixtle, Lechuguilla, Maguey.

Lechuguilla, Maguey, Maguey lechuguilla, Metómetl, Tapamete.

Lechuguilla, Maguey, Tapemete.

Amole de raíz, Ehpuqua.

Acamba, Maguey manso, Metl.

Henequen, Ixcle, Ixtle, Ixtli, Jenequen, Maguey, Maguey de Yucatán, Yaxci.

Maguey manso, Metl, Raíz de maguey, Tlacametl.

Guapilla.

Maguey.

Maguey, Tapemete, Teometl.

Maguey de mezcal, Mezcal.

Amole.

Huila.

Maguey de Tequila.

Mezcal.

Popotule.

Quetzalichtli.

Sotol, Xotol.

Mota morada.

Sereno.

Agrimonia.

Agrimonia.

Ciastillitos.

Siempreviva.

Castarrica.

Hierba del golpe.

Ciebolla, Thumps, Xonácatl.

Cebolleta, Cebollino inglés.

Puerro. 
Allium sativum L

Allium schrenoprasum L.............

Alnus acuminata H. B. K.

Alnus cordifolia 'Ten

Aloe variegata $L$

Aloe vulyaris Lam.

Alternanthera achyrantha $\mathrm{P}$. Brown......

Althrea officinilis $\mathrm{L}$

Altheea rosea Cav....

Alyssum maritimum Lam....

Amanila muscaria L...

Amanita, sp?

Amarantus caudatus

Amarantus hybridus L

Amarantus hypochondriacus L...

Amarantus leucospermus Wats.

Amarantus palmeri Wats...

Amarantus sanguineus L...

Amarantus spinosus L.

Amarantus, sp?

Amarantus, sp?.

Amarantus, sp?.

Amaryllis joseplinæ Red.

Ambrosia artenisizefolia $L$

Amorphophallus rivieri Durieu...........

Amphilophium paniculatum H. B. K......

Anacardium occidentale L...

Anacyclus pyrethrum DC.

Anagallis arvensis L.....

Ananas sativns Schult

Andira excelsa H. B. K

Andropogon cilratus ? DC.

Andropogon condensatus H. B. K........

Angelica mexicana Vatke..................

Anguria trifoliala $L$

Anhalonium fissuratum Engelm..........

Anhalonium lewinii Hennings............

Anhalonium villiamsii Lem......

Anoda cristata Schl....................

Anoda histala Cav

Anoda triangularis DC.

Anona cherinolia Mill.

Anona excelsa H. B. K.

Anona glabra L.

Anona muricala L.......

Anona palustris L.

Anona purpurea DC....

Allona reticulata L....

Anona squamosa L....

Anona, sp?....
Liliáceas.

Liliáceas ....

Gupulíferas.

Cupulíferas.

Liliáceas.

Liliáceas.

Alnarantáceas.....

Nalváceas

Malváceas.

Girucíferas....

Hongos.

Hongos....

Amarantáceas.....

Amarantáceas.....

Amarantáceas....

Amarantáceas.....

Amarantáceas.....

Amarantáceas.....

Amarantáceas......

Amarantáceas......

Amarantáceas......

Amalantáceas......

Amarartáceas....

Amarilídeas.........

Compuestas .........

Aroideas.

Bignoniáceas.

Anacardiáceas.....

Compuestas ........

Primuláceas.

Bromeliáceas

Leguminosas.......

Graníneas ....

Gramíneas

Umbelíferas.......

Cucurbitáceas .....

Cácteas.

Cácteas.

Gácteas.

Malváceas

Malváceas .

Malváceas

Anonáceas ....

Anonáceas

Anonáceas.

Anonáceas

Auonáceas

Anonáceas.

Anonáceas.

Anonáceas.

Anonáceás.
Ajo.

Cebollino común, Cebollino francćs.

Abedul, Aile, Ayle, Olmo del país.

Aile.

Pitasábida, Sábida, Zábida, Zábila.

Pitasábida, Zábida, Zábila.

Tianquiz, Tianquizpepetla, Tumina, Verdolaga de puerco.

Altea, Bimalva, Malvabisco.

Amapola grande, Malva rosa, Vara de San José.

Panal, Panalillo.

Tarequa cahuiqua.

Tarequa varirahpeni.

Cinco de Mayo, Cordón de ó del obispo, Moco de pavo.

Quelite morado.

Alegría, Qnelite, Quelite de espiga, Quelite manchado, Quiltonile.

Alegría.

Quelite.

Amaranto.

Quelite espinoso, Xtez.

Ala de perico.

Parí.

Pistón.

Yuquilla.

Reina de las hermosas.

Altamisa, Altemisa, Ambrosía, Artemisa, Artemisia, Camenzba, Hierba amargosa.

Corona imperial, Imperial.

Pico de pato.

Marañón.

Pelitre, Peritre extranjero.

Anagálide, Anagalis, Curalilla, Coralillo, Hierba del pajaro, Ixcuicuil, Jabonera, Murages, Saponaria.

Matzali, Matzatli, Piña.

Maca colorada, Macallo, Macayo, Moca, Pacay.

Te limón, Zacate limón.

Cola de venado.

Angélica del país.

Sandía de Jalisco.

Peyote, Peyotl.

Hikori, IIo, Peyole, Peyotl, Señi, Wokowi.

Hikori, Ho, Peyote, Peyot, Señi, Wokowi.

Violeta del país.

Altea, Bimalva, Malvabisco.

Arrapolita morada, Violcta del campo, Violeta del país.

Chirimolla, Chirimollo, Chirimoya, Chirimoyo, Matzápott.

Ilana, Ilamazápotl.

Anona.

Anona amarilla, Cabeza de negro, Catuche, Catucho, Guanábana, Guanábano.

Corcho.

Aluate, Anona morada.

Anona, Anona colorada, Quauhtzápotl.

Anona, Anona blanca, Chirimoyo, Saramuya, Saramuyo, Texaltzápoti.

Ahate. 
Anona, sp?

Anthemis nobilis L.

Anthirrinum majus L

Anthriscus cerefolium Hoffm.

Anthurium scandens Engler. var....

Anthurium cordifolium Kunht...

Antigonon cordatum Mart. et Gal.

Antigonon leptopus Hook. et Arn.

Antigonon viride Wats.

Antigonon, sp?

Apeiha tibourbou Aubl...

Apium graveolens L.

Apium petroselinum $\mathrm{L}$

Apocynum cannabinum L

Apocynum, sp?.

Apodanthera? undulata A. Gr.

Aquilegia cærulea Hort

Aquilegia vulgaris L

Arabis mexicana Wats..

Arabis verna $\mathrm{L}$....

Arachis hypogæa L

Aralia humilis Cilv....

Aralia pubescens DC

Arbutus xalapensis H. B. K.

Arbutus, sp?

Arbutus, sp?

Arctostaphylos arguta Zucc.

Arctoslaphylos mucronifera DG.

Arctostaphylos pungens H. B. K

Arctostaphylos tomentosa Dough

Arctostaphylos, sp?

Arctostaphylos, sp?.

Ardisia capollina A. DC

Ardisia revoluta H. B. K

Arethusa oplioglossoidea...

Argemone grandiflora Sweet....

Argemone mexicana L

Argemone ochroleuca Sweet...

Argithamnia heterantha Müil..........

Aristolochia anguicida L.

Aristolochia foetens Lindl.

Aristolochia fotida H. B. K.

Aristolochia grandiflora Swartz

Aristolochia microphylla Willd.

Aristolochia odoratissima L.

Aristolochia, sp?.

Aristolochia, sp?.

Arislolochia, sp?

Aristolochia, sp?

Aristolochia, sp?

Arpophyllum spicatum Llav. et Lex.....

Arracacia atropurpurea Benth. et Hook.
Anonáceas

Compuestas

Escrofularíneas.....

Umbelíferas

A roideas

Aroideas

Poligonáceas.

Poligonáceas.

Poligonáceas

Poligonáceas.....

Tiliáceas.

Umbelíferas.

Umbelíferas ....

Apocináceas..

Apocináceas.

Cucurbitáceas ......

Ranunculáceas...

Ranunculáceas.....

Crucíferas

Crucíferas....

Leguminosas....

Araliáceas.

Araliáceas.

Ericáceas.

Ericáceas

Ericáceas

Ericáceas

Ericáceas

Ericáceas

Ericáceas

Ericáceas

Ericáceas

Mirsineas

Mirsiıea

Orquídeas

Papaveráceas

Papaveráceas

Papaveráceas

Euforbiáceas ....

Aristoloquieas.

Aristoloquieas.

Aristoloquiras.

Aristoloquieas......

Aristoloquieas.....

Aristologuieas.......

A ristoloquieas

Aristoloquieas

Aristoloquieas

Aristoloquiea

Aristologuieas.

Orquídeas

Umbelíferas
Anona silvestre.

Manzanilli, Manzanilla romana.

Perritos:

Perifollo.

Maicillo.

Taramicua.

Flor de San Diego.

Coamecate, Cuamecate, Fulmina, Hierba de Santa Rogil.

Coamecate, Coamecatl.

Cioaniecate verde.

Peine de mico.

Apio.

Perejil, Perejil común.

Cáñamo del Canadá.

Iztacpatli.

Melón loco.

Pajaritos.

Aguileña, Muela de San Cristóbal, Palomas.

Lentejuelilla.

Bola de nieve, Panalillo.

Alfónsigos de tierra, Cucalıuate, Talcacahuate, Talcacáhuatl, Tlalcacáhoatl.

Aralia.

Aralia.

Madroño.

Ayacopal.

Pananex.

Garambullo, Madroño borracho.

Pingüeca, Pingüica.

Gayuba del país, Leño colorado, Palo de pingüica, Pinguieca, Pingüica, Tepeizquitl.

Madroño, Madroño de árbol.

Frutilla.

Madroño chino.

Capulín silvestre.

Ciajulín manso.

Vara de San Miguel.

Chicalote, Chicallotl, Cilicalote grande.

Cardo santo, Cardo santo de Yucitan, Cihicalote, Cilicalliull, C'hichillotl, Chillazotl, Xaté

Chicalote, Chicallotl.

Azafián de bolita.

Raíz del indio.

Palomas.

Hierba del indio.

Flor del pato.

Hierba del indio.

Cocoba, Cocobá, Guaco, Hierba del indio, Tacopate, Tacos patle, Tacopaxtle.

A ristoloquia mata-culebras, Tlacopatli tomáhuac.

Hierba del cuiji.

Pehhuame.

Pipa.

Sombrerito de zote amarillo.

Trauhxilotl.

Acocote, Cominos rústicos, Hierba del ogo. 
Artemisia laciniata Willu.

Artemisia mexicana Willd.

Arundo donax $\mathrm{L}$

Asclepias cornuti? Dcne.

Asclepias curassavica $\mathrm{L}$

Asclepias incarnata $\mathrm{L}$.

Asclepias linaria Cav....

Asclepias nivea L

Asclepias setosa Benth.

Asclepias, sp?

Asimina, sp?

Asparagus officinalis $\mathrm{L} . . . . . . . . . . . . . . . .$.

Aspicarpa, sp?.

Aster pauciflorus Nutt......................

Aster, sp?.

Astragalus adscendens Boiss.

Astragalus humboldtii A. Gr.

Astrocaryum mexicanum Liebm.

Asclepias, sp?.

Atriplex canescens James...

Atriplex hortensis L...

Atriplex, sp?.

Atriplex, sp?

Attalea cohune Mart?

Attalea, sp?.

Avena sativa $\mathrm{L}$

Avicennia nitida Jacq.

Avicennia tomentosa Jacq

Azolla caroliniana Willd...
Compuestas .

Compuestas ....

Gramíneas .....

Asclepiadeas

Asclepiadeas.

Asclepiadeas

Asclepiadeas

Asclepiadeas Asclepiadeas

Asclepiadeas

Anonáceas

Liliáceas.

Malpigiáceas

Compuestas

Compuestas

Leguminosas

Leguminosas...

Palmas......

Asclepiadeas

Quenopodiáceas...

Quenopodiáceas...

Quenopodiáceas...

Quenopodiáceas...

Palmas

Palmas

Gramíneas

Verbenáceas

Verbenáceas

Rizocarpeas.
Ajenjo de México, Estafiate.

Ajenjo del país, Estafiate, Iztauhyatl, Nexmilzi.

Cañaveral, Carrizo,

Huey tochllinacaztli, Solimán vegetal.

Cancerillo, Cocholloxin, Cochinita, Plato y Laza, Ponchishui, Punchuhuiche, Quiebra muelas, Revienta muelas, Señorita.

Algodoncillo, Señorita, Soldadillo.

Mapipilza, Romerillo, Solimán, Teperromero, Tlalayotl, Venenillo.

Coamecate blanco.

Contrahierba, Contrahierba de Julimes.

Pipilote.

Anonilla.

Espárrago.

Coanlecate de cerro.

Escobilla.

Atlacote, Atlacotl.

Tragacanto (goma).

Chinchine, Sonadora.

Palma.

Thalayole.

Cenizo.

Armuelle.

Quelite de puerco.

Ahanico.

Coquilo, Palma.

Coyol real, Palma, Palma coyol.

Avena.

Mangle blanco.

Mingle blanco.

Chilacaxtli.
Baccharis alamani $\mathrm{DC}$

Baccharis conferta H. B. K...............

Baccharis heterophylla H. B. K.

Baccharis mucronata H. B. K...

Baccharis multiflora H. B. K..............

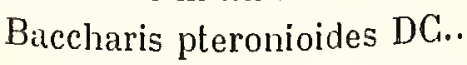

Baccharis, sp?...

Baileya multiradiata Harw. $\&$ Gray.......

Bambusa arundinacea Roxb..............

Bambusa guadua L

Bambusa vulgaris Wendl...................

Bauhinia divaricata Paul Maury...........

Bauhinia pes-capre Cav....................

Bauhinia platypetala Bentl

Bauhinia porrecta $\mathrm{Sw}$.

Baulninia, sp?....
Compuestas.

Compuestas

Compuestas....

Compuestas.

Compuestas

Compuestas

Compuestas

Compuestas

Granıíneas

Gramineas

Gramíneas

Leguminosas

Leguminosas

Leguminosas

Leguminosas

Leguminosas
Hierba del carbonero.

Escobilla, Hierba del carbonero, Tepopote, Tepópotl.

Escobilla.

Hierba del cardonero.

Hierba del carbonero, Limpia-tunas.

Boshi, Jaral blanco, Popotillo, Tepopote, Tepópotl.

Prodigiosa.

Calancapatle, Palancapatli.

Otate.

Jimba

Cupamu, Palamba.

Pata de res.

Pie de cabra.

Timbe.

Calzoncillo.

Papalocuahuite. 
Bauhinia, sp?

Begonia balmisiana Ruiz

Begonia fuchsioides Hook.

Begonia gracilis H. B. K.

Begonia imperialis Lem

Begonia incana Lindl

Begonia tuberosa

Belotia

Berber orefirolia A. Rich

Berberis pinnata Lag.

Berberis, sp?

Bessera elegans Schultes.

Beta cicla L. var.

Beta vulgaris Moq. var..............

Beta vulgaris Moq. var.

Betonica alopecurus I

Betula lenta L.

Bidens angustissima H. B. K

Bidens heliantoides H. B. K.

Bidens heterophylla Ort.

Bidens leucantha Willd...

Bidens ostruthioides Schz. Bip.

Bidens pilosa $\mathrm{L}$

Bidens tetragona DC.

Bigelovia veneta A. Gr.

Bignonia alliacia Lam.

Bignonia buccinatoria Mairet.

Bignonia capensis 'Th.

Bignonia laurifolia Vahl....

Bignonia lindleyi DC......

Bignonia, sp?

Bignonia, sp?

Bignonia, sp?

Bixa orellana I

Bletia campanulata Llav. et Lex

Bletia coccinea Lliv. et Lex

Blitum bonus Henricus Reich.

Bocconia arborea Wats.

Bocconia frutescons L.

Boerhaavia viscosa Lag. et Rodr.

Bœrhaavia, sj?

Bohmeria nivea Hook. et Arnott.

Boletus edulis Bull...

Bomarea hirtella Herb.

Bombax ellipticuin H. B. K

Borago officinalis $L$

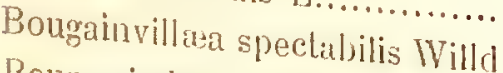

Bourreria huanita Hemsl.
Leguminosas

Begoniáceas .

Begoniáceas

Begoniácens

Begoniáceas

Begoniáceas

Begoniáceas.........

Tiliáceas.

Berberídeas.

Berberideas...

Liliáceas.

Quenopodiáceas ...

Quenopodiáceas ...

Quenopodiáceas ...

Labiadas.

Cupulíferas.

Compuestas

Compuestas

Compuestas

Compuestas

Compuestas

Compuestas

Compuestas.

Compuestas

Bignoniáceas

Bignoniáceas

Bignoniáceas

Bignoniáceas

Bignoniáceas

Bignoniáceas

Bignoniáceas

bignoniáceas.

Bixíneas

Orquídeas

Orquíreas

Quenopodiáceas ...

Pápaveráceas....

Papaveráceas

Nictagineas...........

Niciagínens .........

Urticáceas

Hongos.....

Amarilídeas

Matváceas.

Borragíneas.......

Niclagincas.

Borragíneas...
Pie de venado.

Caslıúrequa.

Corazón de ángel, Corazón de Jesús.

Ala de ángel, Carne de doncella, Hierba de la doncella, Jocoyol color de rosa, Sangre de doncella, Totoncaxoxocoyollin.

Begonia imperial.

Begonia blanca.

Hierba de la doncella.

Capulincillo. Palencano.

Cachisdá, Camisdá, Chachisdá, Palo amarillo, Quisquirindín, Quisquiringuín.

Retamilla.

Arete, Aretillo.

Acelga.

Betabel, Nabo colorado de Jarava, Remolacha.

Acelga.

Betónica.

Abedul.

Cempazuchilito.

Acahualillo, Te de milpa, Girisol de agua.

Capitanejar.

Acahuale blanco, Aceitilla, Acocotli, Mulito, Rosilla del Valle de México, Saetilla, T'e de flor blanca, Te de milpa, 'Te de Veracruz.

Veinle reales.

Acahualillo de flor blanca, Aceitilla, Te de milpa blanco.

Acahualillo, Te amarillo de milpa, Te de nilpa, Te de milpa amarillo.

Boxosdá, Damiana, Falsa damiana.

Bejuco de ajo.

Charín, Palomitas, Yedra-bocina.

Bignonia.

Bejuco de canoa.

Bejuco ties lomos.

Borla de San Pedro.

Sabino.

Bejuco cola de iguana.

Achiote, Achiotillo, Achiotl, Cilincanguarica, Pumacha.

Chautle, Flor de muertos, Silucle, Silutle, Trarcutli, 'Tzilulıtli florida, 'Tzacuxóchitt.

'Tonaloxúchitl, 'Tzacuxóchitl.

Huåzontle, Quauzonletl, Quauzontetle.

Bocconia, Cachisdá, Cocoxiluuitl, Cuatlatlaya, Cuau hichilli, Chicalote de árbol, Enguambo, Engüemba, Inguande, Guachilli, Llora sangre, Palo amarillo, Tlacoxihuitl.

Los mismos nombres vulgares de la anterior.

Coanenepilli.

Guanenepile.

Ramié, Seda vegetal.

Pambazo.

Coyolxóchitl.

Ceiba, Clnicochuchi, Chilochuchi, Itzlámatl, Titiláınall.

Borrina

Azalia de guía.

Huanita, Ixquixócliul, Vanita. 
Boussingaultia baselloides H. B. K........ Bouvardia angustifolia $\mathrm{H}$. B. K........... Bouvardia quaternifolia DC...

Bouvardia triphylla Salisb....

Brachistus stramonifolius Miers.

Brahea dulcis Mart.

Brassica campestris L. var.

Brassica napus L. var

Brassica nigra Koch.

Brassica oleracea L, var.

Brassica oleracea L. var.

Brassica oleracen L. var.

Brassica oleracea L. var....

Brassica oleracea L. var.

Brickellia cavanillesii A. Gr....

Brickellia veronicæfolia A. Gl....

Brickellia, sp?

Bromelia pinguin L.

Bromelia, sp?..

Bromus sterilıs L.

Brongniartia thermoides Spr..............

Brosimum alicastrum Sw

Brosimum, sp?

Brya ebenus DC.

Bryophyllum calycinum Silisb....

Buclinera elongata Sw

Buddleia americana L.

Buddleia humboldtiana Rœm. et Schult. Buddleia lanceolata Benth................ Buddleia marrubiifolia Benth....

Buddleia perfoliata H. B. K...

Buddleia scordioides H. B. K

Buddleia sessiliflora H. B. K...

Buddleia verticillata H. B. K.............

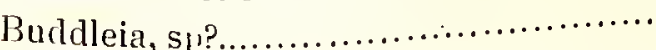

Buddleia, sp?

Buddleia, sp??

Buetheria carthagenensis Jacq...........

Buettneria lanceolata ? DC................

Bryum, sp?

Bumelia lycioides Gertn fil.....

Bunchosia guadalajarensis Wals..........

Bunchosia lanceolata ? Turcz.............

Bunchosia sessilifolial? DC......

Bursera aloexylon Engl....

Bursera bipinnala Engl..

Bursera delpechiana? Poisson.

Bursera filgaroides Engl. ...

Pursera gummifera Jacq
Quenopodiáceas..

Rubiáceas

Rubiáceas .....

Rubiáceas .

Solanáceas

Palmas

Cruciferas.

Ciruciferas.

Cirucíferas.

Cruciferas.

Cirucíferas

Cirucíferas

Cirucíferas....

Ciruciferas

Compuestas

Compuestas

Compuestas

Bromeliáceas

Bromeliáce ıs

Gl'amíneas

Compuestas

Urticáceas

Urticáceas

Leguminosas.......

Crasuláceas...

Escrofularíneas..

Loganiáceas.

Loganiáceas Loganiáceas Lnganiáceas. Loganiáceas. Loganiáceas.

Loganiáceas

Loganiáceas.

Loganiáceas

Loganiáceas.

Loganiáceas.

Ester'culiáceas

Eslerculiáccas.....

Musgos.

Sapotáceas.

Malpigiáceas

Mitlpigiáceas

Malpigiáceas

Burseráceas

Burseráceas.

Burseráceas

Bur'seráceas

Burseráceas.

Anacardiáceas....
Sacasile, Secocile de México.

Trompetilla.

Trompetilla.

Contrahierba, Tlacaxochitl, Tlacoxóchitl, Trompetilla.

Hoja peluda.

Palma apache, Palma dulce, Palma de sombrero, Palma soyal, Soyale, Soyate, Zoyate.

Nabo.

Nabo comestible.

Mostaza, Mostaza negra.

Berza, Col, Nabo, Repollo, Verdal.

Coliflor.

Col enana, Colinabo.

Col de Milán.

Berza colinabo, Berza de Siam.

Atanasia amarga, Hierba del becerro, Hierba dulce de México, Prodigiosa.

Gobernadora de Puebla, Hírba de la Gobernadora de Puebla, Mejorana.

Hierba de la cruz.

Aguava, Cardo, Guamara, Jocuistle, Piñuela, Thumbirichi, Timbirichi.

Cocuite, Cocuiztle.

Bromo, Zacate.

Janacahuate.

Capomo, Nazareno, Ojite, Ox, Ox dulce.

Ox de mico.

Ebano, Granadillo, Paln de fierro.

Admirable, Amor tras de la puerta, Bruja.

Romerillo.

Cayolizán, Cayolozán, Cayoluián, Tepozán, Topoza, Topozán, Zayolizán, Zayolizcán.

Tepozán.

Tepozán del cerro.

Azafrán del campo.

Salvia india, Salvia real de México, Salvia de bolita.

Escobilla.

Tepozancillo.

Hierba de la playa, Mispatle, Silvia común.

Hierlua de San Perlio.

Lengua de huey.

Mispatle.

Zar'za.

Nincenes.

Musgo.

Comil.

Hanzalnito.

Capulincillo, Ciruelillo, Nanche, Zapotito de San Juan.

Ciruelillo.

linaloé.

Giajiote verde.

Copal amargo, Copal amargoso, Copal chino, Incienso del pais, Tellale, Tellatia, Tetlatian, Tetlatin.

Linaloés.

Cuajiote amarillo, Cuajiote colorado.

Archipín (goma), Palo jiote, Palo mulalo, Palo mulato de Chiapas. 
Bursera jorullensis Engl...

Bursera lanuginosa Engl.

Bursera mexicana Engl.

Bursera multijuga Engl.

Bursera trijuga Ramírez

Bursera, sp?

Bursera, sp?

Bursera, sp?

Byrsonima cotinifolia H. B. K

Byrsonima karwinskiana A. Juss

Byrsonima, sp....
Burseráceas. Burseráceas

Burseráceas.

Burseráceas

Burseráceas

Burseráceas

Burseráceas

Burseráceas

Malpigiáceas

Malpigiáceas

Malpigiáceas
Copal blanco, Copal de penca, Copal de santo, Copalli. Copal.

Aceitillo, Copal de santo, Copalxóchitl, Sochicopal ó Suchicopal, Xochicopalli.

Cuajiote amarillo.

Cuajiote chino.

Bálsamo del cirujano, Cirujano.

Tacamaca, Tacamahaca.

Teponaxtle, Teponaxtli.

Nananche, Nance, Nancis, Nanclie, Ninche de perro, Nanzinxocotl.

Nance agrio.

Nanzinguahutl?.
Cabomba aquatica Aubl.

Cæesalpinia bonducella Roxb.

Cæesalpinia cacalaco Humb. et Bonpl....

Cærsalpinia coriaria Willd.

Cirsalpinia echinata ? Lam

Cæsalpinia exostemma DC

Cæsalpinia mexicana A. Gr.

Cæesalpinia pulcherrima Sw.

Cæsaly̧inia, sı̂?

Cirsalpinia, sp?

Cæesalpinia, sp?

Caladium bicolor Vent

Calamintha macrostema Benth....

Calamintha nepeta ? Link. el Hoffm

Calamintha potosina Schaffner...

Calea axillaris $\mathrm{DC}$

Cialea cacosmoides Less....

Calea scabrifolia Benth et Hook

Calea urticæfolia DC.

Calea zacatechichi Schl.

Calendula officinalis

Cialochortus flavus Schull f..

Calophanes linearis A. Gr.

Calophyllum, sp?

Cialliandra formosa Benth....

Calliandra grindiflora Bentl

Calliandra loustoni Bentlı...

Callistephus chinensis Nees.

Camellia japonica I
Leguminosas

Compuestas

Temstrœmiáceas...
Sargazo.

Ninfeáceas

Leguminosas....

Leguminosas...

Leguminosas

Leguminosas....

Leguminosas....

Leguminosas

Leguminosas

Leguminosas

Leguminosas

Leguminosas...

Aroideas.

Labiadas.

Labiadas. .

Labiadas

Compuestas

Compuestas.

Compuestas

Compuestas

Compuestas

Compuestas.

Iiliáceas.

Acantáceas.

Gutíferas.

Leguninosas

Leguminosas...

Haya.

Jarilla.

Ayatilo.
Haba de San Antonio. Taray.

Cacalaco. Cascalote. Nacascul.

Cuscalote. Nacascul.

Huitzquáhuitl. Hotzquáhuitl.

Hoja sen del país. Hojasén. Sen del país.

Chacalxóchitl. Flor del camarón. Sirundaniqua. Tabachín.

Tabachino. T'abachín amarillo ó de Colima. 'Tabaquín. Xiloxóchitl.

Brasil. Palo del Brasil.

Cahuinga.

Tzuraqua.

Papagallo del Brasil.

Nurite. Tabaquillo. Te de monte.

Torongil. Torongil del país.

Orégano de la sierra.

Hierba de la rabia. Ponchishui.

Hierba del becerro.

Hierba de la paloma.

Sacachichic. Sacatechichi. Simonillo. Zacachichi. Zacate annargo. Zacalechichi.

Mercadela. Reinita. Virreina.

Hierba de la vílsora.

Limoncillo de Córdoba.

Ebano de Tabasco.

Cabellitos de ángel. Cirbellitos de una vara. Cilagot. Hierba del ángel. Lele. Paumbolano. Tepachera. Tepexiloxóchitl. Texoxóchitl. Timbrillo. Tlacoxiloxóchitl. Tlamacazcatzoll. T\%onxóchitl. Xiloxóchitl.

Charamusco

Astras. Ester. Esther. Margarita. Reina Margarita. 
Cimpanula medium L....

Campanula rapunculoides ?

Camphorosma mo:ispeliaca L.

Canavalia villosa Benth.

Canna indica L....

Camna paters Ail.

Canna, sp?.

Ciannabis sativa 1 ,

Capsella bursa pastoris L................

Capsicum annuum L

Citusicum annuun acuminatum $\mathrm{F}$.

Capsicum annuum cerasiforme II.

Ciapsicum annuum grossum Sentl....

Capsicum annuum longum Sendt.

Capsicum axi Vell...

Capsicum baccatum I

Capsicum cordiforme Mill..

Capsicum dulce Hort

Capsicum firutescens I.....................

Ciapsicum longum DC....

Capsicum microcarpum DC

Capsicum violaceum H. B. K.

Capsicum, sp:

Ciardiospermum halicacabum $\mathrm{I}$

Cardiospermum molle H. B. K

Gierdiospermum, sp??....

Carduus tenuiflorus DC...

Carica heptaphylla Moe. el Sessé.........

Carica nana $\mathrm{L}$.

Carica papaya $\mathrm{L}$.

Cariea, sp?

Caryophyllus aromáticus L ...............

Carludovica palınata R. el Pav.............

Carthamus tinetor" us $\mathrm{I}$.

Carum carvi L...

Carum petroselinum Benth......

Carya olivæformis Nult.

Ciarya mexicana? Engelm......

Ciarya, sp.....

Casearia nitida Jacq

Ciasearia, sp?

Casearia, sp?

Casimiroa edulis Llav et Lex..............

Casiniroa pubescens Ramz................

Cassia alata

Cassia bacillares L. fil....................

Cassia bicapsularis L.. ..................

Ciassia fistula L.

Cassia floribunda Ciav .....................

Cassia grandis L. fil..

Ciassia lævigata Willd.

Cassia leiophylla Vog.....................

Cassia moschata H. B. K...................

Ciassia occidentalis $\mathrm{L}$.

Ciassia sericea Sw....
Campanuláeeas...

Campanuláceas....

Quenopodiáceas...

Leguminosas.........

Zingiberáceas....

7ingiberáceas

Zingiberáceas.

Urticáceas

Grucíferas..

Solanáceas.

Solanáceas

Solanáceas....

Solanáceas

Solanáceas

Solanáceas ....

Solanáceas ....

Solanáceas

Solanáceas.

Solanáceas

Solanáceas

Solanáceas ....

Solanáceas.

Solanáceas.

Sapindáceas

Sapindáceas

Sapindáceas

Compuestas

Pasifloráceas

Pasifloráceas

Pasifloráceas.

Pasifloráceas..

Mirtáceas

Ciclantáceas.

Compuestas ........

Umbelíferas ......

Umbelíferas

Juglimileas.

Juglarideas

Juglandeas

Samidáceas.

Samidáceas

Samidáceas

Rutáccas

Rutáceas

Leguminosas.......

Leguminosas........

Leguminosas.......

Leguminosas........

Leguminosas........

Leguminosas........

Leguminosas........

Leguminosas........

Leguminosas........

Leguminosas........

Leguminosal.......
Campanilla.

Aguinaldo.

Alcanfor de maceta. Alcanforada.

Frijolillo.

Caña coro. Caña de cuentas. Coyol. Flor del cangrejo. Frutilla. Hierba del rosario. Platanillo.

Bardana del país.

Cliancle.

Cáñamo. Marihuana. Rosa María.

Bolsa del pastor. Comida de pajaritos. Paniquesillo.

Gahúas. Chile. Chiltipiquín de Papantla.

Chile.

Chile piquín.

Chile ancho. Chile poblano.

Chile. Chile cara.

Ghile colorado boludo.

Chillepiquín. Chiltipín.

Chile ancho. Chile mulato.

Chile valenciano.

Chile cuauchilli. Chile piquin. Quauchilli.

Chile pasilla.

Chiltepín. Chiltipiquín. Piquín.

Chile tzincuayo.

Chile mirasol.

Farolitos. Munditos.

Munditos. Ocotillo. Varal.

Moquillo blanco.

Cardo santo.

Bonete.

Bonete.

Melón zapole. Papaya. Papayo. Pul.

Cuaguayote.

Clavo de especie.

Jipijapa de Panamá.

Azafıán. Azafrancillo.

Alcarivea. Comino de los prados.

Perejil.

Nogal morado. Nuez chiquita. Nuez encarcelada. Pecan.

Nogal motudo. Nogal riayado.

Pulo amargo.

Cafelillo.

Capulincitlo.

Pochitoquillo.

Cochilzápotl. Cochiztzápoll. Ixtaclzápotl. Zapote blanco.

Zapote de rala.

Fhor del secreto. Tamalanit.

Quelite.

Alcaparrillo. Bicho. Bricho. Cachiubso.

Cañafistuli. Quauhuayo.

Bricho. Relima.

Ciñullistula grande.

Ciaté del prás. Retama del país.

Hormiguera.

Cañafistula chica.

Bricho. Ecapatli. Mezquitillo.

Xluab. 
Cassia spectabilis DC.

Cassia tagera L

Cassia tomentosa L. fil....

Cassia, sp?

Cassia, sp?

Cassia, sp?

Cassia, sp?..

Castanea vulgaris Lam.

Castela nicholsoni Hook.

Castilleja arvensis Ch. et Schl

Castilleja canescens Benth.

Castilleja lithospermoidt's II. B. K.

Castilleja scorzoneræfolia H. B. K.

Castilleja tenuiflora Benth

Castilleja tenuifolia Mart. et Gal....

Castilloa elastica ('erv....

Cattleya citrina Lindl.

Ceanothus azureus Desf.

Ceanothus cœruleus Lag. . ..

Cecropia mexicana Hemsl.

Cecropia peltata I

Cedrela dugesii Wats.

Cedrela glaziovii C. DC.

Cedrela mexicana Rœm.

Cedrela odorata ? L

Cedrela, sp?

Cedrela, sp?

Cedrela, sp?.

Cedronella mexicana Bent.

Cedronella pallida Lindl.

Celosia cristata I.

Celosia moquini Guillem.

Celtis occidentalis L....

Celtis pallida Torrey.

Cenchrus echinatus L....

Cenchrus tribuloides I

Centaurea americana Nutt

Centaurea cyanus Lindl...

Centaurea grandiflora DC:

Centranthus ruber $\mathrm{DC}$

Centrosema plumieri Benth....

Ceplıelis tomentosa? Willd

Ciephalanthus occidentalis L.

Cerasus caproniana DG

Ceratozamia mexicana Biongn.

Cercocarpus fothergilloides H. B.

Cereus flagelliformis Haw....

Cereus geometrizans Mart.

Ciereus giganteus Engelm.

Cereus marginatus DC:

Cereus senilis Salm. Dyck.

Cereus serpentinus Lagasc
Leguminosas.

Leguminosas.

Leguminosas

Leguminosas.

Leguminosas.

Leguminosas.

Leguminosas.......

Cupulíferas.

Simarrubáceas

Escrofularíneas...

Escrofularíneas...

Escrofularíneas...

Escrofularíneas...

Escrofularíneas...

Escrofularíneas...

Urlicáceas

Orquídeas.

Ramneas

Ramneas

Ulticáceas

Urlicáceas

Meliáccas

Meliáceas

Meliáceas

Meliáceas

Meliáceas

Meliáceas.

Meliáceas...

Labiadas.

Labiadas. . .

Amarantáceas............

Amarantáceas....

Urticáceas .

Urticíceas

Gramíneas.

Gramíneas

Compuestas

Compuesilas

Compueslas

Valerianeas

Jeguminosas

Pubiáceas

Rubiácens

Piosáceas.

Cicadáceas.....

Rosáceas....

Cácteas.

Cáeteas

Cácteas.

Cácteas

Gácteas.

Cácteas
Canchín.

Retama.

Retama de tierra caliente.

Bicho de arbusto.

Hierba del buey.

Junco.

Vainillo.

Castaño.

Amargoso.

Bella Inés. Ciastilleja. Cola de borrego. Enchiluditas.

Cola de borrego. Chnpirín vilzaqua.

Cola de borrego.

Charás petequa.

Hierba del cáncer.

Cialzón de indio.

Aibol del hule. Caoutchouc. Holquálıuitl. Hule. Semilla de luule. Tarantaqua.

Aróraqua. Costicoatexóchitl. Cozticoilzontecoxóchitl. 'T:ıtzingueni.

Chaquira. Chaquirilla.

chaquira. Chaquirilla.

Coilotópalo. Chancilro. Guarumbo. Saruma.

Coilotópalo. Guarima. Gurrumbo. Guarumo. Siruma.

Cedro.

Cedro.

Calicedra. Cedro. Cedro colorado. Cedro de la Habana. Cedro macho.

Ciedro rojo.

Cedro chino.

Cedro liso.

Rosadillo.

Norilen. Torongil. Torongil del país.

Torongil del juás.

Abanic:o. Cinco de Mayo. Cresta de gallo. Mano de león.

Madroño.

Palo blanco.

Granjeno.

Cadillo.

Rosetilla.

Cardo santo.

Botoncillo. Cabezuelit.

Cardo santo.

Va!eriana roja.

Palitos.

Uvero.

Bejuquillo.

Guinda. Guindo.

Palma.

Ramón.

Guerno. Flor del cuerno. Flor del látigo. Floricuerno. Hierba de la alferecía. Junco.

Garambullo.

Pitahaya.

Organo.

Cabeza de viejo.

Junco espinoso. 
Cereus speciosissimus Desf.

Cereus stramineus Engelm

Cereus tetragonus Haw.

Cereus thurberi Engelm...................

Cereus trigontus Haw...

Cereus variabilis Pfeiff.

Cereus, sp?.....

Cereus, sp?

Cereus, sp?

Cestrum aurantiacum Lind..

Cestrum eonfertiflorum Schıl....

Cestrum diurnum L.......

Ciestrum elegans Schil.

Cestrum hartwegii Dun.

Cestrum nitidum Mart. ef Gall..

Cestrum nocturnum L

Cestrum roseum H. B. K.

Ceterach officinarum DC:.

Chanedorea elatior Mant....

Chamedorea ernesti-augusti Went.......

Clammdorea humilis Wals...

Chamedorea tepejilote lichm.............

Chamedorea ?...

Ciliannedorea ?...

Chanmerea ?....

Chamærops humilis L......................

Chaptalia nulans Hemsl....

Cheilanthes lindheimeri Hook.

Cheilanthes myrioplaylla Desv....

Cheiranthus cheiri L.

Cheiranthus seoparius Willd...

Clieirostemon platanoides Humb. et B...

Gheliclonium majus Mill.

Chenopodium album Moq. var.

Chenopodium ambrosioides L....

Cilienoporlium foetirlum Schrad...........

Cillenopodium incisum Poir......

Cilıenopodium mexicanım Moq

Chenopodium vulvaria $\mathrm{L} \ldots . . . . . . . . . . . . . . .$.

Chenopodium, sp?....

Chliococca racemosa Jaq....................

Chlorophora tinctoria Gaud. var............

Cilooisya ternata H. B. K..................

Chrysactinia mexicana A. Gir.............

Ghrysanlhemum coronarium L..........

Chlysantlemum parthenium Snith.....

Chrysobalanus icaco l......................

Chrysophyllum cainito L....

Chusquea galeottina Rupr.................

Cicer arictinum

Cichorium endivia

Cichorium intybus I
Cácteas

Organo. Santa Marta. Xoalácatl.

Cácteas

Cácteas.

Cácteas

Cácteas.

Cácteas

Cácteas

Gácteas

Cácteas

Solanáceas

Solanáceas

Solanáceas

Sol:náceas

Solanáceas

Solanáceas

Solanáceas ....

Solanáceas

Helechos ...

Palmas

Palmas

Palmas

Palmas

Palmas

Palmas

Palmas

Palmas

Compuestas

Helechos

Helechns

Gruciferas.

Gruciferas

Esterculiáceas.....

Papaveráceas

Quenopodiáceas.. Quenopodiáceas ... Qnenopodiáceas...

Quenopodiáceas ... Qnenopndiáceas... Quenopodiáceas... Quenopodiáceas ... Rubiáceas Urticáceas Rutácerts ............ Compuestas........

Compuestas....... Comprestas........ Rosáceas ........... Sapotáceas .......... Gramíneas.......... Leguminosas........ Compuestas........ Compuestas.......

\section{Organo. Pitahaya.}

Xnuntzutzuy.

Pitahaya.

Pitallaya de Yucatán. Pitajaya.

Pilahaya.

Tasajo.

Tasajillo.

Hierba del zopilote.

Hicrba de la mula.

Parqui. Pipiloxóclitl.

Flor del soldado.

Hierba del perro.

Dama de noclie.

Belladona del país. Hediondilla.

Xmuchcoc.

Guaya de cerro. Gilayila.

Guaya de bajo.

Bomaabalsáh.

Tritzambas.

Tacauba. Tacisle.

Xoan.

Palma enana. Palınita.

Motitas.

Hierba de la peña.

Cola de zorra.

Alhelí.

Allelí camaleón. yaqua.

Epazote. Epazoll. Yepazoll.

Epazole del zorrillo.

Quelite cenizo.

Hediondilla.

Armuelle silvestre.

Canica. Caninana. Mariola. Romerillo.

Filipéndula. Flor de oro.

Hierba de Santa Maŕa.

Hicaco. Icaco. Jicaco.

Carrizo.

Garbanzo.

Escarola.

Achicoria. Ahunión. Chirivía.
Gran cuerno.

Hierba hedionda. Huele de noche. Parqui. Pipiloxihuitl.

Tepejilote. Tepepilote. Tepexiloll.

Arbol de las manilas. Flor de la manita. Macpalxóchitî. Te-

Amapola amarilla. Celidonia. Celidueña. Golondrinera. Hierba de la golondrina extranjera.

Quelite. Quelite de trigo. Quilitl.

Epazote cimarrón. Epazote de zorrillo. Quelite apestoso. Quelite de zorrillo. Yepazote le toro.

Moradilla. Moral amarillo. Moral de clavo. Moral hiso.

Clavillo. Clavo de olor. Flor del clavo. Hierba del clavo.

Damiana. Damianita. Hierba de San Nicolás. Llellepaxtle.

Caimito. Caimito verde. Zapote caimito. 
Cinchona calisaya Ruiz et Pav.

Cinchona officinalis $\mathrm{L}$

Cinchona succirubra Pav.

Cissampelos pareira Lamarck.

Cissus tiliacea H. B. K

Citharexylum caudatum L.

Citrullus vulgaris Schrad. var....

Citrus aurantium Risso...

Citrus aurantium Risso var....

Citrus decumana L.

Citrus limelta Risso.....

Citrus limetta Risso var.

Citrus limonum Risso

Citrus limonum Risso var.

Citrus limonum Risso var.

Citrus medica L.

Citrus medica Pisso var.

Citrus nobilis Lour.....

Citrus vulgaris Risso. ....

Citrus vulgaris Risso var.

Clematis dioica L.....

Clematis sericea H. B. K.

Clematis triloba Roth...

Clematis, sp?.

Cleome cardinalis DC.

Cleome heptaphylla L

Cleome spinosa L

Clerodendron fragans Vent. var....

Clerodendron ligustrinum R. Br.

Clethra lanata Mart et Gal.

Clitoria virginiana $\mathrm{L}$.

Cnicus mexicanus Hemsl..

Cnicus pazcuarensis DC....

Cnicus rhaphilepis Hemsl....

Cobæa scandens Cav.

Coccoloba caracasana ? Meisn

Coccoloba uvifera Jacq.

Cochlearia armoracia L.

Cochlospermum hibiscoides Humb.......

Cocos guacoyule Liebm

Cocos nucifera L.

Cocos, sp?

Coffea arabica $L$.

Coix lacryma L.

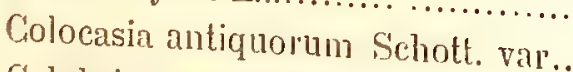

Colubrina alamani DC:

Combretum farinosmm H. B. K.

Combretum jarquini ? Griseb.

Combretum laxum Lofl

Commelina graminifolia II $\mathrm{P} . \mathrm{K} \ldots \ldots \ldots . . .$.

Commelina pallidat Willd.

Commelina tuberosa l....

Condalia mexicana Schl.

Condalia obovata Ruiz of Pav.....................
Rubiáceas.

Rubiáceas.

Rubiáceas.

Menispermáceas...

Ampelídeas

Verbenáceas ........

Cucurbitáceas ......

Rutáceas

Rutáceas

Rutáceas

Rutáceas

Rutáceas

Rutáceas

Rutáceas

Rutácens

Rutáceas

Rutáceas

Rutáceas

Rutáceas

Rutáceas

Ranunculáceas ....

Ranunculáceas....

Ranunculáceas....

Ranunculáceas ..

Caparideas

Caparídeas...

Caparídeas

Verbenáceas

Verbenáceas

Ericáceas

Leguminosas.

Compuestas.

Compuestas.

Compuestas.

Polemoniácens ...

Poligonáceas.....

Poligonáceas

C'rucíferas

Bixíneas

Palmas...

Palmas

Palmas

Pubiáceas

Gramíneas

Aroideas.

Ramneas....

Combretáceas

Combretáceas .

Combretáceas .....

Ciommelináceas ....

Commelináceas...

Commelináceas.

Tiamneas

Jiamneas
Quina calisaya.

Quina.

Quina roja.

Butua. Iztactepetzacuxóchitl. Pareira brava. Pereira brava. Molonqui. Temécatl.

Roble amarillo.

Cidra cayote. Sandía. Zandía.

Naranjo. Naranjo dulce.

Naranjo lima.

Cidro. 'Toronja.

Lima dulce. Limero.

Lima agria. Lima chichona.

Limón. Limonero.

Limón dulce.

Limón real.

Cedro limón. Cidra. Ciidra limón. Ciidrelo. Cidlere.

Limón real. Toronja.

Mandarina.

Naranjo agrio.

Naranjo hoja dr: nirto.

Barbas de galo. Barbas de viejo.

Barbas de chivo. Barbas de viejo. Cillilillo.

Jazmín.

Sacamecate.

Borla de cardenal.

Barbona rosada.

Pata de vaca.

Volcameria.

Musté.

Mamamalhuaztli.

Zapatito de la reina.

Cardo santo. Cardo santo cimarrón. Clıecámequa.

Cardo santo.

Ciardo santo.

Cundeamor. Flor de la campana. Hiedra morada.

Tocó.

Uvas del mar. Uvero.

Piábano rusticano.

Madera de pasta. Pochote. Tecomaxóchill.

Coyol. Coyole. Guacoyul. Haiscoyul. Quacoyul.

Coco. Coco de agua. Coco de caslillo. Cocolero. Palna de coco.

Biscoyol.

Cialé. Caleto.

Acayacoyotl. Lágrimas de Joh ó de San Perlro.

Quequeste.

Cibaquira.

Peinecillo.

Cepillo del diublo.

Cepillo del diablo.

Quesadililus.

Commelina. Hiorbal del pollo. Quesadillas. Posilla.

Coapatli. Hierba del pello. Matladiztic. Quesadillibas. Rosi-

lla. Texcocana. Yoyolxóchitl. Zoyalxóchitl.

Bindó.

Brisil. Capul. 
Condaminea, sp?

Conium maculatum L

Conocarpus ererta L.

Conopholis americana Wallr.

Conostegia xalapensis Don.

Convolvulus arvensis L.

Convolvulus, sp??.

Convolvulus, sp?

Convolvulus, sp?

Conyza filaginoides DC..

Conyza rnaplatioites H. B. K.............

Conyza, sp?

Copaifera officimalis ? L.. .... ..............

Copraifera ?....

Copernicia nana Liebm.

Cordia boissieri A. DC.....

Cordia collococca I

Cordia dodecandra A. DO

Cordia gerascanthoides H. B. K...........

Cordia gerascantlus Jacq..................

Cordia laxiflora H. B. K.............. ..

Gordia, sp?

Cordia, sp?.

Cordia, sp?

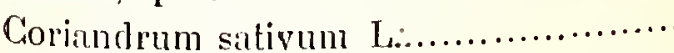

Coriaria tleymifolia H. B. K...............

Cornus disciflora DC.......................

Cornus excelsa H. B. K...................

Cornus tolucensis H. B. K.

Cornutia pyramidata L.

Corypha, sp?.

Cosmos bipinnatus Cav.

Cosmos purpureus Benth.................

Cosmos sulphureus Cav....................

Costus glabratus Sw.......................

Costus spicatus Swartz....................

Cotoneaster denliculata H. B. K...........

Cotyledon caspitosa Haw.................

Lotyledon coccinea Cav....................

Couepia polyandra H. B. K................

Coursetia glandulosa A. Gr...............

Coutarea latiflora DG.......................

Coutarea speciosa Aubl....................

Coutouber spicata Aubl.... ................

Govillea tridentata Vail.....................

Gowania mexicana Don....................

Cranichis tubularis Llav. et Lex...........

Granichis speciosa I.lav. et Lex...........

Cirantzia lineata Nutt

Cratregus clus-galli I.....................

Ciralægus mexicana DCi....................

Cralregus pubescens Stcut,..............
Rubiáceas.......... Umbelíferas ........ Combretáceas......

Orobancáceas .....

Melastomáceas ....

Convolvuláceas..

Convolvuláceas....

Convolvuláceas....

Convolvuláccas....

Compuestas.

Compuestas.

Compuestas.

Leguminosis.......

Leguminosis.......

Pilmas..............

Borragíneas...

Borragineas.

Borrogíneas.

Borragíneas.

Borragíncas...

Borragíneas

Bolragineas.

Borragineas

Borragíneas

Borragíneas

Umbelíferas. ........

Coriarieas.....

Cornáceas

Cornáccas

Cornáceas

Verbenáceas

Palmas ...............

Compueslas........

Compuestas.

Compuestas.

Compuestas.

Zingiberíceas.......

Zingiberáceas......

Rosáceals ...........

Crasuláceas.........

Crasulácens........

Posáceas

Leguminosas .......

Rubiáce:ıs

Rubiáceas....

Geucianeas....

Zigofileas..............

Rosáceas....

Orquídeas

Orquídeas

Umbeliferas ........

Rosáceas ............

Rosáceas ............

Bosáceas
Popiste. Popiste colorado.

Cicuta. Cicuta mayor.

Mangle prieto.

Elotillo.

Capulincillo. Nigua.

Yedra azul.

Chiqueo.

Yedrón.

Tinguitzen.

Falso zacatechichi. Simonillo. Zacachichic.

Saratechichi.

Hierba del histérico.

Tecopalquáhuitı. Tocopalquahuitl.

Quebracho.

Palmilla ó Palmillo.

Anacalıuite. Anacalunitl. Cicuas de Cueramo. Rasca viejo. Siricote. Trompillo.

\section{Candelero.}

Gopite. Copito. Guayabillo. Kopte. Siricote.

Xochiquahuitl. Xochitlquálıuitl.

Barí. Lechemaría. Palo baria.

Bojón blanco. Bojón prieto. Palo María. Tambor.

Azotá caballos.

Cueramo.

Majas grande.

Palo negro.

Cilanlro. Culantro. Perejil. Thantlızanaqua.

Tlalocopetate. Tlalocopétlatl.

Xochilcorona.

Tepeacuílotl.

Tepoza. Topoza.

Pangagé

Guano de sombrero.

Girasol morado. Mirasol. Xaricamala.

Mirasol.

Girasol rojo.

Girasol amarillo. Nirasol amarillo.

Coyol de México.

Caña de jabalí. Cañita agria.

Membrillo cimarrón. Tlaxizqui. Tlaxiztle.

Peyole. Piote.

Siempreviva de nacimienlo.

Zapote amarillo.

Samo prieto.

Copalchi. Falsa quina.

Copalchi de Colima. Copalehi de Miehoncán.

Centaura menor.

Gobernadora.

Romero cedro

Acatlzaulıtli. Tzauhtli arundinacea.

Acatlzautli. Atzahutli. Atzanti. Cilantle. Flor de Corpns.

Tzauhtli. T'zauhtli aquatica.

Sacalillo. Zacatillo.

Texocote. Texócotl.

Tejocote.

Tejocote. 
Cralæva tapia L

Grescentia cujete L

Crescentia macrophylla Seem.......

Crescentia, sp?

Crinum cruentum Ker.

Crocus sativus L

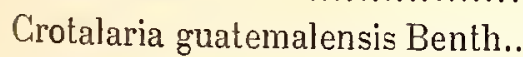

Crotalaria pumila Ort.

Crotalaria, sp?.

Croton ciliato-glandulosus Ort.

Croton corymbulosus Engelm.

Croton dioicus Cav....

Croton fruticulosus Torr

Croton glabellus L.

Croton gossypiifolius Vahl. var.

Croton maritimus Walt....

Croton morifolius Willd. var. sphærocarpus Müller...

Croton niveus Jacq.....

Croton reflexifolius H. B. K.

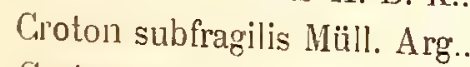

Giroton, sp?

Groton, sp?....

Groton, sp?....

Cucumis anguria L...

Cucumis melo L.

Cucumis sativus L.

Cucurbita ficifolia Bouché....................

Cucurbita fotidissima Kunth.

Cucurbita maxima Duch.

Cucurbita melopepo L.

Cucurbita pepo I

Cucurbita radicans Naud.

Cucurbita, sp?.

Guminum cyminum L.

Cupania akesia Chamb

Cupania americana L....

Cupania ? dentata DC....

Cupania, sp?...

Cupania, sp??

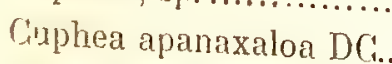

Cuphea lanceolata Ait.

Cuphea, sp?.

Cupressus benthami Endl...........

Cupressus lindleyi Klotsch.....

Cupressus sempervirens I

Cupressus thurifera H. B. K

Capressus, sp?..

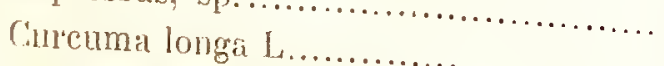

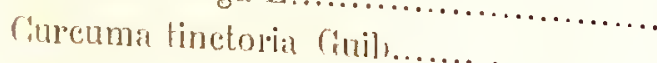

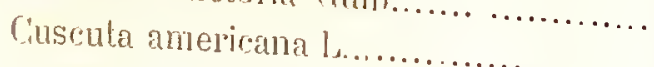

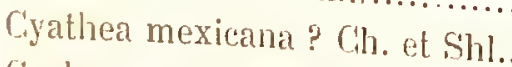

Gyclamen europeum L..

Cyclanen persicum Mill.
Caparideas

Bignoniáceas

Bignoniáceas.......

Bignoniáceas.......

Amarilídeas........

Irideas

Leguminosas

Leguminosas

Leguminosas

Euforbiáceds

Euforbiáceas

Euforbiáceas

Euforbiáceas

Euforbiáceas

Euforbiáceas

Euforbiáceas

Euforbiáceas

Euforbiáceas

Euforbiáceas .. .....

Euforbiàceas ........

Euforbiáceas ........

Euforbiáceas.......

Euforbiáceas

Cucurbitáceas......

Cucurbitáceas

Cucurbitáceas

Cucurbitáceas ...

Cucurbitáceas ...

Cucurbitáceas...

Cucurbitáceas

Cucurbitaceas ....

Cucurbitáceas ...

Cucurbiláceas

Umbelíferas.

Sapindáceas

Sapindáceas

Sapindáceas

Sapindáceas

Sapindáceas .........

Lilrarieas....

Litrarieas.

Litrarieas.

Coníferas

Coniferas

Coníferas

Coniferas

Coníferas

Ziugiberáceas

'Zingiberáceas......

Convolvuláceas....

Helechos

Primulácea

Primuláceas.
Coscorrón.

Arbol de las calabazas. Cuautecomate. Guiiro de Yucatán.

Huás. Jícara. Jícara de Tucatán, Jícaro. Tecomate. Tecómatl.

Huiro de montañas.

Cuahuteshuatl.

Lirio listado.

Azafrán amarillo.

Chipilín cimarrón.

Tronadora.

Cllipilín.

Canelillo. Enchiladora. Picosa. Xunalixase.

Encinilla.

Hierba del zorrillo. Yepaxilhuitl.

Encinilla. Hierba loca.

Caobilla. Copalclii.

Etzquahuitl. Sangre de drago.

Hierba del jabalí.

Palillo. Palillo de Guanajuato.

Copalchi.

Copalchii.

Copalelıi.

Copalillo.

Copalquín.

Zolrillera.

Pepino silvestre.

Melón. Melón blanco.

Pepino.

Cidracayote. Cillilacayote.

Cialabacilla amarga. Cohombrillo.

Ayotli. Calabaza. Semilla de calabaza. Tamalayota. Tecats.

Bonetillos. Cabeza de turco. Calabaza turbante.

Calabaza. Purú. Semilla de calabazi.

Sanacoche.

Calabaza india.

Comino.

Akec.

Cihichón colorarlo.

Sicaxquíhuitl.

Conshuilue.

Chiclón blanco.

Apancholoa.

Atlancán. Atlanclián. Atlanchana.

Yucucaca.

Cedro. Cedro blanco. Ciprés. Ciurés de México. Teotlale.

Cedro blanco. Tlascal. Tlascale. Tlatzcan.

Ciprés. Ciprés común.

Cedro. Ceelro dr: México. Cerho de la sierra. Ciprés.

Cedro amarillo.

rúreuma.

Cińremma.

Tiripu. Zacatlascal Zacatlaxcale. Zacatlaxcalli.

Ocopetale.

Mitra.

Pan de puerco 
Cydonia vulgaris Pers

Gymbopetalon penduliflorum Ball.......

Gynara cardunculus L.....................

Cynara scolymus L....

Cynodon dactylon Per's.

Cyperus articulatus L.

Cyperus esculentus L.

Cyperus flavicomus Michx.....

Cyperus luzulie Rottb.

Cyperus thyrsiflorus Jungh.

Cyperus, sp?

Cyperus, sp?

Cypripedium irapeanum Llav. et Lex.....

Cyrtocaria procera H. B. K.
Rosáceas.

Anonáceas.

Compuestas.

Compuestas.

Gramíneas

Ciperáceas.

Ciperáceas.

Ciperáceas.

Ciperáceas.

Ciperácens.

Ciperáceas.

Ciperáceas.

Orquídeas ....

- Anacardiáceas
Menbrillero. Membrillo.

Guineillo prieto.

Alcabucil. Alcaucil.

Alcachofi.

Acaxacahuiztli. Gallitos. Grama. Grama de pie de polla.

Apoyamatli. Chintul grande. Tule chico.

Peonía de botica. Peonía del prás. Tulillo.

Tule.

Nulinillo.

Peonía. Tulillo.

Chuspala. 'Tuxprata.

Tule grande.

Flor del pelícano.

Copal. Copralcocote. Copalchi. Copaljocole.
Dahlia coccinea Cav

Dilllia imperialis Rœzl

Dalılia varialsilis Desf.

Dahlia, sp?.

Dalea citriodora Willd.

Dalea diffusa Moric.

Dalea gracillima Wat

Dalea lagopus Willd.

Dalea tuberculata Lag.

Daphne laureola L...

Daphnopsis salicifolia Meissn

Dasylirion acrotrichum Zucc...

Dasylirion inerme Wats...

Dasylirion, sp?.

Datura arborea L

Datura sanguinea Ruiz et Pav.

Datura stramonium L.

Datura suaveolens Humb. et Bonpl.......

Ditura tatula L

Daucus carota L.

Delphinium ajacis $\mathrm{L}$

Dendrobium, sp?.

Desmoncus oxyacanthus Mart............

Desmoncus rudentum Mart...............

Deyeuxia, sp?.

Dianthus barbatus L.

Dianthus sinensis L......................

Dianthus, sp?

Dichondra argentea Humb. et Bonpl.....

Digitalis purpurea L

Dioon edule Lindl.
Compuestas

Compuestas

Compueslas

Compuestas

Leguminosas.

Leguminosas.

Leguminosas

Leguminosas..

Leguminosas.:

Timeleáceas..

T'imeleáceas.

Liliáceas.

Liliáceas.

Liliáceas.

Solanáceas

Solanáceas.

Solanáceas

Solanáceas

Solanáceas

Umbelífer'as ... ....

Ranunculáceas....

Orquídeas

Palmas

Palmas

Gramíneas....

Cariofileas

Cariofileas.

Cariofileas .....

Convolvuláceas. ..

Escrofularíneas....

Cicadáceas......
Dalia ó Dahalia. Flor de invierno. Jícama del cólera. Jicamile.

Dalia ó Dahalia.

Charahueso. Dalia ó Dahalia. Flor de invierno. Jicamite.

Charahuesca.

Limoncillo. Ruda cabruna ó capraria.

Escoba colorada. Ratón. Xolteco.

Escoba colorada.

Cóltotl. Terciopelillo.

Engorda-cabras.

Adelfilla.

Hojas de San Pedro.

Cucharilla.

Palma barrigona. Palma culona. Soyate. Zoyate.

Sotol.

Floripondio. Trómbita.

Floripondio rojo.

Ghamico. Flor del muerto. Nacazcul. Quiebra-plato. Tapa-

te. Tlapa. Tlápatl. Toloache. Toloatzin. Xtohcú.

Almizclillo. Campanilla blanca. Floripondio. Floripondio blanco.

Toloache.

Zanahoria.

Espuela de caballero. Miramelindo.

Zaucle. Zautle.

Balli.

Matambilla.

Sirumuta. Surumuta.

Clavel de la nobleza. Glavel del poeta. Clavellina. Minutisa.

Clavel encarnado.

Clavel de manojo.

Oreja de ratón.

Dedalera. Digital. Gualdaperra.

Cabeza de Chamal. Chamal. 
Dioscorea alata L

Dioscorea composita Hemsl...

Dioscorea, sp?...

Diospyros ebenaster Retz.

Diospyros velutina? Hiern....

Diphysa suberosa Wats...

Dipsacus fullonum L...

Dodonæa viscosa $\mathrm{L}$

Dolichos, sp?

Dorstenia contrayerba $\mathrm{L}$

Dorstenia drakena $\mathrm{L}$

Dracocephalum moldavicum L.

Dracæna draco ? L

Dracæna, sp?.

Drimys granatensis H. B. K.

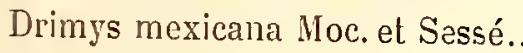

Duranta plumieri Jacq. ...

Dysodia chrysanthemoides Lag.
Dioscoreáceas

Dioscoreáceas

Dioscoreáceas ......

Ebenáceas.

Ebenáceas....

Leguminosas.......

Dipsáceas.

Sapindáceas.

Leguminosas....

Urticáceas .

Urticáceas

i.abiadas.

Liliáceas.

Liliáceas.

Magnoliáceas

Magnoliáceas ......

Verbenáceas

Compuestas
Ignamé ó Iñamé. Yamé.

Corrimiento.

Cuachalalá ó Cuachalalate. Cuauchalalá. Guauchalalate.

Sirvnda vrueta. Tlilzápotl. Zapote negro ó prieto.

Ebano.

Palo santo.

Cardencha. Cardo de cardadores. Cardo de cardar.

Cihapulizctli. Pirimu.

Alampepe.

Barbudilla. Contrahierba. Tuzpatli. Xkanbahau.

Barbudilla. Contrahierba. Tuzpatli.

Torongil.

Palma del desierto.

Palmita.

Corteza de Chichaca, de Chilillo, de Drimis ó de Winter.

Corteza de Chachaca. Chilillo.

Duranta.

Flor del inuerto.
Echeverria coccinea DG

Echeverria pulverulenta Nutt..........

Echinocactus cornigerus DC.

Echinops sphæorocephala L

Echites cimicida Moc. et Sessé.

Echites, sp?

Ehretia elliptica DC.

Eichornia azurea Kunth.

Elæis melanococca Gærtn.....

Elaphrium copalliferum DC

Elephantopus spicatus B. Juss..

Elytraria calicosa Bucks.

Elytraria tridentata Vahl

Encelia mexicana Mart.

Entada polystachya DC

Entada scandens? Benth.

Enterolobium cyclorarpum Griseb.

Enterolobium, sp?.

Ephedra antisy philitir...................

Ephedra trifurca Tors.

Ephedra, sp?.

Epicampes macroura Benth

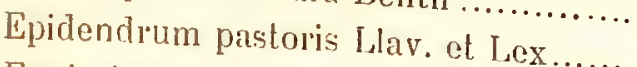

Equisetum mexicanum Milde.............

Equisetum ramosis.imum Desf.............

Equisetum robustum A. Brown.

Eragrostis reptans Nees..

Erblichia odorata? Seen..........

Erigeron affinis DC.

\section{Crasuláceas \\ Grasuláceas........... \\ Cácteas. \\ Compuestas... \\ Apocináceas. \\ Apocináceas........ \\ Borragineas \\ Pontederiáccas. \\ Palmas. \\ Burseráceas}

Compuestas

Acantáceas.

Acantáceas.

Compuestas........

Leguminosas.......

Leguminosas.. .....

Leguminosas.......

Leguminosas.

Gnetáceas.

Gnetáceas

Gnetáceas

Gramíneas.

Orquideas

Equisetáceas .........

Equisetáceas .........

Equiseláceas .......

Gramíneas

T'urneráceas.

Compuestas
Oreja de burro.

Orejita.

Biznaga de clinitos.

Planta de la abeja.

Actimpatli.

Raíz de la cucaracha. Raíz de cuculillo.

Anagua.

Ciamalote. Pico de pato.

Cíoco de aceite. Coquito de aceite.

Arbol del copal. Copal. Copaliquáhuill. Ngedri. Ngidri. Suchicopal.

Lengua de perro.

Anisillo.

Hierba del Sagrado Ciorazón de Jesús.

Acahual.

Bejuco de amole. Bejuco de mondongo

Haba. Haba de la costa. Tacalote.

Hueinacaztli. Parota. Piche.

Nacaxtle. Nacaztle.

Ganastilla. Tepopote.

Tepopote.

Tlalteczolli.

Ráź de zacatón. Zacatón.

Amatzauhtli. Tzacutli.

Cañuela.

Cañuela.

Cañuela. Cola de caballo.

Pan caliente.

Sanjuanero.

Chalchuán. Pelitre. Peritre del país. 
Eriobotrya japonica Lindl

Eriodendron æsculifolium H. B. K...

Eriodendron occidentale Tr. et Pl.

Eritrichium, sı?

Erodium cicutarium Leman...............

Eruca sativa Lamk.

Eryngium beecheyanum Hook. et Arn...

Eryngium comosum Delar.

Eryngium fœtidum L

Eryngium gracile Delar...

Eryngium nasturtiifolium Juss.

Eryngium phyteumatos Delar..........................

Eryngium serratum Cav.

Eryngium, sp?.

Erythræa stricta Schied.

Erythræa tetramera Schiede

Erythrina corallodendron L

Erythrina coralloides DC....

Erythrina, sp?.....

Erythrina, sp?

Erythrina, sp?

Erythroxylon ellipticum Ramz............

Erythroxylon macrophyllum Cav.........

Eschschollzia californica Clıam.

Escobedia linearis Schl...

Escobedia scabrifolia Ruiz et Pavón.....

Eucalyptus globulus Labill.................

Euchlæna luxurians Dur. et Asch........

Eugenia guayaquilensis DG................

Eugenia pseudo-caryopliyllus DC........

Eugenia xalapensis DG....................

Eugenia, sp?

Eupatorium collinum DG...................

Eupatorium conyzoides Vahl..............

Eupatorium deltoideum Jacq..............

Eupatorium glabratum H. B. K..........

Eupatorium macrophyllum L.............

Eupatorium populifolium H. B. K........

Eupatorium sordidum Less....

Eupatorium, sp?.

Eupatorium, sp?.

Eupatorium, sp?...

Eupatorium, sp?

Eupatorium, sp?...

Eupatorium, su?

Eupatorium, sp?...

Euphorbia calyculata H. B. K.............

Euphorbia campestris Cham. et Schll.....

Euphorbia fulgens Karw...................

Euphorbia heterophylla L.................

Euphorbia lathyris L......................

Euphorbia maculata L.....................

Euphorbia mendezii Boiss.
Rosáceas

Malváceas.

Malváceas.

Borragineas.

Geraniáceas

Crucíf ras...

Umbelíleras

Uinbelíferas....

Umbelíferas.

Unubeliferas

Umbelíferas

Umbelíferas

Umbelíferas...

Umbelíferas.

Gencianeas

Gencianeas

Leguminosas........

Leguminosas.......

Leguminosas.......

Leguminosas...

Leguminosas.......

Limeas.

Lineas..

Papaveráceas......

Escrofularíneas.....

Escrofularíneas.....

Mirtáceas...

Graninineas..........

Mirtáceas.

Mirtáceas

Mirláceas.

Mirtáceus.

Compuestas

Conpuestas

Compuestas

Compuestas.....

Compuestas .......

Compuestas ........

Compuestas ........

Conipuestas .........

Compuestas ........

Compuestas .........

Compuestas ........

Compuestas .........

Compu'stas .........

Euforbiáceas ........

Euforbiáceas ........

Eufurbiáceas ........

Euforbiáceas ........

Euforbiáceas ........

Euforbiáceas .......

Euforbiáceas ........
Níspero.

Ceiba ó Ceibo. Pochote. Pochotl. Yaxché.

Arbol del algodón. Ceiba ó Ceibo. Pochote. Pochote de Yucatán. Pochotl. Yaxché.

Cabeza de negro.

Aguja del pastor. Alfilerillo. Peine de bruja.

Mostacilla.

Hierba del sapo. Ocopiaxtli. ?

Hierba del sapo. Raíz del sapo.

Hoitzcololli. Tlipolon.

Hierba del sapo.

Perejilillo.

Hierba del sapo.

Ambástaqua. Checámeti. Hierba del sapo.

Clichicahoatzón.

Cachalohuai. Canchalagua.

Canchalagua. Tlanchalagua.

Culorín. Zumpantle.

Colorin. Peonía de Jalisco. Purénchequa. Puregue. Tzo m pantli. Tzinacanquáhuitl. Zompantle.

Chontal.

Madre brava.

Maidre de cacao.

Zapotillo.

Cuca. Sapotillo.

Amapola amarilliı de la Baja Cúlifornia.

Acaxóchitl. Azafrancillo.

Azafrancilio de México.

Eucalipto. Eucaliptus.

Acecé. Maíz primitivo. Teoxintli.

Escobillo.

Pinienta de México.

Reyán.

Pié de cabra.

Hierba del ángel. Yoloxóchitl.

Mlanzanilla de Jalisco.

Xolochíchitl. Yolochichic.

Mixtlácotl.

Chioplé.

Chioplé.

Xiquite.

Arbol María. Rosa Mirúa. Rosa panal.

Hierba del ángel.

Ilierba buena.

Itavio.

Jarilla.

Tacote.

Tlacamecaxóchitl.

Chupire. Ghupireni. Chupiri. Tencuanete.

Hierba del coyote.

Gorona de Ciristo. Euforbia brillante.

Casalina. Catalina. Nela.

Piñoncillo.

Celedonia. Celidonia ó Celidueña. Hierba de la golondrina. Tlalınenıejan.

Hierba de la golondrina. 
Euphorbia prostrata Ait.

Euphorbia pulcherrima Willd

Euphorbia radians Benth.

Euphorbia splendens Boj...

Euphorbia thymifolia Burm.

Euphorbia, sp?.

Euphorbia, sp?

Euphorbia, sp?

Euphorbia, sp?

Exostemma caribæum Rœm. et Schultz.

Exostemma, sp?.

Exostemma, sp?

Eysenhardlia amorphoides H. B. K.....

Eysenhardtia orthocarpa Wats.
Euforbiáceas ...

Euforbiáceas

Euforbiáceas ..

Eufolbiáceas

Euforbiáceas .

Euforbiáceas

Euforbiáceas ....

Euforbiáceas

Euforbiáceas

Rubiáceas

Rubiáceas

Rubiáceas

Legurninosas

Leguminosas.
Hierba de la golondrina.

Catalina. Flor de nochebuena. Flor de pascua. Paño de Holanda.

Colecitas.

Corona de Cristo.

Celedonia. Hierba de la golondrina. Tlalmemejan.

Espino de Judea.

Papelillo.

Xanabmucuy.

Xococxíhuitl?

Falsa quina.

Hueso.

Quina.

Coate ó Coatl. Guate. Leño nefrítico. Palo dulce. Tillily. Tlapahoaxtli. Urza.

Palo dulce.
Faramea odoratissima DC.

Ficus bonplandiana Miq.

Ficus carica L.

Ficus complicala H. B. K.

Ficus elastica Roxb

Ficus nymphæifolia I

Ficus padifulia H. B. K.

Ficus petiolaris H. B. K

Ficus religiosa L.

Ficus sycomorus L...

Ficus tecolutensis Miq.

Ficus, sp?.

Ficus, sp?.

Ficus, sp?

Ficus, sp?

Ficus, sp?.

Ficus, su?

Ficus, sp?

Ficus, sp?

Ficus, sp?

Flaveria angustifolia Pers

Flaveria contrayerba Per.

Fœniculum vulgare Gærtn

Fouquieria formosa H. B.

Fouquieria spinosa "Torl".

Fouquieria splendens Engelm.

Fourcroya, sp?

Fourcroya, sp?.

Fragaria mexicana Schl

Frankenia gran difolia Gilam. et Schl....

\section{Urticáceas}

Urticáceas

Urticáceas

Ulticáceas

Ulticáceas

Urticáceas

Urticáceas

Urticáceas

Urticáceas

Urticáceas

Urticáceas

Ulticáceas

Ullicáceas

Urticáceas

Compuestas.

Compuestas.

Umbeliferas.

Tamariscineas.......

Tamariscíneas....

'Tamariscineas.

Amarilideas...

Amarilideas.

Rosáceas

Frankeniáceas
Hueso.

Amate blanco.

Higuera. Higuera de higo.

Amesquitea. Samatilo.

Arbol del hule.

Amacoztic. Amale. Higuera. Higuerón. Higurrote. Palo María. Resina tescalama. Tepeamatl. Tescalama. Texcalámatl.

Camichín.

Amate amarillo.

Laurel de India.

Sicomoro.

Amate prieto.

Amate.

Amatito de niontaña.

Capire.

Cuirindal.

Salate. Zalale.

Siranda.

Tepecamichín.

Tepezalate.

Ucpencamichín.

Escoba amarga. Hierbba de la barranc'a.

Contrahierba.

Hinojo.

Palo santo. Rosadillo.

Torole verde.

Ocolilla ú Ocolillo.

Amole de raíz.

Pila.

Fresa.

Hierba de las reumas. 
Fraxinus americana? L.

Fraxinus juglandifolia Lam...

Fraxinus schiedeana Cham. et Schl.

Fraxinus viridis Michx.

Fraxinus, sp?

Fraxinus, sp?

Fraxinus, sp?.

Fraxinus, sp?.

Fuchsia arborescens Sims.

Fuchsia coccinea Ait.

Fuchsia corymbiflora R. et Pav

Fuchsia fulgens DC.

Fucus vesiculosus $\mathrm{L}$

Fumaria parviflora Liam
Oleáceas. .

Oleáceas.

Oleácens

Oleáceas

Oleáceas

Oleáceas

Oleáceas.

Oleáceas.

Onagrarieas....

Onagrarieas

Onagrarieas

Onagrarieas

Algas ....

Fumariáceas
Fresino.

Fresno.

Fresno:

Fresno.

Fresno amarillo.

Fresno azul.

Fresno blanco.

Paramu.

Adelaida. Aretillo. Atexúchitl. Chorros. Don Diego de dia. Flor de arete. Fuscia.

Adelaida. Aretillo. Flor de arete. Fuscia.

Adelaida. Aretillo. Flor de arete. Fuscia.

Adelaida. Aretillo. Flor de arete. Fuscia.

Encina de mar.

Fumaria. Pajarillo.
Galinsoga parviflora Cav

Galium mexicanum H. B. K..............

Galium, sp?.

Galium, sp?.

Galphimia glandulosa Cav.................

Galphimia hirsuta Cav

Galphimia humboldtiana Bart.

Garcinia mangostana L.

G irdenia florida L

Garrya laurifolia Hartw.

Garrya oblonga Benth.

Garrya ovata Benth...

Garrya racemosa Ramz...

Gaudichaudia cynanchoides H. B. K.....

Gaultheria, sp?.

Gaura coccinea Nutt.

Gelsenium sempervirens Ait.............

Genipa caruto H. B. K....................

Gentiana calyculata Llav. et Lex..........

Gentiana spathacea H. B. K.............

Gentiana, sp?.

Gentiana, sp?.

(1)...........................

Geraniun hernanderii DC

Geranium mexicanum H. B. K...........

Geranium robertianum $[$.. ...............

Gerardia purpurea 1 ......................

Gerardia, sp?.

Gladiolus byzantinus Mill.................

Gladiolus comantinus

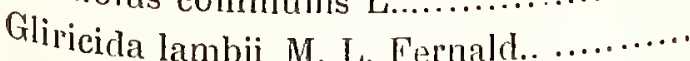

Globularia vulgaris L...................

Glycyrrhiza lepidota Nutt.

Gnaphalium berlandieri Dr.
Compuestas.

Rubiáceas.

Rubiáceas .....

Rubiáceas

Malpigiáceas.

Malpigiáceas

Malpigiáceas.

Gulíferas.

Rubiáceas

Cornáceas

Cornácens

Cornáceas

Gornáceas

Cormáceas ...........

Malpigláceas........

Ericáceas ..

Onngrarieas........

Loganiáceas........

Rubiáceas......

Gencianeas.........

Gencianeas .........

Gencianeas.........

Gencianeas.........

Geraniáceas....

Geraniáceas...

Geraniáceas.

iscrofularíneas....

Escrofularíneas....

lideas ...............

Irideas ..............

Leguminosas.......

Selagineas..

l. egrmininosas.... rompuestas.
Estrellita. Mercurial.

Amor de hortelano. Pegarropa. Presera.

Hierba de la pulga.

Ocoxóchitl.

Grano de oro. Margarita.

Ramo de oro.

Palo del muerto. Vachácata.

Mangostan.

Gardenia.

Chichiquáhuitl.

Cuahuchichi. Chichiquáhuitl.

Cuahuchichi. Chichiquáhuitl. Quauchichic.

Cuahuchichi. Cuauchichic. Chichiquáhuitl. Quauchichic.

Hierba del zorro. Xunequilt ó Xunequiltzio.

Ajocopaque. Axocopaque.

I inda tarde.

Gelsemio.

Jagua. Jagua blanca.

Flor de Santo Domingo. Flor de Nieve.

Flor de hielo.

Genciana.

Tlanoquilixihuitl.

Alquimila del país.

Alquimila. Alquimila del país. Pata de león.

Geranio.

Quanenepile.

Cuanenepile.

Pluma.

Palma gladiola. Pluma.

Cacahuananchi. ?

Globularia.

Raíz del desierto.

Cortolobo del país. Sanguinaria. 
Gnaphalium canescens DG....

Gnaphalium conoideum H. B. K

Gnaphalium oxyphyllum DC.

Gnaphalium sphacelatum H. B. K........

Gnaphalium, sp?.

Gomphrena decumbens Jacq....

Gomphrena globosa L

Gomphrena nilida Rothr.

Gonolobus nummulinius Hemsl...

Gonolobus pedunr.ulatus Hemsl....

Gonolobus uniflorıs H. B. K. .

Gonolobus, sp?

Gonolobus, sp?

Gossypium arboreum L

Gossypium barbadense L

Gossypium herbaceum L

Gossypium religiosum L

Govenia Tiliacea Lindi

Govenia superba Lind

Grindelia inuloides Willd

Gronovia scandens I

Guadua amplexifolia Presl....

Guaiacum coulteri A. Gr.

Guaiacum officinalis $L$

Guaiacum sanctum $\mathrm{L}$

Guaiacum verticale Ort

Guaiacum, sp?

Guarea trichilioides

Guarea, sp?

Guazuma polybotrya Cav

Guazuma tomentosa H. B. K

Guazuma ulmifolia Lamk.

Guilleminea illecebroides H. B. K

Gymnosperma inultiflorum DC.

Gymnosperna corymbosum Di:

Gymnosperma, sp?

Gynandropsis speciosa DC

Gynerium saccharoides Humb. el. Bonpl.

Gyrocarpus jacquini Roxb.
Compuestas

Compuestas.

Compuestas.

Compuestas

Ciompuestas

Ainarantáceas .

Amarantáceas .

Amarantáceas

Asclepiadeas ...

Asclepiadeas.

Asclepiadeas .

Asclepiadeas .

Asclepiadeas

Malváceas.

Malváceas

Malváceas

Malváceas

Orquídeas

Orquídeas

Compuestas.

Loaseas

Gramíneas.

Zigofileas....

Zigofileas.

Zigofileas

Zigofileas....

Zigofileas

Meliáceas....

Meliáceas.

Esterculiáceas..........

Esterculiáceas...

Esterculiáceas....

Amarantáceas

Compuestas

Compuesta ........

Compuestas ..........

Caparideas .........

Gramíneas.

Combretáceas
Gordolobo. Papaconi. Tlacochichic. Tzopotonic.

Gordolobo del país.

Manzanilla del río.

Canelillo. Gordolobo.

Verbasco.

Amor seco. Cabezona. Madroño del campo ó raslrcro. Siempreviva silvestre.

Amor seco. Chacmol. Flor de San Francisco. Hierlua del cáncer. Inmortal. Madroño. Permanente. Sempiterni.

Cordón de San Francisco.

Talayote.

Talayote. Tlalayote ó Tlislayotl.

Rosa verde.

Pancncolote.

Tecuampatle ó Tecuampatli.

Algodón. Algodón de árlıol. Algodonero arbóreo.

Algodón común. Xurata.

Algoclón. Algodón coyuche. Algodonero herbáceo. Algodón.

Azucena del monte. Icohueyo. Itztactepetzacuxóchill. Petzanxóchitl.

Azucena amarilla. Coztic\%acatzacuxóchitl.

Palancapatli de Puebla.

Chichicaxlli.

Otate.

Guayacán.

Guayacán. Hoayacán. Palo de rosiı.

Guayacán. Pallo santo.

Arbol santo. Gunyacán. Hoaxacán. Matlalquáhuitl. Palo santo.

Quiebra-hacha.

Cedrillo cimarrón.

Trementino.

Cuahulote. Guácima. Guázima ó Guázuma.

Guácima ó Guácimo. Guazima ó Guazuma. Pixoy.

Guayacán. Guázima. Vácima.

Rosa de Castilla cimarrona.

Hierba de Tata Lencho. Tatalencho.

Hierba de Tata-Lenclio.

Cola de zorra.

Volantín.

Caña brava.

Palu hediondo. Quillacoctli ó Quitlacotli.

\section{$\mathrm{H}$}

Hematoxylon boreale Wats

Hrematoxylon campectriinum I

Hampea integerrina Sch .

Hechtia ghiesbreghtii Lemaine...........

Leguminosas....... Brasil.

Leguminosas............

Mal váceas.......... Brasil.

Jechtia glomerata zuec.

Bromeliáces....... Majahua.

Bromeliáceas ...... Guapilla.

Bromeliáceas ...... Guipilla. 
Hedeoma piperita Bentl

Hedera helix L

Hedwigia balsamifera ? Sw

Helenium autumnale L..

Helenium integrifolium Benth. et Hook..

Helenium mexicanum H. B. K

Helenium ooclinium A. Gr...

Helenium quadridentatum Labill.........

Heleocharis geniculata Rœm....

Helianthemum glomeratum Lag....

Helianthus annuus L...

Helianthus cornifolius H. B. K

Helianthus ovatus Lelım

Helianthus, sp?.

Helianthus, sp?

Helianthus, sp?.

Helichrysum orientale Gærtn.............

Heliconia bihai DG

Heliconia caribea Lamk ..................

Helicteres guazumæfolia H. B. K...........

Helietla parvifolia Benth..................

Heliocarpus arborescens Seem ............

Heliocarpus americanus L

Heliopsis buplıthalmoides Dun

Heliotropium curassavicum L.............

Heliotropium corymbosum ? K. et Pav...

Heliotropium indicum L

Heliotropium limbatum Benth.............

Heliotropium parviflorum L...............

Heliotropium peruvianum $\mathrm{L}$

Hemerocallis flava $\mathrm{L}$

Hernandia sonora $\mathrm{L}$

Heteranthera graminea Vahl...

Heteroloma lobelioides Zucc..............

Hibiscus abelmoschus $\mathrm{L}$................

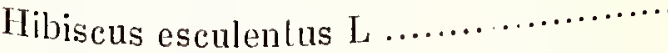

Hibiscus manihot L

Hibiscus mutabilis

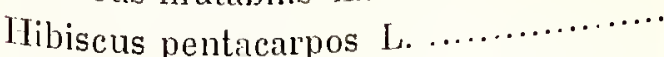

Hibiscus rosa-sinensis $\mathrm{L}$.................

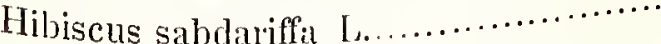

Hibiscus tiliaceus $\mathrm{L}$.......................

Hibiscus tubifforus $\mathrm{DG}_{1} \ldots \ldots \ldots \ldots \ldots . . . . . . .$.

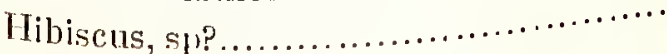

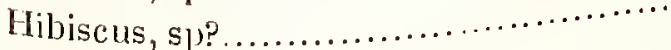

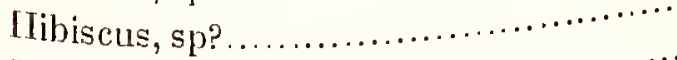

Hieracium mexicanum Less..............

Hieracium premorsiforme Scliz. Bip.....

Hippeastrum equestre Herb

Hippeastrum, s|?
Labiadas.

Araliáceas..

Anacardiáceas .....

Compuestas .

Compuestas.

Compuestas.

Compuestas.

Compuestas.

Ciperáceas..

Cistineas.

Compueslas.

Compuestas.

Compuestas.

Compuestas

Compuestas

Compuestas

Compuestas

Musáceas.

Musáceas.

Esterculiáceas....

Rutáceas

Tiliáceas.

Tiliáceas

Compuestas

Borragineas...

Borragineas

Borragíneas

Borragín eas....

Borragineas....

Borragíneas

Liliáceas.

Lauríneas....

Pontederiáceas....

Compuestas

Compuestiss

Lobeliáceas...

Malváceas

Malváceas

Malváceas

Malváceas

Malváceas

Malváceas

Malváceas

Malváceas

Malváceas

Malváceas

Malvíceas

Malváceas

Compuestas

Compueslas ........

Amarilídeas........

Amarilídeas........
Hierba buena. Piperita de México. Tabaquillo oloroso.

Hiedra exlranjera. Yedra exlranjera.

Incienso.

Chapuz. Manzanilla de Yucatán.

Chapuz.

Arnica del país. Cabezona. Chapuz. Ecxoxóchitl. Hierba de las ánimas. Rosilla. Rosilla de Puebla.

Hierba cabezona. Hierba de la víbora.

Florecilla. Manzanilla.

Junco.

Juanila.

Acahual. Andani. Chimálatl ó Cliimalitl. Gigantón. Lampote. Maíz de Texas. Mirasol.

Lampote.

Mirasol anaranjado.

Acaute.

Tlacopatli.

TlalpopolotI.

Sempiterna.

Camelina. Plátano de flır.

Hoja blanca.

Majaguiilla. Saratrapo.

Barreta.

Jolocín.

Copal de Veracruz. Cuahualahua. Cuaulaláhuac blanco. Cuaulote. Jolocín. Jolocín blanco. Jonote. Majahua.

Hierba anıarilla.

Cola de mico. Hediondilla. Heliotropo cimarrón. Quiebraplatos. Rabo de mico.

Heliotropo del cerro.

Alacrancillo. Rabo de mico.

Heliotropo cimarrón.

Neman. Rabo de mico ó de Yucitán.

Heliotropo.

Azucena amarilla ó azafrimada.

Mago.

Sargazo.

Acalıuatl. Arnica del país. Cuauteleco. Falsa árnica.

Alcanfor de mata. Falsa áruica.

Cardenal.

Abelmosco. Algalia.

Chimbombó. Quimgombo.

Pajiza. Viudas.

Amistad. Amor de estos tiempos. Amor al uso. Cortejo.

Atlalzopillin ó Atlatzompillin.

Bis. Obelisco. Rosa china. Súchil. Tulipán.

Flor de Jamaica. Jamaica.

Majagua. Masahua.

Monacillo del rín.

Ambar.

Encantadora.

Monacillo blanco.

Lechuguilla.

Lechuguilla.

Azucena encarnada ó roja.

Azucena matizarlir. 
Hippocratea obcordala Lamk

Hippocratea ovata Lamk.

Hippocratea, sp?.

Hippomane mancinella $L$.

Hirtella acayacensis ? DC

Hirtella dodecandra DC

Hoffmanseggia stricta Benth.

Hordeum distichon L

Hordeum hexastichon L....

Hordeum vulgare $\mathrm{L}$.

Hosackia, sp?

Houstonia longiflora A. Gr.

Houstonia, sp?

Houtthuyna californica Benth et Hook..

Hoya carnosa R. Br

Humulus lupulus $\mathrm{L}$.

Hunnemannia fumariæf „li Sweet

Hura crepitans L

Hyacinthus orientalis L

Hydrangea hortensia DC.

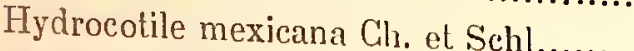

Hydrocotile ranunculoides L.

Hydrocotile umbellata L.

Hydrocotile, sp?.

Hydrolea spinosa L.

Hymenæa candolleana H. B. K

Hymenæa courbaril L.

Hy menæa, sp?

Hymenocallis rotata Herb.

Hyoscyamus niger L

Hypericum denticulatum H. B. K

Hypericum fasligiatum H. B. K

Hypericum formosum $H$. $\mathrm{K}$

Hyptis spicata Poit.

Hyptis suaveolens Prif

Hyptis verticillata Jacq.
Hipncrateáceas ..... Coanabichi.

Hipocrateáceas ..... Coanabichi.

Hipocrateáceas ..... Bejuco colorado.

Euforbiáceas ....... Manzanillo.

Rosácear ........... Icaquillo.

Rosáceas .......... Pío. Uspio.

Leguminosas....... Gamote del ratón.

Gramíneas.......... Cebada.

Gramíneas.......... Cebada.

Graníneas......... Cebada.

Leguminosas....... Coachipilín.

Rubiáceas.......... Flor de San Juan.

Rubiáceas .......... Tlacoxóclhill.

Piperáceas.......... Raíz del manso.

Asclepiadeas....... Flor de cera.

Urticáceas.......... Hombrecillo. Lúpulo.

Papaveráceas...... Amapola amarilla de la Baja California.

Euforbiáceas ....... Arbol del diablo. Haba de San Ignacio. Haba de indio. Habilla. Pepita de San Ignacio. Quauhtlatlatzin.

Liliáceas............ Jacinto.

Saxifragáceas....... Hortensia.

Umbelíferas........ Sombrerillo de agua.

Umbelíferas........ Omblign de Venus.

Umbelíferas........ Ombligo de Venus.

Umbelíferas ... ..... Sombrerillo. Sombrerito de agua.

Hidrofiláceas....... Barba de juil. Tabaquillo.

Leguminosas....... Cuapinol ó Cuapinole.

Leguminosas....... Guapinole ó Cuapinoli.

Leguminosas....... Quiebra-hacha.

Amarilideas........ Barbas de gato. Gebolla albarrana. Escila del país. Estrella de San Nicolás. Flor de estrella. Lirio blanco.

Solanáceas

Hipericíneas..... Beleño negro. Hierba de la punzada.

Hipericineas....... Hipericón.

Hipericíneas....... Hipericón.

Labiadns........ Hipericón.

Labiadas.......... Cía.

Labiara......... Confitura.

Hierba Martín.

Iberis intermedia Guer.

Iberis umbellata

llex dugesii M. L. Fernald................

Ilex, sp?.

Illicium anisatum $\mathrm{L}$

Impatiens balsamina 1

Indigofera anil L.

Indigofera argentea L

Indigofilat mucronatal Spreng.

Crucifuras.

Cirucíferas........ Charraspique.

Ilicineas.......... Charraspique.

Ilicineas........... Naranjiilo.

Magnoliácen....... Acebo.

Geraniáceas ...... Raíz de Abadiana.

Leguninosas..... Belén ó Belenes. Chinos. Miramelindos.

L_eguminosas....... Añil.

Leguminosas....... Añil. 
Indigofera linctorea L....

Inga foetida Willd.

Inga jinicuil Sclil.

Inga spuria IIumb. el Bonpl.

Inga unguis-cati ? Willd.

Inga, sp?

Inga, sp?

Inga, sp?

Ingn, sp?.

Inga, sp?.....

Inga, sp??

Inga, sp?

Inga, sp?

Inga, sp?.

Ingenlousia triloba Moc....

Iochroma tubulosum Benth...

Ionidium angustifolium H. B. K

Ionidium polygalefolium Vent.....

Iostephane heteroplyylla Benth.....................

Ipomail arborescens Don.

Iponnoa batatis Lim

Ipomoa bona-nox L.

Iponıca capillacea Don....

Ipomoa coccinea $L$....

Ipomosa emetica Choisy

Ipomøea glabratia A. Gl.

Ipomoa hederifolia L...

Ipomoa jalapa Pursh...

Ipomœa longepedunculata Hems ..

Inomøal murucoides Rœm. et Schult....

Ipomœa pes-capre Sweet

Ipomœa purga Hayne.

I pomœa purpurea Lan

Ipomœa quamoclit L....

Iponca sidrefolia Choi

Ipomœa simulans Hanbury...

Ipomca stans Cav...

Ipomœa versicolor Meissn

Ipomca, sp?.

Ipomoea, sp?.

Ipomoca, sp?.

Ipomce, sp?

Ipomcea, sp?

Ipomca, sp?.

Iresine sp?............................

Iresine, sp?

Iris germanica.
Leguminosas....

Leguminosas.

Leguminosas........

Leguminosas ........

Leguminosas.

Leguminosas ....

Leguminosas

Leguminosas

Leguminosas

Leguminosas

Leguminosas

Leguminosas

Leguminosas

Leguminosas

Malváceas

Solanáceas

Violarieas.

Violarieas.

Compuestas.

Convolvuláceas....

Convolvuláceas....

Convolvuláceas....

Convolvuláceas....

Convolvuláceas.....

Convolvuláceas.....

Convolvuláceas.....

Convolvuláceas.....

Convolvuláceas....

Convolvuláceas....

Convolvuláceas....

Convolvuláceas....

Convolvuláceas....

Convolvuláceas....

Convolvuláceas....

Convolvuláceas....

Convolvuláceas....

Convolvuláceas....

Convolvuláceas....

Convolvuláceas....

Convolvuláceas....

Convolvuláceas....

Convolvuláceas....

Convolvuláceas....

Convolvuláceas....

Convolvuláceas....

A.marantáceas......

Amarantáceas......

Irideas
Añil blanco. Xiuhquílitl.

Coyuacate.

Guajuinicuil. Jinicuil.

Bitze. Guatope.

Pintzan.

Algodoncillo.

Caracolillo.

Chelele.

Hulándini.

Mezquite.

Mezquite amarillo.

Mezquite colorado.

Nisquicopalli.

Quauhxonequillin.

Encantadora.

Yocroma.

Hierba de San Nicolás.

Ipecacuana de México.

Quauhtolotlanenci. Riúz del manso. Tlacopatli.

Casahuate blanco. Ciazahuate blanco.

Camote ó Camotic.

Nicua.

Campanilla silvestre. Tlalcocotl.

Cundeamor. Melindre de monja. Yedra colorada.

Temécatl.

Palo santo.

Chiqueo de monjas.

Michoacán. Puscua. Tacuache. Tlallantlacacuitlapilli.

Manto de la Vírgen. Quiebra plato.

Arbol del muerto. Casahuate prielo. Cazahuate prieto. Micaquáhuitl. Pa!o bobo. Palo del muerto. Palo santo.

Pata de vaca.

Jalapa. Jalapa limoncillo. Limoncillo. Purga. Tolonpatl.

Aurora. Campanilla. Manto de la Virgen. Mecapatli. Metlancasis. Quiebra plato. Xhail. Yedra morada.

Hiedra roja.

Ololiuhqui. Pascua.

Jalapa de Tampico.

Espanta-lobos ó Espanta-vaqueros. Limpiatunas. Pegajosa.

Tanibata. Tlaxcapan. Tumba-vaqueros.

Bandera española. Palmira.

Atzoyátic. Campanilla silvestre.

Chupiqua.

Oreja de liebre.

Pusqua.

Tetoyexill. Tetoyoxitl.

Tlaxcala.

Purga de las ánimas.

Pié de paloma.

Tlitlancuaya. Tlatlincuaye.

Lirio. Omixóchitl. 
Krameria secundiflora DC

Krameria, sp?.

Krynitzkia floribunda A. Gr
Poligaleas

Poligaleas

Borragíneas.
Crameria.

Encinilla.

Cabeza de negro.
Lactuca sativa $\mathrm{L}$

Lactuca sativa L. var.

Laelia antumnalis Lind

Lelia grandiflora Lindl.

Lælia grandifolia Lindı...

Læelia majalis Lindl...

Lagascen mociniana DC...

Lagenaria vulgaris Ser

Lagerstromia indica L

Lantana camira L.

Lantana hirsuta Mart. et Gal.

Lantana liorrida H. B. K.

Lantima, sp?

Larrea mexicana Moric

Latania horbonica Willd....

Lathyrus odoratus I

Lathyrus, sp?

Laurus nobilis L.........

Lemna gibba L.

Lemna minor L

Lennoa corulea Fourn....

Lennoa madreporoides Llav. et Lex......

Lens esculenta Monch...

Lepidium latifolium L...

Lepidium virginicum L

Leucrena esculenta Benth...

Leucena, sp?

Licania arborea Seem....................

Ligustrum japonicum Thunb.

Lilium auratum Lindl.

Lilium candichum L

Lilium tigrinum Ker...

Limnanthem Ker.......................

Linum hemum humboldtianum Griseb.

Linum, sp?

Lippia berlandieri Schane

Lippia callicarpæfolia I. B. K

Lippia citriodora H. B. K

Lippia dulcis Trevir.

Lippia geminata H. B. K

Lippia graveolens H. B. K...

Lippia graveolens H. B. K................

Lippia origanoides

lippia palmeri spionta Rose......
Compuestiss........ Lechuga.

Compuestas........ Lechuga romanita.

Orquídeas ....... Flor de los santos. Lirio de San Francisco. Lirio parásito.

Orquídeas.......... Hierba de Corpus. Itzamaqua. Itzmaqua.

Orquídeas.......... Flor de Corpus.

Orquídeas.......... Itzmaqua.

Compnestas........ Casanaca. Zazanaca.

Cucurbitáceas ..... Acocote. Alacate. Bule. Calabazale.

Litrarieas........... Astronómica. Atmosférica.

Alantana. Lampana. Lantana. Matizadilla. Siete colores.

Sonoba. Tozisquint. Tozizquiuh.

Verbenáceas Cinco negritos. Flor de San Cayetano.

Alfombrilla hedionda.

Mejorana.

Falsa alcaparra. Gobernadora de México. Guamis.

Verbenáceas

Zigofileas

Palmas.

Leguminosas........

Leguminosas........

Lauríneas......

Lemnáceas

Lemnáceas.

Lennoáceas...

Lennoáceas.....

Leguminosas.......

Crucíferas....

Crucíferas.

Leguminosas........

Legruminosas.......

Rosáceas ...........

Oleáccas....

Liliáceas

Liliáceas.

Liliáceas.

Gencianeas........

Lineas....

Lineas

Verbenáceas........

Verbenáceas........

Verbenáceas........

Verbentíceas........

Verbenáceas.........

Verbenáceas.........

Verbenáceas........

Terbenácess........

Verbenáceas.........
Huano. Palma abanico.

Chícharo de olor.

Frijolillo.

Laurel de poeta.

Lentejilla.

Lentejilla de agua.

Angiolote.

Flor de San Andrés. Tlalchiólotl.

Lenteja.

Coclearia del país.

Coclearia del país. Comida de pajarito. Lentejilla. Lepídio.

Guaje. Hoaxin. Huaje.

Tepeguaje.

Cacahoanantzin. Cacahuananche. Caña dulce.

Troeno. Trueno.

Lirio dorado.

Azucena blanca.

Lirio del Japón.

Niufa. Hoja de pescarlo. Ilojis de pescado. Pan de manteca.

Linaza. Lino.

Lino rojo.

Orégano.

Salvia poblana. Salvia real de Puebla.

Cedrón. Hierba Luisa. Hierba de la princesa.

Hierba dulce. Neuctixihuitl. Orozúz del país

Hierba buena cimarrona. Te del país.

Hierba dulce. Neuctixíhuitl. Orozúz del país.

Caneiilla.

Orégano del país. Tehmacín.

Orégano. 
Lippia purpurea Jacq.

Lippia umbellata Cav.

Lippia, sp?

Lippia, $s p$ ?

Lippia, sp?.

Liquidambar styraciflua L.

Lithospermum, sp?

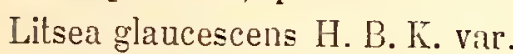

Lobelia cardinalis L....

Lobelia fenestralis Gav

Lobelia fulgens Willd.

Lobelia laxiflora H. B. K. var

Lobelia tupa I

Lobelia, sp?.

Lobelia, sp?

Lœselia cœrulea Don.

Loselia coccinea Don.

Joeselia, sp?

Lolium perenne L.

Lonchocarpus seriteus H. B. K..

Lonchocarpus, sp?.

Lonicera caprifolium L

Lonicera sempervirens L

Lopezia mexicana Jacq.

Loranthus americanus Jacq

Loranthus calyculatus DG.

Loranthus mexicanus Presl

Loranthus venetus H. B. K.

Loranthus, sp?.

Lucuma capiri A. DC

Lucuma manmosa Gærtn. fil

Lucuma salicifolia H. B. K

Lucuma sphærocarpa A. DC

Lucuma?.

Lucuma, sp?

Luffa cylindrica Roem.

Luffa operculata Cogn

Luhea platypetala A. Pich..........

Lupinus elegans H. B. K....

Lychnis chalcedonica I. ...

Lycoperdon bovista L. .

Lycoperdon?.

Lycopersicum cerasiforme Dun

Lycopersicum esculentum Mill.

Lycopersicum pyriforme Dum.

Lyeoprodienn clavatum L....

Lychinis coronitlia Lenn....
Verbenáceas......... Orégano.

Verbenáceas........ Salvia de México. Salvia poblana.

Verbenáceas........ Mastranzo.

Verbenáceas......... Salvia real.

Verbenáceas........ Te.

Hamamelídeas..... Bálsamo copalme. Copalme. Corteza de liquidámbar. Estoraque. Liquidámbar. Maripenda. Ocoxotl. Xochiocotzoquáhuitl. Xochiocótzotl.

Borragíneas........ Acacóyotl. Hierba de las perlas.

Laủríneas........... Laurel. Sufricago.

Lobeliáceas ........ Ciurdenal de maceta. Lobelia.

Lobeliáceas........ Cola de zorra. Gusanillo. Lobelia morada. Talclote.

Lobeliáceas........ Cardenal de la laguna. Lobelia. Pajaritos del Norte.

Lobeliáceas........ Acaxóchitl. Aretitos. Ciúralo todo. Chilpantlozolli. Chilpanxóchitl. Hoitzitzilteutli. Jarritos. Lobelia. Panxócliill. Pipiloxóchitl. Toxcuitlapilxóchitl. Xochipipile.

Lobeliáceas........ Zal’cillos.

Lobeliáceas ......... Cúralo todo.

Lobeliáceas ......... Jara.

Polemoniáceus...... Banderilla. Jarritos.

Polemoniáceas..... Guachile. Chuparrosa. Espinosilla. Hierba de la virgen. Huicicile. Huichichile. Iuitzitziltzin. ILuitzitzilxóchill. Mirto silvestre. Quachichil.

Polemoniáceas..... Hinseseli.

Gramíneast......... Pasto inglés. Rai grass.

Leguminosas....... Gusano prieto.

Leguminosas....... Gusano amarillo.

Caprifoliáceas...... Madreselva.

Caprifoliáceas...... Madreselva.

Onagrarieas........ Mitlatzíhuitl. Perlilla. Zancudo.

Lorantáceas........ Injerto. Xkeu.

Lorantáceas........ Liga. Mal de ojo. Malojo. Muérdago. Quauhtzictli. Quaulzi-

Lorantáceas. Hi. Visco. Visco cuercino.

Lorantáceas...... Muérdago. Quauhchitle.

Cabellera.

Sapotáce

Sapotáceas........ Cápiri. Tempixque.

Atzapotlquáhuitl. Mamey. Sapote colorado. Tezontzápotl. Zapote mamey.

Sapotáceas

Sapotáceas

Sapotáceas

Sapotáceas

Cucurbitáceas.................

Gucurbitáceas ......

Tiliáceas. ....

Leguminosas

Ciariofileas

Hongos.

Hongos.

Solanáceas ....

Solanáceas.

Solanáceas ....

Licopodiáceas .....

Cariofileas
Huicumo. Zapote amarillo. Zapote borracho.

Comíngalo.

Huacux.

Zapote de niño.

Estropajos.

Esponjilla.

Garbancillo.

Cruz de Jerusalen.

Hongo de huevo.

Pedo del diablo.

Gitomate redondo.

Gitomate. Jitomate. Xitomame Xitómat Xitómatl. Xitotómatl. Xucúpara.

Gitomale guajito.

Licopodio.

Sangre de Cristo.
Pataxtillo. 
Lysiloma acapulcensis Benth...

Lysiloma candida Brandegee...

Lythrum alatum Pursh...

Lythrum album H. B. K

Lythrum gracile Benth...

Lythrum kennedianum II. B. K.
Leguminosas

Leguminosas.

Litrarieas.

Litrarieas.

Litrarieas

Litrarieas.
Tepeguaje. Tepehoaxin.

Palo blanco.

Hierba del cáncer.

Hierba del cáncer.

Jaralillo amarillo.

Atlanchane.
Maclura aurantiaca Nult.

Macrosiphonia brachysiphon A. Gr.

Macrosiphonia hypoleuca Miull. Arg......

Magnolia glauca Moc. et Sessé..

Magnolia grandiflora L

Magnolia, su?

Malachra palmata Moench.

Malpighia glabra L

Malpighia urens L...

Malpighia, sp?

Malpighia, sp?

Malpighia, sp?

Malpighia, sp?

Malpighia, sp?..

Malva americana $\mathrm{L}$.

Malva borealis Wall.

Malva leprosa Ort

Malva parviflora $L$

Malva rotundifolia I

Malva scoparia L'Herit.

Malva, sp?

Malva, sp?

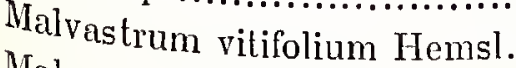

Malvaviscus arboreus Cav.

Malvaviscus pentacarpus DC.

Mammea americana

Mamillaria coronaria Haw

Mamillaria pusilla DG.

Mamillaria sphærica Dietrick.

Mamillaria, sp?

Mangifera indica L.

Manilıot resculifolia Pohl....

Manihot aipi Poln...

Manilıot foticla Pohl....

Manihot utilissima, Pohl...

Maranta arundinacea L

Maranta metallica Lind

Maranta zebrina Sims.

Marrubium vulgare L.

Martynia fragrans Lindl.......
Urticáceas ........... Maclura.

Apocináceas........ Cacaloxóchitl.

Apocináceas........ Flor de San Juan. Hierba de la cucaracha.

Magnoliáceas ....... Flor del corazón.

Magnoliáceas ...... Anonilla. Flor del corazón. Magnolia. Semíramis.

Magnoliáceas ....... Corpus.

Malváceas .......... Malva.

Malpigiáceas ....... Escobillo. Nancenes.

Malpigiáceas........ Ahualtzocotl. Ahualtzocotlque. Palo bronco.

Malpigiáceas ........ Bejuco vaquero.

Malpigiáceas ........ Changungo.

Malpigiáceas ....... Escobillo colorado.

Malpigiáceas ........ Guayabillo.

Malpigiáceas ....... Laurelillo.

Malváceas .......... Malva de Yucatán.

Malváceas .......... Malva.

Malváceas .......... Malva rastrera.

Malváceas .......... Malva.

Talváceas ....... Malva. Malva de Castilla. Malva medicinal.

Malváceas ........... Huinari.

Malváceas .......... Apitzatlpatli.

Malváceas .......... Huriata erauche.

Malváceas .......... Malva.

Malváceas ........... Civil. Monacillo.

Malváceas .......... Monacillo.

Sapótáceas..........

Cácteas.............

Cácteas.

Gácteas

Cácteas...

Anacardiáceas.....

Anacardiaceas.....

Euforbiáceas ...

Euforbiáceas ........

Euforbiáceas ........

Euforbiáceas ........

Zingiberáceas......

Zingiberáceas......

Zingiberáceas.....

Labiadis..

Pedalíneas..........
Mamey. Mamey de Santo Domingo. Zapole de Santo Domingo.

Junco.

Biznaga.

Biznaga.

Biznaguita.

Mango.

Yuca cimarrona.

Iuca dulce.

Ayotequeli. Yuca cimarrona.

Huacamote. Huacamotl. Huacamotli. Pan de tierra caliente. Tapioca. Yuca. Yuca brava.

Sagú cimarrón.

Maranta.

Maranta.

Marrubio. Marrubio blanco. Vitsiqua.

Cuernito de hormiguero. Toritos. Tolonche. 
Martynia triloba Cham. et Schl.

Mathiola annua Sweet....

Matricaria chamomilla L

Matricaria pyrethroides DC

Maurandia barclayana Lindl...

Maurandia semperflorens Ort. . .

Medicago denticulata Willd.

Medicago lupulina L.

Medicano sativa L

Melampodium americanum L...............

Melampodium perfoliatum H. B. K........

Melanthera deltoidea Rich

Melastoma, sp?.

Melastoma, sp?

Melia azederach $\mathrm{L}$

Melia sempervirens Sw

Melicocca bijuga L.

Melilotus officinalis Willd....

Melilotus parviflora Desf....

Melissa officinalis L.

Melothria scabra Naud....

Mentha pulegium L.

Mentha rotundifolia L.....

Mentha silvestris L

Mentha viridis L.

Mentha, sp?......

Mentzelia hispida Willd

Mentzelia scabra H. B. K

Mentzelia strigosa H. B. K.

Mesembryanthemum crassifoliuın L......

Mesembryanthemum crystallinum L.....

Mespilus germanica L

Miconia argentea DC.

Micromeria brownei Benth....

Micromeria douglasii Benth.

Micromeria xalapensis Benth

Microsechiuı helleri Cogn....

Mikania denticulata WVilld.

Mikania gonoclada DC..

Mikania guaco Humb. et Bonpl.........

Mikania houstonis Willd.

Mikania scandens Willd.

Milla biftora Cav.

Milleria linearifolia $\mathrm{DC}$.

Nilleria quinqueflora L

Milleria, sp?.

Mimosa biuncifera Benth....

Mimosa ervendbergii A. Gr.........

Mimosa pudica L.

Mimosa, sp?.

Mimosa, sp?

Mimosa, sp?

Mimosa, sp?

Mimosa, sp?

Mimosa, sp?
Pedalíneas....

Crucíferas

Compuestas

Compuestas.

Escrofularíneas....

Escrofularíneas....

Leguminosas...

Leguminosas.

Leguininosas.

Compuestas

Compuestas

Compuestas

Melastomáceas ..

Melastomáceas .

Meliáceas

Meliáceas

Sapindáceas.

Leguminosas

Leguminosas

Labiadas.

Cucurbitáceas

Labiadas.

Labiadas

Labiadas.

Labiadas.

Labiadas.

Loaseas

Loaseas

Loaseas

Ficoideas

Ficoideas

Rosáceas

Melastomáceas

Labiadas.

Labiadas.

Labiadas.

Cucurbitáceas

Compuestas

Compuestas

Compuestas.

Compueslas

Compuestas

Liliáceas.

Compuestas.

Compuestas

Compuestas

Leguminosas.

Leguminosas.

Leguminosas.

Leguminosas.

Leguminosas.

Leguminosas

Leguminosas.

Leguminosas...........

Leguminosas
Uña del diablo.

Alhelí blanco y encarnado.

Manzanilla de Alemania. Manzanilla común.

Sayulquelite.

Cundeamor.

Cundeamor. Manguila del Niño Jesús.

Carretilla.

Carretilla.

Alfalfa.

Xcantumbul.

Ojo de perico.

Canilla de mulito. Mulito.

Catarrillo.

Teshuate.

Paraíso. Paraíso morado. Pioclıa.

Lila.

Guayo.

Trébol oloroso.

Meliloto.

Melisa. Toronjil extranjero.

Sandiita de ratón ó del campo.

Poleo.

Hierba buena. Mastranzo. Sacui.

Mastranzo.

Hierba buena. Te de olor.

Andúmaqua.

Pega-ropa amarilla. Pegajosa. Zazale. Zazalic.

Pegajosa de las paredes.

Pegajosa del suelo.

Cortina.

Gristalillo. Hierba escarchada. Rocío.

Níspero.

Cenizo. Sabano.

Hierba del espanto.

Hierba buena.

Tomillo de Jalapa.

Amolquelite. Chichicamole ó Chichicamolli.

Guaco de Jalapa.

Guaco.

Guaco. Guaco de Guatemala ó de Tabasco.

Palo guaco.

Guaco.

Estrella.

Centaura

Escobilla.

Escoba amarga.

Uña de gato.

Sierrilla.

Dormilona. Pinahuihuixtle. Quececupatli. Sensitiva. Ten vergüuenza. Vergonzosa. Xmutz.

Arí. Chaparro prieto. Gabia. Trinacancuitlaquahuitl.

Glachalcahuite.

Cuatante.

Ebano de Nuevo León.

Escoba colorada.

Espino blanco. 
Mimosa, sp?.

Mimosa, sp?.

Mimosa, sp?

Mimosa, sp?

Mimosa, sp?

Mimosa, sp?...

Mirabilis dichotoma $\mathrm{L}$

Mirabilis jalapa L.

Mirabilis longiflora L......

Mirabilis, sp?

Mirasolia diversifolia Hemsl.

Misanteca capitata Cham et Schl........

Mocinna helerophylla Llav et Lex.......

Modiola carolineana Don.

Momordica balsamina L...

Momordica charantia L

Monnina ocampii Horre et Vill...

Monnina xalapensis H. B. K.

Monstera deliciosa Liebm...

Montanoa arborescens DG

Montanoa florilunda DC.

Moritanoa tomentosa Llav, et Lex.

Montanoa, sp?.

Morisonia americana $\mathrm{L}$

Morus alba $L$

Morus celtidifolia ? H. B. K . .

Morus nigra $L$

Mucuna urens DC.

Muehlenbergia dislichophylla Kuntl......

Muntingia calabura L.

Musa cavendishii Lambert.

Musa coccinea Andr.

Musa ensete Gmelin

Musa paradisiaca $I$

Musa regia Rumph

Musa rosacea Jacq

Musa sapientum I

Musa textilis Nees.

Musa, sp?

Musa, sp?

Myoporum ellipticum R. Br

Myosotis palustris With.

Myrica xalapensis H. B. K

Myrica, sp?

Myristica fragrans Houtt.

Myrodia funebris Benth.

Myrospermum frutesen.................

Myrospermum frutescens ? Jacq

Myroxylon peruifermm DC

Myroxylon, sp?

Myrtus arayan H. B. K.

Myrtus ehrenbergii Berg.
Leguminosas........ Leguminosas....... Leguminosas................. Leguminosas... Leguminosas.... Leguminosas Nictagineas....

Niclagineas.

Niciagineas.

Niclagineas.

Compuestas.

Lauríneas.

Pasifloráceas.

Malváceas

Cucurbitáceas

Cucurbitáceas

Poligaleas

Poligaleas

Aroideas.

Compuestas.

Compuestas.

Compuestas.

Compuestas

Caparideas

Urticáceas

Urticáceas

Urticáceas.

Leguminosas........

Gramíneas.... ......

Tiliáceas.

Musáceas

Musáceas

Musáceas

Musáceas...

Musáceas

Musáceas...

Musáceas....

Musáceas.

Musáceas.

Musáceas...

Miopormeas.......

Borragíneas.........

Miricáceas.........

Miricáceas..........

Miricáceas ..........

Esterculiáceas......

Leguminosas.......

Leguminosas.......

L.eguminosas........

Leguminosas........

Mirtáceas...........

Mirtáceas...........

virtáceas...........
Garabato.

Huizache.

Tapincerán. Tapinlzirán.

Tenaza.

Tepame.

Timbe.

Arrebolera. Maravilla. Maravilla silvestre.

Arrebolera. Don Diego de noche. Trompetilla.

Alzoyatic. Alzoyatl. Maravilla de jardín. Pebete. Suspiros.

Tlaquilin.

Tanchichi.

Laurel. Palo misanteco.

Jarilla.

Yedra terrestre.

Avellana de guía. Cundeamor.

Cundeamor. Cundeamor de Yucatán.

Hierba de la mula. Yayaquáhuitl.

Palo de la mula.

Piña anona.

Tacote de flor.

Zoapatle. Zoapatli. Zuapatli.

Cihoacpatli. Sinhuapastle. Zilnoapatli. Zihuapatli. Zoapatle.

Zoapatli. Zuapatli.

Cerbatana.

Arbol del diablo.

Moral blanco.

Palo moral.

Moral negro.

Ojo de borrico. Ojo de venado. Pica-pica.

Zacatón.

Capulín.

Plátano. Plátano de Costa Rica, costeño ó domínico.

Plátano. Plátano chico.

Plátano de Abisinia. Plátano gigante.

Plátano. Plátano grande. Plátano hembra. Plátano largo. Zapalote.

\section{Costeño.}

Plátano morado.

Camburí. Plátano. Plátano chico. Plátano guineo.

Plátano de Manila.

Plátano enano.

Plátano manzano.

Mióporo.

No me olvides.

Arbol de la cera. Huacanalá.

Guacanalá.

Nuez moscada.

Cacahuaxóchitl. Cacaoxóchitl. Flor de cacao.

Nabá.

Bálsamo del Perú.

Bálsamo. Bálsamo de la Unión. Chucte. Hoitzilóxitl. Palo de bálsamo. Semillas de bálsamo ó del obispo.

Bálsamo.

Arrayán.

Arrayán.

Guayabo agrio. 


\section{N}

Nama palmeri A. Gr.

Nama undulatum H. B. K.

Narcissus biflorus Cart.

Narcissus poeticus L.

Narcissus pseudo-narcissus L

Nasturtium officinale Brow...

Nectandra mollis Nees.

Nectandra polita Nees. var

Nectandra, sp?.

Negundo mexicanum DC.

Nepeta cataria ? L

Nephrodium filix-mas Rich.

Neptunia oleracea Lour...

Nerium oleander L....

Nesæa salicifolia H. B. K.

Nesæa syphilitica DG.

Nicandra physaloides Gærtn

Nicotiana glauca Grah.

Nicotiana mexicana Schl.

Nicotiana pilosa Dun.

Nicotiana plumbaginifolia Viv.

Nicotiana pusilla L.

Nicotiana rustica $\mathrm{L}$

Nicotiana tabacum L.

Nicotiana trigonophylla Dun

Nicotiana, sp?.

Nigella damascena L.

Nolina, sp?..

Nymphoea ampla DC.

Nymphœea elegans Hook. ...

Nymphœa gracilis Zucc.

Nymphœa mexicana Zucc

Nymphoea odorata Ait.

Nymphœa, sp?

Nymphoca, sp?
Hidrofiláceas.

Hidrofiláceas.

Amarilídeas.

Amarilídeas.

Amarilídeas.

Cruciferas

Lauríneas.

Lauríneas....

Lauríneas.

Aceráceas.

Labiadas.

Helechos

Leguminosas.

Apocináceas

Litrarieas.

Litrarieas

Solanáceas

Solanáceas

Solanáceas.

Solanáceas

Solanáceas

Solanáceas

Solanáceas.

Solanáceas

Solanáceas

Solanáceas

Ranunculáceas

Liliáceas.

Ninfeáceas

Ninfeáceas

Ninfeáceas

Ninfeáceas

- Ninfeáceas

Ninfeáceas

Ninfeáceas
Hierba de la punzada.

Hierba de la punzada.

Narciso.

Narciso.

Narciso.

Berros.

Laurel pimienta. Laurel pimiento.

Laurel de chile.

Laurelillo agrio.

Acezintle.

Toronjil de China.

Helecho macho.

Dormilona de agua.

Adelfa. Laurel rosa. Rosa laurel.

Granadillo. Hauchinal. Hauchinol ó Huachinoli. Sinicuiche.

Sinicuilche. Sinicuil.

Hachinal ó Hauchinal. Hauchinol ó Hauchinoli.

Belladona del país. Tomate de culebra.

Tabaco amarillo. Tabaquillo. Tronadora.

Tabaco cimarrón.

Tabaco cimarrón.

Tabaquillo.

Tabaco cimarrón.

Andúmuqua. Picietl. Tabaco de macuchi.

Kuutz. Picietl. Quauhyetl. Tabaco.

Tabaco cimarrón.

Tabaquito.

Araña. Ciabellos de Venus. Estrella del mar. Nigela.

Soyate.

Sol del agua.

Cabeza de negro. Ninfa.

Cabeza de negro. Ninfa.

Cabeza de negro. Ninfa.

Cumbaquare. Lampazo.

Chachamole.

Chúmbahua.
Ocimum basilicum L.

Ocimum micranthum Willd.

Ocinum minimum $L$

Ocotea, sp?
Lúbiadas.

Labiatas....

Labiadas.

Lauríneas.
Albaca. Albacarón. Albahaca.

Albahaca de la tierra.

Albaca fina.

Huacalillo. 
Ocotea, sp?

Odontoglossum, sp?

Enothera grandiflora Ait

Enothera rosea Ait

Enothera simsiana Ser.

Enothera suaveolens Desf....

Enothera tetraptera Cav....

Olea europea I

Olyra latifolia $L$

Oncus esculentus l.our.

Oncidium tigrinum Llav. et Lex...

Oplismenus crus-galli Kunth...

Oplismeıus holciformis H. B. K

Opuntia engelmannii Salm Dyck.

Opuntia ficus-indica Mill.

Opuntia hernandezii DC.

Opuntia microdasys Lehm. var....

Opuntia pseudo-tına Salm Dyck

Opuntia Luna Mill.

Opuntia tunicata Lehm

Opuntia vulgaris Mill.

Opuntia, sp?

Oreodoxa regia H. B. K

Origanum majorana $\mathrm{L}$

Origanum vulgare $\mathrm{L}$

Oryza sativa $\mathrm{L}$

Ostrya virginica Willd...

$\mathrm{O}_{\text {xalis }}$ angustifolia H. B. K

$\mathrm{O}_{X a l i s}$ corniculata $\mathrm{L}$

$\mathrm{O}_{x a l i s}$ crenata Jacq

OXalis decaphylla H. B. K

Xalis latifolia H. B. K

$\mathrm{O}_{\mathrm{X}}$ alis tetraphylla Cav............

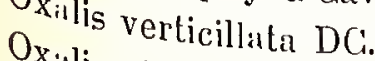

Oxalis violacea? L

Oxalis, sp?.

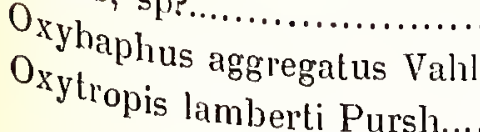

Lauríneas......

Orquídeas

Onagrarieas

Onagrarieas.

Onagrarieas.

Onagrarieas

Onagrarieas

Oleáceas.

Gramíneas

Dioscoreáceas

Orquídeas.

Gramíneas.

Gramíneas

Cácleas

Cácteas

Cácteas.

Gácteas.

Cícteas

Gácteas.

Cácteas

Cácteas....

Cácteas.

Palmas.

Labiadas.

Labiadas.

Gramíneas.

Cupulíferas

Geraniáceas

Geraniáceas...

Geraniáceas

Geriniáceas.

Geraniáceas.

Geraniáceas.

Geraniáceas

Geraniáceas

Geraniáceas.

Nictagineas.

Leguminosas.
Laurel de la playa.

Parácala.

Capa de San José.

Agua de azahar. Tharepeni.

Hierba del golpe.

Viuda.

Linda tarde.

Aceituno. Olivo.

Garrizo verde.

Camote de cerro.

Flor de muertos. Parácata.

Grama de agua. Zacate de manatí.

Camelote. Zacate camelote.

Nopal.

Tuna.

Nocheznopalli. Nopal común. Nopalnocheztli.

Nopal real.

Nopal.

Alquitira del país. Culhua. Flor de tuna blanca. NochtliNopal. Nopal de cochino. Paré. Parí. Tuna.

Abrojo.

Culhua. Nochtli. Nopal. Parí. Tragacanto del país (goma). Tuna.

Xoconochtli.

Palma real. Palmacte. Pumú.

Mejorana. Orégano.

Orégano.

Arroz.

Guapaque.

Jocoyol. Socoyol. Xarimpscua. Xocoyol. Xoxocoyollin. Acedera. Agritos de maceta. Jocoyol. Socoyol. Xarimpscua Xocoyol. Xoxocoyollin.

Oca del Perú.

Agritos color de rosa.

Agritos.

Ghirlo. Ghirlos. Jocoyol.

Jocoyol. Socoyol. Xarimpscua. Xocoyol. Xoxocoyollin.

Xoxocoyollin.

Agritos:

Maravillita.

Hierba loca.

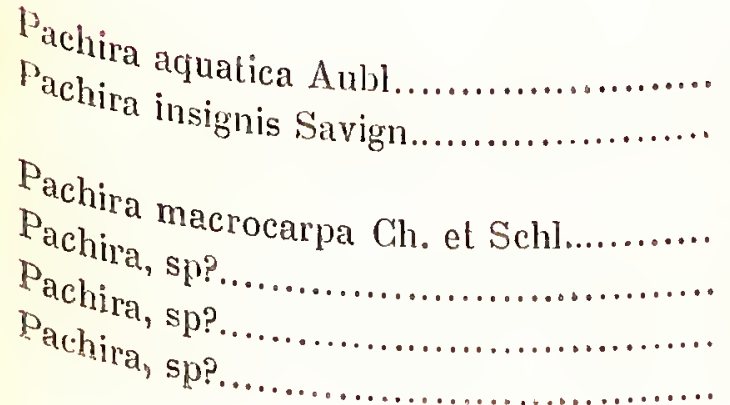

Malváceas Malváceas

Malváceas

Malváceas

Malváceas

Malváceas
Sapote bobo. Zapole de agua.

Apompo. Cabellos de angel. Clavellina. Clavellina roja. Pitón. Thurá. Xcuiche. Xiloxóchitl.

Apompo.

Clavellina blanca.

Coquito.

Tumbile. 
Pachyrhizus angulatus Rich

Pachyrhizus palmatilobus Bent. et Hook

Pæonia montana Sims

Pancratium illiricum L

Paparer rhœas L.

Papaver somniferum $L$

Parietaria pennsylvanica Mülıl

Parkinsonia aculeata L.

Parmentiera alata Miers....

Parmentiera edulis DC

Parmentiera, sp?

Parthenium hysterophorus L....

Parthenium incanum H. B. K...............

Paspalum pusillum Vent.

Passiflora ciliata Ait.

Passiflora dictamo DC

Passiflora difformis $\mathrm{H} . \mathrm{B}, \mathrm{K}$

Passiflora edulis Sims

Passiflora foetida L

Passiflora mexicana Juss

Passiflora serratifolia L

Passiflora sexflora Juss.

Passiflora, sp?

Passiflora, sp?

Paullinia barbadensis Jacq.

Paullinia pinnata $\mathrm{L}$

Paullinia pteropoda DC

Paullinia, sp?.

Pectis difusa Hook. et Arn

Pedilanthus pavonis Boiss.

Pedilanthus tithymaloides Poit.

Pedilanthus tomentellus Rob. et Green.

Pedilanthus, sp?

Pelargonium hybridum Ait.

Pelargonium inquinans Ait.

Pelargonium odoratissimum Ait

Pelargonium speciosum Willd...

Pellæa cordata J. Sm

Pentstemon barbatus Nutt.

Pentstemon imberbis Trauty.

Peperomia umbilicata Ruiz et Pay.

Pepernmia, sp?.

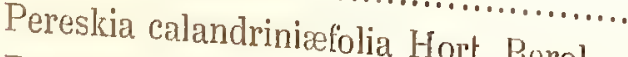

Pereskia, sp?

Perezia alamani Hems

Perezia adnata A. Gr.

Perezia hebeclada A. Gr.

Perezia moschata Llav. et Lex.................

Perezia thyrsoidea A. Gr.

Pernettya ciliaris Don.

Persea drimyfolia Cham.................

Persea gratissima Gærtn.
Leguminosas.....

Leguminosas

Ranunculáceas :...

Amarilídeas

Papareráceas.....

Papaveráceas

Urticáceas.

Leguminosas

Bignoniáceas

Bignoniáceas

Bignoniáceas

Compuestas

Compuestas

Gramíneas

Pasifloráceas

Pasifloráceas

Pasifloráceas

Pasifloráceas

Pasifloráceas.

Pasifloráceas.

Pasifloráceas

Pasifloráceas.

Pasifloráceas

Pasifloráceas

Sapindáceas

Sapindáceas

Sapindáceas

Sapindáceas

Compuestas

Euforbiáceas

Euforbiáceas

Euforbiáceas

Euforbiáceas

Geraniáceas

Geraniáceas

Geraniáceas

Geraniáceas .......

Helechos.

Escrofularíneas

Escrofularíneas...

Piperáceas.

Piperáceas

Cácteas

Cácteass.

Compuestas.

Compuestas

Compuestas

Compuestas

Compuestas

Ericáceas

Lauríneas.

Laurineas.
Cazotl. Jicama. Xícama.

Jícama. Xícama.

Peonía de jardines.

Araña.

Ababol. Adormidera. Amapola.

Adormidera china.

Parietaria.

Guacóporo.

Cirial. Cirian. Cuastecomate. Cuautecomate. Cuautecomatl.

Güiro. Huajerician. Huiro. Quautecómatl. Tecomate. Tecómatl.

Cuajilote. Guajilote. Quauhxílotl.

Cirucetillo.

Altamisa. Cicutilla. Cionfitilla. Zacate amargo.

Mariola.

Grama.

Jujito colorado.

Díctamo real. Itamo real.

Murciélago.

Clavos del Señor. Flor de la pasión. Granada de China. Granadita de China. Pasionaria. Túnica de Cristo.

Jujito peludo.

Díctamo real.

Jujito amarillo.

Granadita fétida.

Coanenepilli.

Trepadora hoja de murciélago.

Ojo de perro.

Barbasco.

Barbasquillo.

Monilla.

Limoncillo.

Gandelillo. Canelillo. Jumete.

Yaxhalalché.

Cordobán.

Pericos.

Malva rubí.

Bola de fuego. Malva bouquet. Malva geranio. Malva Luisa. Malvón.

Geranio de olor. Geranio rosa. Malva de olor.

Geranio de la reina.

Itamo real.

Cebadilla falsa. Chilpantlácol. Jarritos.

Cebadilla falsa. Chilpantlácol. Jarritos. Perritu silvestre.

Pimienta de tierra. Pimientilla.

Cordoncillo.

Patilón. Pititache

Pitayita de agua.

Papalohuiteconi.

Hojarasca. Pipitzáloac. Pipitzáhuac.

Xararo.

Cóla de zorra. Hierba del zopilote.

Ciola de zorra. Hierba del zorrillo.

Capulincillo.

Aguacate oloroso.

Aguacate. Aguacatillo. Ahoacaquáhuill. Ahuacate. Ahuac quáhuitl. Ciupanda. Cupandra. Palto. Tonalahuate. 
Persea, sp?

Persea, sp?

Persea, sp?

Persea, sp?

Persea, sp?

Peteria scoparia A. Gr.

Petiveria alliacea

Petrea volubilis Jaco.

Petunia nyctaginiflor: Juss.

Peucedanum anethum Bn...

Phalaris canariensis L...

Pharbitis violacea Boj...

Phaseolus caracalla L..

Phaseolus formosus H. B. K

Phascolus lunatus L

Phaseolus multiflorus Willd.

Phaseolus vulgaris L. var.....

Phascolus, sp?

Phaseolus, sp?

Phaseolus, sp?

Phaseolus, sp?

Phascolus, sp?.

Plaseolus, sp?

Pliascolus, sp?.

Philadelphus coronarius $\mathrm{L}$

Philibertia cumanensis Hemsl.

Philibertia, sp?

Philodendron, sp?

Phlomis pungens Willd...........

Phœnix dactylifera L

Phoradendron rubrum Griseb.

Phoradendron tomentosum D............

Phoradendron, sp?

Phormium tenax Forst

Phragmites communis Trin

Phyllocactus angulifer Lem

- n......

Phyllocactus latifrons Salm Dyck.........

Phyllocactus phyllanthoides Salm Dyck.

Phyllocactus, sp?

$\mathrm{Physalis} \mathrm{angulata,} \mathrm{L}$

Physalis coztomatl Moc. et Sessé

Physalis mollis Nutt.

Physalis nicandroides Schl.

Physalis peruviana $L$

Physalis pubescens

Physalis, sp?

Phytolacca icosandra...

Phytolacca octandra

Picridium vulgare Desf...

Pilea falcata Liebm.

Pilea microphylla I.iebm
Lauríneas.

Laurineas.

Laurineas.

Laurineas.

Lauríneas.

Leguminosas

Fitolacáceas.

Verbenáceas

Solanáceas

Umbelíferas ... ....

Gramíneas.

Convolvuláceas.....

Leguminosas.

Leguminosas

Leguminosas.

Leguminosas

Leguminosas

Leguminosas........

Leguminosas........

Leguminosas........

Leguminosas........

Leguminosas.......

Leguminosas.......

Leguminosas.......

Saxifragáceas... ..

Asclepiadeas........

Asclepiadeas........

Aroideas............

Labiadas............

Palmas

Lorantáceas........

Lorantáceas........

Lorantáceas.

Liliáceas............

Gramíneas..........

Cácteas.............

Cácteas.

Cácteas

Gácteas.

Solanáceas ..........

Solanáceas

Solanáceas ....

Solanáceas..........

Solanáceas ..........

Solanáceas..........

Solanáceas.........

Fitolacáceas........

Fitolacáceas.........

Compuestas.........

Urticáceas ...........

Urticáceas
Aguacachile. Ahuacachile.

Aguacatillo.

Aguacatillo blanco.

Aguacatillo colorado.

Chinín.

Camote del monte.

Hierba de las gallinitas. Zorrillo.

Flor de Santa Rita. Jazmín.

Petunia. Tabaquillo oloroso.

Encldo.

Alpiste. Alpistera.

Manto de la Virgen. Yedra terrestre.

Caracol. Caracolillo.

Yeguas.

Frijol blanco.

Frijol ayocote blanco. Frijol ayocote morado. Frijolillo. Patoles. Yeguas.

Frijol amarillo. Frijol blanco. Frijol colorado. Frijol colorado bolita. Frijol negro. Frijol parraleño. Frijol prieto. Habichuela. Judía. Thatzin.

Frijol almendro.

Frijol ayocote.

Frijol judío.

Frijol meco.

Frijol moro.

Frijol prieto garrapata.

Tlaljícama.

Jazmín mosqueta. Mosqueta.

Petaquilla.

Lengua de víbora.

Hoacaxóchitl.

Salvia amarilla.

Datil. Palma común. Zoyacapulín.

Cabellera.

Liga. Visco cuercino.

Ghachahua.

Lino de Nueva Zelanda.

Carricillo. Carrizo. Piquaremu.

Jarana de pitahayita.

Pitayita de agua. Reina de la noche.

Nopalillo color de rosa. Nopalxochicuezaltic.

Pitahaya.

Pakmul. Tlemolli. Tomate. Tomatl. Tomate de culebra.

Tomatillo.

Coscomate. Costomate. Costómatl. Tomate de comer.

Jaltomate. Xaltómatl.

Tomate de culebra.

Tomate. Tomatl.

Tomate. Tomatl.

Chichi de gato.

Amolquelite. Amolquilitl. Cóngora. Conguerán. Tarasca dis: los negros.

Conguarán. Jabonera. Mazorquilla. Namole. Verbachina.

Escorzonera. Pulmonaria de herbolario.

Hierba de la viruela.

Golondrina. Hierba de la viruela. 
Pimenta officinalis Berg. var

Pinaropappus roseus Less

Pinus arizonica Engelm.

Pinus ayacahuite Ehrenb.

Pinus cembroides Zucc.

Pinus chihuahuana Engelm

Pinus edulis Engelm

Pinus engelmanni Carr.

Pinus filifolia Lindl.

Pinus flexilis James

Pinus greggii Engelm.

Pinus hartwegii Lindı

Pinus lambertiana Dougl....

Pinus lawsoni Roezl.

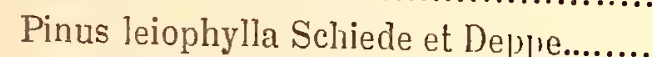

Pinus montezumæ Lamb. vir

Pinus oocarpa Schiede.

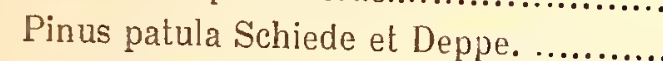

Pinus pinea L.......................

Pinus pinceana Gordon.

Pinus ponderosa Dougl....

Pinus pseudo-strobus Lind...............

Pinus teocote Cham. et Schl...

Pinus, sp?

Pinus, sp?

Piper amalago $\mathrm{I}$

Piper angustifolium Ruiz et Pav.

Piper auritum H. B. K.

Piper longum L.

Piper palmeri C. DC.

Piper peltatum $\mathrm{L}$.

Piper sanctum Schl.

Piper tuberculatum dacq...

Piper, sp?

Piptadenia fotida Benth .................

Piqueria trinervia Cav

Piscidia erythrina L

Piscidia mollis Rose.

Piscidia, sp?.

Piscidia, sp?.

Pisonia aculeata L

Pisonia hirtella H. B. K.

Pistacia mexicana H. B. K

Pistia stratioles L

Pisum sativum L

Pithecoctenium hexagonum DC:

Pithecoctenium, sp? ...

Pithecolobium albicans Be..............

Pithecolobium tuicans Bentl

Pithecolobium ligustrinum K!...
Mirtáceas.

Compuestas

Coníferas

Coníferas

Coníferas

Coníferas

Coniferas

Coníferas

Coniferas

Coníferas

Coníferas

Coníferas

Coníferas

Coníferas

Coníferas

Coníferas

Coníferas

Confferas

Coníferas

Coníferas

Coníferas

Coniferas

Coníferas

Coniferas

Coníferas

Piperáceas.

Piperáceas....

Piperáceas.

Piperáceas

Piperáceas.

Piperáceas

Piperáceas

Piperáceas

Piperáceas

Leguminosas

Compuestas

Leguminosas.

Leguminosas.

Leguminosas.

Leguminosas.

Nictagineas.

Niclagineas.

Anacardiáceas...

Aroideas..

Leguminosas.....

Bignoniáceas.....

Bignoniáceas

Leguminosas.

Leguminosas.

Leguminosas......
Pimienta gorda. Pimienta de Tabasco. Pimienta de la tierra. Xocoxóchitl.

Escorzonera. Motita. Motita morada.

Pino.

Acalocahuite. Ayacahuite. Ayacahuite colorado. Ocote blanco. Pino. Pino real. Sacalacahuite.

Pino. Pino piñón. Piñón.

Pino.

Pino.

Pino.

Pino.

Pino.

Pino.

Pino.

Pino.

Pino.

Ocote chino. Pino.

Ocote hembra. Pino. Pino blanco. Pino común.

Pino.

Ocote. Ocote macho. Pino.

Picea.

Pino.

Pino.

Pino.

Jalocote. Ocote. OcotI. Pino. Teocote. Teocotl. 'Tos'arza. Tzat'adi. Xalocotl.

Ocote amarillo.

Trivireni.

Mecaxóchitl.

Achiotlín. Cordoncillo. Mático. Soldadillo.

Momo.

Cordoncillo.

Hachogue.

Momo de zopilote.

Acuyo. Hierba santr. Hoja de aján. Tlamapaquelile. Tlanepaquelite. Tlanepraquillitl.

Cordoncillo.

Tlalacuaje.

Coyacale. Huizache. Yóndiro.

Empueshte. Hierba de San Nicolás. Hierba del tabardillo. Xoxonitzal. Xoxonitztac. Xoxonitzal. Yoloxiltic.

Cocuile. Colorín de peces. Chijol. Flor de papagallo. Jalbí. Jabín. Mata pez de México.

Palo blanco.

Amargoso.

Cocuite.

Beeb. Uña de gato.

Jazmincillo.

Lentisco de Nuevo León.

Lechuguilla de agua.

Alverja. Alverjón. Chícliaro. (:híclaro de comer. Guisante.

Palomitas.

Bejuco de huico.

Hoizachinquáhuitl. Huisache. Huizache. Tzurumbeni?

Guamachi. Huamúchil. Pinzan. Quanóchitl. Tucuy. 
Pithecolobium mexicanum Rose

Pithecolobium multiflorum Benth.

Pilhecolobium, sp?

Pittosporum tobira Ait.

Plantago galeotliana Dcne...

Plantago major L

Plantago mexicana Link.

Plantago psyllium I

Plantago, sp?

Platanus lindeniana Mart. et Gal...

Platanus mexicana Moric...

Platanus occidentalis L.

Platanus, sp?.

Plumbago capensis Thunb.

Plumbago europæa L

Plumbago pulchella Boiss...

Plumbago scandens L

Plumeria bicolor R. \& Pav

Plumeria pudica Jacq.

Plumeria purpurea R. \& Pav...

Plumeria rubra L

Plumeria rubra L. var.

Plumeria tricolor R. \& Pav

Plumeria, sp?

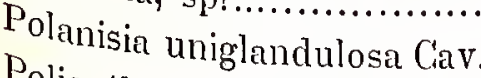

Polianthes tuberosa L.

Polygala galioides Poir.

Polygala mexicana DC

Polygala myrtifolia L

Polygala scoparia H. B. K

Polygonum acre H. B. K

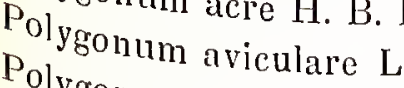

Polygonum hydropiper L....

Polygon hepiper L.................

Polygonum hydropiperoides Michx.......

Polygonum pennsylvanicum L

Polgonum persicarioides H. B. K........

Polygonum, sp?....

Polypodium angustum Mett...

Polyodium aureum $\mathrm{L}$

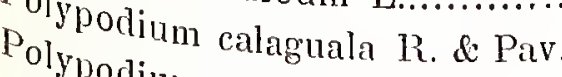

Polyodium crassifolium L...

Polyodiun decumanum Willd

Polypodium lanceolatum L....

Polypodium lycopodioides L

Polypodium neriifolium Scllkuhr...

Polypodium plebeium Ch. et Schl........

Polypodium vulgare I

Polypodium, sp?.

fomentarius Fries...
Leguminosas.

Leguminosas.

Leguminosas.

Pitosporeas....

Plantagíneas

Plantagíneas

Plantagineas

Plantagíneas

Plantagíneas

Platanáceas.

Platanáceas

Platanáceas

Plitanáceas

Plumbagíneas.

Plumbagineas

Plumbagíneas

Plumbagíneas ......

Apocináceas

Apocináceas

Apocináceas.

Apocináceas

Apocináceas

Apocináceas....

Apocináceas

Caparideas...

Amarilídeas...

Poligaleas

Poligaleas

Poligaleas....

Poligaleas

Poligonáceas.......

Poligonáceas ........

Poligonáceas........

Poligonáceas........

Poligonáceas........

Poligonáceas.......

Poligonáceas.......

Helechos

Helechos

Helechos....

Helechos

Helechos

Helechos

Helechos

Helechos

Helechos

IIelechos...

Helechos

Hongos
Chino.

Granadillo.

Tucuy liso.

Clavo. Lila.

Lantén. Llanlén. Plataxíhuitl. Totoncapatli.

Lantén. Llantén. Vilzaqua sipiati.

Lantén.

Zaragatona.

Chía gorda.

Alamo.

Alamo blanco.

Alamo. Plátano de Occidente.

Haya.

Plúmbago.

Plúmbago.

Cola de iguana. Cola de pescado. Curiqua. Chilillo. Hierba del alacrán. Hierba lumbre. Jiricua. Pañati. Pañete. Tianquiz. Tlepatli.

Hierba del alacrán. Plúmbago błanco. Tlalchichinolli. Tlepatli.

Flor de Nayo.

Flor de Mayo. Xtuhuy.

Flor de Mayo.

Alejandría. Cacaloxóchitl. Cacaloxúchil. Campechana. Campotonera. Cuauxúchil. Cundá. Flor del cuervo. Flor de Mayo. Jacalozúchil rojo. Sabacnicté.

Jacalozúchil blanco.

Ensalada. Flor de Nayo.

Súchil.

Hierba del coyole.

Amiga de noche. Amole. Amolle. Flor de hueso. Margarita blanca. Margarita olorosa. Nardo. Omixóchitl. Vara de Jessé. Vara de San José.

Poligala.

Polígala de México. Xochipitzáhoac

Polígala de jardines.

Polígala medicinal. Polígala de México.

Chilillo.

Lengua de pájaro.

Chilillo.

Chilillo.

Moco de guajolote.

Moco de guajolote. Persicaria.

Cordón de cardenal.

Calaguala.

Calaguala. Polipodio.

Calaguala.

Calaguala.

Calaguala.

Lengua de ciervo. Mananepile.

Calaguala.

Calaguala.

Calaguala.

Calahuala.

Calaguala. Palmitas.

Yesca. 
Polyporus igniarius? Pers.

Polytrichum species.

Pontederia cordata L.

Populus alba L.

Populus fremonti Wats.

Populus heterophylla L....

Populus nigra L

Populus tremula L.

Populus, sp?

Porophyllum coloratum DC.

Porophyllum macrocephalum DC..

Portulaca oleracea L

Posoqueria latifolia Røem. et Schultz....

Posoqueria, sp?.

Potamogeton natans L....

Potentilla comaroides Nestl.

Potentilla candicans Humb. et Bonpl....

Potentilla ranunculoides H. B. K.

Potentilla tormentilla ? Nestl. var..........

Potentilla, sp?.

Primula auricula

Primula sirensis Lindl.

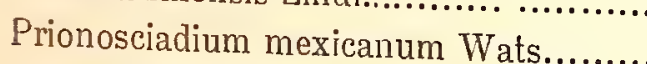

Priva echinata Juss

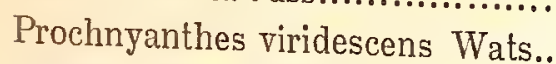

Prosopis cinerascens A. Gr.

Prosopis juliflora DC.

Prosopis pubescens Benth

Prosopis, sp?

Prunus amygdalus Hook fil

Prunus armeniaca L

Prunus capuli Gav

Prunus domestica L

Prunus persica Bent. et Hook

Pseudosmodingium perniciosum Engl...

Pseudotsuga douglasii Carr.

Psidium pomiferum L.

Psoralea pentaphylla L

Psychotria excelsa H. B. K.

Psychotria mexicana Willd

Pteris aquilina $\mathrm{L}$.

Pterocarpus draco L.

Pterocarpus, sp?

Punica granatum L

Pyrethrum parthenium Smith

Pyrus communis L. var.

Pyrus malus L. var.
Hongos .............. Yesca.

Musgos............. Musgo.

Pontederiáceas..... Tule.

Salicineas........... Alamo blanco.

Salicineas........... Chopo.

Salicineas.......... Alamo plateado.

Salicineas........... Alamo. Alamo negro. Chopo.

Salicineas........... Alamo temblón.

Salicineas........... Alamo.

Compuestas........ Chivatillo. Papaloquelite.

Compuestas ........ Hierba del venado.

Portulacáceas...... Acahuecaxaqua. Quauquílitl. Verdolaga. Xucul.

Rubiáceas........... Palo de peine blanco.

Rubiáceas........... Palo de peine amarillo.

Nayadáceas........ Acaxaxán.

Rosáceas ........... Tormentila.

Rosáceas .......... Atlanchana. Coanenepilli. Sínfito. Tormentila.

Rosáceas ........... Tormentila.

Rosáceas ........... Tormentila.

Rosáceas .......... Itamo real.

Primuláceas........ Prirnavera.

Primuláceas........ Primavera.

Umbelíferas ........ Jalocote.

Verbenáceas......... Cadillo de bolsa.

Amarilídeas........ Amole. Amolilla.

Leguminosas........ Mezquite.

Leguminosas....... Chachaca. Ghucata. Mezquite. Mezquitl. Misquicopalli. Tziritzequa.

Leguminosas....... Mezquite. Tornillo.

Leguminosas....... Tepemezquite.

Rosáceas........... Almendro.

Rosáceas ........... Albaricoque. Chabacano.

Rosáceas.......... Capolín. Capulín. Xeugua.

Rosáceas.......... Ciruelo de España.

Rosáceas .

Anacardiáceas.........

Coníferas

Mirtáceas.

Leguminosas.

Albérchigo. Durazno. Melocotón. Melocotonero. Prisco.

Copaljiote. Cuajiote. Cuajiote blanco. Guajilote.

Abeto. Jaboncillo. Pino de corcho.

Guayaba. Guayabo. Guayabo de las sabanas. Guayabo rojo y blanco. Xalxócotl.

Contrahierba aromática. Gontrahierba blanca. Contrahier pa del interior.

Rubiáceas

Pubiáceas

Helechos

Ipecacuana de Jalapa.

Ipecacuana de México.

Leguminosas.

Helecho hembra. Polipodio.

Leguminosas..... Huahuauchi. Sangre de drago.

Litrariegs ........ Palo de rosa.

Compuestas ..... Granado. Granado dulce. Raíz de granado.

Rosáceas ........ Altamisa.

Rosáceas ........ Pera. Peral. Peral común. Manzano. Perón. 
Quassia amara L

Quassia cedron H. Bn.

Quassia, sp?

Quercus acutifolia Nee

Quercus agrifolia Nee.

Quercus barbinervis Benth.

Quercus castanea Nee...

Quercus chrysophylla Humb. et Bonpl...

Quercus confertifolia Humb. et Bonpl....

Quercus cortessi Liebm.

Quercus crassifolia Humb. et Bonpl......

Quercus crassipes Humb. et Bonpl........

Quercus depressa Humb. et Bonpl........

Quercus glaucescens Humb. et Bonpl....

Quercus insignis Mart. et Gal.

Quercus lanceolata Humb, et Bonpl......

Quercus laurina Humb, et Bonpl.........

Quercus microphylla Nee.................

Quercus obtusata Humb. et Bonpl........

Quercus polymorpha Gham. et Schl.....

Quercus pulchella Humb. et Bonpl........

Quercus repanda Humb. et Bonpl.........

Quercus reticulata Humb. et Bonpl.......

Quercus rugulosa Mart. et Gal

Quercus sideroxyla Humb. et Bonpl.....

Quercus skinneri Benth.

Quercus splendens Nee.

Quercus strombocarpa Liebm...

Quercus tomentosa Willd.

Quercus virens Ait.

Quercus xalapensis Humb. et Bonpl......

Quercus, sp?...

Quercus, sp?

Quercus, sp?

Quercus, sp?

Quercus, sp?

Quercus, sp?

Quercus, sp?

Quercus, sp?

Quercus, sp?

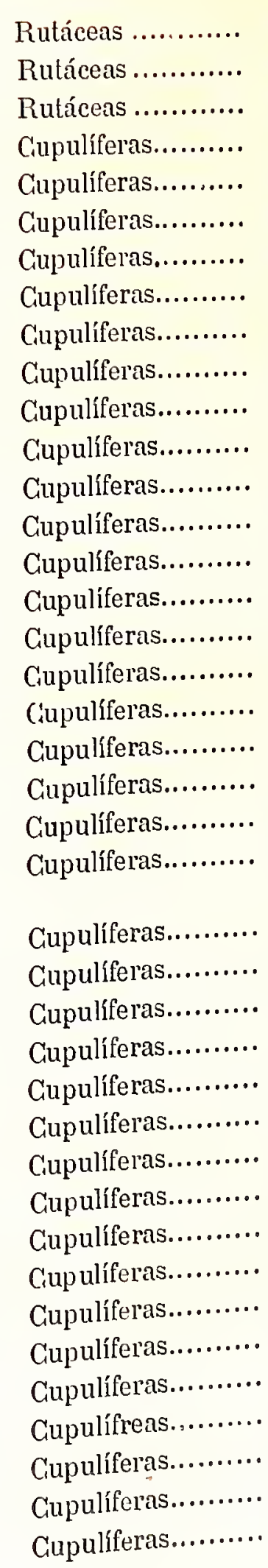

Cuasia.

Cedrón de Oaxaca.

Tinco.

Aguatle. Ahoatl. Ahuatle. Encina. Encina memelito.

Encina memelito.

Encina.

Bellota. Encina. Encina memelito.

Encina memelito.

Encina.

Encian.

Encina.

Encina. Encina negra.

Encina.

Encina memelito.

Ahoaquálıuitl. Aoatl. Encina.

Encina memelito.

Ahucepitzáhuac. Encina memelito. Encino delgado.

Encino capulincillo.

Encina. Encina memelito.

Encina.

Encina.

Encina. Encina negra.

Ahoaquáhuitl. Aoatl. Encina. Encina memelito. Encina de miel.

Encina.

Encina memelito.

Encina memelito.

Encina.

Ahoaquáhuitl. Aoatl. Encina.

Encina.

Encino negro. Maculi. Maquilíhuatl. Roble. Roble serrano.

Encino roble. Roble de duelas.

Ahuatezón.

Alcornoque.

Encino aguacapuli.

Encino blanco.

Encino colorado.

Encino trompillo.

Qualıutomatl. Quauhtómatl.

Tarecuen.

Texmole.
Plandia tetracantha DC.

Randia, sp?

Randia, sp?
Rubiáceas

Rubiáceas.

Rubiáceas
Arbol de las cruces.

Espino blanco.

Espino herrero. 
Randia, sp?

Ranunculus hookeri Schl....

Ranunculus hydrocharis Spenner.

Ranunculus napellifolius DC....

Raphanus sativus L. var

Reseda luteola L

Reseda odorata L......

Rhamnus humboldtianus Rœm. et S....

Rhamnus serratus Willd

Rhamnus, sp?

Rhamnus, sp?

Rhizophora mangle I

Rhododendron indicum Sweet...

Rhus copallina $\mathrm{L}$

Rhus microphylla Engelm.

Rhus mollis H. B. K.

Rhus sempervirens Scheele..

Rhus toxicodendron $\mathrm{L}$

Rhus, sp?

Rhus, sp?

Rhus, sp?

Rhus, sp?

Rhus, sp?

Rhus, sp?....

Rhynchosia phaseoloides DC

Rhynchosia precatoria DC

Rhynchospora aurea Vahl

Ribes affine $\mathrm{H} . \mathrm{B}$. K

Ribes multiflorum H. B. K

Richardia africana Kunth

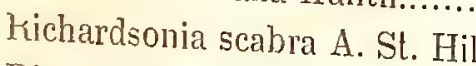

Ricinus comunis L. var.

Rivina hunilis

Robinia pseudo-acacia

Robinia, sp?.

Robinia, sp?

Roccella fusiformis ? Ach.............

Poccella tinctoria Ach

Rollinia mucosa Baill

Rosa alba L. var.

Rosa bengalensis Pers...

Posa canina L var

Prosa centifolia I

Rosa cinnamomea L. var.........

Piosa damascena Mill.

Rosa gallica L, var. ...

Rosa indica I. var.....

Rosa montezumse Red.........

Rosa mulliflora Thunb. va

Rosa parviflora Ehrb.

Rosa pimpinellifolia I.

Rosa sempervirens L. vap.................
Rubiáceas

Ranunculáceas....

Ranunculáceas ....

Ranunculáceas....

Crucíferas

Resedáceas

Resedáceas

Ramneas

Ramneas.

Ramneas.

Ramneas

Rizoforáceas

Ericáceas

Anacardiáceas

Anacardiáceas

Anacardiáceas

Anacardiáceas

Anacardiáceas

Anacardiáceas

Anacardiáceas

Anacardiáceas

Anacardiáceas

Anacardiáceas

Anacardiáceas

Leguminosas...

Leguminosas

Ciperáceas.

Saxifragáceas

Saxifragáceas

Aroideas.

Rubiáceas.

Euforbiáceas

Fitolacáceas

Leguminosas....

Legumino=as

Leguminosas...

Liquenes

Líquenes

Anonáceas

Rosáceas

Rosáceas

Rosáceas

Riosáceas

Rosáceas

Riosáceas

Rosáceas

Rosáceas

Rosáceas

Rosáceas

Rosáceas

Rosáceas

Rosáceas
Puro Cecilia.

Pata de león.

Panúnculo.

Ranúnculo amarillo.

Rábano.

Gualda.

Miñoneta. Resedá.

Capulincillo. Capulincillo cimarrón. Margarita del cerro. Tlalcapolín. 'Tullidora.

Capulincillo,

Amole. Amolli.

Azufaifo.

Candelón. Mangle. Mangle colorado. Manglero.

Azalea. Azalea de arbusto.

Copalcuaquitle de México. Copalquáhuitl. Sumaque.

Correosa.

Zumaque.

Lambrisco.

Bembériqua. Guardalagua. Guau. Mala mujer. Vembérequa. Zumaque.

Ciraroque.

Copalquahujílotl.

Hincha huevos.

Palo tambor.

Timbe.

Xoxoco.

Negritos. Ojitos de picho. Peonía.

Atecuixtli. Colorín chiquito. Negritas. Ojo de cangrejo. Peonía de niños. Pulguitas. Purensapichu.

Navajuela.

Capulincillo. Ciruelillo.

Capulincillo. Ciruelillo.

Alcartaz. Alcatraz. Aro. Tonalxóchitl.

Ipecacuana blanca.
Higuera del diablo. Higuerilla. Higuerilla pequeña. Palma Christi. Ricino. Tläpatl. Xkoch. Coral.

Acracia. Robinia.

Cocoite. Cocuite. Cocuit].

Chiopilcoite.

Toje.

Orchilla.

Anona.

Rosa blanca. Rosa cambriy.

Rosa de Bengala.

Rosa de Jericó.

Prosa de Ciastilla.

Rosa canela.

Rosa de todos los meses.

Piosa de Jericó. Rosa del Noríe.

Rosa de Bengala. Rosa te.

Garambullo. Posa de Moctezuma uña de galo.

Rosa enredadera.

Rosa miniatura.

Rosa pimpinela.

Trepadora. 
Posa sulphurea Ait........................

Rosa, sp?.

Rosa, sp?

Rosa, sp?

Rosa, sp?.

Rosilla lutea Less.

Rosmarinus officinalis $\mathrm{L}$

Rourea oblongifolia Hook. et Arn

Rubia levigrsta DC.

Rubia tinctorum L.

Rubus fruticosus L

Rubus stıigosus Miclix.

Rudbeckia mexicana $W_{i l}$

Ruellia macroplyylla ? Valıl.

Rumex acetosa L

Pumex acetosella $\mathrm{L}$

Pumex hymenosepalus 'Torr

Rumex mexicanıs Meissn

Rumex obtusifolius L.

Pummex obtusifolins L.......................

Runf patientia L..........................

Runifordia floribunda DC.

Russelia juncea Zucc..

Ruta gravenlens L..
Rosáceas

Rosáceas

Rosáceas

Rosáceas

Rosíceas

Compuestas.

Labiadas....

Connaráceas.

Rubiáceas.....

Rubiáceas

Rosíceas

Rosáceas

Compuesias

Acantáceas.

Poligonáceas

Poligonáceas

Poligonáceas.

Poligonáceas.

Poligonáceas

Poligonáceas.

(ompuestas

Escrofularíneas.....

Riulácens
Canaria. Rosa amarilla.

Rosa anglicana.

Trepadora amarilla.

Trepadora blanca.

Trepadora color de rosa.

Rosilla.

Romero. Romero extranjero.

Ghilillo de la Huasteca. Chlilillo venenoso. Palo del chilillo.

Rubia.

Rubia.

Situni. Zarzamora.

Coatlamitl.

Honoba.

Puxcanxenec.

Acedera. Vinagrera. Xoxoyolpapatla?

Acederilla. Vinagrerita.

Cannagria. Canaigre. Cañagre. Cañagria. Gañaigre. Raíz dlel indio.

Amamastla. Lengua de vaca.

Izquia. Lengua de vaca.

Lengua de vaca. Romoza. Ruibarho de frailes.

Catiriqua.

Coral de la playa.

Acuitze varikua. Ruda.
Saccharum officinarum L

Sagittaria lancifolia L. var.

Sagiltaria sagittifolia L

Salıagunia mexicana Liebm?

Six bliptica Peyr....

Silix babylonica $L$

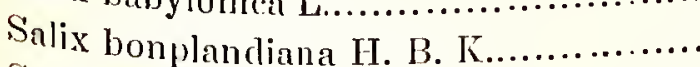

Salix cana Mart. et Gal....................

Salix lautwegi Bentl....

Salix humboldtiana Willd....

Salix irrorata And

Salix lasiolepis Benth....

Salix latifolia Mart. et Gal.

Salix longifolia Mulıl.

Salix mexicana Seem......

Salix nigra Marslı.

Salix paradoxa H. B. K.

Salix pringlei Rowlee....

Salix stipulacea Mart. et Gat

Salix laxifolia H. B. K

Salix, sp?

Salix, sp?.

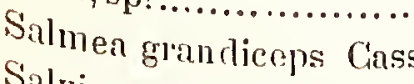

Salvia angrustifolia Cav.
Gramíneas...

Alismaceas.

Alismáceas

Utticáceas

Cielastrincas

Salicineas.

Salicineas.

Salicineas.

Salicineas.

Salicineas.

Salicineas.

Salicineas

Salicineas.

Salicineas.

Salicineas.

Salicineas.

Salicincas

Salicineas.

Salicineas.

Salicineas

Salicin eas

Salicineas

Compuestas........

Labiadas
Caña de azúcar. Caña criolla.

Cola de pato.

Colomo delgado. Flecha de agna. Sagitaria.

Arbol del pan.

Goco dulce. Guoguo.

Sauce llorón.

Sauce. Sauz.

Sauce. Sauz.

Sauce. Sauz.

Sauce. Sauz.

Sauce. Sauz.

Ahuejote. Huejocote. Huéxotl. Sauce. Siluz. Tepehuexotr.

Sauce. Sauz.

Sauce. Sauz.

Siluce. Sauz.

Sauce. Sauz.

Sauce. Saurz.

Sauce. Siuz.

Siluce. Siluz.

Sauce. Sillt\%

Sittzo.

Tarimu.

Hierba de Sam Antonio.

chía cimarronis. 
Salvia axillaris Moc. et Sessé ....

Salvia ballotæflora Bentlı....

Salvia coccinea I

Salvia cyanea Benth...

Salvia eriocalyx Bert.

Salvia hispanica L

Salvia indica $\mathrm{L}$

Salvia leucantha Cav....

Salvia longispicata Mart. et Gal.

Salvia microphylla H. B. K.

Salvia patens Gav....

Salvia polystachya Ort

Salvia tiliæfolia Vahl

Salvia, sp?

Salvia, sp?

Salvia, sp?

Sambucus mexicana Presl

Sanvitalia procumbens DC....

Sapindus marginatus Willd

Sapindus, sp?

Sapindus, sp?

Sapindus, sp?.

Sapindus, sp?....

Saracha jaltomiata Schl

Saracha umbellala G. Don

Sassafras officinale Nees

Saxifraga crassifolia L

Scabiosa atropurpurea L

Schinus molle L....

Schinus, sp?

Schkuhria abrotanoides Poth

Schkuhria virgala $\mathrm{DC}$.

Schonocaulon officinale A. Gr.

Schœenocaulon, sp?

Scirpus, sp?

Seoparia dulcis L.

Sebastiania pilmeri Rose.

Sechium edule Swartz

Sedum acre $\mathrm{L}$

Sedum dindroideum Moc. et Șessé

Sedum quitense H. B. K...

Sedum roseum Stev.

Sedun saxutile Willd

Sedum, spe?

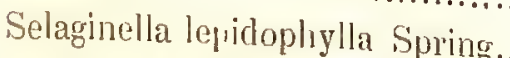

Selaginella rupestris Spring...

Selaginella, sp?

Senecio barba-johanis DC

Senecio calcareus H. B. K.

Senecio calophyllus Hemsl.

Senecio canicilit Mor. ot Sessé
Labiadas.

Labiadas

Labiadas.

Labiadas

Labiadas.

Labiadas

Labiadas

Labiadas.

Labiadas....

Labiadas....

Labiadas....

Labiadas

Labiadas

Labiadas...

Labiadas...

Labiarlas.

Caprifoliáceas.

Compueslas

Sapindáceas

Sapindáceas

Sapindáceas

Sapindáceas

Sapindáceas

Solanáceas ...

Solanáceas

Lauríneas

Saxifragáceas

Dipsáceas ....

Anacardiáceas

Anacardiáceas

Compuestas

Compuestas

Liliáceas

Liliáceas.

Ciperáceas.

Escrofularíneas

Euforbiáceas.

Cucurbiláceas.

Cirasuláceas

Crasuláceas

Cirasuláceas

Crasuláceas

Cirasuláceas

Crasuláceas

Licopodiáceas

Licoporliáceis

Licopodiáceas

Compuestas

Compuestas.

Compuestas

Gomprustas
Hisopo del país. Hisopo de Pucbla.

Mejoraná. Mejorana del país.

Mirto.

Chía azul grande.

Pluma de Santa Teresiı.

Chía.

Hierba del cáncer.

Hierba de la playa. Pluma de Santa T'eresil. Sinnta María.

Cantueso.

Mirto. Pabellón mexicano. Salvia del monte. Torongil.

Almoraduj. Almoraduz. Flor del gillito. Mejorina del país. Quiquiriquí.

Chía. Hisopo. Hisopo de Pueblia. Romerillo. Salvia de GuaIlalajara.

Chía. Chiantzozolli. Quicbra-plato. .

Orégano.

Payenaltzi.

Silvia de San Juan de Los Lagos.

Cundemba. Sauco. Xúmetl.

Ojo rle gillo. Ojo negro. Xkaritnmluul.

Amole de bolita. Jilonncillo.

Amole de bolila.

Copalxocotr.

Iyamolle.

Tehoitztli. Telunixlle.

Jaltomate. Xaltómatl.

Jaltomate. Tinguara.

Navanché de Yucaláı.

Oreja de oso.

Ambar. Ambarilla. Ambarina. Escabiosa. Rscobilla. Viudas.

Arbol del Perú. Copalastle. Copalquálıuitl. Corteza del Perú. Molle. Pelonquáhuitl. Perú. Pimienta de Anérica. Pirú. Pirul.

Palo mulato.

Anisillo. Anisillo cimarrón. Escobilla. Putzuleni.

Anisillo.

Cebadilla. Cebolleja. Cunicho. Etzemo. Itzcuimpalli. Qnimichpati.

Hierla del burro.

Tule.

Escobilla amarga.

Hierba de la flecha. Palo de la flecha.

Alupu. Camochayote. Chaiotl. Gihayote. Cillinchayole.

Chismes. Jaspalache. Xalitche.

Siempreviva amarilla.

Itzmell. Siempreviva.

Itzmetl. Siempreviva.

Hierba de San Nicolás.

Asmodeo.

Siempreviva.

Doradilla. Flor de piectra.

Lama.

Barbas de Juan de Dios.

Jarilla.

Mano de león. Pejole. Piote.

Clarincillo. Hierba de la Pucbla. Ilzcuimpatli. 
Senecio cardiophyllus Hemsl...

Senecio cervariefolius Hemsl.

Senecio cinerarioides H. B. K.

Senecio grayanus Hemsl....

Senecio hartwegii Bentlı...

Senecio helodes Benth

Senecio petasitis DG.

Senecio precox DC.

Senecio roldana DC....

Senecio salignus DC

Senecio sanguisorbo DC.................

Senecio sinuatus H. B. K.

Senecio tolucanus DC.

Senecio vulneraria DC

Senecio, sp?.

Serjania mexica a Willd.

Serjania racemosa Schum...

Sesamum indicum L

Sesbania picta Pers

Sesuvium revolutifolium Ort

Setaria glauca Kuntl.

Sicjo; angulatus L....

Sida carpinifolia L

Sida perduncularis DC.

Sida rhombifolia L.

Sida triloba Cav.

Sida, sp?.

Siegesbeckia orienlalis I

Silene catesbrei Wallh

Simaba cedron Planch.

Simaruba, sp?.

Siparuna riparia A. DG

Sisymbrium canescens Nutt.

Sisymbrium nasturtium $\mathrm{L}$

Sisymbrium, sp?.

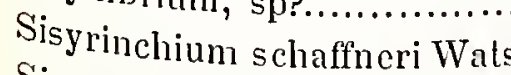

Sium angustifolium

Smilax bona-nox L. var.

Smilax cordifulia Humb. et Bonpl. var...

Smilax glauca Walt

Smilax jalapensis Schl

Smilax medica Cham. et Sclıl

Smilax mexicana Griseb.

Smilax rolundifolia $L$

Solandra grandiflora Sw...

Solandra guttata Don.

Sol anum amictum Moric.

Solanum asperun Vialıl...

Solanum bulbocastanum Dum....

Solanum candidum Lindl.

Solanurn cervantesii Lag.

Solanum cornulum Lam

Solanum cuneifolium Dun

Solanum dulcamara L. var.

Solanum suranifolium ('av.
Compuestas

Compuestas

Compuestas

Compuestas.

Compuestas.

Compuestas

Compuestas.

Compuestas

Compuestas

Compueslas

Compuestas

Conpuluestas

Compuestas

Compuestas

Compuestas

Sipindáceas

Sajpindáceas

Pedalíneas.

Leguminosas...

Ficoideas.

Gramíneas.

Cucurbiláceas ...

Malváceas...

Milváceas ...

Malváceas

Malváceas

Malvácens

Compuestas

Cariofileas

Rutáceas

Simarrubáceas.....

Monimiáceas ........

Crucíferas.

Grucíferas

Grucíferas....

Irideas...

Umbelífelas.

Liliáceas.

Liliáceas

Liliáceas.

Liliáceas.

Liliáceas.

Liliáceas

Liliáceas

Solanáccas.........

Solanáceas ..........

Solanáceas ..........

Solanáceas.........

Solanáceas ..........

Solanáceas ........

Solanáceas

Solanácens

Solanáceas

Solanáceas

Solanáceas .........
Peyote. Piote.

Mataril. Matarique. Pichichagua.

Rosa de San Juan.

Güereña. Matarique.

Peyote cimarrón.

Moradilla.

Peyote.

Palo loco. 'Tezcapatli.

Burro.

Jara. Jaral amarillo. Jarilla.

Pabanillo.

-Hediondilla.

Rabanillo.

Calomópotl. Palancapatli.

Damiana.

Cuahuhuecatl. Quaumécatl. Quirote-culebra.

Contra ranilla de bestias. Nueve lıojas.

Ajonjolí.

Zacale de aguil.

Cenicilla.

Zacale sedoso.

Acarino. Chayolillo.

Malvavisco.

Vara de San Joaquín.

Axocatzin. Huinar. Huinari.

Violeta de México.

Chichibé.

Siegesbequia.

Pipiloxóchill.

Cedrón de Oaxaca.

Palo ịulato de Córdoba.

Limoncillo.

Pamita.

Berros.

Shorure.

Zacate de la muela.

Barrera. Berro. Berros.

Mecapatli. Zarzaparrilla.

Cocolmecan. Cozolmécatl. Olcacatzín. Palıcas. Raíz de China.

Zarzaparrilla.

Nanahuapalle.

Nanahuapatli. Quauhmecapatli. Zirzi. Zarzaparrilla.

Bejuco de chiquilıuite.

Cocolmecan. Cocolmécatl. China.

Cuítaqua.

Gopa de oro. Tecomaxóclill.

Hierba de rosa.

Candelero.

Papas cimarronas. Patalas cimarronas.

Hierba del perro.

Hierba del perro.

Acayocahuiztle. Ayolunislle. Quiebri-plato. Vacquerillo.

Vaquerillo.

Dulcamara. Flor de gloria. Gloria. Gúa de Jazmincillo.

Trompillo. 
Solanum esculentum Dun

Solanum hernandezii Moc. el Sessé..

Solanum macrophyllum Dun

Solanum mammosum L...

Solanum muricatum Ait. var.

Solanum nigrum L

Solanum ovigerum Dun

Solanum pseudocapsicum L

Solanum rostratum Dun

Solanum tectum Pers

Solanum torvum Sw

Solanum tuberiferum Don

Solanum tuberosum L

Solanum, sp?

Solanum, $\mathrm{sp}$ ?

Solanum, sp?....

Solanum, sp?

Solanum, sp?

Solanum, sp?

Solidago mexicana L

Solidago paniculata DC..

Solidago velutina $\mathrm{DC}$

Solidago virga aurea DG

Solidago, sp?

Solidago, sp?

Solidago, sp?.

Sonchus ciliatus L.

Sonchus oleraceus L

Sophora secundiflora Lag.

Sorghum saccharatum Pers

Spartium junceum L....

Spathiphyllum, sp?

Spermacoce diversifolia H. B. K

Sphæralcea angustifolia St. Hil...

Spigelia anthelmia L

Spigelia longiflora Mart. et Gal.

Spilanthes becabunga DG.

Spilanthes corymbosus Moc. et Sessé...........

Spilanthes mutisii H. B. K

Spinacia oleracea I

Spiranthes aurantiacus Hemsl...

Spiranthes cinnabarinus Hemst.

Spiræa hypericifolia DG

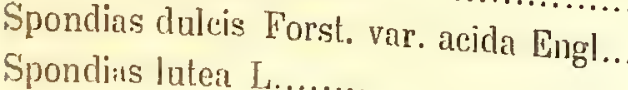

Spondias purpurea L.

Spondias, $s p$ ?

Spondias, $\mathrm{sp}$ ?

Spondins, sp?
Solanáceas......... Berengena.

Solanáceas......... Huitztomatzín.

Solanáceas......... Tzopilotláquatl.

Solanáceas......... Berenjenita peludita.

Solanáceas......... Pepino de maceta.

Solanáceas ......... Chichiquelite. Chichiquílitl. Hicrba mora. Solino nogro.

Thucupache xaqua. Tropilotláquatl.

Solanáceas ......... Flor de huevo. Maná.

Solanáceas......... Coral de jardines. Manzanila de amor.

Solanáceas......... Mala mujer.

Solanáceas .......... Tomate cinıarrón.

Solanáceas ......... Cinoca. Sosa.

Solanáceas......... Papas cimarronas. Patatas cimarronas.

Solanáceas........ Papa. Papas. Patata ó Patalas.

Solanáceas.......... Chinchiligua.

Solanáceas ......... Hierba del buey.

Sol.náceas......... Hierba del zopilote.

Solanáceas......... Pitzequa.

Solináceas......... Tinguarn.

Solanáceas......... Vaquerillo.

Compuestas ........ Calancapatle. Cislincapalli. Lechuguilla. Nanahuapalli. Palancapatli.

Compuestas ........ Amamalócoll.

Compuestas........ Calancapatle. Escobilla. Lechuguilla. Niınalıuapatli. Palancapatli. Perú cinnarrón.

Compuestas........ Vara de oro.

Compueslas........ Cineraria azul.

Compuestas........ Cineraria común. Gordolobo de la siurriı. Gulajaje.

Compuestas........ Huaxaten.

Compuestas........ Achicoria iulce. Chichicaquilill. Lechnguilla. Tlamatzalin.

Compuestas....... Achicoria dulce. Chichicaquílitl. Leclugruilla. Mitiluaaxaqua. Tlamatzaliı.

Leguminosas........ Frijolillo.

Gramíneas........ Maíz de Cihina.

leguminosis....... Retama delgadia ó de Ciluiniı. Relimma de escolnas.

Aroideas........... Hierba del manso.

Rubiácens........... Polígala filsa.

Malváceas .......... Hierba del negro. Tlilxihuill.

Loganiáceas........ Lombricera blanca.

Loganiáceas........ Hierba del burro. Raíz de yorba del burro. Sangre de loro.

Compuestas ........ Botón de oro. Perilre. Tripa de pollo.

Compuestas ........ Limpia tunas. Vara cenizat.

Compuestas ........ Grano de oro.

Quenopodiáceas... Espinaca.

Orquideas ........... Cutzis.

Orejuídeas ........... Cutzis.

Rosáceas ........... Rosa japonesa.

Anacardiáceas ...... Cirial. Cirueto. Mo!nbiı. Anacardiáceas ...... Cirnela amarillo. Costilxócoti. Ilobo. Jolyo. Mondo espi-

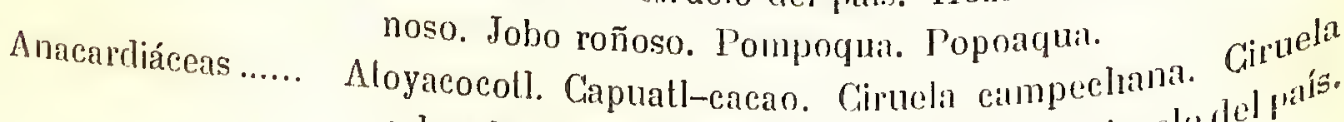
colorada. Ciruela roja. Cirucho de México. Ciruelo del láto Liruelo rojo. Costixocoll. Cimpr. IIobo. Jolno.

Anacardiáceas ...... Ciruela.

Anacardiáceas ..... Ciruelo cumarillo de Cuba.

Anacardiáceas ..... Cirmelo chico. 
Spondias, sp?

Sporobolus wrightii Munro.....

Sprekelia formosissima Herl)

Stanhopea oculata Lindl...

Stanhopea ligrina Batem

Stegnosperına halimifolia Bentlı...

Stenanthimm frigidum Kuntlı...

Sterculia carthagenensis Cav

Sterculia, sp?.

Stevia clinopodia DC.

Stevia salic.ifolia Cav.

Stevia viscida H. B. K.

Stevia, sp?.

Stevia, sp?..

Sticla pulmonacea Aclı....

Stigmaphyllon humboldtianmm Ad. Juss.

Stipa jarava Beauv.

Strelitzia reginn Ait

Stryehnos triplinervia Mar'...

Slyrax argenteun Presl.

Styrax ramirezii Greenm.

Suæda diffusa Wats

Sureda torreyana Wats.

Swietenia luumilis Zucc

Swictenia mahogani L.

Swietenia, sp?.

Sivictenia, sp?.

Symphoricarpus microphyllus H. B. K...

Symplocos citrea

Symplocos martinicensis Jacq

Symplocos, sp?

Symplocos, sp?

Symplocos, sp?
Anacardiáceas ...... Jobo liso.

Gramíneas.......... Zacatón.

Amarilídeas........ Amacayo, Atzcalxóchitl. Azcalxóchitl. riruz. Flor de Mayoo.

Flor de Santiago. Venera de Santiago. Zagalejo.

Orquídeas.

Orquídeas

Torito.

Fitolacáccas........ Amole.

Liliáceas ............ Cebadilla. Cebolleja.

Esterculiáceas..... Bellota.

Esterculiáceas ...... Dongón. Dungón.

Compuestas ........ Roselina. Rosillita.

Compuestas ........ Jarilla. Zazale de olor.

Compuestas ........ Hierba de la pulga. Mala pulgas.

Compuestas ......... Hierba del zopilote.

Compuestas ........ Pesto.

Liquenes ........... Pulmonaria.

Malpigiáceas ....... Chinaca.

Gramineas.......... Sumic-Ichu. Yurac-Ichlıu.

Nusáceas........... Pájaro azul.

Loganiáceas........ Cabalonga. Cabalonga de Tiabseo.

Estiráceas........... Chilacuate.

Estiráceas........... Chilacuale.

Quenopodiáceas... Jaujas.

Quenopodiáceas... Romerillo. Romeritos.

Meliáceas ........... Gateado. Flor de renadillo.

Teliáceas.......... Caoba. Caobo. Rosadillo. Tezopilotlzontecómatl. Tropilotlzontecómatl. Zopiloquáhuitl. Zopilote. Zopilotl.

Meliáccas........... Zopilote colorado.

Meliáceas........... Zopilote negro.

Caprifoliáceas...... Perlilla. Perlitas.

Estiráceas........... Jaboncillo.

Estiráceas.......... Garrapata. Garrapatilla. Limoncillo.

Estiráceas......... Limoncillo amarillo.

Estiráceas......... Limoncillo blanco.

Tstirácess......... Limoncillo coloratlo.
Tabebıia guayacan Hemsl..............

Tabebuia leucoxyla DC...

Tabebuia palmeri Rose.

Tabebuia pentaphylla Hemsl...

Tabebuia rosea DC:

Tabernæmontana acapulcensis Miers....

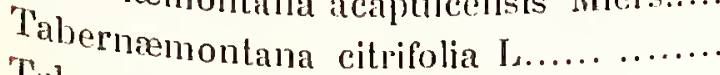

Tibernemontana grandiflora Jacq....

Tabernemontana litoralis H. B. R.

Tabernæenontana, sp?

Tabernemonlana, sp?
Bignoniáceas.

Bignoniáceas.......

Bignoniáceas.......

Bignoniáceas.. ....

Bignoniáceas

Apocinácens.

Apocináceas.

Apocináceas

Apocináceas...

Apocináceas

Apocináceas........
Guayacán.

Maculiz. Maculiz priel.o. Zapote.

Amapa.

Vaquile. Roble blanco. Roble blanco de San Luis. Roble de

San Luis.

Palo de rosa.

Tuevos de toro. Jazmín de perro.

Sictillo.

Lecherillo.

Sicte. Sictillo.

Chapón.

Rosa. 
Tagetes erechi, L

Tagetes lucirla Cinv....

Tagetes lunuliata Orl...

Tagetes micranth:ı Giv

Tigetes multiseta DC:

Tagetes palula L.

Tagetes pusil'a H. B. K.

Tagetes, sp?

Tirgetes, sp?

Tagetes, sp?

Talauma macrocaria Zucc.

Talauma mexicanil Don....

Tamarindus indica 1

Taraxacum officinale WVigg.

Taxodinm mueronatum Ten...

Teconna mollis H. B. K.

Tecoma radicans Juss

Tecoma sambucifolia H. B. K

Tecoma stans Juss.

'Tecoma viminalis H. B. K.

Tecoma, sp?

Tecoma, sp?

Tecoma, sp?

'Tecoma, sp?

Tecoma, sp?.

Tephrosia toxicaria Pers

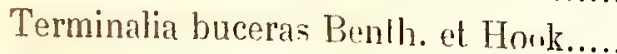

'Terminalia catappa I,

Terminalia excelsa Liebm.

Ternstromia altamininia Schiede.

Ter'nstr'emia sylvatica (:I. et Schl....

Ternstrøenia tepezapote ${ }^{\circ} \mathrm{Ch}$. et Schl.....

Ternstrœmia, sp?

Tetracera erecta Moc. et Sessé.

Tetracera ovalifolia DC.

Tetracera volubilis L....

Thalia geniculata $\mathrm{I}$.

Thalictrum hernandezii Tausch....

Thenardia florifunda H. B. K ....

Theobroma angustifolia DC.

Theobroma bicolor Humb. et Ponjs......

Theobroma cacao L

Theobroma ovalifolia DC.

Tluespesia populnea Cors.

'I'hevetia nerifolia Juss

Thevetia ovata A. DC...

Thevelia yoyolli A. DC:

Thuibergia alala Hook
Compnestas.

Compuestas

Compuestas

Compuestas

Compuestas.

Compuesias

Compuestas

Compueslas.

Compuestas

Compueslas

Magnoliáceas

Magnoliáceas

Leguminosas

Compuestas.

Coníferas

Bignoniáceas

Bignoniáceas

Bignoniáceas

Bignoniáceas

Bignoniáceas

Bignoniáceds...

Bignoniáceas ...

Bignoniáceas

Bignoniáceas

Bionnnliáceas

Leguminosas

Combretáceas

Combreláceas .....

Ciombretáceas

Ternstrœmiáceas..

Ternstrœmiáceas..

Ternstrœmiáceas..

'Ternstrœmiáciras..

Dileniáceas

Dileniáceas

Dileniáceas

Zingiberáceas.

Ranunculáceas...

Apocináceas.

Esterculiácens.....

Esterculiácens .....

Esterculiáceas .......

Esterculiáceas

Malváceas

Apocináceas

Apocináceas.

Apocinácens.

Acantáceas
Apátzequa. Compaxúchill. Cempazúchil. Cempoalxóchitl. Clavel de las Indias. Cilavelón. Copetes. Sempasńchil. Tepezempoalxóchill. Zempaxúchill. Zempoalxóchitl.

Guhrúcumin. Falso liipericón. Flor de Siunta María. Irierba añil. Hierba de Santa María. Periquillo.

Cinco Ilagas. Flor de las cinco lligras.

Anisillo.

Cempazúchil chiquito.

Clemole. Cilemolilos. Exprujuj. Iscoque. Pastora. Pissloral. Pastoreilla. Tlemole. Themolilos. Zempoala.

Anisillo.

Aceitilla.

Tlapatecrcáyall.

Xpuluue.

Corleza de yoloxóchitl. Yoloxóchill.

Flor del corazón. Hicrha de lis mitaduras. Mntilluma. Lanrel tulipán. Magnolia. Yoloxóchill.

Hoaxinus. Tamarindo.

Achicoria amarga. Cierrija. Diente de león.

Ahoéhuetl. Ahuchuete. Ahuehnetl. (:iprés de Moctezmma.

Pentamón. Pentamu. Sabinio. Sabino de Oaxica.

Flor de San Pedro.

Monapeslo.

Xkanlol.

Bignonia. Borla de San Pello. Huachacita. Iehculili. Mazorca. Nixtamaxóchitl. Tromadores.

Sabino de arrojos.

Agiirjote. Ahuejole.

Flor de día.

Macuile.

Palo marleria.

Palo de rosa.

Barbaseo.

Pucté.

Almendra.

Pucté.

Hierba del cura.

Hierba del cura.

Ulerba del cura.

Limoncillo de Mextillán.

Tachicón.

Bejuco de tachicón. Tachicón de sabana.

Bejuco re tachicón.

Kentó.

Cozlicpatli.

Pelalillo.

Ciacao. Cacaoquálhuill.

Pilaste ó Pataxle.

Cacao. Cacaorpuáhnill.

Cacao,

Majahua.

Acitz. Campanilla. Naranjo anarillo. Yoyote. Yoyolli.

Naranjo amarillo. Narciso amarillo. Yoyole. Yoyolli.

C'abrilo. Godo de fraile. Hueso de fraile. Narciso amarillo.

T'zinacanytlácuatl. Yoyole. Yoyolli.

Oio de Venus. 
Thuya occidentalis L...

Thlyınus vulgatris I

Tigridia dugesii Wats.

Tigridia pavonia Ker.

Tilia heleroplyylia Vent.

Tilia mexicana Benth...

Tilia Inexicana Sehl.

Tillandsia brachycaulos Schl

Tillandsia limbata? Sclıl

Tillandsia recurvata $]$

Tillandsia usneoides [.

'Tillandsia, sp?.

'Tinantia fugax s.heidw

Tithonia lagetiflora Desf.

'Tithonia tubæformis Cass

'Tilhonia, sp?...

'tournefortia hirsutissima L.

Tourneforlia mexicana Vatke...

Tourneforlia, sp??

Tourneforlia, sp??

Traclielospermum stans A. Gr....

Tradescantia geniculata Jacq.

'Tragopogon porrifolium L.

Trema micrantha Bl...

Trianosperma attenuata? Hemsl...

Trianosperma racemosa? Hemsl

Trianthema monogyna L...

Tribulus cistoides

Trichilin cistoides L.......................

Triel spondioides Swartz....

Tridax trifida A. Gr.

Trifolium arvense I

'Triticum repens $\mathrm{L}$....

Triticum vula

gare Villars. vars...

Tritoma uvaria Ker.

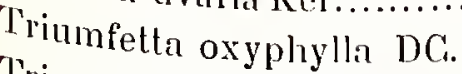

Triumfetla semitriloba $L$

'Tliumfetta, sp?

Trixis angustifolia DC.

Trixis cal)reriana Schaffn

Trixis corymbosi Don.

Tropieolım majus L.

Tropreolum peregrinum I

Prophis americana L.

'Prophis mexicana Bureau.

Tulipa gesneriana L.

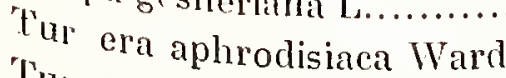

'Turnera humifusa Endl...

'Turnera ulmifolia

Typha angustifolia

Typlar latifolia
Coniferas...........

Labiadas

Irideas

Irideas

Irideas

Iridleas

Iridcas

Bromeliáceas

Bromeliáceas

Bromelińcéts

Bromeliáceas

Bromeliáceas ....

Commelináceis.....

Compueslas

Compuestas

Compuestas

Borlagíneas

Bolragineas....

Borragineas.

Borragíneas

Apocináceas

Commelináceas....

(ompuestas.

Urticáceas ............

Cucurbiláceas ...

Cucurbitáceas ......

Ficoideas ............

Zigofileas...

Meliáceas

Labiatdas....

Compuestas

Leguminosas........

Gramíneas.

Gramíneas.

Liliáceas

Tiliáceas.

T'iliáceas .

Tiliáceas.....

Compuestas

Compuestas.

Compuestas

Geraniáceas........

Geraniáceas.........

Urticáceas..........

Urticáceas ..........

Jiliáceas...........

'Tuneráceas.........

Turneráceas........

Turneráceas.........

Tifáceas.... ........

Tifáceas....
Tuya.

Tomillo.

Salıuiqui.

Cacomite. Flor del tigre. Hierba de la Trinidad. Oceloxóchitl. Trinitaria. Xahuiqui.

Sirinı.

Cirimo. Tila. Tilia.

Tilia. Tiliı. Tilo sirimo. Tírimo.

Gallitos.

Flor del incienso. Saripara.

Agave palo. Gallitos. Heno pequeño. Magueyitos parásitos. Salvagina macho.

Biuba española. Heno. Heno largo. Pastle. Patzueni. Salvagina. Tícari.

Flor de encino.

Felvira. Hierba del pollo. Plateido.

Acaute de flor naranjada.

Acalunale. Acaute.

Arnica.

Orliga de hoja grande. Ortiguilla.

Tlachichinoa. Tlepatli.

Hierba del mulato.

Hachichinoa. Patlahuac.

Hierba de la cucaracha.

Hicrba del pollo. Malaliz.

Salsifi. Salsifis.

Ixpepe.

Aqualacti.

Ahuichichi. Totolonchi.

Verdoliga blanca.

Abrojo de tierra caliente. Chanxnuc.

Cabo de hacha.

Romero del pais.

Molitas de liı playa.

Tréhol.

Acazacahuistli.

Trigo.

Tritonia.

Cajete.

Cadillo. Huizapotillo.

Giiizapol de borrego.

Falsa árnica. Plumilla.

Pichına. Pichaguilla.

Matatera.

Capuchina. Cuitziquiendas. Mastuerzo. Pelón. Pelonchili. Pelonmexixquílitl.

Pajaritos. Palomillis.

Ramón. Ramoncillo.

Ramón.

Tulipán.

Damiana de California.

Damiana.

Marilópez.

Espadaña.

Espadiña. Maza de agua. 


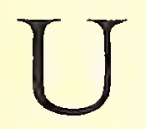

\begin{tabular}{|c|c|c|}
\hline Ulmus mexicana Planch................... & Urticáceas ........... & Cempoaleliuatl. Negrillo. Olmo. Pipalote. \\
\hline Urera caracasana Griseb.... & Urticáceas ............ & Ortiga. \\
\hline Urtica dioica $L$. var......... & Urticáceas ........... & Dominguilla. Ortiga. Solimán. \\
\hline Urtica mexicana Liebm...... & Urticáceas........... & Yesgos. \\
\hline Urtica urens L............................. & Uılicáceaș ........... & Aparequa. Ortiga pequeñil. \\
\hline Urtica, sp?............... & Urticáceas ........... & Ortiguilla. \\
\hline Usnea barbata L.......... & Líquenes ............ & Barbas de vicjo. \\
\hline Ustilago maydis DC....... & Hongos............... & $\begin{array}{l}\text { Cuernecillo de maíz. Cuitlacoche. Huillacoche. Hustlacoche. } \\
\text { Popoiol. Tizón de máx. }\end{array}$ \\
\hline
\end{tabular}

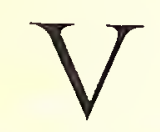

Vaccinium micranthum Dun.

Valeriana ceratophylla H. B. K

Vacciniáceas.

Cahuichi. Cahuitzi.

Valeriana mexicana DC.

Valerianeas ........ Jícama contra el tifo. Raíz del oso. Valeriana.

Valeriana tolucana DC.

Valerianeas ........ Hierba del gato. Raíz del gato. Valeriana.

Valeriana, sp?.

Valerianeas ....... Canónigos. Hierba del galo. Mazaletes. Ucuares. Vauar.

Vanilla planifolia Andrews

Valerianeas

'Teperonxóchitl.

Vanilla pompona Schierle.

Orquídeas

Orquídeas .

Flor negra. Vainilla.

Vanilla sativa Schiede.

Vanilla sylvestris Schiede.......

Orquídeas

Verbena andrieuxii Schaner.

Orquídeas

Plátano vainilla. Segue xeuté. Vainilla boba. Vainilla pompona. Vainillón.

Verbena canescens H. P. K

Verbenáceas

'Tilixóchitl. Tlilxóchill. Vainilla escarchıdil, Vainilla mansa.

Verbena caroliniana L

Verbenáceas.

Vainilla cimarrona.

Verbena ciliata Bentl.

Verbenáceas....

Verbena de Puebla.

Verbena corymbosa R. et Pav

Verbenáceas.

Verbenáccas...

Verbena de Gunnajuato.

Verbena.

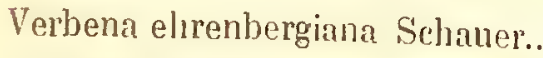

Verbenáceas....

Alfombrilla del campo. Verlona.

Verbena officinalis I

Verbenáceas.

Alfombrilla de jardín.

Verbena teucriifolia Mart. et Gal

Verbenáceas....

Verbena trifida H. B. K.

Verbenáceas....

Verbena.

Verbena, sp?.

Verbenáceas....

Verbena, sp?

Verbenáceas....

Verbesina alata L.

Compuestas.

Verbesina capitaneja Nees.

Compuestas

Verbesina crocala Less.

Compuestas

Verbesina pinnatifida (av. . .

Compuestas

Verbesina serrata Cav

Compuestas

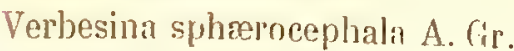

Verbesina virgatra Cav

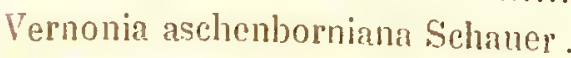

Compuestas

Vernonia karwinskiana DC.

Compuestas

Comprestas

Verbena.

Verbena de Orizaba.

Cierrón.

Hierba de Sin Juan.

Palo de la vida.

Capitaneja.

Capitaneja. Capilaneja de Tonila.

Capitaneja. Nalınitiput.

Capitana. Huichín.

Palo cenizo.

Cípilaneja.

Gordolobo del monte. Ocolillo.

Vara gruesa de San Miguel.

Vara de San Franciseo. 
Vernonia salicifolia Schz

Vernonia schiedeana Less

Vernonia serratuloides H. B. K.

Veronica peregrina L.

Veronica speciosa Hook.

Veronica spicata L.

Viburnum elatum Benth.

Viburnum glabratum H. B. K.

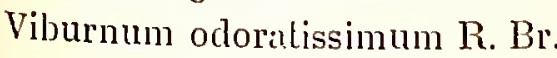

Viburnum prunifolium L.

Viburnum tinus L

Vicia faba $L$

Vicia sativa L.

Viguiera excelsa Benth. et Hook ..........

Viguiera linearis Schz.

Vinca minor L

Vinca rosea L.

Viola odorata L

Viola tricolor L. var.

Vitex mollis H. B. K

Vitis acapulcensis H. B. K.

Vitis caribae DG.

Vitis incisa Nutt.

Vitis indica L

Vitis quinquefolia Michx.

Vitis rotundifolia Michx.

Vitis sicyoides Baker.

Vitis tiliacea H. B. K.

Vitis tiliæefolia Willd...

Vitis tuberosa DC...

Vitis vinifera L. val...

Vitis, sp?.....

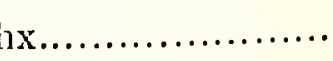

.....................

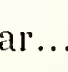

.
Compuestas

Compuestas

Compuestas.

Escrofularineas...

Escrofularíneas...

Escrofularíneas....

Gaprifoliáceas...

Caprifoliáceas.....

Caprifoliáceas..

Caprifoliáceas

Caprifoliáceas.

Leguminosas

Leguminosas........

Compuestas.

Compuestas.

Apocináceas...

Ayocináceas.

Violarieas.

Violarieas.

Verbenáceas

Ampelídeas

Anıpelídeas...

Ampelídeas

Ampelídeas

Ampelídeas

Ampelídeas

Ampelídeas

Ampelídeas

Ampelídeas

Ampelídeas

Ampelídeas

Ampelídeas
Ahuitule.

Flor de borla.

Vara de San Francisco. Vara de San Miguel.

Verónica.

Verónica de jardines.

Nueva reforma. Verónica.

Tlamahuácatl.

Achichil. Sundá.

Sardonia.

Chinchín.

Viburno.

Haba. Haba amarilla. Haba blanca mediana. Haba cochinela amarilla. Veza.

Algarrobilla. Jara amarga.

Raiz del manso.

Romerillo.

Cieló riso. Vinca. Vinca pervinca.

Maravilla de España.

Hierba del aguardiente. Violeta española. Violeta extranje-

ra. Violeta de jardín. Violeta de olor.

Pensamiento. Trinitaria.

Ahuilote. Atulo. Coyolomate. Cuyotomate. Uvalano.

Vid silvestre.

Parra silvestre.

Hierba del buey.

Uva cimarrona.

Gualt.

Vid silvestre.

Bejuco loco. Parra silvestre. Vid silvestre.

Tripa de Judas. Tumba-vaqueros.

Uvilla cimarrona.

Coral de Colima.

Parra. Pasas. Uva. Vid.

Siruri.
Wedelia hispida H. B. K

Wigandia caracasana H. B. K

Wigandia kunthii Choisy

Wigandia scorpioides Choisy

Wigandia urens Choisy.
Compuestas

Hidrofileas.

Hidrofileas

Hidrofileas.

Hidrofileas...
Clemolillo.

Ortiga de tierra caliente.

Ortiga grande. Ortiga de tierra caliente. Sosa. Tabaco cimarrón.

Consuelda mayor. Ortiga de tierra caliente. Ortiga de tierra caliente.

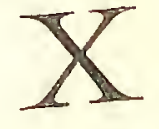

Xanthium strumarium L

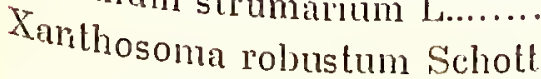

Compuestas
Aroideas. ..
Abrojo. Arámicua. Cacalacacaxtli. Carámiqua. Mafafa. 
Yucca aloifolia $L$

Yucra baccata Torr.

Yucca filamentosa L....

Yucca filifera Chabaud

Yucca treculeana Carr.
Liliáccas

Liliáceas

Liliácca.s.

Liliáceas

Liliáccas.
Iczotli. Izote. Yuca.

Amole. Izote. Palma criolla.

Izote. Izotl. Yuca.

Palma loca.

Isote. Izote. Izotl.
Zaluzania angusta Schz

Zamia spartea A. DC.

Zanthoxylon affine H. B. K.

Zanthoxylon pentanome DC

Zanthoxylon pterota L...

Zanthoxylon, sp?

Zanthoxylon, sp?

Zea mays L. var.

Zephyranthes carinata Herb.

Zingiber officinalc Roscoc.

Zinnia elegans Jacq.

Zinnia linearis Benth.

Zinnia multifiora L

Zinnia pauciflora $\mathrm{L}$

Zinnia tenuiflora Jacq.

Zizyphus mexicana Rose.

Zornia diphylla Pers...

Zornia tetraphylla? Michx.

Zygadenus mexicanus Hemsl
Amarilídeas

Zingiberáccas

Compuestas

Compuestas

Compuestas

Compuestas

Compuestas

Ramneas

Leguminosas.

Leguminosas.

Liliáccas.

\section{Z}

Cenicilla. Limpia tunas.

Trukix.

- Palo mulato de Miclıoncálı.

. Palo mulato de MÍ́xico.

Colima. Palo mulato.

. Flor de encinillo.

Palo mulato de Mazatlán.

Detha. Maíz. Maíz amarillo, apastillado, blanco ancho, cacahuazintli, colorado, cruzado, cruzado fino, chalcayote, chino, dulce, de clote rojo, forrado, liso, mala cara, momochite, pepitilla cruzado, pinto, prieto, rojo, temitzi amarillo, temitzi blanco, tolonti blanco, tolonti rojo, veteado. Milpa. Tlaolli. Tlauli. Tziri. Atzcalxóchitl. Flor de Mayo.

Ancoas. Gengibre. Jengibre. Labitz. Sillii.

Mal de ojo. Pecetas. Virginia.

Hierba del toro.

Virginia.

Malojo.

Mal de ojo.

Amole.

Raíz de la víbora.

Viperina.

Cebadilla. 


\section{APÉNDICE.}

Por motivos que se explican en el Prólogo de esta obra, se omitieron en la primera y segunda parte muchos de los nombres vulgares, con sus respectivas clasificaciones botánicas, consignados en las memorias de los Sres. Coulter, Watson, Rose, Seler, Brandegee, Conzatti y Smith, que se habían acopiado con objeto de completar nuestra sinonimia.

En el curso de la impresión, que ha sido muy lenta, fué posible reunir olra vez aquellos nombres que, con otros, recogidos posteriormente, con las nuevas clasificaciones hechas en el Instiluto Médico y, con los que se rectifican de los ya publicados, forman bastante material para que merezca se agregue como apéndice.

Con esta adición, suponemos que ya pocos nombres quedarán omitidos, de los que corresponden á clasificaciones seguras, y que se puede considerar casi como completa la sinonimia vulgar y científica de las plantas mexicanas.

En este apéndice se ha seguido el misno orden, sin variación alguna, que el que se aceptó en la primera y segunda parte de la obra.

José Ramíres.

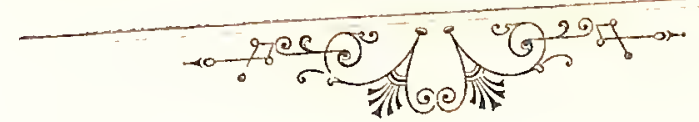




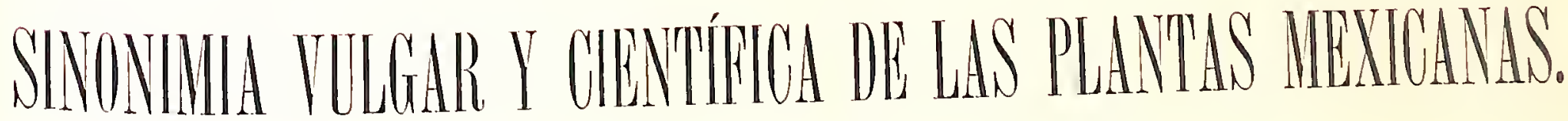

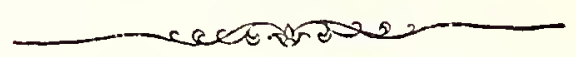

\section{APIÉNDICE Á I.A PRIMERA PARTE.}

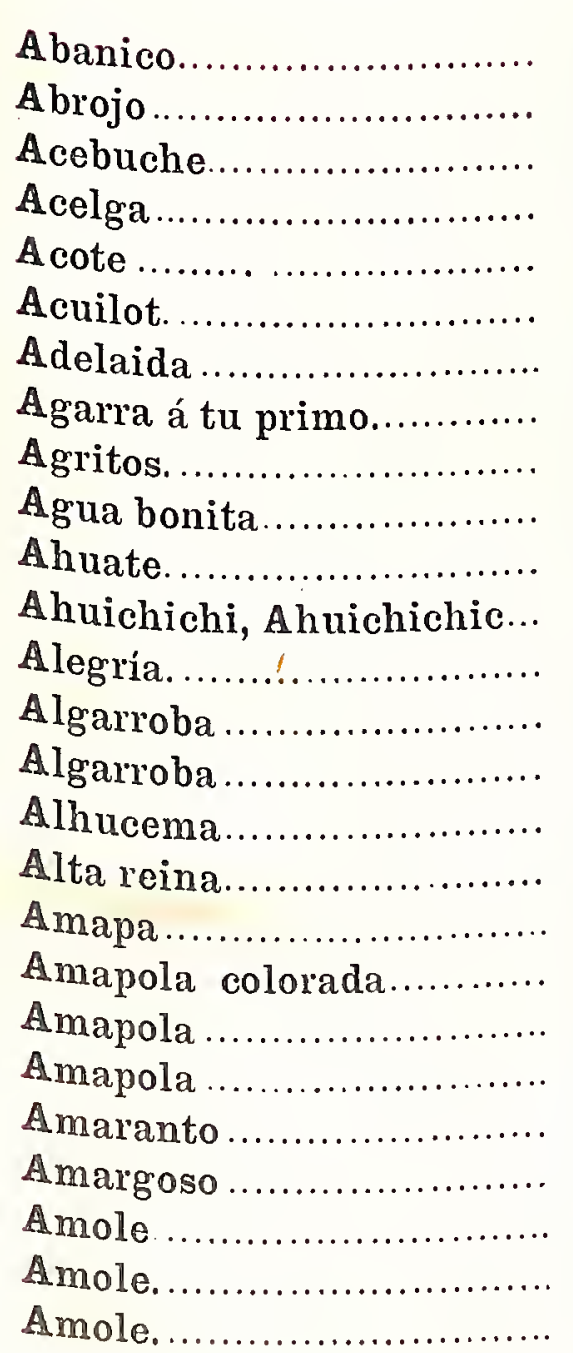

Atriplex, sp? Triunfetta scmitriloba L.

Forestiera sp. nov.

Beta vulgaris Moq. var. Cicla.....

Convolvuláceas ...

Ipomoe wolcottiana Rose...........

Philadelphus mexicanus Schl......

Fuchsia corymbiflora Ruiz et Pav.

Solanum enoplocalyx Dum....

Begonia (Knesebeclia) discolor WV Gilia tenuifolia Gray....

Ruprechtia cumingii Meissn...... Cayriponia racemosa? Cogu. var. Amarentus leucospcrmus Watson. Acacia pennatuta Benth... Prosopis juliflora Dr:

Lavandula vera $\mathrm{D}$ (:.

Piqueria trimervia Cav

Tabcbuia palmeri Giay.

I'achime insigmis Savign.

P'assiftore foetida L. var.

Passifthina serratifolia L

Tumere ulmifolice $\mathrm{L}$....

C'tsetele micholsoni Hook.

Proclunganthes vivideseens. Walson Srpindus saponaria Linn.......... Stegnosperma halimifolia Benth...
Quenopodiáceas... Esterculiáceas

Oleáceas.

Quenopodiáceas ...

Convolvuláceas ....

Saxifragáceas.......

Solanáceas

Begoniáceas

Polemoniácens......

Poligonáceas ........

Cucurbitáceis .....

Amarantáceas......

Leguminosas.......

Leguminosas........

Labiadas............

Compueslas........

Bignoniáceas........

Malváceas ...........

Pasifloríceas........

Pasifloríceas.......

Turnerríceas........

Simarubáceas.....

Amarilícleis.

Simpindáceas ....

Fitolacáceas.
Onagrarieas........
Michoacán........ M. Tena.

Manzanillo...... Palmer y Rose.

Tacámbaro...... Rose, Ramírez.

Cultivada......... Alcocer.

Manzanillo...... Palmer y Rose.

Veracruz, Méx... Ramírez.

C (ullivadi......... A'cocer.

Tancanhuitz..... Seler.

Jalisco........... Palmer y Wats.

Bajı California... Brandegee.

O\%uluama......... Seler y Lœs.

Regiones temp. Alcocer.

Guadilajara..... Palmer y Wats.

Sonora........... Palmer y Rose.

Manzanillo...... Palmer y Rose.

Art de comercio Alcocer.

Ayacapixtla...... Seler y Loes.

Alamos, Sonola. Palmer y Rose.

Yucatán.......... Dondé.

Iruejutla.......... Seler y Los.

T'ancanhuil\%...... Seler y Los.

Tucatán.......... Dondé.

Río Crande..... Coulter.

Río Blinco, Jal.. Palmer y Wats.

Chihuihua...... Palmer y Wats.

Raja Califormia.. Palmer y Rose. 
Amole (raiz).

Amole (frutos)

Amole de raíz

Anana.

Anaqua

Anis, A. común, A. verde.

Anis estrellado ó de China

A vellana.

Arocopaconi.

Ayal.
Yucca baccata Torr

Zizyphus mexicana Rose.

Fourcroya, sp? .

Ananas sativus Schult

Ehretia elliptica DC.

Pimpinella anisum L.

Illicium anisatum $\mathrm{L}$....

Corylus avellane L. var.

Gantheric, sp?.

Crescentia alata H. B. K.
Liliáceas.

Ramneas

Amarilídeas

Bromeliáce as...

Borragíneas.

Umbelíferas.

Magnoliáceas

Cupuliferas.

Ericáceas

Bignoniáce ıs.
Jal., Fronl. N....

Armeria, Colima

Jalisco

Yucitán.

Río Grande......

Cullivada...

Art. de comercio

Art. de comercio

Pueblil....

Guaymas
Rose $d$ Coulter.

Palmer y Rose.

B. $T$. et Urbina.

Dondé.

Coulter.

Farm. Mex.

Palmer y Wats.
Balsamito

Barbasco....

Barbudilla..

Bejuco de agua.

Bejuco de caballo.

Bejuco colorado.

Belladona

Bequilla

Berengena.

Bicho.

Binorama. .

Biznada (biznaga),

Biznaga

Bizmaga.

Biznaga

Bledo .

Bola yaqui

Bolontibi.

Bonete

Bonete.

Brasil.

Brasil...

Buul (maya)
Myrospermum peruiferum $\mathrm{DC}$

Dorstenia drakena $\mathrm{L}$

Dorstenia crispata Watson.

Tetracera volubilis $\mathrm{L}$....

Petrea arborea H. B. K.

Hippocratea utilis Rose...

Atropa belladonna 1.

Sesbania nacrocarpa MI uhl. var....

Cyphomandra betacea Sendt.

Cassies sericea Swartz.

A cacia furnesiana Willd.

Echinocactus wislizeni lecontei Eng.

Echinocactus wislizeni lecontei Eng.

Echinocactus horizonthalonius Lem

Echinocactus wislizeni Engelm.....

Amarentus spinosus L.

Montanoa, sp?

Titis, sp?

Jacaratia mexicana A. DC

Pilers heptrphyllus Ram...

Conclalin obovuta Hook.

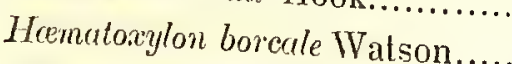

Phaseolus vulgaris 1.
Leguminosas........ Yucatán

Urticáceas ....

Urlicáceas .

Dileniáceas...

Verbenaceas

Hipocrileáceas....

Solanáceas

Leguninosas..

Solanáceas

Leguminosus.

Leguminosas.....

Cácteas

Cácteas

Cácteis

Cácleas

Amarantíceas

Compuestas

Ampelíileas

P'asifloráceas....

Pasifloráceis

Ramneas

Leguminosas...

Lerguminosas ...
Jalisco.

Río Blanco, Jal..

Manzanillo.......

Hucjulla...

1 :olomas, Sinaloa

Cultivardi....

Guaymas

Guadalijar

Manzanillo.

Guaymas...

B. Cal., Sonora..

B. Cill., Sonora..

El Paso, Chilı...

S. Gregorio, B. C.

Yucitán.

Alianos, Sonori.

Yucitán.

Yucitán.

Morelos..

Río Grande.....

Chill., Guaymas.

Yucillán...
Dondé, Ramı.

Palmer.

Palmer y Wats.

Palmer y Rose.

Seler y Los.

Rose.

Conzatti.

Palmer y Wals.

Palmer y Wals.

Palmer y Rose.

Palmer y Wils.

Coulter.

Couiter.

Coulter.

Biandegee.

Dondé.

Pilmer y Rose.

Dondé.

Doudé.

Ramírez.

Coulter.

Palmer y Wats.

Dondlé.

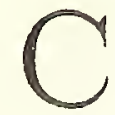

Cabalonga de la Iuasteca. Cabalsit (maya).

Cabello de angel.

Cabeza de viejo.

Cabeza de viejo

Cacachila.

Cacahuananche.

CacaloxóchitI.

Cacanaguasle.
Strychnos ignatti Ait

Commelina, sp?....

Calliandre houstoni

Cereus pectinatus rigidissimus Eng.

Cerens schotlii Engelm.

Karwinstiva humboldtiana Zuec...

Cliricidia lambii Fernald.

I'lumeria aculifolie Poir?

Apoplunesia qreniculata Pres]....
Loganiáceas.

Commeìnáceas...

Leguminosas.......

Cácteas.

Cácteas

Ramneas.

Leguminosas.

Apocináceas.

T.eguminosas.....
Costa del Golfo..

Yucalán

Ciudad del Maíz. Chihuahma, Son. B. Cal., S. L. P. Chilı., B. Cal.... Ros:, Sin., Tepic S.J. de Cabo, B.C Manzanillo.
Conzalti.

Dondé.

Seler y LœS.

Coulter.

Coulter.

Palmer y Wats.

Lamb. y Fern.

Brandegee.

Palmer y Rose. 
Cacomite

Café cimarrón.

Calabacilla....

Calabacilla loca

Calabazo

Caléndula

Callules?.

Cambustera

Camelote

Camichín.

Camote amarillo, c. blanco, c. morado...

Campanilla encarnada.....

Camuesa (tuna)

Canela

Canzuuc (maya).

Caña de javalí.

Cañoto

Capnlín....

Cardo

Cardo

Cardo santo

Cardón

Cardón.

Cardón pelón

Cascalote.

Castaña (frutos)

Catena

Cayules (coyoles)

Cebolleja.....

Cedro

Cempoalxóchitl cimarón.

Cerote.

Cicioin (maya).

Cidra cayote.

Ciis (maya).

Cilantrillo de pozo...... ....

Cihuapatli ?

Cirio.

Ciruela

Ciruela.

Ciruelo.

Clavo.....

Clusia.

Coacollutillo....

Coamecatl ó Cualmecatl...

Cochal (nombre de los inclios)

Cochinilla.....

Cohombro

Cojón de gato.

Colima

Confituria

Confiturea

Contrayerba

Copa de oro

Copal.

Copal.
Nemastylis versicolor Watson......

Cassic leiophylla Vog. var.

Cucurbita fotitlissima H. B. K.....

Cucurbita fetidissime II. B. K.....

Iagenaria vulgaris Ser. var........

Tirmera ulmifolia L.

Bignonia bicolor Watson.............

Ipomeer quemoclit L.

P'anienm mynrum Meyer....

Ficus fuseiculate Watson.

Ipomece butatas Lam. vars...

Datura sangninea Ruiz et Par....

Opuntia larreyi Weber.

Pluchea eamphorata DC..

Tititicum repens I.

Costus spicatus Swarlz.....

Pluragmites commumis 'Trin.........

Arclisia escallonioides Gh. et Schl.

Argemone mexicana L...

Argemone albiflora Hornem.......

Cnicus rhaphilepis Watson........

Cereus pringlei Watson.............

Cereus pecten-aboriginum Engelm.

Cerens calvus Engclm...

Pitherelobium (?)

Castanea vulguris Lam.

Heliocarpus americamus L...........

Begonia (Knesebeelia) bicolor IV.. Stenanthinm frigidum Kunllı...... Cedrela odorata L...

Tagetes patula L.

Viguiera buddleiceformis B. et H...

Mrenilot aipi Pohl....

Cueurbita ficifolic Bouché.

Gyroearpus jaequini Roxb...

Adiantum tricholepis Fúe.

Eupatorium odorctum L...

Fouquieria splendens Engelm.....

Cyrtocarpa proeera H. B. K........

Spondias mexicana IVatson.

Spondias purpuren I...

Pittosporum tobira $\Lambda$ it.

Chusin pulchella (?).

Mabu albens Hiern.

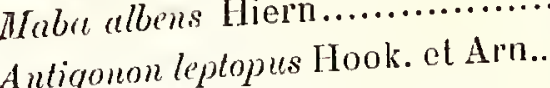

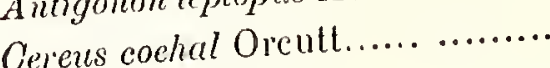

Pluchea borealis Gray.

Cucumis sativus L

Taberncmontuna paisavalensis $\mathrm{L}$ Zunthoxylon pterota H. B. K.......

Hyptis semanmi Gray...

Lantane cemara Limn.............. Euphorbire (Poinsetia) tuberosa R. Sulandra selere Dammer............ Bursera hinelsiana Engl.. Bursera lariflora Watson...
Irideas

Leguminosas....

Cucurbitáceas .....

Cucurbiláceas .....

Cucurbitaceas ......

Turneráceas........

Bignoniáceas.......

Convolvuláceas....

Gramíneas....

Urlicáceas....

Convolvuliceas.....

Solináceaıs..........

Cácteas....

Compuestas .........

Gramíneas.........

Zingiberáceas.......

Gramíneas.

Mlirsineas.

Palpaveráccas......

Paipilveráceas.......

Compueslas.

Ciácteas.

Cácteas.

Cáctcas.

Leguminosas........

Cupulíferas.

Tiliáceas.

Begoniáceas.....

Liliáceas .

Meliáceas............

Compuestas........

Compuestas.........

Euforbiáceas .........

Cucurbitáceas ......

Combreláceas .....

Helechos ...........

Compuestas........

Timariscincas .....

Anacardiácias .....

Anacardiáceas .....

Anacardiáceas .....

Pitosporeas........

Gulíferas............

Ebenáceas ..........

Poligonáceas........

Cácters..............

Compuestas ........

Cucurbiláceas ......

Apocináceas........

Rutáceas ............

Labiadis...........

Verbenáce:ıs ........

Euforbiáceas........

Solınáceas.........

Burseráccas .........

Burseráceas........
Río Blanco, Jal... Palmer y V Vats. Tancanhuitz..... Seler y Lœs.

Frontera Norte.. Coulter.

San Luis Potosí. Schaff. y Wats.

Gultivada......... Alcocer.

Yucatán.......... Dondé.

Jalisco............. Watson.

Yucatái........... Dondé.

Miehoacán........ Dodge.

Colima........... Palmer y Rose.

Gultivada......... Alcocer.

Oixaca............ Conzatli.

Querétaro........ IVeber.

I.S.Agueda. B.C. Vas., Rose.

Yucalán.......... Dondé.

Veracruz..........

Yucalán........... Dondé

Tancanhuitz...... Seler y Lœs.

Baja (alifornia... Vas., Rose.

Mulegé........... Palmer y Wats.

Gualalajara..... Palmer y Tiats.

I. S. Pedro, B.C. Palmer, Coult.

Chihuahua...... Palmer y Wals.

Baja California... Engelm., Coult.

Armeria, Colima Palmer y Rose.

Cullivada......... Alcocer.

Varios lugares... Dodge.

Guadalıjara...... Palmery Wats.

Guanajuato,Mćx.

Yucatán........... Dondé

Morclos........... Seler y Lœes.

Hidalgo ........... Villada.

Yucatán.......... Dondé.

Guadalajara...... Palmer y Wals.

Yucatán........... Dondé.

Tequila, Jalisco.. Palmer y Wals.

Tancanhuilz...... Seler y Locs.

El Rosario, B. C.. Brandegee.

Bajal California... Eisen.

'Tequila........... Palmer y Wats.

Colima........... Palmer y liose.

Cultivarla......... Aleocer.

Yucalán........... Dondé.

Acapulco........ Fernaldy l'alm

Varios lugares... Seler y Los.

Baja California... Coulter.

Río Grande...... Coulter.

Yucatán.......... Dundé.

Ozuluama........ Seler y Locs.

Río Grande..... Coulter.

Alamos, Sonora. Palmer y Rose.

Guaynas ......... Palmer y Wals.

Somora........... Palmer y Rose.

Cullivada........ Seler y Dam.

Isla Magd., B. C. Brandegee.

Guaymas ........ Palmer y Mats. 
Copalquien (Copalchi ?)..... Copó (maya)

Coquistle.

Cordoncillo

Cornezuelo cimarrón.........

Corpus.....

Costomate ó Coxtomatl.....

Coyolróchitl....

Crucita.

Cuanchalala, Cuanchalalate.

Cuatz chté gueeco

Cuchilxíu, Cuchilloxíu......

Cum (maya).
Veatchia discolor Benth.

Ficus mubiginosa Desf.

Nemastylis tenuis Benth. et Hook Elytraria tridentata Vahi

Jussiena suffruticosa L.

Jacquinia macrocarpa Cav.........

Plysalis pubescens L

Tecoma sp. aff T. pentaphylla Juss

Eupatorium odoratum L.

Dioscorea?

Asclepias longicornu Benth........

Asclepias curassavica L.

Cucurbita pepo I.
Liliáceas.

Urticáceas

Irideas

Acantáccas

Onagrarieas

Mirsineas.............

Solanáceas

Bignoniáceas.....

Compuestas ......

Dioscoreáceas......

Asclepiadeas...

Asclepiadoas.

Cucurbitáceas ....
I. Sta. Marg., B G Brandegee.

Yucatán........... Dondé.

Río Blanco, Jal.. Palmery Wats.

Guaymas, B. C... Pal., Wat., Vas.

Morelos ........... Seler y Los.

Ozuluama......... Seler y Loes.

Varios lugares... Pl. Nov. Hisp.

Huejulla.......... Seler y Locs.

Tancanhuitz..... Seler y Locs.

Oaxaca............ Frm. Mex. ant.

Oaxaca............ Seler y Lœs.

Yucatál........... Dondé.

Yucatán........... Dondé.

\section{$\mathrm{CH}$}

Chacah (maya).

Chacalhaas (maya).

Chacloco

Chacmol (maya).

Chacsinkin (maya).

Chaile.

Chala

Chalahuitl.

Chalate.

Chalché (maya)

Chana.

Chaparral.

Chapote amarillo (Zapote?)

Chi.....

Chía grande

Chicoria

Chicoyote

Chiican (Jicama, maya)....

Chilchaca.

Chilenola.

Chilicote.

Chilicoyote

Chinquapin

Chirinole.

Chiteuuc (maya).

Chom (maya)

Chote.

Choven

Choyer.

Chufas
Bursera gummifera Jacq.

Lucuma mammosa Gærln. fil.

Hrmelia palens Jacq

Gomphrena globosa L.

Ccesalpinia pulcherrima Sw.

Parthenium hysterophorus L

Ficus, sp?..

Inga ingoides Willd

Ficus, sp?

Eupalorium, s»?

Inyptis suacenlens Poit.

Condalire oborate Hook.

Sargentia greggii Watson

Malpighia glabra L.

Hyptis suaveolens Poit.

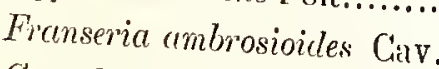

Cucurbita redicans Naud.

Pachyrlizus?

Galea axilluris DC

Cereus eruca Brandegee.

Erythrina coralloides DC.

Cucurbila foetidissima H. B. K....

Nelumbo lulea Pers

Cereus eruca Brandegee.

Cypripedium calceolus L.?

Bromelia pinguin $\mathrm{L}$.

Parmentierce edulis $\mathrm{DC}$

Mimosa asperata L.

Opuntia, sp?.

Cyperus eseutentus L.............
Burscráceas........ Yucalán........... Dondé.

Sapotáceas......... Yucatán........... Dondé.

Rubiáceas .......... Pánuco, Tam.... Seler y Locs.

Amarantáceas...... Yucatán.......... Dondé.

Leguminosas........ Yucalán........... Dondé.

Compuestas ......... Chih., Ver......... Seler y Los.

Urticáceas ............ Chilhuahua........ Palmer y Rose.

Leguminosas........ San Luis Potosí, Seler y Lœs.

Urlicáceas.. ....... Chihuahua....... Palm. y Wals.

Compuestas ......... Yucatán........... Dondé.

Labiadas........... Manzanillo, Col.. Palmer y Rose.

Ramneas...... ... Río Grande, Jal. Coulter.

Rutáceas ........... Monterrey ....... Gregg y Wats.

Malpigiáceas ........ Yucatán........... Dondé.

Labiadas............. Varios lugares... Palmer y Rose.

Compuestas ........ I. Ia Agueda, B.C Vasey, Rose.

Cucurbitáceas...... Sinaloa, Chih..... Palm. y Rose.

Leguminosas........ Yucalán........... Dondé.

Compuestas........ Trencanhuilz...... Seler y Loes.

Cácteas.............. I. Magd., B. C.... Brand., Coult.

Leguminosas........ (hihuihua...... Palm. y Wats.

Cucurbitáceas ..... Chihuilhua........ Palm. y Wals.

Ninfeáccas.......... Río Grande, Jal. Coulter.

Cácteas.............. Baja Cialifurnia... Brand., Coult.

Orquíd as.......... Yucalán........... Dondé.

Bromeliáceas ....... Yucalán........... Dondé.

Bignoniáceas........ Tancanhuilz...... Seler y Lœs.

Leguminosas......... Ozuluama......... Seler y Lœes

Cácteas ............. I. S. Pedro, B.C.

Ciperáceas.. ........ Yucatán..........
Paln. y Wats.

Donlé. 


Damiana...$\ldots \ldots \ldots \ldots \ldots \ldots \ldots$
Damiana .......................
Damiana .....................
Don Juan ...................
Doradilla...................
Doradilla ...................

Lippia fastigiata Brandegee. Turnera diffusa Willd....

Turnera sp. nov.

Nicotiana glauca Graham

Ceterach officinarum DC.

Selaginclla pilifera A. Braun.......
Verbenáceas....

Turneráceas....

Turneráceas.

Solanáceas.

Helechos

Selagineláceas......
I. S? Agueda, B.C Vasey, Rose. Baja California... Eisen. Michoacán....... Ramírez. Guaymas......... Palmer y Rose. Yucatán.......... Dondé. Guanajuato...... Dugès,

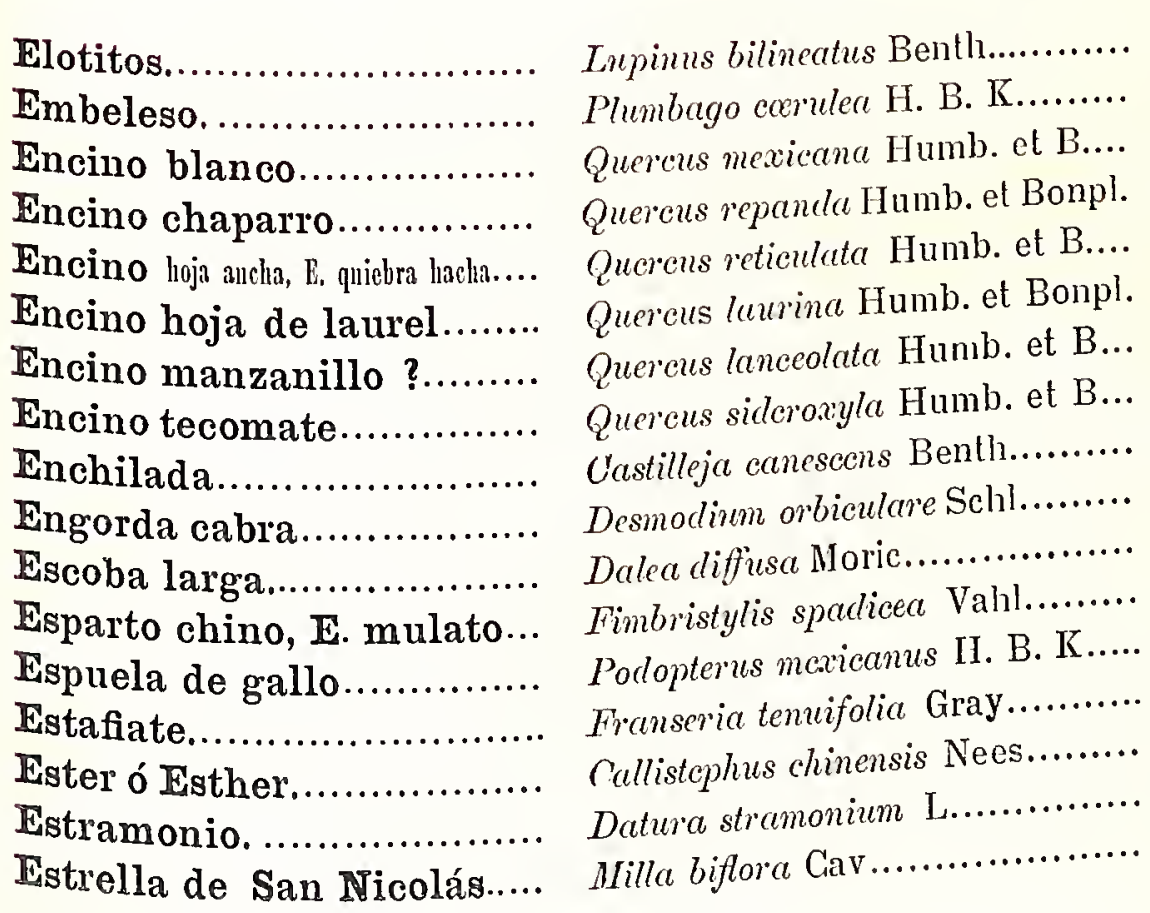

Elotitos.

Embeleso

Encino blanco

Encino chaparro.

Encino hoja ancha, E. quiebra lacka....

Encino hoja de laurel.

cino manzanillo

cino tecomate.

Espuela de gallo..

Estafiate.

Estramonio.

Fstrella de San Nicolás....
Leguminosas........

Plumbagíneas ......

Cupulíferas....

Cupulíferas.........

Cupulíferas.........

Cupuliferas..........

Cupuliferas..........

Gupulíferas..........

Escrofularíneas.....

Leguminosas.......

Leguminosas.......

Ciperáceas...........

Poligonáceas........

Compuestas

Compuestas

Solanáceas

Liliáceas.
Guanajuato ......

Yucatán.......... Dondé.

Hidalgo ........... Villada.

Hidalgo ........... Villada.

Hidalgo ........... Villada.

Hidalgo ........... Villada.

Hidalgo ........... Villada.

Hidalgo ........... Villada.

Guanajuato...... Dugès.

Guanajuato ...... Dugès.

Morelos ........... Seler y Lœs.

Veracruz......... Dodge.

Ozuluama......... Seler y Lœs.

Mulegé, B. C..... Palm. y Wats.

Cultivada......... Alcocer.

Yucatán.......... Dondé.

Guanajuato..... Dugès.

\section{F}

Farolito

Fiñisachi.

Flor de calavera

Flor de la chuparrosa....................

Flor de milpa.

Flor del muerto.

Flor de piedra.

Flor de sol.

Floripondio encarnado.....

Friega platos espinoso...

Frijol del monte

Frijolillo .
Solanáceas ..........

Leguminosas........

Orquídeas...........

Labiadas ...........

Escrofularíneas....

Asclepiadeas:........

Selagineláceas......

Compuestas .........

Solanáceas ..........

Solanáceas ..........

Leguminosas........

Leguminosas........
Yucatán........... Dondé.

Guanajuato ...... Dugès.

Guanajuato ...... Dugès, Alcocer.

Guanajuato...... Dugès.

Guanajuato ...... Dugès.

Guanajuato ...... Dugès.

Manzanillo....... Palmer, Rose.

Yucatán........... Dondé.

Oaxica........... Conzatti.

Tancanluitz...... Seler y Loes.

S.Miguelito, SLP Schaff. y Wats.

Morelos ........... Seler y Loes.
Solanum torvum Sw................

Phaseolus multiflorus Willd......... Tigna huteola Bentlu... 


\begin{tabular}{|c|c|}
\hline Gallinita & Hircea macroptera DG.............. \\
\hline Gallinita ...... & Tillandsia achyrostachys Ed. Mor. \\
\hline Gallitos........ & Tillandsia vestita Benth..... \\
\hline Gamalote.. & Fimbristylis spadicea Vahl.......... \\
\hline abatillo. . & Mimosa distachya Cav............... \\
\hline Garbancillo... & Astragalus humboldtii A. Gr........ \\
\hline atilla.. & Trichilia haranensis spatulata Ros. \\
\hline duna... & Tribulus maximus L.. \\
\hline Grama........ & Cathesticum erectum Vas. \& Hack.. \\
\hline a........... & hroides H. B. K.. \\
\hline ..... & Triticum repens L............... \\
\hline dina. & Dodonate riscosa L.......... \\
\hline ...... & Potamogeton pectinatus L..... \\
\hline Guacamote........................ & Manihot utilissima Pohl....... \\
\hline co mexicano.. & Aristolochia pardina Duch...... \\
\hline Guaco de San Cristóbal...... & Aristolochia pentandra I.......... \\
\hline Guaje, Guajito........ & Lagenaria vulgaris Ser. var..... \\
\hline теоиi & Maximowiczia sonore Watson... \\
\hline Guayacán. & Guaiacum angustifolium Engelm \\
\hline & Viscainoa geniculata Greene.... \\
\hline Guayule (huayule?). & Parthenium argentatum A. Gr... \\
\hline yun (ma) & Melicocea bijuga L. \\
\hline
\end{tabular}

Malpigiáceas.....

Bromeliáceas .......

Bromeliáceas .......

Ciperáceas...........

Leguminosas........

Leguminosas........

Meliáceas.

Zigofileas.

Gramíneas.... ......

Gramíneas.

Gramíneas.

Sapindáceas

Nayadáceas.

Euforbiáceas........

Aristoloquieas.....

Aristoloquieas...

Cucurbitáceas .....

Cucurbitáceas .....

Zigofileas

Zigofileas.

Compuestas

Sapindáceas
I. S: Agueda, B.G Vasey, Rose. Morelos.. ......... Suler y Loss. Guanajuato ...... Dugès. Veracruz......... Dodge. Oaxaca ............ Seler y Los. Guanajuato ...... Dugês. Colima........... Palm. y Rose. Agiabampo, Sin. Palmer, Rose. Guadalajara..... Palm. y Wats. Guadalajara..... Palm. y Wats. Yucatán.......... Dondé, San Pablo, B. G. Brandegee. Mulegé........... Palmer. Cultivada......... Alcocer. Ciolima ........... Hems. y Rose. Yucatán.......... Dondé. Guerrero ......... Alcocer. Guaymas....... Palm. y Wats. Río Granter. S.Raimundo, BC Brandegee. S. Raimundo, BC Alt., Alcocer. Yucatán........... Dondé.

\section{$\mathrm{H}$}

Háas (maya)

Halal (maya).

Musa, sp?.

Phragmiles communis Trin.

Eecho (nombre apache?)...

Heno.

Cereus pecten-aborigimum Engelm

Tillandsia recurivata $\mathrm{I}$.

Hierba del aire.

Hierba colorada.

Hierba colorada.

Hierba de la cucaracha.

Hierba del cuervo.

Fierba de la flecha ........

Eierba del golpe...

Hierba del golpe

Hierba hedionda

Hierba del indio.

Hierba de la lagun

Hierba de la muela.

Hierba de San Marcos.....

Fierba del pasmo

Hierba del pasmo.

Hierba del pasmo.
Stevia glutinosa II. B. K. var....

Rumex hymenosepalus Torr...

Haplophylum cimicidum A. DC...

Crotalaria pumila Ort

Sebastiania bilocularis Wats.......

Dinothera rosea Ait.

Enothera tetraptera Cav............

Datura stramonium L

Aristolochia brevipes Benth.........

Ruellia, sp?

Stevia sulicifolia Cav.

Lupinus bilineatus Benth.

Buccharis sarothroides Gray.......

Cordia palmeri Wats...
Eriogonum atrorubens Engelm....

Ligelovia diffusa Gray.
Musáceas.

Gramíneas

Cácteas.

Bromeliáceás.....

Compuestas ...

Poligonáceas.

Poligonáceas

Apocináceas

Leguminosas.

Euforbiáceas

Onagrarieas.

Onagrarieas.

Solunáceas

Aristoloquicas

Acantáceas.

Compuestas.

Leguminosas.....

Compuestas.

Compuestas.

Borragíneas.
Yucatán......

Yucatán...........

Ghihuahua .......

Guanajuato ......

Guanajuato ......

Norogachi, Chih.

Baja Culifornia...

Morelos....

Guanajuato......

Guaymas.........

Guanajuato ......

Tancanhuitz......

México.

Guaymas

Ozuluama...

Guanajuato ......

Guanajuato ......

I. S. Pedro, B.G.

Sonora, B. Cal...

Guaymas...
Dondé.

Dondé.

Paln. y Wats.

Dugès.

Dugès.

Palm. y Wats.

Vasey, Rose.

Altamirano.

Dugès.

Palmer.

Dugès.

Seler y Los.

Dondé.

Palmer.

Seler y Loes.

Dugès.

Dugès.

Palm. y Wals.

Palnier y Rose.

Palm. y Wats. 


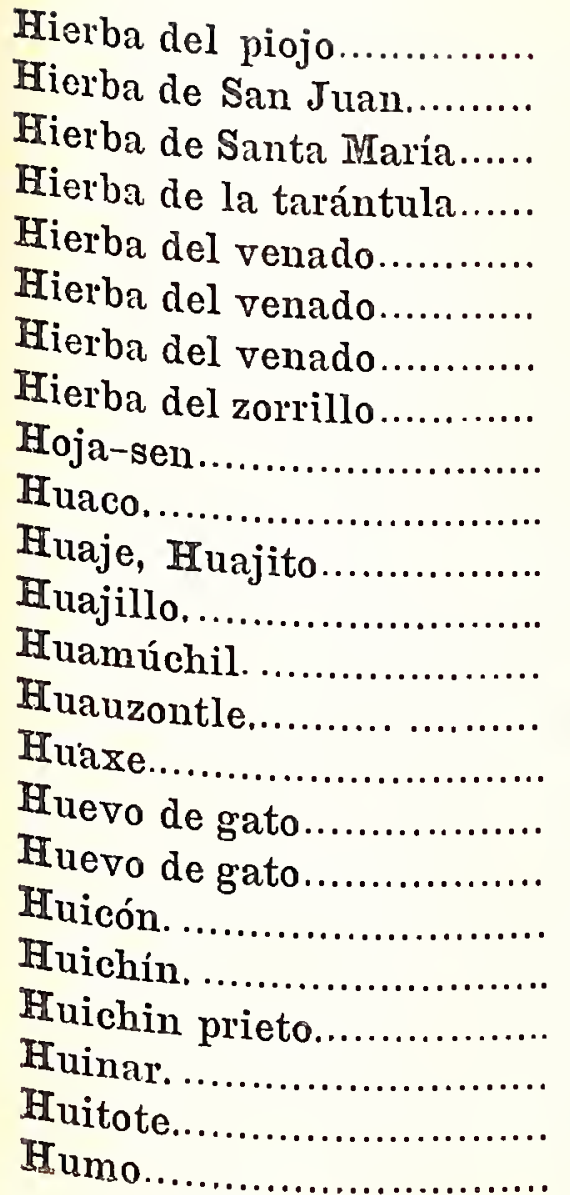

Galphimia gracilis Bartl. Bouvardia flos Joannis $K$. Sch..... Pluchea odlorata (L.) Cass.......... Ipomea sinuata Ort Porophyllum coloratum DC......... Porophylhum gracilc Benth......... Porophyllum obtusifolium DC..... Polanisia uniglandulosa Cav....... Cassia coresii A. Gr. Aristolochia pardina Duch... Lagenaria vulgaris Ser. var....... Pithecolobium brevifolium Benth... Bauhinia divaricata $\mathrm{L}$ Blitum bonus Henricus Reich..... Leucana pulverulenta Benth....... Cardiospermum hulicacabum L..... Thevetia cuneifolia A. DC.

Incuma palmeri Fernald........... Sclerocarpus uniserialis B. et H... Baccharis rhexioirles H. B. K..... Malva scoparia I'Herit Calliandrce houstoni Benth......... Pithccolobium oblongum Benth...

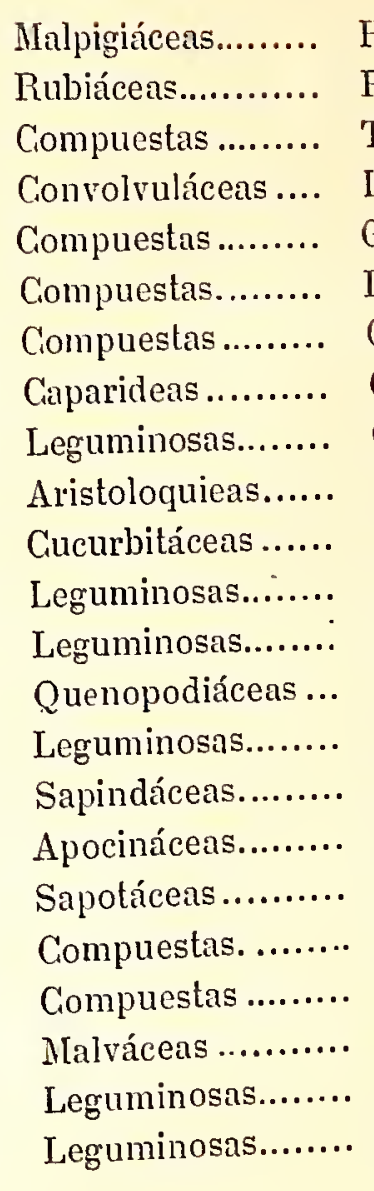

Huejutla......... Seler y Los. Puebla............ Seler y Lœs. Tancanhuitz..... Seler y Lœs Lug. cálidos...... Conzatti. Guanajuato ...... Dugès. Islas de la B. C... Pal., Ros., Vas. Guanajuato...... Dugès. Guanajuato...... Dugès. Guay.,Son., B.C. Palm. y Wats. Colima........... Dugès, WVatson. Guerrero......... Alcocer. Río Grande, Jal. Coulter. Ciudad del Maíz. Seler y Lœs. Naturalizada ..... Alcocer. Ciudad del Maíz. Seler y Lœs. Guanajuato ...... Dugès. Chapala .......... Pa!m. y Wats. Acapulco........ Fernald y Palm. Veracruz, S.L.P. Seler y Lœs. Tancanhuitz...... Seler y Lœes. Lug. lemplados.. Farm. Mex. Ciudad del MIáz. Seler y Lœs. Ozuluama......... Seler y Los.

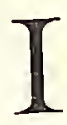

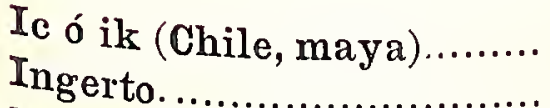
Ixin (maya)
Capsicum, sp?. Loranthus calyculatus DC.... Zea mays L.

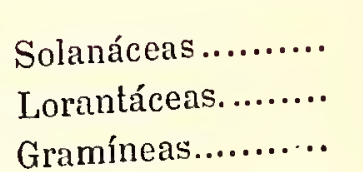

Yucatán Guanajuato ...... Yucatán.
Dondé. Dugès. Dondé.

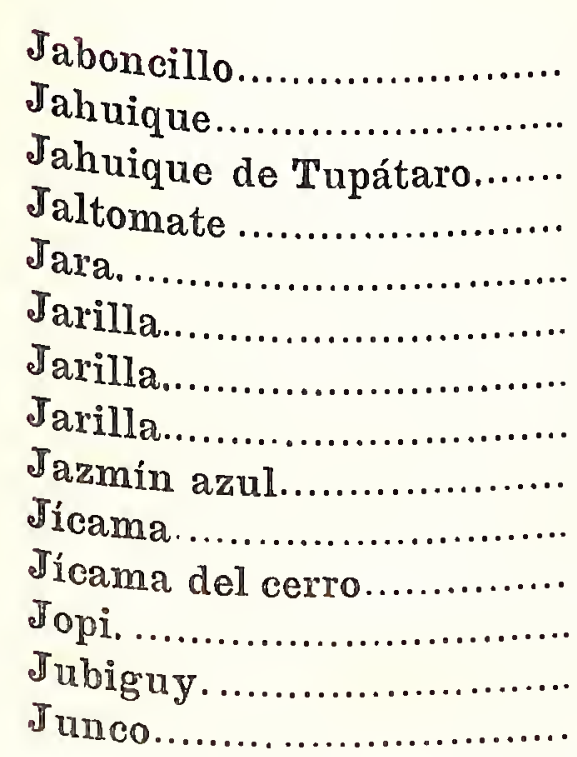

Bursera bipinnata Engl..

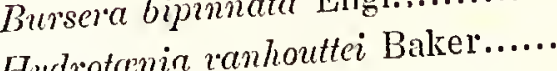
Tigridia lugesii Wals. Bellinia umbellata Rom. et Sch.. Buccharis glutinosa Pers. Dodoncea viscosa $\mathrm{L}$ Nescea salicifolia H. B. K. Solanum cnoplocalyx Dun... Shago conce H. B. K........ Tpomeca jicama Brandegee........ Phascolus heterophyllus Willd..... Ochroma lagopus Sw.............. Ochroma lagopus Sw.... Parkinsonia aculectat I
Burseráceas........ Irideas ............... Irideas Solanáceas Compuestas. Sapindáceas Litrarieas... Solanáceas .......... Plumbagíneas ...... Convolvuláceas..... Leguminosas....... MIalváceas .......... Malváceas ........... Leguminosas.......
Chihuahua....... Guanajuato...... Guanajuato ...... Guadalajara...... Guanajuato ...... Oaxaca, Mor.... Tancanhuitz..... Tancanhuilz..... Yucatán........... Baja California... Granajuato ...... Tabasco.......... Tabasco........... Guanajuato .....
Palm. y Wats. Rose. Dugès y Wats. Palm. y Wats. Dugès. Seler y Lœs. Seler y Los. Seler y Lœs. Dondé.

Dugès.

Rovirosa.

Rovirosa. Dugès. 


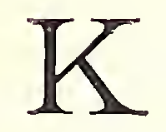

Tuché (maya)

Kunché (maya)

Kuyché (maya)
Cedrela odorata L

Jacaratia mexicana A. DG.

Pachira insignis Savign.
Meliáceas

Pasifloráceas

Malváceas
Yucatán.

Yucatán

Yucatán.
Dondé.

Dondé.

Dondé.

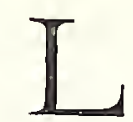

Laal (maya)

Iadilla.

Lageara

Lampotillo

Laurel.

Laurel blanco

Lebon.

Iechuguilla mezcal.

Lengua de vaca.

Lengua de vaca

Iiga..

Limoncillo

Lindrilla...

tuch (maya)
Urica urens $\mathrm{L}$

Cenchrus tribuloides L

Ficus, sp?.

Heterotheca leptoglosa DC.

Nectandra sanguinea Rottb.

Taberncemontana grandiflora Jaca.

Parkinsonia microphylla Torr.

Agare aurea Brandegee.

Buddleia verticillata $\mathrm{H}$. B. K

Rumex crispus L

Viguiera excelsa Benth. et Hook.

Pectis filipes A. Gr.

sporobolus indicus R. Br.

Crescentia cujete L...
Urticáceas

Gramíneas

Urlicáceas

Compuestas

Lauríneas.

Apocináceas...

Leguminosas.

Amarilídeas

Loganiáceas

Poligonáceas

Compuestas

Compuestas.

Gramíneas.

Bignoniáceas
Yucatán.

Tehuacán.

Ghihuahua......

Guanajuato .....

Tancanhuitz.....

Yucatán

I. Sa Rosalia, B.C

Baja California...

Oaxaca.

Guanajuato......

Valle de México.

Xochicalco

Río Blanco, Jal.

Yucatán.
Dondé.

Seler y Lœs.

Palm. y Wats.

Dugès.

Seler y Los.

Dondé.

Palm., Vas., R.

Dodge.

Conzatti.

Dugès.

Farm. Mex.

Seler y Lœs.

Palm. y Wats.

Dondé.

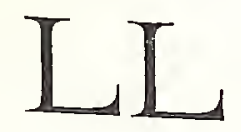

Llavina

Mamillaria goodridgii Scheer.

Cácteas

Isla Magd., B. G. Brandegee.

\section{M}

Macal (maya)

Maguapas

Maíz de coyote..

Mal de ojos.

Mal de ojos

Malacatillo.

MaIva de Castilla

Mangle dulce.

Manto de la reina.

Manzana...
Colocasia antiquorum Schott. var.

Erechlhites runcinata DG

Zea canina Wats.

Tribulus grandiflorus Benth. et $\mathrm{H}$

Zinnia pauciflora I

Zinnia pauciflora I

Malva parviflora L.

Maytenus phyllunthoides Benth................

Xanthosoma sagittifolium Schott.

Pyrus malus L. vars.
Aroideas

Compuestas

Gramíneas

Zigofileas

Compurstas

Compuestas

Malváceas

Celastrineas

Aroideas.

Rosáceas
Yucatán.

Colima....

Guanojuato

Guaymas .........

Guanajuato

Puebla, Oaxaca.

Acaponela, Tep.

I. Sta. Marg. B C

Yucatán.

Cullivada.
Dondé, Alcocer. Palmer y Rose. Dugès.

Palm. y Wats.

Dugès.

Seler y Los.

Rose.

Brandegee.

Dondé, Alcoeer.

Alcocer. 
Míanzanilla

Manzanita (fruto de moluaillo)......

Maraquiana

Maravilla .....

Maravilla

Margarita, Margarita reina

Margarita pequeña.

Mariola

Matamuchachos

Matanene

Maxocotl.

Mayitos....

Mayitos, Mayo.

Menta.

Mezquite, goma de

Mezquitillo.

Miramelindo

Mirto.

Mizquitl.

Moco de pavo.

Mora, Moras.

Mora del campo.

Moradilla.

Moro (frutos).

Mostaza montés

Muela de caballo
Helenium autumalc L.............. Compuestas......

Mralcaviscus arboreus Cav....

Nicotiana glauca Graham.

Porophyllum seemanni Schultz. B.

Mirctbilis Jalapa L....

Callistephus chinonsis Nees....

Bellis perennis L.

Horsfordia palmeri Wats

Jatropha cordata Nüll. Ary.

Hircea macroptcra DC.

Mascagnia seicriana Lœs....

Zephyranthes sessilis Herb.

Zephyranthes carinata Herb.

Mentha viridis $\mathrm{L}$

Prosopis juliftora DO

Trameria canescens Gray

Delphiniuñ ajacis L.

Stachys coccinea Jacq.

Prosopis juliffora DC

Polygonum mexicanum (Misn?)...

Morrus nigra $\mathrm{L}$. var.

Bunchosia, sp?.

Ageratum stellare Schultz Bip......

Lantana camara L

Nicotiana glauca Graham.

Sonchus oleraceus L
Malváceas.

Solanáceas

Compueslas.

Nictagineas.

Compuestas.

Compuestas

Malváceas

Euforbiáceas

Malpigiáceas

Malpigiáceas

Amarilídeas

Amarilídeas

Labiadas.

Leguminosas.......

Poligaleas

Ranunculácens....

Labiadas.

Leguminosas.......

Poligonáceas.......

Urticáceas.....

Malpigiáceas

Compuestas.

Verbenáceas

Solanáceas

Compuestas
Cultivada........ Dondé.

Manzanillo...... Palmer y Rose.

Alamos; Sonora. Palmer y Rose.

Guaymas ........ Palm. y Wats.

Guanajuato:..... Dugès.

Cultivada......... Alcocer.

Cultivada::....... Alcocer.

Baja California... Palm., Vasey.

Chihuahua....... Palm. y Wats.

Mulegé, B. C..... Palm. y Wats.

Tlacolula........ Seler y Lœs.

Distrito Federal. Alcocer.

Guanajuato ..... Dugès.

Cultivada........ Farm. Mex.

Varios lugares... Farm. Mex.

I.S.Agueda, B.C. Palm., Vasey.

Cultivada........ Dondé.

Guanajuato ...... Dugès.

Varios lugares...

Guanajuato ...... Dugès.

Cultivada......... Alcocer.

Manzanillo...... Palmer y Rose.

Tancanhuitz...... Seler y Los.

Manzanillo...... Palmer y Rose.

Oaxaca........... Conzatti.

Guanajuato ...... Dugès.

$\mathrm{N}$

Nacapuli

Nanche.

Nemax (maya).

Nenufar.

Nep-cha-ga(indios cocopa)

Nesco

Nesco
Urticáceas ............

Malpigiáceas ........

Borragíneas

Ninfeáceas......

Orobancácea.....

Levuminosas......

Leguminosas........

Aphyllon cooperi Gray..............

Coursetia (?) mexicana Wats.....

Villardia mexicana Rose.

Guaymas........ Palmer.

Oaxaca........... Seler y Lœs.

Yucatán.......... Dondé.

Cultivada......... Alcocer.

Lerdo, B. C...... Palm., Vasey.

Chihuahua....... Palm. y Wats.

Alamos, Sonora. Palmer y Rose.

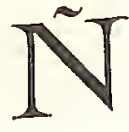

Namé

Colocasia antiquor um Schott. var. Aroideas..

Yucatán.

Dondé,Alcocer.

$P$

Ococotillo

Deotillo
Prionosciatium, sp?.

Dodoncen viscosa L.

Umbelíferas....

Valle de México. Palm. y Wats.

Sapindáceas........ Guanajuato ..... Dugès. 
Ocoxihuitl.

Ojacen (Hoja-sen).

Ojo de pollo.

Ojo de venado.

Ojo de Venus.

On (maya).

Orégano.

Orégano del cerro, Orégano del campo..

Oreja de liebre.

Oreja de ratón....

Orozuz blanco........

Orozuz colorado

Orozuz morado.

Ortiga.

Ortiguilla

Oyutch (indios cocopa).......
Spilanthes, sp?.

Cassia covessii Gray....

Sanvitalia procumbens Lam..

Thevetia nitida A. DG....

Thunbergia alata Boj.

Persea gratissima Gærtn.

Lippia palmeri Wats.

Brickelia veroniccefolia A. Gr...... Asclepias glaberrima Noc. et S....

Phaseolus atropurpureus DC.....

Lantana lilacina Desf. ?

Lantana trifolia. I.

Lippirs geminata H. B. K

Jatropha herbacea L

Tragia nepetcefolia Calv.

Ammobroma sonorce Torr.
Compuestas ....

Leguminosas.....

Compuestas ....

Apocináceas.

Acantáceas.

Lauríneas......

Verbenáceas.

Compuestas.

Asclepiadeas.

Leguminosas.

Verbenáceas

Verbenáceas.

Verbenáceas.

Euforbiáceas.....

Euforbiáceas

Lennoáceas.
Amecameca.....

Baja California...

Varios lugares...

Tabasco, Yuc....

Cultivada.

Yucatán..........

Sonora, B. C.....

Varios lugares...

Ayacapixtla......

Metzlitlán.........

Tancanhuitz.....

Huejutla.

Ozuluama........

Hostotipaquillo..

Guanajuato ......

Lerdo, B. C......
Pl. Nov. Hisp.

Palm., Vas.

Seler y Lœs.

Conzatti.

Alcocer.

Dondé.

Palm. y Wits.

Farm. Mex.

Pl. Nov. Hispl.

Seler y Los.

Seler y Lœs.

Seler y Lws.

Seler' y Lœes.

Farm. Mex.

Dugès.

Palm., Vas.,

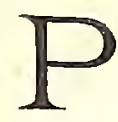

Pac (maya).

Pacasquihuit (totonaco).....

Paccanil (maya).

Pájaro bobo.....

Pájaros azules.

Palman (totonaco)

Palmilla.

Palo de Adam..

Palo Alejo.

Palo amargo.

Palo de arco.

Palo de asta.

Palo colorado.

Palo dulce.

Palo fierro.

Palo fierro.

Palo de hierro

Palo de hojas de manteca.

Palo piojo.

Palo piojo.

Palo de rosa.

Palo verde.

Panoquera.

Papachi.

Papachi.

Papaloquilitl

Papaya de pájaro

Parrilla ...........................

Pata de vaca.

Patitos.

Patzahumacachil (totonaco)

Pavito cimarrón.

Paxtle.
Lycopersicum esculentum Mill......

Paullinia arborea Hoc. et Sessé..

Physalis angulata $\mathrm{L}$.

Ipomcea intrapilosa Rose.

Salvia patens Cav.

Muntingia calabura...

Agave angustissima Engelm....

Fouquieria spinosa H. B. K.

Ccesalpinia eriostachys Bentl......

Hippophä mexicana Moc. et S...

Tecoma stans Juss.

Cordia (Sebestenoides) sonorce R... Cessalpinia (?) platiloba. Wats..

Eysenhardtia orthocarpa Wals...

Acacia, sp?

Cesalpinia (Iibidibia) sp?...........

Prosopis pulmeri Wats.

Solenum verbascifolium $\mathrm{L}$

Willurdia mexicana Rose...

Coursetia (?) mexicana Wats.....

Tecoma, sp? aff. T. pentaphyllce J..

Parkinsonia torreyana Wats....

Paullinia fusccsecns Kunth

Randia thitrberi Wals.

Randia, sp?

Porophyllum tagetoides DC.......

Carica papaya $\mathrm{I}$

Loperia mexicanra Jaeq.

Bauhinia divaricata L.

Astragalus potosencis Rol.

Callicarpa emericana Moc. et..... Astragalus humboldtii A. Gr. Tillandsia recurrata I
Solanáceas

Sapindáceas.

Solanáceas....

Corıvolvuláceas...

Labiadas.

Tiliáceas.

Amarilídeas.

Tamariscineas .....

Leguminosas.

Eleagneas.

Bignoniáceas

Borragíneas.

Leguminosas.

Leguminosas.

Leguminosas.

Leguminosas

Leguminosas.......

Solanáceas

Leguminosas

Leguminosas

Bignoniáceas.

Leguminosas

Sapindáceas

Rubiáceas

Rubiáceas.

Compuestas

Pasiflorácea:.

Onagrarieas.

Leguminosas....

Leguminosits.

Verbenáceat......

Leguininosas....

Bromeliáceas
Yucatáin

Tenampulco,Pb?

Yucatán..........

Oaxaca...........

Guanajuato ......

Huehuetla, Pue.

Manzanillo........

Isla Magd., B. C.

Manzanillo........

Desierto de Cuaj.

Sonora

Alamos, Sonora.

Chihuahua........

Alamos, Sonora.

Baja California...

Armeria, Colima

Pura. y Com. B.C.

Reg. templadas..

Alamos, Sonora.

Chihuahua.......

Huejutla.........

Baja California...

'Tancanhuitz......

Guaymas, Ures..

Comondú, B. C..

Xochicalco.......

Yucatán.

Distrito Fed.....

Ciudad del Maíz.

S. L. P., V. de M.

El Espinal, Oax..

dalgo ............

Guanajuato ...
Dondé.

Flora Mex.

Dondé.

Conzatti.

Dugès.

Flora Niex.

Engel. Greggi.

Brandegee.

Palmer y Rose.

Flora Mex.

Conzatti.

Palmer y Rose.

Palm. y Wals.

Palmer y Rose.

Brandegee.

Palmer y Rose.

Brandegee.

Conzatti.

Palmer y Rose.

Palm. y Wats.

Seler y Loes.

Palm., Vissey.

Seler y Loes.

Palm. y Watr.

Brandegee.

Seler y Loes.

Dondé.

Seler y Loes.

Seler y Lœs.

Ramirez.

Flora Hex.

villada.

Dugès. 


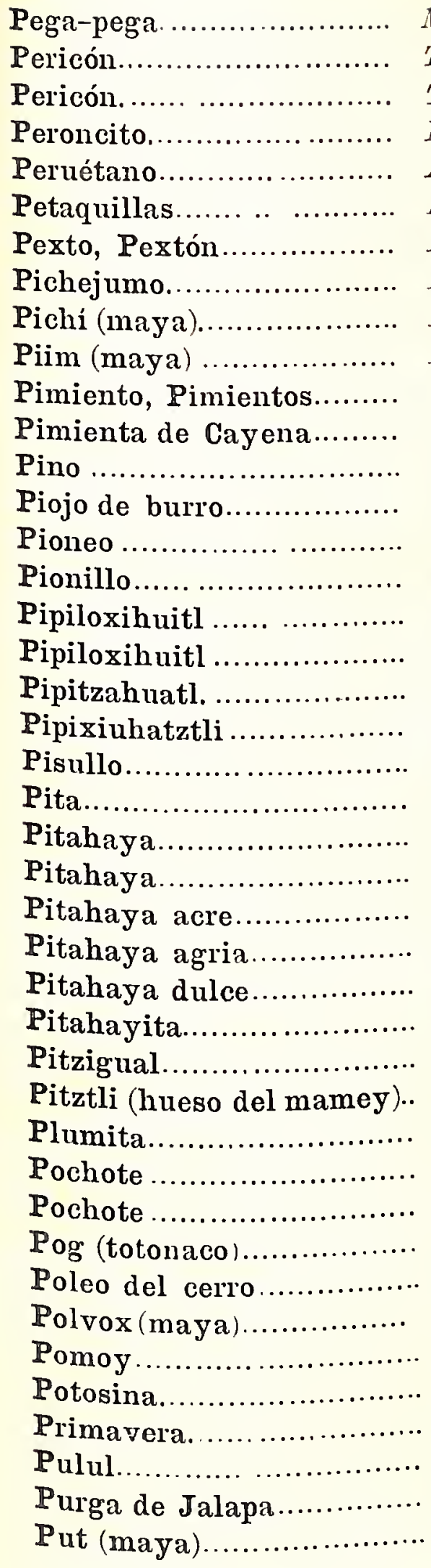

Mentzelia adherens Benth. Tribulus cistoides L

Tragetes lucida Gav.

Lopezia triehota Schl.

Achras sapota L.

Pithecoctenium murieatum $\mathrm{M}$. el S.

Brickelia veronicefolia A. Gr.....

Pithecolobium ligustrinum Kl. var..

Psidium pomiferum L..............

Eriodendron occidentalc $\mathrm{Tr}$. et $\mathrm{Pl}$.

C'apsicum, sps. \& vars..

Capsicum breeatum L.

Cupressus semperrirens L.

Xanthium canarlense Mill.

Erythrina coralloides DC...

Erythrina coralloides DC....

Cestrum diurnum L...

Cestrum noeturnum L

Stevia viscida H. B. K..............

Cypripedium turgidum Mloc. et S..

Rondeletia spinosa K. Sch...........

Agave americana $\mathrm{L}$

Cereus gummosus Engelm.

Cereus straminens Engelm.........

Cereus gummosus Engelm.

Cereus flexuosus Engelm.

Coreus thurberi Engelm.

Cereus striatus Brandegee...........

Lobelia cliffortiana L...............

Lucuna mammosa Gærtn. fil......

Ipomoca versicolor Meissn........... Ceiba (Erione) grandiflora Rose..

Eriodendion acuminatum Wats...

Crescentia cujete L...

Cunila secunda Wals.

Anona nurvicata L...

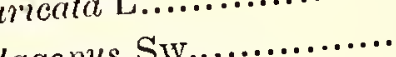

Ochroma lagopus Sw.........

Calliandra nitida Wats.............

Tabebuia donnell-smithii Rose.....

Nelumbium, sp?.

Ipomoer purga Hayne.

Carica papaya L.
Loaseas

Zigofileas

Compuestas

Onagrarieas.

Sapotáceas .

Bignoniáceas.

Compuestas.

Leguminosas........

Mirtáceas...........

Malváceas

Solanáceas

Solanáceas

Coníferas.

Compuestas

Leguminosas........

Leguminosas........

Solanáceas ...

Solanáceas.

Compuestas

Orquídeas..

Rubiáceas

Amarilídea

Cácteas..

Cácteas....

Cácteas.

Cácteas

Cácteas

Cácteas.

Lobeliáceas

Sapotáceas.

Convolvuláceas....

Malváceas ... .......

Malváceas ...........

Bignoniáceas........

Labiadas.... .......

Anonáceas..........

Malváceas ...........

Leguminosas........

Bignoniáceas........

Ninfeáceas.........

Convolvuláceas....

Pasifloráceas.......
Ba Los Ang., BC. Palm. y TVats.

Oaxaca........... Seler y Lœs.

E. de México... . Alcocer.

Guanajuato...... Dugès.

Yuc., Col., Tam.. Urbina.

Cuernava, Mor... Pl. Nov. Hisp.

Varios lugares... Farm. Mex.

Ozuluama......... Seler y Lœs.

Yucatán.......... Dondé.

Yucatán.......... Dondé, Alcocer.

Cultivadas........ Alcocer.

Reg. tropicales.. Conzatti.

Yucatán.......... Dondé.

Guanajuato ...... Dugès.

Agiabampo...... Palmer y Rose.

Chihuahua...... Palı. y Wats.

Amecameca..... Pl. Nov. Hisp.

Varios lugares... Pl. Nov. Hisp.

San Angel, D. F. Pl. Nov. Hisp.

Chiluancingo..... Pl. Nov. Hisp.

Huejulla......... Seler y Lœs.

Varios lugares... Dodge.

Baja California... Parry. Coult.

Coah., S. L. P... Coulter.

I. Carmen, B. C.. Palmer y Rose.

Baja California... Engelm., Coult.

S.Fernando, BC. Palm., Coult.

Baja California... Brand., Coult.

Ciudad del Maíz. Seler y Lœs.

Lug. calientes... Pl. Nov. Hisp.

Varios lugares... Conzatti.

Manzanillo...... Palmer y Rose.

Chihuahua...... Rob. y Fernald.

El Espinal, Oax.. Flora Mex.

Guanajuato..... Dugès.

Varios lugares... Urbina.

Tabasco.......... Rovirosa.

Río Blanco, Jal.. Palm. y Wats.

Colima........... Rose.

Tamaulipas..... Maury.

Veracruz......... Conzatti.

Yucatán.......... Dondé.
Quapionchí.

Quauhayohuacht1 ...........

Quauhtlatlatzin.

Quauhtzapot1

Quauhtrapotl.
Leguminosas........

Euforbiáceas ........

Rhynchosia, spo

Jatropha cureas L,

Hura crepitans L.................

Anona cherimolia Mill.

Anona squamosa L................

Euforbiáceas.......

Anonáceas..........

Anonáceas .........
Morelos.

Guerrero

Sinaloa.

Ver., Mor., Pba.

Lug. calientes..
Seler y Los.

Pl. Nov. Pisp.

Rose.

Urbilla.

Unbina. 


\section{$\mathrm{R}$}

Rabo de iguana......

Rabo de mico.

Rabo de mico.

Rabo de mico.

Raíz de chayote.

Raíz de fregar

Raíz del indio

Rama del tecolote.

Raspa sombrero

Rosa de Castilla del campo

Rosal, Rosales
Solanum rostratum Dun.

Heliotropium curassavicum L.

Heliotropium indicum L

Heliotropium parviflorum L.

Sechium edule Sw.

Cyclanthera dissecta Arn... Aristolochia foetida H. B. K.

Iillandsia utriculata L.

Petrcea arborea H. B. K.

Lippia callicarpifolia H. B. K.

Rosa, sps. \& vars.
Solanáceas

Borragineas

Borragineas

Borragíneas..

Cucurbitáceas .

Cucurbitáceas ....

Arist oloquieas.

Bromeliáceas

Verbenácess

Verbenáceis

Rosáceas .
Todo el país.... Conzatti.

Mesa central.... Herrera.

Tabasco......... Rovirosa.

Yucatán.......... Dondé.

Varios lugares... Herrera.

Guanajuato..... Dugès.

Nichoacán........ Farm. Mex.

Ozuluama......... Seler y Lœs.

Huejulla......... Seler y Lœs.

Cuernavaca...... Seler y Los.

Cultivadas....... Alcocer.
Sabacnicté (maya)

Sábila.

Sacci (maya)

Sacziu (maya)

Saguaro (nombre indigena)

Sal sieso.

Salvia.

Salvia.

Salvia.

Salvia para la misa

Sandía de la Pasión

Sangregrado

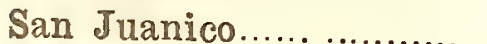

Santa María...

San Miguelito

San Nicolás

Santa María.

Sapote blanco.

Sapote de rata.

Saramuya. Saramuyo....

Sayas

Say unsay .......................

Segne Xanté (totonaco)......

Señora de noche

Sisote

Sosa

Sosquil.

Sosquil.

Subin (maya)...

Suopakal (maya)..............

Suponite.
Plumeria rubra L

Aloe barbadensis Mill.

Agave rigida Mill. var. elongata...

Sida, sp?.

Cereus giganteus Engelm

Lycium carinatum Wats.

Hyptis emoryi Torr

Hyptis laxiflora Benth.

Hyptis palmeri Wats.

Hyptis collina Brandegec

Passifora palmeri Rose.

Jatropha canescens Müll. Arg.......

Jacquinia pungens A. Gr.

Tagetes tenuifolia Cav.

Antigonon leptopus Hook. el Aru. Milla biflora Ciav.

Eupatorium, sp?.

Casimiroc edulis Llav. et Lex.............

Casimiroc pubescens Ram.

Anona squamosa $\mathrm{I}$

Amoreuxia palmatificla Mo......... Mentzelia aspera $\mathrm{I}$.

Vanilla pompona Schiede.

Clerodendron ligustrinum R. Br...

Bursera palmeri Wats.

Solanum revbascifolium .........

Agave rigida Mill. var. elongata...

Agave rigida Mill. var. sisulana.

Mimosa, sp? .....

Citrus vulyuris Risso

Melochia py.amidata
Apocináceas

Liliáceas.

Amarilídeas

Malváceas

Cácteas

Solanáceas.

Labiadas.

Labiadas.

Labiadas....

Labiadas.

Pasifloráceas ........

Euforbiáceas .........

Mirsineas.

Compuestas ............

Poligonáceas .........

Liliáceas

Compuestas.

Rutáceas .

Rutáceas

Anonáceas

Poligaleas.....

Loaseas....

Orquídeas

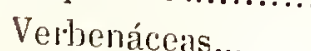

Burseráceas..........

Solanáceas ..........

Amarilídeas........

Amarilídeas........

Leguminosas.......

Rutáceas ...........

Esterculiáceas ......
Yucatán.

Guanajuato .....

Yucatán.

Yucatán.

Sonora...

Guaymas

B? Los Ang., BC.

Baja California...

Guaymas

S. José del C., BC:.

I. del Carm., B.C.

Guaymas

Guaymas

Colima...

Guaymas...

Río Blanco, Jal.

Yucatán...

Dist.Fed., Sin., \&

Querétaro....

Yucatán.

Guaymas

Yucatán.

Oaxaca, Ver.....

Ozuluama.

Guanajuato

Mor. Ver. S.I. P.

Yucatán

Yucatán.

Yucatán.

Yucatán.

Huejulla.
Dondé.

Dugès.

Dondé, Alcocer.

Dondé.

Coulter.

Palm. y Wals.

Palm. y Wats.

Palm., Vas., R.

Palm. y Wats.

Brandegee.

Palmer y Rose.

Palm. y Wals.

Palm. y Wats.

Palmer y Rose.

Palm. y Wats.

Palm. y Wals.

Dondé.

Herrera.

kamírez.

Dondé.

Palm. y Wats.

Dondé.

Farm. Mex.

Seler y Los.

Dugès.

Seler y Loes.

Dodge.

Dodge.

Dondé.

Dondé.

Seler y l,œs. 
Tabaco cimarrón.

Tabaco coyote.

Tabaco de coyote

Tabaco de perro.

Tabkanil (maya)

Talajote (Talayote?)

Talayote

Talay ote

Taman (maya).

Tapona (tuna).

Tarahumara

Tauch (maya).

Teclatilla

Tecuampatli.

Tempixque, Tempixtle......

Tempixtle, Tempizquixtli.

Tempixtle, Tempizquixtli.

Tepehuaje.

Tepetzapotl, Tepezapote.....

Tepozán blanco.

Tepozán verde

Tetazo

Texaltzapotl

Thumbirichi.

Tilapo.

Tilapos.

Tilimi

Tijerilla.

Tivinagua.

Tizón de maíz

Tlacazochitl....

Tlacopatli quauhtotollanensi.

Tlalnochtli...

Tlamalacatlacotli

Tlaquilin.

Tlatlauhcapatli

Tlzehuite.

Tomate de burro

Tomate capotillo

Tomate de la paz.

Tomate de perro.

Torito

Torote.

Torote.

Torote.

Torote amarillo

Torote blanco.

Torote común.
Wigandia caracasana II. B. K..... Nicotiana trigonophylla Dun....

Nicotiana ipomopsiftara Dun

Nicotiana clevelandi Gray.

Vitis sicyoitles? Baker. var......

Marsdenia edulis Wits

Rothrockia cordifolia A. Gr.

Gonolobus erianthus Dene.

Gossypium barbadense L.

Opuntia tapona Engelm.

Asclepias setosa Benth....

Diospyros ebenaster Retz.

Comocladia engleriana Lœs

Marsdenia zimapanica Hemsl.....

Lucuma sphcerocarpa A. DC.

Bumelia lcete-vivens, Hemsl.........

Bumelia subsessitiflora Hemsl......

Lysiloma acapulcensis Benth. var.

Dupinia tcpezapote Durand.........

Buddleia humboldtiana Rœm. etS.

Buddleia verticillata $\mathrm{H}$. B. K,......

Cereus tetazo Weber..

Anona cherinolia Mill..

Karatas plumieri E. Morr.

Bumelia lote-virens Hemsl........

Bumelia subsessiliftora Hemsl.....

Erysimum tilimi Gray.

Echites montana (?).

Eriogonum inflatum Torr.

Ustilago maydis DG.

Bouvardia triphylla Salisb.........

Viguiera excelsa Bent. et Hook...

Asclepias linaria Cav..

Ternonia liatroides DG..............

Hirabilis jalapa L..................

Gercanirm alchimilloides? L.....

Tibouchina longifolia Baill......

Nicandira physaloides Gærtn.......

Physalis crassifolia Benth ...........

Cyphomandra betacea Sendt......

Physalis nicandroides Schl........

Asclepias linaria Cav................

Burserca odorata Brandegee........

Bursera fragilis Wats..............

Bursera microphylla Gray.......... Jatropha spatulata Müll., Arg. var. Burserca microphylla Gray......... Jatropha cardiophylla Miill., Arg.
Hidrofiláceas........ Cuautla............ Seler y Loes. Solnnáceas......... Baja California... Pal,, Vas., Ros. Solanáceas .......... S. Gregorio, B.C. Brandegee. Solanáceas .......... I.Magdalena,B.C Brandegee. Ampelídeas ......... Yucatán........... Dondé, Alcocer. Asclepiadeas....... Sonora.......... Palm. y Wats. Asclepiadeas........ S.José del C., BC. Brandegee. Asclepiadeas ........ Guanajuato ...... Dugês. Yucatán........... Dondé. Cácteas............. Baja Galifornia... Engelm., Coult。 Asclepindeas ........ Guanajuato ...... Dugès. Ebenáceas .......... Yucatán........... Dondé, Alcocer. Anacardiáceas ...... Morelos ........... Seler y Los. Asclepiadeas ....... Jojutla, Mor...... Ramírez. Asclepiadeas....... Lugares cálidos.. Urbina. Sapotáceas......... Oaxaca, Ver...... Urbina. Sapotáceas .......... Oaxaca, Ver...... Urbina. Sapotácens......... Jalisco............ Alamos, Sonora. Palmer y Rose. Leguminosas........ Alam de México. Urbina. Ternstrœmiáceas... Guanajuato ...... Dugès. Loganiáceas ......... Guanajuato ...... Dugès. Loganiáceas........ Guanotlán, Jalisco Weber, Coult. Cácteas.............. Zer., Mor., Pueb. Urbina. Anonáceas.......... Ver., Mor., Pueb. Uichoacán........ N. León. Bromeliáceas ....... Michoacan....... Ramírez. Sapotáceas.......... Oaxaca, Jalisco.......... Urbina. Sapotáceas......... Jáxico, D. F..... Hemsley. Cruciferas........... Guanajuato ...... Dugès. Apocináceas........ Go Angeles, B C. Palm. y Wats. Poligonáceas........ Hongos............... Rubiáceas ......... Compuestas ......... Asclepiadeas........ Compuestas......... Nictagineas .......... Geraniáceas......... Melastomáceas.... Solanáceas.... Solináceas

Solanáceas .

Solanáceas ..........

Asclepiadeas....... Burseráceas........ Burseráceas ......... Burseráceas ......... Euforbiáceas ........ Burseráceas ......... Euforbiáceas ....... Parásita del maíz Distrito Federal. Valle de México. Tetzcotzinco..... Yautepec... Lug. templados. Varios lugares... Huejutla.......... Guanajuato ...... Baja California... Oaxaca ?......... Río Blanco, Jal. Tetzcotzinco..... S. Gregorio, BC. Chiluuahua...... Palm. y Wats.

I. Carmen, B. C.. Palmer y Rose. Sonora........... Guaymas ......... Sonora........... Alcocer.

Farm. Mex.

Farm. Mex. Seler y Lœs. Seler y Las. Pl. Nov. Hisp. Pl. Nov, Hisp. Seler y Las. Dugès. Palm., Vas. Conzatti. Palm. y Wats. Seler y Loes.

Brandegee.

Palm. y Wats. 
Torote priete.

Torote prieto.

Torote verde.

Torotito

Totocuitlatzapotl

Tototzapotl...

Toxichec cimarrón.

Tripa de vaca.

Trompetilla.

Tronador...

Tronadora

Tronadora.

Tronadora de España...

Tule.

Tule.

Tule.

Tuna camuesa

Tuna cardona.

Tuna chaveña.

Tzinacanatlapatli.
Burserca laxiflora TVats. Jatropha spatulata Müłl. var....... Fouquiera spinosa H. B. K......... Jatropha canescens Mï̈ll., Arg...... Diospyros ebenaster Retz. Sideroxylon mexicanum Hemsl..... Mikania cordifolia Willd. Vitis tiliacea H. B. K. Ipomcea llaveana Meissn. Ruellia albiflora Fernald.. Abutilon incanum (Link.) Sweet.. Tecona mollis H. B. K. Nicotiana glauca ? Grah. Scirpus californicus Britton.... Scirpus lacustris L.

Typha angustifolia L

Opuntia larreyii TVeber..

Opuntia, sp?

Opuntia, sp?.

Passiflora maximiliana? Bory....
Burseráceas

Euforbiáceas

Tamariscineas.....

Euforbiáceas

Sapotáceas.

Sapotáceas

Compuestas

Ampelídeas

Convolvuláceas....

Acantáceas..

Malváceas .....

Bignoniáceas........

Solanáceas

Ciperáceas.

Ciperáceas.

Tífáceas...

Cácteas.

Cácteas.

Cácteas.

Pasifloráceas.
Sonora.

Ba Los Ang

Alamos, Sonora. Palmer y Rose.

Sonora, B. C.....

Morelos ...........

Michoacán, Gro.

Tancanhuitz...

Guanajuato ......

Guanajuato .....

Acapulco ........

Manzanillo, Col..

Guanajuato ......

Guanajuato ......

Lerma, México..

Baja California...

Sla. Agueda, B.C.

Querétaro

San Luis Potosí.

San Luis Polosí.

Tamaulipas......
Urbina.

Urbina.

Seler y Lœs.

Dugès, Alcocer.

Dugès.

Fernald y Palm.

Palmer y Rose.

Dugès.

Dugès.

Rose.

Dodge.

Palnı., Vas.

Weber.

Coulter.

Cioulter.
Dict. d'H.N.
Ubi?

Ubi ?

Jcuares

Toupek (maya).

Uña de gato

Uña de gato

Uña de gato

Uvalama
Gilia tenuifolia Gray.

Gilia gloriosa Brandegee.

Phyllactis pratensis Bent. et Hook

Taberncemontana acapulcensis M..

Mimosa monancistra Benth.

Olneya tesota A. Gr.

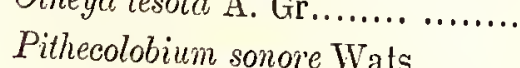

Vitex mollis $\mathrm{H}$. B. K.
Polemoniáceas.....

Polemoniáceas.....

Valerianeas

Apocináceas.

Leguminosas........

Leguminosas........

Leguminosas........

Verbenáceas.......
Baja California... Baja California... Guanajuato ...... Yucatán Guanajuato ...... Comondú, B. C.. Guaymas, Mule..

Mulegé, B. C..... Palm. y Wats.
Brandegee.

Brandegee.

Dugès.

Dondé, Alcocer.

Dugès.

Brandegee.

Palm. y Wats.
Vara blanca...

Vara prieta.

Vela de coyote.

Verbena.

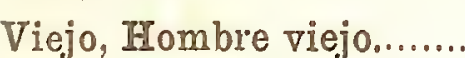

Vinorama

Violeta cimarrona

Violeta cimarrona.

Volador
Verbesina serrata Cav.

Casalpinia gracilis Benth

Opuntic fulgida Engelm

Mentha rotundifolia L.

Cereus schottii Engelm.

Acasia farmesiana Willd...

Anoda dilleniana C'av.

Eustoma exaltatum Griseb.

Gyrocarpus jacquini Roxb.
Compuestas .....

Leguminosas........

Cácteas.

Labiadas

Cácteas.

Leguminosas

Malváceas ...

Gencianeas. .

Combretáceas
Guanajuato ......

Sonora....

Baja Cialifornia...

Guadalajara.....

S. Quintín, B. C.

Baja California...

Ayacapixtla......

Seler y Lœs.

Yucatán.....
Dugès.

Coulter.

Coulter.

Palm. y Wats.

Brandegee.

Palm., Vas.

Seler y Loes.

Dondé, Alcocer. 
Xilitl

Ximacol.

Xocoyolli
Lobelia cliffortiana $\mathrm{L}$

Sicyos elcppei Gr. Don Oralis albicans H. B. K....
Lobeliáceas

Cucurbitáceas

Geraniáceas.
Giudad del Máz. Seler y Lœs.

Zacualtipán ...... Seler y Lœs.

Amecameca...... Seler y Loes.
Yamete

Yaxci

Yelera

Yoyochichil.

Yumete.
Asclepias subulata Dene. Agave rigida Mill. var. sisalana.. Gentiana spathacea H. B. K Eupatorizm petiolare Moc... Asclepias subulata Done.
Asclepiadeas Amarilídeas. Gencianeas. Compuestas Asclepiadeas
Baja California... Palmer, Vas. Yucatán.......... Dondé. Guanajuato .... . Dugès. Texcoco.......... Seler y Lœs. Guaymas ........ Palm. I Wats.

\section{Z}

Zacatlazcal

Zacatlazcal.

Zapatillo amarillo

Zapote de abejas.

Zapote de ave.

Zapote de viejas

Zapotillo

Zaragoza

Zarzamora

Zicana

Zina ó Sinita (nombre indíg.)
Cuscuta odlontolepis Engelm.

Crateva, sp?

Achras sapota L

Anona muricata $\mathrm{L}$

Lantana polyacantha Schauer....

Natas major All.

Rubus aff. $R$. occidentalis

Ipomea bracteata Cav

Cereus schottii Engelm. Cuscuta tinctoria Mart...

Sideroxylon mexicanum Hemsl...
Corivolvuláceas.....

Convolvuláceas....

Caparideas

Sapotáceas

Sapotáceas

Anonáceas

Verbenáceas

Nayadáceas.

Rosáceas .........

Gonvolvuláceas ....

Cácteas.
Guanajuato ...... Dugès.

Guanajuato ...... Dugés.

Manzanillo....... Palmer y Rose.

Yuc., Col., Tam.. Urbina.

Mich., Guerrero. Urbina.

Varios lugares... Urbina.

Oaxaca............ Seler y Lœs.

Mulegé, B. G..... Palmer.

Zacualtipán...... Seler y Lœes.

Alamos, Sonora. Palm. y Rose.

Son., S. Luis P... Goulter.

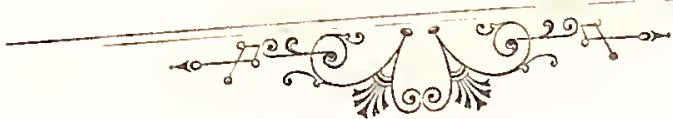





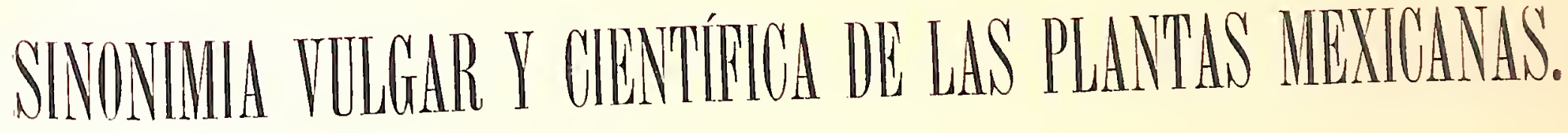

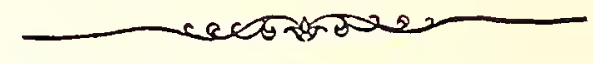

\section{APÉNDICE Á TAA SEGUNDA PARTE.}

\section{A}

Abutilon incanum (Link) Sweet

Acacia farnesiana Willd.

Acacia pennatula Benth.

Acacia, sp?.

Achras sapota L....

Adiantum tricholepis Fée.

Agave americana $\mathrm{L}$

Agave angustissima Engelm

Agave aurea Brandegee.

Agave rigida Mill. var. elongata...

Agave rigida Mill. var. sisalana

Ageralum stellare Schultz Bip...

Aloe barbadensis Mill.

Amarantus leucospermus Watson...

Amarantus spinosus $\mathrm{L}$

Ammobroma sonoræ Torr...

Amoreuxia palmatifida Moc. et Sessé....

Ananas sativus Schult.

Anoda dilleniana Cav.

Anona cherimolia Mill.

Anona muricata $\mathrm{L}$

Anona squamosa L

Antigonon leptopus Hook. et Arn.

Aphyllon cooperi A. Gr.

Apoplanesia paniculata Presl.

Ardisia escallonioides $\mathrm{Ch}$. et Schl....

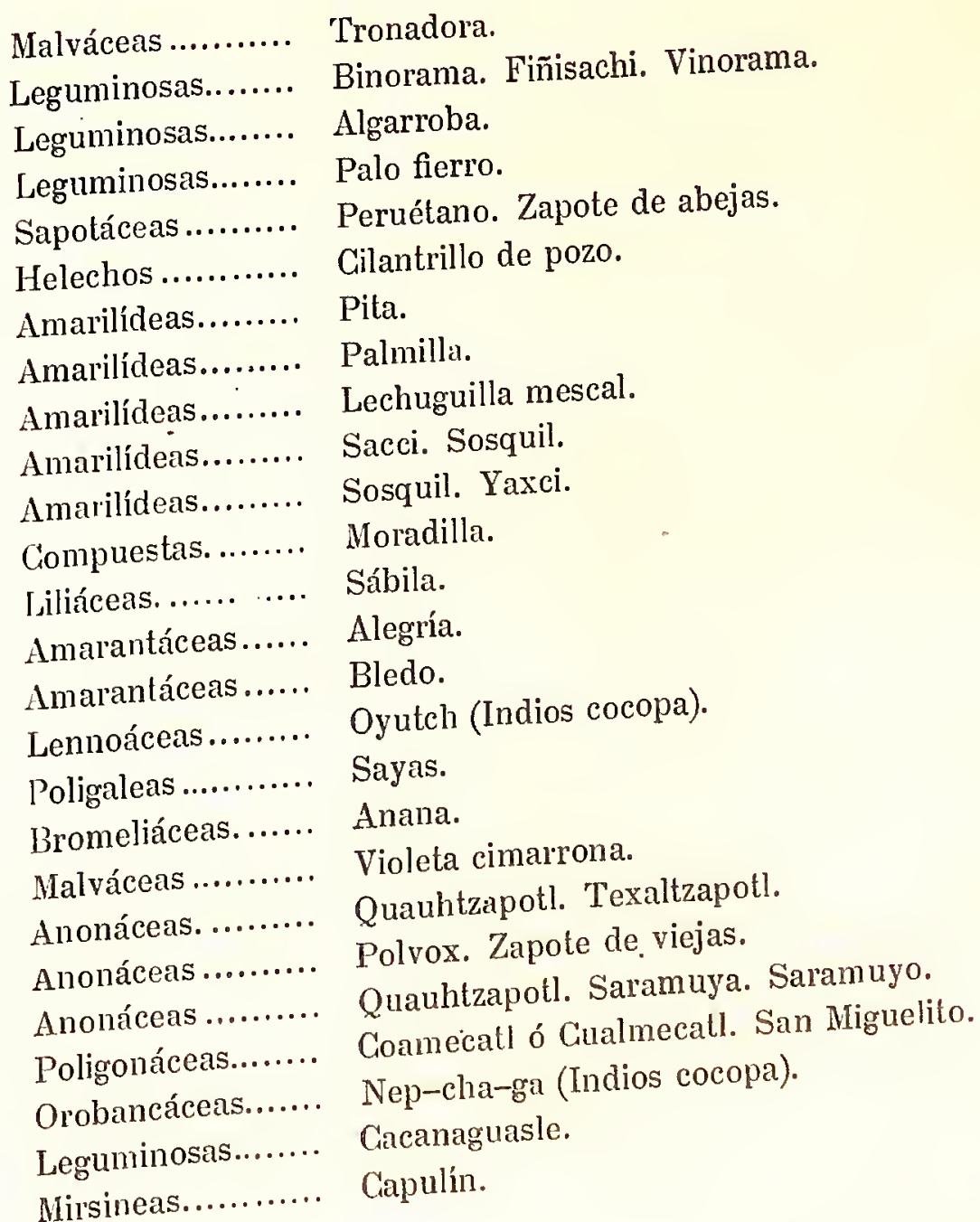


Argemone albiflora Hornem.

Argemone mexicana L...

Aristolochia brevipes Benth.

Aristolochia fœtida H. B. K.

Aristolochia pardina Duch

Aristolochia pentandra L.

Asclepias curassavica L.

Asclepias glaberrima Moc. et Sessé

Asclepias linaria Cav....

Asclepias longicornu Benth.

Asclepias setosa Benth.

Asclepias subulata Dcne.

Astragalus humboldtii A. Gr.

Astragalus potosencis Rob.

Atriplex, sp?.

Atropa belladonna L.
Papaveráceas...... Cardo.

Papaveráceas...... Cardo.

Aristoloquieas..... Hierba del indio.

Aristoloquieas..... Raíz del indio.

Aristoloquieas..... Guaco mexicano. Huaco.

Aristoloquieas..... Guaco de San Gristóbal.

Asclepiadeas ....... Cuchilxíu. Cuchilloxíu.

Asclepiadeas ....... Oreja de liebre.

Asclepiadeas ........ Tlalnochlli. Torito.

Asclepiadeas ....... Cuatz chté gueeco (zapoteco ?)

Asclepiadeas ....... Tarahumara.

Asclepiadeas ....... Yamete. Yumete.

Leguminosas....... Garbancillo. Pavito cimarrón.

Leguminosas....... Patitos.

Quenopodiáceas... Abanico.

Solanáceas.
Baccharis glutinosa Pers....

Baccharis rhexioides H. B. K.

Baccharis sarothroides Gray.

Bauhinia divaricata $\mathrm{L}$

Begonia (Knesebeckia) bicolor Watson...

Begonia (Knesebeckia) discolor Watson..

Bellinia umbellata Rom. et Schultz.

Bellis perennis L.

Beta vulgaris Moq. var. Cicla

Bigelovia diffusa Gray.

Bignonia bicolor Watson

Blitum bonus Henricus Reich

Bouvardia flos Joannis K. Sch.....

Bouvardia triphylla Salisb.

Brickelia veronicæfolia A. Gr.

Bromelia pinguin L.

Buddleia humboldtiana Rœem. et Schultz

Buddleia verticillata $\mathrm{H}$. B. K

Bumelia læte-virens Hemsl.

Bumelia subsessiliflora Hemsl.

Bunchosia, sp?.

Bursera bipinnata Engler.

Bursera fragilis Walson.

Bursera gummifera Jacq. .

Bursera hindsiana Engler.

Bursera laxiflora Wats.

Bursera microphylla Gray.

Bursera odorata Brandegee.

Bursera palmeri Wats...
Compuestas.

Compuestas.

Compuestas

Leguminosas

Begoniáceas

Begoniáceas

Solanáceas

Compuestas

Quenopodiáceas...

Compuestas

Bignoniáceas....

Quenopodiáceas...

Pubiáceas

Rubiáceas

Compuestas

Bromeliáceas.

Loganiáceas.

Loganiáceas.

Sapotáceas ..... Leınua de vaca. Tepozán verde.

Sapotáceas......... Tempixtle. Tempizquixtle. Tilapo.

Malpigiáceas ....... Tempixtle. Tempizquixtle. Tilapos.

Burseráceas....... Mora del campo.

Burserácea...... Jaboncillo.

Bursera..... Torote.

Burseraceas........ Chacah.

Burseráceas ........ Copal.

Burseráceas ........ Copal. Torote prieto.

Burseráceas........ Torote. Torote blanco.

Burseráceas........ Torote.

Burseráceas....... Sisote. 


\section{C}

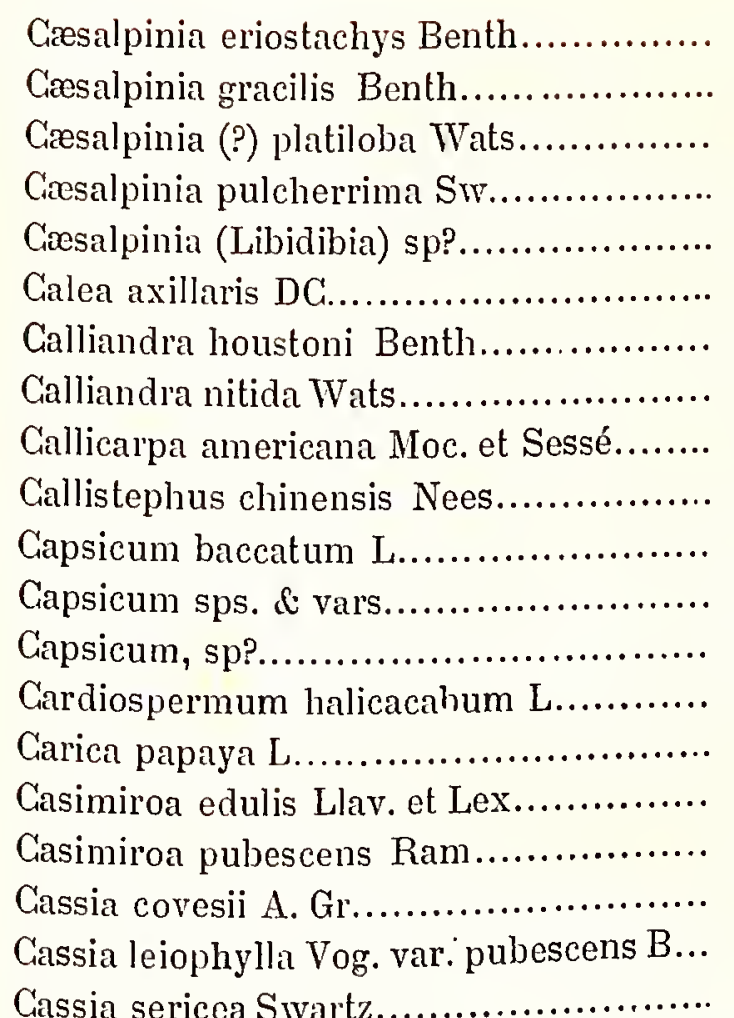

Castanea vulgaris Lam.

Castela nicholsoni Hook...

Castilleja canescens Benth................

Castilleja scorzoneræfolia H. B. K.........

Cathesticum erectum Vasey \& Hackel....

Cayaponia racemosa ? Cogn. var...........

Cedrela odorata L.

Ceiba (Erione) grandiflora Rose...........

Cenchrus tribuloides L...

Cereus calvus Engelm

Gereus cochal Orcutt.

Ciereus eruca Brandegee...................

Cereus flexuosus Engelm..................

Cereus giganteus Engelm.

Cereus gummosus Engelm ...............

Cereus pecten-aboriginum Engelm........

Cereus pectinatus rigidissimus Engelm...

Cereus pringlei WVats

Cereus schottii Engelm.

Cereus stramineus Engelm.................

Cereus striatus Brandegee..................

Cereus tetazo Weber.......................

Cereus thurberi Engelm..................

Cestrum diurnum L..........

Cestrum nocturnum L.

Geterach officinarum DC...................

Citrus vulgaris Risso...

Glerodendron ligustrinum R. Br..........

Clusia pulchella (?)

\author{
Leguminosas....... Palo Alejo. \\ Leguminosas....... Vara prieta. \\ Leguminosas....... Palo colorado. \\ Leguminosas....... Ghacsinkin. \\ Leguminosas....... Palo fierro.
}

Compuestas ........ Chilchaca.

Leguminosas....... Cabello de angel. Huitote.

Leguminosas....... Potosina.

Verbenáceas ....... Patzahuma cachil (totonaco).

Compuestas ........ Ester ó Esther. Margarita. Margarita reina.

Solanáceas......... Pimienta de Cayena.

Solanáceas......... Pimiento. Pimientos.

Solanáceas.......... Ic ó Ik.

Sapindáceas......... Huevo de gato.

Pasifloráceas....... Papaya de pájaro. Put.

Rutáceas ........... Sapote blanco.

Rutáceas .......... Sapote de rata.

Leguminosas....... Hoja-sen. Ojacen.

Leguminosas....... Gafé cimarrón.

Leguminosas........ Bicho.

Cupulíferas......... Castaña (frutos).

Simarrubáceas..... Amargoso.

Escrofularíneas.... Enchilada.

Escrofularíneas.... Flor de milpa.

Gramíneas......... Grama.

Cucurbitáceas ...... Ahuichichi. Ahuichichic.

Meliáceas........... Gedro. Kuché.

Malváceas .......... Pochote.

Gramíneas.......... Ladilla.

Cácteas............ Cardón pelón.

Cácteas............. Cochal.

Cácteas............. Chilenola. Chirenole.

Cácteas............. Pitahaya agria.

Cácteas............. Saguaro.

Cácteas............. Pitahaya. Pitahaya acre.

Cácteas ............. Ciardón. Hecho.

Cácteas............. Cabeza de viejo.

Cácteas............. Cardón.

Cácteas............. Cabeza de viejo. Viejo. Hombre viejo. Zina ó sinita.

Cácleas............. Pitahaya.

Cácteas............. Pitahayita.

Cácteas............. Tetazo.

Cácteas............. Pitahaya dulce.

Solanáceas......... Pipiloxihuitl.

Solanáceas ......... Pipiloxihuitl.

Helechos ........... Doradilla.

Rutáceas .......... Suopakal (maya).

Verbenáceas........ Señora de noche.

Gutíferas........... Cilusia. 
Cnicus rhaphilepis Wats.

Colocasia antiquorum Schott. var.........

Commelina, sp?.

Comocladia engleriana Lœs.

Condalia obovata Hook.

Cordia palmeri Wats.

Cordia (Sebestenoides) sonoræ Rose.....

Corylus avellana L. var.

Costus spicatus Swartz.

Coursetia (?) mexicana Wats.

Cratæva, sp?.

Crescentia alata H. B. K.

Crescentia cujete L.

Crotalaria pumila Ort.......................

Cucumis sativus L.....

Cucurbita ficifolia Bouché.

Cucurbita fœlidissima H. B. K

Gucurbita pepo L

Cucurbita radicans Naud.

Cunila secunda Wats.

Cupressus sempervirens $\mathrm{L}$

Cuscuta odontolepis Engelm...............

Cuscuta tinctoria Mart.

Cyclanthera dissecta Arn.

Cyperus esculentus L.

Cyphomandra betacea Sendt...

Cypripedium calceolus L. ?

Cypripedium irapeanum Llav, et Lex....

Cypripedium turgidum Moc. et Sessé.

Cyrtocarpa procera H. B. K.
Compuestas ........

Aroideas.

Commelináceas....

Anacardiáceas .....

Ramneas.

Borragíneas........

Borragíneas........

Cupuliferas.

Zingiberáceas......

Leguminosas.......

Caparídeas

Bignoniáceas.

Bignoniáceas

Leguminosas

Cucurbitáceas

Cucurbitáceas .

Cucurbitáceas ....

Cucurbitáceas.

Cucurbitáceas .....

Labiadas.

Coníferas

Convolvuláceas..

Convolvuláceas.

Cucurbitáceas

Giperáceas.

Solanáceas

Orquídeas

Orquídeas.

Orquídeas.

Anacardiáceas
Cardo santo.

Macal. NTamé.

Cabalsit (maya).

Teclatilla. (Tetlatia, Tetlatziam.)

Brasil. Chaparral.

Hierba del pasmo.

Palo de asta.

Avellana.

Caña de javalí.

Nesco. Palo piojo.

Zapatillo amarillo.

Ayal.

Luch (maya). Pog (totonaco).

Hierba del cuervo.

Cohombro.

Cidra cayole.

Calabacilla. Calabacilla loca. Chilicoyote.

Cum (maya).

Chicoyote.

Poleo del cerro.

Pino.

Zacatlazcal.

Zacatlazcal.

Raíz de fregar.

Chufas.

Berengena. Tomate de la paz.

Chitcuuc (maya).

Flor de calavera.

Pipixiuhatztli.

Ciruela.
Dalea diffusa Moric

Datura sanguinea Ruiz et Pav.

Datura stramonium L

Delphinium ajacis L

Desmodium orbiculare Schl.

Dioscorea ?.

Dodonæa viscosa L.

Dorstenia crispata Wats.

Dorstenia drakena L

Diospyros ebenaster Retz.

Dupinia tepezapote Durand.

\author{
Leguminosas........ \\ Solanáceas \\ Ranunculáceas.... \\ Leguminosas. \\ Dioscoreáceas...... \\ Sapindáceas..... \\ Urticáceas \\ Urticáceas \\ Ebenáceas. \\ Ternstrœmiáceas. \\ Escoba larga. \\ Campanilla encarnada. Floripondio encarnado. \\ Estramonio. Hierba hedionda. \\ Miramelindo. \\ Engorda cabra. \\ Cuanchalala, Cuanchalalate. \\ Granadina. Jarilla. Ocotillo. \\ Barbudilla. \\ Barbasco. \\ Tauch (maya). Totocuitlatzapotl. \\ Tepetzapotl. Tepezapote.
}

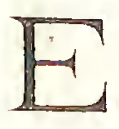

Echinocactus horizonthalonius Lem

Echinocactus wislizeni Engelm.

Cácteas. Bisnaga.

Echinocactus wislizeni lecontei Engelm.. 
Echites montana (?)

Ehretia elliptica DG

Elytraria tridentata Vahl.

Erechthites runcinata DC.

Eriodendron acuminatum Wats.

Eriodendron occidentale $\mathrm{Tr}$. et $\mathrm{Pl}$

Eriogonum atrorubens Engelm.

Eriogonum inflatum Torr.

Erysimum tilimi Gay.

Erythrina coralloides DC

Eupatorium odoratum L.

Eupatorium petiolare Moc...

Eupatorium, sp?

Eupatorium, sp?

Euplorbia (Poinsettia) tuberosa Rose.....

Eustoma exaltatum Griseb.

Eysenhardtia orthocarpa Wats...
Apocináceas........ Tijerilla.

Borragíneas........ Anaqua.

Acantáceas......... Cordoncillo.

Compueslas ......... Maguapas.

Malviceas .......... Pochote.

Malváceas .......... Piim.

Poligonáceas....... Hierba colorada.

Poligonáceas....... Tivinagua.

Crucíferas........... Tilimi.

Leguminosas........ Chilicote. Pioneo. Pionillo.

Compuestas ........ Cihuapatli ? Crucita.

Compuestas....... Yoyochichil.

Compuestas ........ Chalché.

Compuestas....... Santa María.

Euforbiáceas....... Contrayerha.

Gencianeas......... Violeta cimarrona.

Leguminosas....... Palo dulce.
Ficus fasciculata Wats.

Ficus rubiginosa Desf.

Ficus sonoræ Wats...

Ficus, sp?....

Ficus, sp?.

Ficus, sp?

Fimbristylis spadicea Vall

Forestiera, sp. nov.

Fouquiera spinosa H. B. K

Fouquiera splendens Engelm.

Fourcroya, sp?

Franseria ambrosioides Cav...

Franseria tenuifolia A. Gr...

Fuchsia corymbiflora Ruiz et Pav

\section{sing}

Con
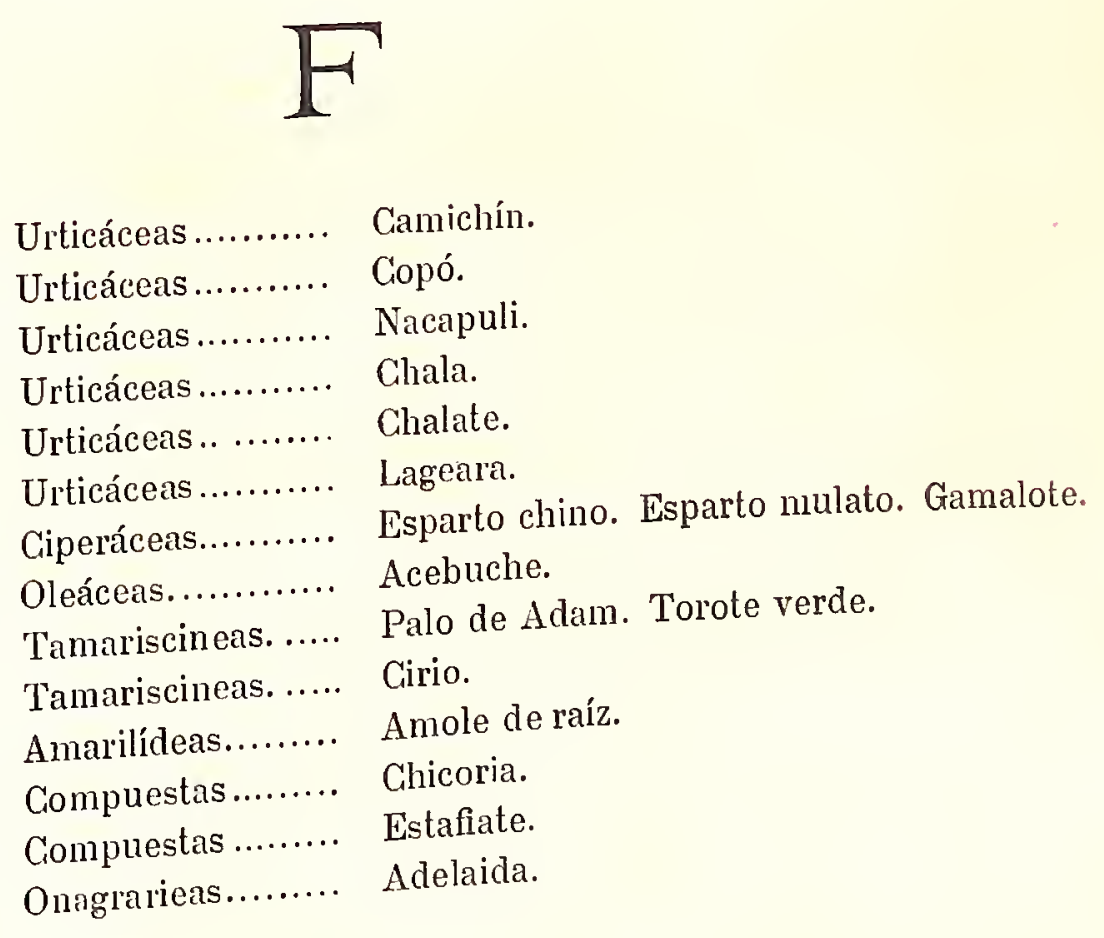

\section{G}

Galphimia gracilis Bartl...

Gaultheria, sp?.

Gentiana spathacea H. B. K.

Geranium alchemilloides ? L...

Gilia gloriosa Brandegee....

Gilia tenuifolia A. Gr..

Gliricidia lambii Fernald.

Gomphrena globosa L...

Gonolohus erianthus Dene.

Gonolobus pilosus Benth.

Gossypium barbadense L...
Malpigiáceas..

Ericáceas ..........

Gencianeas.........

Geraniáceas........

Polemoniáceas.....

Polemoniáceas.....

Leguminosas........

Amaranláceas.....

Asclepiadeas.......

Asclepiadeas.......

Mirlváceas.
Hierba del piojo. Axocopaconi.

Yelera.

Tatlauhcapatli.

Ubi ?

Agua bonila. Ubi ?

Gacahuananchi.

Chacmol.

Talayote.

Flor del imuerto.

Taman. 
Guaiacum angustifolium Engelm

Gyrocarpus jacquini Roxb.
Zigofileas

Combretáceas
Guayacán.

Giis. Volador.

\section{$\mathrm{H}$}

Hæmatoxylon boreale Wats

Hamelia patens Jacq.

Haplophytum cimicidum A. DC.

Helenium autumnale L.

Heliantus annuus L.

Heliocarpus americanus L

Heliotropium curassavicum L.

Heliotropium indicum $\mathrm{L}$

Heliotropium parviflorum L.

Heterotheca leptoglosa DG.

Hilaria cenchroides H. B. K

Hiræa macroptera DC.

Hippocratea utilis Rose

Hippophaë mexicana Moc. et Sessé..

Horsfordia palmeri Wats.

Hura crepitans L.

Hydrotænia vanhouttei Baker

Hyptis collina Brandegee.

Hyptis emoryi Torr...

Hyptis laxiflora Benth.

Hyptis palmeri Wats.

Hyptis seemanni Gray

Hyptis suaveolens Poit.

\author{
Leguminosas....... Brasil. \\ Rubiáceas .......... Chacloco. \\ Apocináceas........ Hierba de la cucaracha. \\ Compuestas. ....... Manzanilla. \\ Compuestas........ Flor de sol. \\ Tiliáceas........... Catena. \\ Borragíneas........ Rabo de mico. \\ Borragineas........ Rabo de mico. \\ Borragíneas....... Nemax. Rabo de mico. \\ Compuestas ........ Lampotillo. \\ Gramíneas........ Grama. \\ Malpigiáceas....... Gallinita. Matanene. \\ Hipocrateáceas.... Bejuco colorado. \\ Eleagneas........... Palo amargo. \\ Malváceas ......... Mariola. \\ Euforbiáceas....... Quaulitlallatzin. \\ Irideas . \\ Labiadas........... Salvia para la misa. \\ Labiadas........... Salvia. \\ Labiadas ........... Salvia. \\ Labiadas........... Salvia. \\ Labiadas........... Confituria. \\ Labiadas. ... \\ Chana. Chía grande.
}

Illicium anisatum L....

Inga ingoides Willd.

Ipomcea batatas Lam. vars.

Ipomcea bracteata Cav.

Ipomœea intrapilosa Rose.

Ipomoa jicama Brandegee.

Ipomœa llaveana Meissn.

Ipomœa purga Hayne.

Ipomœa quamoclit L.

Ipomoea sinuata Ort.

Ipomœa versicolor Meissn.

Ipomœa wolcottiana Rose.
Magnoliáceas

Leguminosas

Convolvuláceas..

Convolvuláceas

Convolvuláceas.

Corivolvuláceas.

Convolvuláceas..

Convolvuláceas.

Convolvuláceas...

Convolvuláceas...

Convolvuláceas.

Convolvuláceas.....
Anis estrellado ó de China.

Cibalahuill.

Camote amarillo. Camote blanco. Camote morado. Zicana.

Pájaro bobo.

Jícama.

Trompetilla.

Purga de Jalapa.

Cambustera.

Hierba de la tarántula.

Plumila.

Acote. 
Jacquinia macrocarpa Cav....

Jacquinia pungens A. Gr...

Jatropha canescens Müll. Arg...

Jatropha cardiophylla Müill. Arg.

Jatropha cordata Müll. Arg...

Jatropha curcas L.

Jatropha herbacea L

Jatropha spatulata Mïll. Arg. var.

Jussieua suffruticosa L
Mirsineas

Mirsineas.

Euforbiáceas

Euforbiáceas

Euforbiáceas

Euforbiáceas

Euforbiáceas.

Euforbiáceas

Onagrarieas.
Corpus.

San Juanico.

Sangregrado. Torotito.

Torote común.

Matamuchachos.

Quauhayohuachtli.

Ortiga.

Torote amarillo. Torote prieto.

Cornezuelo cimarrón.
Karatas plumieri E. Morr

Karvinskia humboldtiana Zuce.

Krameria canescens Gray
Bromeliáceas

Ramneas.

Poligaleas
Thumbirichi.

Cacachila.

Mezquitillo.

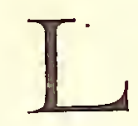

Lagenaria vulgaris Ser. var

Lantana camara L.

Lantana lilacina Desf. ?

Lantana polyacantha Schauer.

Lantana trifolia L

Lavandula vera $\mathrm{DC}$.

Leucæna pulverulenta Benth...

Lippia callicarpifolia H. B. K

Lippia fastigiata Brandegee....

Lippia geminata H. B. K

Lippia palmeri Wats

Lobelia cliffortiana $\mathrm{L}$

Lopezia mexicana Jacq

Lopezia trichota Schl.

Loranthus calyculatus DC.

Lucuma mammosa Gærtn. fil...

Lucuma palmeri Fernald.

Lucuma sphærocarpa A. DC...

Lupinus bilineatus Benth...

Lycium carinatum Wats...

Lycopersicum esculentum Mill

Lysiloma acapulcensis Benth. var.

\section{K}

Cucurbitáceas ....

Verbenáceas.

Verbenáceas.

Verbenáceas

Verbenáceas

Labiadas....

Leguminosas.......

Verbenáceas.........

Verbenáceas........

Verbenáceas........

Verbenáceas........

Lobeliáceas .....

Onagrarieas

Onagrarieas

Lorantáceas.

Sapoláceas

Sapotáceas...

Sapotáceas.

Leguminosas

Solanáceas

Solanáceas

Leguminosas
Calabazo. Guaje. Guajito. Huaje. Huajito.

Confiturea. Moro (frutos).

Orozuz blanco.

Zapotillo.

Orozuz colorado.

Alhucema.

Huaxe.

Rosa de Castilla del campo.

Damiana.

Orozuz morado.

Orégano.

Pitzigual. Xílitl.

Parrilla.

Peroncito.

Ingerto.

Chacalhaas. Pitztli (hueso).

Huicón.

Tempixque. Tempixtle.

Elotitos. Hierba de San Marcos.

Sal sieso.

Pac (maya)

Tepehuaje. 
Malpighia oaxacana Niedz....

Malva parviflora L.

Malva scoparia L'Herit.

Malvaviscus arboreus Cav....

Mamillaria goodridgii Scheer.

Manihot aipi Pohl.

Manihot utilissima Pohl.

Marsdenia edulis Wats.

Marsdenia zimapanica Hemsl.

Mascagnia seleriana Lœs.

Maximowiczia sonoræ Wats..

Maytenus phyllanthoides Benth.

Melicocca bijuga L.

Melochia pyramidata L

Mentha rotundifolia L

Mentha viridis $\mathrm{L}$.

Mentzelia adherens Benth.

Mentzelia aspera L.

Mikania cordifolia Willd.

Milla biflora Cav.

Mimosa asperata $\mathrm{L}$.

Mimosa distachya Cav...

Mimosa monancistra Benth.

Mimosa, sp?.

Mirabilis jalapa $\mathrm{L}$

Montanoa, sp?.

Morus nigra L. var.

Muntingia calabura L

Musa, sp?.....

Myrospermum peruiferum $\mathrm{DC}$.

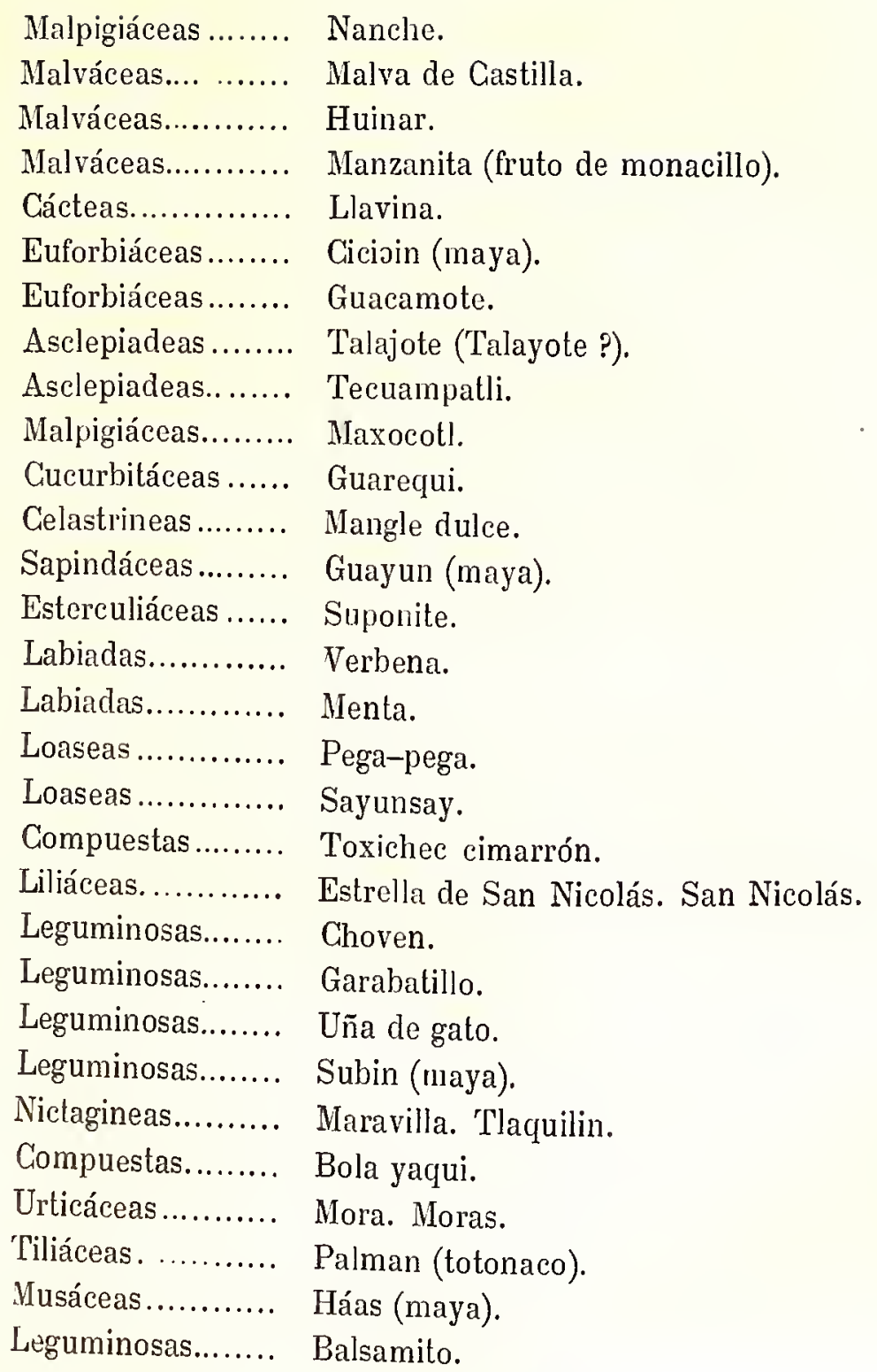

Naias major All

Nectandra sanguinea Rottb.

Nelumbo lutea Pers.

Nelumbo, sp??

Nemastylis tenuis Benth. \& Hook

Nemastylis versicolor Wats.

Nesæa salicifolia H. B. K

Nicandra physaloides Gærtn.

Nicotiana clevelandi Gray.

Nicotiana glauca Grah.

Nicotiana ipomopsiflor'a Dun.

Nicoliana trigonophylla Dun.

Nymphæa alba I.
Nayadáceas

Lauríneas.

Ninfeáceas..

Ninfeáceas.

Irideas

Irideas

Litrarieas

Solanáceas.

Solanáceas....

Solanáceas.

Solináceas

Solanáceas....

Ninfeáceas.
Zaragoza.

Laurel.

Cihinquapin.

Pulul.

Coquistle.

Cacomite.

Jarilla.

Tomate de burro.

Tabaco de perro.

Don Juan. Maraquiana. Mostaza montés. Tronadora de España.

Tabaco de coyote.

Tabaco coyote.

Nenufar. 
Ochroma lagopus Sw...

Enothera rosea Ait.

Enothera tetraptera Cav.

Olneya tesota A. Gr.

Opuntia fulgida Engelm.

Opuntia larreyi Weber.

Opuntia tapona Engelm

Opuntia, sp??.

Opunlia, sp?.

Opuntia, sp?.

Oxalis albicans H. B. K.
Malváceas

Onagrarieas

Onagrarieas.

Leguminosas

Cácteas.

Cácteas.

Cácteas....

Cácteas....

Cácleas.

Cácteas.....

Geraniáceas.
Jopi. Jubiguy. Pomay.

Hierba del golpe.

Hierba del golpe.

Uña de gato.

Vela de coyote.

Camuesa. Tuna camuesa.

Tapona (tuna).

Choyer.

Tuna cardona.

Tuna cliaveña.

Xocoyolli.
Pachira insignis Savign.

Pachyrhizus?.

Panicum myurum Meyer.

Parkinsonia aculeata $L$

Parkinsonia microphylla Torr

Parkinsonia torreyana Wats.

Parmentiera edulis DC.

Parthenium argentatum A. Gr...

Parthenium hysterophor us L.

Passiflora fotida L. var. hirsuta Mart...

Passiflora maximiliana? Bory.

Passiflora pralmeri Rose.

Passiflora serratifolia $L$

Paullinia arborea Moc. et Sessé.

Paullinia fuscescens Kunth.

Pectis filipes A. Gr...

Persea gratissima Gærtn

Petræa arborea H. B. K.

Phaseolus atropurpureus DC

Phaseolus heterophyllus Willd...

Pliaseolus multiflorus Willd.

Phaseolus vulgaris L...

Philadelphus mexicanus Schl...

Phragmites communis Trin...

Phyllactis pratensis Benth. et Hook.....

Physalis angulata $\mathrm{L}$

Physalis crassifolia Benth....

Physalis nicandroides Schl.

Physalis pubescens L...

Pileus heptaphyllus Ram

Pimpinella anisum $\mathrm{L}$

Piqueria trinervia Cav
Malváceas

Leguminosas....

Gramíneas

Leguminosas.......

Leguminosas.......

Leguminosas........ Palo verde.

Bignoniáceas........ Chote.

Compuestas........ Guayule (Huayule?).

Compuestas ........ Chaile.

Pasifloráceas....... Amaprola.

Pasifloráceas....... Tzinacanatlapatli.

Pasifloráceas....... Sandía de la Pasión.

Pasifloráceas....... Amapola.

Sapindáceas ........ Pacasquihuit (totonaco).

Sapindáceas........ Panoquera.

Compuestas......... Limoncillo.

Lauríneas........... On.

Verbenáceas........ Bejuco de caballo. Raspa sombrero.

Leguminosas........ Oreja de ratón.

Leguminosas....... Jícama del cerro.

Leguminosas....... Frijol del monte.

Leguminosas....... Buul (maya).

Saxifragáceas.. . Acuilot.

Gramíneas.......... Cañoto. Halal.

Valerianeas ......... Ucuares.

Solanáceas......... Farolito. Paccanil.

Solanáceas......... Tomate capotillo.

Solanáceas ......... Tomate de perro.

Solanáceas ......... Costomate ó Coxtomatl.

Pasifloráceas....... Bonete.

Umbeliferas ... .... Anis. Anis común. Anis verde.

Compuestas........ Alta reina. 
Pithecoctenium muricatum Moc. et Ses.. Bignoniáceas.

Pithecolobium brevifolium Benth...

Pithecolobium ligustrinum $\mathrm{Kl}$, var.

Pithecolobium oblongum Benth....

Pithecolobium sonore Wats

Pithecolobium (?)

Pittosporum tobira Ait

Pluchea borealis Gray....

Pluchea camphorata DC

Pluchea odorata (L.) Cass....

Plumbago cœrulea H. B. K

Plumeria acutifolia Poir ?.

Plumeria rubra $]$

Podopterus mexicanus H. B. K

Polanisia uniglandulosa Cav.

Polygonum mexicanum (Meisn. ?)

Porophyllum coloratum DG

Porophyllum gracile Benth....

Porophyllum obtusifolium DG...

Porophyllum seemanni Schultz. Bip....

Porophyllum tagetoides DC

Potamogeton pectinatus $\mathrm{L}$.

Prosopis juliflora DC

Prosopis palmeri Wats.

Prionosciadium, sp?.

Prochnyanthes viridescens Wat

Psidium pomiferum L

Pyrus malus L. vars.

Amarilídeas...
Leguminosas.

Leguminosas.....

Leguminosas.......

Leguminosas.......

Leguminosas.......

Pitosporeas .........

Compuestas.

Compuestas

Compuestas.

Plumbagíneas

Apocináceas.

Apocináceas.....

Poligonáceas........

Caparideas

Poligonáceas

Compuestas.

Compuestas

Compuestas

Compuestas

Compuestas

Nayadáceas

Leguminosas.

Leguminosas

Umbelífel'as....

Mirtáceas.

Rosáceas.
Petaquillas.

Huajillo.

Pichejumo.

Humo.

Uña de gato.

Cascalote.

Glavo.

Cochinilla.

Canela.

Hierba de Santa María.

Embeleso. Jazmín azul.

Cacaioxóchitl.

Sabacnicté (maya).

Espuela de gallo.

Hierba del zorrillo.

Moco de pavo.

Hierba del venado.

Hierba del venado.

Hierba del venado.

Maravilla.

Papaloquilitl.

Granza.

Algarroba. Mezquite (goma de).

Palo de hierro.

Ococotillo.

Amole.

Pichí (maya).

Manzana.
Quercus lanceolata Humb. et Bonp

Quercus laurina Humb. et Bonpl

Quercus mexicana Humb. et Bonp

Quercus repanda Humb. et Bonpl.

Quercus reticulata Iumb. et Bonp

Quercus sideroxyla Humb. et Bonpl.....
Cupulíferas.

Cupulíferas.

Cupulíferas.

Cupulíferas....

Cupulíferas.

Gupulíferas.
Eneino manzanillo.

Encino hoja de laurel.

Encino blanco.

Encino chaparro.

Encino hoja ancha. Encino quiebra hacha.

Encino tecomate.

\section{$\mathrm{R}$}

Randia thurberi Wats....

Randia, sp?

Phynchosia, sp?

Rondelelia spinosa K. Sch.

Rosa, sus. \& valrs

Rothrockia cordifolia A. Gr.

Rubus aff. R. occidentalis I

Ruellia albiflor'a Fernald.
Pubiáceas

Rubiáceas

Leguminosas

Rubiáceas

Riosáceas

Asclepiadeas

Rosáceas

Acantáceas.
Papachi.

Papachi.

Quapionchi.

Pisullo.

Rosal. Rosales.

Talayote.

Zarzamora.

Tronador. 
Ruellia, sp?

Rumex crispus L

Rumex hymenosepalus Torr

Ruprechtia cumingii Meissn.
Acantáceas.

Poligonácens.

Poligonáceas.

Poligonáceas.
Hierba de la laguna.

Lengua de vaca.

Hierba colorada.

Ahuate.
Salvia mexicana $\mathrm{I}$

Salvia patens Cav

Sanvitalia procumbens Lam

Sapindus saponaria $L$

Sargenlia greggii Wats.

Scirpus californicus Britton.

Scirpus lacustris L.

Sclerocarpus uniserialis Bentl. et Hook..

Sebastiania bilocularis Wats.

Sechium edule Sw.

Selaginella lepidophylla Spring...........

Selaginella pilifera A. Braun...............

Sesbania macrocarpa Muhl. var. picta.....

Sicyos deppei G. Don.

Sida, sp?

Sideroxylon mexicanum Hemsl

Solandra seleræ Dammer..

Solanum enoplocalyx Dun

Solanum rostratum Dun...

Solanum torvum Sw...

Solanum verbascifolium L

Sonchus oleraceus L.

Spilanthes, sp?...

Spondias mexicana. WVats.

Spondias purpurea $\mathrm{L}$

Sporobolus indicus $\mathrm{R}$. Br.

Stachys coccinea Jacq

Stegnosperma halimifolia Benth...

Stenanthium frigidum Kunth...

Stevia glutinosa H. B. K. var. oaxacana DC

Stevia salicifolia Cav.

Stevia viscida H. B. K

Strychnos ignatti Ait.
Labiadas

Labiadas.

Compuestas

Sapindáceas.........

Rutáceas

Ciperáceas

Ciperáceas.

Compuestas

Euforbiáceas

Cucurbitáceas ......

Selagineláceas......

Selagineláceas......

Leguminosas........

Cucurbitáceas ......

Malváceas

Sapotáceas.

Solanáceas

Solanáceas

Solanáceas.

Solanáceas

Solanáceas

Compuestas.

Compuestas

Anacardiáceas .....

Anacardiáceas .....

Gramíneas

Labiadas....

Fitolacáceas

Liliáceas

Compuestas

Compuestas

Compuestas

Loganiáceas
Flor de la chuparrosa.

Pájaros azules.

Ojo de pollo.

Amole.

Chapote amarillo (Zapote ?).

Tule.

Tule.

Huichín.

Hierba de la flecha.

Raíz de chayote.

Flor de piedra.

Doradilla.

Bequilla.

Ximacol.

Sacxiu (maya).

Totolzapotl. Zapote de ave.

Copa de oro.

Agarra á tu primo. Jarilla.

Rabo de iguana.

Friega platos espinoso.

Palo de hojas de manteca. Sosa.

Muela de caballo.

Ocoxihuitl.

Ciruela.

Ciruelo.

Lindrilla.

Mirto.

Amole.

Gebolleja.

Hierba del aire.

Hierba de la muela.

Pipitzahuatl.

Cabalonga de la Huasteca.
Tabebuia domnell-smithii Rose

Tabebuia palmeri Gray

Tabernæmontana acapulcensis Miers...

Tabernæmontana grandiflora Jacq...

Tabernemontana paisavalensis Losn...
Bignoniáceas..... Bignoniaiceas. Apocináceas Apocináceas Apocináceas.
Primavera.

Amapa.

Usupek (maya).

Laurel blanco.

Cojón de gato. 
Tagetes lucida Cav.

Tagetes patula L...

Tagetes tenuifolia Cay...

Tecoma mollis H. B. K.

Tecoma stans Juss

Tecoma, sp? aff. T. pentaphylla Juss

Tetracera volubilis $L$

Thevetia cuneifolia A. DC.

Thevetia nitida $A$. DC

Thunbergia alata Boj.

Tibouchina longifolia Baill

Tigridia dugesii Wats

Tillandsia achyrostachys Ed. Morr.

Tillandsia recurvata L.

Tillandsia utriculata L.....

Tillandsia vestita Benth,

Tragia nepetæfolia Cav.

Tribulus cistoides $\mathrm{L}$

Tribulus grandiflorus Benth. et Hook....

Tribulus maximus $\mathrm{L}$.

Trichilia havanensis spatulata Rose......

Triticum repens $\mathrm{L}$

Triumfelta semilriloba $\mathrm{L}$

Turnera diffusa Willd...

Turnera ulmifolia $\mathrm{L}$

Turnera sp. nov.

Typha angustifolia L.

\begin{tabular}{|c|c|}
\hline Compuestas ......... & Pericón. \\
\hline Compuestas......... & Cempoalxóchitl cimarrón. \\
\hline Compuestas ......... & Santa María. \\
\hline Bignoniáceas........ & Tronadora. \\
\hline Bignoniáceas........ & Palo de arco. \\
\hline Bignoniáceas........ & Coyolxóchitl. Palo de rosa \\
\hline Dileniáceas.......... & Bejuco de agua. \\
\hline Apocináceas......... & Huevo de gato. \\
\hline Apocináceas......... & Ojo de venado. \\
\hline Acantáceas.......... & Ojo de Venus. \\
\hline Melastomáceas ..... & Tlzehuite. \\
\hline Irideas ............... & Jalıuique de Tupátaro. \\
\hline Bromeliáceas....... & Gallinita. \\
\hline Bromeliáceas ....... & Heno. Paxtle. \\
\hline Bromeliáceas....... & Rama del tecolote. \\
\hline Bromeliáceas....... & Gallitos. \\
\hline Euforbiáceas........ & Ortiguilla. \\
\hline Zigofileas............ & Pericón. \\
\hline Zigofileas............ & Mal de ojos. \\
\hline Zigofileas............ & Geonduna. \\
\hline Meliáceas............ & Garrapalilla. \\
\hline Gramíneas........... & Carrzue. Grama. \\
\hline Tiliáceas............. & Ahrojo. \\
\hline Turneráceas......... & Damiana. \\
\hline Turneráceas......... & Amaranto. Caléndula. \\
\hline Turneráceas......... & Damiana. \\
\hline Tifá & Tule \\
\hline
\end{tabular}

Compuestas ........ Pericón.

Cempoalxóchitl cimarrón.

Palo de arco.

Huevo de gato.

Ojo de venado.

Ojo de Venus.

Tlzehuite.

Gallinita.

Heno. Paxtle.

Rama del tecolote.

Gallitos.

Ortiguilla.

Pericón.

Garrapalill:

Ciarzuue. Grama.

Ahrojo.

Damiana.

Damiana.

Tule.
Urtica urens L

U:tilago maydis DG.
Urticáceas Hongos
Laal (maya).

Tizón de maíz.

\section{V}

Vallesia dichotoma Ruiz et Pavon...

Vanilla pompona Schiede.

Veatchia (Brodiæa) discolor Brandegee...

Verbesina serrata Cav.

Vernonia liatroides DC....

Vigna luteola Benth....

Viguiera buddleiæformis Benth el Hook..

Viguiela excelsa Bentli. et Hook.

Viscainua geniculata Greene.

Vitex mollis H. B. K.

Vitis sicyoides? Baker. var.

Vitis tiliacea H. B. IT.

Vitis, sp?.

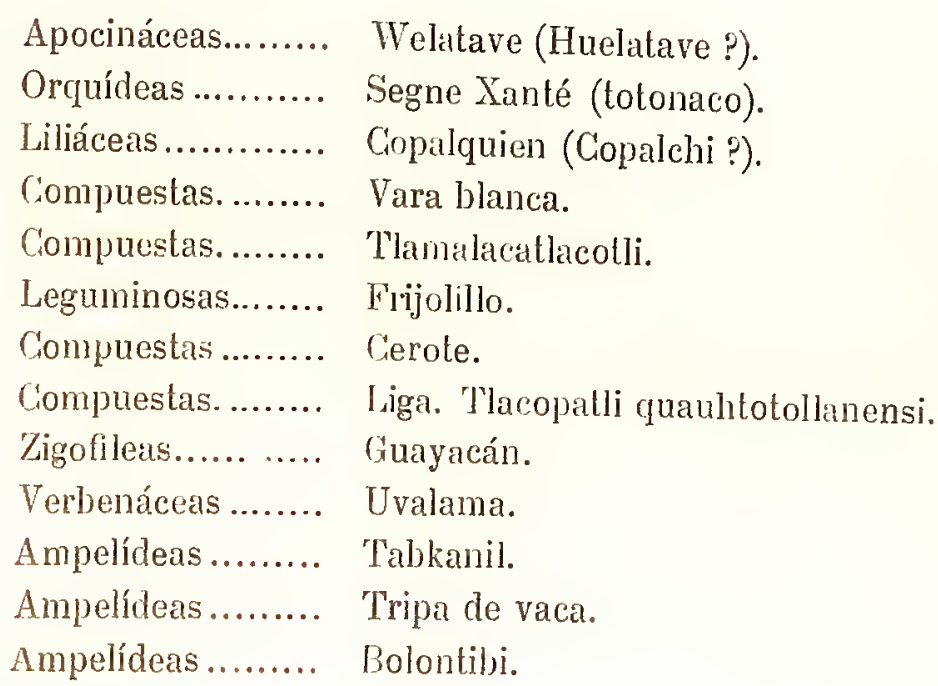

Welatave (Huelatave?). Segne Xanté (totonaco). Copalquien (Copalchi?). Vara blanca.

Tlamalacatlacotli. Frijolillo. Cierote.

Liga. T'lacopatli quauhtotollanensi. Guayacán.

Uvalama.

Tabkanil.

Tripa de vaca.

Bolontilsi. 


\section{W}

Wigandia caracasana H. B. K. Willardia mexicana Rose.
Hidrofiláceas....... Tabaco cimarrón.

Leguminosas....... Nesco. Palo piojo.

\section{X}

Xanthium canadense Mill.

Xanthosoma sagillifolium Schott...

Compuestas ......... Piojo de burro. Aroideas........... Manto de la reina.

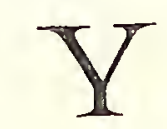

Yucca baccata Torr.

Liliáceas............ Amole (raíz).

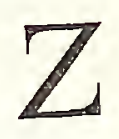

Ruláceas ........... Colima.

Gramíneas......... Maiz de coyote.

Gramíneas.......... Ixin (maya).

Amarilídeas........ Mayo. Mayitos.

Amarilídeas........ Mayilos.

Colacatillo. Mal de ojos.

Ramneas........... Amole (frutos).

Zephyranthes sessilis Herb...

Zinnia pauciflora L.... 



\section{MEMORTAS DEI DR. JOSE IAMIRIZ.}

1.-Origen teratológico de las variedades, razas y especies.- "La Naturaleza," periódico de la Sociedad Mexicana de Historia Natural. Tomo IV, página 235. 1871.

2.-Las semillas brincadoras._-"La Naturaleza." Segunda serie. Tomo I página 54 y Tomo II páginas 403 y 408.

3.-Descripción microscópica de las raíces de las Lobelia laxiflora y L. fenestralis._-"El Estudio," semanario de ciencias médicas, órgano del Instituto Médico Nacional Tomo IV, página 8, con dos láminas. 1890.

4.-Sinonimia vulgar y científica de varias de las "Plantas de Nueva España" de M. Sessé y José Mociño.-Op. cit. Tomo IV, página 226. 1892.

5.-Estudio bacteriológico de las aguas potables de la ciudad de México.-Op. cit. Tomo IV, página 339.1893

6.-Lista de las plantas recogidas en la Expedición á las Mixtecas, Oaxaca, en el mes de Enero de 1893.-Oj. cit. Tomo IV, página 452.

7.-Descripción de una nueva especie de Pterostemon.--La Naturaleza." Segunda serie. Tomo II, página416. 1893. Con lámina.

8.-Vegetación de Pátzcuaro.-Anales del Instituto Médico Nacional. Tomo I, página 56. 1891.

9.-La Mocinna heterophylla. Nuevo género de las papayáceas.-Op. cit. Tomo I, página 205. 1894. Con cuatro láminas. 10.-Raíces de vid atacadas por la filoxera.-Informe á la Secretaría de Fomento.-1893. Op. cit. Tomo I, página 259.

11.-Cafeto atacado por un parásito (Dactylopius destructor). 1894. Op. cit. Tomo I, página 262.

12. - Lista de nombres vulgares y botánicos de árboles y arbustos propios para repoblar los bosques de la República.-Imprenta de la Secretaría de Fomento. 1894. En colaboración con el Dr. F. Altamirano.

13.-Datos para el estudio de los Cuajiotes. Descripción de tres especies nuevas del género Bursera. 1895.-Anales del Instituto Médico Nacional. Tomo II, página 14. Con cuatro láminas.

14.-Una nueva especie de Casimiroa. (Casimiroa pubescens.)-Op. cit. página 18. 1895. Con láminas.

16.-Discurso pronunciado por el Dr. Jose Ram Trata de los fundamentos de Horticultura. de 1895. Op. cit. Tomo II, página 29 Trán y algunos otros granos.-Informe al Director del Instiluto Médico Nacio-

17.-Medidas para preservar del gorgojo 120.1895.

las montañas del Ajusco y Sierra de las Cruces. En el informe

18.-Enumeración de las principales plantas colectadas en las montituto Médico Nacional, sobre algunas excursiones á las que rinde á la Secretaría de Fomento el Director del En colaboración del Dr. F. Altamirano. montañas del Ajusco y Sierra de las Cruces. México. (Halenia cándida y Passiflora eslavensis). Op. cit.

19.-Descripción de dos nuevas especies del Valle de Médico Nacional en la Exposición de Coyoacán. Oficina Tipográfica 20. - Catálogo de los productos que exhibe el Institulo néción con los señores Dr. F. Altanirano, G. Alcocer é Ing. José N. Rovirosa.

21.-Una especie nueva de Erythroxylon y un dat
Tomo III, página 36. Con una lámina.

22.-El picudo del algodonero. 1897. Op. cit. página 164. Tomo IlI. 
23.--Tres monstruosidades en ovarios ínferos. 1898. Op. cit. Tomo III; página 223. Con tres cromo-litografías.

24.--Los escritos inéditos de Martín Sessé y José Mariano Mociño. 1899. Op. cit. Tomo IV, página 24.

25.- - Tres mazorcas anómalas. 1898. Op. cit. Tomo IV, página 32. Con cuatro cromo-litografías.

26.--El Peyote. Anhalonium Lewini y Anhalonium Williamsii. Cactáceas. 189u. Op. cit. Tomo IV, página 233.

27.--El Ololiuhqui.-Ipomœa sidæfolia. Convolunláceas. Memoria leída en la XIII reunión del Congreso Internacional de Americanistas verificada en Paris en 1900.

28.--La papaya voladora. 1901._-"La Naturaleza." Segunda serie. Tomo III, página 548. Con lámina.

29.--El Pileus heptaphyllus. Nuevo género de las Papayáceas. 1901._-"La Naturaleza." Segunda serie. Tomo III, página Con una litografia yo tres cromo-litografías.

30.--La hierba de la Cucaracha. Haplophyton cimicidum y Trachelospermun stans. Op. cit. T. III, página 549. Segunda serie. Con dos láminas.

31--La vegetación de México.-Recopilación y análisis de las principales clasificaciones propuestas. 1899. Ofic. Tip. de la Secretaría de Fomento. Esta obra contiene además: Las regiones botánico-geográficas de la República y una introducción para una flora del Valle de México.

32.--La hierba de la Puebla, el Yoloxochitl, el Pipitzaoac, el Zoapatle, la Hierba del Tabardillo y el Chicalote; artículos de la primera parte de los Datos para la Materia Médica Mexicana. Tip. de la Secretaría de Fomento. 1894. Cion seis láminas.

33.--Descripción botánica de las cincuenta y una plantas que forman los tres primeros tomos de los Datos para la Materia Médica Mexicana. 1884-1900. Con 51 láminas.

3t.--La sección histórica de los reintiún artículos que forman los tomos segundo y tercero de los Datos para la Materia Médica Mexicana.

35.-Descripción de una especie nueva de Garrya. Opera cit. Primera parte, página 184, con lámina.

36.--Sinonimia vulgar y botánica de las plantas mexicanas. Ofic. Tip. de la Secretaría de Fomento. 1902. En colaboración del Sr. G. Alcocer. Contiene los nombres de más de cuatro mil plantas, la mayor parte de ellos rectificados.

37.--El Herbario de Cervantes.--Abril de 1902. Anales del Instituto Médico Nacional. Tomo V. 



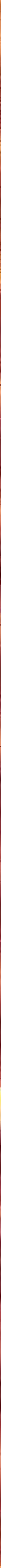




\section{2}

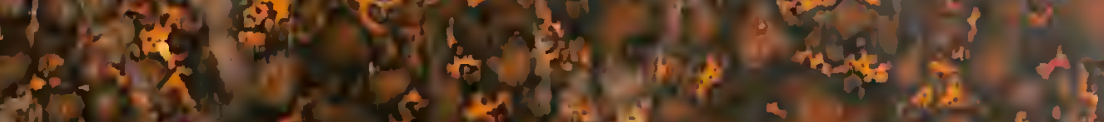

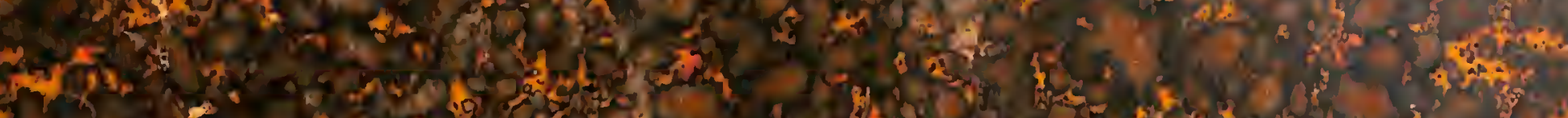

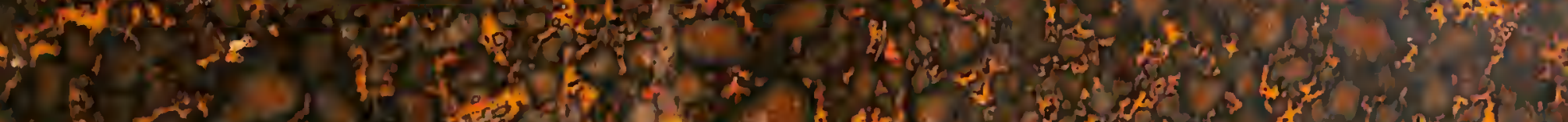

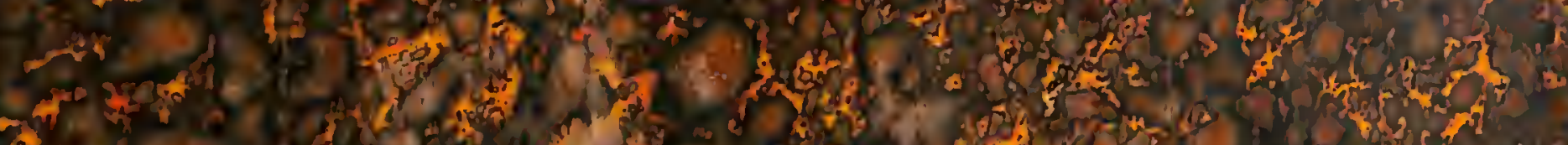

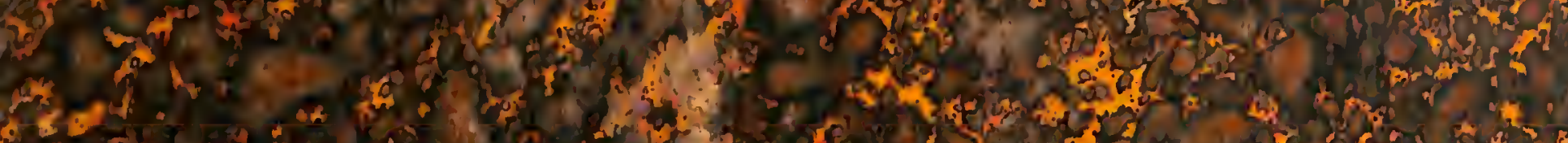

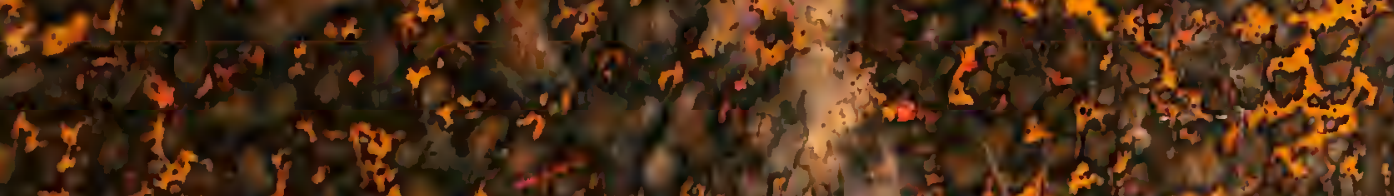

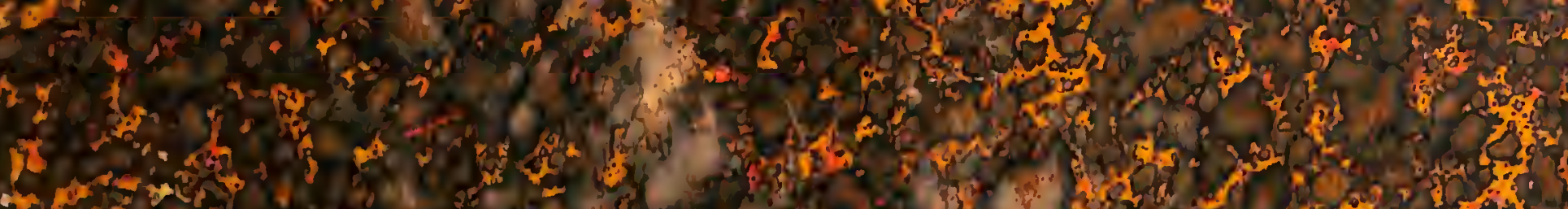

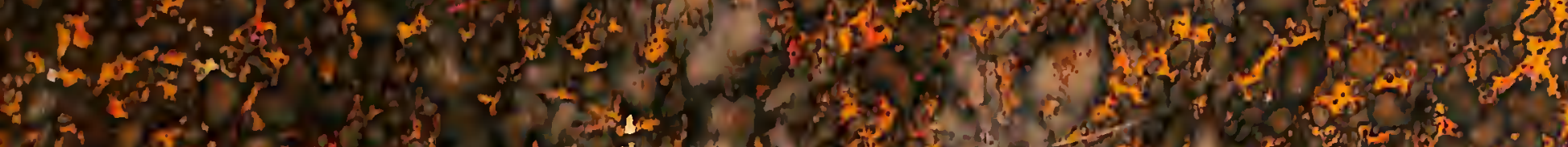

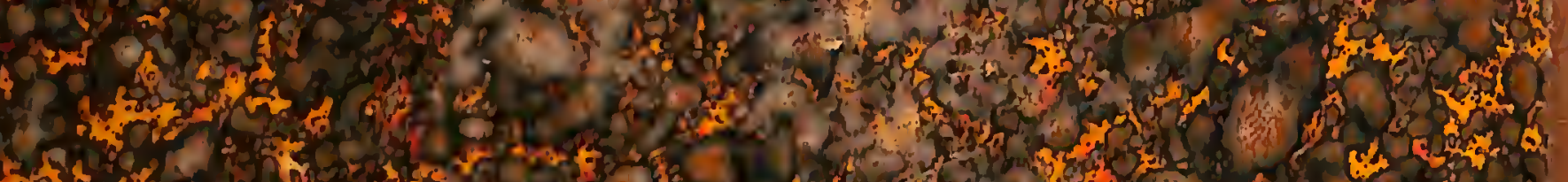

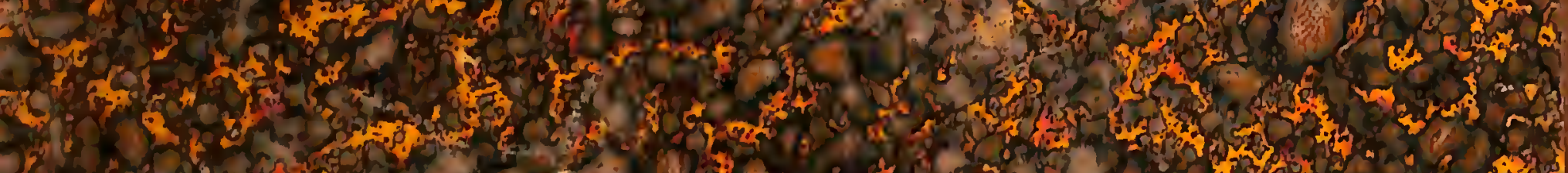

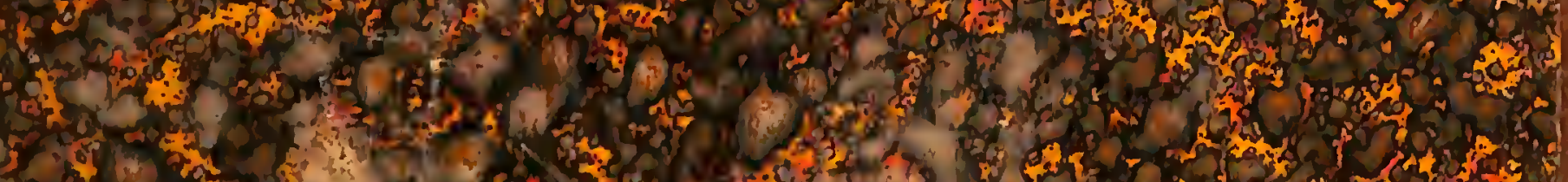

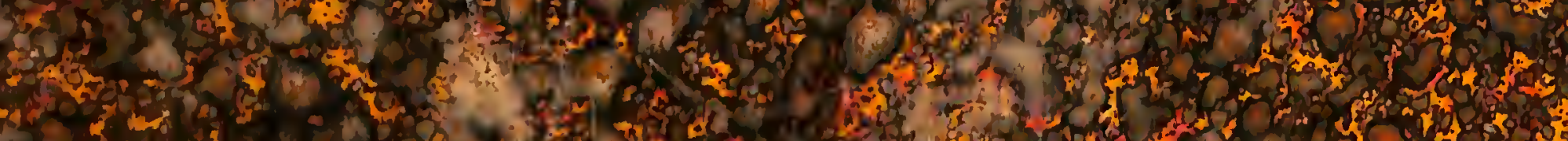

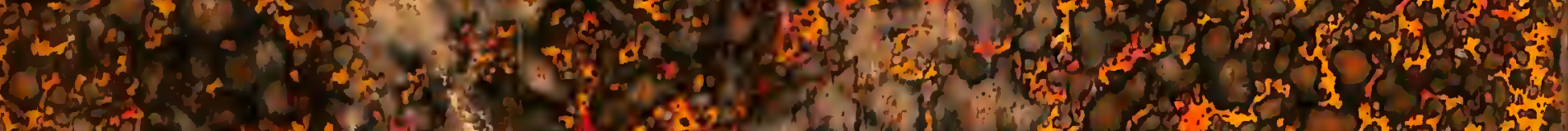

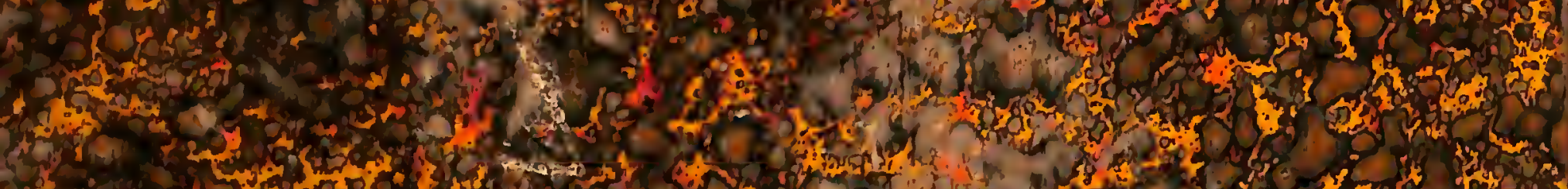

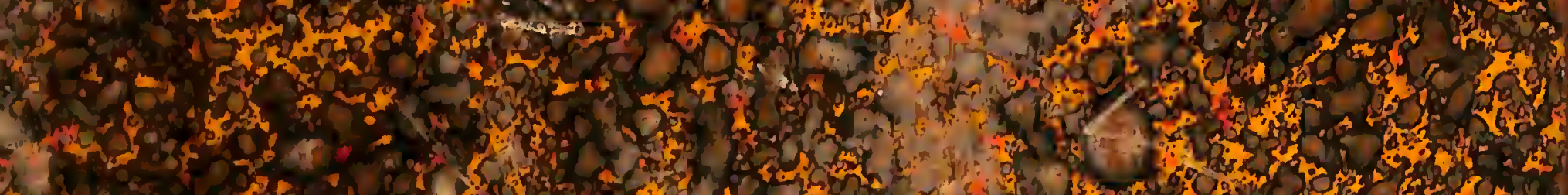

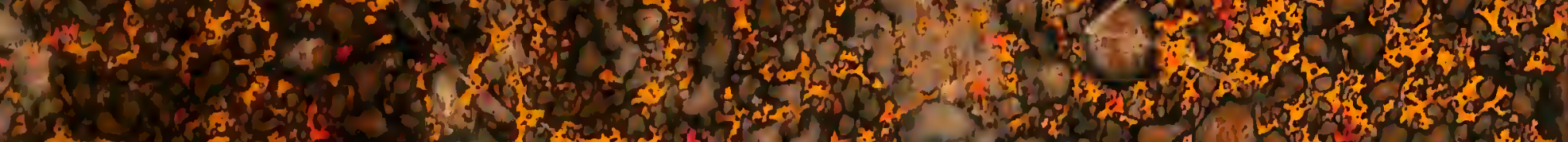

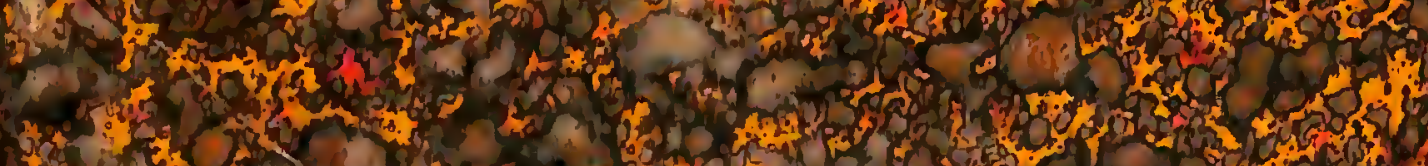

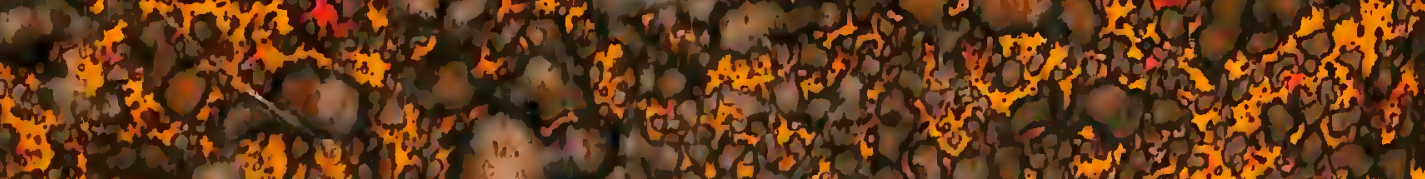

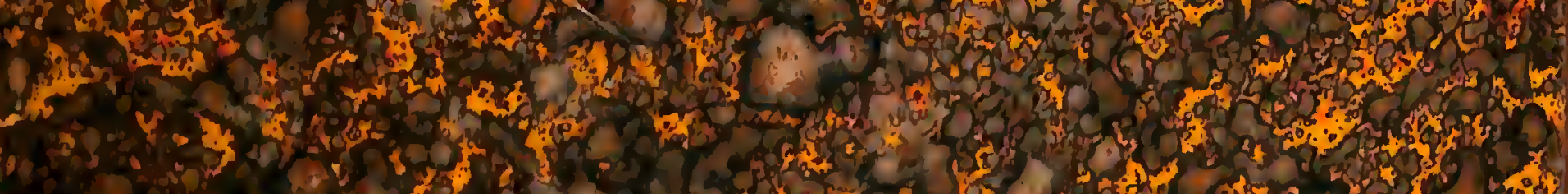

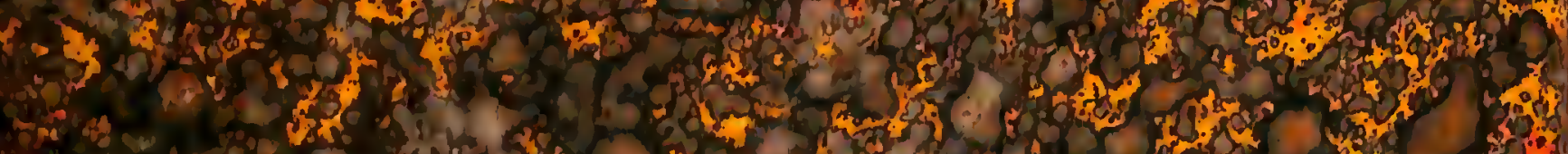

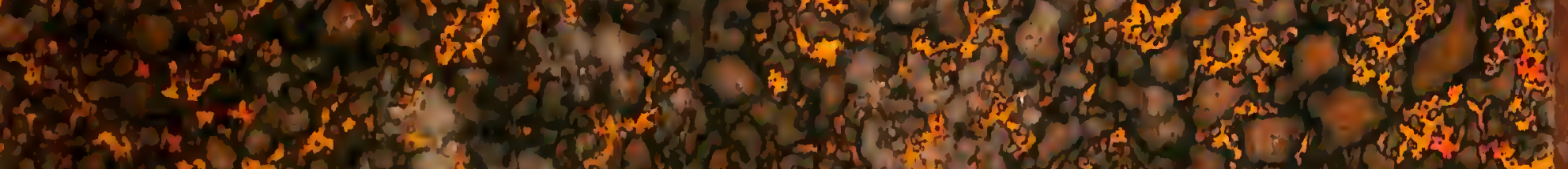

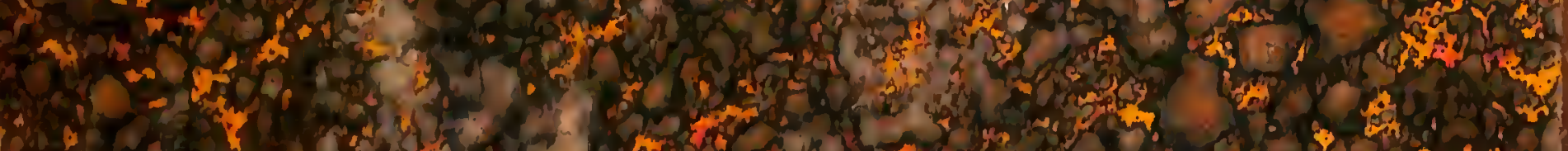
He (1)

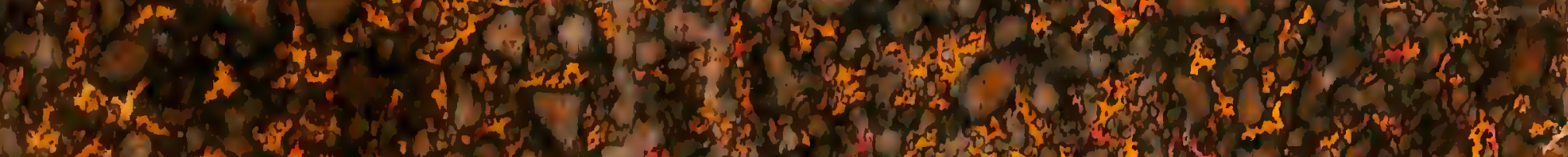

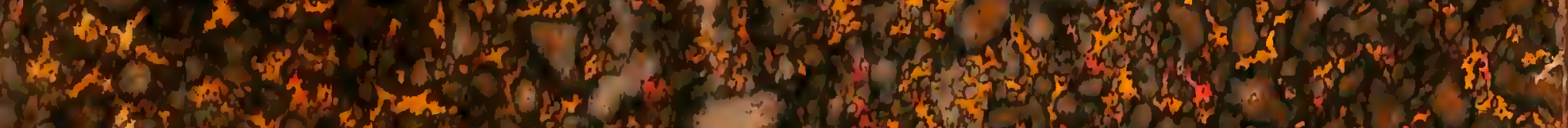
(5) 